Florida International University FIU Digital Commons

$11-9-2017$

\title{
Bidirectional Electric Vehicles Service Integration in Smart Power Grid with Renewable Energy Resources
}

\author{
Ahmed A S Mohamed Mr \\ Florida International University, amoha070@fiu.edu
}

DOI: $10.25148 /$ etd.FIDC004037

Follow this and additional works at: https://digitalcommons.fiu.edu/etd

Part of the Electrical and Electronics Commons, and the Power and Energy Commons

\section{Recommended Citation}

Mohamed, Ahmed A S Mr, "Bidirectional Electric Vehicles Service Integration in Smart Power Grid with Renewable Energy Resources" (2017). FIU Electronic Theses and Dissertations. 3529.

https://digitalcommons.fiu.edu/etd/3529 


\title{
FLORIDA INTERNATIONAL UNIVERSITY
}

Miami,

\section{BIDIRECTIONAL ELECTRIC VEHICLES SERVICE INTEGRATION IN SMART POWER GRID WITH RENEWABLE ENERGY RESOURCES}

\author{
A dissertation submitted in partial fulfillment of \\ the requirements for the degree of \\ DOCTOR OF PHILOSOPHY \\ in \\ ELECTRICAL ENGINEERING \\ by
}

Ahmed Abdelfatah Abdelaal Said Mohamed 
To: Dean John Volakis

College of Engineering and Computing

This dissertation, written by Ahmed Abdelfatah Abdelaal Said Mohamed, and entitled Bidirectional Electric Vehicles Service Integration in Smart Power Grid with Renewable Energy Resources, having been approved in respect to style and intellectual contents, is referred to you for judgment.

We have read this dissertation and recommend its approval.

Sakhrat Khizroev.

Armando Barreto.

Bilal El-Zahab.

Kemal Akkaya.

Osama A. Mohammed, Major Professor.

Date of Defense: November 9, 2017

The dissertation of Ahmed Abdelfatah Abdelaal Said Mohamed is approved.

Dean John Volakis
College of Engineering and Computing

Andrés G. Gil

Vice President for Research and Economic Development and Dean of the University Graduate School

Florida International University, 2017 
Copyright (C) 2017 by Ahmed Abdelfatah Abdelaal Said Mohamed All rights reserved. 


\section{DEDICATION}

I dedicate this dissertation work to my wife, Asmaa, who has been a constant source of support and encouragement during the challenges of graduate school and life. I am truly thankful for having you, our sons, Osama and Mohamed, and our daughter, Sarah, in my life.

I also dedicated this work to my parents, brother and sisters, who I did not see them much during my Ph.D. A special feeling of gratitude to my loving parents, Abdelfatah and Fathya who have always loved me unconditionally and whose good examples have taught me to work hard for the things that I aspire to achieve. My brother, Yasser, whose words of encouragement and push for tenacity ring in my ears. My sisters Hanan and Mervat have never left my side and are very special. 


\section{ACKNOWLEDGMENTS}

I owe my deepest gratitude to my advisor, Professor Osama A. Mohammed, for his invaluable guidance and support throughout my Ph.D. studies. He has by far been the most influential and inspiring teacher and advisor I have ever had the privilege of working with. Indeed he has been the predominant source of the academic and professional expertise I have acquired. Throughout my Ph.D., Professor Mohammed provided me with endless support, guidance, expert technical assistance and significant contributions through the period of the research and dissertation development. I will always remember his belief in

my abilities to work hard and to produce at a high level. I do appreciate his many constructive suggestions while the material was being developed and tested at the energy systems research laboratory. I am indebted for his willingness to accept me in his laboratory and for providing significant research facilities to develop this work in addition to his expert guidance and continued assistance, without which, I wouldn't have been able to successfully accomplish my experimental work. I am also grateful to Dr. Sakhrat Khizroev, Dr. Armando Barreto, Dr. Kemal Akkaya and Dr. Bilal El-Zahab for serving on my dissertation advisory committee and for the many constructive discussions.

I am also thankful to the Cultural Affairs and Missions Sector, Ministry of Higher Education, Government of Egypt, which provided me with the financial support as a doctoral student at Florida International University. I also thank the graduate school at Florida International University for providing me with the Doctoral Evidence Acquisition (DEA) Fellowship and the Dissertation Year Fellowship (DYF) during my last year to complete my doctoral work. 
I would also such as to acknowledge all the members and students of the Energy Systems Research Laboratory, Florida International University for their assistance during the experimental testing of this research. 


\title{
ABSTRACT OF THE DISSERTATION \\ BIDIRECTIONAL ELECTRIC VEHICLES SERVICE INTEGRATION IN SMART \\ POWER GRID WITH RENEWABLE ENERGY RESOURCES
}

by

\author{
Ahmed Abdelfatah Abdelaal Said Mohamed \\ Florida International University, 2017 \\ Miami, Florida \\ Professor Osama A. Mohammed, Major Professor
}

As electric vehicles $(\mathrm{EVs})$ become more popular, the utility companies are forced to increase power generations in the grid. However, these EVs are capable of providing power to the grid to deliver different grid ancillary services in a concept known as vehicle-to-grid (V2G) and grid-to-vehicle (G2V), in which the EV can serve as a load or source at the same time. These services can provide more benefits when they are integrated with Photovoltaic (PV) generation. The proper modeling, design and control for the power conversion systems that provide the optimum integration among the EVs, PV generations and grid are investigated in this thesis.

The coupling between the PV generation and integration bus is accomplished through a unidirectional converter. Precise dynamic and small-signal models for the grid-connected PV power system are developed and utilized to predict the system's performance during the different operating conditions. An advanced intelligent maximum power point tracker based on fuzzy logic control is developed and designed using a mix between the analytical model and genetic algorithm optimization. 
The EV is connected to the integration bus through a bidirectional inductive wireless power transfer system (BIWPTS), which allows the EV to be charged and discharged wirelessly during the long-term parking, transient stops and movement. Accurate analytical and physics-based models for the BIWPTS are developed and utilized to forecast its performance, and novel practical limitations for the active and reactive power-flow during $\mathrm{G} 2 \mathrm{~V}$ and $\mathrm{V} 2 \mathrm{G}$ operations are stated. A comparative and assessment analysis for the different compensation topologies in the symmetrical BIWPTS was performed based on analytical, simulation and experimental data. Also, a magnetic design optimization for the double-D power pad based on finite-element analysis is achieved. The nonlinearities in the BIWPTS due to the magnetic material and the high-frequency components are investigated rely on a physics-based co-simulation platform. Also, a novel two-layer predictive powerflow controller that manages the bidirectional power-flow between the EV and grid is developed, implemented and tested. In addition, the feasibility of deploying the quasidynamic wireless power transfer technology on the road to charge the EV during the transient stops at the traffic signals is proven. 


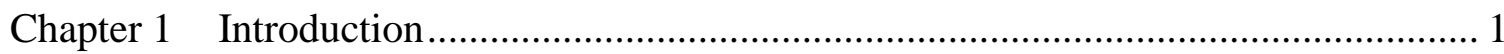

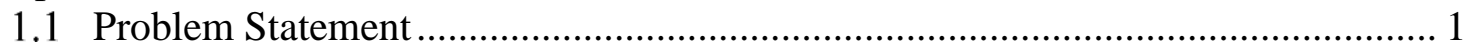

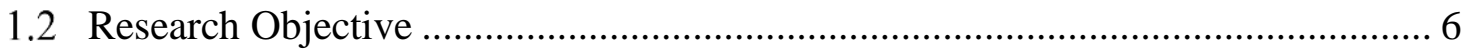

1.3 Original Contributions of the Dissertation....................................................... 9

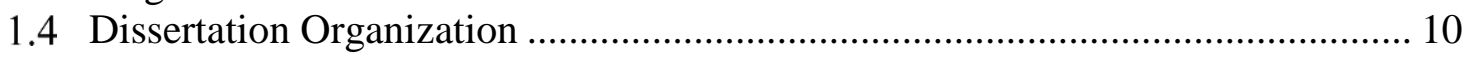

Chapter 2 Dynamic Modeling of Photovoltaic Power System.................................... 15

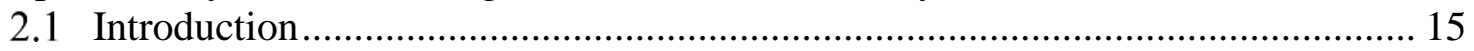

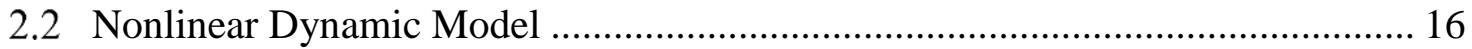

2.2.1 PV Panel Dynamic Model ................................................................... 17

2.2.2 DC-DC Converter with DC Bus Model................................................... 20

2.3 Small Signal Analysis of PV Power System..................................................... 21

2.3.1 PV Panel Small Signal Model....................................................................... 21

2.3.2 DC-DC Converter and DC Bus Small Signal Model................................... 25

2.4 Small Signal Perturbation Analysis ……………............................................ 26

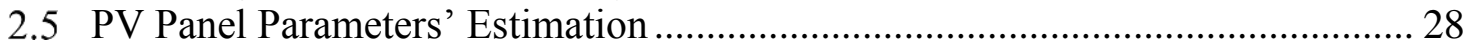

2.5.1 Analytical Parameter Estimation ............................................................... 29

2.5.2 GA-Based Parameters Estimation............................................................... 30

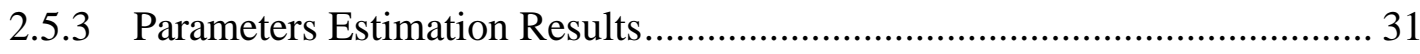

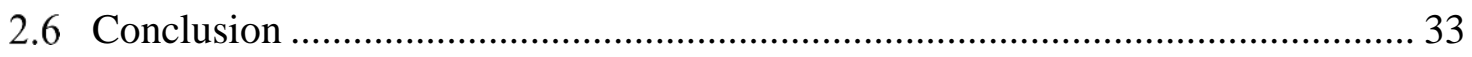

Chapter 3 Design and Implementation of Fuzzy MPPT for PV Power System............. 34

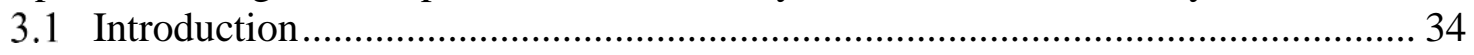

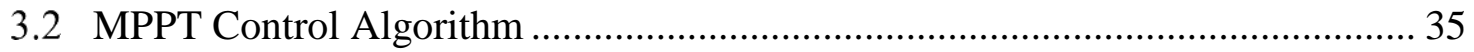

3.2.1 Classical Direct P\&O MPPT Algorithm................................................... 36

3.2.2 Proposed Fuzzy Direct MPPT Algorithm...................................................... 38

3.3 Design Methodologies of Fuzzy-MPPT Controller.............................................. 40

3.3.1 Analytical Fuzzy Logic Design Strategy ...................................................... 41

3.3.2 Genetic Algorithm-Based Design Optimization........................................... 42

3.4 Hardware Implementation of Fuzzy-MPPT Controller .......................................... 46

3.5 Experimental and Simulation Results and Discussion.......................................... 47

3.5.1 Assessment of the Proposed FL Design Strategies ........................................ 47

3.5.2 Experimental and Simulation Results of Fuzzy MPPT ................................. 49

3.5.3 Comparison between the Proposed Fuzzy-MPPT, P\&O and INC ............... 51

3.6 Conclusion .............................................................................................. 54

Chapter 4 Analytical Modeling of Different Topologies of BIWPTS in EVs

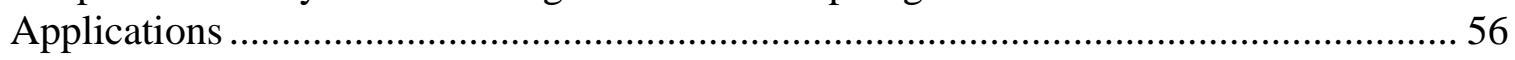

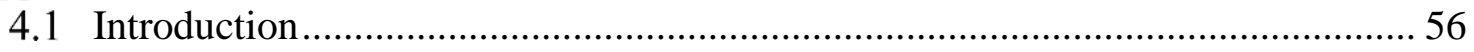

4.2 V2G Scheme Description ......................................................................... 58 


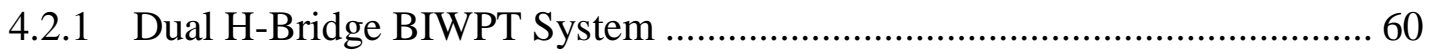

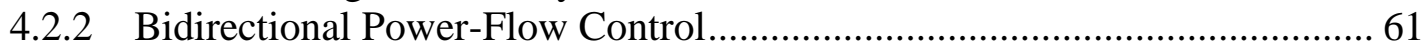

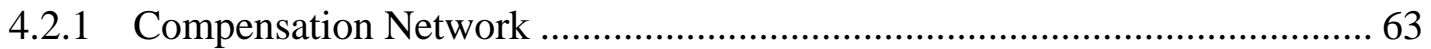

4.3 Steady-State Circuit representation of BIWPTS's Components ........................... 65

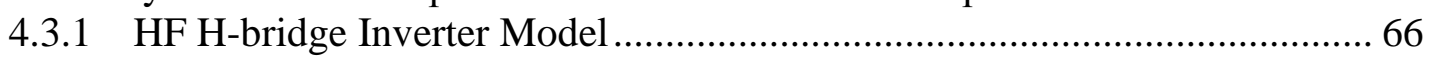

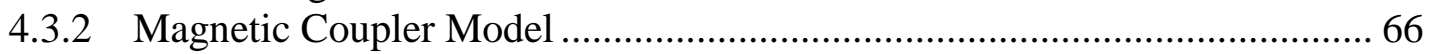

4.4 Steady-State Mathematical Model of Different Topologies of BIWPTS .............. 69

4.4.1 LC-Series Compensation Network .......................................................... 71

4.4.2 LC-Parallel Compensation Network ……………....................................... 72

4.4.3 LCL Compensation Network …………………........................................ 73

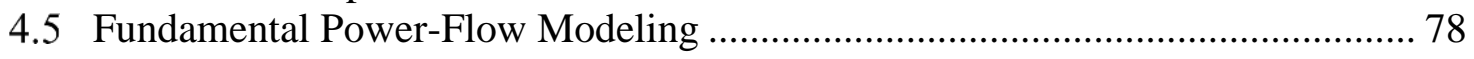

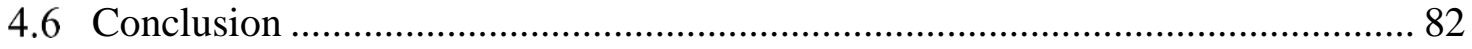

Chapter 5 Hardware Implementation of a Dual H-Bridge BIWPTS Prototype ……..... 83

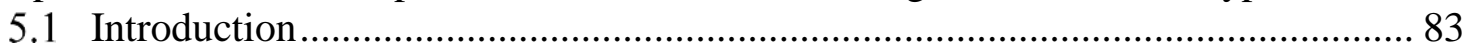

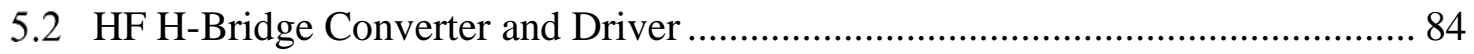

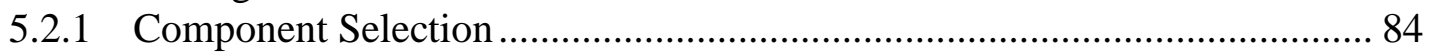

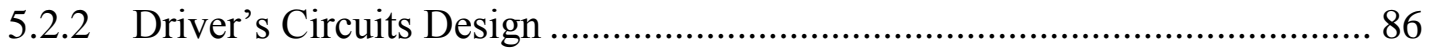

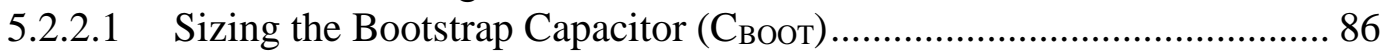

5.2.2.2 Sizing the Turn-on Gate Resistance $\left(\mathrm{R}_{\mathrm{g}(\mathrm{on})}\right)$......................................... 87

5.2.2.3 Sizing the Turn-off Gate Resistance $\left(\mathrm{R}_{\mathrm{g}(\mathrm{off})}\right)$.......................................... 88

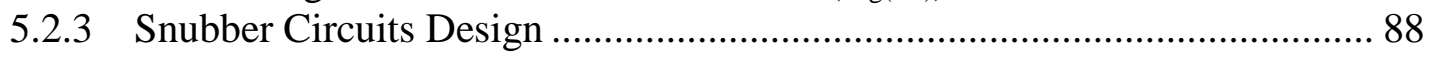

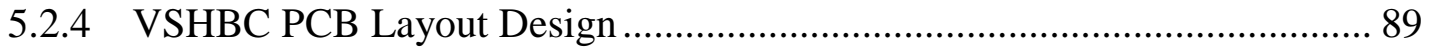

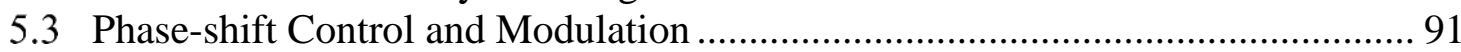

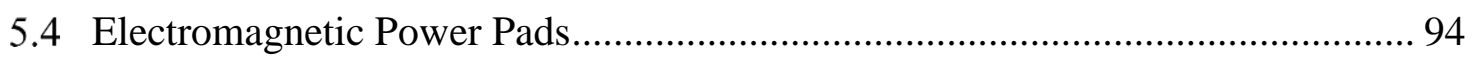

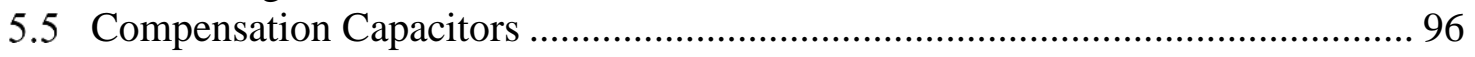

5.6 Design Verification and Results ....................................................................... 97

5.6.1 Evaluating the Phase-shift Circuit Performance ........................................... 99

5.6.2 Evaluating the Driving Circuits Performance ............................................. 101

5.6.3 The Converter's PCB Layout Performance Assessment .............................. 103

5.6.4 The VSHBC's Circuit Performance Assessment........................................ 105

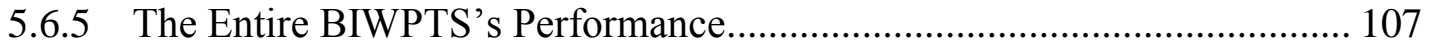

5.6.5.1 Full Supply Capacity Test............................................................... 109

5.6.5.2 Reduced Supply Capacity Test ........................................................... 112

5.6.5.3 Practical Considerations during Testing the BIWPTS......................... 113

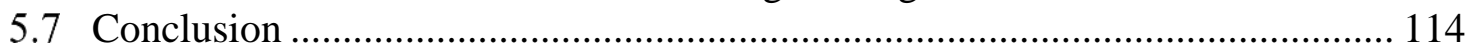

Chapter 6 Assessment Analysis of Different Compensation Topologies of BIWPTS 116

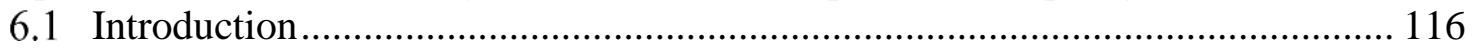

6.2 Assessment Criteria of Compensation Topologies in BIWPTS. ......................... 117

6.3 Performance Evaluation for Different Topologies. .......................................... 119

6.3.1 Assessment of LC-Series Topology........................................................ 120

6.3.2 Assessment of LC-Parallel Topology ……………..................................... 124

6.3.3 Assessment of LCL Topology.............................................................. 125

6.3.4 Power-Flow Assessment for Different Configuration................................ 127 
6.4 Experimental Evaluation for Different Topologies. ....................................... 129

6.4.1 Experimental Validation of LCL Topology Model. .................................. 130

6.4.2 Evaluating the Sensitivity to Misalignment for all Topologies. ................. 133

6.4.3 Summary for the Assessment Analysis.................................................. 138

6.5 Conclusion ..................................................................................... 140

Chapter 7 Dynamic and Physics-based Models of LCL BIWPTS ............................. 142

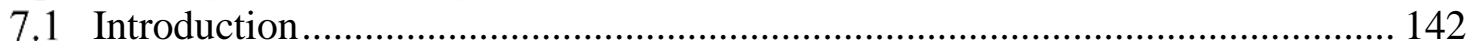

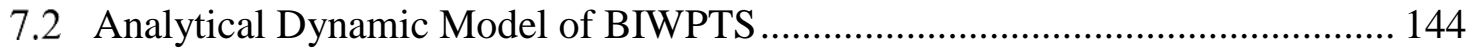

7.2.1 HF VSHBC Dynamic Model ................................................................. 145

7.2.2 Magnetic Coupler and Compensation Network Dynamic Model............... 148

7.3 Physics-Based Co-simulation Platform ......................................................... 150

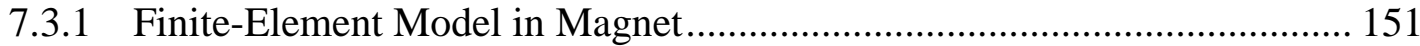

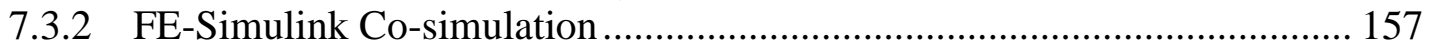

7.4 Model Verification and Discussion ............................................................. 158

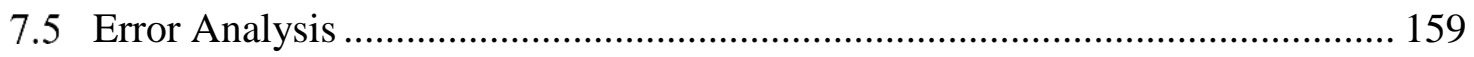

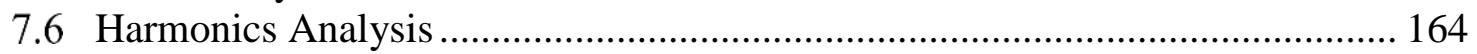

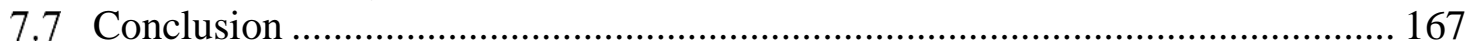

Chapter 8 Magnetic Design Strategy of High-Power Symmetrical LCL BIWPTS .... 168

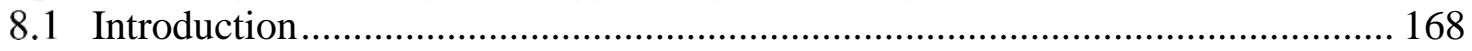

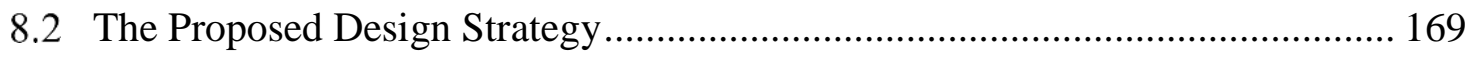

8.3 Magnetic Design of the Power Pads ............................................................ 173

8.3.1 3D Finite Element Model of DD Power Pad ............................................ 174

8.3.2 3D Finite Element Analysis and Optimization of DD Power Pad.............. 176

8.3.3 Design of LCL Compensation Configuration ........................................... 181

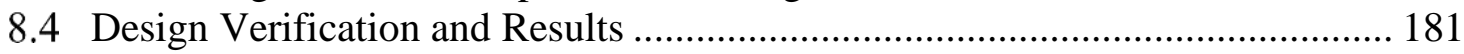

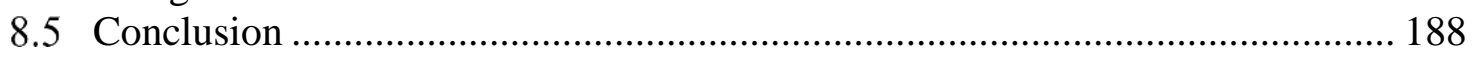

Chapter 9 Coil Design Optimization in the DD Power Pad Structure....................... 189

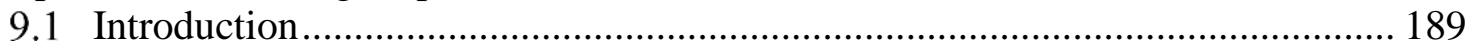

9.2 A Detailed 2D FEM of the DD Power Pad .................................................... 190

9.3 The Multi-objective Optimization Problem...................................................... 192

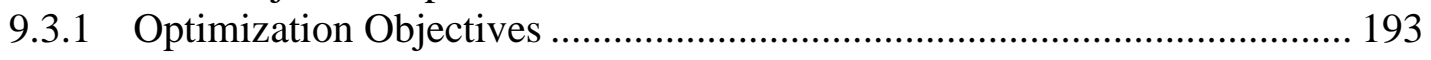

9.3.1.1 Coupling Performance with Misalignment ....................................... 194

9.3.1.2 Material Cost of the DD Power Pad ................................................. 195

9.3.2 Multi-objective Cost Function and Constraints ........................................ 197

9.4 Solving of the Optimization Problem ............................................................ 197

9.4.1 The Proposed Improved Tabu Search (ITS) Algorithm ........................... 198

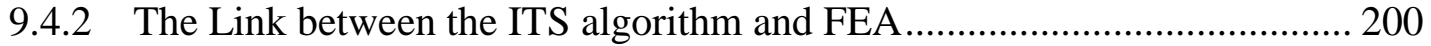

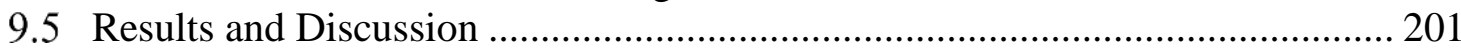

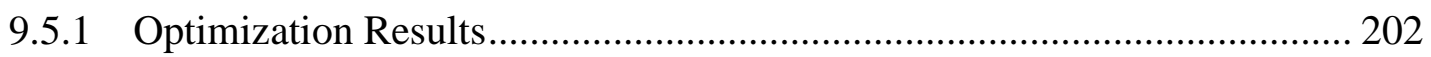

9.5.2 Assessment of the IPT System's Performance ..................................... 205

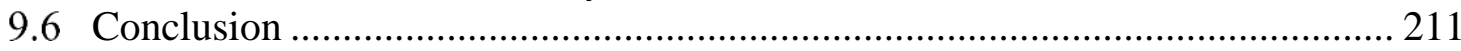

Chapter 10 Autonomous Two-Layer Predictive Power-Flow Control for BIWPTS .... 212 


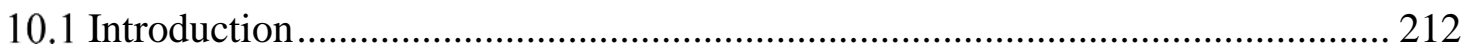

10.2 Detailed Power-Flow Modeling..................................................................... 214

10.2.1 Analytical Models of Active and Reactive Power-Flow ……………….... 215

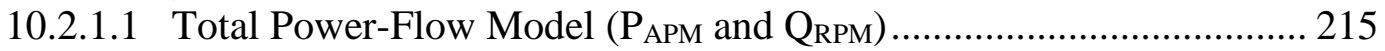

10.2.1.2 Fundamental Power-Flow Model (P PAPM and Q QRPM) ........................ 216

10.2.1.3 Approximate Fundamental Power-Flow Model (PAFAPM and QAFRPM)216

10.2.1.4 Approximate Power-Flow Model (QARPM) ……................................. 217

10.2.2 Models Verification and Comparison ...................................................... 217

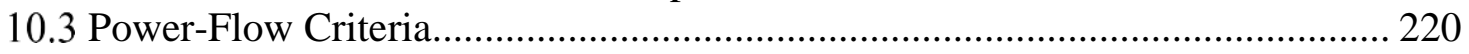

10.3.1 Active Power-Flow Criteria ……………………................................. 222

10.3.2 Reactive Power-Flow Criteria................................................................... 222

10.3.3 Verification for the Power-Flow Criteria.................................................. 223

10.4 Proposed Two-Layer Predictive Active Power-Flow Controller.......................... 226

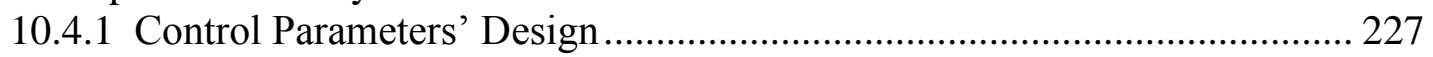

10.4.2 Online Mutual Inductance Estimation ..................................................... 228

10.4.3 First Control Layer............................................................................. 229

10.4.4 Second Control Layer ............................................................................ 234

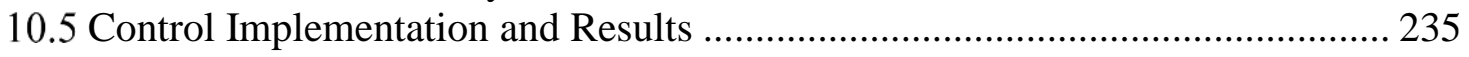

10.5.1 Testing the Proposed Mutual Inductance Estimation .................................. 237

10.5.2 Verifying the Proposed Controller Performance ………………................. 238

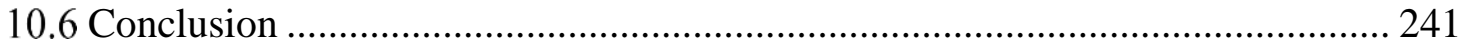

Chapter 11 Feasibility Analysis of Quasi-dynamic BIWPTS for EV Applications...... 243

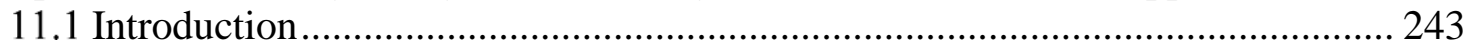

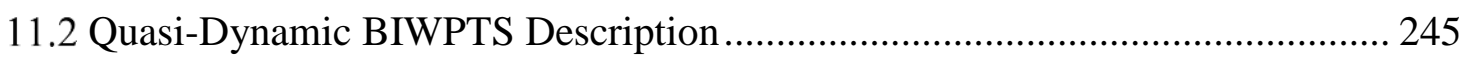

11.3 Modeling of Wirelessly Connected Light-Duty EV .......................................... 248

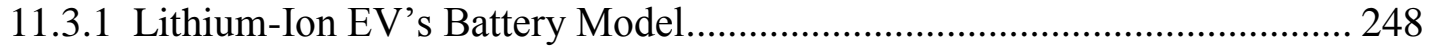

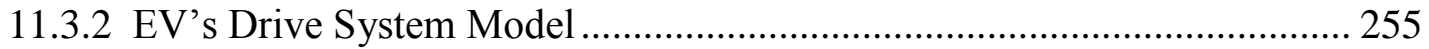

11.3.3 Model Verification............................................................................... 258

11.4 Feasibility Analysis of Implementing QDWPT Systems at Traffic Signals....... 261

11.4.1 Fixed Power Charging Scenario ………………….................................. 261

11.4.2 Variable Power Charging Scenario........................................................... 264

11.4.3 Fixed Power Charging and Discharging Scenario ...................................... 266

11.4.4 Driving Cycle Performance Evaluation ..................................................... 268

11.5 Impact of Misalignment on the Feasibility Analysis .......................................... 271

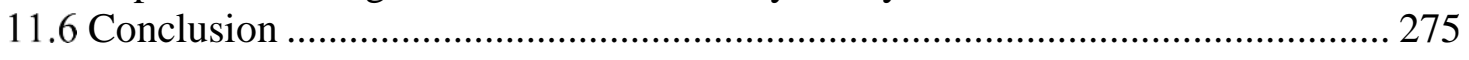

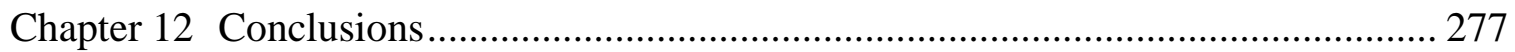

Chapter 13 Recommendations and Future Works ........................................................ 281

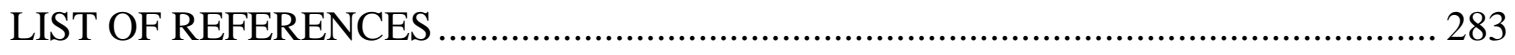

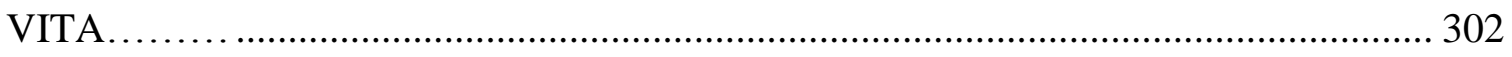




\section{LIST OF TABLES}

TABLE

PAGE

Table 2.1. BP 4175T PV Module Specifications at STC ............................................

Table 2.2. Extracted Parameters of BP 4175T PV Module...........................................32

Table 3.1. Results of Design Parameters from Analytical Design Method......................41

Table 3.2. GA Setup for the Design Optimization Algorithm.......................................44

Table 3.3. Results of Design Parameters from GA Design Method...............................45

Table 3.4. Specifications of Hardware Components. ...................................................47

Table 3.5. MAPE for the Proposed FL-MPPT, P\&O and INC Algorithms. ....................54

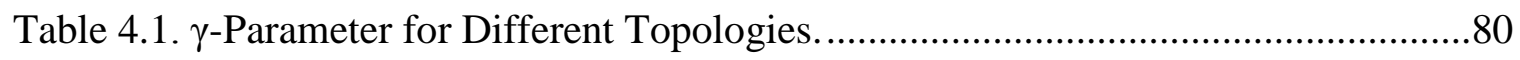

Table 5.1. Final Converter Design Parameters. .............................................................99

Table 5.2. Performance Parameters during Step Response. ......................................104

Table 5.3. Design Parameters of LCL BIWPTS.......................................................109

Table 5.4. NRMSE between Simulation and Experimental Results............................113

Table 6.1. Power-Flow Performance for all Topologies under Full-Loading Conditions.

Table 6.2. Power-Flow Performance for all Topologies under Light-Loading Conditions. .128

Table 6.3. Large and Small-Scale Power-Flow Analysis. ........................................133

Table 6.4. Summary of the Assessment Analysis..................................................139

Table 7.1. Switching States of the Primary HF VSHVC ..........................................147

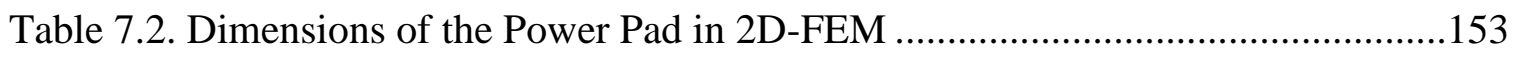

Table 7.3. Design Parameters of the BIWPTS Model ................................................159

Table 7.4. \%NRMSE at Full and Light Loading Conditions......................................164

Table 7.5. \% THD during G2V Operation for Full and Light Loading ..........................166 
Table 7.6. \% THD during V2G Operation for Full and Light Loading 167

Table 8.1. Final Design Parameters of the DD Power Pad...............................................180

Table 8.2. Design Parameters of LCL BIWPTS with DD pads structure.........................184

Table 8.3. The Power Flow of the Large and Small Scale Models ..................................187

Table 9.1. Dimensions of the 2D FEM for the DD pad structure....................................192

Table 9.2. The Misalignment Parameters and the Cost Coefficients during the Optimization.

Table 9.3. Boundaries of the Optimization Variables......................................................203

Table 9.4. Settings of the ITS Optimization Algorithm's Parameters.............................204

Table 9.5. The Optimum Design Parameters.................................................................205

Table 9.6. Cost of the Wireless Coupler (Two Pads) in all Scenarios..............................209

Table 10.1. Numerical Power-Flow Evaluation using the Different Models..................220

Table 10.2. Worst Power-Flow Criteria $\left(\alpha=\beta=180^{\circ}\right)$ for LCL BIWPTS. ......................224

Table 10.3. Different Modes of Power-Flow $\left(\alpha=\beta=180^{\circ}\right)$ in BIWPTS. ........................225

Table 11.1. Charging and Discharging Coefficients of the Battery Module's Analytical Model. ...................................................................................................25

Table 11.2. Design Parameters of an $11.1 \mathrm{~kW}$ LCL BIWPTS at 40 and $85 \mathrm{kHz} \ldots \ldots \ldots \ldots . .259$

Table 11.3. Benefit Factor Evaluation for all Scenarios (miles/kWh)............................270

Table 11.4. Driving Performance Considering Misalignment........................................275 


\section{LIST OF FIGURES}

FIGURE

PAGE

Figure 1.1. DC-bus configuration for G2V and V2G services with wirelessly connected

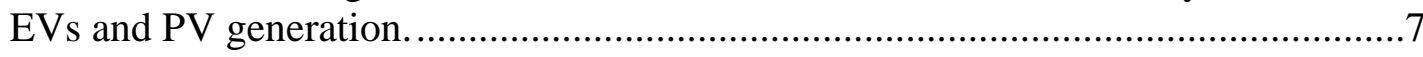

Figure 2.1. Block diagram of grid-connected PV power system..................................16

Figure 2.2. Equivalent circuit of a grid-connected PV power system through a DC-DC Cuk converter.

Figure 2.3. Effect of $R_{p}$ on I-V characteristics of PV module at different climatic conditions.

Figure 2.4. Comparison between I-V characteristic for BP 4175T PV module at STC from measurement and simulated using GA and analytical based parameters. 32

Figure 3.1. Block diagram of grid-connected PV power system including MPPT control.

Figure 3.2. Flow-chart of the classical direct P\&O MPPT algorithm. .37

Figure 3.3. Block diagram of the proposed fuzzy logic system tracking algorithm

Figure 3.4. Flowchart of GA optimization procedure.

Figure 3.5. Progression of the GA Fitness function...................................................45

Figure 3.6. Experimental setup of PV power system...............................................46

Figure 3.7. Simulation results for the proposed FL system designs .............................48

Figure 3.8. Experimental and simulation results for FL-MPPT control .........................50

Figure 3.9. Experimental and simulation results for FL-MPPT controller under fast

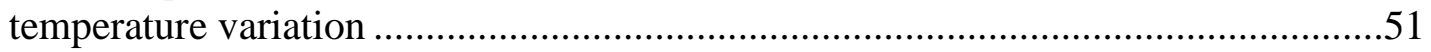

Figure 3.10. Comparison between the proposed fuzzy-MPPT and INC algorithm ...........52

Figure 3.11. Comparison between the proposed fuzzy-MPPT and $\mathrm{P} \& \mathrm{O}$ algorithm .........53

Figure 3.12. Experimental and simulation results for $\mathrm{P} \& \mathrm{O}$ algorithm ..........................54

Figure 4.1. Wired and wireless connected EV through a DC-bus................................58 
Figure 4.2. Wired and wireless connected EV though an AC-bus.

Figure 4.3. Schematic circuit diagram of BIWPTS .................................................61

Figure 4.4. Phase-shift control of the HF inverters...................................................62

Figure 4.5. Legs and total inverter output voltages in terms of the phase-shift control

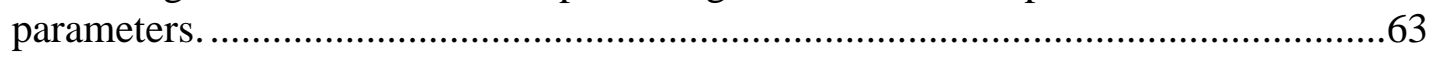

Figure 4.6. Different compensation topologies in BIWPTS.........................................64

Figure 4.7. T-model representation of wireless coupler ..........................................68

Figure 4.8. $\pi$-model of BIWPTS for inverter's currents in terms of inverter's voltages...69

Figure 4.9. $\pi$-model of BIWPTS for coil's currents in terms of inverter's voltages. ........70

Figure 4.10. BIWPTS equivalent circuits using T-model mutual coupling for LC-series

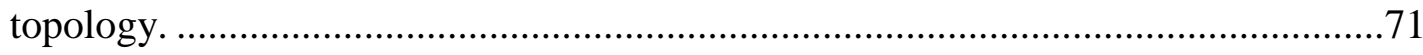

Figure 4.11. BIWPTS equivalent circuits using T-model mutual coupling for

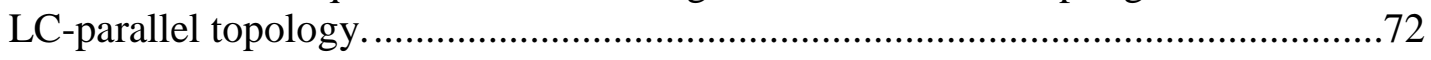

Figure 4.12. BIWPTS equivalent circuits using T-model mutual coupling for LCL

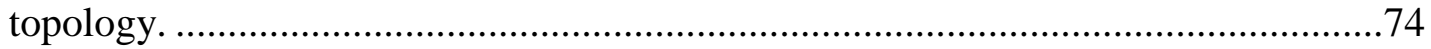

Figure 4.13. Effect of filters inductances on the fundamental impedance frequency response for LCL-parallel design .76

Figure 4.14. Effect of system inductances on the fundamental impedance frequency response for LCL-parallel design $(40 \mathrm{kHz})$...................................................... 77

Figure 4.15. Effect of system resistances on the fundamental impedance frequency

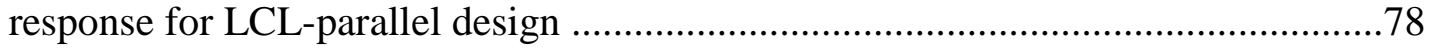

Figure 5.1. Forward-bias safe operating area of IXFB110N60P3 Si power MOSFET....85

Figure 5.2. Bootstrap driving circuit components using FAN7391 IC driver. .................86

Figure 5.3. Layout of the optimized design converter's PCB ....................................90

Figure 5.4. Layouts of an arbitrary design converter's PCB. .....................................90

Figure 5.5. Switching signals and output voltage of VSHBC under phase-shift control. .91 Figure 5.6. Hardware implementation of adjustable dead-time circuit. ..........................92

Figure 5.7. Logic block diagram of phase-shift control circuit. .93 
Figure 5.8. Phase-shift circuit schematic diagram....................................................93

Figure 5.9. The hardware of phase-shift circuit's PCB. .............................................94

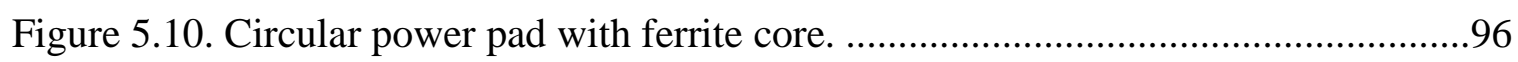

Figure 5.11. The hardware implementation of VSHBC's PCB ..................................98

Figure 5.12. Measured switching signals (0-5V) of one inverter (4 MOSFETs) during

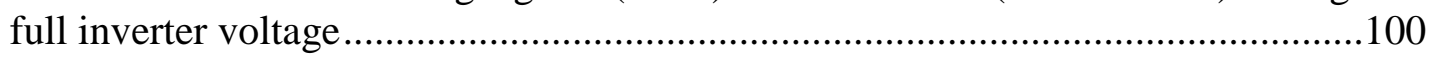

Figure 5.13. Measured switching signals of one leg (2 MOSFETs) during full inverter

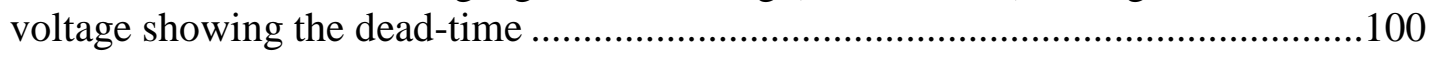

Figure 5.14. Measured switching signals (0-5V) of one inverter (4 MOSFETs) during reduced inverter voltage.

Figure 5.15. Measured gate signals (0-15V) of one inverter (4 MOSFETs) during full inverter voltage

Figure 5.16. Measured gate signals (0-15V) of one inverter (4 MOSFETs) during reduced inverter voltage.

Figure 5.17. Measured ringing effect of the optimized and non-optimized PCB designs.

Figure 5.18. Measured output inverter voltage with and without snubber circuit.....

Figure 5.19. Measured unit-step response of inverter output voltage.

Figure 5.20. Measured inverter output voltage during full output test

Figure 5.21. Measured inverter output voltage during reduced output test 106

Figure 5.22. Measured inverter output voltage at different frequencies .107

Figure 5.23. Experimental setup of LCL BIWPTS ...............................................108

Figure 5.24. Simulink model of LCL BIWPTS ...................................................108

Figure 5.25. Experimental and simulated BIWPTS's performance under full supply voltage for $\mathrm{G} 2 \mathrm{~V}$ operation $\left(\alpha=\beta=180^{\circ}, \delta=-90^{\circ}, \mathrm{V}_{\mathrm{dc}}=60 \mathrm{~V}\right)$

Figure 5.26. Experimental and simulated BIWPTS's performance under full supply voltage for $\mathrm{V} 2 \mathrm{G}$ operation $\left(\alpha=\beta=180^{\circ}, \delta=90^{\circ}, \mathrm{V}_{\mathrm{dc}}=60 \mathrm{~V}\right)$.

Figure 5.27. Experimental and simulated BIWPTS performance under reduced supply voltage for $\mathrm{G} 2 \mathrm{~V}$ operation $\left(\alpha=\beta=90^{\circ}, \delta=-90^{\circ}, \mathrm{V}_{\mathrm{dc}}=60 \mathrm{~V}\right)$ 
Figure 6.1. LC-series topology performance under full supply capacity for G2V operation $\left(\alpha=\beta=180^{\circ}, \delta=90^{\circ}, V_{d c}=240 \mathrm{~V}\right.$ and $\left.V_{b}=200 \mathrm{~V}\right)$..

Figure 6.2. LC-series topology performance under reduced supply capacity for charging operation $(\mathrm{G} 2 \mathrm{~V})\left(\alpha=\beta=90^{\circ}, \delta=90^{\circ}, \mathrm{V}_{\mathrm{dc}}=240 \mathrm{~V}\right.$ and $\left.\mathrm{V}_{\mathrm{b}}=200 \mathrm{~V}\right)$.

Figure 6.3 LC-series topology performance under reduced supply capacity for discharging operation (V2G) $\left(\alpha=\beta=90^{\circ}, \delta=-90^{\circ}, V_{d c}=240 \mathrm{~V}\right.$ and $\left.V_{b}=200 \mathrm{~V}\right)$

Figure 6.4. LC-parallel topology performance under reduced supply capacity for G2V operation $\left(\alpha=\beta=90^{\circ}, \delta=-90^{\circ}, \mathrm{V}_{\mathrm{dc}}=240 \mathrm{~V}\right.$ and $\left.\mathrm{V}_{\mathrm{b}}=200 \mathrm{~V}\right)$

Figure 6.5. LCL-topology performance under reduced supply capacity for charging operation (G2V) $\left(\alpha=\beta=180^{\circ}, \delta=-90^{\circ}, V_{d c}=240 \mathrm{~V}\right.$ and $\left.V_{b}=200 \mathrm{~V}\right)$

Figure 6.6. Experimental and theoretical LCL-topology performance under full supply capacity for $\mathrm{G} 2 \mathrm{~V}$ operation $\left(\alpha=\beta=180^{\circ}, \delta=-90^{\circ}, \mathrm{V}_{\mathrm{dc}}=60 \mathrm{~V}, \mathrm{~V}_{\mathrm{b}}=50 \mathrm{~V}\right)$.

Figure 6.7. Experimental and theoretical LCL-topology performance under full supply capacity for $\mathrm{V} 2 \mathrm{G}$ operation $\left(\alpha=\beta=180^{\circ}, \delta=90^{\circ}, \mathrm{V}_{\mathrm{dc}}=60 \mathrm{~V}, \mathrm{~V}_{\mathrm{b}}=50 \mathrm{~V}\right)$.

Figure 6.8. Experimental and theoretical LCL-topology performance under reduced supply capacity for $\mathrm{G} 2 \mathrm{~V}$ operation $\left(\alpha=\beta=90^{\circ}, \delta=-90^{\circ}, \mathrm{V}_{\mathrm{dc}}=60 \mathrm{~V}, \mathrm{~V}_{\mathrm{b}}=50 \mathrm{~V}\right)$.

Figure 6.9. Experimental and theoretical LCL-topology performance under reduced supply capacity for $\mathrm{V} 2 \mathrm{G}$ operation $\left(\alpha=\beta=90^{\circ}, \delta=90^{\circ}, \mathrm{V}_{\mathrm{dc}}=60 \mathrm{~V}, \mathrm{~V}_{\mathrm{b}}=50 \mathrm{~V}\right)$.

Figure 6.10. Angle misalignment in IPT system

Figure 6.11. Measured and estimated normalized primary coil current under misalignment for all topologies $\left(\mathrm{G} 2 \mathrm{~V}, \alpha=\beta=180^{\circ}\right)$.

Figure 6.12. Measured and estimated normalized power under misalignment for all topologies $\left(\mathrm{G} 2 \mathrm{~V}, \alpha=\beta=180^{\circ}\right)$

Figure 6.13. Theoretical fundamental equivalent impedance under misalignment for all topologies $\left(\mathrm{G} 2 \mathrm{~V}, \alpha=\beta=180^{\circ}\right)$

Figure 7.1. Power electronics circuit diagram of LCL BIWPTS. 146

Figure 7.2. Switching activities and output voltage waveform of primary inverter. 146

Figure 7.3. Dynamic electric equivalent circuit of symmetrical LCL BIWPTS. 149

Figure 7.4. Double-D power pad structure using sheet of ferrite core.

Figure 7.5. Characteristics of N87 ferrite material 
Figure 7.6. 2D-FEM of one power pad of the wireless coupler.

Figure 7.7. Instantaneous wireless pads currents waveforms..........................................155

Figure 7.8. Magnetic flux density distribution of 2D-FEM. ...........................................156

Figure 7.9. Co-simulation Simulink-Magnet Platform...................................................157

Figure 7.10. Coupling between Simulink and Magnet software. ……..............................158

Figure 7.11. Introduced dynamics in BIWPTS $\left(\mathrm{V}_{\mathrm{dc}}=240 \mathrm{~V}\right.$ and $\left.\mathrm{V}_{\mathrm{b}}=200 \mathrm{~V}\right) \ldots \ldots \ldots \ldots \ldots \ldots . . .160$

Figure 7.12. $\mathrm{RMS}$ values of BIWPTS's variables $\left(\mathrm{V}_{\mathrm{dc}}=240 \mathrm{~V}\right.$ and $\left.\mathrm{V}_{\mathrm{b}}=200 \mathrm{~V}\right) \ldots \ldots \ldots \ldots \ldots . . .161$

Figure 7.13. Error between analytical and FE co-simulation platform results ................162

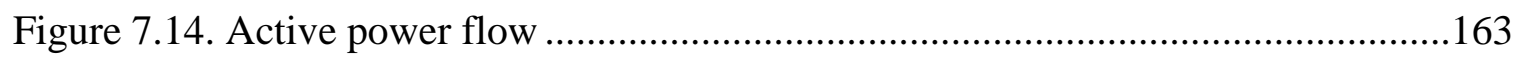

Figure 7.15. FFT analysis during full $\mathrm{G} 2 \mathrm{~V}$ operation $\left(\mathrm{V}_{\mathrm{dc}}=240 \mathrm{~V}, \mathrm{~V}_{\mathrm{b}}=200 \mathrm{~V}, \alpha=\beta=180^{\circ}\right.$

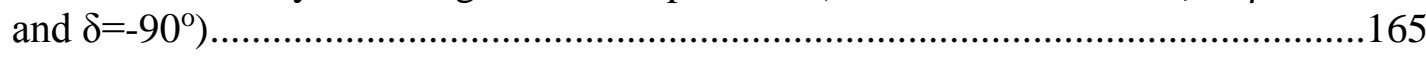

Figure 7.16. FFT analysis during full $\mathrm{V} 2 \mathrm{G}$ operation $\left(\mathrm{V}_{\mathrm{dc}}=240 \mathrm{~V}, \mathrm{~V}_{\mathrm{b}}=200 \mathrm{~V}, \alpha=\beta=180^{\circ}\right.$

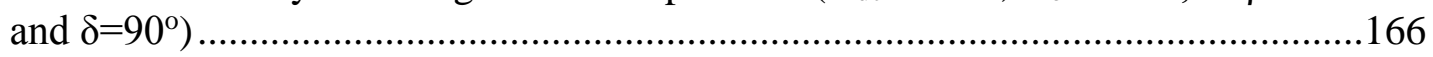

Figure 8.1. Flow-chart for the proposed design methodology ........................................170

Figure 8.2. Effect of the pad self-inductance on the BIWPTS's performance $\left(\mathrm{V}_{\mathrm{dc}}=\right.$ $420 \mathrm{~V}$ and $\mathrm{V}_{\mathrm{b}}=350 \mathrm{~V}$ ).

Figure 8.3. Different structures of power pads in EVs applications ................................173

Figure 8.4. 3D-FEM for DD wireless coupler based on ferrite bars................................175

Figure 8.5. Different views of DD wireless coupler ..................................................175

Figure 8.6. Magnetic field distribution across a vertical section in the middle of the 3D

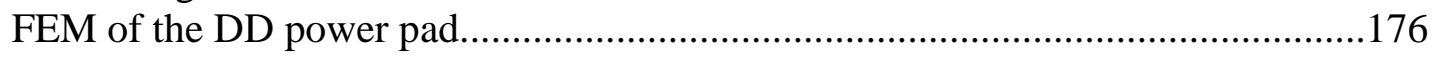

Figure 8.7. Magnetic flus density distribution in the ferrite bars of the DD power pad model.

Figure 8.8. Effect of coil-width on the evaluation parameters $\left(\mathrm{V}_{\mathrm{dc}}=420 \mathrm{~V}\right.$ and $\mathrm{V}_{\mathrm{b}}=350$ V) .178

Figure 8.9. Effect of $\mathrm{Df}$ on the evaluation parameters $\left(\mathrm{V}_{\mathrm{dc}}=420 \mathrm{~V}\right.$ and $\left.\mathrm{V}_{\mathrm{b}}=350 \mathrm{~V}\right)$......179

Figure 8.10. Effect the pitch on the evaluation parameters $\left(\mathrm{V}_{\mathrm{dc}}=420 \mathrm{~V}\right.$ and $\mathrm{V}_{\mathrm{b}}=350$ V). 180 
Figure 8.11. Ferrite bars of N87 material from EPCOS for one DD power pad. 182

Figure 8.12. Final shape of the developed $8 \mathrm{~kW}$ DD power pad.

Figure 8.13. Test-bed for a symmetrical LCL BIWPTS based on the DD power pads...184

Figure 8.14. Performance of an $8 \mathrm{~kW}$ LCL BIWPTS, during G2V operation $(\alpha=\beta=$ $180^{\circ}, \delta=-90^{\circ}, V_{\mathrm{dc}}=420 \mathrm{~V}$ and $\mathrm{V}_{\mathrm{b}}=350 \mathrm{~V}$ ), using Simulink and analytical models ....185

Figure 8.15. Performance of the small scale LCL BIWPTS, during G2V operation $\left(\alpha=\beta=180^{\circ}, \delta=-90^{\circ}, \mathrm{V}_{\mathrm{dc}}=60 \mathrm{~V}\right.$ and $\left.\mathrm{V}_{\mathrm{b}}=50 \mathrm{~V}\right)$, using experimental and analytical models

Figure 8.16. Performance of the small scale LCL BIWPTS, during V2G operation $\left(\alpha=\beta=180^{\circ}, \delta=90^{\circ}, \mathrm{V}_{\mathrm{dc}}=60 \mathrm{~V}\right.$ and $\left.\mathrm{V}_{\mathrm{b}}=50 \mathrm{~V}\right)$, using experimental and analytical models

Figure 9.1. A 2D-FEM of the DD power pad, showing the optimization variables........191

Figure 9.2. Different positions of misalignments

Figure 9.3. Top view of DD power pad with components' dimensions.

Figure 9.4. Flowchart of the proposed ITS algorithm.

Figure 9.5. Block diagram for the link between ITS algorithm in MATLAB and FEA in Maxwell. .201

Figure 9.6. Progression of ITS optimization algorithm. .204

Figure 9.7. Four different design scenarios 206

Figure 9.8. Magnetic flux distribution for all scenarios $(\mathrm{g}=130 \mathrm{~mm}$ and $\mathrm{v}=\mathrm{h}=\theta=0) \ldots \ldots .207$

Figure 9.9. Coupling factor vs. air gap length with perfect alignment $(\mathrm{h}=\theta=0)$. .208

Figure 9.10. Coupling factor vs. horizontal misalignment with $\mathrm{g}=130 \mathrm{~mm}$ and $\theta=0 \ldots . .210$

Figure 9.11. Coupling factor vs. rotational misalignment with $\mathrm{g}=130 \mathrm{~mm}$ and $\mathrm{h}=0 \ldots \ldots .210$

Figure 10.1. Comparison between the different active power models $\left(\alpha=\beta=180^{\circ}\right.$, and $\mathrm{P}_{\mathrm{S}_{-} \max }$ is the base).

Figure 10.2. Comparison between the different reactive power models $\left(\alpha=\beta=180^{\circ}\right.$ and $\mathrm{Q}_{\text {s_max }_{-}}$is the base).

Figure 10.3. BIWPTS's active and reactive power-flow criteria. .221 
Figure 10.4. Power flow criteria with different control parameters $\left(\mathrm{V}_{\mathrm{dc}}=60 \mathrm{~V}\right)$.

Figure 10.5. The proposed two-layer predictive active power-flow control. .227

Figure 10.6. First layer control principle .231

Figure 10.7. The proposed first layer control algorithm. .233

Figure 10.8. The proposed second control layer algorithm. .235

Figure 10.9. Cmod S6 FPGA board. .236

Figure 10.10. Implementation of FPGA-based PPM including the dead-time and the driver. .236

Figure 10.11. Mutual inductance estimation performance $\left(\mathrm{V}_{\mathrm{dc}}=60 \mathrm{~V}\right)$

Figure 10.12. PAPF control performance during Abstain and Discharge modes..... .238

Figure 10.13. Comparison between the performance of PI and the proposed PAPF control during Abstain and Discharge modes......

Figure 10.14. PAPF control performance during Discharge and Charge modes. .240

Figure 10.15. Comparison between the performance of PI and the proposed PAPF control during Discharge and Charge modes.

Figure 11.1. The proposed implementation of Quasi-dynamic WPT system at traffic signal.

Figure 11.2. Block diagram of a wirelessly connected EV through the BIWPTS...........248

Figure 11.3. Lithium-ion test battery

Figure 11.4. $2^{\text {nd }}$ order dynamic battery equivalent circuit model with EV traction system. .250

Figure 11.5. Curve fitted battery model coefficients .251

Figure 11.6. Battery model verification tests. .255

Figure 11.7. FTP-72 Driving profile. .257

Figure 11.8. BIWPTS's performance at $40 \mathrm{kHz}$ resonant frequency, during G2V operation $\left(\alpha=\beta=180^{\circ}, \delta=-90^{\circ}\right)$.

Figure 11.9. BIWPTS's performance at $85 \mathrm{kHz}$ resonant frequency, during G2V operation $\left(\alpha=\beta=180^{\circ}, \delta=-90^{\circ}\right)$. .260 
Figure 11.10. Driving performance under fixed power charging WPT2 $\left(\mathrm{SOC}_{\mathrm{i}}=80 \%\right)$.

Figure 11.11. Driving performance under fixed power charging WPT4 $\left(\mathrm{SOC}_{\mathrm{i}}=80 \%\right)$.

Figure 11.12. Driving performance at different charging levels $\left(\mathrm{SOC}_{\mathrm{i}}=70 \%\right) \ldots \ldots \ldots \ldots . .264$

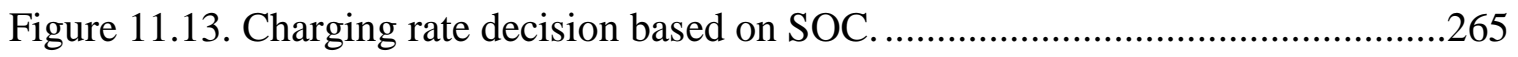

Figure 11.14. Driving performance under variable power charging level $\left(\mathrm{SOC}_{\mathrm{i}}=\right.$

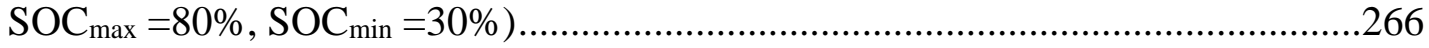

Figure 11.15. Driving performance under charging and discharging operation (WPT4,

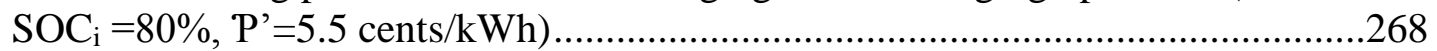

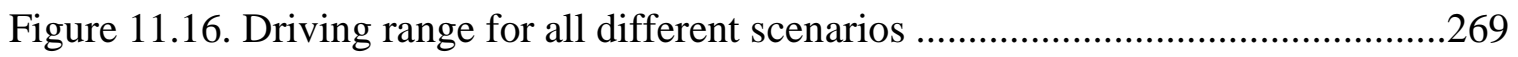

Figure 11.17. Long track primary coupler.........................................................2

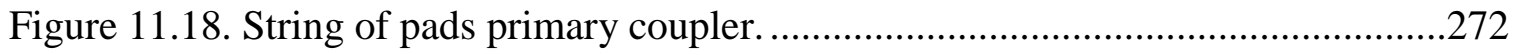

Figure 11.19. Driving performance considering a misalignment (WPT4, $\mathrm{SOC}_{\mathrm{i}}=80 \%$ ). 


\section{LIST OF ABBREVIATIONS AND ACRONYMS}

\begin{tabular}{ll} 
2D FEM & Two Dimensional Finite Element Model \\
3D-FEM & Three Dimensional Finite Element Model \\
A.M & Air Mass \\
AC & Alternating Current \\
ADC & Analogue to Digital \\
Ah & Ampere-Hour \\
b.f & Benefit Factor \\
BIWPTS & Bidirectional Inductive Wireless Power Transfer System \\
BMS & Battery Management System \\
BWPT & Bi-directional Wireless Power Transfer \\
DC & Direct Current \\
DD & Double-D \\
DDQ & Double-DQ \\
DWPT & Dynamic Wireless Power Transfer \\
EC & Equivalent Circuit \\
EMI & Electromagnetic Interference \\
EPRI & Electric Power Research Institute \\
ESL & Equivalent Series Inductance \\
ESS & Energy Storage System \\
eV & Electron Volts \\
\hline
\end{tabular}




\begin{tabular}{|c|c|}
\hline EVs & Electric Vehicles \\
\hline FCT & Full Cycle Time \\
\hline FE & Finite Element \\
\hline FEA & Finite Element Analysis \\
\hline FEM & Finite Element Model \\
\hline FFT & Fast-Fourier Transform \\
\hline FIS & Fuzzy Inference System \\
\hline FL & Fuzzy Logic \\
\hline FLC & Fuzzy Logic Control \\
\hline FPGA & Field-Programmable Gate Array \\
\hline FTP & Federal Test Procedure \\
\hline G2V & Grid-to-Vehicle \\
\hline GA & Genetic Algorithm \\
\hline $\mathrm{HF}$ & High Frequency \\
\hline IC & Integrated Circuit \\
\hline ICE & Internal Combustion Engine \\
\hline ICNIRP & International Commission on Non-Ionizing Radiation Protection \\
\hline IEC & International Electrotechnical Commission \\
\hline IEEE & Institute of Electrical and Electronics Engineers \\
\hline IGBT & Insulated-Gate Bipolar Transistor \\
\hline INC & Incremental Conductance \\
\hline IPT & Inductive Power Transfer \\
\hline
\end{tabular}




$\begin{array}{ll}\text { ITS } & \text { Improved Tabu Search } \\ \text { ITSs } & \text { Intelligent Transportation Systems } \\ \text { kHz } & \text { kilo-Hertz } \\ \text { kW } & \text { kilo-Watt } \\ \text { kWh } & \text { kilo-Watt-Hour } \\ \text { LEM } & \text { Liaisons Electroniques et Mecaniques } \\ \text { LHS } & \text { Latin Hypercube Sampling } \\ \text { Li } & \text { Lithium } \\ \text { MAP } & \text { Maximum Active Power } \\ \text { MAPE } & \text { Mean Absolute Power Error } \\ \text { MMF } & \text { Magneto-Motive Force } \\ \text { MnZn } & \text { Manganese and Zinc } \\ \text { MOF } & \text { Multi-Objective Optimization Function } \\ \text { MOSFET } & \text { Metal-Oxide-Semiconductor Field-Effect Transistor } \\ \text { MP } & \text { Maximum Power } \\ \text { mpc } & \text { miles per cycle } \\ \text { MPP } & \text { Maximum Power Point } \\ \text { MPPT } & \text { Maximum Power Point Tracking } \\ \text { MRP } & \text { Maximum Reactive Power } \\ \text { NHTSA } & \text { National Highway Traffic Safety Administration } \\ \text { NMSE } & \text { Normalized Mean Square Error } \\ \text { NRMSE } & \text { Normalized Root Mean Square Error } \\ & \\ \text { MA } & \end{array}$




\begin{tabular}{ll} 
OCV & Open Circuit Voltage \\
ODE & Ordinary Differential Equation \\
P\&O & Perturb and Observe \\
PAPF & Predictive Active Power-Flow \\
PCB & Printed Circuit Board \\
PF & Power Factor \\
PFC & Power Factor Correction \\
PI & Proportional-Integral \\
PID & Proportional-Integral-Derivative \\
PLL & Phase-Locked Loop \\
PPM & Pulse-Phase Modulation \\
PV & Photovoltaic \\
PWM & Pulse-Width Modulation \\
QDWPT & Quasi-Dynamic Wireless Power Transfer \\
RESs & Renewable Energy Sources \\
RK4 & Runge-Kutta \\
RMS & Root-Mean Square \\
SAE & Society of Automotive Engineers \\
SOC & State of Charge \\
STC & Standard Test Conditions \\
THSL & Single-Turn Single-Layer \\
THD & Total Harmonic Distortion \\
\hline
\end{tabular}




$\begin{array}{ll}\text { T-S } & \text { Takagi-Sugeno } \\ \text { UPF } & \text { Unity Power Factor } \\ \text { UTS } & \text { Universal Tabu Search } \\ \text { V2G } & \text { Vehicle-to-Grid } \\ \text { VA } & \text { Volt-Ampere } \\ \text { VAR } & \text { Volt-Ampere-Reactive } \\ \text { VSHBC } & \text { Voltage-Source H-Bridge Converter } \\ \text { WPT } & \text { Wireless Power Transfer } \\ \text { WPTAC } & \text { Wireless Power Transfer Automatic Charging } \\ \text { ZCS } & \text { Zero-Current Switching } \\ \text { ZPF } & \text { Zero Power Factor Operation } \\ \text { ZVS } & \text { Zero-Voltage Switching }\end{array}$




\section{Chapter 1 Introduction}

\subsection{Problem Statement}

Volatile fuel prices, coupled with an increased emphasis on reducing greenhouse and carbon dioxide emissions, have fostered significant growth in the electric vehicles' (EVs') market over the last ten years. Even though EVs have not been widely adopted, in part because of technical limitations, social obstacles, and cost compared to conventional internal combustion engine (ICE) vehicles [1], based on moderate expectations, by 2020 up to $35 \%$ of the total vehicles in the U.S. will be EVs, according to the Electric Power Research Institute (EPRI) [2]. With the large-scale introduction of EVs, the power grid will face a major challenge to satisfy the load demand. The increasing number of EVs will put an additional stress on the existing distribution system's components, such as transformers and cables, and may perturb their operation, particularly during the peak demand periods.

Several studies have been carried out to investigate the impact of EVs on the power system in terms of load capacity, power quality, economy and environment [1], [3]-[5]. The impact of EV charging on the grid load capacity was discussed in [6]-[12]. The studies concluded that the disorder charging will increase grid peak load, so it needed additional investment on generating electricity and transmission capacity. In terms of power quality, EVs' charging behavior will bring the voltage out-of-limits of the distribution network, reduce the power quality, increase the line loss, reduce the life span of distribution transformers and increase the harmonics and current faults [3], [13]-[15]. In [16], a review for the impact of the EVs charging on the residential distribution systems was presented in 
terms of electricity generation adequacy [11], [17]-[20], transformer aging [14], [21]-[23] and distribution system power quality [24]-[28]. According to the literature, numerous techniques are proposed to mitigate the impacts of EVs' charging on the power grid. These techniques can be categorized into two groups [16]. In the first approach, the utilities indirectly manage EVs' charging using the Time-of-Use pricing [29]-[35]. The low offpeak electricity rates in a Time-of-Use pricing scenario stimulates EVs' owners to charge their vehicles during the off-peak hours. This scheme significantly decreases the peak load demand and mitigates transformer overloading and heating concerns. In the other technique, the utilities directly control EVs' charging rates and charging start time using smart charging algorithms [24], [7], [36]-[46].

Traditionally, these vehicles have been considered as nonlinear loads for the power grid, whose impacts on supply's stability and quality have been well studied. However, as the penetration rate of EVs into the transportation increases, the idea of utilizing them not just as loads but also as energy storage systems (ESSs) is explored. This is a very promising technology since most the light-duty vehicles spend significant time not being operated and there may be opportunities to utilize their stored energy to support the power grid. This concept is known as vehicle-to-grid (V2G) and grid-to-vehicle (G2V) service [1], [47][50]. In this concept, an EV can operate in discharge (V2G) mode, as a source, to inject energy to the grid, or in charge (G2V) mode, as a load, to suck energy from the grid. This concept has attracted the attention of the grid operators and vehicles' owners, as EVs are considered an indispensable component in both "living and mobility" and sustainable living in the near future [51]. In the V2G concept, EVs as ESSs can provide peak load shaving and act as a reserve resource against unexpected outages [52]. As the size of the 
EV fleet increases, the bulk energy storage available can become considerable in size, which adds to flexibility of actions taken by the power system operator.

The benefits of V2G technology can be maximized if it is being associated with renewable energy sources (RESs), such as solar and wind generations [53]-[55]. The intelligent integration between the EVs and RESs can provide an ideal solution for the issues due to both of them. The RES can be designed to supply the bulk power demand of EVs charging with minor support from the power grid. On the other hand, EVs (as ESSs) can be utilized to smoothen the power generation for the intermittent RESs and mitigate their bad impacts on the power grid, such as voltage and frequency instability issues [56][61]. Unlike conventional loads, the energy in EVs is sizable enough to maintain all solar and most of the wind generation. Among all RESs, Photovoltaic (PV) power systems are expected to play a vital role in the future energy efficient and zero emission society. Several factors have been boosting this: improved generation efficiency of PV modules, flexibility of implementation and governmental subsidies [62]. However, PV power fluctuates depending on the environmental conditions, season, and geographic location, and causes problems, such as voltage fluctuation and large frequency deviation in an electric power system operation [63]. In order to mitigate these impacts, it is recommended that equipment with storage capability (such as EVs) should be controlled to compensate the variation in PV generation [60], [64]-[67]. The integration between EVs and PV generation is promising due to the massive similarities between them [68]-[71]. The most important match between them is that EVs will probably be connected to the grid in the same highly dispersed manner, at the same voltage levels, and in the same range as PV systems. For example, EVs at residential homes are similar to those typical residential PV systems in 
parameter values [72]. Also, both PV and EV are integrated to the grid though power electronic interface with the potential to create intelligent nodes in the system. One vital difference is that the EV, as an ESS, is bidirectional and can act as either a source or a load, whereas the PV behaves as a source only. Another dissimilarity is that EVs have both power and energy limitations, but PV does not have an explicit energy limitation. Due to these common features between PV and V2G from the grid's perspective, the grid-support functions requested for PV will be similar to those required for V2G. In fact, it is possible that codes and standards presently applied to the PV systems, such as IEEE-1547 and IEC62116, may apply to V2G systems with small modification [68]. Therefore, in this study, the integration between the EVs and PV systems is considered.

As of now, the size, complexity and economics of utility-scale centralized energy storage control and management might not have been generally acknowledged and supported. Notwithstanding, with moderate assumptions, the required power and energy available in the EVs, even with today's battery technologies, can become available in the very near future [73]. However, what distinguishes the energy stored in EVs from other conventional ESSs is their mobile nature. Like other ESSs, accessibility of these resources relies upon the availability of the primary source of energy (the batteries in this case). Although unlike other ESSs, accessibility of EVs' energy depends likewise on the locations of the vehicles. This complicates matters further by adding another constraint to the problem. Traditionally, people have been looking at extracting the stored energy, either from locations where a relatively large number of vehicles exist (e.g., charging stations, parking lots) [65], [74], or from individual charging devices at residential units [69]. The common factor in both lies in the fact that the vehicles should be stationary (i.e., parked). 
While this is a reasonable assumption in many cases, it undermines a great potential source of energy in the system: vehicles in motion. Furthermore, in order to inject energy back into the grid, it is necessary for the vehicle to be driven to the charging station/device, and parked for the duration of the power exchange [75]-[77]. Over those, the driver may need to change his normal route to get to the charging station. All these could inconvenience the driver(s) and hence reduce the tendency (or participation level) for participating in V2G services. Additionally, when the source of energy is needed immediately (e.g., fast reserves) the distances of the vehicles to the charging stations would serve as a restricting factor. Finally, for the vehicle to be able to transfer power to the grid, the driver (or an operator) needs to be physically present to set up an electrical connection between the vehicle and the station [78], [79]. This obstructs the implementation of supervisory level control schemes that are managed remotely.

Wireless charging stations (based on inductive power transfer (IPT) technology) have been proposed as a mean for energy transfer without physical contacts [80]-[91]. Without the need for establishing an actual electrical connection, this technology enables transfer of energy from the power grid to the vehicle and possibly in the reverse direction through the usage of magnetic circuits operating at resonance. This power transfer can occur while the vehicle is in long-term parking (stationary), transient stops (quasi-dynamic) or moving even close to normal speed (dynamic) [92]-[97]. Utilizing this technology can turn the potential energy stored in the EVs' batteries into an accessible source of energy distributed across the power grid [98]. In addition, a supervisory control scheme can be designed and implemented to remotely control these individual sources of energy in order to provide a considerable source of energy storage for the power grid [93]-[95]. Several 
studies have been conducted, which show comparative analyses for the percentage of EVs interactivity between the conductive and inductive connections [77], [99], [100]. The studies emphasized that the conductive connection guarantees about $10 \%$ of EVs to interact successfully with the power grid, while the inductive connection provides about 65\% [75], [100]-[102]. Thus, the bidirectional IPT technology is considered in this study to accomplish the integration between the EVs, PV generation and power grid, and achieve G2V and G2V services.

\subsection{Research Objective}

The main objective of this dissertation is to provide a comprehensive study for wirelessly connected EVs to achieve G2V and V2G services with the large-scale penetration of PV generation. The system's configuration under consideration is indicated in Figure 1.1. In this structure, it is proposed that all the resources should be integrated through a common direct current (DC)-bus. The alternating current (AC) power grid connection is established using a bi-directional grid-tied converter, which is responsible for regulating the DC-link voltage. Each EV is coupled to the DC-bus through its own bidirectional inductive wireless power transfer system (BIWPTS) to facilitate charging (G2V) and discharging (V2G) operation. In the G2V mode, control of the BIWPTS can provide a mechanism to regulate multiple charging scenarios. In the V2G mode, EV's energy can be injected back to the AC grid to provide support during peak loading periods or an outage. As an added benefit, the introduction of a localized DC-bus would help ease the integration of PV generation, which is accomplished through a unidirectional DC-DC converter, connected directly to the DC-bus. In addition, the surrounding DC loads can be 
supplied using another unidirectional DC-DC converter. Through the inclusion of PV generation, the bulk load demand can reduce grid stress when EVs are in the G2V mode, while in the V2G mode, the EV's battery array can aid in smoothening the PV generation.

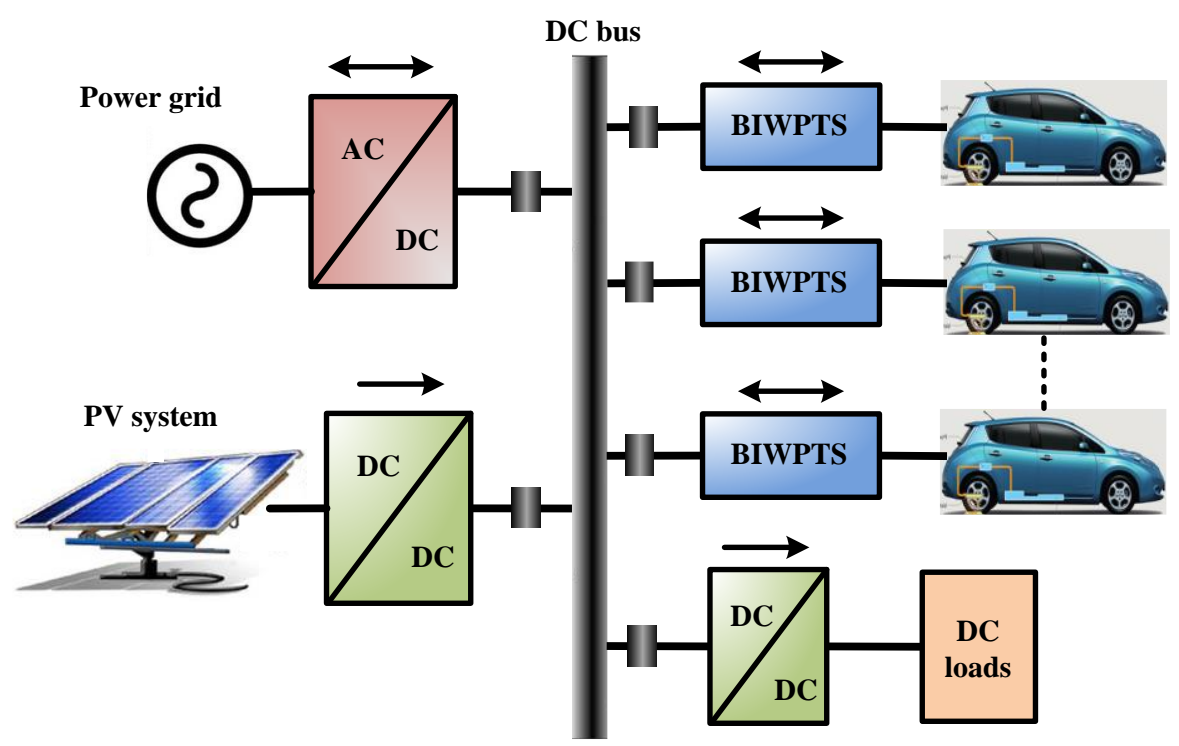

Figure 1.1. DC-bus configuration for G2V and V2G services with wirelessly connected EVs and PV generation.

This architecture is flexible and can be physically implemented in several positions, in which EVs are in long-term parking, such as charging stations, public and private parking lots and car parks, and short-term parking, such as bus stops and road traffic signals. Furthermore, the same configuration can be deployed in the powered roads to provide $\mathrm{G} 2 \mathrm{~V}$ and $\mathrm{V} 2 \mathrm{G}$ services for the in-motion EVs, through the dynamic wireless charging.

This study is crucial and provides valuable solutions for many issues that impede the penetration of EVs in transportation and RES in electric energy generation. The study 
presents modeling, design and control analyses for the power interfaces among the EV, PV system and utility grid. These interfaces have the capability to provide the necessitate power-flow between the power grid and the other resources (EVs and PV panel) to achieve G2V and V2G services. For the PV power system, a unidirectional DC-DC converter is considered as the power interface to extract the maximum power from the PV generator, regardless of the environmental conditions, by implementing an intelligent maximum power point tracking (MPPT) algorithm. For the EV, the duel-side full-bridge BIWPTS is utilized to manage the two-way power-flow between the EV and DC-bus. Several aspects have been investigated, analyzing practical issues in the BIWPTS, including developing accurate analytical and physics-based models, performing effective design optimizations, developing and implementing new closed-loop controllers, and building prototypes and testing them.

The outcomes of this study are useful for many organizations, such as utility companies, automotive companies and consumers. For the power grid, the results help to minimize the impact of EVs penetration, increase the penetration of PV generation, provide predictable energy resources, enhance the power grid stability and mitigate its power quality issues. For EVs manufacturers, it aids to reduce the battery size required by EVs, improve the operation of EVs through utilizing the energy sources available from other vehicles and make the vehicle become an energy source to provide energy services to the grid. Moreover, it is helpful for the consumers by reducing the EV's price, providing flexible, reliable and automatic bidirectional interface. Also, it enables the vehicle's owner to participate in V2G services, making the vehicle a micro-grid that can be used during power interruptions and storms. 


\subsection{Original Contributions of the Dissertation}

The proper modeling, design and control for the power interfaces among the EVs, and PV generation, and the power grid through a common DC-bus, is investigated in this work. The main findings and contributions in this thesis are summarized as follows:

- Developing analytical dynamic modeling and small signal analysis for a grid-connected PV power system, through a DC-bus.

- Achieving design and optimization analysis for an intelligent maximum power point tracker in PV systems, based on fuzzy logic, genetic algorithm and analytical models. The proposed controller is implemented and verified by means of simulation and experimentally, using the DSPACE microcontroller board.

- Developing accurate steady-state mathematical and computer-based models for the different topologies of a symmetrical BIWPTS in EV applications. The models were able to predict the systems' performance and power-flow; and novel practical limitations and criteria for the active and reactive power-flow during G2V and V2G operations are stated.

- Performing a comprehensive performance assessment analysis for the main configurations of the symmetrical BIWPTS, which aims to define the merits and demerits of each structure and the most appropriate one for G2V and V2G services.

- Building experimental prototypes for the BIWPTS, including power electronic converters, compensation networks, power pads and power-flow controller, to verify the proposed theoretical analyses. 
- Developing an accurate nonlinear physic-based co-simulation platform that links the model of the power electronic converters and controllers in Simulink, to the finiteelement model (FEM) of the electro-magnetic coupler.

- Performing magnetic design multi-objective optimization for the power pads in the wireless interface, based on finite element analysis (FEA) and experimental tests.

- Developing a new, autonomous, two-layer predictive power-flow controller to manage the bidirectional active power-flow between the EV and DC-bus. The controller is designed to be placed on the vehicle's side, and is implemented, using a FieldProgrammable Gate Array (FPGA) board.

- Developing a comprehensive simulation platform for a light-duty wirelessly connected $\mathrm{EV}$, including the BIWPTS, Lithium (Li)-ion battery and traction system. The platform was utilized to study the feasibility of implementing the quasi-dynamic wireless charging systems at the traffic signals on the road, to charge the EVs during the transient stops at the intersections.

\subsection{Dissertation Organization}

This dissertation is organized as follows:

Chapter 1 introduces the integration between EVs, PV power system and the power grid, and identifies the G2V and G2V concept based on the wirelessly connected EVs. In addition, the new findings and contributions in this study are stated.

Chapters 2 and 3 present modeling and control analysis for a grid-connected PV power system through a DC-bus. In chapter 2, a detailed mathematical nonlinear dynamic model for the PV power system is developed. Then, this dynamic model is linearized to 
state the small-signal model for the same system, which is utilized to state the design considerations for the MPPT algorithm's parameters. The developed models were verified by means of simulation and experimental tests. The optimum design for the fuzzy-based MPPT for the PV system is investigated in chapter 3. Two different design methodologies are studied in this chapter: the analytical and genetic algorithm (GA)-based method. The proposed design was implemented and tested by means of simulation and experimentally in comparison with the existing techniques in the literature.

The following 3 chapters provide assessment analysis for the main topologies of the symmetrical BIWPTS for EV's integration. Chapter 4 presents modeling analysis for the steady-state performance of BIWPTSs based on Fourier series analysis. The analysis is achieved for the three main compensation configurations of a symmetrical BIWPTS: LCseries, LC-parallel and LCL-topology. Moreover, the steady-state equivalent circuit-based mathematical models for all topologies are developed. Finally, general formulas for the fundamental power-flow (active and reactive) between the EV and DC-bus are developed.

Chapter 5 discusses design and implementation considerations for the entire BIWPTS's components. It presents more details about the high frequency (HF) converter design and optimization, including component selections, driving circuits, snubber circuits, protection elements and the converter's printed circuit board (PCB). Additionally, the hardware implementation of the phase-shift control based on the analog phase-locked loop (PLL) circuit is investigated and tested. Also, the chapter presents the details of design and building two symmetrical circular power pads with the compensation network to form the wireless coupler. The outcomes form the experimental prototype and the simulated model are presented. 
Chapter 6 presents verification analysis for the developed theoretical models in chapter 4 by means of simulation and using the experimental prototype discussed in chapter 5. Then, these models are utilized to assess the performance of the different topologies during the bidirectional operation, and present the merits and demerits of each structure. Also, it presents comparative analysis for the BIWPTS's performance parameters (power, power factor and efficiency) among all topologies, under both full and light loading conditions. Finally, the sensitivity of the different topologies against the misalignment in the system, based on theoretical models and measurements, is investigated

The next 3 chapters are investigate the nonlinear physics-based model and magnetic design for the symmetrical LCL BIWPTS. Chapter 7 presents a physics-based cosimulation platform for LCL BIWPTS in EVs applications. The platform is established through the coupling between finite element and circuit analysis. In addition, a state-space dynamic mathematical model for the same system is developed and implemented in the MATLAB environment. A $1.2 \mathrm{~kW}$ LCL BIWPTS is analyzed under different dynamics by both models, and the results are compared and presented in this chapter. Finally, the effect of the nonlinearities and the magnetic material characteristics on the system's performance is assessed in terms of error and harmonics analysis.

Chapter 8 presents detailed analysis for the choice and design of the passive elements in a BIWPTS. A clear methodology for achieving the proper design for all the passive elements in a symmetrical LCL BIWPTS is discussed. A three dimensional finite element model (3D-FEM) for an $8 \mathrm{~kW}$ polarized double-D (DD) power pad is developed and optimized intuitively. Moreover, the choice of the power factor correction (PFC) capacitors and the impedance matching coils was investigated. The recommendations from the 
proposed design methodology are considered to build an entire prototype for a BIWPTS to meet with WPT2 level and Z2 class in the J2954A standard. The system's performance is evaluated based on experimental, simulation and analytical tests.

In chapter 9, an automatic multi-objective optimization for the different design parameters of the DD power pad structure is performed. A detailed two dimensional finite element model (2D FEM) for the power pad, considering the separations among the turns, is developed and linked with an improved Tabu search (ITS) algorithm, for optimization purposes. The proposed design optimization results are evaluated based on the system's coupling performance and cost, in comparison with three other designs that are presented in the literature.

Chapter 10 presents a new two-layer predictive active power-flow control for the LCL BIWPTS in EVs applications. The design analysis for the proposed controller is presented in detail, based on an accurate power-flow analytical model, which is developed and verified in this chapter. The procedures for executing the two control layers and the link between them are presented and explained. In addition, a real time mutual inductance estimation technique that needs to be applied one step before implementing the proposed controller is discussed. Finally, the proposed controller is implemented and tested using the FPGA board, and its performance is compared with the classical proportional-integral (PI) controller.

In chapter 11 , the feasibility of implementing the quasi-dynamic wireless power transfer (QDWPT) system at the traffic signals is explored. A comprehensive modeling platform for a wirelessly connected EV, including the BIWPTS, EV's battery, and traction system, is developed and investigated. The feasibility of implementing a QDWPT system 
at traffic signals is investigated under three distinct wireless power transfer (WPT) scenarios: fixed power charging, variable power charging and fixed power charging and discharging, based on the maximum driving range, duration per cycle, and the additional distance gained for each consumed $\mathrm{kWh}$. In addition, the effect of coil misalignment in the WPT system over the driving performance is investigated.

Finally, the conclusions and recommendations for further research are presented in chapter 12 . 


\section{Chapter 2 Dynamic Modeling of Photovoltaic Power System}

\subsection{Introduction}

Considering the DC-bus configuration in Figure 1.1 for integrating the PV generations with the wirelessly connected EVs, this chapter is investigating the first source in the structure, which is the grid-connected PV power system. PV utilization is getting more attention due to the increased progress in power electronics and semiconductor technologies. A first step to analyze and study a PV power system is to develop an accurate model that has the ability to predict the system performance. It is also very useful for control and design purposes. Thus, in this chapter a detailed mathematical nonlinear dynamic model for grid-connected PV power system is developed. The dynamic model is used to simulate and predict the entire system performance under different dynamics, such as variation in environmental and load conditions. It is also helpful to evaluate the proposed control and maximum power point tracking performance under different irradiance and temperature conditions. Then, the developed dynamic model is linearized to state the small signal model for the same system. This small signal model is utilized to state some design considerations for MPPT algorithm's parameters. The developed models were verified and tested by means of simulation and experimental tests.

The PV power system under consideration consists of a PV array connected to a DC bus through a unidirectional DC-DC converter, as shown in Figure 2.1. In this case, the DC-DC converter is responsible for boosting the array voltage to the DC bus voltage level. Also, it is in charge of extracting the maximum power from the PV panel at different 
environmental and load conditions. The DC bus is coupled to the power grid through a bidirectional three-phase grid-tie converter. This converter is taking charge of managing the power flow between the DC bus and the power grid, in addition to regulating the DC bus voltage to a certain level.

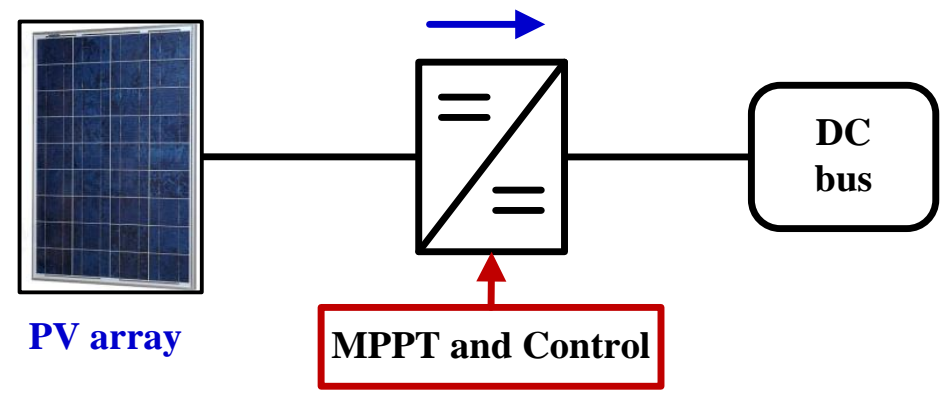

Figure 2.1. Block diagram of grid-connected PV power system.

\subsection{Nonlinear Dynamic Model}

In this section, the details of the non-linear dynamic equations for the PV power system are presented. The formulas are derived based on the electric circuit model of each component in the system. The individual equivalent circuits are lumped together in one circuit that represents the entire PV power system, as shown in Figure 2.2. The system contains a PV array, DC-DC Cuk converter and DC bus. The PV panel is represented by the single diode (four parameters) model equivalent circuit. The Cuk converter is modeled by its ideal passive components with neglecting the resistive losses. Since the DC bus voltage is kept fixed by the grid-tie converter, the DC bus is denoted in the model by a fixed DC source $\left(V_{d c}\right)$ with a series resistance $\left(R_{d c}\right)$ that emulates its resistive losses. The details for the dynamic equations of each component are presented in the following sections. 


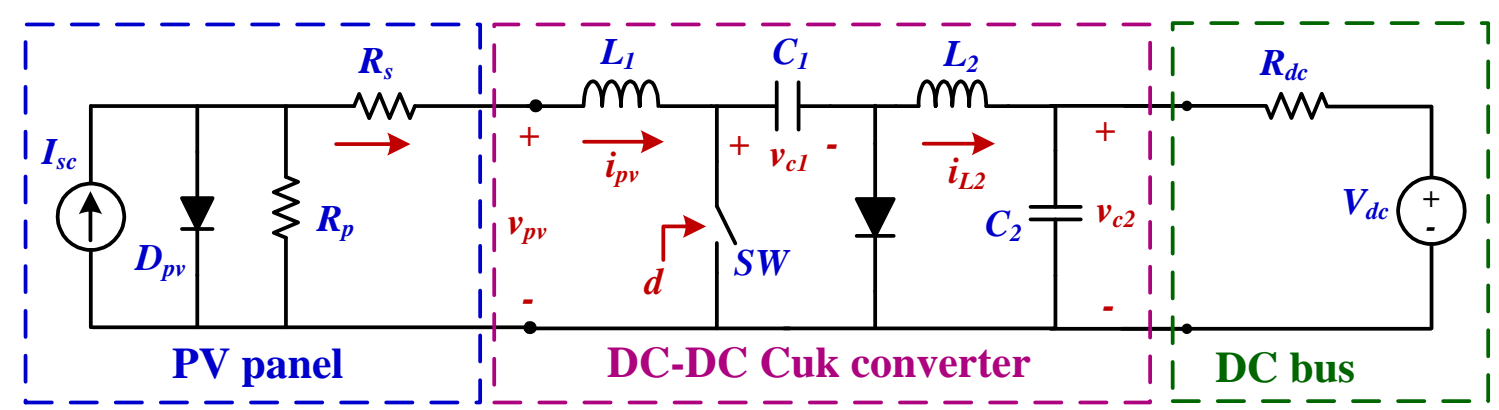

Figure 2.2. Equivalent circuit of a grid-connected PV power system through a DC-DC Cuk converter.

\subsubsection{PV Panel Dynamic Model}

Two advanced equivalent circuit PV models are proposed in the literature: singlediode and double-diode model. The double diode model features high accuracy since it considers the carriers' recombination. The first diode accounts for carriers diffusing across the P-N junction and recombining in the bulk or at surfaces. The second diode is sometimes attributed to carrier recombination by traps within the depletion region, or recombination at an unpassivated cell edge. Even though, this model is rarely used due to its complexity, high computational cost, and inability to be parameterized based solely on data sheet information [103]. Single-diode model is the most commonly used in PV systems' studies, because it offers reasonable tradeoff between simplicity and accuracy. Also, it has the possibility to be parameterized based on the provided information on the manufacture datasheet [104]. A comparative analysis between the performance of the single and double diode models has been conducted in [105]. The single-diode model shows an average absolute error $=0.0085$ and root-mean square $(\mathrm{RMS})$ error $=1.67 \%$. By using the twodiode model, it introduced some singular solutions. After solving the issue by adding more 
complexity to the analysis, it gave an average absolute error $=0.0080$ and $\mathrm{RMS}$ error $=$ $1.35 \%$. Both the errors are slightly reduced compared with the one-diode model results. Also, the study showed that, the reverse saturation current of the second diode is indeed extremely close to zero, whereas other parameters are comparable to their counterparts in the one-diode model. It was concluded that the one-diode model is good enough to represent the whole $I-V$ characteristic accurately. Thus, in this work, the PV panel is modeled based on the single diode equivalent circuit model, as shown in Figure 2.2 and its $I-V$ characteristic is presented in Equation (2-1).

$$
i_{p v}=I_{s c}-I_{r}\left(e^{\frac{v_{p v}+i_{p v} R_{S}}{n V_{T}}}-1\right)-\left(\frac{v_{p v}+i_{p v} R_{S}}{R_{p}}\right) ; \quad V_{T}=\frac{k T}{q}
$$

where, $i_{p v}$ and $v_{p v}$ are the PV output current and voltage, respectively, $I_{s c}$ is the photon (short circuit) current, $q$ is the charge of electron (C), $k$ is Boltzmann constant $\left(\mathrm{kg} \cdot \mathrm{m}^{2} / \mathrm{s}^{2} \mathrm{~K}\right), T$ is cell temperature $(\mathrm{K}), n$ is the diode ideality factor, $R_{S}$ is the series resistance, $R_{p}$ is the parallel resistance, and $I_{r}$ is reverse saturation current.

The series resistance $\left(R_{S}\right)$ stands for the lumped resistive losses in the current path through the semiconductor material, the metal grid, contacts and current collecting bus. The parallel resistance $\left(R_{p}\right)$, represents the losses associated with a small leakage of current through a resistive path in parallel with the intrinsic device. These are due to crystal damage and impurities in and near the junction. Considering $R_{p}$ allows the model to provide a logic behavior under the impact of shading on a string of cells connected in series. The effect of $R_{p}$ on the PV array performance is less conspicuous compared to the series resistance, but it will become noticeable when a number of PV modules are connected in parallel for a larger system. The recombination in the depletion region of PV cells provides non-ohmic 
current paths in parallel with the intrinsic PV cell. This is represented by a single diode with unknown diode ideality factor $(n)$ [106]. This factor $(n)$ ranges from 1 to 2 , where 1 ideality factor means ideal diode behavior.

For the purposes of performing small signal analysis, it is better to express the PV panel output voltage in terms of its current. Thus, by rearranging Equation (2-1), a second formula for the PV panel $I-V$ characteristic is obtained, as indicated in Equation (2-2).

$$
v_{p v}=n V_{T} \ln \left[\frac{I_{r}+I_{s c}-i_{p v}-\left(\frac{v_{p v}+i_{p v} R_{S}}{R_{p}}\right)}{I_{r}}\right]-i_{p v} R_{S}
$$

By applying the open circuit conditions on the PV panel $\left(v_{p v}=V_{o c}\right.$ and $\left.i_{p v}=0\right)$, an expression for reverse saturation current in terms of the panel parameters can be obtained, as in Equation (2-3).

$$
I_{r}=\frac{\left(R_{p} I_{s c}+V_{o c}\right)}{\left[R_{p}\left(e^{\frac{V O C}{n V_{T}}-1}\right)\right]}
$$

where, $V_{o c}$ is the open circuit voltage of the PV module.

By inserting Equation (2-3) into Equation (2-2), a third accurate formula for the $I-V$ characteristic of PV panel is given in Equation (2-4).

$$
v_{p v}=n V_{T} \ln \left[1+\left(e^{\frac{V_{o c}}{n V_{T}}}-1\right)\left(\frac{R_{p}\left(I_{s c}-i_{p v}\right)+i_{p v} R_{S}+v_{p v}}{R_{p} I_{s c}-V_{o c}}\right)\right]-i_{p v} R_{S}
$$

By applying the approximation of $\left(e^{\frac{-V_{o c}}{n V_{T}}} \ll 1\right)$, a simple formula for PV output voltage is obtained in Equation (2-5).

$$
v_{p v}=V_{o c}+n V_{T} \ln \left[\frac{R_{p} I_{s c}-i_{p v}\left(R_{p}-R_{S}\right)+v_{p v}}{R_{p} I_{s c}-V_{o c}}\right]-i_{p v} R_{S}
$$




\subsubsection{DC-DC Converter with DC Bus Model}

DC-DC Cuk converter is selected in this work to boost up the PV panel voltage to the DC link level. It is also controlled to extract the maximum power form the PV panel regardless of the irradiance level and cell temperature. The circuit diagram of Cuk converter is shown in Figure 2.2. It consists of an input inductor $\left(L_{l}\right)$ and capacitor $\left(C_{l}\right)$, power electronic switch $(S W)$ [metal-oxide semiconductor field-effect transistor (MOSFET) or insulated-gate bipolar transistor (IGBT)], which is controlled by the duty cycle $(d)$, power semiconductor diode $\left(D_{c}\right)$, and output filter capacitor $\left(C_{2}\right)$ and inductor $\left(L_{2}\right)$ to smooth the output voltage and current. Under the assumption that the inductor current is always positive, when the switch $S W$ is ON, the diode is OFF and the capacitor $C_{1}$ is discharged by the inductor $L_{2}$ current. When the switch is OFF, the diode conducts the current of the inductors $L_{1}$ and $L_{2}$ whereas capacitor $C_{1}$ is charged by the inductor $L_{1}$ current. The Cuk converter offers several advantages, such as providing capacitive isolation, which protects against switch failure, continuous input current, and almost ripplefree output current, which is important for efficient systems. The passive components $\left(L_{1}\right.$, $L_{2}, C_{1}$ and $C_{2}$ ), are designed based on the switching frequency, and the output voltage and current ripple.

Following the state-space representation theory, the dynamic performance of the Cuk converter and the DC bus is represented by four non-linear ordinary differential equations (ODEs) that describe the inductors' current $\left(i_{L I}=i_{p v}\right.$ and $\left.i_{L 2}\right)$ and capacitors' voltage ( $v_{C l}$ and $v_{C 2}$ ). The final set of nonlinear ODEs are stated in Equation (2-6). 


$$
\begin{aligned}
& p i_{p v}=\frac{1}{L_{1}}\left[v_{p v}-(1-d) v_{c 1}\right] \\
& p v_{C 1}=\frac{1}{C_{1}}\left[d i_{L 2}+(1-d) i_{p v}\right] \\
& p i_{L 2}=\frac{1}{L_{2}}\left(-d v_{C 1}-v_{C 2}\right) \\
& p v_{C 2}=\frac{1}{C_{2}}\left(i_{L 2}-\frac{v_{C 2}}{R_{d c}}+\frac{V_{d c}}{R_{d c}}\right)
\end{aligned}
$$

where, the symbol $p$, represents the differential operator $\left(\frac{d}{d t}\right)$.

Equations (2-2) and (2-6) represent the non-linear state-space dynamic model for the entire PV power system. These equations are implemented and analyzed in MATLAB environment to simulate the PV system performance. Equation (2-6) is analyzed by means of the numerical integration technique (Runge-Kutta) and Equation (2-2) is solved by the iterative technique (Newton-Raphson).

\subsection{Small Signal Analysis of PV Power System}

For proper converter control system design, it is necessary to model the system dynamic behavior. In particular, it is required to determine how the variations in the converter duty cycle affect the PV power and voltage. Unfortunately, understanding converter dynamic behavior is hampered by the nonlinear time-varying nature of the switching and pulse-width modulation process. These difficulties can be overcome through the use of waveform averaging and small-signal modeling techniques [107]. The dynamic model that is presented in section 2.2 is linearized to state the small signal model for the PV power system.

\subsubsection{PV Panel Small Signal Model}

The main function of MPPT in PV system is to keep the PV panel working at the 
maximum power point (MPP) regardless of the irradiance level and temperature. Thus, in the PV systems that contain MPPT, it can be considered that the operating point is the MPP. When the PV panel is operating at MPP, the variation of the operating point around the MPP due to the fast control action $(10-20 \mathrm{kHz})$ can be linearized using the small-signal analysis. This analysis can be performed by assuming small variations in the different dynamics that the PV panel is usually subjected to. The disturbance in PV panel output is mainly due to the variation of temperature, radiation and load conditions. Thus, the small variation in the PV output voltage $\left(\hat{v}_{p v}\right)$ will be affected by the small variation in PV current $\left(\hat{\imath}_{p v}\right)$, radiation level $(\hat{g})$, and cell temperature $(\hat{t})$ [108]. If these conditions slightly change around the operating point, it can be written as: $G=G_{Q}+\hat{g}, T=T_{Q}+\hat{t}$ and $i_{p v}=I_{p v_{Q}}+$ $\hat{\imath}_{p v}$, where, $G, T$ and $i_{p v}$ are the dynamic irradiance level, temperature and PV current, respectively, $G_{Q}, T_{Q}$ and $I_{p v_{Q}}$ are the average of these variables at the quiescent point $(\mathrm{Q})$, and $\hat{g}, \hat{t}$ and $\hat{l}_{p v}$ are their small variations. Thus, the small signal representation of the PV voltage at any operating point (e.g. MPP) is described in Equation (2-7).

$$
\hat{v}_{p v}=K_{i v} \hat{\imath}_{p v}+K_{g v} \hat{g}+K_{t v} \hat{t}
$$

where, $K_{i v}$ is the variation of the PV voltage with respect to the variation of PV current, which represents the slope of $I-V$ characteristic at the operating point; $K_{g v}$ is the variation of the PV voltage with respect to the variation of the irradiance level; and $K_{t v}$ is the variation of the PV voltage with respect to the variation of the cell temperature.

These small signal coefficients $\left(K_{i v}, K_{g v}\right.$ and $\left.K_{t v}\right)$ can be evaluated using an explicit PV voltage equation as function of current, radiation and temperature. Small approximation needs to be applied to Equation (2-5) to put it in an explicit form. This form can be obtained 
by neglecting the shunt resistance effect in Equation (2-5) by assuming very large resistance $\left(R_{p} \approx \infty\right)$. Typically, the PV power systems are operating at MPP due to the MPPT in the system. Thus, the small signal analysis of PV panel is typically applied at the MPP. In [109], the effect of $R_{p}$ on the MPPT control performance is studied for a certain PV module. It shows that at high values of $R_{p}(\geq 601.34 \Omega)$, its effect is negligible. Based on this study, we conduct a study for the effect of $R_{p}$ on the $I-V$ characteristics of the used PV module (BP 4175T PV array) in this work at different climatic conditions, as shown in Figure 2.3. It can be noticed that for high values of $R_{p}(\geq 100 \Omega)$ its impact on the slope at MPP is negligible, even at different climatic conditions. Thus, its effect on the small signal analysis will be unnoticeable. The estimated $R_{p}$ for the used PV module in the work is 2.8 $\mathrm{k} \Omega$, as will be demonstrated in section 2-5, which is much larger than $100 \Omega$ [109].

By applying this approximation on Equation (2-5), an explicit PV model that is appropriate for the small signal analysis is obtained in Equation (2-8).

$$
v_{p v}=V_{o c}+n V_{T} \ln \left[1-\frac{i_{p v}}{I_{s c}}\right]-i_{p v} R_{s}
$$

where, $V_{o c}, I_{s c}$ and $V_{T}$ are function of the cell temperature and irradiance level as described in Equations (2-9), (2-10) and (2-11), respectively.

$$
\begin{gathered}
V_{o c}(T, G)=V_{o c_{o}}\left[1+\beta\left(T-T_{o}\right)\right]+V_{T_{o}} \ln \left(\frac{G}{G_{o}}\right) \\
I_{s c}(T, G)=I_{s c_{o}}\left(\frac{G}{G_{o}}\right)\left[1+\alpha\left(T-T_{o}\right)\right] \\
V_{T}(T)=V_{T_{o}}\left(\frac{T}{T_{o}}\right)
\end{gathered}
$$




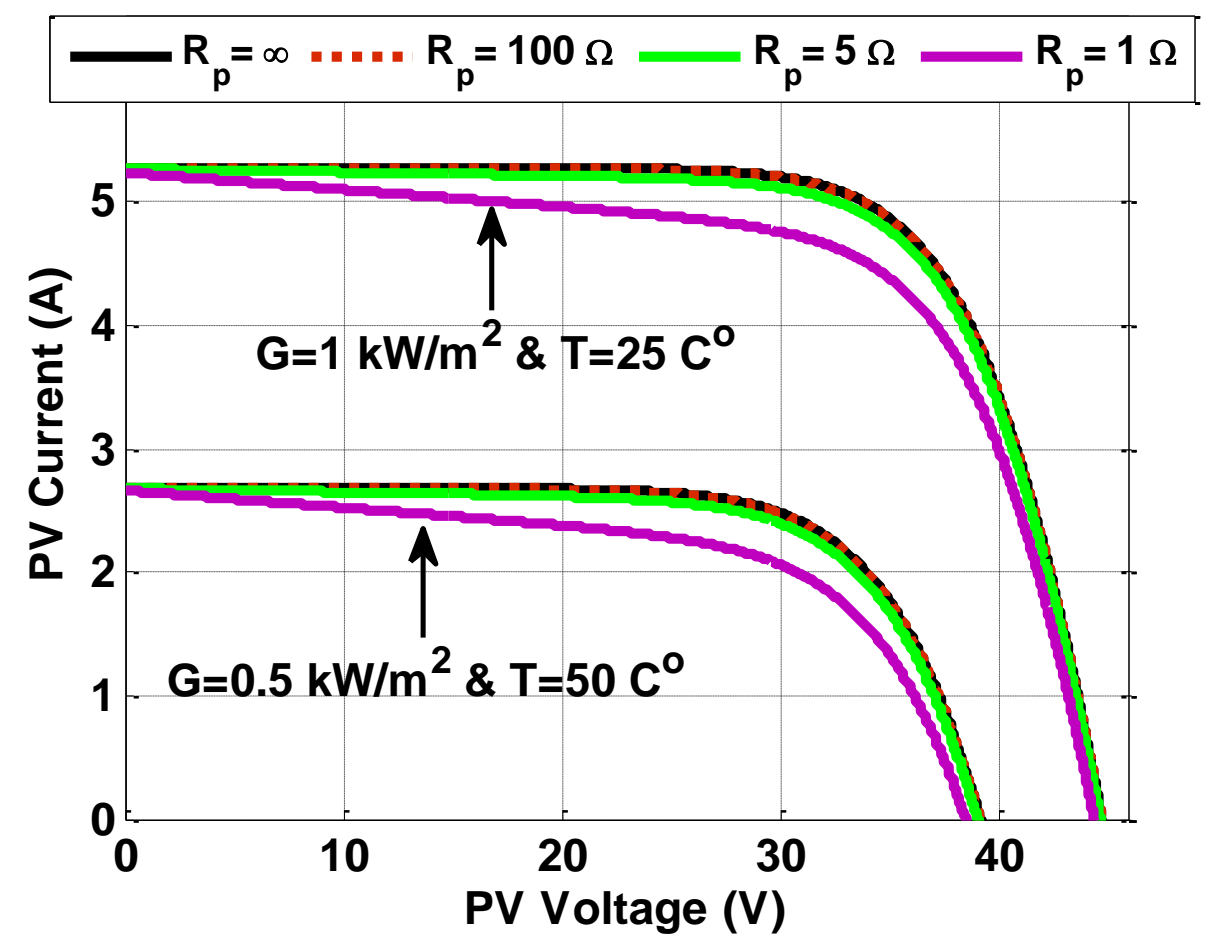

Figure 2.3. Effect of $R_{p}$ on I-V characteristics of PV module at different climatic conditions.

By differentiating Equation (2-8) with respect to the PV current, an expression for the coefficient $K_{i v}$ at Q-point, is obtained in Equation (2-12).

$$
K_{i v_{Q}}=\left.\frac{d v_{p v}}{d i_{p v}}\right|_{Q}=-\frac{n V_{T Q}\left(1-e^{-\frac{V_{o c Q}}{n V_{T Q}}}\right)}{\left[I_{s c_{Q}}-I_{p v_{Q}}\left(1-e^{-\frac{V_{o c}}{n V_{T}}}\right)\right]}
$$

where, the subscript $(\mathrm{Q})$ denotes the variables at the operating point (Q-point).

For the radiation coefficient, $K_{g v}$, Equation (2-8) is differentiated with respect to the radiation level $(G)$ as given in Equation (2-13). 


$$
K_{g v_{Q}}=\left.\frac{d v_{p v}}{d G}\right|_{Q}=\left.\frac{d v_{O C}}{d G}\right|_{Q}+\left.n V_{T_{Q}} \frac{d}{d G}\left[\ln \left(1-\frac{i_{p v}}{I_{s c}}\right)\right]\right|_{Q}-\left.R_{S} \cdot \frac{d i_{p v}}{d G}\right|_{Q}
$$

Using Equations (2-9), (2-10) and (2-11), with the assumption of, $\left.\left.\frac{d i_{p v}}{d G}\right|_{Q} \approx \frac{d i_{s c}}{d G}\right|_{Q}$ at MPP, the final formula for the radiation coefficient at Q-point is given in Equation (2-14). Similarly, the temperature coefficient at Q-point, $K_{t v}$, is presented in Equation (2-15).

$$
\begin{gathered}
K_{g v_{Q}}=\left.\frac{d v_{p v}}{d G}\right|_{Q}=\frac{V_{T_{o}}}{G_{Q}}-\left(\frac{I_{s c_{o}}}{G_{o}}\right)\left[1+\alpha\left(T_{Q}-T_{o}\right)\right]\left(\frac{n V_{T_{Q}}}{I_{s c_{Q}}}-R_{s}\right) \\
K_{t v_{Q}}=\left.\frac{d v_{p v}}{d T}\right|_{Q}=\beta V_{o c_{O}}-\left(\frac{\alpha G_{Q} I_{s c_{o}}}{G_{o}}\right)\left(R_{S}+\frac{n V_{T_{Q}}}{I_{s c_{Q}}}\right)+\frac{n k}{q} \ln \left(1-\frac{I_{p v_{Q}}}{I_{s c_{Q}}}\right)
\end{gathered}
$$

where, the subscript (o) distinguishes the variables at standard test conditions (STC), which are available in the PV module manufacture datasheet.

\subsubsection{DC-DC Converter and DC Bus Small Signal Model}

The DC-DC converter and DC bus are modeled based on the state-space averaging technique in [107]. Each variable in Equation (2-6) is replaced by the superposition of the average value and the variations; $f=F_{Q}+\hat{f}$, where $f=\left\{i_{p v}, v_{p v}, v_{c 1}, i_{L 2}, v_{c 2}, D\right\}, F_{Q}$ is the average value of the variable $f$ at the Q-point and $\hat{f}$ is the variation of $f$ around the Qpoint. For example, $i_{p v}=I_{p v_{Q}}+\hat{\imath}_{p v}$. In this analysis, the DC components and the secondorder small variation terms are neglected. Thus, final small signal representation of the DCDC Cuk converter with the DC bus is given in Equation (2-16). 


$$
\begin{aligned}
& p \hat{\imath}_{p v}=\frac{1}{L_{1}}\left[\hat{v}_{p v}-\left(D_{Q}-1\right) \hat{v}_{c 1}+V_{c 1} \hat{d}\right] \\
& p \hat{v}_{C 1}=\frac{1}{C_{1}}\left[D_{Q} \hat{\imath}_{L 2}+\left(1-D_{Q}\right) \hat{l}_{p v}+\left(I_{L 2}-I_{p v_{Q}}\right) \hat{d}\right] \\
& p \hat{\imath}_{L 2}=\frac{1}{L_{2}}\left(-D_{Q} \hat{v}_{C 1}-\hat{v}_{C 2}-V_{c 1} \hat{d}\right) \\
& p \hat{v}_{C 2}=\frac{1}{C_{2}}\left(\hat{\imath}_{L 2}-\frac{\hat{v}_{C 2}}{R_{d c}}\right)
\end{aligned}
$$

By mixing the PV array in Equations (2-7), (2-12), (2-14), and (2-15), with the Cuk converter and the DC bus models in Equation (2-16), the final small signal model for gridgrid connected PV system is obtained. Based on these equations, the state-space representation for the system is given Equation (2-17).

$$
\dot{\mathrm{x}}=\mathrm{Ax}+\mathrm{Bu} ; \quad \mathrm{y}=\mathrm{C} \mathrm{x}+\mathrm{Du}
$$

where, $\mathbf{x}=\left[\begin{array}{llll}\hat{\imath}_{p v} & \hat{v}_{C 1} & \hat{\imath}_{L 2} & \hat{v}_{C 2}\end{array}\right]^{T} ; \mathbf{u}=\left[\begin{array}{lll}\hat{d} & \hat{g} & \hat{t}\end{array}\right]^{T} ;$ and $\mathbf{y}=\left[\begin{array}{ll}\hat{\imath}_{p v} & \hat{v}_{p v}\end{array}\right]^{T}$.

$$
\begin{array}{cc}
\mathbf{A}=\left[\begin{array}{cccc}
\frac{K_{i v}}{L_{1}} & \frac{D_{Q}-1}{L_{1}} & 0 & 0 \\
\frac{1-D_{Q}}{C_{1}} & 0 & \frac{D_{Q}}{C_{1}} & 0 \\
0 & \frac{-D_{Q}}{L_{2}} & 0 & \frac{-1}{L_{2}} \\
0 & 0 & \frac{1}{C_{2}} & \frac{-1}{R_{d c} C_{2}}
\end{array}\right] ; & \mathbf{B}=\left[\begin{array}{ccc}
\frac{V_{c 1 Q}}{L_{1}} & \frac{K_{g v}}{L_{1}} & \frac{K_{t v}}{L_{1}} \\
\frac{I_{L 2}-I_{p v}}{C_{1}} & 0 & 0 \\
\frac{-V_{c 1}}{L_{2}} & 0 & 0 \\
0 & 0 & 0
\end{array}\right] \\
\mathbf{C}=\left[\begin{array}{cccccc}
1 & 0 & 0 & 0 & 0 & 0 \\
K_{i v} & 0 & 0 & 0 & 0 & 0
\end{array}\right] ; \quad \mathbf{D}=\left[\begin{array}{ccc}
0 & 0 & 0 \\
0 & K_{g v} & K_{t v}
\end{array}\right]
\end{array}
$$

\subsection{Small Signal Perturbation Analysis}

The maximum power point tracker in PV system is responsible for adjusting the duty cycle of the DC-DC converter to force the operating point to match with the MPP. Thus, the main effect of the controller in MPPT is applying a small variation in duty cycle. Thus, by considering fixed temperature and irradiance level, the effect of the small variation in 
duty cycle on the PV output (voltage, current and power) at steady-state can be investigated by following the state-space averaging technique in [107]. By considering the variation in duty cycle only as an input in Equation (2-17), the state-space model can be rewritten as in Equation (2-18).

$$
\begin{gathered}
\dot{\mathbf{x}}=\grave{A} \mathbf{x}+\grave{B} \hat{d} \\
\text { where, } \quad \grave{\boldsymbol{A}}=\left[\begin{array}{cccc}
\frac{K_{i v}}{L_{1}} & \frac{D_{Q}-1}{L_{1}} & 0 & 0 \\
\frac{1-D_{Q}}{C_{1}} & 0 & \frac{D_{Q}}{C_{1}} & 0 \\
0 & \frac{-D_{Q}}{L_{2}} & 0 & \frac{-1}{L_{2}} \\
0 & 0 & \frac{1}{C_{2}} & \frac{-1}{R_{d c} C_{2}}
\end{array}\right] ; \quad \grave{\boldsymbol{B}}=\left[\begin{array}{c}
\frac{V_{c 1 Q}}{L_{1}} \\
\frac{I_{L 2}-I_{p v}}{C_{1}} \\
\frac{-V_{c 1 Q}}{L_{2}} \\
0
\end{array}\right]
\end{gathered}
$$

By applying the averaging technique in the model in Equation (2-18), the average small variation in state-space variables can be evaluated as in Equation (2-19).

$$
\mathbf{x}=-\boldsymbol{A}^{-1} \mathbf{B} \hat{d}
$$

By solving Equation (2-19), the average small variation of the PV current $\left\langle\hat{\imath}_{p v}\right\rangle$ with respect to the perturbation in the duty cycle is stated in Equation (2-20).

$$
\left\langle\hat{\imath}_{p v}\right\rangle=I_{f}\langle\hat{d}\rangle
$$

where, $I_{f}=-\frac{D_{Q} V_{c 1}+R_{d c}\left(1-D_{Q}\right)\left(I_{p v_{Q}}-I_{L 2 Q}\right)}{K_{i v} D_{Q}{ }^{2}-R_{d c}\left(1-D_{Q}\right)^{2}}, D_{Q}, V_{c 1_{Q}}, I_{L 2_{Q}}$ and $I_{p v_{Q}}$ are the steady-state values of the duty-cycle, capacitor $\left(C_{1}\right)$ voltage, inductor $\left(L_{2}\right)$ current and PV current at the MPP, respectively.

The average PV voltage perturbation is obtained by combining Equations (2-7) and (2-20), as in Equation (2-21). 


$$
\left\langle\hat{v}_{p v}\right\rangle=K_{i v} I_{f}\langle\hat{d}\rangle
$$

By applying the small-signal concept and neglecting the second order variation terms, the PV power small variation $\left\langle\hat{p}_{p v}\right\rangle$ is deduced in Equation (2-22) [110].

$$
\left\langle\hat{p}_{p v}\right\rangle=\left\langle\hat{v}_{p v}\right\rangle I_{p v_{Q}}+V_{p v_{Q}}\left\langle\hat{l}_{p v}\right\rangle
$$

Thus, the perturbation in the PV power with respect to the perturbation in the duty cycle is obtained by inserting Equations (2-20) and (2-21) into Equation (2-22), as given in Equation (2-23).

$$
\left\langle\hat{p}_{p v}\right\rangle=\left(K_{i v} I_{p v_{Q}}+V_{p v_{Q}}\right) I_{f}\langle\hat{d}\rangle
$$

Equations (2-20), (2-21) and (2-23) represent the key design formulas that can be used to choose the proper perturbation size in the classical perturb and observe (P\&O) algorithm. Equation (2-21) is useful when the control is based on the voltage observation, while Equation (2-20) is used for the current observation. These formulas are utilized in this work to achieve the proper design for MPPT algorithm based on fuzzy logic control, as will be discussed in detail in the next chapter.

\subsection{PV Panel Parameters' Estimation}

In order to solve the equivalent circuit model of the PV panel, the model parameters need to be known. The single diode model has four parameters that need to be estimated to match with the practical $I-V$ characteristics [111]. These parameters are the series resistance $\left(R_{s}\right)$, parallel resistance $\left(R_{p}\right)$, diode ideality factor $(n)$, and reverse saturation current $\left(I_{r}\right)$. In this work, two different parameter estimation techniques are used and compared to extract 
the PV module parameters. The first one is based on mathematical equations and the measurements of the $I-V$ characteristic's slope at open-circuit voltage and short-circuit current [112]. The second technique is performed by using GA optimization procedure. The details of these methodologies and results are discussed in this section.

\subsubsection{Analytical Parameter Estimation}

This methodology is mainly based on measuring the slope of the $I-V$ characteristic of the PV module (given in the datasheet) and some analytical formulas. The first parameter $\left(I_{r}\right)$ is estimated at STC by neglecting the parallel resistance effect in Equation (2-3), as given in Equation (2-24). Since, $I_{r}$, is a temperature-dependent parameter, its value at a given temperature $(T)$ is updated using Equation (2-25) [113].

$$
\begin{gathered}
I_{r_{o}}=\frac{I_{S c_{O}}}{\left(e^{\left.V_{O c_{O}} / n V_{T-1}\right)}\right.} \\
\left.I_{r}\right|_{T}=\left.I_{r}\right|_{T_{o}} \cdot\left(\frac{T}{T_{o}}\right)^{\frac{3}{n}} \cdot e^{\frac{-E g}{n}\left(V_{T}-V_{T_{o}}\right)}
\end{gathered}
$$

where, $E_{g}$ is the band gap energy; it is the energy that an electron must acquire to jump across the forbidden band to the conduction band, and it is equal $1.12 \mathrm{eV}$ for Silicon.

Until this point, the diode ideality factor is unknown and must be estimated. Ideal diode $(n=1)$ is assumed in this stage until a more accurate value is estimated later by trial and error. The series resistance of the PV module has a large impact on the slope of the $I$ $V$ curves near the open-circuit voltage. Hence, the value of $R_{s}$ is calculated by evaluating the slope $\left(\frac{d V}{d I}\right)$ of the $I$ - $V$ curve at $V_{o c}$, as indicated in Equation (2-26) [114]. 


$$
R_{S}=-\left.\frac{d V}{d I}\right|_{V_{O C}}-\left(\frac{n V_{T}}{I_{r} e^{\left(\frac{V_{O C}}{n V_{T}}\right)}}\right)
$$

The parallel resistance of the PV module has a large impact on the slope of the $I-V$ curves near the short circuit current. Thus, the value of $R_{p}$ is calculated by evaluating the

slope $\left(\frac{d V}{d I}\right)$ of the $I-V$ curve published on the datasheet at $I_{s c}$ and substituting in Equation (2-27) [114]. The last parameter is the diode ideality factor, which is estimated by trail and error such that its value attains the best match with the $I-V$ curves on the datasheet.

$$
R_{p}=\frac{-\left(\left.\frac{d V}{d I}\right|_{I_{S C}}+R_{S}\right)}{1+\frac{I_{r}}{n V_{T}} \cdot\left(\left.\frac{d V}{d I}\right|_{I_{S C}}+R_{S}\right) \cdot e^{\left(\frac{R_{S} \cdot I_{S C}}{n V}\right)}}
$$

\subsubsection{GA-Based Parameters Estimation}

GA is a stochastic search algorithm that emulates biological evolutionary theories to solve optimization problems. It enables parallel search from a population of points. Based on the literature, GA shows massive success in estimating the system parameters in many different applications due to the following reasons [115], [116]:

- It enables parallel search from a population of points. Therefore, it has the ability to avoid being trapped in a local optimal solution, unlike traditional methods, which search from a single point.

- It supports probabilistic selection rules, not deterministic ones.

- It behaves well in the case of noisy or stochastic objective function.

- It does not require explicit definitions.

- It is very efficient in case of cheap cost function (which is our case). 
Therefore, GA has been used in this study to extract the best combination of the parameters such that the error between the measured (from the datasheet) and the simulated $I-V$ characteristics is minimized.

\subsubsection{Parameters Estimation Results}

The two techniques of parameters estimation are utilized to extract the parameters of the commercial BP 4175T PV module [117]. The electrical characteristics and specifications of this module at STC $\left[G_{o}=1000 \mathrm{~W} / \mathrm{m}^{2}\right.$ at the air mass $($ A.M $)=1.5$ and $T_{o}=$ $\left.25^{\circ} \mathrm{C}\right]$ are given in Table 2.1 .

Table 2.1. BP 4175T PV Module Specifications at STC.

\begin{tabular}{ll}
\hline \hline Electrical characteristic & Value \\
\hline Open circuit voltage $\left(V_{o c}\right)$ & $43.6 \mathrm{~V}$ \\
Short circuit current $\left(I_{S c}\right)$ & $5.45 \mathrm{~A}$ \\
Voltage at maximum power $\left(V_{m p @ S T C}\right)$ & $35.4 \mathrm{~V}$ \\
Current at maximum power $\left(I_{m p} @ S T C\right)$ & $4.94 \mathrm{~A}$ \\
Maximum power $\left(P_{\max } @ S T C\right)$ & $175 \mathrm{~W}$ \\
Temperature coefficient of $I_{s c}$ & $(0.065 \pm 0.015) \% /{ }^{\circ} \mathrm{C}$ \\
Temperature coefficient of $V_{o c}$ & $-(0.5 \pm 0.05) \% /{ }^{\circ} \mathrm{C}$ \\
Module efficiency & $14 \%$ \\
\hline
\end{tabular}

The two techniques are implemented in MATLAB environment and applied for BP 4175T PV module. The final extracted parameters from each technique are presented and 
compared in Table 2.2. Moreover, the $I-V$ characteristic of PV module is plotted at STC using the parameters estimated by each technique and compared with the measured $I-V$ characteristic in Figure 2.4. It can be observed that GA technique shows more accurate results than the analytical one. GA-based parameters give $4.77 \%$ of current error, while the analytical technique presents $6.45 \%$. The parameters that provide the least error (GAbased) are considered in this work to model the performance of the PV module.

Table 2.2. Extracted Parameters of BP 4175T PV Module.

\begin{tabular}{llllll}
\hline Method & $\boldsymbol{R}_{\boldsymbol{s}}(\mathbf{\Omega})$ & $\boldsymbol{R}_{\boldsymbol{p}}(\boldsymbol{\Omega})$ & $\boldsymbol{n}$ & $\boldsymbol{I}_{\boldsymbol{r}}(\mathbf{A})$ & Current Error $\mathbf{\%})$ \\
\hline Analytical & 0.0068 & 1000.8 & 1.6 & $1.45^{*} 10^{-6}$ & 6.45 \\
GA & 0.00657 & $2.8 * 10^{3}$ & 1.51 & $7.0685^{*} 10^{-7}$ & 4.77 \\
\hline
\end{tabular}

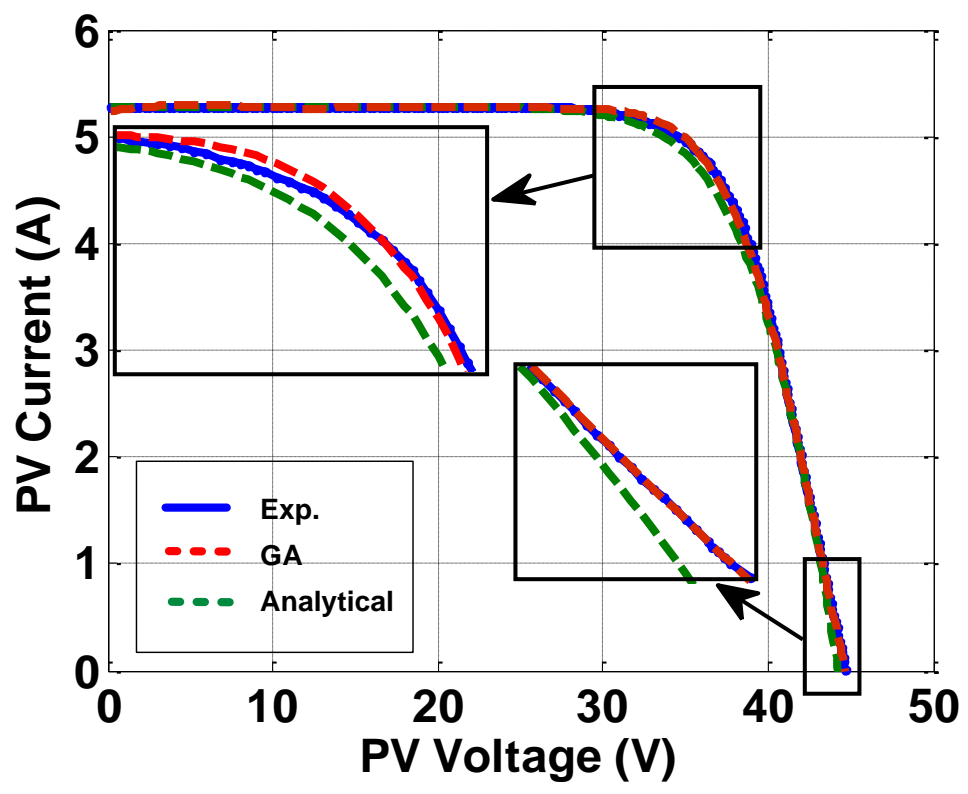

Figure 2.4. Comparison between I-V characteristic for BP 4175T PV module at STC from measurement and simulated using GA and analytical based parameters. 


\subsection{Conclusion}

In this chapter detailed dynamic model for grid-connected PV power system is presented. Four ODEs are developed to represent the entire system's dynamics including a PV generator, DC-DC Cuk converter and DC bus. Moreover, the dynamic model is linearized to develop a small-signal model for the same system. The small-signal model is utilized to investigate the effect of duty-cycle perturbation on the performance of the PV generator in terms of power, current and voltage. Final design formula for MPPT algorithm parameters are derived, which will be utilized to design the proposed MPPT algorithm in the next chapter. 


\section{Chapter 3 Design and Implementation of Fuzzy MPPT for PV Power System}

\subsection{Introduction}

When a PV array is directly connected to a load, the operating point will be at the intersection of their I-V curves. This point may be anywhere on the curve, and it is seldom to be at MPP of PV array, thus the PV array will not be able to produce its maximum power. Even if the system was designed to match the operating point with MPP, this operation is not guaranteed since the MPP changes as the module temperature and the solar irradiance change. This mismatch in the maximum power causes further over-sizing of the PV array and the whole system as a result. To mitigate this problem, a MPPT must be added to the system to follow up the MPP of PV panel regardless of changing climatic conditions and/or loads.

MPPT consists of hardware (DC-DC converter) and software (MPPT algorithm) parts. Several MPPT algorithms were presented, experimentally tested and compared in the literature [118], [119]. The most widely used in PV power applications is the Perturb and Observe $(\mathrm{P} \& \mathrm{O})$ algorithm, due to the simplicity, ease of implementation, and not requiring previous knowledge for the system characteristics [120]. In this technique, the perturbation action can be applied directly [120], [121] or indirectly [122]. The direct perturbation algorithm is preferred, as it does not require PI controller, and provides higher energy utilization efficiency and less noise and oscillations [123]. The main drawbacks of $\mathrm{P} \& \mathrm{O}$ algorithm include its failure under fast variation in climatic conditions and the steadystate swinging around the MPP [124]. Some solutions were presented in literature to deal with these issues. Variable perturbation size or adaptive techniques are proposed to 
enhance the tracking response and minimize the steady-state mismatch [125]-[127]. Most of these techniques are based on analytical models and need deep knowledge about the system. Other nonlinear tracking techniques based on fuzzy logic (FL) are presented in [128]-[131]. These techniques suffer from complexity due to the lack of the design strategy, which is mostly done by experience.

This work presents an optimum design for fuzzy-based MPPT controller to solve the classical P\&O drawbacks. Two different design methodology are considered in this work: analytical method and GA-based methods. In both techniques, the small signal analysis that was presented in the previous chapter are utilized during the design. The proposed design was implemented and tested by means of simulation and experimentally. The system performance was studied at different environmental conditions and compared with the existed techniques in the literature.

\subsection{MPPT Control Algorithm}

MPPT algorithm is the software part of the power conditioning component, which is responsible for calculating the converter duty-cycle to control the PV voltage and current. The main objective of MPPT algorithm is to extract the maximum power from the PV panel regardless of the fluctuations of environmental and/or load conditions. The entire system block diagram showing the MPPT control is depicted in Figure 3.1. The controller measures the output voltage and current of the PV panel, which are converted to digital signals using analogue to digital converters (ADCs). The digital measurements are passed to the MPPT algorithm to estimate the converter duty cycle that achieves the maximum 
power operation. The duty cycle is translated to switching signal using a pulse-width modulation (PWM) generator. The output of the PWM generator is applied to the driver circuit. The driver circuit boosts the switching signal to the voltage and power level to drive the semiconductor switch of the DC-DC converter.

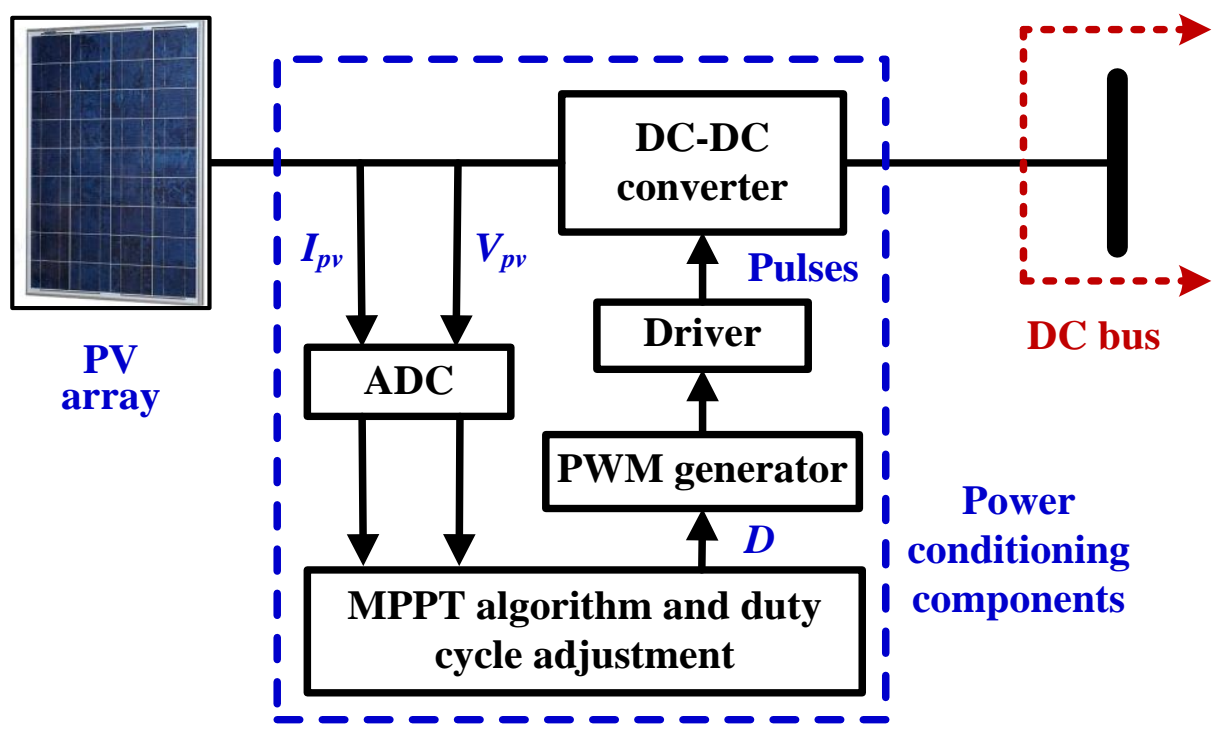

Figure 3.1. Block diagram of grid-connected PV power system including MPPT control.

\subsubsection{Classical Direct P\&O MPPT Algorithm}

In the direct $\mathrm{P} \& \mathrm{O}$ method, two measurements $\left(v_{p v}\right.$ and $\left.i_{p v}\right)$ are required to calculate the PV power $\left(p_{p v}\right)$. The duty cycle is perturbed with a fixed step size $\left(D_{\text {step }}\right)$ and the variation of power $\left(\Delta p_{p v}\right)$ and voltage or current $\left(\Delta v_{p v}\right.$ or $\left.\Delta i_{p v}\right)$ are observed. If the power increases, the algorithm continues to perturb the system in the same direction; otherwise the system is perturbed in the opposite direction until it reaches the MPP. The voltage or current observation are utilized to define the direction of the power increase. The classical 
direct $\mathrm{P} \& \mathrm{O}$ MPPT algorithm is indicated in Figure 3.2. At the MPP, the algorithm cannot stay at that point but keeps swinging around it, which causes steady-state error. Moreover, under the fast fluctuation of climatic conditions, the algorithm fails to track the MPP, since it cannot identify the reason of the power variation, either it is due to the normal control action or the radiation variation. Thus, wrong actions are taken in this situation and the tracking will be lost. This represents a serious problem, especially in inclement weather conditions [120].

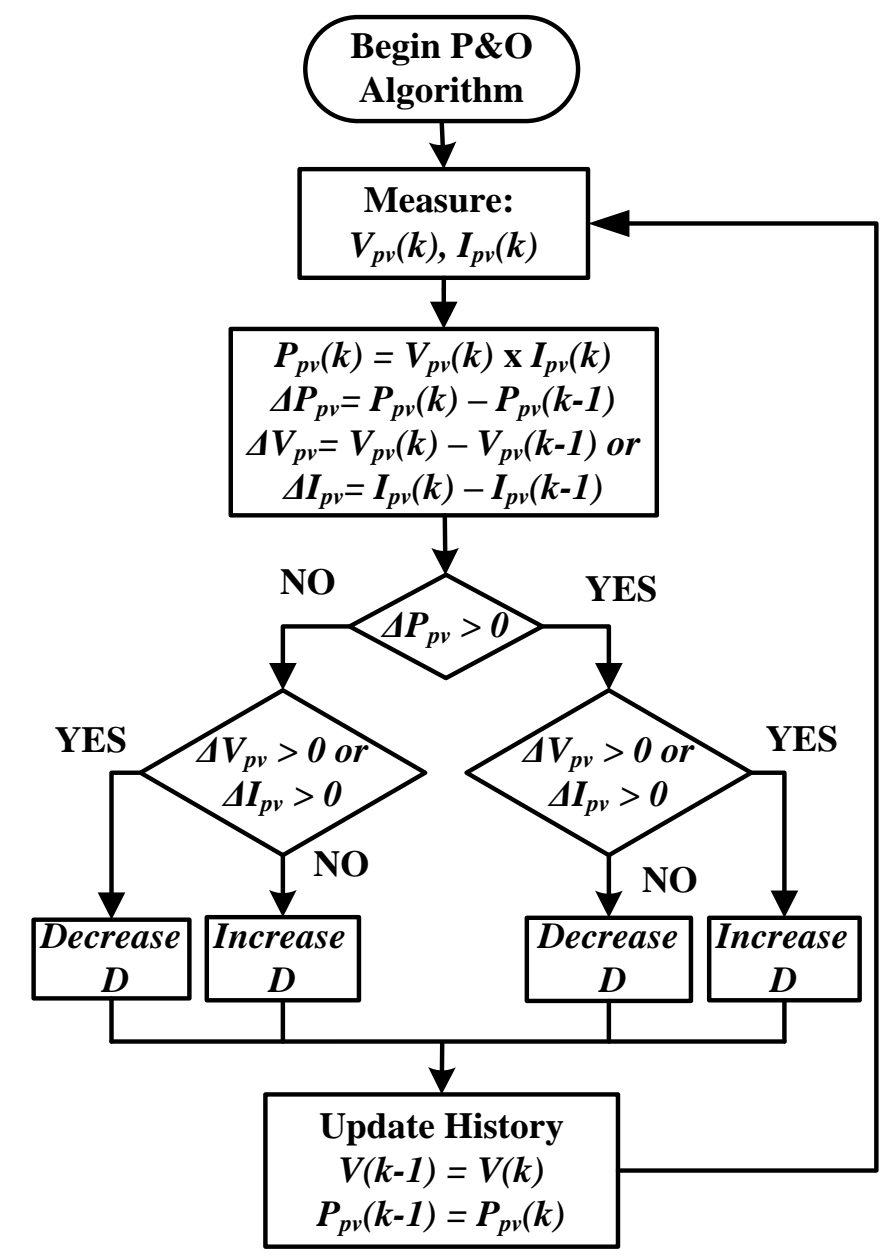

Figure 3.2. Flow-chart of the classical direct P\&O MPPT algorithm. 


\subsubsection{Proposed Fuzzy Direct MPPT Algorithm}

An effective solution for mitigating the problems of the classical $\mathrm{P} \& \mathrm{O}$ algorithm is to apply the nonlinear fuzzy logic for successful tracking [123]. The FL-based MPPT provides an adaptive variation of the duty-cycle based on the location of the operating point with respect to the MPP. This helps to minimize the steady-state error and the malfunction problem during the fast variations of climatic conditions. The main limitation of the fuzzy logic control (FLC) is the requirement of high-speed microprocessor ability with large memory size [123]. In addition, the design of FLC system parameters shows tradeoff between complexity and accuracy [132]. The contributions in this work appear in the design strategy of the fuzzy inference system (FIS).

The proposed design aims to deal with the abovementioned drawbacks of the classical MPPT algorithm without losing the simplicity of the control. The proposed FIS is indicated in Figure 3.3. It consists of two inputs $\left(\Delta p_{p v}\right.$ and $\left.\Delta v_{p v}\right)$ and one output $\left(D_{s t e p}\right)$. The universe of discourse of each input is described by three membership functions [Negative (N), Zero $(\mathrm{Z})$ and Positive $(\mathrm{P})$ ]. The proposed FIS design outlines to achieve both simplicity and efficiency are summarized as follows:

- A minimum number of membership functions for each input variable was considered (three functions; $\mathrm{N}, \mathrm{Z}$, and $\mathrm{P}$ ).

- Linear shapes of membership functions were used (Triangle and Trapezoidal).

- The linear Z and $\mathrm{S}$ shapes were assigned to $\mathrm{N}$ (red) and $\mathrm{P}$ (blue) membership functions, respectively, to define the limits of the universe of discourse.

- A trapezoidal function was allocated to Z-membership function (green), which is responsible for minimizing the swinging around MPP and improving the steady-state 
tracking performance. Using the trapezoidal shape increases the region at which Zmembership function equals unity around the MPP. Thus, the controller will not take action in this region, which is desirable since the system is working already at the MPP.

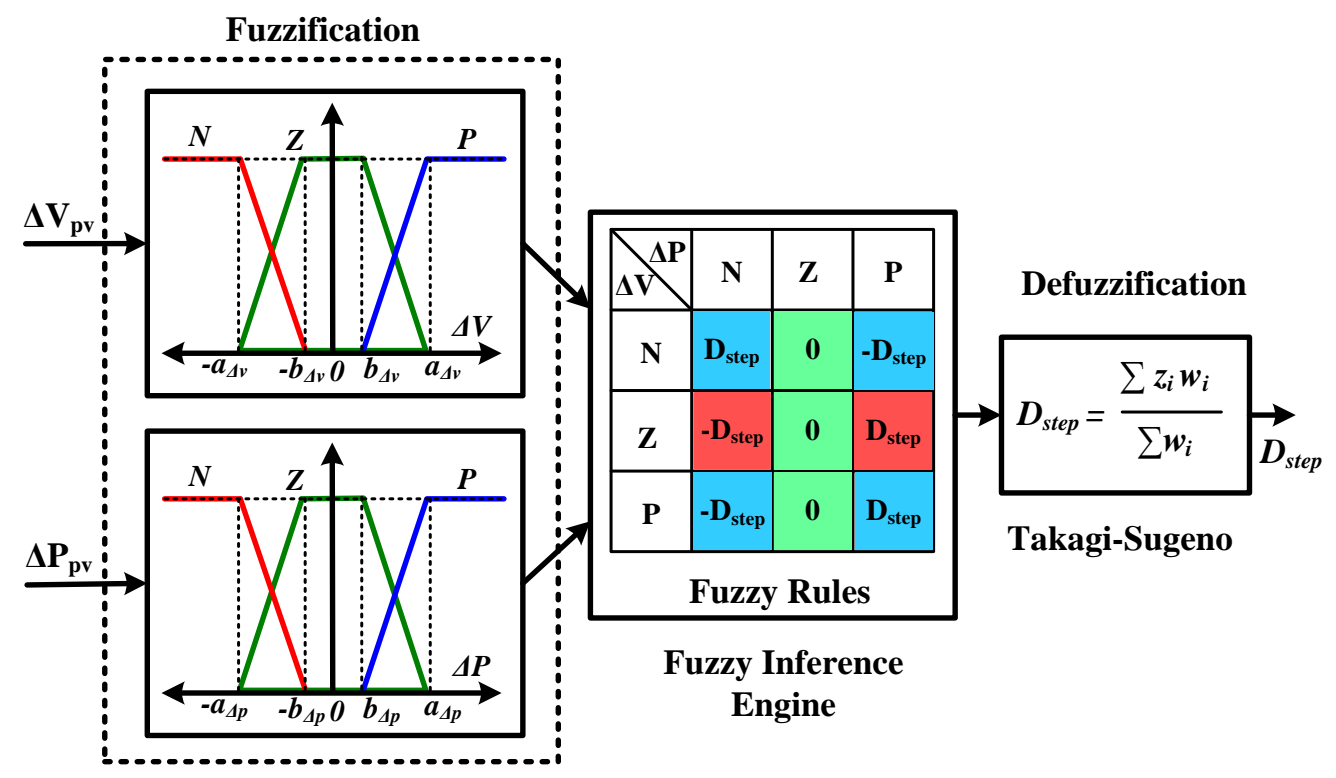

Figure 3.3. Block diagram of the proposed fuzzy logic system tracking algorithm.

- The fuzzy rules were set to achieve the tracking in all possible modes of operation as follows:

1. The blue rules are set to achieve the tracking through the $I-V$ characteristic of the PV panel during the starting and variation of the load conditions, while the climatic conditions are fixed.

2. The red rules are designed for tracking during the variation of climatic conditions (radiation and temperature). 
3. The green rules are for keeping the system working at the MPP when there are no variations.

- Based on the inputs and the rules table, the output of the fuzzy system was calculated by the Takagi-Sugeno (T-S) formula, which requires the least computation effort among all other FIS [133].

- Moreover, in order to keep the control efficient enough, the locations and the boundaries of the input membership functions and T-S function coefficients are assigned using the developed small-signal model and GA optimization procedure. The details of the parameters' generation procedure are presented in the next section.

\subsection{Design Methodologies of Fuzzy-MPPT Controller}

One of the major drawbacks of FLC is the lack of the design strategies. Most of fuzzy system parameters are determined intuitively based on the designer and/or the operator experience [134]. In this section, general design considerations for any MPPT controller are deduced. In our particular case, the Cuk converter is used, however, these considerations can be extended to any other converter while the dynamic model is developed and linearized, as was presented in chapter (2). For the proposed fuzzy-MPPT controller, the most effective parameters on the tracking performance are the locations and the boundaries of the input membership functions $\left(a_{\Delta v}, b_{\Delta v}, a_{\Delta p}\right.$ and $\left.b_{\Delta p}\right)$, and the coefficient of T-S function $\left(D_{\text {step }}\right)$. Thus, for accurate design parameters, two different approaches are proposed to choose these parameters. The first one is pure analytical based on the small-signal model presented in chapter (2). The other one depends on the same the small-signal model with a combination of a stochastic searching technique based on GA. 


\subsubsection{Analytical Fuzzy Logic Design Strategy}

This design technique is based on formulas in Equations (2-21) and (2-23), in which the FL design parameters $\left(a_{\Delta v}, b_{\Delta v}, a_{\Delta p}, b_{\Delta p}\right.$ and $\left.D_{s t e p}\right)$, are estimated as following:

- Assume maximum $\left(\Delta P_{\max }\right)$ and minimum $\left(\Delta P_{\min }\right) \mathrm{PV}$ power perturbation such that, $a_{\Delta p}=\Delta P_{\max }$ and $b_{\Delta p}=\Delta P_{\text {min }}$

- Evaluate the corresponding variation of duty cycle $D_{s t e p_{\max }}$ and $D_{s t e p_{\text {min }}}$, respectively based on Equation (2-23).

- Assign, $D_{\text {step }}=D_{\text {step }}$ min , since the smaller step size provides more accuracy.

- Estimate the maximum $\left(\Delta V_{\max }\right)$ and the minimum $\left(\Delta V_{\min }\right)$ voltage perturbation using Equation (2-21).

- Assign, $a_{\Delta v}=\Delta V_{\max }$ and $b_{\Delta v}=\Delta V_{\min }$.

Based on the intuitive assumption that, $\Delta P_{\max }=0.1 \mathrm{~W}$ and $\Delta P_{\min }=0.02 \mathrm{~W}$, the design parameters are estimated and presented in Table 3.1.

Table 3.1. Results of Design Parameters from Analytical Design Method.

\begin{tabular}{cc|cc}
\hline \hline Parameter & Value & Parameter & Value \\
\hline $\boldsymbol{D}_{\text {step }}$ & 0.0015 & $\boldsymbol{a}_{\Delta P}$ & 0.1 \\
$\boldsymbol{a}_{\Delta \boldsymbol{V}}$ & 0.01 & $\boldsymbol{b}_{\Delta P}$ & 0.02 \\
$\boldsymbol{b}_{\Delta \boldsymbol{V}}$ & 0.002 & & \\
\hline \hline
\end{tabular}




\subsubsection{Genetic Algorithm-Based Design Optimization}

The second proposed strategy for generating the FL design parameters is based on combination between analytical and artificial intelligent techniques. GA is used in this work to optimize the proposed FL system design parameters and reduce time consumption comparatively to the trial and error method. GA is a random search methodology based on population genetics, and is used for optimization purposes. Based on the literature, using GA for tuning FL system parameters exhibited huge success in many different applications [115], [116]. Such an optimal FLC could provide ideal control performance and achieve the desired MPPT performance. GA was used to select the optimum combination of the FL system design parameters $\left(a_{\Delta v}, b_{\Delta v}, a_{\Delta p}, b_{\Delta p}\right.$ and $\left.D_{s t e p}\right)$ based on the optimization procedure described in Figure 3.4.

GA generates an initial random population within the given ranges of variables. Then, it calls and runs the PV power dynamic simulation to calculate the integral absolute PV power error, as given in Equation (3-1), which represents the fitness function $(F)$. The optimization constraints are defined in Equation (3-2).

$$
\begin{gathered}
F=\text { Min. }\left\{\int_{t_{o}}^{t_{f}}\left|p_{p v_{-} t h}(t)-p_{p v_{-} a c t}(t)\right| d t\right\} \\
\text { Constraints }=\left\{\begin{array}{l}
a_{\Delta v_{\text {min }}}<a_{\Delta v}<a_{\Delta v_{\text {max }}}<b_{\Delta v}<b_{\Delta v_{\text {max }}} \\
b_{\Delta v_{\text {min }}}<a_{\Delta p}<a_{\Delta p_{\text {max }}} \\
a_{\Delta p_{\text {min }}}<b_{\Delta p}<b_{\Delta p_{\text {max }}} \\
b_{\Delta p_{\text {min }}}<b_{\Delta t e p}<D_{\text {step }} \text { max } \\
D_{\text {step }}<D_{\text {step }}
\end{array}\right.
\end{gathered}
$$

where, $p_{p v_{-} t h}$ is the theoretical maximum power (MP) that can be extracted at certain irradiance, and $p_{p v_{-} a c t}$ is the actual PV power extracted by the proposed MPPT. 


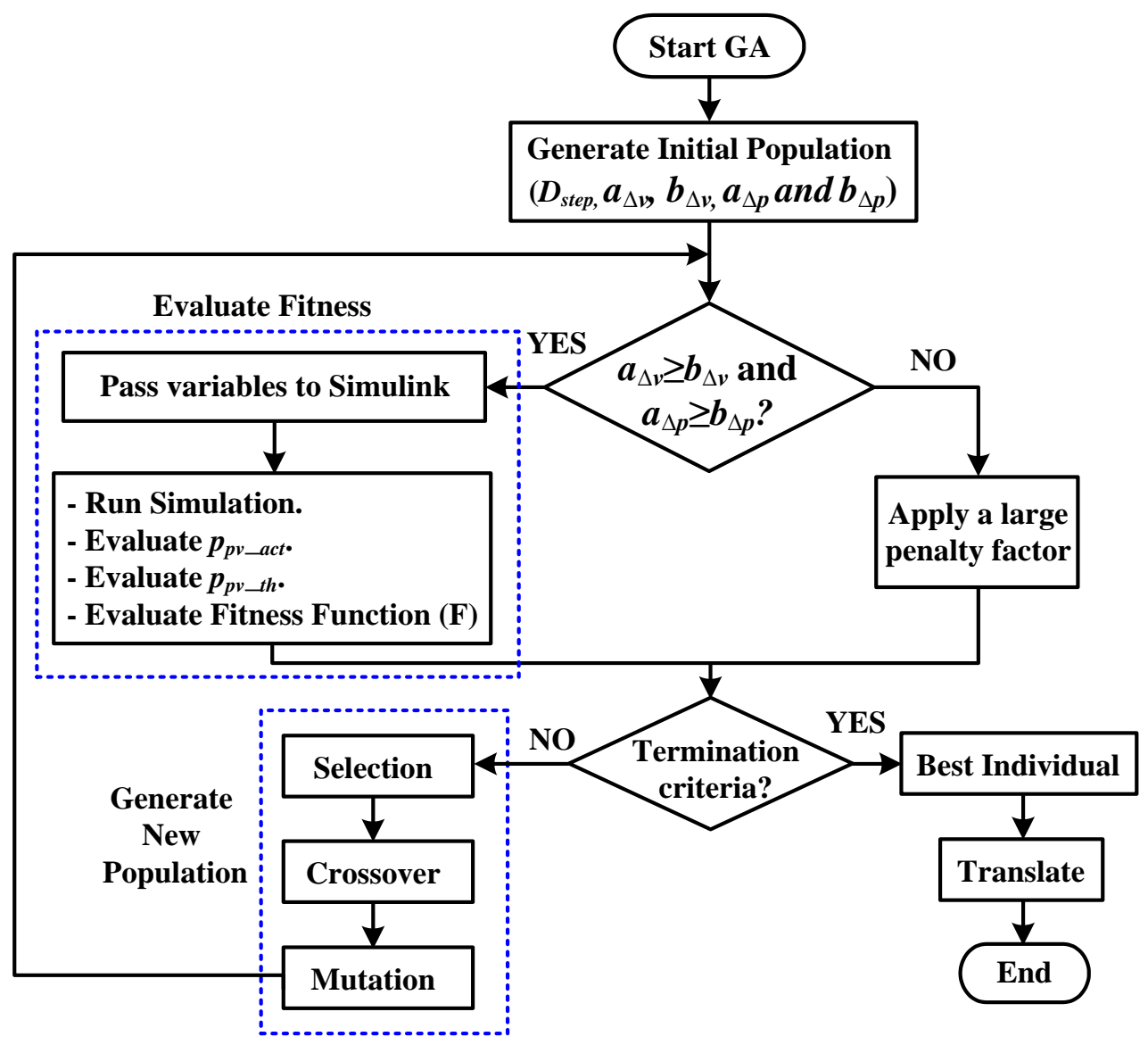

Figure 3.4. Flowchart of GA optimization procedure.

The objective of GA optimization is to minimize the cost function by accurately choosing the design parameters of the FL system. GA setup for the proposed design optimization procedure is presented in Table 3.2.

The selection of optimization constrains in Equation (3-2) (lower and upper boundaries of the optimization variables) are typically assigned by experience. However, these boundaries can be better configured using the developed formulas (2-21) and (2-23). The step size $\left(D_{\text {step }}\right)$ when $\Delta p_{\text {Upper }}=0.3 \mathrm{~W}$ is $\Delta d_{@ 0.3 \mathrm{~W}}=3.87 \times 10^{-4}$, and the change of 
the voltage is $\Delta v_{@ 0.3 W}=0.0195$. Thus, the upper boundaries of $D_{\text {step }}$, and $\Delta v$ are calculated as in Equation (3-3).

$$
\begin{aligned}
& \Delta d_{\text {Upper }}=5 . \Delta d_{@ 0.3 W} \approx 0.002 \\
& \Delta v_{\text {Upper }}=5 . \Delta v_{@ 0.3 W} \approx 0.1
\end{aligned}
$$

These values are used to set the upper boundaries for the GA optimization variables $a_{\Delta v}, a_{\Delta p}$ and $D_{\text {step }}$. The lower boundaries of the variables are set to be very close to zero for considering the maximum range of the design optimization. The optimization upper boundaries of $b_{\Delta v}$ and $b_{\Delta p}$ are heuristically set, as indicated in Table 3.2.

Table 3.2. GA Setup for the Design Optimization Algorithm.

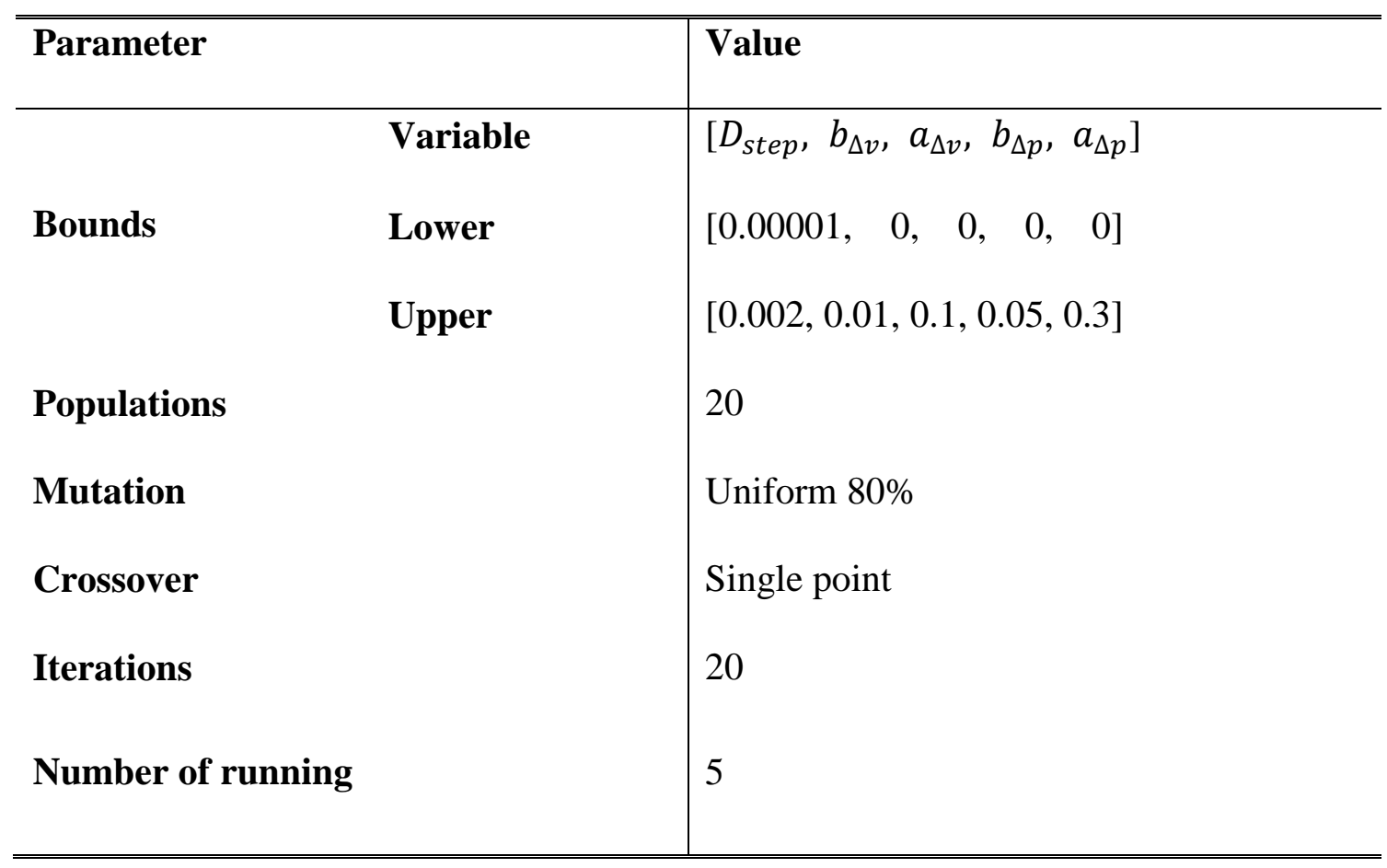

In order to avoid the infeasible optimization solutions, the parameters $a_{\Delta v}$ and $a_{\Delta p}$ should always be greater than or equal to $b_{\Delta v}$ and $b_{\Delta p}$, respectively. A large penalty factor 
is appended to the cost function if the solution does not satisfy these criteria, as indicated in Figure 3.4. This penalty factor helps to guide the next iterations to the feasible region after evaluating the fitness, as indicated in the results presented in Figure 3.5. The fitness function shows smooth descending progress without any infeasible solution. The final optimum FL system design parameters using GA optimization are presented in Table 3.3.

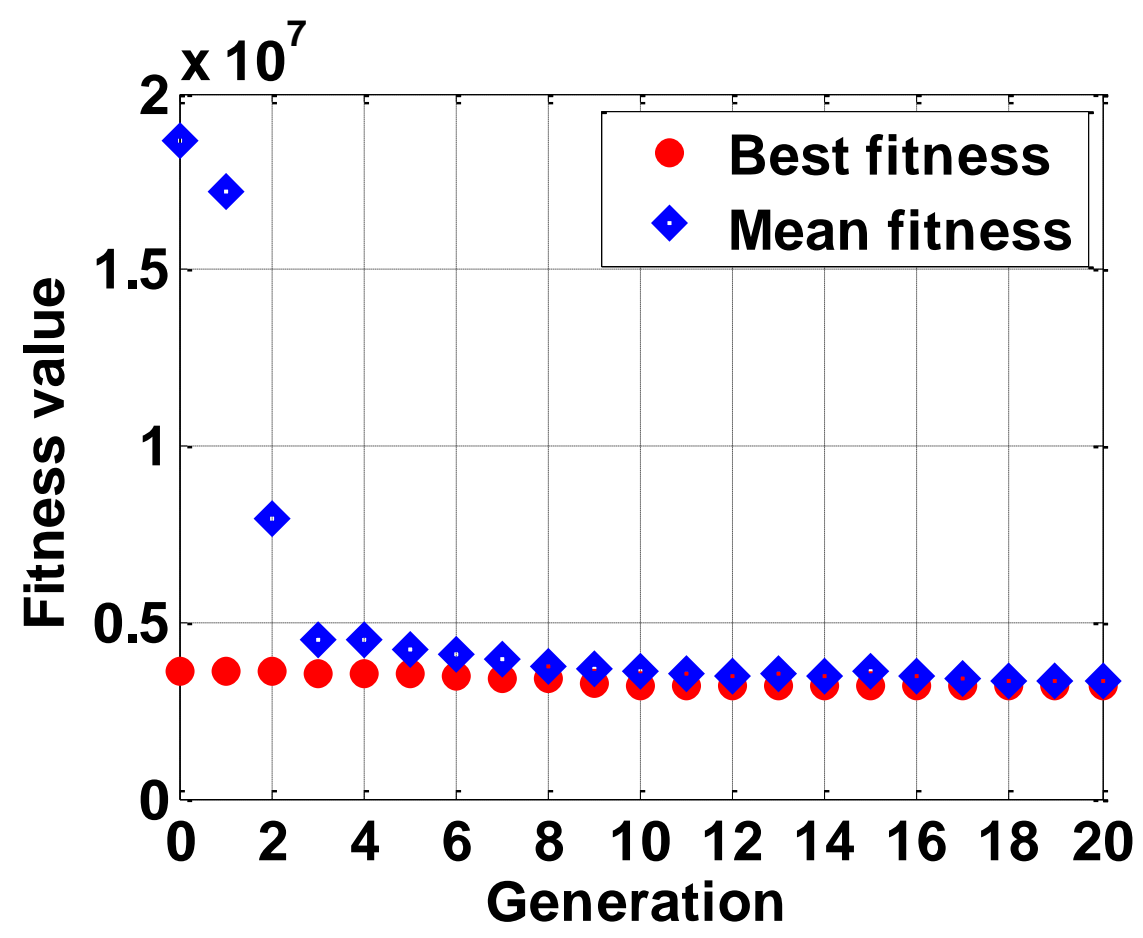

Figure 3.5. Progression of the GA Fitness function.

Table 3.3. Results of Design Parameters from GA Design Method.

\begin{tabular}{cc|cc}
\hline \hline Parameter & Value & Parameter & Value \\
\hline $\boldsymbol{D}_{\text {step }}$ & $9.7353 \times 10^{-4}$ & $\boldsymbol{a}_{\Delta \boldsymbol{P}}$ & 0.08417 \\
$\boldsymbol{a}_{\Delta \boldsymbol{V}}$ & 0.00779 & $\boldsymbol{b}_{\Delta \boldsymbol{P}}$ & 0.01271 \\
$\boldsymbol{b}_{\Delta \boldsymbol{V}}$ & $3.1832 \times 10^{-4}$ & & \\
\hline
\end{tabular}




\subsection{Hardware Implementation of Fuzzy-MPPT Controller}

The system described in Figure 3.1 was simulated and experimentally tested under fast variations of climatic conditions. The simulations were performed in Simulink MATLAB and compared with the mathematical model presented in chapter (2). The experimental test setup is shown in Figure 3.6. It consists of a commercial BP 4175T PV array connected to a DC bus through a DC-DC Cuk converter and its control. The PV array was emulated by XR375-15.9 Programmable DC MAGNA power supply. The proposed FL MPPT algorithm was implemented using the commercial microcontroller DSpace 1104. The DC-DC converter is built using HGTG30N60C3D IGBT power switch and FFPF15S60S power diode. The commercial SKHI 22A H4 R gate driver was considered to drive the IGBT. Hall effect LEM voltage and current sensors are utilized to measure the PV voltage and current. The nominal values for the chosen system's components are given in Table 3.4. The converter switching frequency is $10 \mathrm{kHz}$ and the perturbation rate is 50 variations/sec.

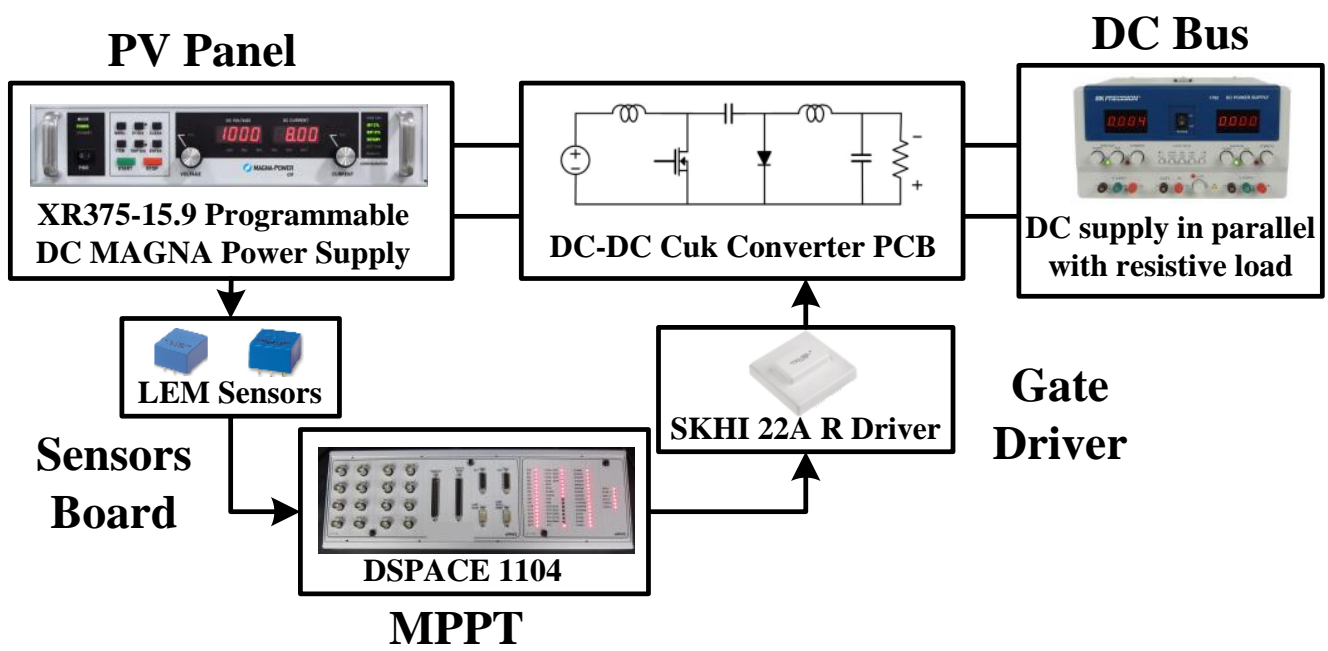

Figure 3.6. Experimental setup of PV power system. 
Table 3.4. Specifications of Hardware Components of the DC-DC Converter.

\begin{tabular}{ll}
\hline Component & Model/Rating \\
\hline Power IGBT & HGTG30N60C3D, 600 V, 63 A \\
Power Diode & FFPF15S60S, 600 V, 15 A \\
Gate Driver & Hybrid Dual SKHI 22 A / B H4 (R) \\
Current Sensor & LA25-NP 713194 \\
Voltage Sensor & LV25-P 712100 \\
\hline \hline
\end{tabular}

\subsection{Experimental and Simulation Results and Discussion}

The PV power system with the FL MPPT is evaluated in this section. Several tests were applied to the system for verification purposes. The first category of tests are made to validate the proposed design analysis. The second class of measurements are achieved to verify the robustness of the proposed FL MPPT under different environmental conditions. Finally, comparative analysis is realized between the performance of the proposed MPPT and the most common techniques in the literature.

\subsubsection{Assessment of the Proposed FL Design Strategies}

The effect of the two design techniques presented in section 3.3 on the MPPT performance is indicated in this section. The FL system is analyzed and compared using the parameters resulting from the analytical and GA-based procedure. The comparison results are described in Figure 3.7. It shows the PV variables $\left(p_{p v}, i_{p v}\right.$ and $\left.v_{p v}\right)$ under 
certain irradiance profile during $4.5 \mathrm{sec}$ in which two step changes were applied: from 1 to $0.6 \mathrm{~kW} / \mathrm{m}^{2}$, and then turns back to $1 \mathrm{~kW} / \mathrm{m}^{2}$. Figure 3.7 (a) presents the $\mathrm{PV}$ power using the two combinations of design parameters (analytical and GA-based) and compared with the theoretical MP, which is supposed to be generated from the PV panel. Both design techniques exhibit successful tracking performance but the analytical one shows slower transient response, as indicated in Figure 3.7(c). By evaluating the Mean Absolute Power Error (MAPE) for both combinations, GA-based provides better accuracy with 2.36\% than the analytical one, which shows $3.15 \%$. Thus, the parameters from GA optimization are considered for the rest of the results.

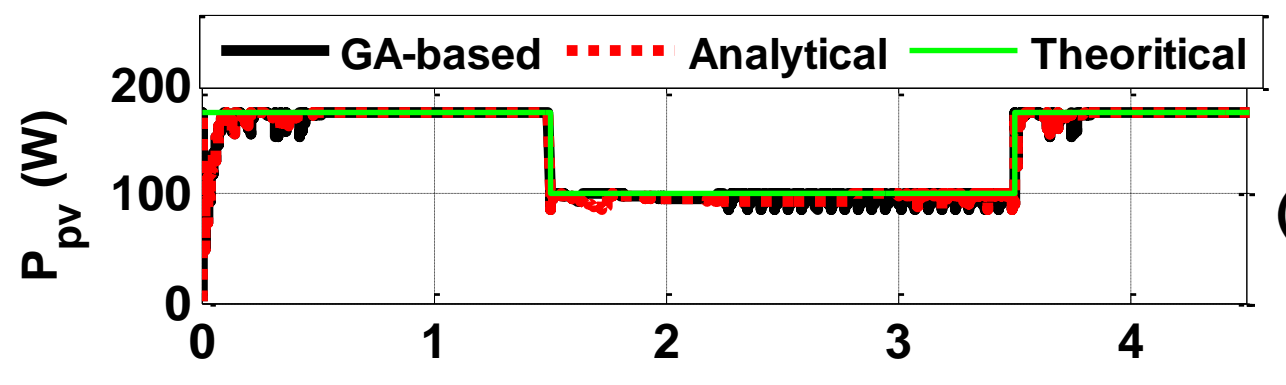

(a)
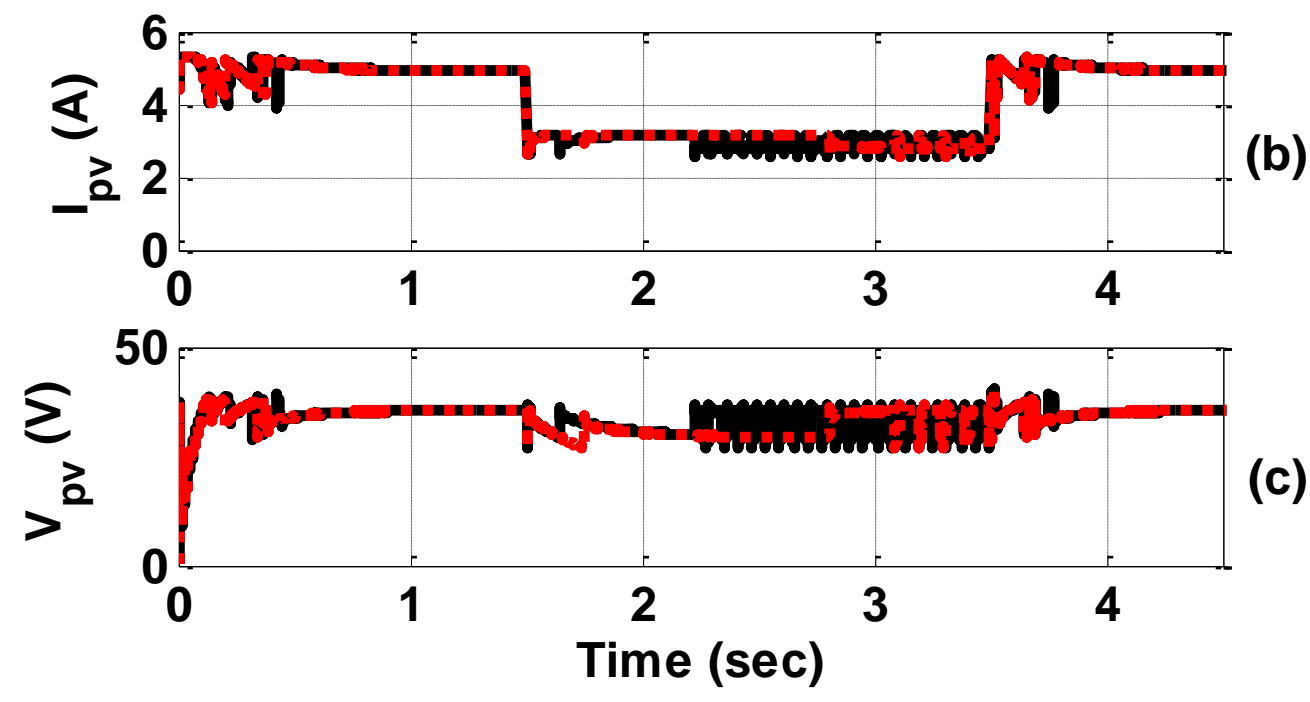

Figure 3.7. Simulation results for the proposed FL system designs. (a) PV power. (b) PV current. (c) PV voltage. 


\subsubsection{Experimental and Simulation Results of Fuzzy MPPT}

In this section, a comparison between the theoretical, experimental and simulation results are presented to evaluate the dynamic and steady-state performance of the proposed FL MPPT controller. The entire system performance is analyzed by simulation and compared with the experimental results. A pulse irradiance profile is applied for $10 \mathrm{sec}$, which shows a step change from 1 to $0.6 \mathrm{~kW} / \mathrm{m}^{2}$ and turns back to $1 \mathrm{~kW} / \mathrm{m}^{2}$.

The PV variables are indicated in Figure $3.8\left(p_{p v}, v_{p v}\right.$ and $\left.i_{p v}\right)$. The figure shows how the control successfully tracked the MPP regardless of the irradiance variation. Generally speaking, the figures show very good correlation between the experimental, simulation and theoretical results. Larger ripples in the simulation results than the experimental can be noticed. This is due to using digital low-pass filters after the voltage and current transducers to minimize the noise and disturbances due to the PV emulator. These filters help the MPPT control algorithm to work properly, however, they smooth the normal ripple due to the swinging around the MPP. That is why these oscillations appear in the experimental results during the whole operating period, as shown in Figure 3.8, unlike the simulation results, which show oscillations during the transient region only. Also, another reason for the experimental oscillations is the low acquisition resolution of the experimental tests $(5 \mathrm{kHz})$ compared with the simulation $(0.5 \mathrm{MHz})$. The slow dynamic performance of the PV emulator can be observed during the irradiance step down and up transient response of the experimental results in Figure 3.8. 


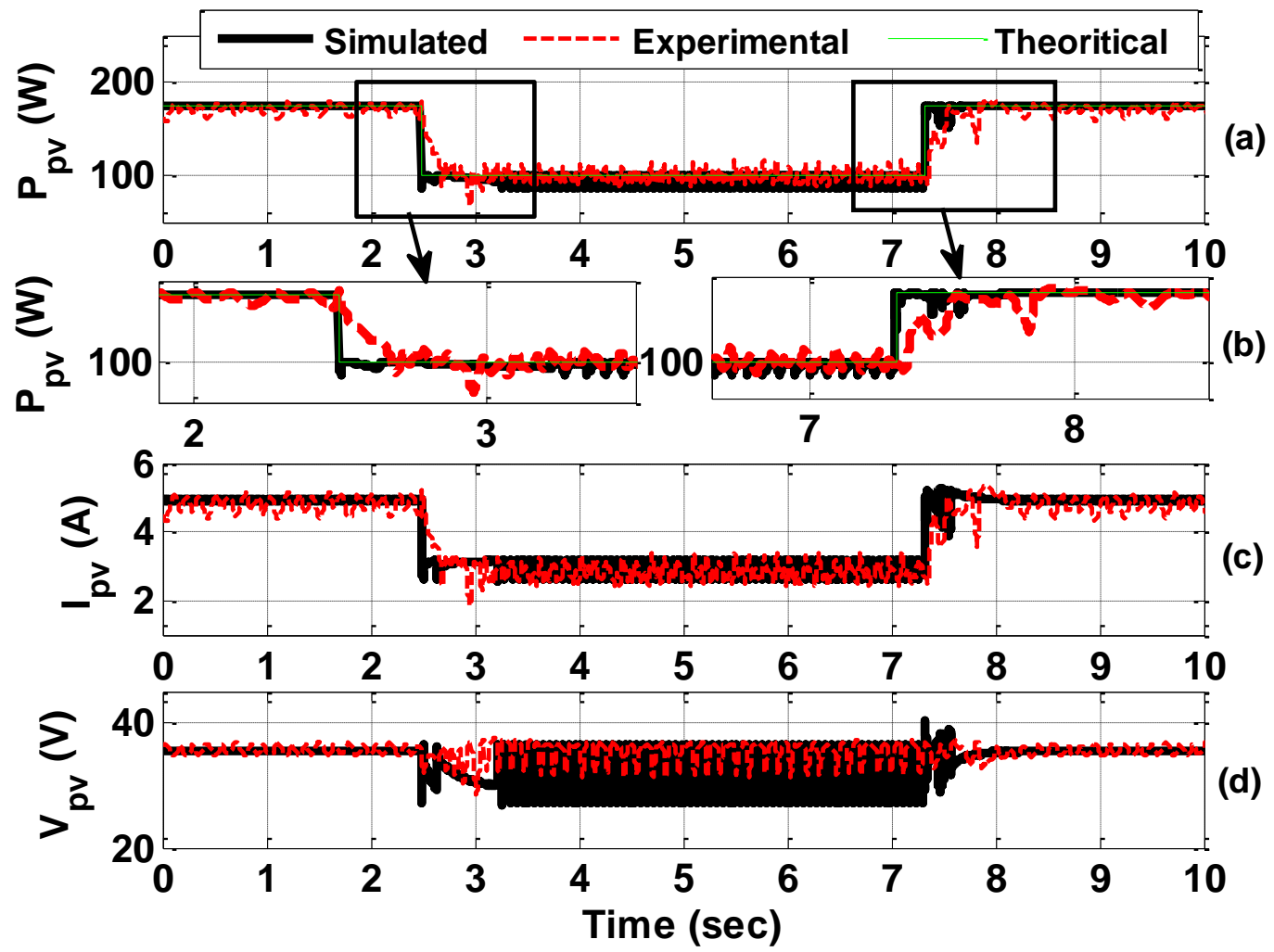

Figure 3.8. Experimental and simulation results for FL-MPPT control. (a) PV power. (b) PV power zoomed. (c) PV current. (d) PV voltage.

The effectiveness of the proposed FL controller under the fast temperature variation is investigated in Figure 3.9. Typically, the variation of the temperature is slow compared to the radiation variation. However, a dramatic temperature variation is assumed in this test to confirm the robustness of the proposed controller. The temperature stepped from $25^{\circ} \mathrm{C}$ to $50{ }^{\circ} \mathrm{C}$, then returned back to $25{ }^{\circ} \mathrm{C}$. As it can be noticed, increasing the temperature slightly increases the PV current and significantly decreases the PV voltage, which results in a small reduction of the PV power. The effect of the temperature on the PV power is small compared with the radiation impact. The proposed MPPT shows robust tracking 
performance under the fast temperature variation with good agreement between experimental, simulated and theoretical results.

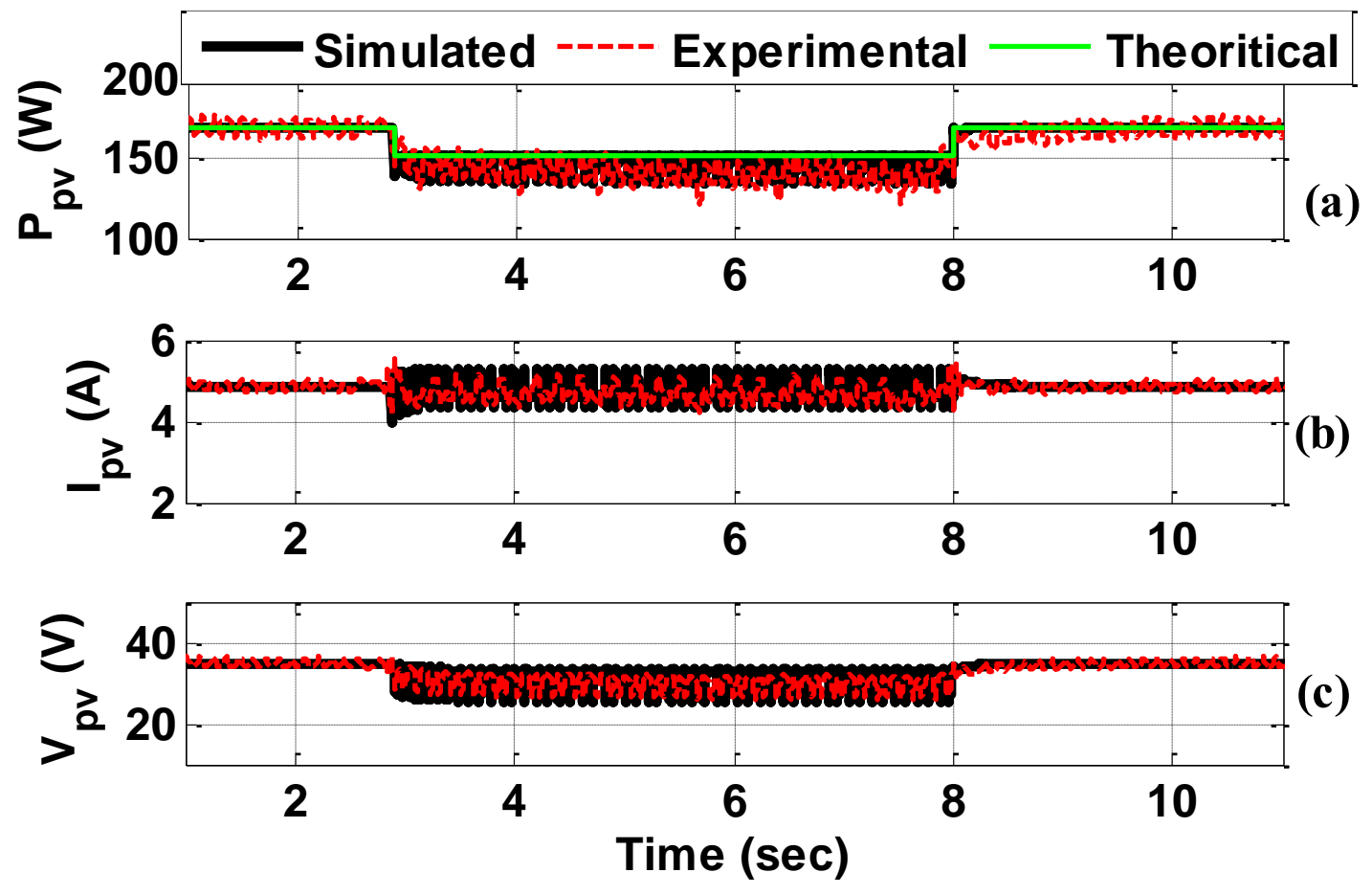

Figure 3.9. Experimental and simulation results for FL-MPPT controller under fast temperature variation. (a) PV power. (b) PV current. (c) PV voltage.

\subsubsection{Comparison between the Proposed Fuzzy-MPPT, P\&O and INC Algorithm}

In order to verify the effectiveness of the proposed FL-MPPT technique, a comparative study has been conducted between the proposed algorithm and the two most commonly used hill-climbing MPPT algorithms: P\&O and Incremental Conductance (INC) [135]. Figure 3.10 presents the tracking performance of the PV power system under 
the proposed FL-MPPT and INC using the same irradiance profile used in Figure 3.8. The proposed technique shows faster transient response and less steady-state error. Also, losstracking is noticed in INC performance during the low irradiance level, which results in less tracking efficiency.

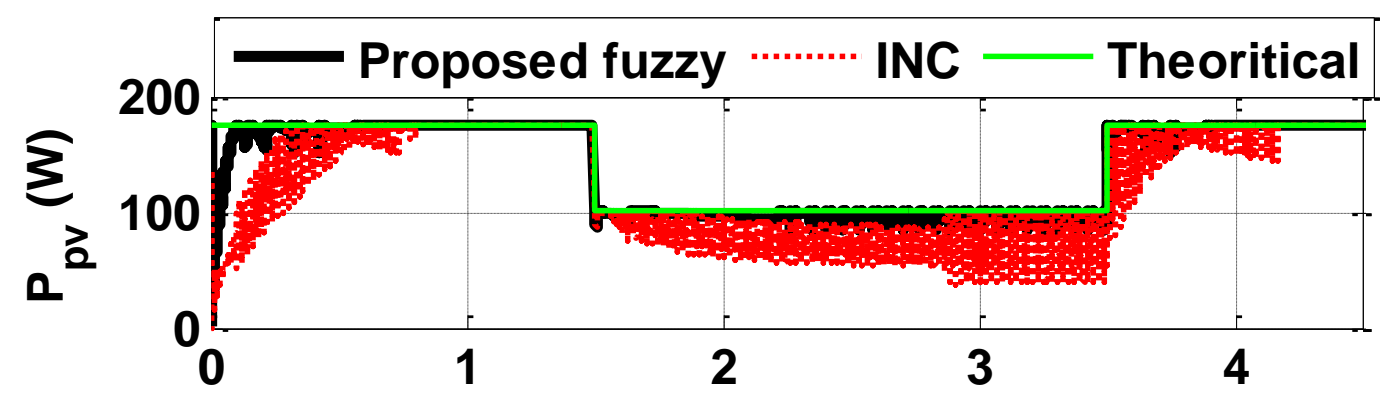

(a)

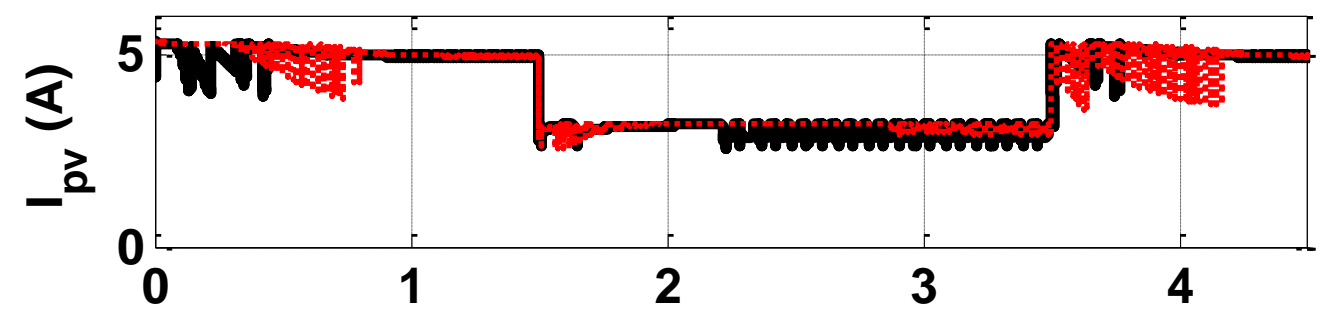

(b)

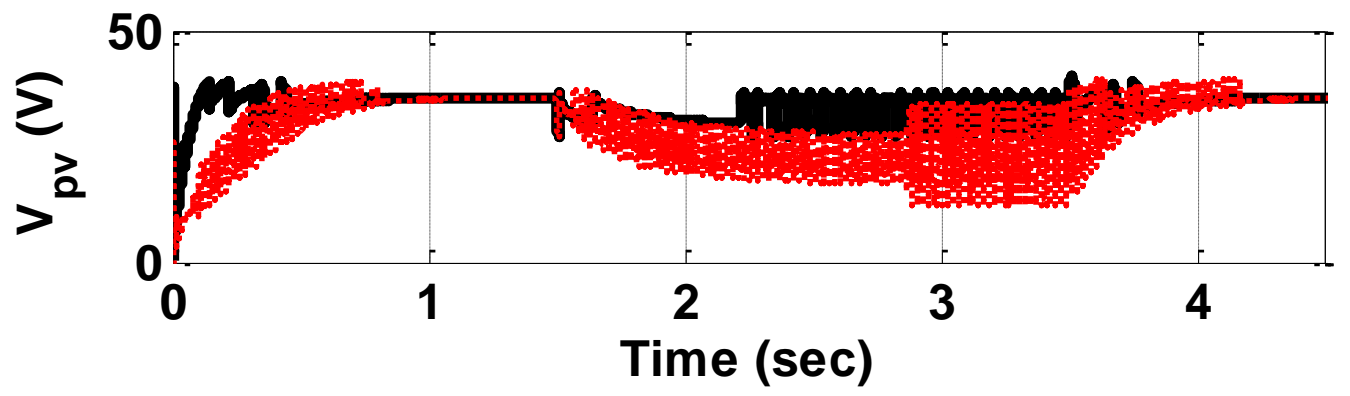

(c)

Figure 3.10. Comparison between the proposed fuzzy-MPPT and INC algorithm. (a) PV power. (b) PV current. (c) PV voltage.

Figure 3.11 describes a comparison between the proposed FL-MPPT and the conventional $\mathrm{P} \& \mathrm{O}$ algorithm. $\mathrm{P} \& \mathrm{O}$ algorithm exhibits steady-state error due to swinging 
around the MPP during the whole operating period, unlike the proposed algorithm, in which the error appears only during the transient. Also, longer transient can be noticed with $\mathrm{P} \& \mathrm{O}$ during the step variation of irradiance due to the wrong control actions. The MAPE has been evaluated for the three algorithms, as presented in Table 3.5. The proposed algorithm shows the least power error among all techniques. The conventioal P\&O algorithm is implemented experimentally and the results are presented in Figure 3.12. Good agreement is noticed between simulation and experimental results.

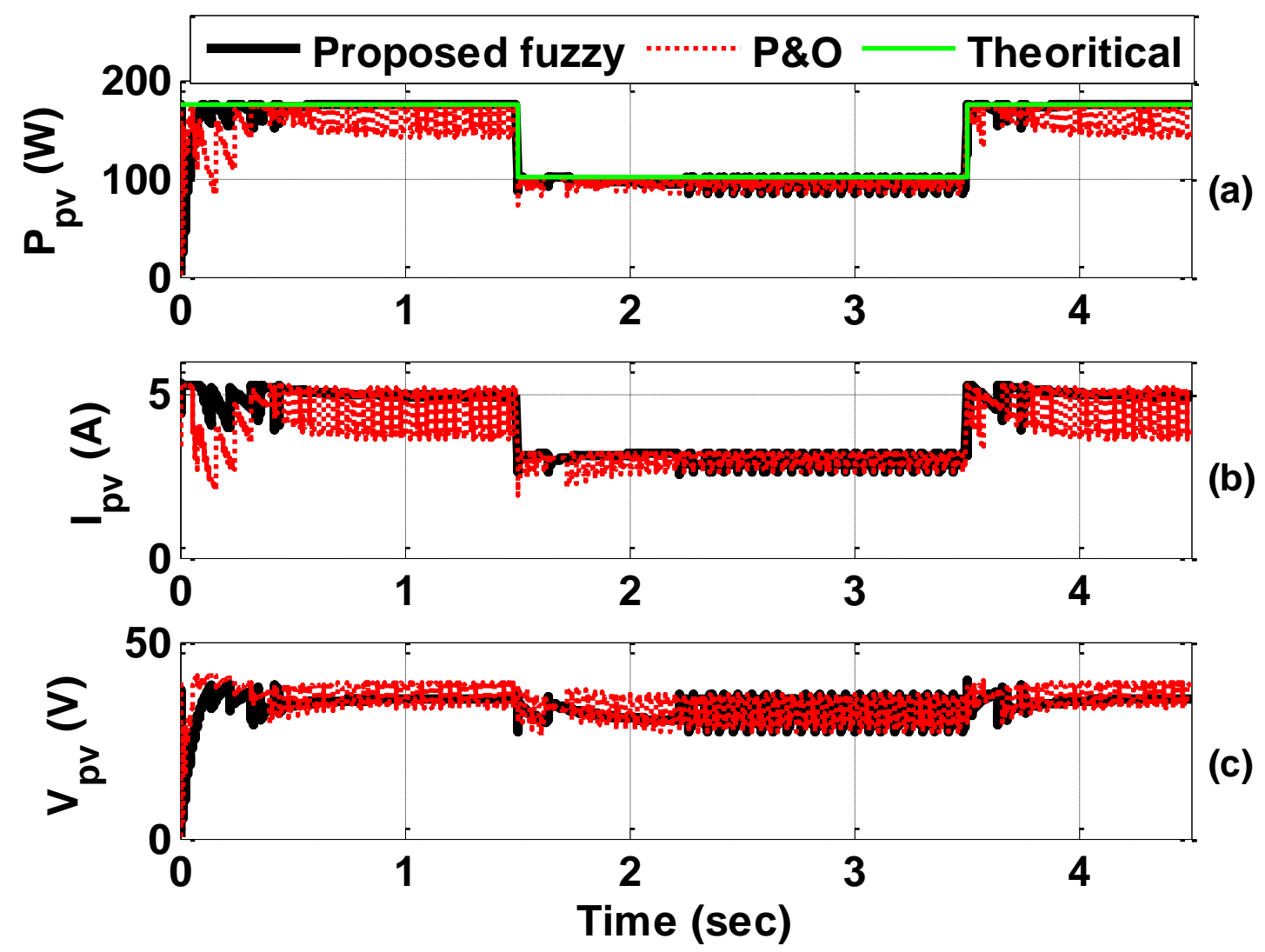

Figure 3.11. Comparison between the proposed fuzzy-MPPT and P\&O algorithm. (a) PV power. (b) PV current. (c) PV voltage. 
Table 3.5. MAPE for the Proposed FL-MPPT, P\&O and INC Algorithms.

\begin{tabular}{llll}
\hline Variable & P\&O & INC & Fuzzy-MPPT \\
\hline MAPE \% & 7.1951 & 13.5513 & 2.3605 \\
\hline \hline
\end{tabular}

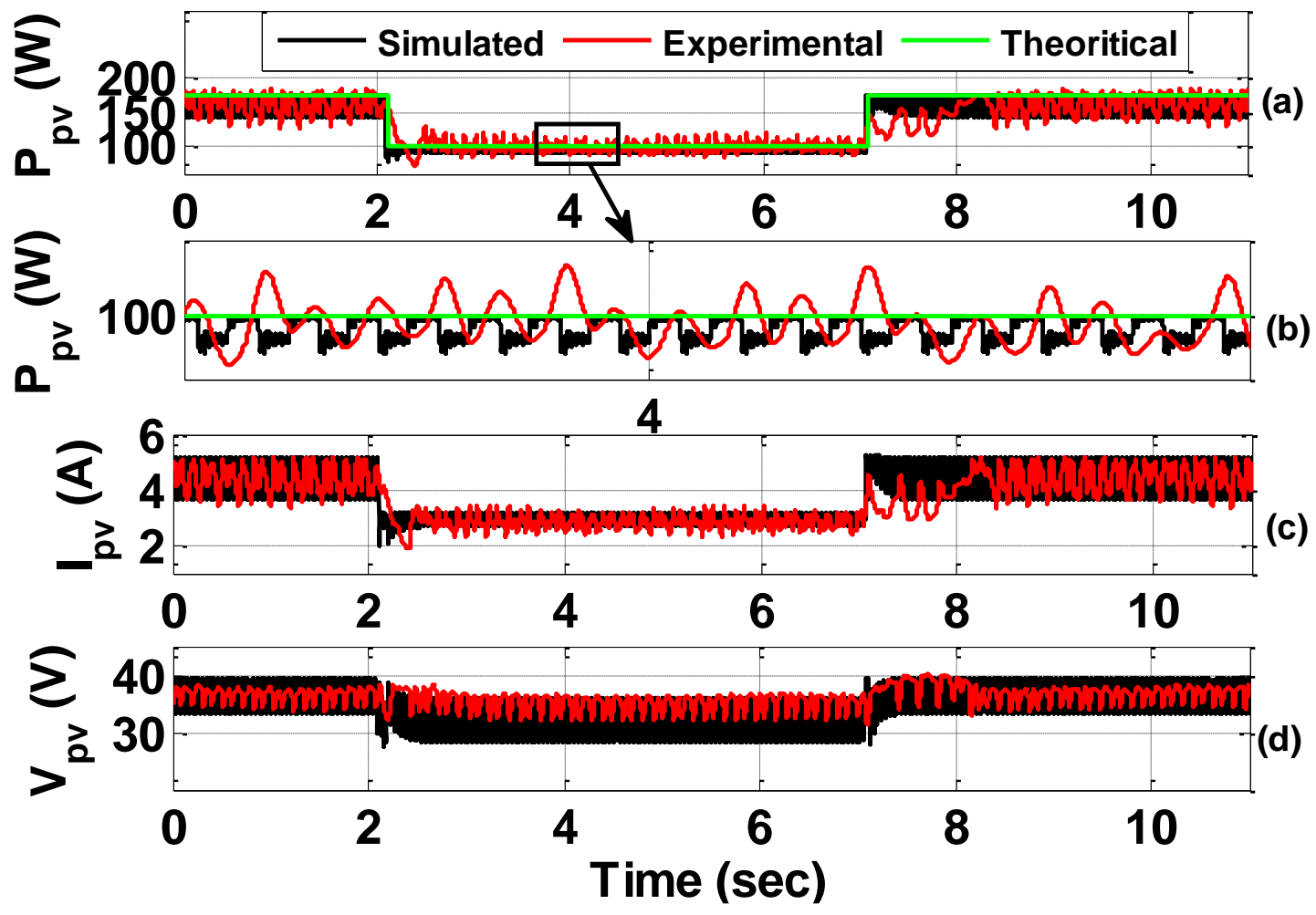

Figure 3.12. Experimental and simulation results for P\&O algorithm. (a) PV power. (b) PV power zoomed. (c) PV current. (d) PV voltage.

\subsection{Conclusion}

In this chapter, a comprehensive study for grid-connected PV power system is presented. The system dynamics are accurately modeled by integrating $4^{\text {th }}$ order ODE (for 
DC-DC Cuk converter and DC bus) with a non-linear equation representing the PV panel. General MPPT control design considerations were developed based on the small signal analysis. Then, proper FL-MPPT control design was performed by means of combining GA and the analytical design formulas. This technique is used for determining the location and the boundaries of the FL membership functions. The proposed intelligent MPPT control algorithm was simulated and experimentally tested under fast variation of irradiance and temperature conditions, and compared with the $\mathrm{P} \& \mathrm{O}$ and INC algorithms. The proposed control shows faster transient response, less steady state error and robust tracking performance. In all the tests, a comparative analysis was conducted between the simulation, experimental and theoretical results. The results show very good agreement between all of them. 


\section{Chapter 4 Analytical Modeling of Different Topologies of BIWPTS in EVs Applications}

\subsection{Introduction}

The Smart grids are converting the traditional power system into more efficient and reliable networks. Using EVs as active elements to serve the power grid is a promising service in smart grid applications, which is knowing as V2G service [136]. This kind of application requires a flexible, automatic, safe and reliable interface between the EVs and the power grid. The inductive wireless power technology is an ideal choice for these services as [92], [137]: 1) the vehicle does not need to be parked in a certain location for a long time, 2) avoiding the manual plug provides safe operation in the presence of water, rain or dust, 3) it is reliable during the environmental disaster such as hurricanes, storms and earthquakes, and 4) it is automatic and does not need the driver intervention. This wireless interface needs to be bidirectional to allow two-way power flow between the EV and power grid.

According to the literature, the BIWPTS has been proposed in [51], [138]. These systems have been produced for aircraft application, in which the leakage inductance of a transformer forms a resonant circuit with a series capacitor to allow the bidirectional power transfer while operating as a voltage source. Such a system would not be appropriate for V2G applications, where a fleet of EVs needs to be supplied at the same time [139]. A dual-side full-bridge BIWPTS for charging and discharging EVs was proposed in [137], [140]. Several analytical models and assessment analyses for IPT systems can be found in the literature. Most of these analyses were developed for unidirectional IPT systems, such 
as in [82], [86], [89], [141]. These studies cannot be simply applied for bidirectional operation, since in this case the system is designed such that each side should be able to work as a source and a load at the same time. Moreover, in most of these models, only the fundamental frequency component was considered, which leads to inaccurate analysis especially in the topologies where both the voltage and the current waveforms are nonsinusoidal. For BIWPTS, steady-state mathematical models were presented in [75], [101], [142], a dynamic analytical model was developed in [143], and a physics-based model was proposed in [144], [145]. In these models, the harmonics components were considered, however, they were developed for LCL topology only and no assessment analysis was presented. Simple mathematical models for LC-series and LCL topologies were presented in [146]. In this study, only the fundamental frequency component was considered, and brief sensitivity analysis was performed. Up to the author knowledge, a comprehensive modeling and evaluation analysis for the different compensation topologies in BIWPTS has not been presented yet.

This chapter presents modeling analysis for the steady-state performance of BIWPTS in EVs implementations based on Fourier series analysis considering the harmonic contents in the system's performance. The analysis is presented for the three main compensation configurations of a symmetrical BIWPTS: LC-series, LC-parallel and LCL-topology. Moreover, the steady-state equivalent circuit-based mathematical models for all topologies are developed. These models were used to precisely determine the system's response during V2G and G2V operations based on Fourier series. Generalized fundamental power flow formulas (active and reactive), to evaluate the power flow performance in the different topologies, are developed. 


\subsection{V2G Scheme Description}

The description of G2V and V2G concepts including the wireless power interface are depicted in Figure 1.1. The figure demonstrates a DC-bus integration arrangement, in which all the elements are connected together through a common DC-link. Typically, the integration between EVs and the power grid can be achieved through a DC or an AC-bus. The DC-bus configuration offers more benefits than the AC-bus structure, whether the EV was connected by wires or wirelessly, or both of them (which is the typical case in commercial EVs that support wireless chargers nowadays) [147]. In this sense, two block diagrams are prepared to indicate the wired and wireless connection of an EV to a DC and an AC-bus. In Figure 4.1, which shows the DC-bus connection, the EV carries a wireless power pad, high-frequency inverter (for wireless) and DC-DC converter (for wired). Also, during the installation of the primary side of the wireless system (which is still the responsibility of the automotive company), the company does not need to worry about the grid-tie inverter, since it will work directly with the available DC-bus.

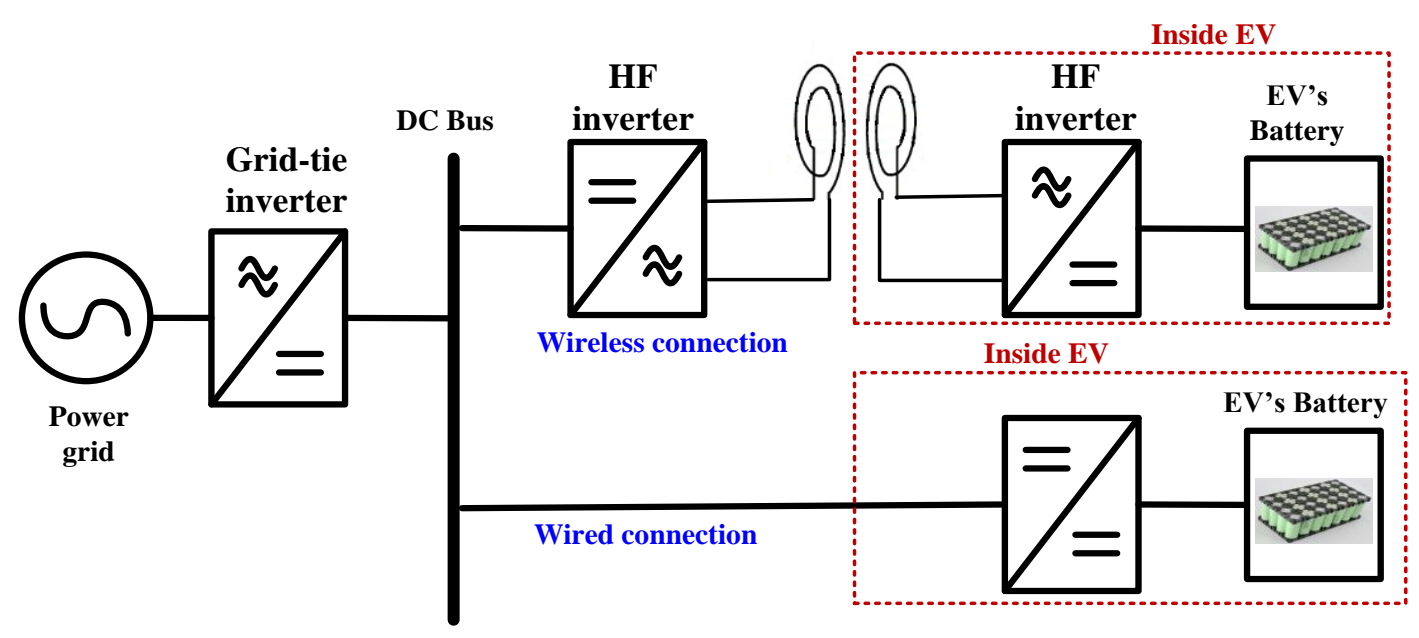

Figure 4.1. Wired and wireless connected EV through a DC-bus. 
On the AC-bus connection, which is shown in Figure 4.2, the EV carries the same devices as those that are in the DC-bus connection, in addition to a grid-tie inverter for the wired connection, which means more EV's weight and cost, and less utilization efficiency. Moreover, during the installation for the primary side of the wireless system, the company will be responsible for installing a second grid-tie inverter for the wireless option. This means more cost and effort for the automotive companies, which will be reflected in the price of $\mathrm{EV}$.

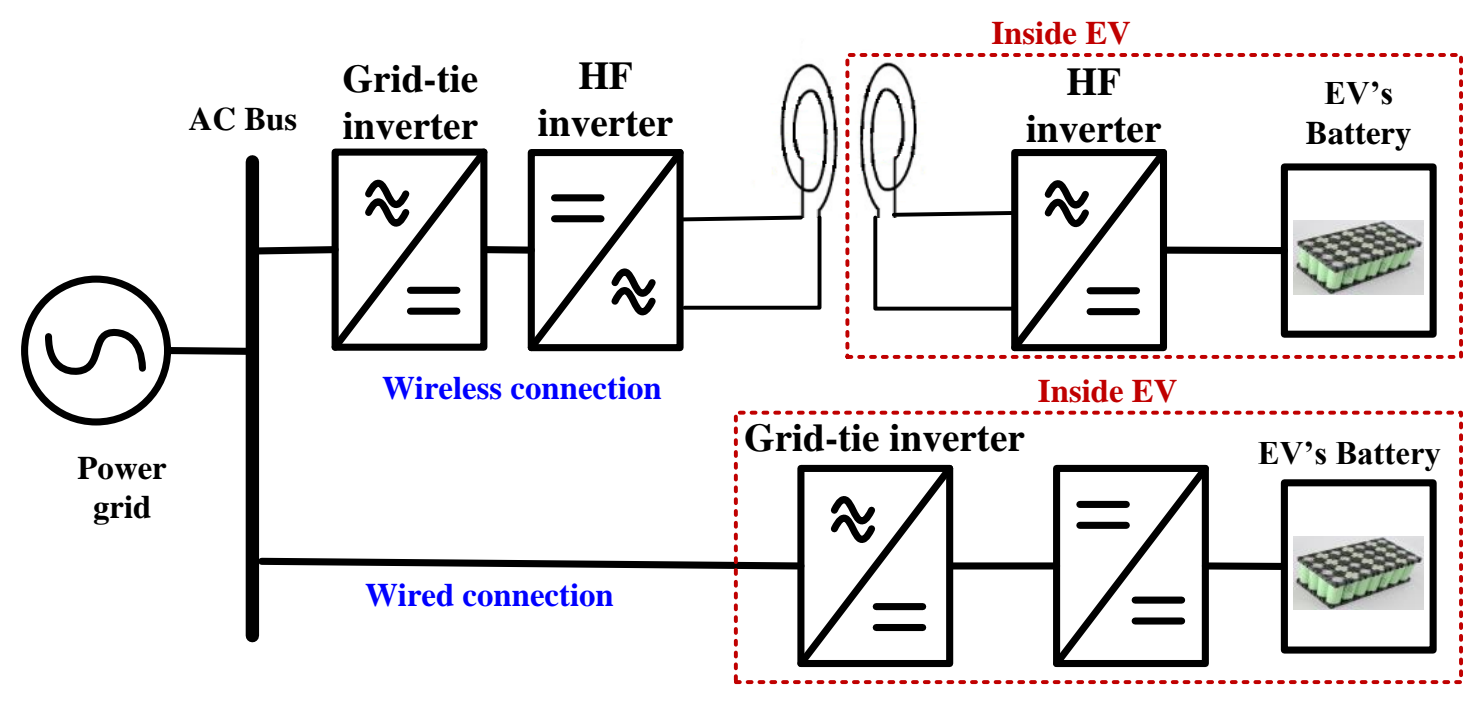

Figure 4.2. Wired and wireless connected EV though an AC-bus.

Based on this discussion, we can conclude that the DC-bus architecture provides more benefits for EVs manufactures and users, in both the wired and wireless connection. It needs less components inside the EV in the wired connection and on the road for the wireless integration. This results in less EV weight and cost, more utilization efficiency, 
and EV manufacturing companies can get rid of installing the grid-tie converter. Also, it is more convenient for integrating RESs and energy storage systems [148].

\subsubsection{Dual H-Bridge BIWPT System}

Typically, the dual H-bridge BIWPTS consists of two sides: primary (grid or ground) and secondary (vehicle). The former is connected to the DC-bus and is implemented on the road underneath the vehicle. The latter is coupled to the EV battery and is placed inside the vehicle. Each side consists of a HF H-bridge inverter, controller, compensation circuit and the wireless pad, as shown in Figure 4.3. The two sides are weakly coupled by magnetic induction through a large air gap (100-200 mm), according to the Society of Automotive Engineers (SAE) J2954A standard [149]. During G2V (charging) operation, the power flows from the DC-bus to charge the EVs. The DC power is converted to HF AC (81.38$90 \mathrm{kHz}$ ) by the primary inverter of the wireless entity to supply the primary circuit. The primary power is transferred by magnetic induction to the secondary circuit through the air-gap with the same frequency. Then, the secondary power is converted to DC by the secondary converter to supply the EV battery. The compensation capacitors are essential to resonate with the wireless coupler coils and provide reactive power compensation and unity power factor (UPF) operation in the primary and secondary sides. These capacitors result in minimizing the required VA from the supply and maximizing the utilization efficiency [150]. In the discharging mode (V2G), the power is transferred from the EVs and the DC-bus through the same path. 


\section{Primary inverter}

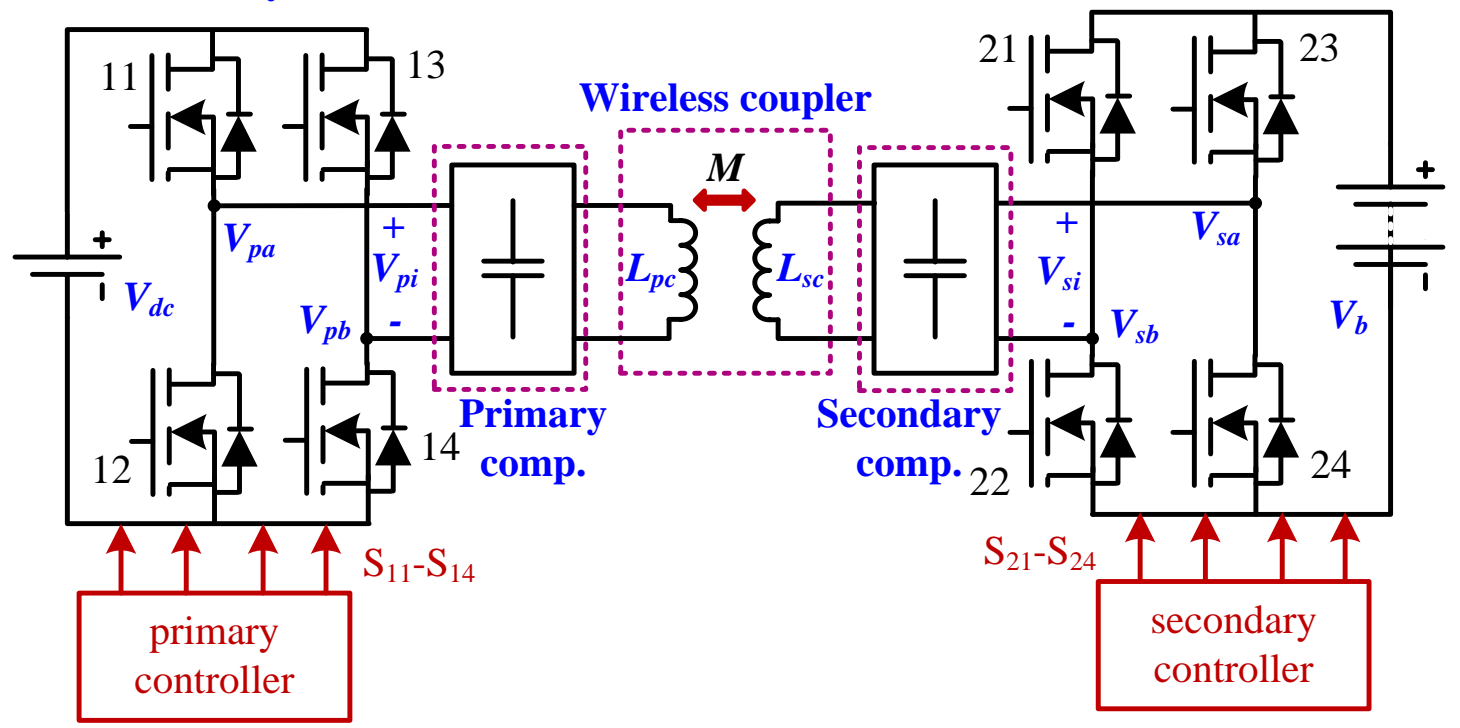

Figure 4.3. Schematic circuit diagram of the dual-side full-bridge BIWPTS.

\subsubsection{Bidirectional Power-Flow Control}

The bidirectional power-flow operation between the EVs and DC-bus is managed by the two HF H-bridge inverters in the system. These inverters are driven by two synchronized controllers, as indicated in Figure 4.3. In this work, both the primary and secondary controllers are utilizing the phase-shift technique. In this technique, the system's currents and power are managed by adjusting both the magnitude and phase of the two inverters' voltage. This voltage control is achieved by changing the phase-shift between legs' voltage for the same inverter and the two inverters' voltage. The primary controller generates the phase-shift between the two primary inverter's legs $(\alpha)$. This parameter $(\alpha)$ is used to adjust the magnitude of the primary inverter output voltage based on the reference signal, which is typically the rated current that the primary circuit can support [see Figure 
4.4(a)] [137]. The secondary controller produces the phase-shift between the secondary inverter's legs $(\beta)$, and the phase shift between the primary and secondary inverters' output voltage $(\delta)$. Typically, the secondary control parameters $(\beta$ and $\delta)$ are adjusted to achieve the required power flow magnitude and direction, respectively [Figure 4.4(b)] [139]. The phase-shift parameters, from both the controllers $(\alpha, \beta$ and $\delta)$, are then translated to lowlevel switching signals based on the pulse-phase modulation (PPM) technique, as indicated in Figure 4.4. These signals are boosted up using the driver circuits to drive the eight switches of the two HF inverters. By applying these switching signals, the inverter voltage magnitude and phase are adjusted to achieve the required power-flow in the system. The variation of the inverter voltages in terms of the phase-shift control parameters is described in Figure 4.5.

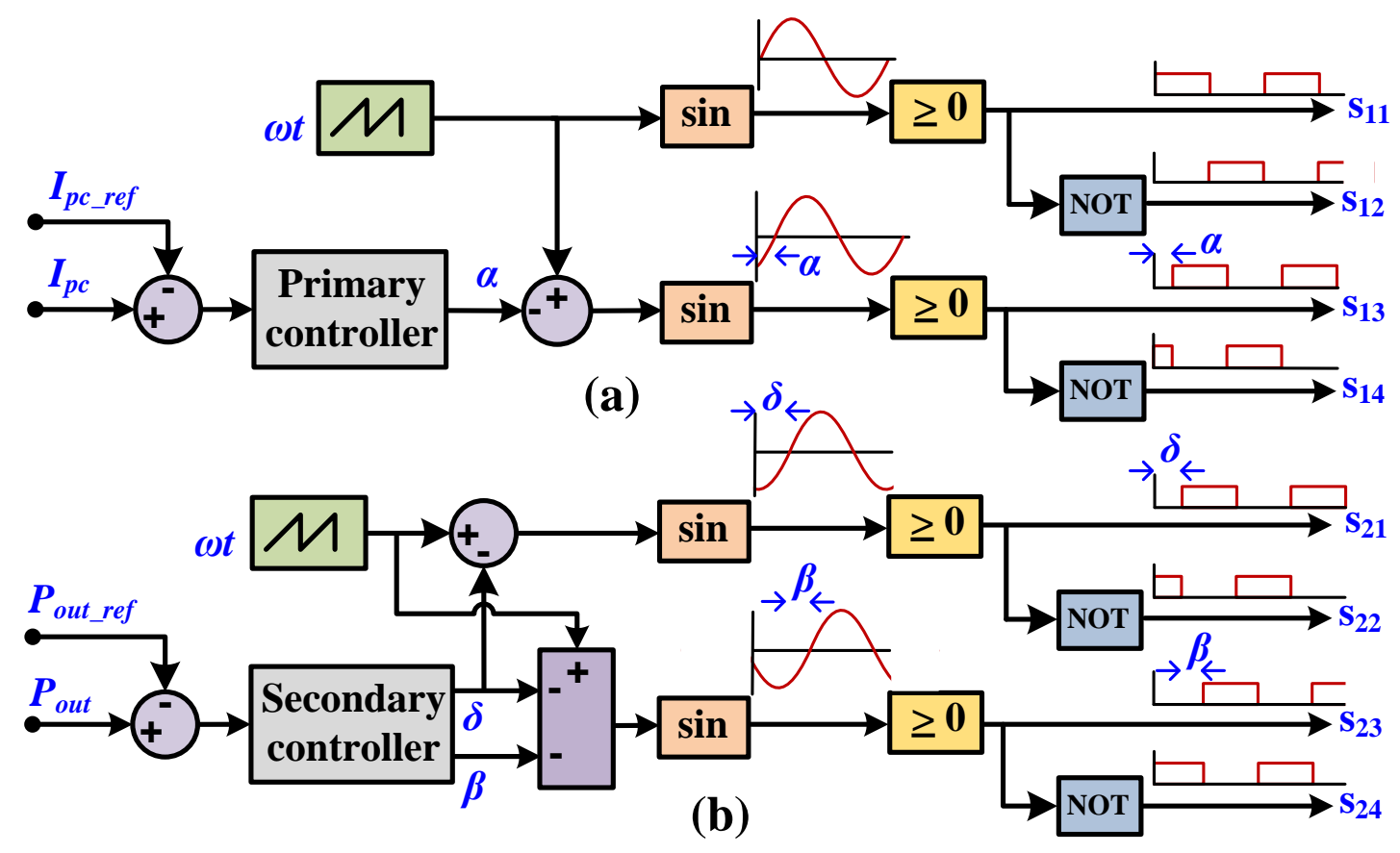

Figure 4.4. Phase-shift control of the HF inverters. (a) Primary controller. (b) Secondary controller. 


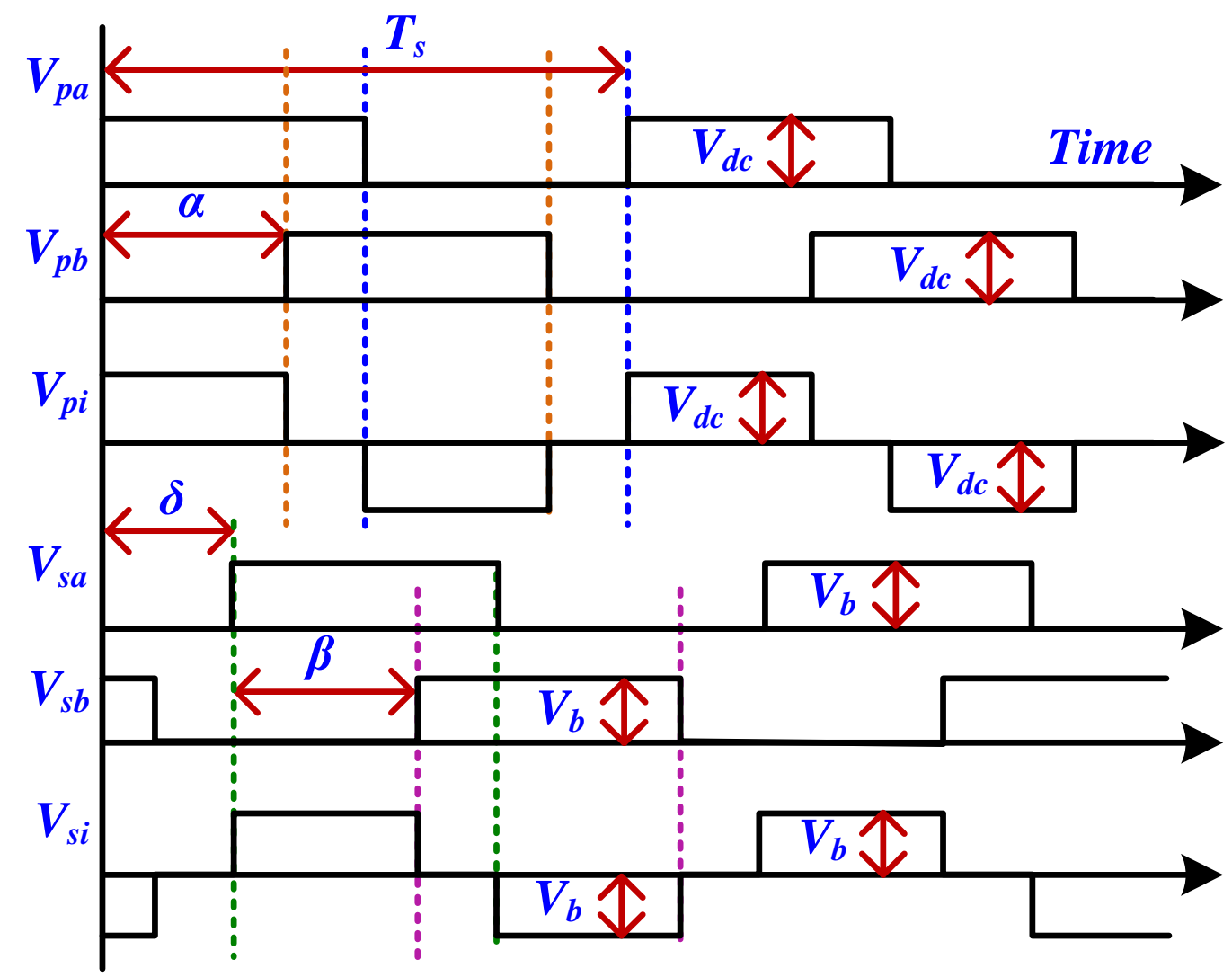

Figure 4.5. Legs and total inverter output voltages in terms of the phase-shift control parameters.

\subsubsection{Compensation Network}

The large air-gap between the two power pads in the IPT system decreases the system magnetizing reactance and increases the leakage reactance. Therefore, large magnetizing current will be drawn from the supply in terms of reactive power. Compensation PFC capacitors are typically used in BIWPTS to provide reactive power restitution and improve the operating power factor in the primary and secondary circuits. Improving the power factor (PF) leads to higher utilization efficiency and power transfer capability [151]. These 
capacitors can be connected either in series or in parallel based on the applications. In BIWPTS, each side may act as a supply or a load at the same time, thus the two sides should be treated equally. Therefore, in this study it is assumed that, the BIWPTS under consideration is symmetrical. Therefore, there is no a specific side that needs to be designed to work as a source or a load. Both sides should be able to supply and collect the power at the same time.

Based on this assumption, there are four compensation topologies for symmetrical BIWPTS in the literature: LC-series, LC-parallel, LCL-topology and CLCL-topology, as indicated in Figure 4.6. In LC-series structure, the capacitors $\left(C_{p}\right.$ and $\left.C_{s}\right)$ are connected in series with the pad coils ( $L_{p c}$ and $L_{s c}$ ) [Figure 4.6(a)], and in parallel for LC-parallel structure [Figure 4.6(b)]. In LCL-topology, L-filters $\left(L_{p i}\right.$ and $\left.L_{s i}\right)$ are added between the inverters and the resonance tank in LC-parallel topology, as shown in Figure 4.6(c).

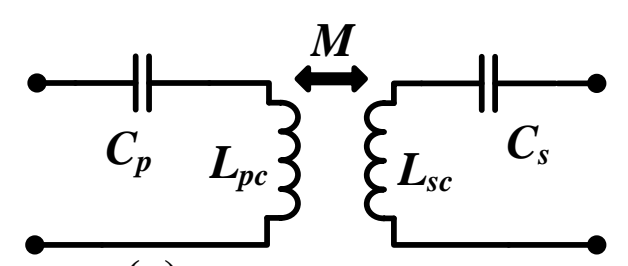

(a)

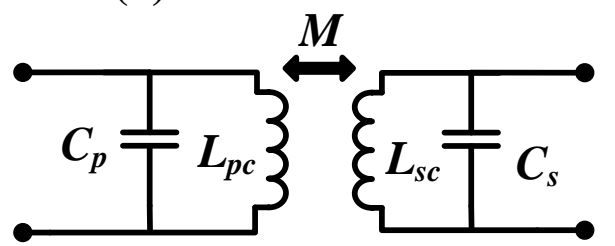

(b)

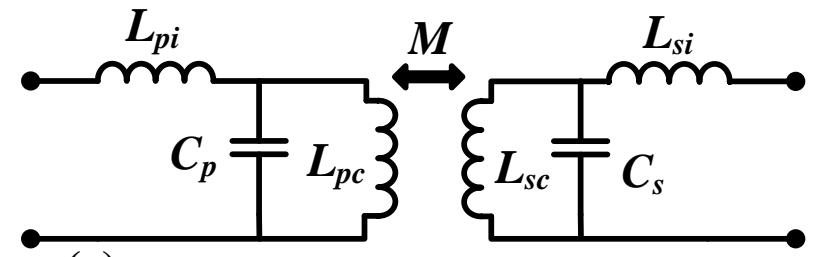

(c)

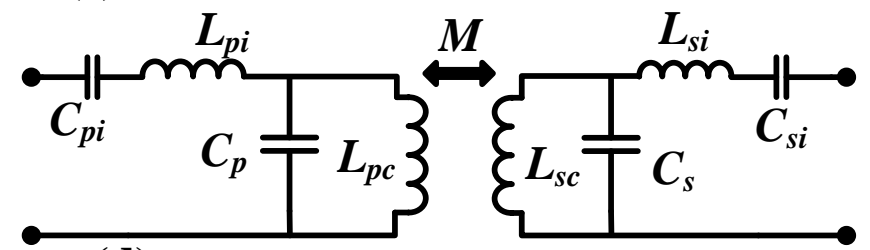

(d)

Figure 4.6. Different compensation topologies in BIWPTS. (a) LC-series. (b) LC-parallel. (c) LCL-topology. 
In CLCL structure, shown in Figure 4.6(d), DC blocking capacitors $\left(C_{p i}\right.$ and $\left.C_{s i}\right)$ are inserted in series with the filter inductances, in order to remove the DC current component. The best design for these capacitors is such that the equivalent reactance of the capacitors and the filters' inductance in each side is equal to the filter reactance in LCL-topology. In this case, the mathematical model and the performance of CLCL-topology is equivalent to that of LCL architecture, as was discussed in [142]. Hence, in this work, the authors decided to focus on the three main topologies: LC-series, LC-parallel and LCL-topology.

\subsection{Steady-State Circuit representation of BIWPTS's Components}

BIWPTS in EV applications consists of three main elements: EV's battery, HF inverters and the wireless coupler, which includes the power pads and the compensation capacitors. As indicated in Figure 1.1, a grid-tie converter is used in the framework to keep the DC-bus voltage level fixed and to manage the power-flow between the EV and the grid. Hence, the DC-link is represented mathematically as a fixed DC voltage source $\left(V_{d c}\right)$. Also, due to the huge difference between the dynamics of the charging circuit and Li-ion battery, which is commonly used in EVs, the battery side is ideally represented as a second fixed DC voltage source $\left(V_{b}\right)$. The steady-state mathematical model for each component in the system based on its equivalent circuit (EC), including HF inverters, power pads and compensation network, are presented in this section. In addition, the mathematical link between these components is stated to predict the entire system's performance. 


\subsubsection{HF H-bridge Inverter Model}

Two HF inverters are used in the system, to supply the wireless coupler with controlled square waves at the resonant frequency, as depicted in Figure 4.3. Both inverters generate periodic square or quasi-square wave voltages $\left(V_{p i}\right.$ and $\left.V_{s i}\right)$. The steady-state inverter output voltages are represented, in terms of the system design and control parameters $(\alpha, \beta$, and $\delta)$, based on Fourier series analysis, as given in Equation (4-1) [75]. Only odd harmonics appear in the equations due to the symmetry around the origin.

$$
\begin{aligned}
V_{p i} & =\frac{4}{\pi} V_{d c} \sum_{n=1,3, \ldots}^{\infty} \frac{1}{n} \cos \left(n \omega_{s} t-\frac{n \alpha}{2}\right) \sin \left(\frac{n \alpha}{2}\right) \\
V_{s i} & =\frac{4}{\pi} V_{b} \sum_{n=1,3, \ldots}^{\infty} \frac{1}{n} \cos \left(n \omega_{s} t-\frac{n \alpha}{2}+n \delta\right) \sin \left(\frac{n \beta}{2}\right)
\end{aligned}
$$

where, $n$ is the number of harmonics, $\alpha$ is the PPM parameter of the primary inverter, $\beta$ and $\delta$ are the PPM parameters of the secondary inverter, and $\omega_{s}$ is the switching frequency for both primary and secondary circuits, which is equal to the resonant frequency $\omega_{r}$.

The resonant frequency is chosen based on the self-inductance of the coupler coils to be independent on the mutual inductance and the misalignment between the two sides during the operation, as given in Equation (4-2).

$$
\omega_{s}=\omega_{r}=1 / \sqrt{L_{p c} C_{p}}=1 / \sqrt{L_{s c} C_{s}}
$$

where, the subscript ' $p$ ' stands for the primary side and ' $s$ ' for the secondary circuit, $L_{p c}$ and $L_{s c}$ are the pad self-inductances, and $C_{p}$ and $C_{s}$ are the compensation capacitors.

\subsubsection{Magnetic Coupler Model}

Typically, for high power applications (e.g. EVs), the power pads consists of copper coils and magnetic ferrite martials. The magnetic coupling between the primary and 
secondary power pads is demonstrated in this work by the T-model representation of the mutual coupling. In this representation, the effect of the magnetic material losses and nonlinearities are neglected, and only the resistive losses of the coils are considered. These are accepted assumptions for inductive power transfer systems, due to the following reasons [147]:

- Typically, the magnetic material used in the design of the IPT system is a composition of different soft ferrites. The most popular combinations are manganese and zinc (MnZn) magnetic material. This compound exhibits good magnetic properties and has a rather high intrinsic electric resistivity. These materials can be used up to very high frequencies without laminating (up to $10 \mathrm{MHz}$ ) [152]. As an example that is considered in this work, the industrial type ferrite N87, which has very soft behavior and nearinsulating character, makes it ideal for uses in $10 \mathrm{kHz}-10 \mathrm{MHz}$ range of frequencies in the design of high-power applications. This kind of material shows low hysteresis and eddy current losses [152].

- The operating frequency of IPT systems for EV applications is less than $100 \mathrm{kHz}(81.38$ $90 \mathrm{kHz}$ ) [149], which is relatively low compared with the maximum operating frequency that this material can handle $(10 \mathrm{MHz})$. Thus, this relatively low-frequency range leads to insignificant magnetic losses.

- The magnetic losses mainly depend on the volume of the magnetic core. The core that is typically used in IPT systems is small compared with the entire volume of the system. In contrast with the conventional transformer, the core is huge and it introduces large magnetic losses, which cannot be neglected. This small core results in negligible magnetic losses compared with the other losses in the system. 


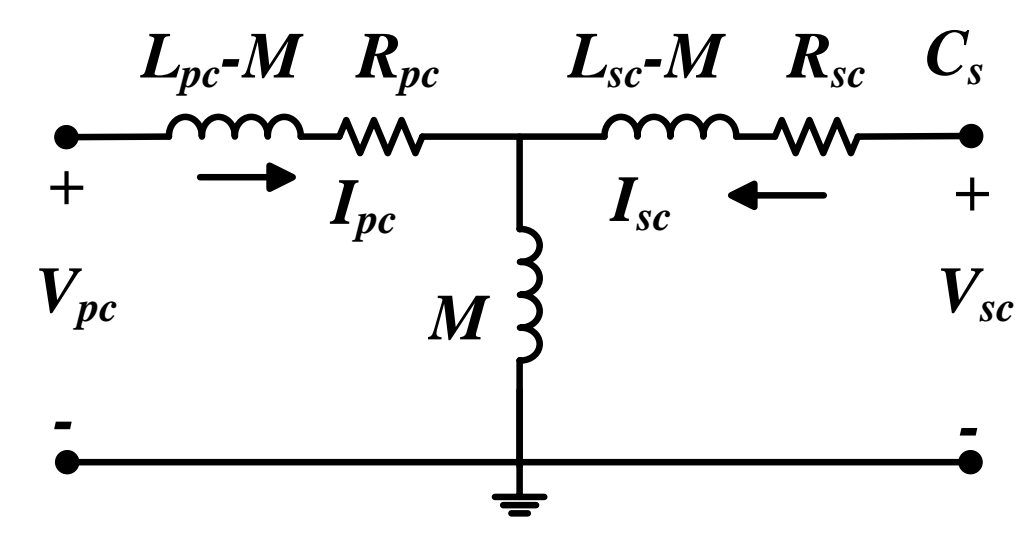

Figure 4.7. T-model representation of wireless coupler.

The mutual inductance is described in terms of the circuit parameters and the coupling factor $(k)$, as given in Equation (4-3).

$$
M=k \sqrt{L_{p c} L_{s c}}
$$

Although the analysis are achieved for symmetrical BIWPTS, in which the two sides are identical, the two sides are represented in the mathematical model by different parameters to present the general unsymmetrical case. The T-model equivalent circuit, as a recognized model, is used to represent the mutual inductance in the system and represent the system with one equivalent circuit instead of two isolated circuits with mutual inductance. In the T-model representation, although the direct correlation with turns-ratio is missing, its effect on the system is included in the model parameters $\left(L_{p c}, L_{p c}\right.$ and $\left.M\right)$ [153]. These parameters are considered as constant in the model and were measured using the RLC meter. Thus, general turns ratio (other than one) was internally considered in the model by using different symbols for each side. A different number of turns will be reflected on the values of self and mutual inductances of the wireless coupler. The T-model 
representation is a general structure that can be used to represent unsymmetrical coils with mutual coupling. It has been used in the literature for unsymmetrical IPT system, such as in [154].

\subsection{Steady-State Mathematical Model of Different Topologies of BIWPTS}

In the steady-state equivalent circuit, the two HF inverters are represented as two controlled AC voltage sources with output voltages given in Equation (4-1). The DC-bus and the EV's battery are represented by two fixed DC sources ( $V_{d c}$ and $V_{b}$, respectively). These sources are linked with the coupler model equivalent circuit and the compensation elements to develop the whole steady-state equivalent circuit model. This circuit is deduced for the three main compensation topologies: LC-series, LC-parallel and LCL topology. Using electric circuits' analysis, the equivalent circuit of each topology is analyzed and two $\pi$-models are developed to investigate the system's currents in terms of the inverter voltages. The first model is shown in Figure 4.7, which represents inverters' currents $\left(I_{p i}\right.$ and $\left.I_{s i}\right)$ in terms of their voltages $\left(V_{p i}\right.$ and $\left.V_{s i}\right)$. The admittance matrix of this model is stated mathematically in Equation (4-4).

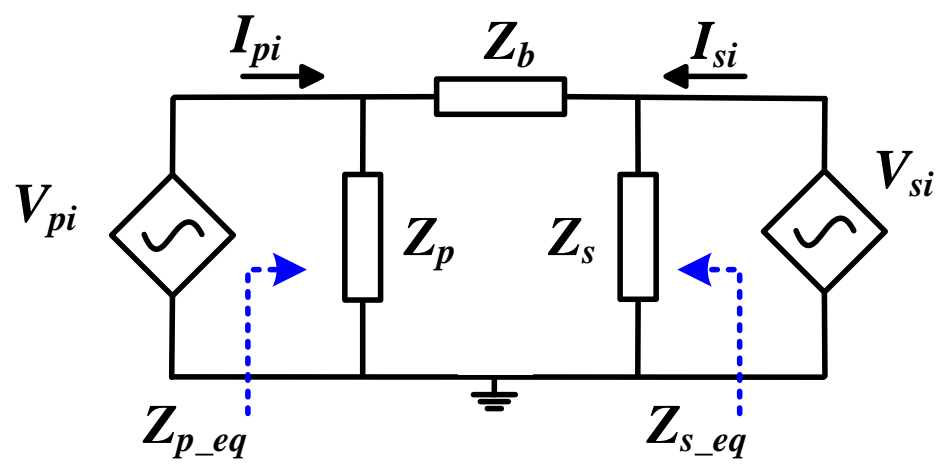

Figure 4.8. $\pi$-model of BIWPTS for inverter's currents in terms of inverter's voltages. 


$$
\left[\begin{array}{l}
I_{p i} \\
I_{s i}
\end{array}\right]=\left[\begin{array}{cc}
\frac{\left(z_{p}+Z_{b}\right)}{Z_{p} Z_{b}} & -1 / Z_{b} \\
-1 / Z_{b} & \frac{\left(Z_{s}+Z_{b}\right)}{Z_{s} Z_{b}}
\end{array}\right]\left[\begin{array}{c}
V_{p i} \\
V_{s i}
\end{array}\right]
$$

where, $Z_{p}, Z_{b}$ and $Z_{s}$ are the model parameters which are different for each configuration, as is explained in detail in the next sections.

The second $\pi$-model demonstrates the pad currents $\left(I_{p c}\right.$ and $\left.I_{s c}\right)$ as functions of $V_{p i}$ and $V_{s i}$, as indicated in Figure 4.8 and represented mathematically in Equation (4-5).

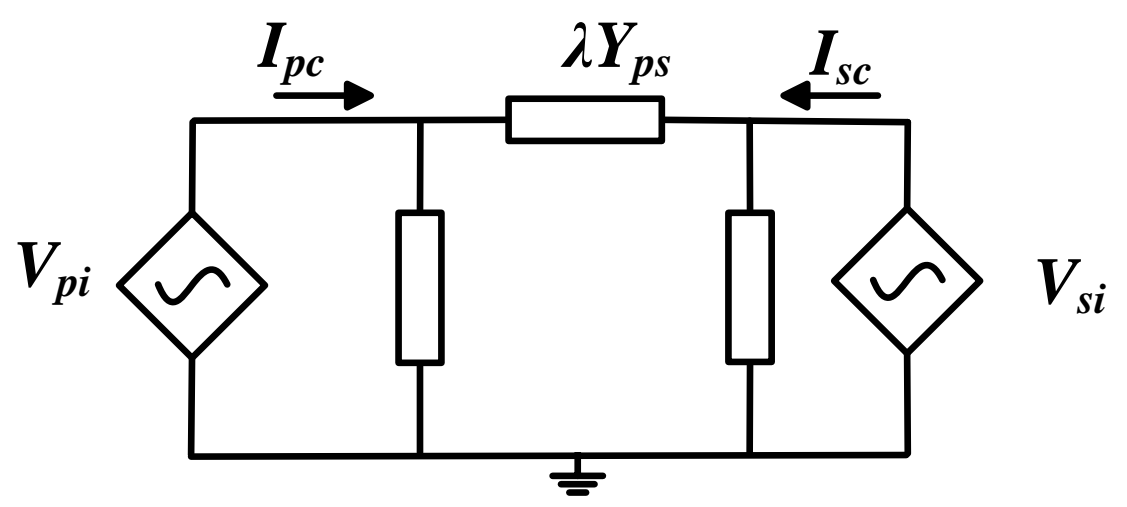

Figure 4.9. $\pi$-model of BIWPTS for coil's currents in terms of inverter's voltages.

$$
\left[\begin{array}{l}
I_{p c} \\
I_{s c}
\end{array}\right]=\lambda\left[\begin{array}{ll}
Y_{p p} & Y_{p s} \\
Y_{s p} & Y_{s S}
\end{array}\right]\left[\begin{array}{l}
V_{p i} \\
V_{s i}
\end{array}\right]
$$

where, $\lambda, Y_{p p}, Y_{s s}, Y_{p s}$ and $Y_{s p}$ are the model parameters which are different in each structure.

This model is based on the harmonic contents, and all the presented parameters and variables need to be solved at each harmonic component and added together. By knowing the admittance matrix models, the system steady-state response can be evaluated and many 
variables can be calculated, such as the active and reactive power, the charging and discharging efficiency, and primary and secondary PF. Also, the equivalent impedance can be easily obtained from the $\pi$-model in Figure 4.7, by applying Thevenin's theory, as given in Equation (4-6). This impedance model is very useful for tuning and evaluating the compensation capacitors [102].

$$
\begin{aligned}
Z_{p_{-} e q} & =Z_{p} Z_{b} /\left(Z_{p}+Z_{b}\right) \\
Z_{s_{-} e q} & =Z_{s} Z_{b} /\left(Z_{s}+Z_{b}\right)
\end{aligned}
$$

\subsubsection{LC-Series Compensation Network}

In this arrangement, the capacitor is connected in series with the pad on both the primary and secondary sides. The steady-state equivalent circuit is shown in Figure 4.9. The inverter and pad currents are the same and are represented by the first model given in Equation (4-4). The $\pi$-model parameters for this configuration are described in Equation $(4-7)$.

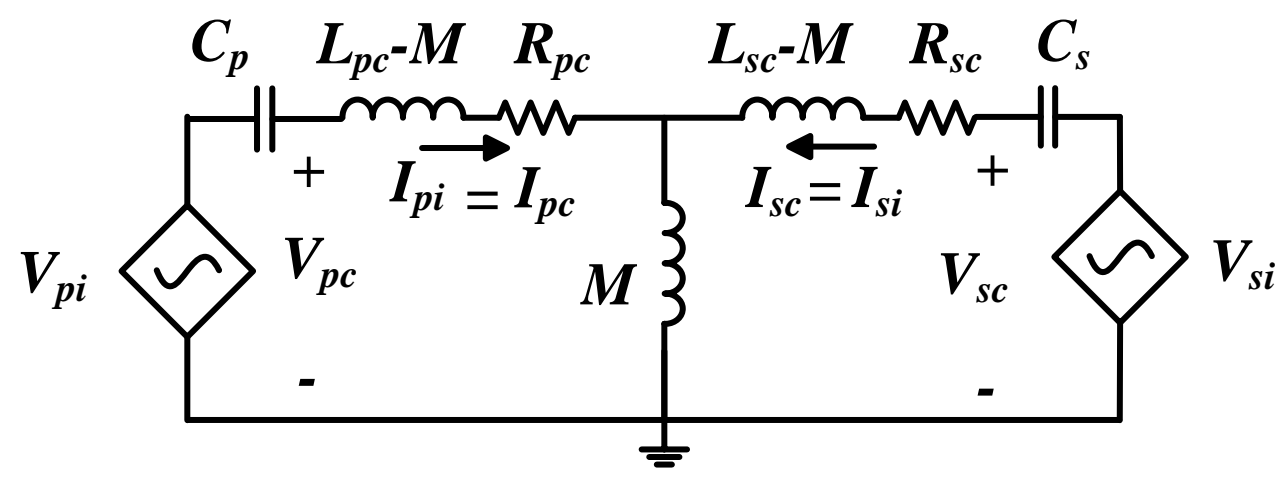

Figure 4.10. BIWPTS equivalent circuits using T-model mutual coupling for LC-series topology. 


$$
\begin{aligned}
& Z_{b}=\left(\omega_{s}{ }^{4} M^{2} C_{p} C_{s}-Z_{p 1} Z_{s 1}\right) /\left(j \omega_{s}{ }^{3} M C_{p} C_{s}\right) \\
& Z_{p}=\left(Z_{p 1} Z_{s 1}-\omega_{s}{ }^{4} M^{2} C_{p} C_{s}\right) /\left[j \omega_{r} C_{p}\left(\omega_{s}{ }^{2} M C_{s}+Z_{s 1}\right)\right] \\
& Z_{s}=\left(Z_{p 1} Z_{s 1}-\omega_{s}{ }^{4} M^{2} C_{p} C_{s}\right) /\left[j \omega_{r} C_{s}\left(\omega_{s}{ }^{2} M C_{p}+Z_{p 1}\right)\right]
\end{aligned}
$$

where,

$$
\begin{gathered}
Z_{p 1}=1-\omega_{s}^{2} L_{p c} C_{p}+j \omega_{s} C_{p} R_{p i} \\
Z_{s 1}=1-\omega_{s}^{2} L_{s c} C_{s}+j \omega_{s} C_{s} R_{s i} .
\end{gathered}
$$

By knowing the currents, the voltage across the coils can be estimated using Equation (4-8).

$$
\begin{aligned}
V_{p c} & =V_{p i}+\frac{j}{\omega_{s} C_{p}} I_{p i} \\
V_{s c} & =V_{s i}+\frac{j}{\omega_{s} C_{s}} I_{s i}
\end{aligned}
$$

\subsubsection{LC-Parallel Compensation Network}

In this structure, the PFC capacitors are attached in parallel with the power pads. The steady-state equivalent circuit is given in Figure 4.10. In this case, the inverter and pad currents are different. They are described by the two admittance-matrix model parameters given in Equations (4-9) and (4-10).

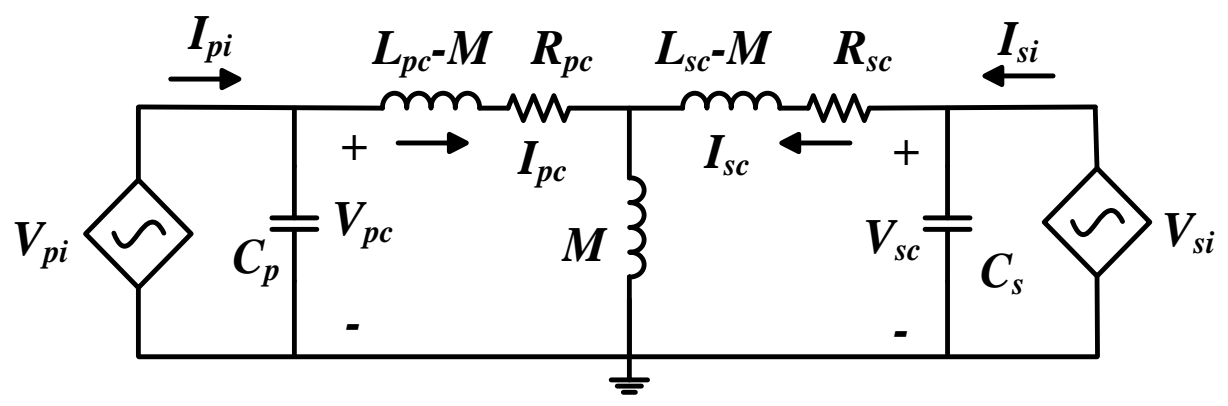

Figure 4.11. BIWPTS equivalent circuits using T-model mutual coupling for LC-parallel topology. 


$$
\begin{gathered}
Z_{b}=\left[\omega_{s}^{2} M^{2}+\left(j \omega_{s} L_{s c}+R_{s c}\right)\left(j \omega_{s} L_{p c}+R_{p c}\right)\right] /\left(j \omega_{s} M\right) \\
Z_{p}=\left(j \omega_{s} M Z_{b}\right) /\left[j \omega_{s}\left(L_{s c}-M\right)+R_{s c}-\omega_{s}^{2} M C_{p} Z_{b}\right] \\
Z_{s}=\left(j \omega_{s} M Z_{b}\right) /\left[j \omega_{s}\left(L_{p c}-M\right)+R_{p c}-\omega_{s}^{2} M C_{s} Z_{b}\right] \\
Y_{p p}=\frac{-j \omega_{s} M V_{s i}+\left(j \omega_{s} L_{s c}+R_{s c}\right)}{\omega_{s}^{2} M^{2}+\left(j \omega_{s} L_{s c}+R_{s c}\right)\left(j \omega_{s} L_{p c}+R_{p c}\right)} \\
Y_{s s}=\frac{-j \omega_{s} M V_{p i}+\left(j \omega_{s} L_{p c}+R_{p c}\right) V_{s i}}{\omega_{s}^{2} M^{2}+\left(j \omega_{s} L_{s c}+R_{s c}\right)\left(j \omega_{s} L_{p c}+R_{p c}\right)}
\end{gathered}
$$

where, $\lambda=1, Y_{s p}=Y_{p s}=0$.

In this case, the coil voltages are the same as the inverter voltages. The direct

connection of the voltage source inverters leads to very large $\left(\frac{d v}{d t}\right)$ across the capacitors, which results in large current spikes to be drawn from the inverter. These spikes are very damaging for the inverter and lead to poor system's performance. Thus, L-filters need to be added to each inverter output to block the HF harmonics in the currents and remove the current spikes. This modification leads to the LCL-compensation network, which is analyzed in the next section.

\subsubsection{LCL Compensation Network}

In this configuration, a second coil is inserted in series to each inverter output in the LC-parallel topology (Figure 4.11). This filter converts the voltage-source inverter into a current source supply, which is desirable for EV's battery operation. The filter inductance is chosen to reduce the harmonics in the inverter current and allow a certain amount of active and reactive power transfer. The admittance matrix model parameters for LCL-entity are given in Equations (4-11) and (4-12). 


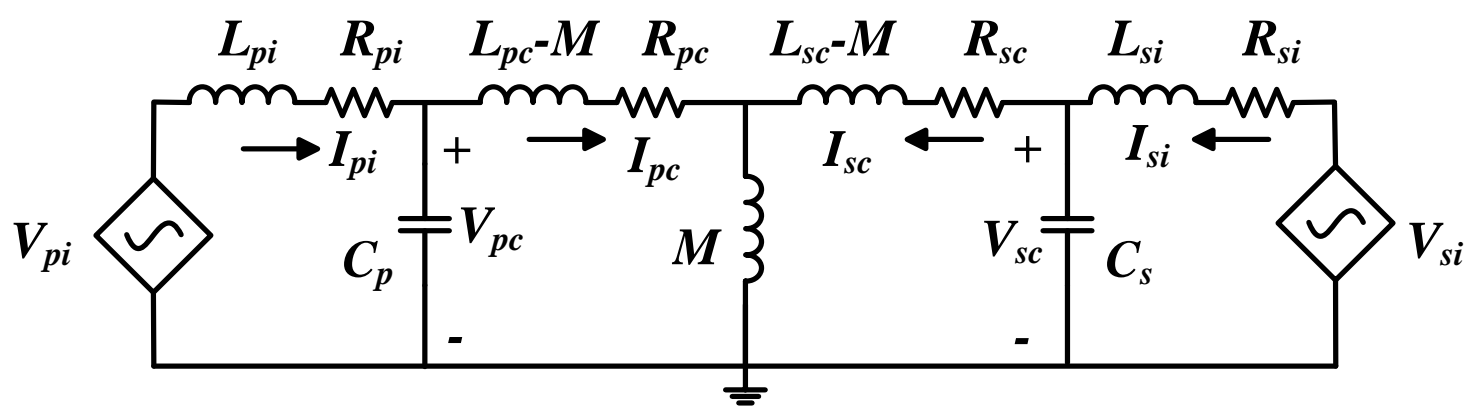

Figure 4.12. BIWPTS equivalent circuits using T-model mutual coupling for LCL topology.

$$
\begin{aligned}
& Z_{b}=\left(Z_{p m} Z_{s m}\right) /\left(j M \omega_{s}\right)+Z_{p m} Z_{s 2}+Z_{s m} Z_{p 2} \\
& Z_{p}=\left(Z_{p m} Z_{b}\right) /\left(Z_{b}\left(\omega_{s}^{2} M C_{p}+Z_{p 2}\right)\right)-Z_{s m}-Z_{p m} \\
& Z_{s}=\left(Z_{s m} Z_{b}\right) /\left(Z_{b}\left(\omega_{s}^{2} M C_{s}+Z_{s 2}\right)\right)-Z_{p m}-Z_{s m}
\end{aligned}
$$

where,

$$
\begin{gathered}
Z_{p 2}=1-\omega_{s}^{2} L_{p i} C_{p}+j \omega_{s} C_{p} R_{p i} \\
Z_{s 2}=1-\omega_{s}^{2} L_{s i} C_{s}+j \omega_{s} C_{s} R_{s i} \\
Z_{p m}=Z_{p 1}\left[j \omega_{s}\left(L_{p c}-M\right)+R_{p c}\right]+j \omega_{s} L_{p i}+R_{p i} \\
Z_{s m}=Z_{s 1}\left[j \omega_{s}\left(L_{s c}-M\right)+R_{s c}\right]+j \omega_{s} L_{s i}+R_{s i} \\
\lambda=1 /\left[Z_{b}\left(\omega_{s}^{2} M{ }^{2}+Z_{s c}+Z_{p c}\right)\right] \\
Y_{p p}=Z_{s c}\left[Z_{b}-\left(1-Z_{b} / Z_{p}\right) Z_{p i}\right]-j \omega_{s} M Z_{s i} \\
Y_{p s}=Z_{p i} Z_{s c}+j \omega_{s} M\left[Z_{s i}\left(1+Z_{b} / Z_{p}\right)-Z_{b}\right] \\
Y_{s p}=Z_{s i} Z_{p c}+j \omega_{s} M\left[Z_{p i}\left(1+Z_{b} / Z_{p}\right)-Z_{b}\right] \\
Y_{s s}=Z_{p c}\left[Z_{b}-\left(1-Z_{b} / Z_{p}\right) Z_{s i}\right]-j \omega_{s} M Z_{p i}
\end{gathered}
$$

where,

$$
Z_{p c}=R_{p c}+j \omega_{s} L_{p c}
$$




$$
\begin{aligned}
& Z_{s c}=R_{s c}+j \omega_{s} L_{s c}, \\
& Z_{p i}=R_{p i}+j \omega_{s} L_{p i}, \\
& Z_{s i}=R_{s i}+j \omega_{s} L_{s i} .
\end{aligned}
$$

In this case, the coil's voltage is different from the inverter's voltage, due to the voltage drop on the filter. By knowing the inverter current, the voltage across the coils can be estimated using Equation (4-13).

$$
\begin{aligned}
& V_{p c}=V_{p i}-j \omega_{s} L_{p i} I_{p i} \\
& V_{s c}=V_{s i}-j \omega_{s} L_{s i} I_{s i}
\end{aligned}
$$

In this topology, the compensation capacitors are designed based only on the resonance frequency and the coupler self-inductances, as given in Equation (4-2). The filter parameters $\left(L_{p i}, L_{s i}, R_{p i}\right.$ and $\left.R_{s i}\right)$ are assigned based on the power transfer capabilities. Even though, the BIWPTS performance is very sensitive to the variation of the filter inductances with respect to the coupler inductances, as indicated in Figure 4.12. The figure shows the fundamental equivalent impedance seen from the primary side $\left(Z_{p-e q}\right)$ with respect to the operating frequency at different filter designs. In this study, the system is designed to resonate at $40 \mathrm{kHz}$. As it can be noticed, the resonance frequency matches the design frequency only when the filter and the coil inductances are equal $\left(L_{p i}=L_{p c}\right)$ and by assuming a symmetrical system $\left(L_{s i}=L_{s c}\right)$. In this case, the system exhibits zero impedance angle which means UPF operation. Any deviation of $L_{p i}$ from $L_{p c}$ results in resonance frequency deviation. In this sense, the operating frequency needs to be adjusted to make the system work at resonance. For more clear analysis, the effect of the variation of $L_{p i}$ 
and $L_{p c}$ on the system impedance is investigated in Figure 4.13. As it can be observed, the resonance always occurs at the operating frequency $(40 \mathrm{kHz})$ when $L_{p i}=L_{p c}$. Thus, in order to keep the operating frequency of BIWPTS the same as the resonant design frequency, the filter inductances should match with the pad inductances (i.e $L_{p i}=L_{p c}$ and $L_{s i}=L_{s c}$ ) [76], [102]. In addition, this synthesis allows the system to behave as a current source topology, supplying a fixed current regardless of the loading conditions [137]. This characteristic is very desirable for EV charging operation, in which the battery is charged at the constant current mode.

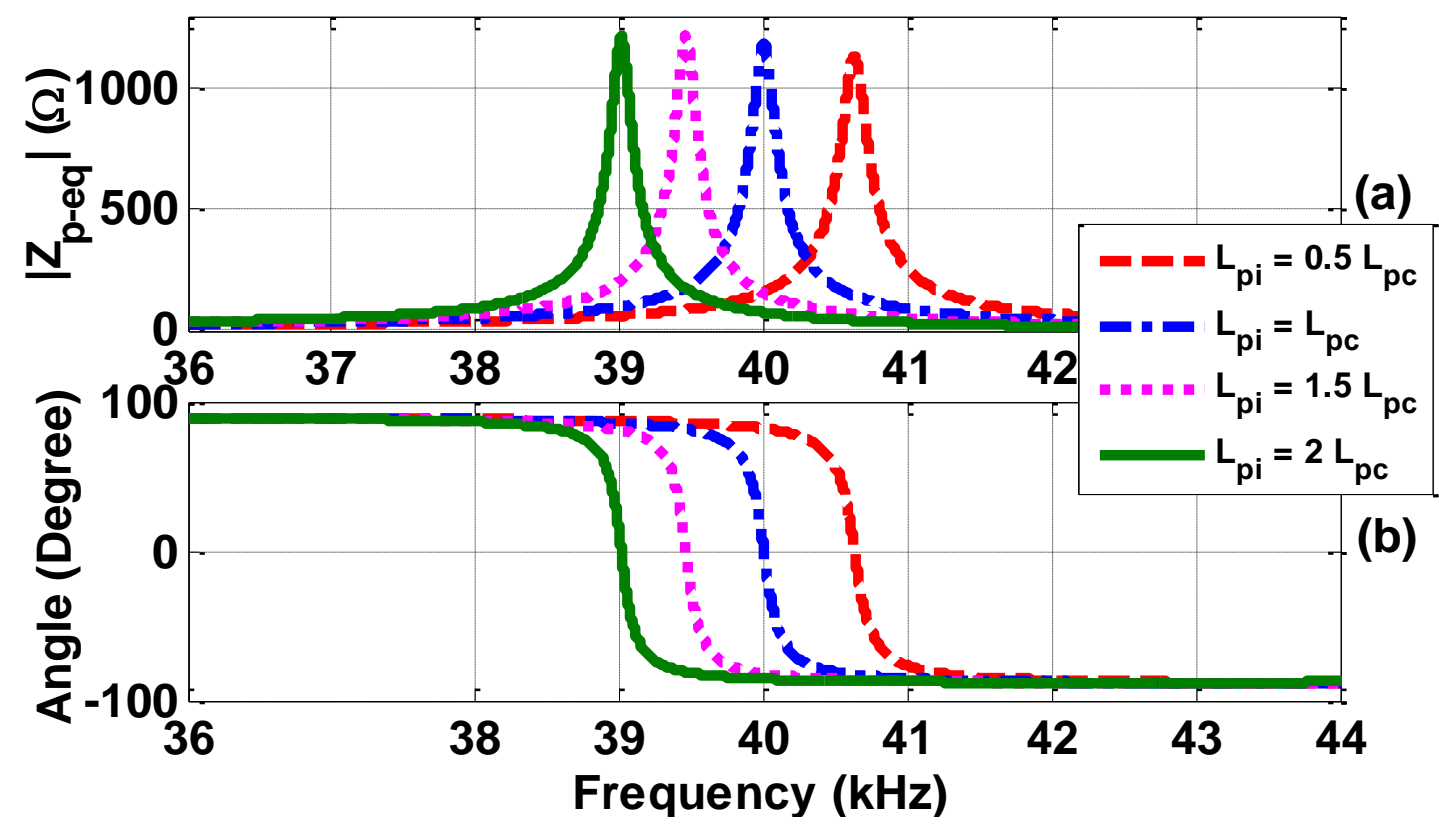

Figure 4.13. Effect of filters inductances on the fundamental impedance frequency response for LCL-parallel design. (a) Impedance magnitude. (b) Impedance phase angle. 

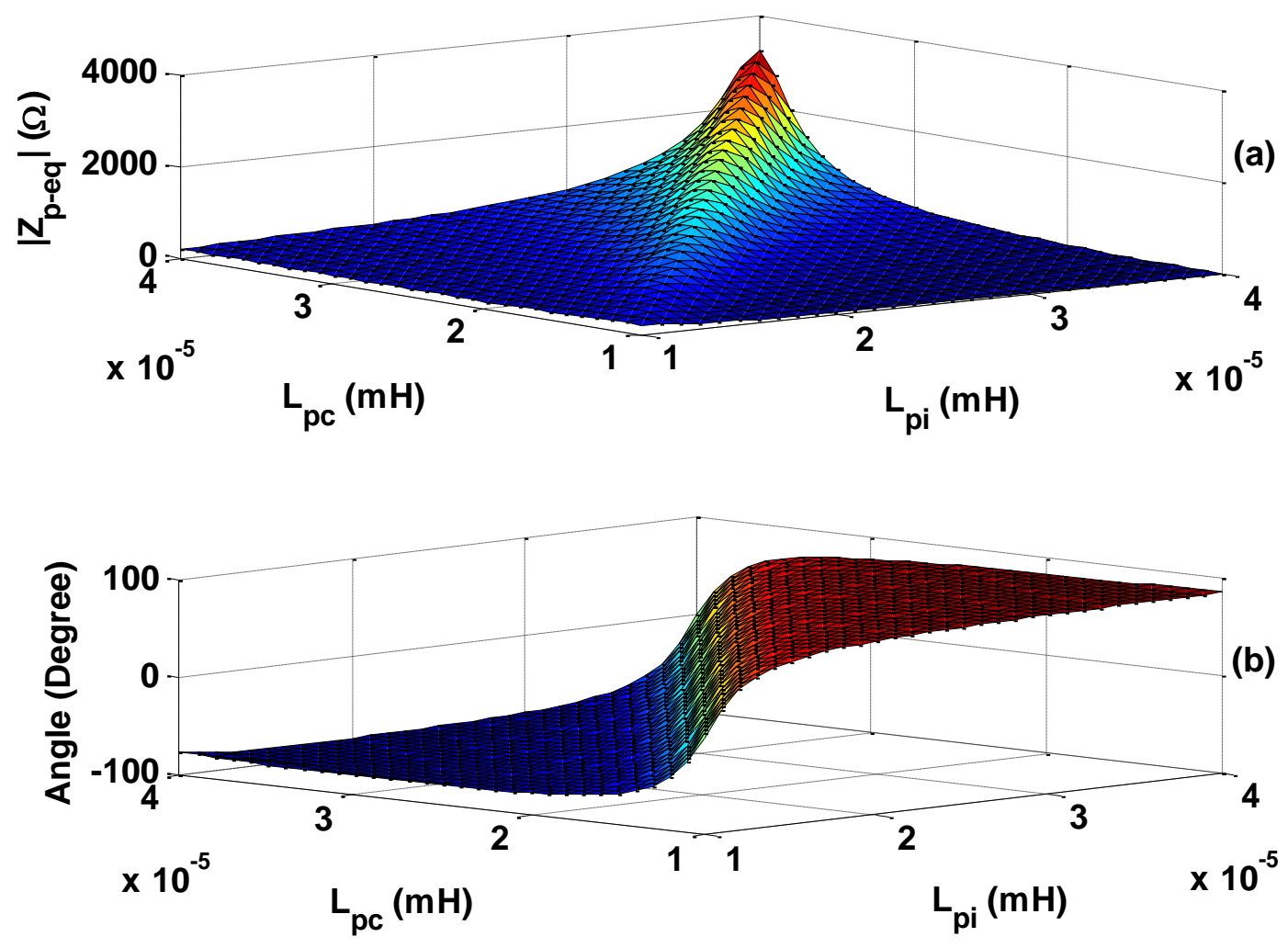

Figure 4.14. Effect of system inductances on the fundamental impedance frequency response for LCL-parallel design $(40 \mathrm{kHz})$. (a) Impedance magnitude. (b) Impedance phase angle.

The effect of the filters' resistances $\left(R_{p i}\right.$ and $\left.R_{p c}\right)$ on the system performance is indicated in Figure 4.14. Generally speaking, the filters' resistances impact on the resonance performance is negligible compared with the pads' resistances. Varying the system resistances affects the impedance magnitude but does not affect the location of the resonance (resonant frequency). It also has a slight effect on the impedance phase angle [Figure 14(b)]. 

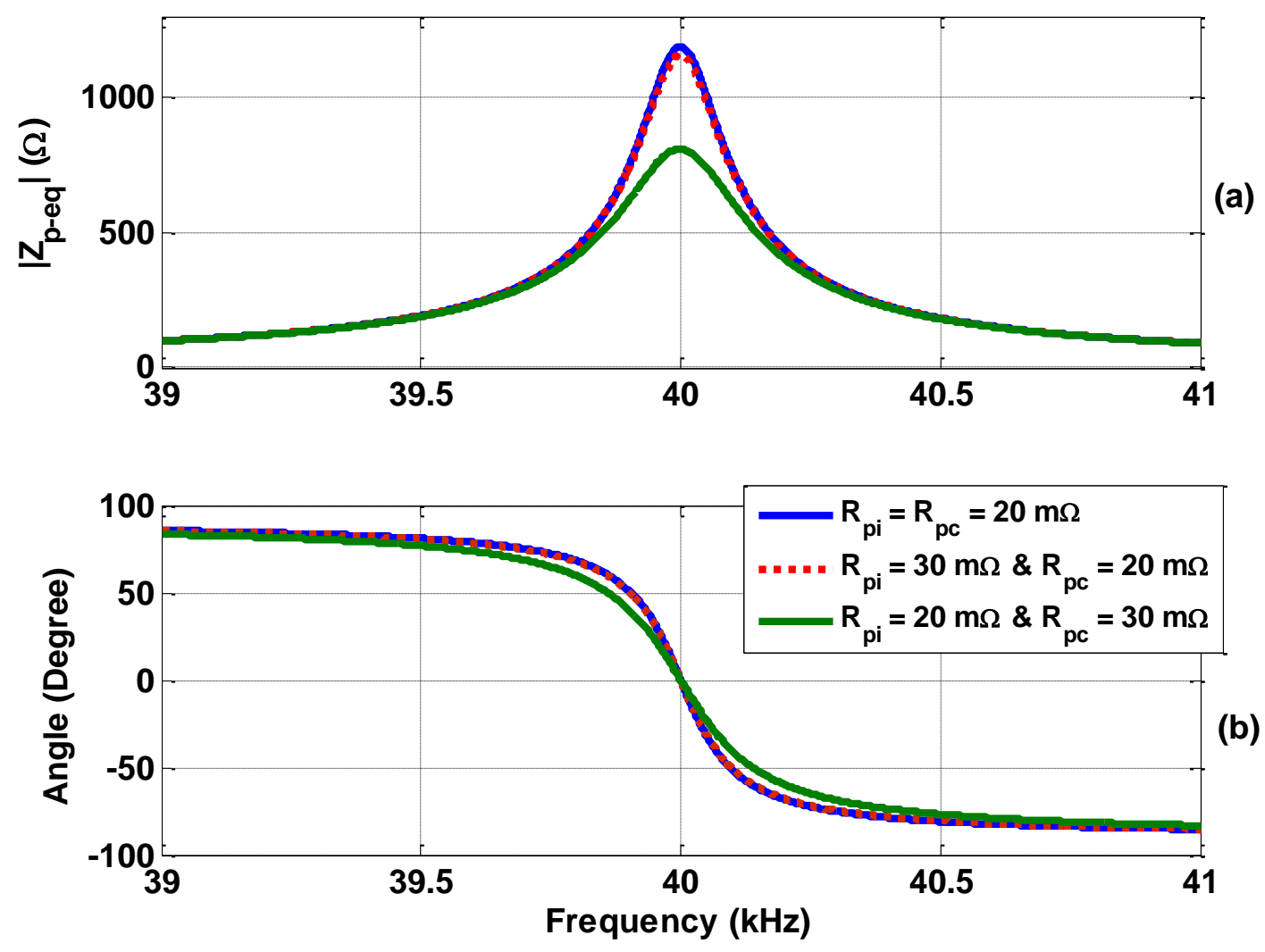

Figure 4.15. Effect of system resistances on the fundamental impedance frequency response for LCL-parallel design. (a) Impedance magnitude. (b) Impedance phase angle.

\subsection{Fundamental Power-Flow Modeling}

The main feature of the BIWPTS is the ability to transfer power from EV to the grid and vice versa, such that EVs can be used to inject or absorb active and reactive power to and from the grid, respectively. Generalized simple formulas for active and reactive powerflow in all structures of BIWPTS are presented in this section. Moreover, the effect of the control parameters $(\alpha, \beta$, and $\delta)$ on the energy-flow between EV and the grid, is described. The power-flow formulas were derived for the fundamental primary active $\left(P_{p_{-} l}\right)$ and 
reactive $\left(Q_{p_{-}}\right)$power. Harmonic components in Equation (4-1) are neglected and formulas for the fundamental component of $V_{p i}\left(V_{p i-1}\right)$ and $V_{s i}\left(V_{s i-1}\right)$ are obtained in Equation (4-14). The $\pi$-model in Equation (4-4) is solved for $I_{p i}$ in terms of $V_{p i}$ and $V_{s i}$ for each system's topology. Then, a simple formula for the fundamental component of $I_{p i}\left(I_{p i-1}\right)$ is obtained, by neglecting the higher order harmonics' components and the resistive losses, and applying the resonance condition given in Equation (4-2). This formula is the same for all topologies, as given in Equation (4-15). By substituting Equations (4-14) and (4-15) in the basic powerflow formulas in Equation (4-16), the final mathematical model for the fundamental primary active and reactive power is described in Equation (4-17).

$$
\begin{gathered}
V_{p i_{-} 1}=\left(\frac{4}{\pi}\right) V_{d c} \cos \left(\omega_{s} t-\frac{\alpha}{2}\right) \sin \left(\frac{\alpha}{2}\right) \\
V_{s i_{-} 1}=\left(\frac{4}{\pi}\right) V_{b} \cos \left(\omega_{s} t-\frac{\alpha}{2}+\delta\right) \sin \left(\frac{\beta}{2}\right) \\
I_{p i_{-} 1}=\frac{j M \gamma}{\omega_{s} L_{p} L_{s}} V_{s i_{-} 1} \\
P_{p_{-} 1}=\operatorname{Real}\left(V_{p i_{-} 1} I_{p i_{-} 1}{ }^{*}\right) \\
Q_{p_{-} 1}=\operatorname{Imag}\left(V_{p i \_1} I_{p i \_}{ }^{*}\right) \\
P_{p_{-} 1}=-\left(\frac{8}{\pi^{2}}\right) \frac{M \gamma}{\omega_{s} L_{p} L_{s}} V_{d c} V_{b} \sin \left(\frac{\alpha}{2}\right) \sin \left(\frac{\beta}{2}\right) \sin (\delta) \\
Q_{p_{-} 1}=-\left(\frac{8}{\pi^{2}}\right) \frac{M \gamma}{\omega_{s} L_{p} L_{s}} V_{d c} V_{b} \sin \left(\frac{\alpha}{2}\right) \sin \left(\frac{\beta}{2}\right) \cos (\delta)
\end{gathered}
$$

where, $\gamma$ is a real value which is a function of the system parameters and is defined for each composition in Table 4.1, $L_{p}$ and $L_{s}$ are the primary and secondary inductances, which are accurately defined in the same table for each structure. 
Table 4.1. $\gamma$-Parameter for Different Topologies.

\begin{tabular}{lll}
\hline \hline Configuration & Inductances & $\gamma$ - equation \\
\hline$L C$-series & $L_{p}=L_{p c} ; L_{s}=L_{s c}$ & $-L_{p c} L_{s c} / M^{2}=-1 / k^{2}$ \\
$L C$-parallel & $L_{p}=L_{p c} ; L_{s}=L_{s c}$ & $L_{p c} L_{s c} /\left(L_{p c} L_{s c}-M^{2}\right)=1 /\left(1-k^{2}\right) \approx 1$ \\
$L C L$-topology & $L_{p}=L_{p i} ; L_{s}=L_{s i}$ & 1 \\
& & \\
\hline \hline
\end{tabular}

As it can be noticed in Equation (4-17), the power-flow in BIWPTS depends on the DC voltage level on both sides and the system design parameters (resonant frequency, coil inductances and mutual coupling between coils). After the design stage, these parameters become fixed except the mutual inductance, which varies based on the vehicle position. Also, the power-flow is a function of the control parameters of the primary and secondary inverters $\left(\alpha, \beta\right.$ and $\delta$ ). Typically, the angle $\delta$ is kept fixed at $\pm 90^{\circ}$ to minimize the reactive power-flow and achieve UPF operation. The sign of $\delta$ is decided to control the power-flow direction to charge or discharge the EV and switch between the various modes of operation (G2V and $\mathrm{V} 2 \mathrm{G}$ ). When $\delta$ is negative, the power flows from the primary to the secondary for charging the $\mathrm{EV}(\mathrm{G} 2 \mathrm{~V})$. However, when $\delta$ is positive, the power transfer occurs from the secondary to the primary for supplying the grid (V2G). This $\delta$ sign notation is applicable for LC-parallel and LCL structures, but for the LC-series, the opposite notation is the correct, due to the negative sign in $\gamma$-equation (see Table 4.1).

The power-flow magnitude can be controlled by varying either the primary side voltage magnitude $V_{p i}$ (using $\alpha$ ) or the secondary side voltage magnitude $V_{s i}$ (using $\beta$ ) while 
keeping the relative phase angle $(\delta)$ at $\pm 90^{\circ}$. However, controlling $V_{p i}$ to regulate the amount of power delivery to a particular secondary is not possible in multiple secondary systems as it affects the power delivered to other EVs. In single secondary IPT systems, the power-flow can be controlled through the modulation of either $V_{p i}$ or $V_{s i}$ but the former is preferable, as IPT systems are usually designed for a given primary current. Moreover, the modulation of $V_{p i}$ changes the primary current and, hence, the induced voltage in the secondary. Consequently, low primary currents at light loads will result in a very high quality factor, Q, (the ratio between the effective AC output voltage and induced voltage) for a given secondary output voltage. Such an operation with high and variable Q is undesirable as the system becomes too sensitive. Therefore $V_{s i}$ of the secondary side converter is modulated to control both forward and reverse power flow while using $V_{p i}$ to maintain a constant primary coil current [137]. Thus, the parameter $\beta$ is used to control the value of the power-flow, which ranges from 0 to maximum when $\beta$ changes from 0 to $180^{\circ}$, respectively. The relative power-flow value between different configurations can be observed from the $\gamma$ parameter equations in Table 4.1. For the same design parameters, in LC-parallel and LCL assembly, the power are almost equal, since $\gamma$ is almost the same and very close to unity. However, for $\mathrm{LC}$-series arrangement, $\gamma$ is inversely proportional to the square of the coupling factor. When the coupling factor changes from 0.3 to 0.1 (which is the typical range in the IPT system), $\gamma$ varies from $11.11(\approx 10)$ to 100 , respectively. Therefore, the power of the LC series network ranges from 10 to 100 times the power of the other two structures as well. This indicates that LC-series structure is very sensitive to the coupling factor and the misalignment, which is one of the main limitations of this topology. 


\subsection{Conclusion}

In this chapter, a comprehensive steady-state performance analysis for various configurations of the bidirectional inductive power transfer system in EVs applications is presented. The analysis was based on theoretical harmonics representation for each topology, using Fourier series analysis. For each topology, two different $\pi$-models are

developed, which represent the system's currents in terms of the inverter's voltages. The developed models provide information for inverter currents, inverter voltages, coil currents and coil voltages on both system's sides. It also provides a simple derivation for the system's equivalent impedance, which is very useful to evaluate the design parameters. In addition, the analytical models are utilized to derive simple generic formulas for fundamental active and reactive power-flow in terms of the design and control parameters. The utilization of these models to evaluate the different compensation configurations and compare them is demonstrated in chapter (6). 


\section{Chapter 5 Hardware Implementation of a Dual H-Bridge BIWPTS Prototype}

\subsection{Introduction}

The dual H-bridge BIWPTS under consideration consists of four main components; HF H-bridge converters, power pads, compensation network and controllers, as depicted in Figure 4.3. Building such a kind of system for EVs' applications presents huge challenges. All the system's components require robust and reliable design to be able to handle the high-power and high-frequency operation. The semiconductor switches need to be carefully chosen to support the high-voltage and current stresses during the resonance operation. The power pads must be designed to provide maximum coupling performance and less sensitivity for the misalignment in the system. This chapter discusses design considerations for the entire BIWPTS's component. It presents more details about the HF converter design and optimization. An effective design strategy for the HF H-bridge resonant converter is investigated. The driving and power component selections and design issues are discussed in detail. The driver and the inverter circuits were implemented together in one PCB. The PCB layout was optimized for reducing the electromagnetic interference (EMI). Moreover, two snubber circuits are developed and added to the inverter PCB to minimize the output voltage ringing phenomenon. Also, the proposed structure includes voltage and current protection for both the DC and AC sides. Additionally, the hardware implementation of phase-shift control based on the analog PLL circuit is investigated and tested. In addition, two symmetrical circular power pads are built to form the wireless coupler. The practical design considerations for the power pad and the 
compensation network are discussed as well. All the components are linked together and tested during both the charging and discharging operation.

\subsection{HF H-Bridge Converter and Driver}

One of the most critical components in IPT system is the HF inverter. Many inverter topologies were proposed in the literature for the power supply design [87]. The most popular one is the voltage-source H-bridge converter (VSHBC) structure. VSHBC is a bidirectional topology, which has the capability to operate in both inverting and rectifying modes. It provides flexible management for the power-flow in the system. Design aspects of the HF inverter for induction heating applications are evaluated in [155], [156]. These works presented some design notes without going into detail about the inverter circuit design such as component selection, driver circuit, PCB layout, etc., especially under the hard switching activities. Typically, VSHBC consists of four semiconductor power switches. Power MOSFETs are still preferred for automotive manufactures due to the positive temperature coefficient and their ability to handle high switching frequency, as well as considerations related to the breakdown voltage, cost and reliability requirements. The entire inverter design process is investigated in this section.

\subsubsection{Component Selection}

All the inverter's components are selected based on the power handling and switching requirements of the inverter. Also, the cost and the size are considered during 
the selection process. IXFB110N60P3 Si power MOSFET is chosen for this design. It supports up to the absolute maximum $600 \mathrm{~V}$ and $110 \mathrm{~A}$ with $56 \mathrm{~m} \Omega \mathrm{ON}$ resistance [157]. It provides very wide safe operating area in terms of voltage and current capability, as shown in Figure 5.1. To drive the gates of the four switches of VSHBC, two FAN7391 IC drivers are used. They are monolithic high and low-side gate drive IC, which can drive high-speed MOSFETs that operate up to 600V. They have high current capabilities up to $4.5 \mathrm{~A}$ [158]. Surface mounted ceramic capacitors and the hyper-fast diodes are selected for the driver circuit design to handle the HF operation and the size limitations.

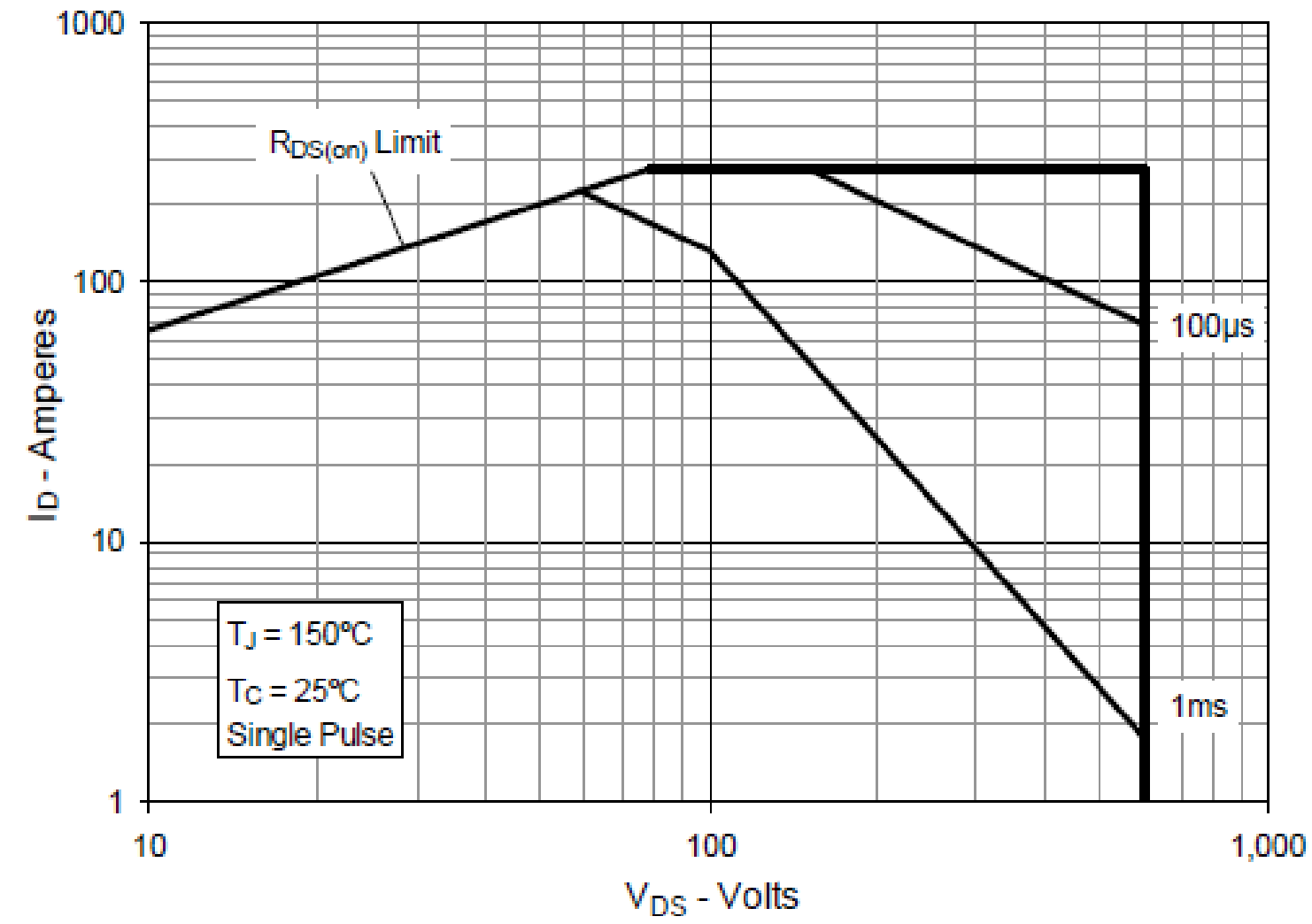

Figure 5.1. Forward-bias safe operating area of IXFB110N60P3 Si power MOSFET [157]. 


\subsubsection{Driver's Circuits Design}

The FAN7391 IC driver is attached to the Bootstrap circuit to drive the power MOSFETs. Two driving circuits are built to drive the entire VSHBC. Each circuit is managing one converter's leg (two MOSFETs). The components of the driving circuit for one leg are shown in Figure 5.2, and the design steps are indicated below [159]. The details of sizing each component in the circuit are discussed in this section.

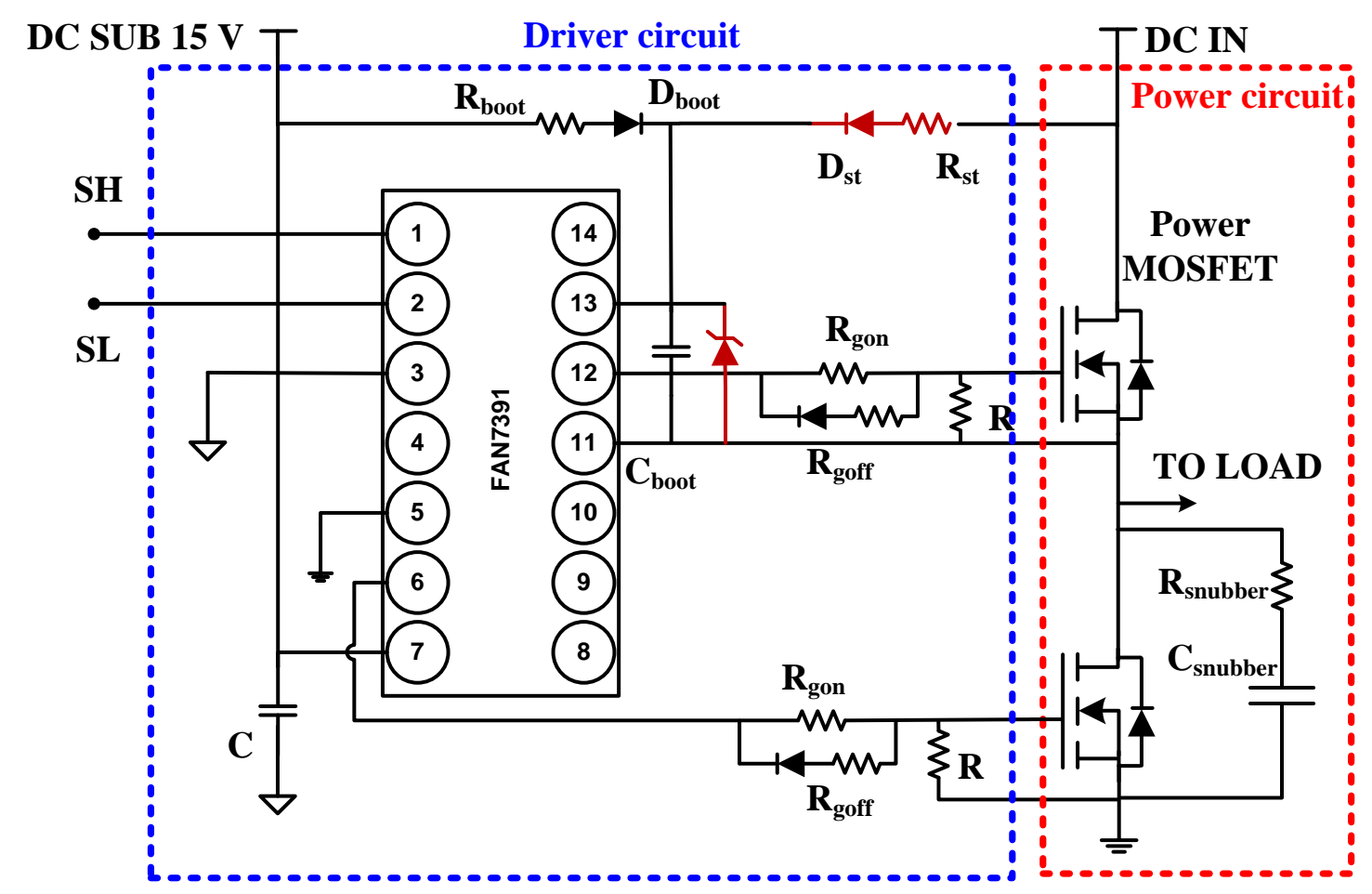

Figure 5.2. Bootstrap driving circuit components using FAN7391 IC driver.

\subsubsection{Sizing the Bootstrap Capacitor (Своо)}

$\mathrm{C}_{\text {воот }}$ is designed based on the maximum allowable voltage drop $\left(\Delta V_{\text {Воот }}\right)$, which depends on the minimum gate-drive voltage for the upper-side switch. Considering the minimum gate-source voltage $\left(V_{G S M I N}\right), \Delta V_{\text {BOоT }}$ is calculated using Equation (5-1). 


$$
\Delta V_{B O O T}=V_{D D}-V_{F}-V_{G S M I N}
$$

where, $V_{D D}$ is the supply voltage of the gate driver and $V_{F}$ is the bootstrap diode forward voltage-drop.

The bootstrap capacitance is estimated using Equation (5-2).

$$
\begin{gathered}
C_{B O O T}=\frac{Q_{T O T A L}}{\Delta V_{B O O T}} \\
Q_{T O T A L}=Q_{G A T E}+\left(I_{L K C A P}+I_{L K G S}+I_{Q B S}+I_{L K}+I_{L K D I O D E}\right) t_{O N}+Q_{L S} Q_{G A T E}
\end{gathered}
$$

where, $Q_{T O T A L}$ is the total charge supplied by the capacitor, $Q_{G A T E}$ is the total maximum gate-charge of the MOSFET switch, $I_{L K G S}$ is the switch gate-source leakage current, $I_{L K C A P}$ is the bootstrap capacitor leakage current, $I_{Q B S}$ is the bootstrap circuit quiescent current of the driver, $I_{L K}$ is the bootstrap circuit leakage current and $Q_{L S}$ is the charge required by the internal level shifter, which is usually $3 \mathrm{nC}$ for all high voltage gate drivers.

\subsubsection{Sizing the Turn-on Gate Resistance $\left(\boldsymbol{R}_{g(o n)}\right)$}

This parameter is very critical and can be determined based on the required output voltage rate of variation $\left(d V_{\text {out }} / d t\right)$, as indicated in Equation (5-3).

$$
\begin{aligned}
& R_{g(o n)}=R_{\text {Total }}-R_{R D V(o n)} \\
& R_{R D V(o n)}=\frac{V_{D D}}{I_{\text {SoURCE }}}, R_{\text {Total }}=\frac{\left(V_{D D}-V_{G S(t h)}\right)}{\left(c_{g d(o f f)} * \frac{d V_{\text {out }}}{d t}\right)}
\end{aligned}
$$

where, $I_{\text {SOURCE }}$ is the source-driver current, $V_{G S(t h)}$ is the gate-drive voltage of the switch, $R_{D R V(o n)}$ is the equivalent ON resistance of the gate-driver, and $C_{g d(o f f)}$ is the miller effect capacitor of the switch. 


\subsubsection{Sizing the Turn-off Gate Resistance $\left(\boldsymbol{R}_{g(o f f)}\right)$}

This element is calculated at the worst case scenario, i.e. when the drain of the MOSFET is in turn-off state, as given in Equation (5-4).

$$
\begin{aligned}
& R_{g(o f f)} \leq \frac{V_{g s(t h)}}{C_{g d^{*} \frac{d V_{0 u t}}{d t}}}-R_{D R V(o f f)} \\
& R_{D R V(o f f)}=\frac{V_{D D}}{I_{S I N K}}
\end{aligned}
$$

\subsubsection{Snubber Circuits Design}

During the HF operation with hard switching activities, a snubber $R C$ circuit is required to minimize the ringing caused by the recovery-induced oscillations and the conducted EMI due to the stray inductances and capacitances. Two $R C$ snubber circuits are designed and added to the inverter circuit, as depicted in Figure 5.2. By knowing the parasitic capacitance $\left(C_{p}\right)$ from the MOSFET data sheet, the snubber capacitance $\left(C_{\text {snubber }}\right)$ will be within the range of (0.5-2) $C_{p}$ [160]. The ringing frequency $\left(F_{p}\right)$ is measured and used in Equation (5-5) to estimate the snubber resistance $\left(R_{\text {snubber }}\right)$. This resistance needs to be carefully designed to handle the high power flow through it. The wattage of $R_{\text {snubber }}$ is calculated based on the switching frequency $\left(F_{s w}\right)$ and the snubber voltage $\left(V_{\text {snubber }}\right)$, as given in Equation (5-6).

$$
\begin{gathered}
R_{\text {Snubber }}=\frac{1}{4 \pi F_{p} C_{p}} \\
P_{\text {Snubber }}=\frac{1}{2} C_{\text {Snubber }} V_{\text {Snubber }}{ }^{2} F_{\text {SW }}
\end{gathered}
$$




\subsubsection{VSHBC PCB Layout Design}

In the proposed design, all the inverter's components and circuits are implemented in one PCB, including the power switches, snubber, driver and protection elements. This design helps to minimize the inverter size and weight, and the EMI effect. The details of a double-layer converter PCB are presented in this section.

PCB layout design is a critical stage in the inverter circuit planning process. It is important to maximize the power transfer capability for all the stages and minimize the switching losses. Moreover, it can significantly reduce the magnitude and the duration of the ringing on the inverter outputs. In the proposed design, the components are arranged in a way to minimize the tracks' length and avoid creating loops and junctions between the top and bottom layers. The components of each inverter's leg are equally distributed to achieve symmetrical square output voltage waveforms. The power tracks are assigned to be straight, short and continuous to minimize the conducted and radiated EMI in the circuit. The node length between the negative terminal of the input capacitor and the source pin of the MOSFET is diminished to reduce the parasitic elements' effect. Also, ceramic input capacitors are considered to provide low equivalent series inductance (ESL). A comparison between the optimized PCB layout and an arbitrary (non-optimized) one is presented in Figures 5.3 and 5.4, respectively.

Two protection schemes are implemented in this design: over-current and over-voltage

protection. The former is achieved by using current-limiting fuses in the DC power input ports. The latter is performed by using a varistor in series with a current-limiting fuse across the AC output terminals. 


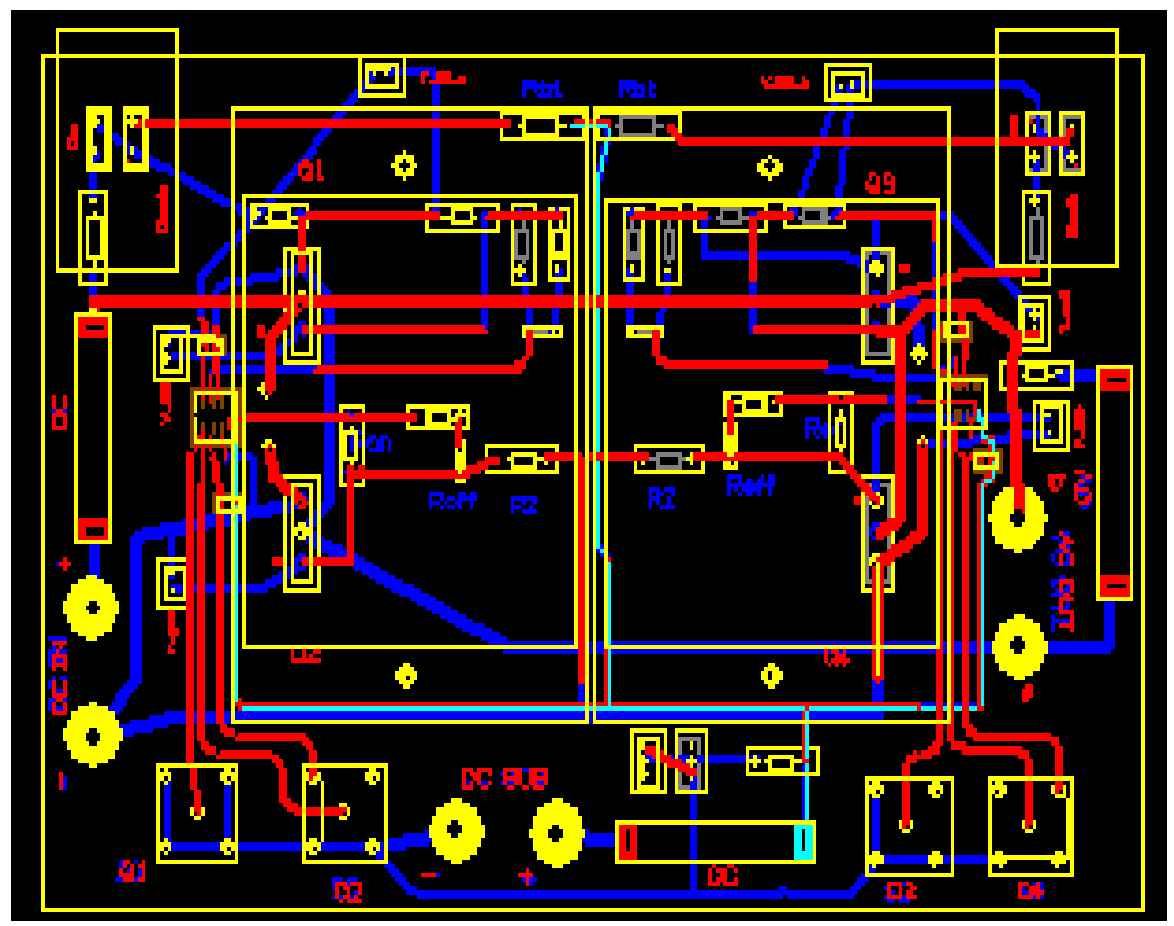

Figure 5.3. Layout of the optimized design converter's PCB.

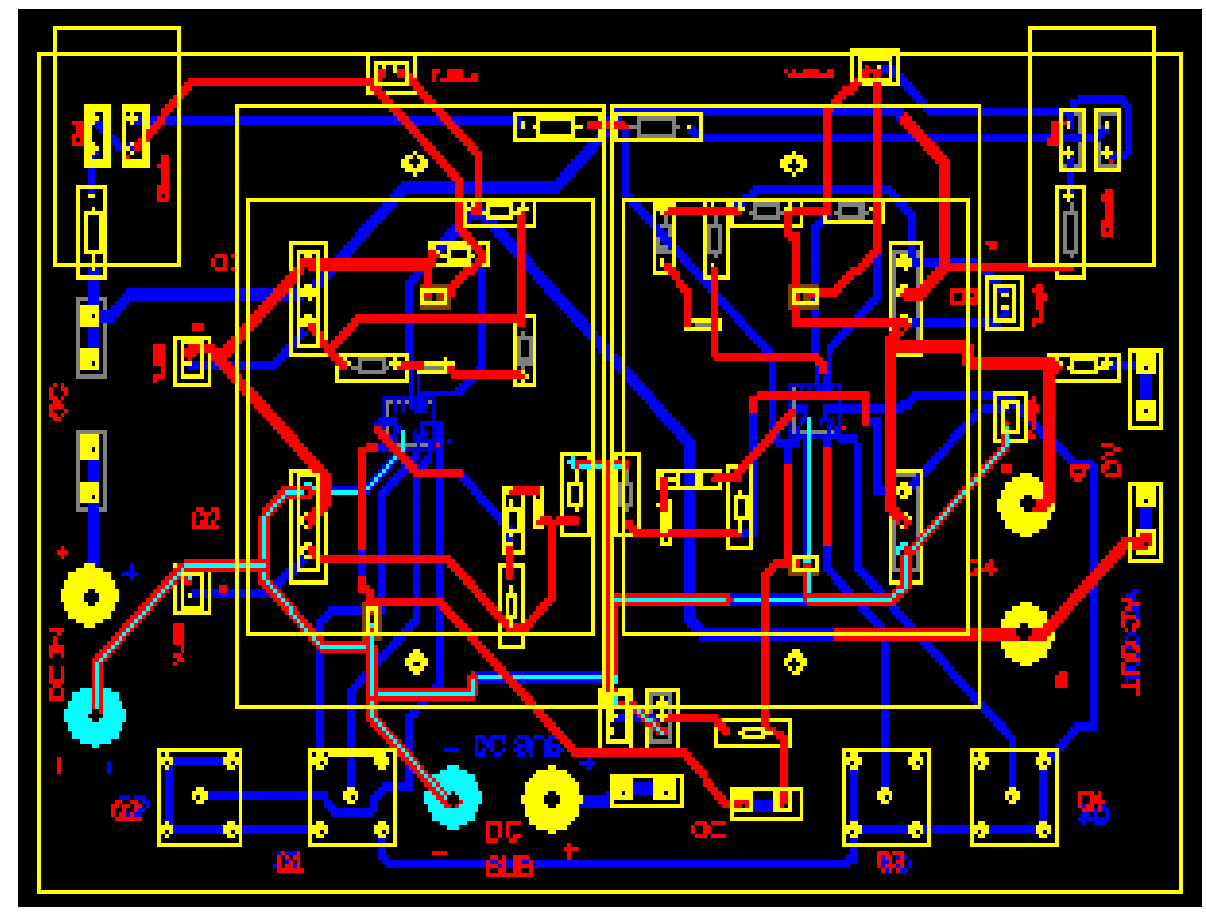

Figure 5.4. Layouts of an arbitrary design converter's PCB. 


\subsection{Phase-shift Control and Modulation}

In HF applications, like IPT, it is difficult to use the conventional modulation techniques to generate the switching signals of the power MOSFETs. Typically, other modulation techniques, in which the switching frequency is the same as the power frequency, are considered [161]-[166]. The most commonly used approach in IPT systems is the pulse-phase modulation (phase-shift) technique [102], [162], [165]. In this procedure, the switching signals have a fixed 50\% duty cycle at the resonant frequency, as indicated in Figure 5.5. The inverter output voltage is controlled by adjusting the phase-shift between the switching signals of the two legs $(\alpha)$. The figure also shows the dead-time periods $\left(t_{d}\right)$ that need to be introduced between the switching of two MOSFETs in the same leg.

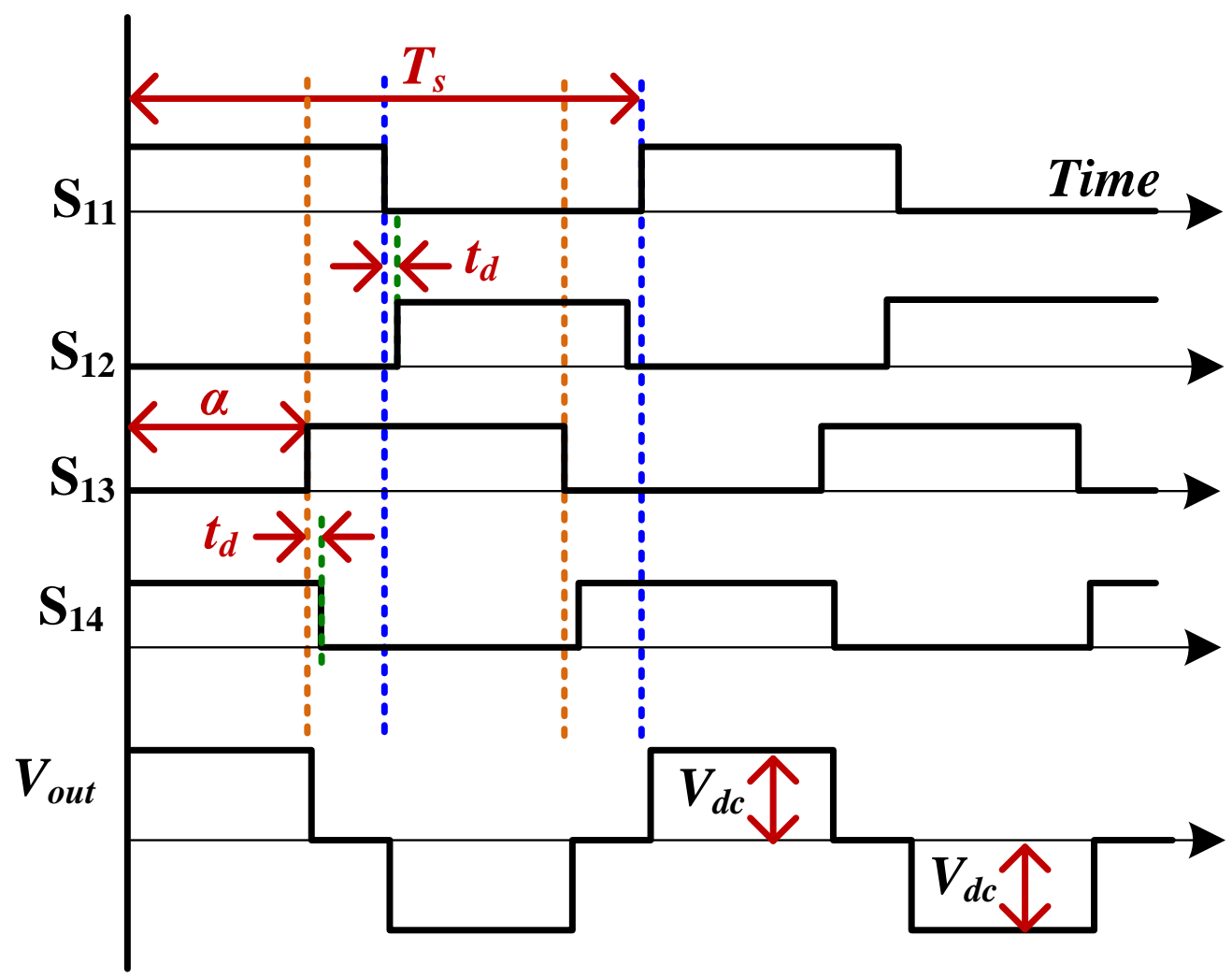

Figure 5.5. Switching signals and output voltage of VSHBC under phase-shift control. 
An analog circuit is designed and implemented in this work to achieve the phase-shift control algorithm. The circuit is able to supply the switching signals for driving two synchronized inverters at the same time. It is able to provide variable modulation parameters $(\alpha$ and $\beta$ ) for each inverter. Also, it supplies the phase-shift between the two inverters' output voltages $(\delta)$. By varying these parameters, the power flow magnitude and direction can be controlled, as was indicated in chapter (4). Moreover, the circuit includes four adjustable dead-time circuits, to assign the dead-time between the switches in the same leg. Each dead-time circuit is simply variable $R C$ branch with AND gate, as shown in Figure 5.6 .

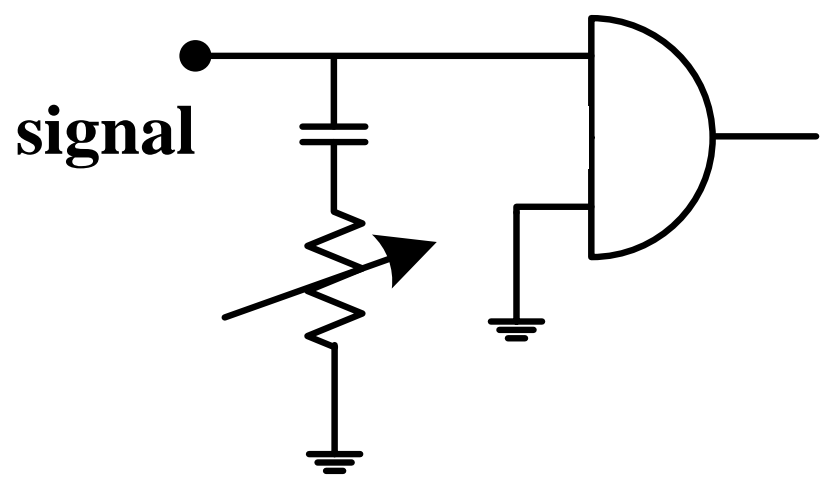

Figure 5.6. Hardware implementation of adjustable dead-time circuit.

A logic block diagram for the phase-shift analogue circuit is depicted in Figure 5.7. It consists of four dead-time, two NOT circuits and one PLL circuit. The NOT gates are used to generate $180^{\circ}$ phase shift between two trains of pulses. The PLL circuit is used to provide the $90^{\circ}$ phase-shift ( $\delta$-parameter). It is designed to lock with frequency ranges from $0-40$ kHz. The cascaded NOT gates, shown in Figure 5.7, are used for isolation purpose. The 
schematic diagram that shows the physical connections and components of the phase-shift circuit is described in Figure 5.8; and the implemented PCB of the same circuit is depicted in Figure 5.9.

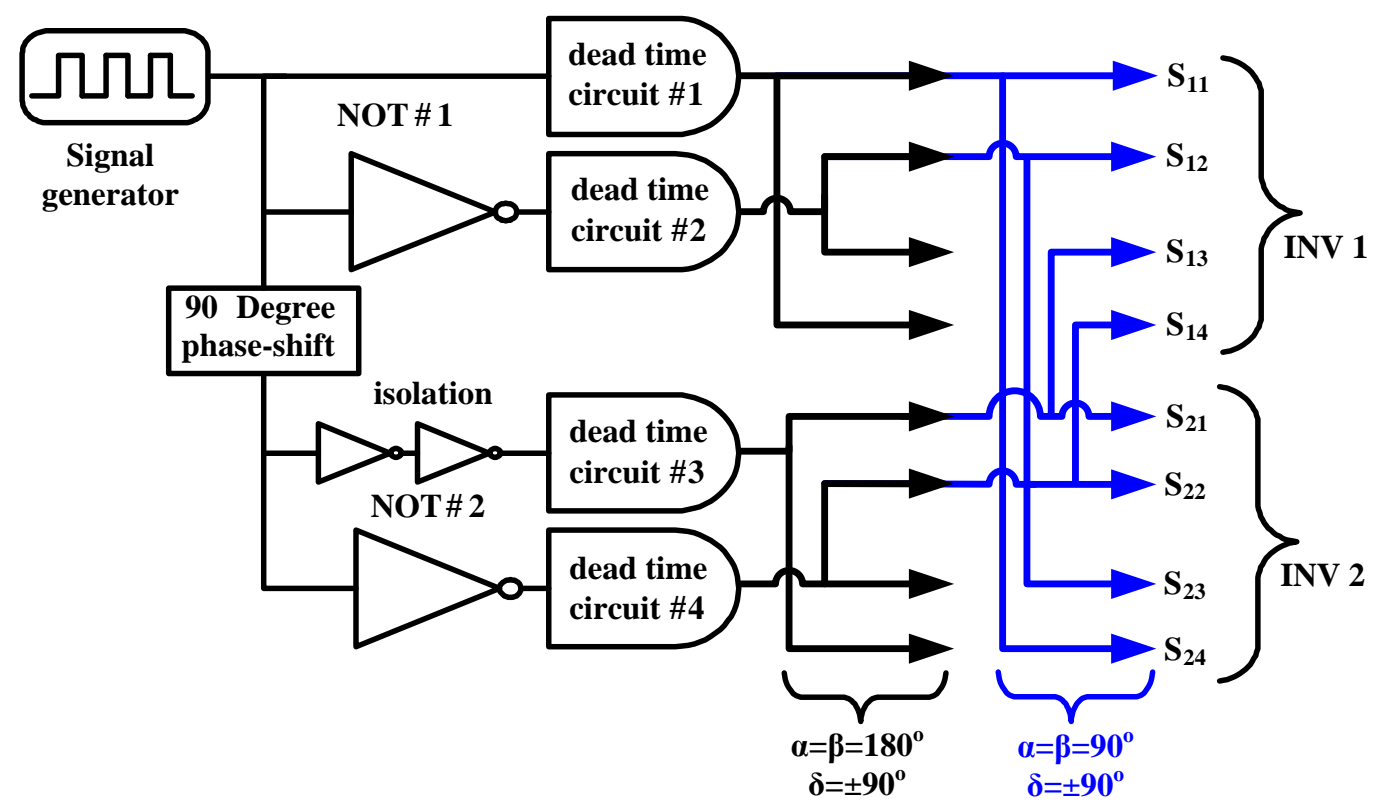

Figure 5.7. Logic block diagram of phase-shift control circuit.

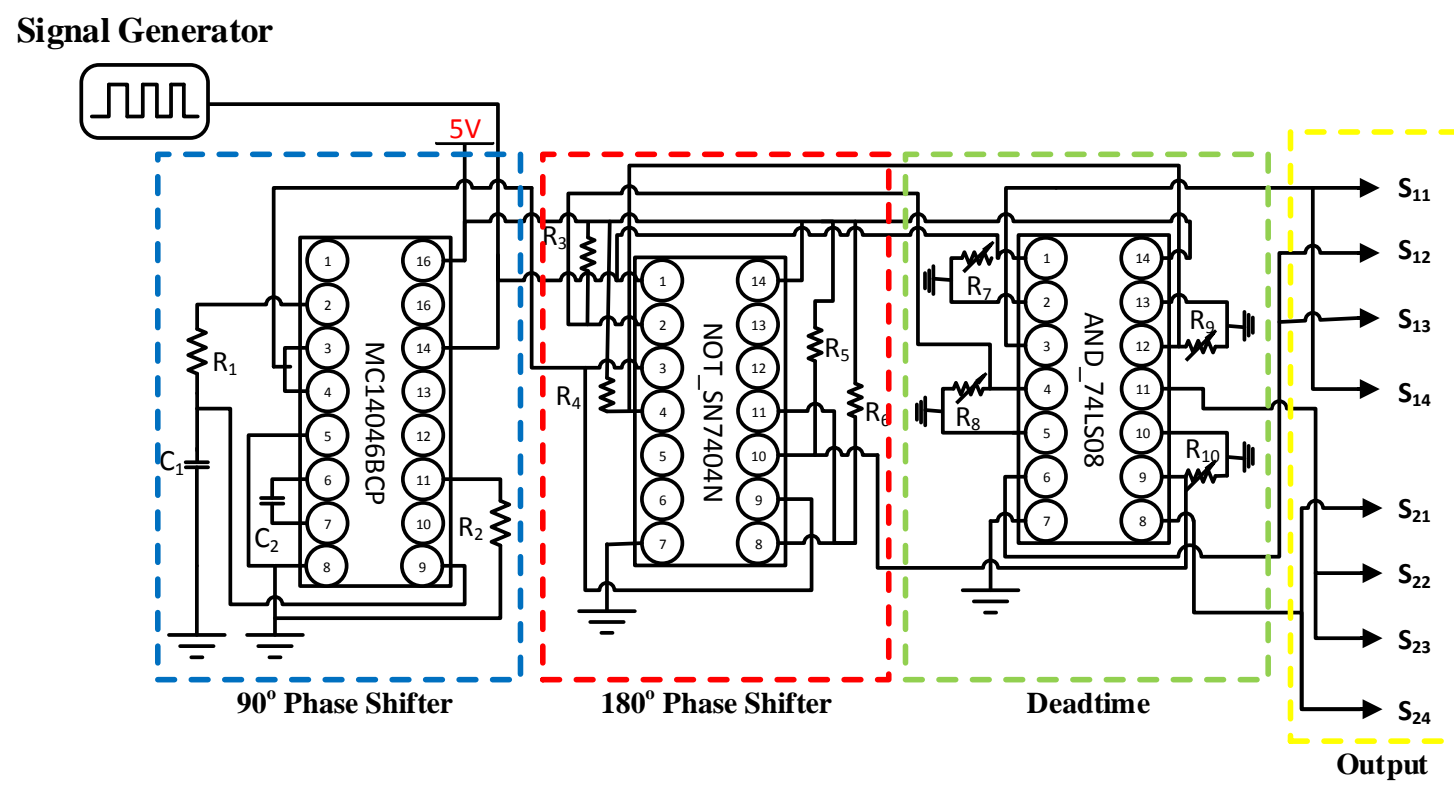

Figure 5.8. Phase-shift circuit schematic diagram. 


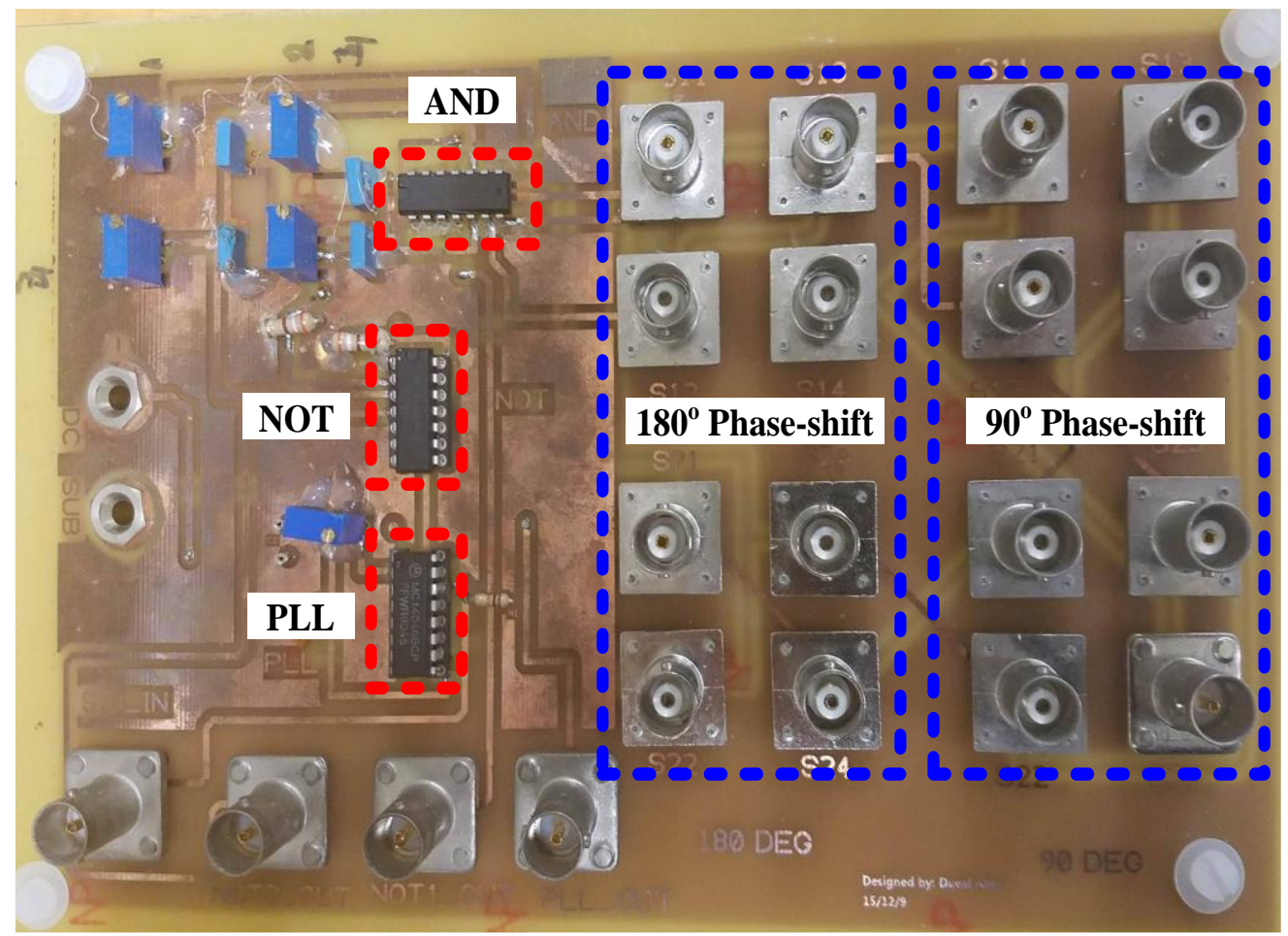

Figure 5.9. The hardware of phase-shift circuit's PCB.

\subsection{Electromagnetic Power Pads}

Magnetic power pad is the most important component in an IPT system, since it dominates the design of the power electronics, maximum power, efficiency, and power transfer distance. Each IPT system contains two power pads. The first one in placed in the ground and is called the primary, ground or transmitter pad. The second pad exists in the vehicle and is called the secondary, vehicle, pick-up or receiver pad. The two pads can be symmetrical or unsymmetrical. Although, the symmetrical pads provide better coupling performance, the international standard SAE J2954A recommends smaller size for the vehicle pad due to space limitations inside the vehicle [149]. The primary pad generates the HF AC magnetic flux that is linked to the secondary pad. Secondary coil, capturing the 
applied field, induces voltage that is then converted to a regulated DC voltage to charge the EV's battery. Typically, the design of magnetic pads targets high coupling factors, smaller size and cost, confining the magnetic field between pads without having considerable fringe/stray magnetic and electric fields, and achieving inductance values that requires reasonable DC-bus voltage [167]. The typical design of power pads in EVs' applications contains copper coils, ferrite magnetic material and aluminum shield. The copper coils are responsible for transmitting and picking up the magnetic field between the two system's sides. Each pad may contain one or more coils that are electrically and magnetically connected. Litz wire is preferred for the coils in order to minimize the internal resistance, skin effect losses, and increase the quality factor.

Magnetic materials are typically used in order to shape and direct the magnetic field, and maximize the coupling performance. They also help to minimize the leakage flux in the system and decrease the reluctance of the magnetic field path. All these effects lead to maximizing the magnetic coupling coefficient $k$, which is the most important factor in an IPT system. An IPT system with a higher coupling coefficient means a system that can transfer power more efficiently than others. There may, however, be a cost penalty, as magnetic materials such as ferrite are expensive and fragile. In circumstances where a large lateral tolerance to misalignment is needed, the cost may be excessive [84]. Aluminum shield is used in the system to minimize the stray field around the power pads to comply with the standard limits defined by the International Commission on Non-Ionizing Radiation Protection (ICNIRP) guidelines [168].

Several studies for different planar pad configurations for EV charging, such as circular, DD, bi-polar, double-DQ (DDQ) and tri-polar are presented in the literature [84], 
[85], [169]. In this prototype, the circular design is considered. Two symmetrical power pads are built. Each pad consists of one stranded copper coil of radius $200 \mathrm{~mm}$, attached to seven ferrite rods with a pole shoe. Each rod consists of three standard ferrite cores (I93x28x16) of N87 magnetic material from EPCOS, as indicated in Figure 5.10. More details about the design process and how to optimize this design are presented in chapters (8) and (9).

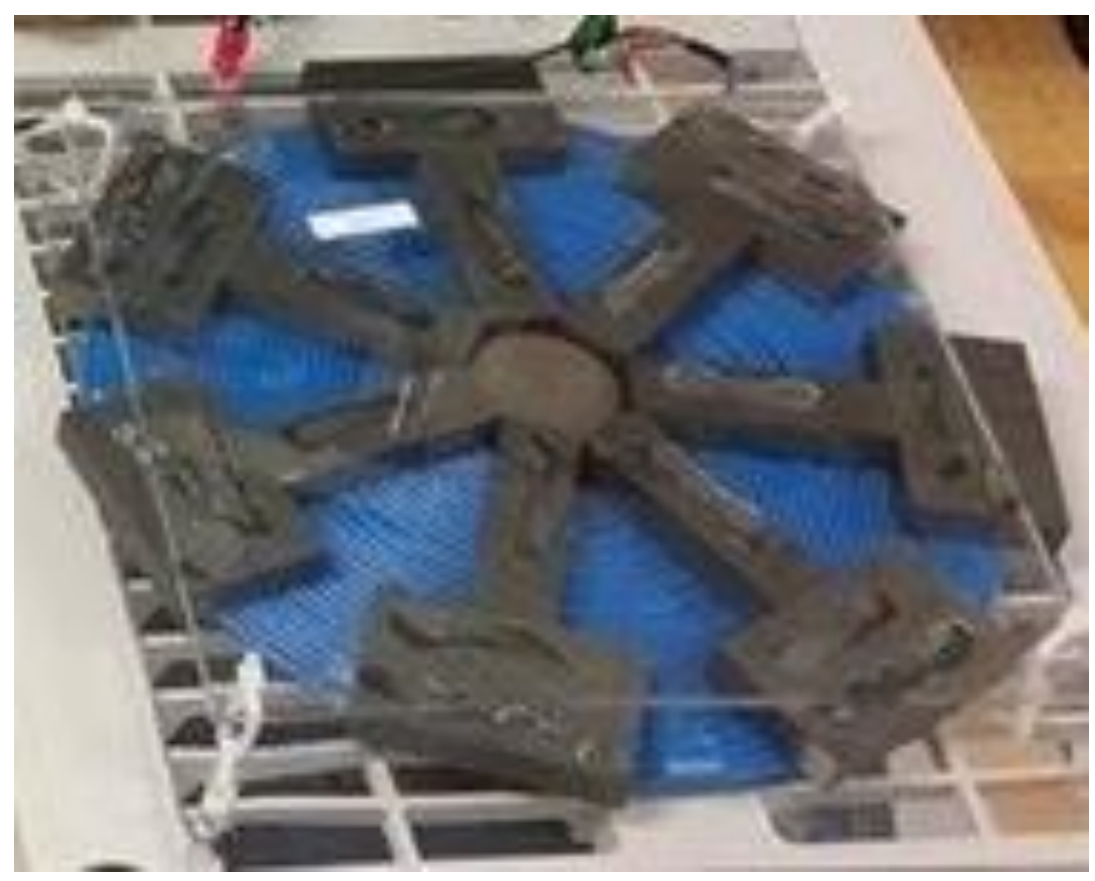

Figure 5.10. Circular power pad with ferrite core.

\subsection{Compensation Capacitors}

As discussed in chapter (4), the compensation capacitor plays a very important role in the operation of the IPT system. It is responsible for creating the resonance in the system. 
The design of these capacitors is challenge, since they must be able to handle the highvoltage and current stresses. The theoretical value of the capacitance is estimated based on the self-inductance of the power pad and the resonant frequency using Equation (4-2). Typically, this calculated value is close to the required one but not exactly the same. This deviation is due to errors in measurements and parameters' estimation. Thus, the estimated capacitance must be corrected to achieve the desired resonance performance. In addition, the resonance network is affected by the parasitic elements of the wires and HF converter's components. Thus, in this work, the value of the compensation capacitor is decided through two steps. The first step is to calculate the capacitance value using Equation (4-2), and find a capacitor or a combination of capacitors that match or are near this value. The second step is achieved by connecting one side of the system (HF inverter, power pad and the compensation network) and operating it at the resonant frequency. Then, the value of the capacitor is modified, such that the system hits the resonance by showing pure resistive performance (the inverter's current and voltage are in phase). Even in the symmetrical system, small differences are expected between the two sides. Thus, these steps must be

repeated for the other side as well. Metallized Film Capacitors are typically used in IPT system applications, since they provide high operating voltage (700 $\left.\mathrm{V}_{\mathrm{RMS}}\right)$, current (800 $\mathrm{A}_{\mathrm{RMS}}$ ) and power (400 KVA). They also support HF operation, up to $700 \mathrm{kHz}$, and show good conduction cooling [170].

\subsection{Design Verification and Results}

For design verification purposes, two different designs of PCB for VSHBC were developed, built, tested and compared. A snubber circuit is developed and included in the 
optimum inverter design and tested. The final shape and dimensions of the proposed inverter, including drivers, protections, snubber circuits and cooling, are indicated in Figure 5.11 [171]. The details of the used components and the estimated design parameters are presented in Table 5.1. The proposed inverter performance is studied at different modulation and operating conditions. The different components are linked together to form a LCL BIWPTS. The entire system is tested and compared with a Simulink model results.

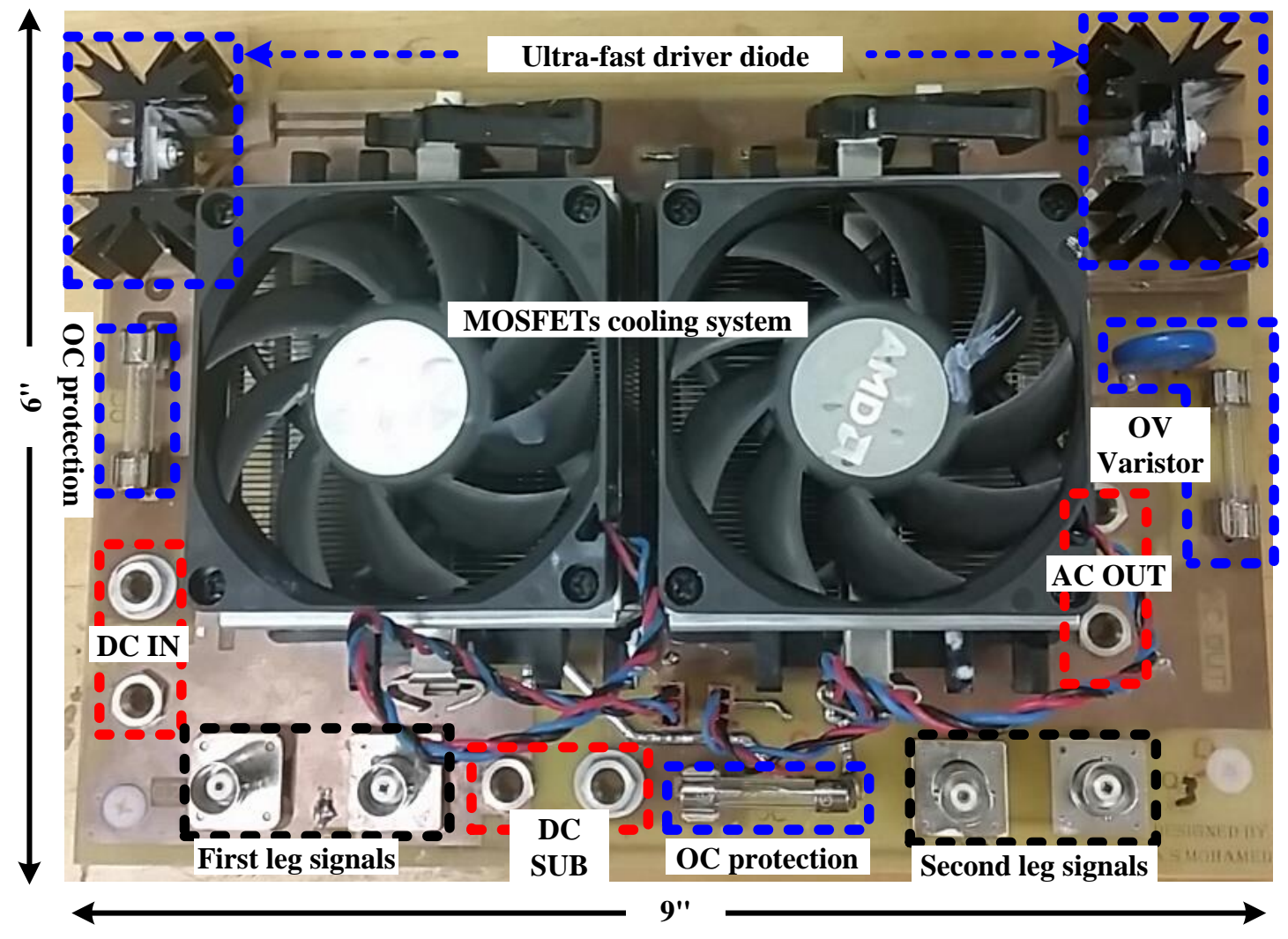

Figure 5.11. The hardware implementation of VSHBC's PCB. 
Table 5.1. Final Converter Design Parameters.

\begin{tabular}{llll}
\hline Component & Value & Commercial name & Type \\
\hline Power MOSFET & $600 \mathrm{~V}, 110 \mathrm{~A}$ & IXFB110N60P3 & Si Power MOSFET \\
IC Driver & $4.5 \mathrm{~A}$ & FAN7391 & High \& Low side \\
Diodes $\left(\boldsymbol{D}_{\text {boot }} \& \boldsymbol{D}_{\text {st }}\right)$ & $8 \mathrm{~A}, 600 \mathrm{~V}$ & VS-ETX0806-M3 & Hyper fast \\
$\boldsymbol{R}_{\text {boot }}$ & $9 \Omega$ & ------ & $(5-10 \Omega), 1-2 \mathrm{~W}$ \\
$\boldsymbol{C}_{\text {boot }}$ & $470 \mathrm{nF}$ & ----- & Ceramic \\
$\boldsymbol{R}_{\text {g-on }}$ & $10 \Omega$ & ------ & $1-2 \mathrm{~W}$ \\
$\boldsymbol{R}_{\text {g-off }}$ & $33 \Omega$ & ----- & $1-2 \mathrm{~W}$ \\
$\boldsymbol{R}$ & $2.2 \mathrm{k} \Omega$ & ------ & $1-2 \mathrm{~W}$ \\
$\boldsymbol{C}$ & $8 \mu \mathrm{F}$ & ----- & Ceramic, $>10 C_{\text {boot }}$ \\
$\mathbf{C}_{\text {snubber }}$ & $100 \mathrm{nF}, 630 \mathrm{~V}$ & R0614 & MMKP \\
\hline \hline
\end{tabular}

\subsubsection{Evaluating the Phase-shift Circuit Performance}

The main objective of the phase-shift circuit is to generate the switching signals ( 0 $5 \mathrm{~V}$ ) of the inverters with the necessary dead-times. Two tests are performed: in the first, the inverter supplies the full capacity with a phase-shift parameter $(\alpha)$ of $180^{\circ}$, while in the second test, the phase-shift parameter is adjusted to $90^{\circ}$ to reduce the RMS inverter's output voltage. The switching signals for the 4 MOSFETs of one inverter during the first test are depicted in Figure 5.12. As it can be noticed, the phase-shift between S11 and S13 is $180^{\circ}$. Also, there is dead-time between the switches in the same leg (S11, S12 and S13, S14), which is clarified in Figure 5.13. The assigned time in this case is $250 \mu \mathrm{sec}$ at each 
transition. The switching signals during the reduced inverter's output voltage are described in Figure 5.14. As it can be observed, the phase phase-shift between S11 and S13 is $90^{\circ}$.

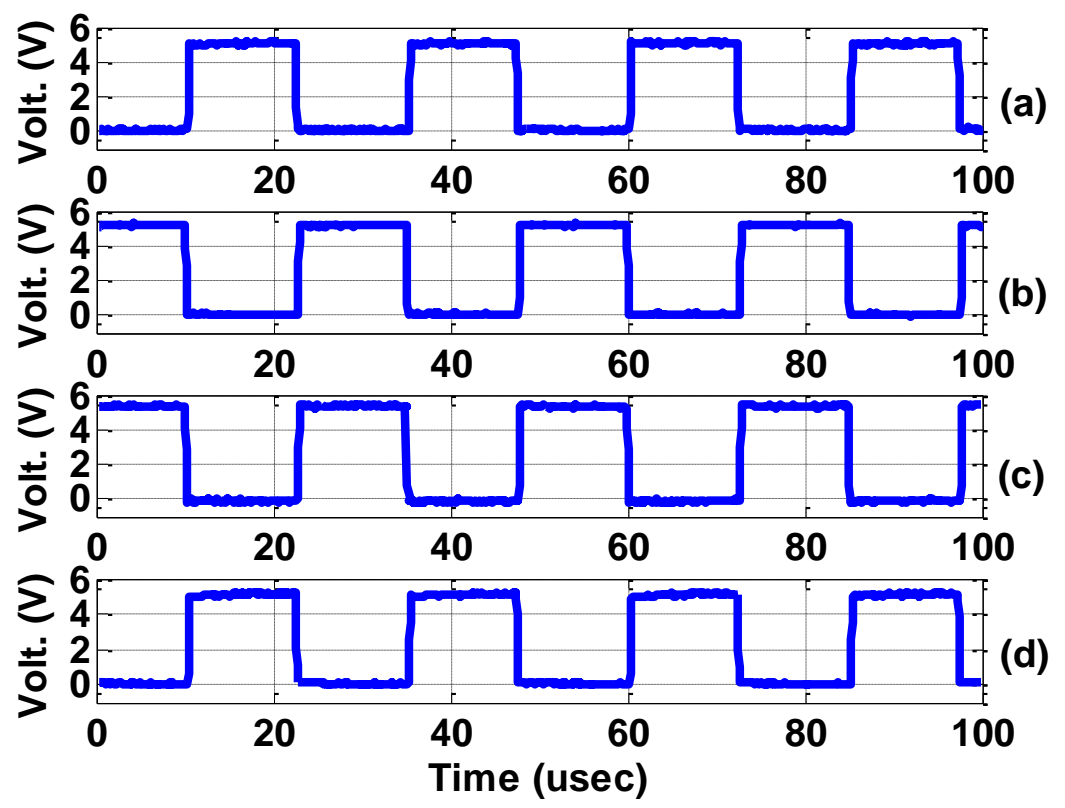

Figure 5.12. Measured switching signals (0-5V) of one inverter (4 MOSFETs) during full inverter voltage. (a) S11. (b) S12. (c) S13. (d) S14.

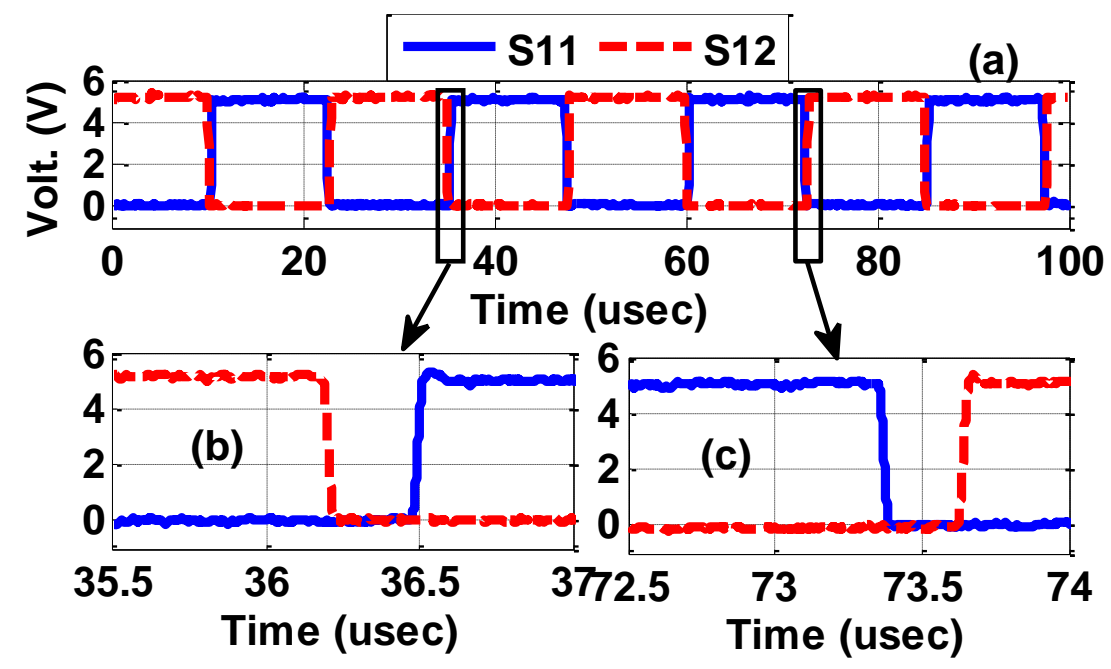

Figure 5.13. Measured switching signals of one leg (2 MOSFETs) during full inverter voltage showing the dead-time. (a) S11 and S12. (b) Left dead-time. (c) Right dead-time. 


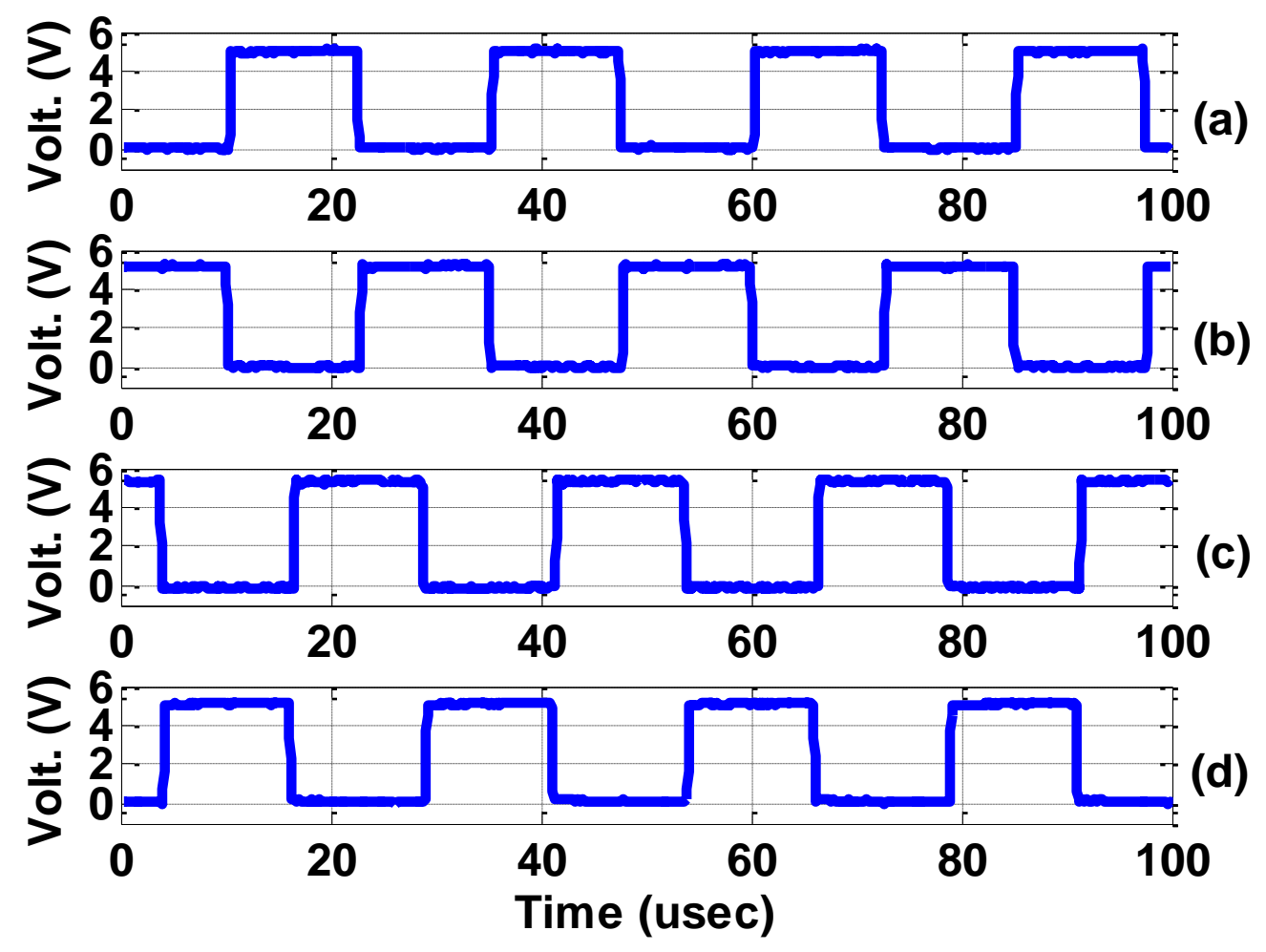

Figure 5.14. Measured switching signals (0-5V) of one inverter (4 MOSFETs) during reduced inverter voltage: (a) S11. (b) S12. (c) S13. (d) S14.

\subsubsection{Evaluating the Driving Circuits Performance}

The next step, after generating the switching signals, is to boost them to the voltage and the power levels of the power MOSFETs. This is achieved using the bootstrap circuit with FAN7391 integrated circuit (IC) gate drivers. The driving circuits receive the signals with $0-5 \mathrm{~V}$ level and generate $0-15 \mathrm{~V}$, which represent the gate-voltage level of the IXFB110N60P3 Si power MOSFET. Also, the driving circuits provide the required currents to drive the gate of the MOSFETs. The gate signals (output of drivers), during the full and the reduced output inverter voltage tests, are presented in Figure 5.15 and 5.16, respectively. 


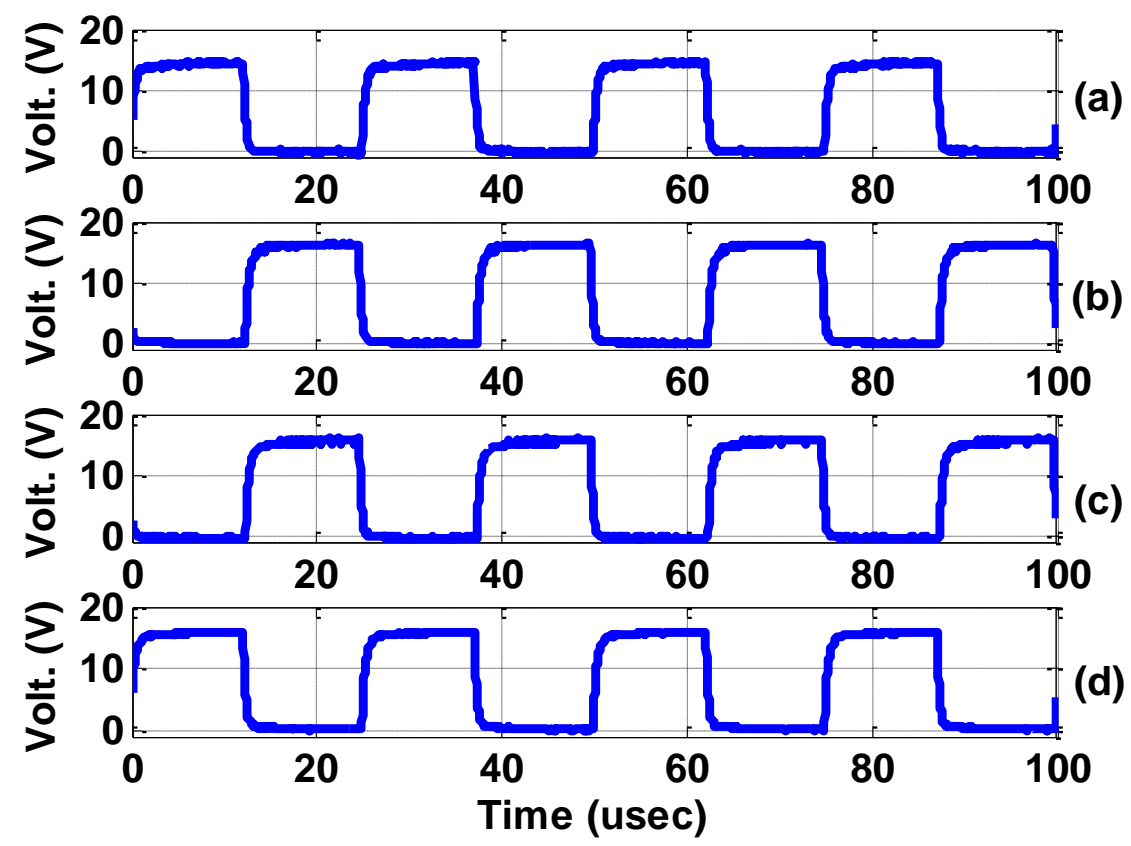

Figure 5.15. Measured gate signals $(0-15 \mathrm{~V})$ of one inverter (4 MOSFETs) during full inverter voltage. (a) S11. (b) S12. (c) S13. (d) S14.
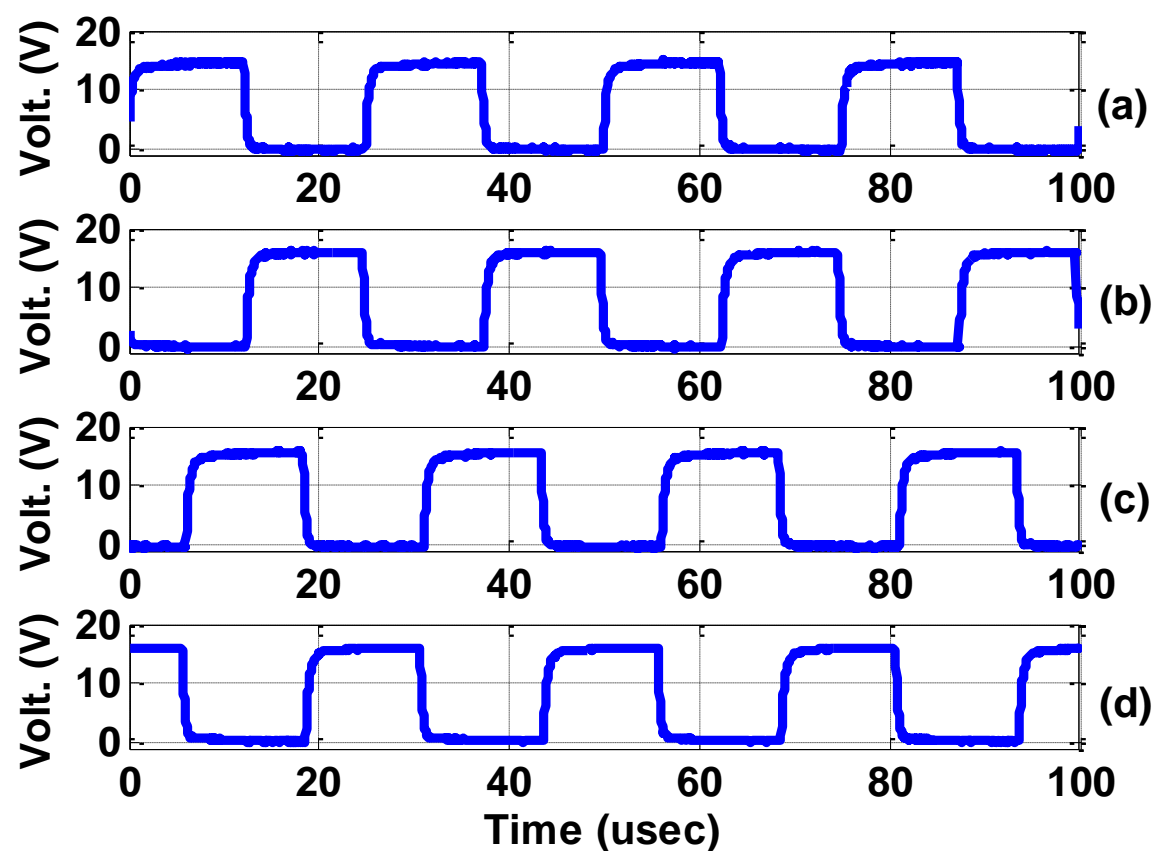

Figure 5.16. Measured gate signals (0-15V) of one inverter (4 MOSFETs) during reduced inverter voltage. (a) S11. (b) S12. (c) S13. (d) S14. 


\subsubsection{The Converter's PCB Layout Performance Assessment}

The effect of the PCB layout design optimization on the ringing of the inverter output voltage is shown in Figure 5.17. As it can be noticed, the ringing amplitude is reduced and also the voltage reaches to the steady-state faster in the optimized design compared with the non-optimized one. The voltage profile of the optimum design is improved during both the rising and falling edges. The effect of the snubber circuit design on the inverter performance is indicated in Figure 5.18. It can be observed that, the snubber circuit cleaned the ringing in the inverter's output without affecting the rise or the fall time.

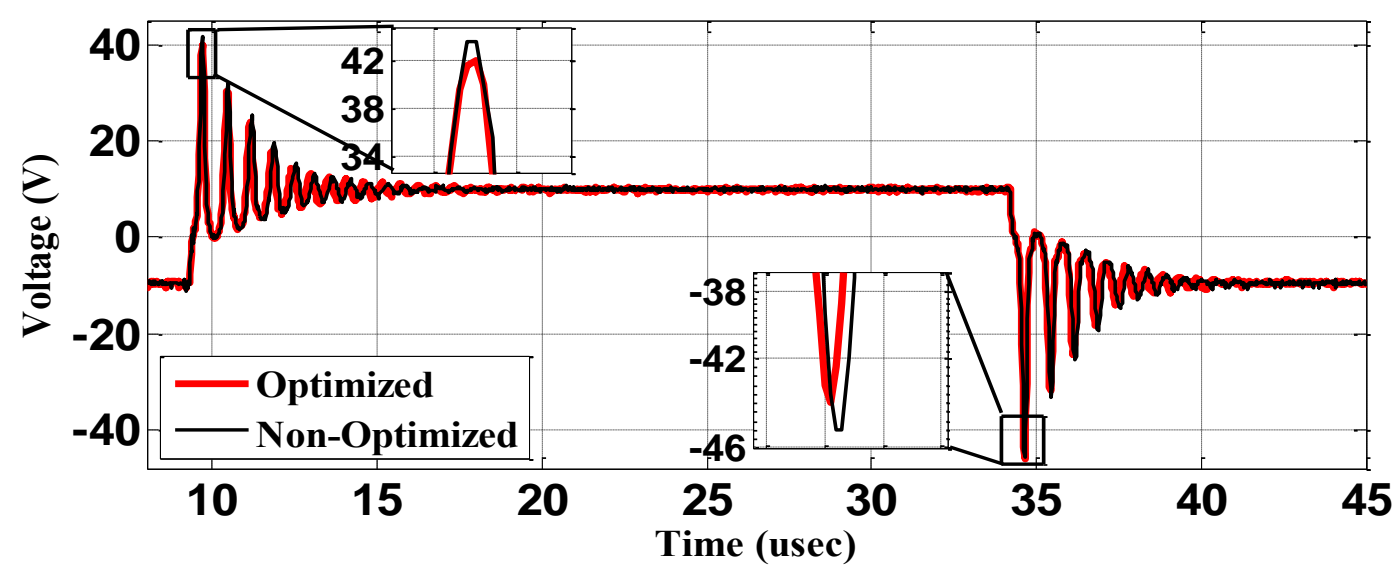

Figure 5.17. Measured ringing effect of the optimized and non-optimized PCB designs.

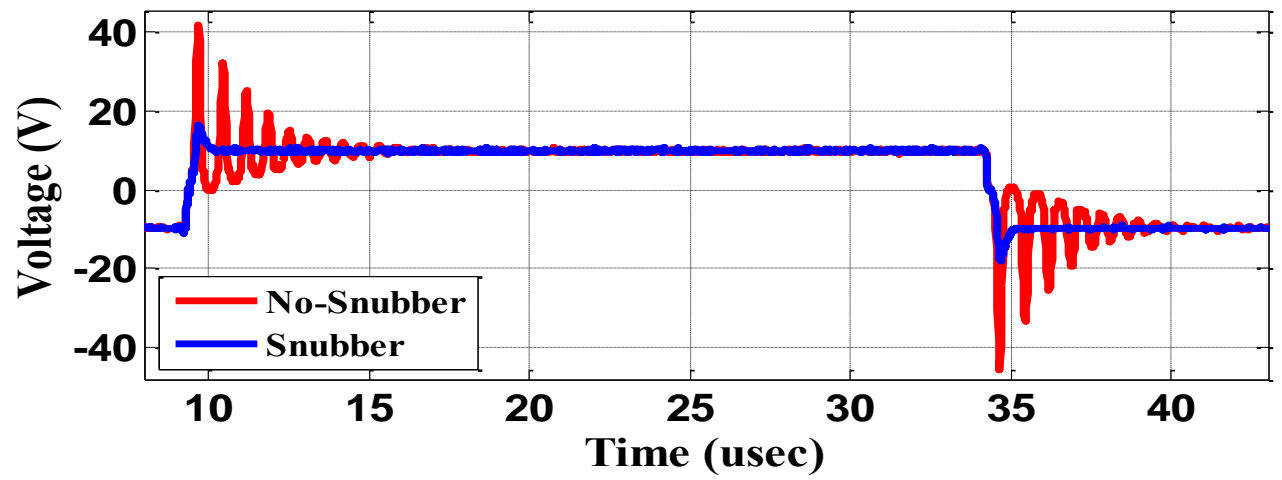

Figure 5.18. Measured output inverter voltage with and without snubber circuit. 
Transient performance analysis is conducted for a step variation of the output voltage of each inverter design: the non-optimized, optimized without snubber and the optimized with snubber. The unit step output voltage response for each case is described in Figure 5.19. The performance evaluation parameters, such as settling time, rise time, overshoot and undershoot are estimated and compared for all cases in Table 5.2. It can be noticed the improvement of the inverter performance by following the proposed design, which leads to less transient oscillations amplitude and time.

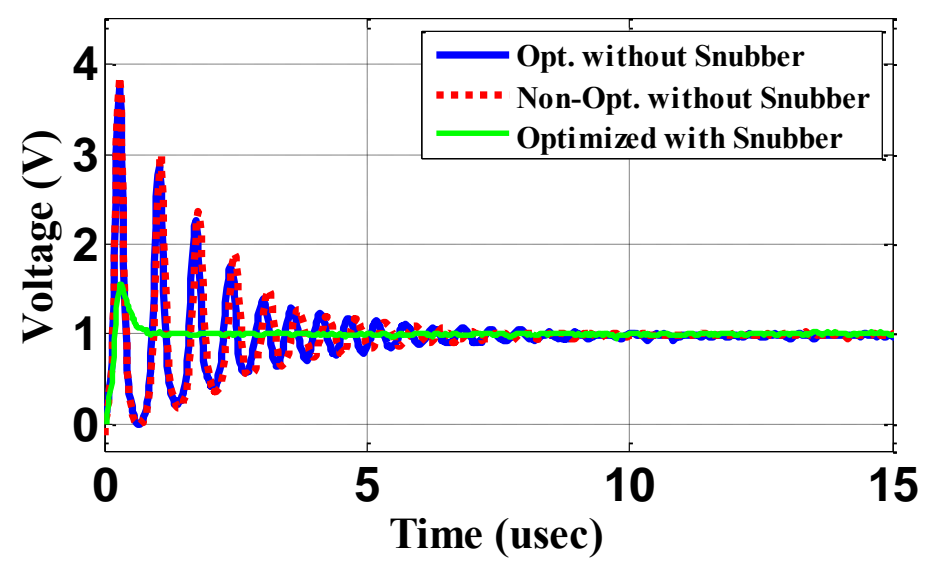

Figure 5.19. Measured unit-step response of inverter output voltage.

Table 5.2. Performance Parameters during Step Response.

\begin{tabular}{llll}
\hline Parameters & $\begin{array}{l}\text { Non-opt. } \\
\text { without Snubber }\end{array}$ & Opt. without Snubber & Opt. with Snubber \\
\hline Rise time $(\boldsymbol{\mu} \mathbf{s e c})$ & 0.15 & 0.1336 & 0.1437 \\
Settling time $(\boldsymbol{\mu s e c})$ & 6.71 & 6.52 & 0.8 \\
Overshoot $(\%)$ & 285 & 274 & 55.2 \\
Undershoot $(\%)$ & 64 & 64 & 0 \\
Peak (pu) & 3.86 & 3.736 & 1.55 \\
Peak time ( $\mu \mathrm{sec})$ & 0.3 & 0.28 & 0.32 \\
\hline \hline
\end{tabular}




\subsubsection{The VSHBC's Circuit Performance Assessment}

The output voltage of each inverter's leg $\left(V_{a}\right.$ and $\left.V_{b}\right)$ and the final AC output voltage $\left(V_{a b}\right)$ are measured during the full and reduced output tests and investigated in Figure 5.20 and 5.21. As it can be noticed, each leg generates train of pulses with an amplitude equal to the DC input level and a specific phase-shift based on the PPM parameters. The AC output voltage represents the subtraction of the two legs' voltages $\left(V_{a b}=V_{a}-V_{b}\right)$. In Figure 5.20, the phase-shift is $180^{\circ}$, thus the AC output voltage is full-square waveforms, as given in Figure 5.20(c). In Figure 5.21, the phase-shift is $90^{\circ}$, therefore the inverter generates quasi-square waveforms [see Figure 5.21(c)]. In this case, a zero voltage level appears in the voltage profile to reduce the RMS voltage values, which is useful for power-flow control in IPT systems.
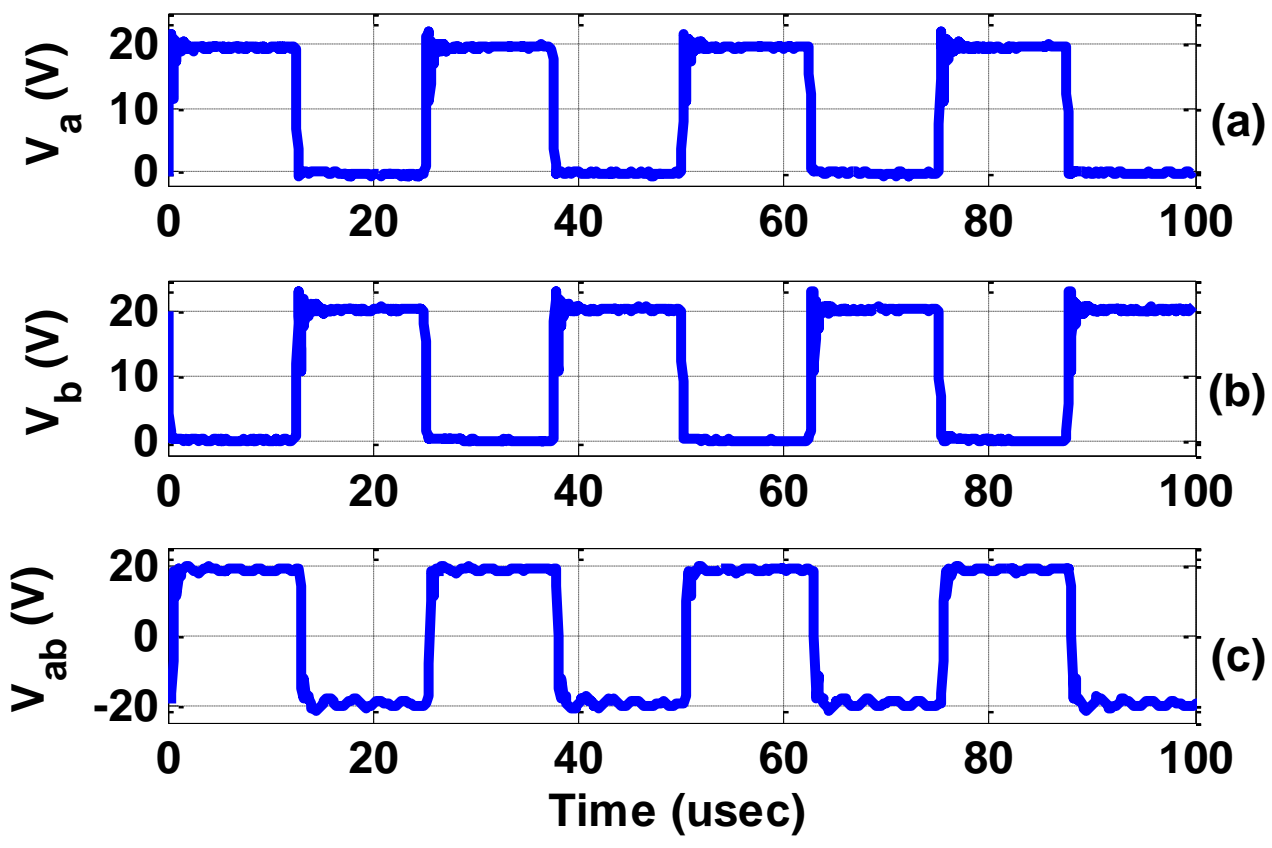

Figure 5.20. Measured inverter output voltage during full output test: (a) Voltage of the first leg. (b) Voltage of the second leg. (c) AC inverter voltage. 

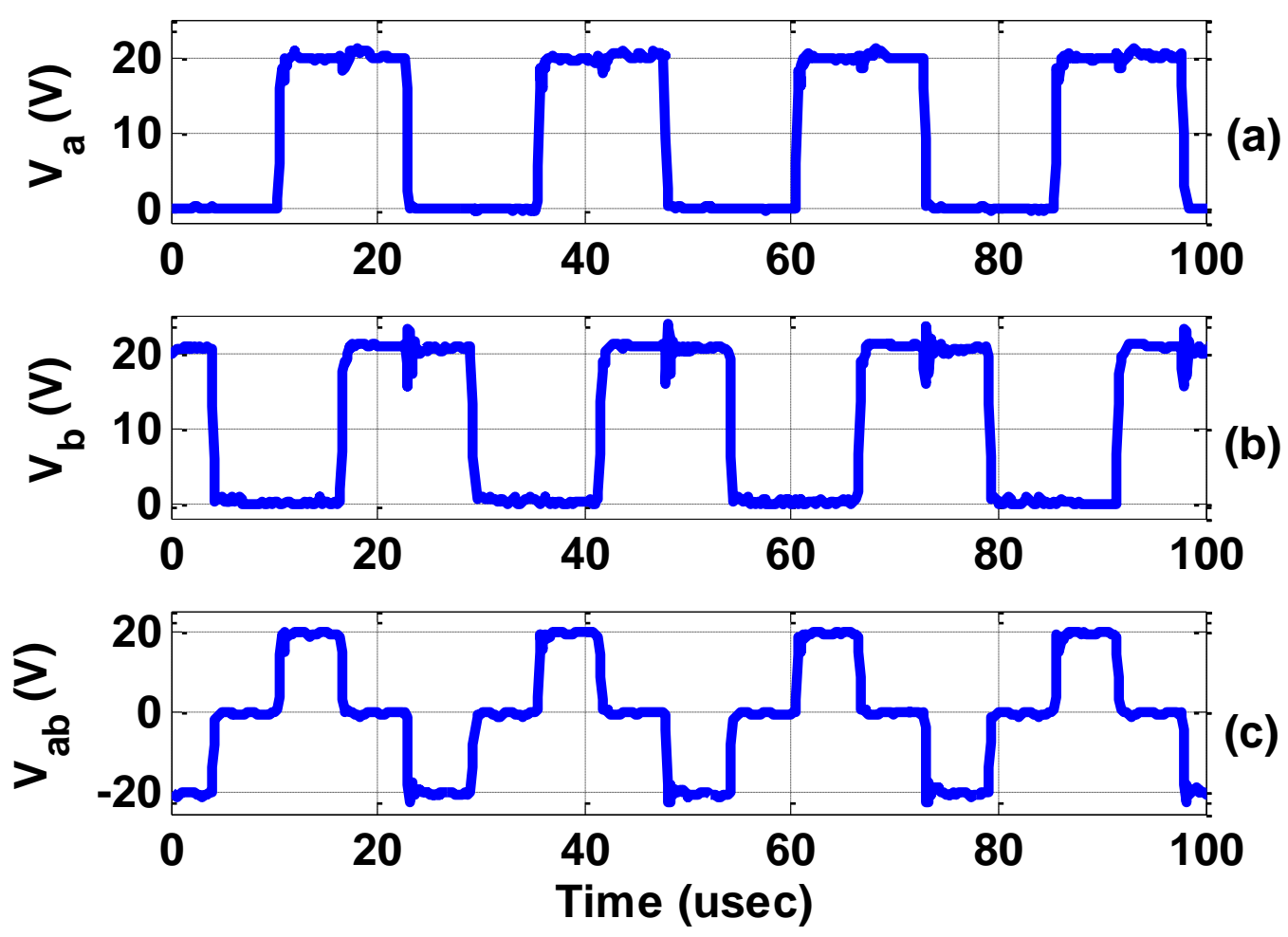

Figure 5.21. Measured inverter output voltage during reduced output test: (a) Voltage of the first leg. (b) Voltage of the second leg. (c) AC inverter voltage.

Another test is performed to check the inverter behavior under different operating frequencies. The inverter is tested with $180^{\circ}$ phase-shift at $20 \mathrm{kHz}$ and $40 \mathrm{kHz}$ and the results are indicated in Figure 5.22(a) and (b), respectively. As it can be noticed, a clean full square wave is generated from the inverter with different operating frequency. Also, the inverter is tested at $40 \mathrm{kHz}$ with $90^{\circ}$ phase shift between the inverter legs, as shown in Figure 5.22(c). 


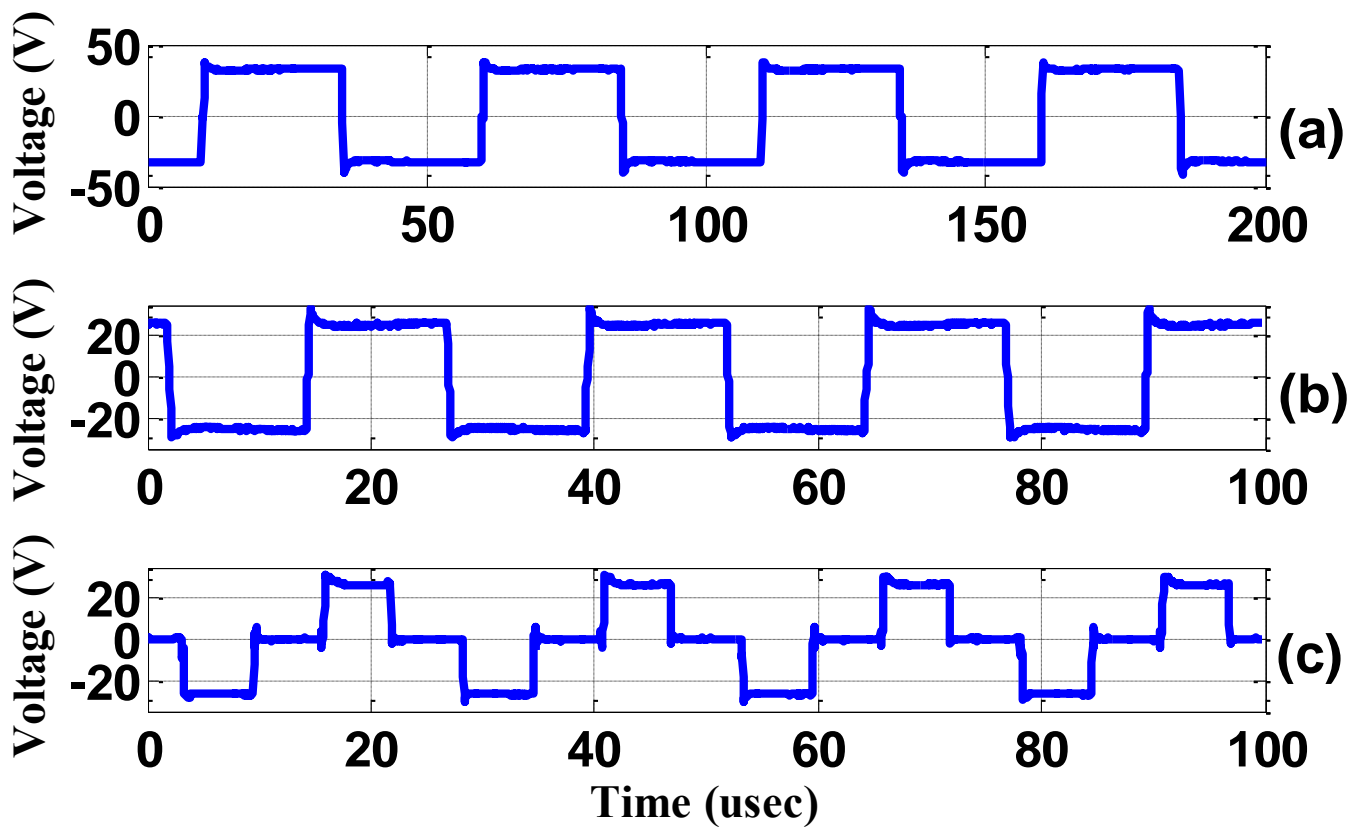

Figure 5.22. Measured inverter output voltage at different frequencies. (a) Full-square voltage at $20 \mathrm{kHz}$. (b) Full-square voltage at 40kHz. (c) Quasi-square voltage at 40kHz.

\subsubsection{The Entire BIWPTS's Performance}

The VSHBCs, power pads and LCL compensation networks are linked together to form a LCL BIWPTS, as shown in Figure 5.23 [171]. The system consists of a DC power supply for DC-bus emulation; a $21 \mathrm{Ah}, 53 \mathrm{~V}$ Polymer Li-ion battery pack to emulate EV behavior; two HF VSHBCs; two identical circular pads with Ferrite cores\; two parallel compensation capacitors; and two $L$-filters to form the LCL compensation topology. The same system is simulated in MATLAB Simulink, as depicted in Figure 5.24. The two model are analyzed and compared in this section. The system is designed to resonate at 40 $\mathrm{kHz}$ and the air-gap length between the primary and secondary coils is $150 \mathrm{~mm}$, which is 
the common case in EV situation. The design parameters of the LCL BIWPTS are indicated in Table 5.3.

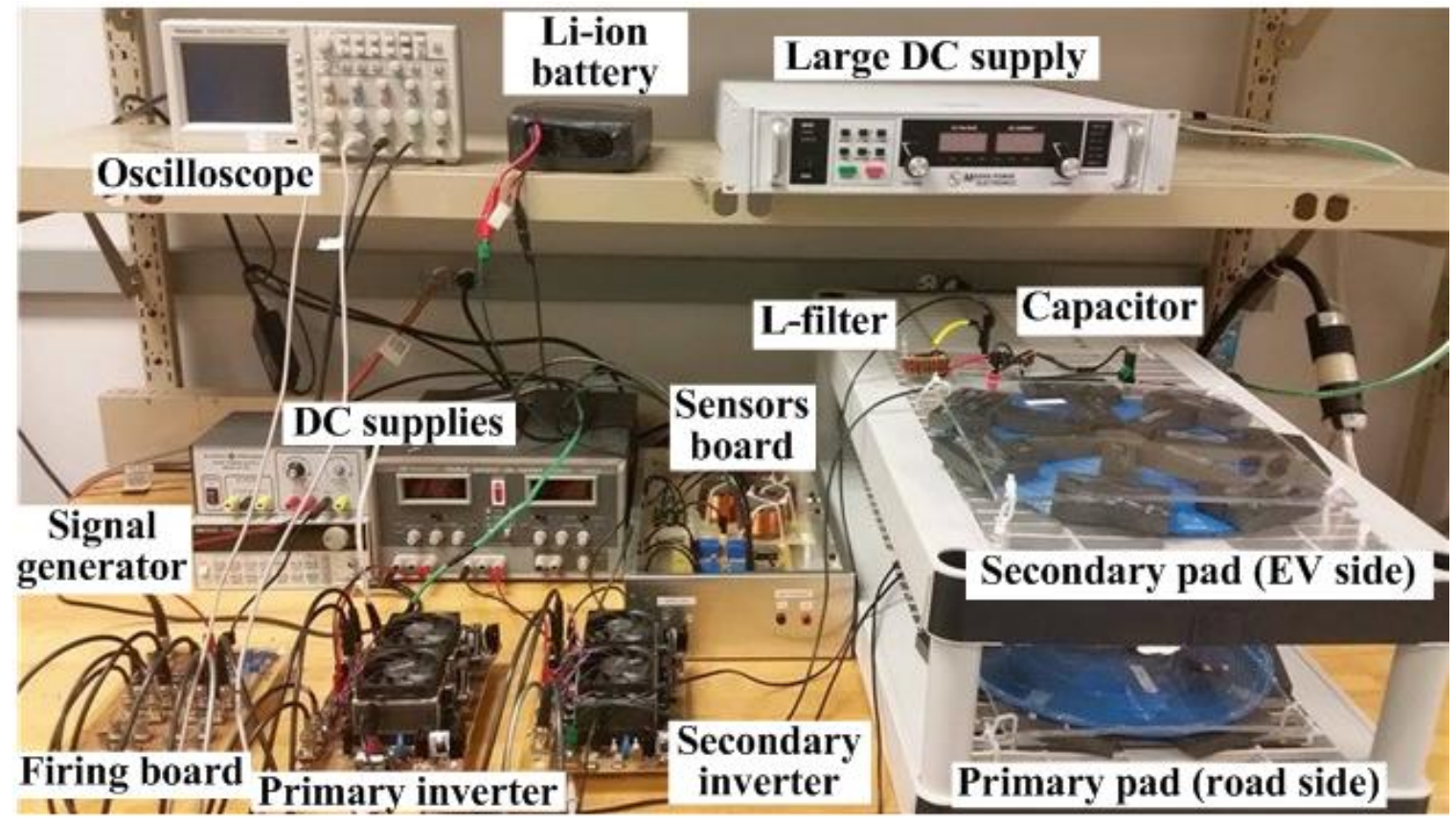

Figure 5.23. Experimental setup of LCL BIWPTS.

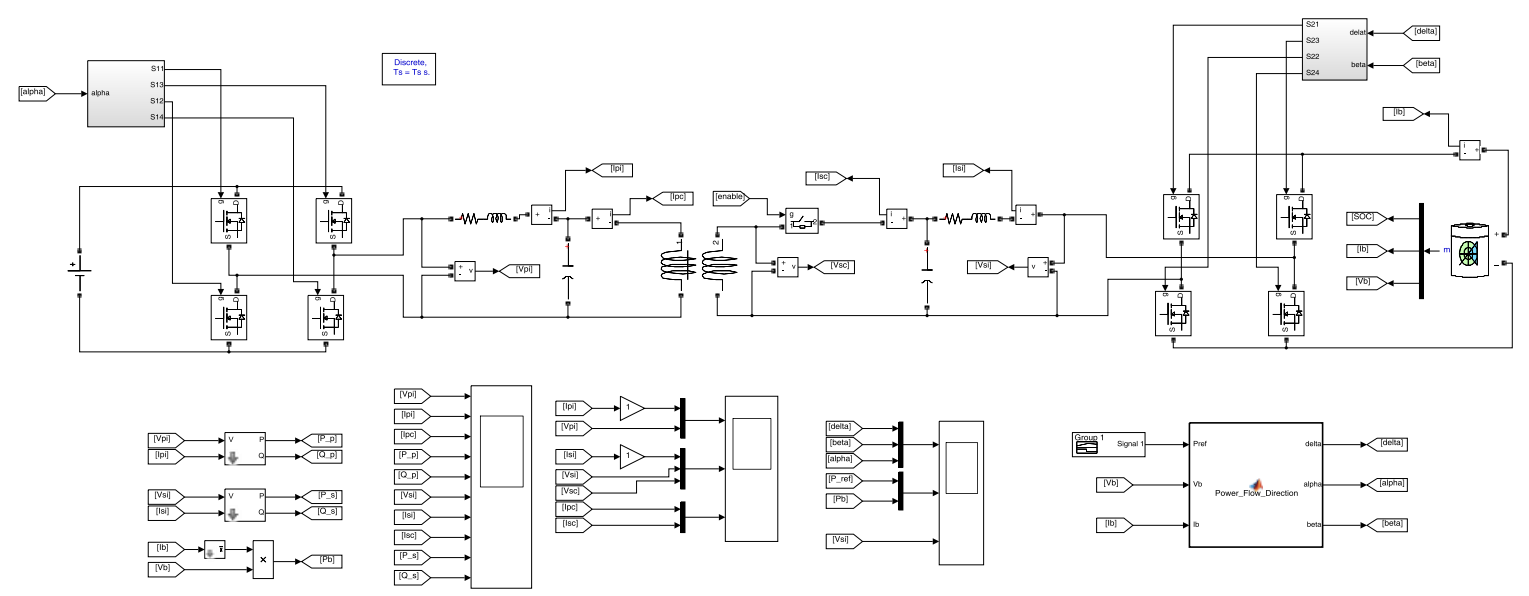

Figure 5.24. Simulink model of LCL BIWPTS. 
Table 5.3. Design Parameters of LCL BIWPTS.

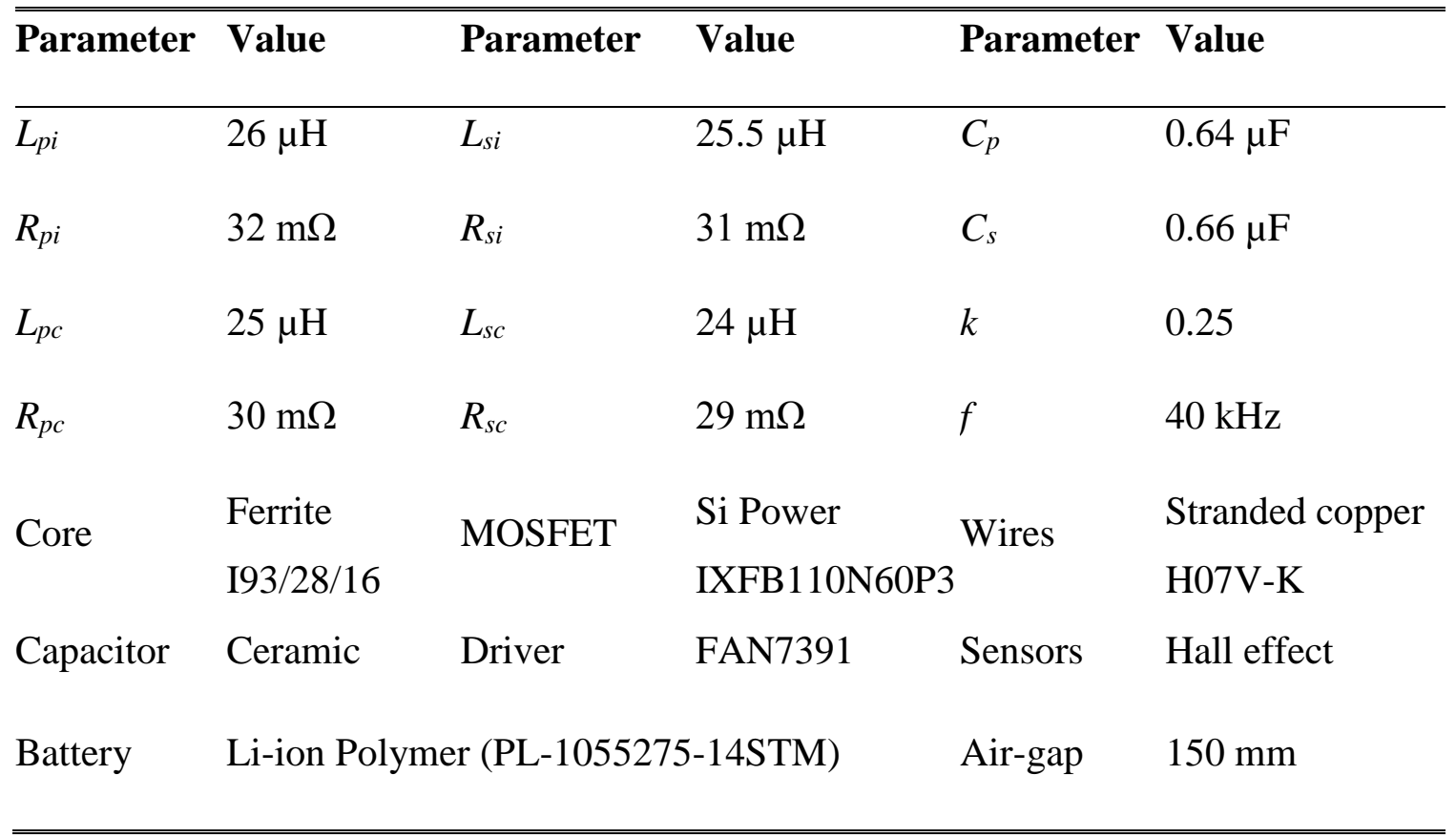

\subsubsection{Full Supply Capacity Test}

In this section, the system is analyzed during the full operation of supply capacity. In this mode, the phase-shift between the two inverters' legs ( $\alpha$ and $\beta$ ) are set to $180^{\circ}$, to provide the maximum available power on the supply. The BIWPTS is tested during G2V (charging) mode of operation, as given in Figure 5.25. In this test, the phase-shift between the two inverters' voltages $(\delta)$ is set to $-90^{\circ}$, to allow the power transfer from the DC-bus to the EV's battery. The figure shows the voltage and current waveforms in the primary and secondary circuits. Figure 5.25(a) and (b) describe the primary and secondary inverters' variables, respectively. It can be noticed that, the two inverters generate full square voltages with $90^{\circ}$ phase-shift $\left(V_{s i}\right.$ lags $\left.V_{p i}\right)$. Also, it can be observed that, $V_{p i}$ and $I_{p i}$ are almost in phase, however $V_{s i}$ and $I_{s i}$ are anti-phase, which means that the system is 
hitting the resonance and the primary and secondary power-flow are in opposite direction (one side is transmitting the energy and the other side is receiving it). The primary and secondary coils' currents and voltages are described in Figure 5.25(c) and (d), respectively. The inverters' currents $\left(I_{p i}\right.$ and $\left.I_{s i}\right)$ are not pure sinusoidal, but the coils' currents ( $I_{p c}$ and $\left.I_{s c}\right)$ are almost pure sinusoidal, due to the filtering effect of the circuit impedance. Good agreement can be observed between the simulation and experimental results.
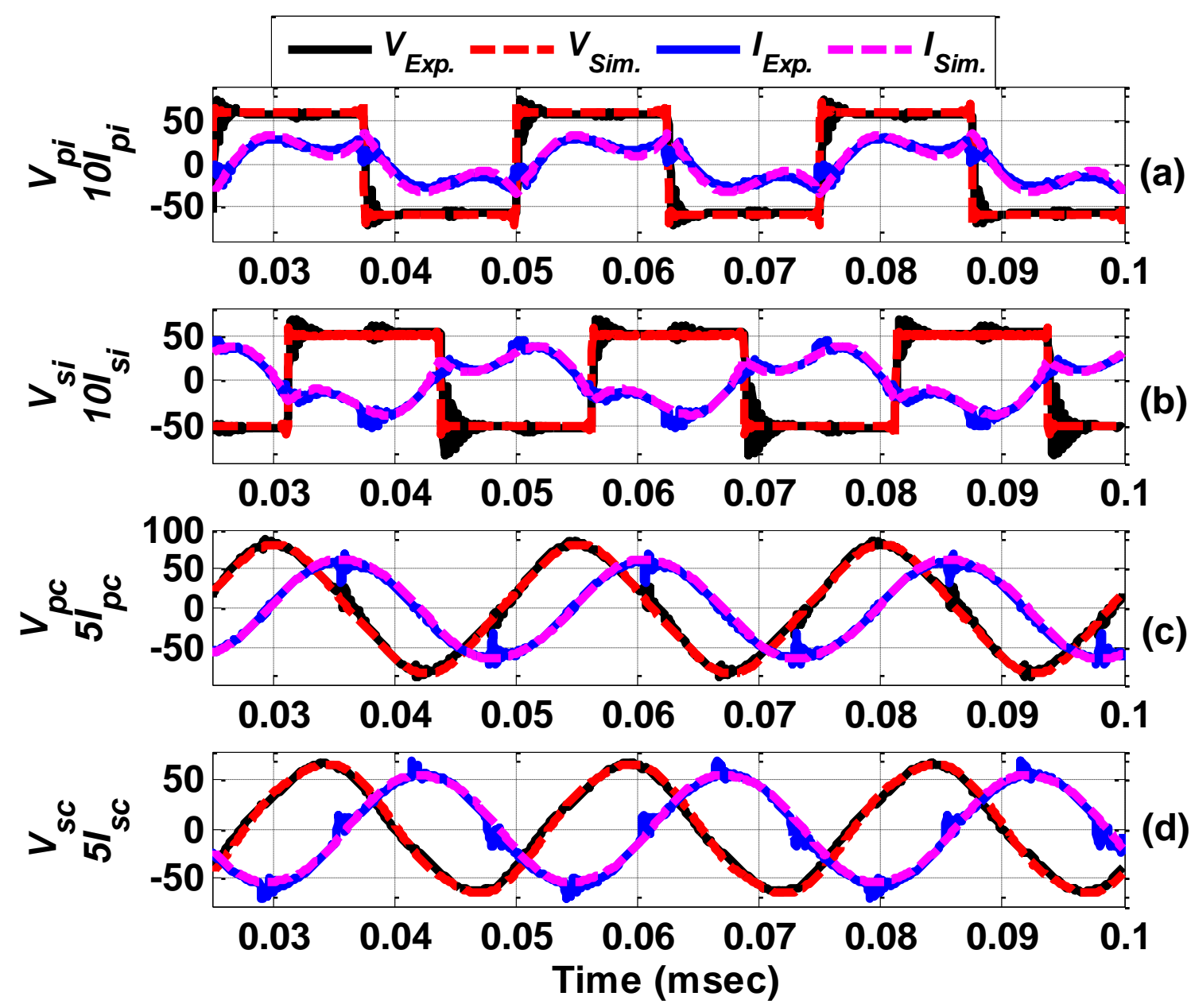

Figure 5.25. Experimental and simulated BIWPTS's performance under full supply voltage for G2V operation ( $\alpha=\beta=180^{\circ}, \delta=-90^{\circ}, V_{d c}=60 \mathrm{~V}$ ). (a) Primary inverter variables. (b) Secondary inverter variables. (c) Primary coil variables. (d) Secondary coil variables. 
The BIWPTS is analyzed during V2G (discharging) operation. In order to achieve this mode, the secondary inverter's voltage is set to lead the primary one by $90^{\circ}$ phase-shift $\left(\delta=90^{\circ}\right)$. The parameters $\alpha$ and $\beta$ are kept fixed at $180^{\circ}$ for supplying the full system capacity. The discharging operation results are presented in Figure 5.26. As it can be noticed, the secondary voltage leads the primary one by $90^{\circ}$, which allows the power to flow from the EV to the DC-bus. In this case, $V_{p i}$ and $I_{p i}$ are anti-phase and $V_{s i}$ and $I_{s i}$ are in-phase, which means that the system is still working at UPF but with the reverse power flow direction.
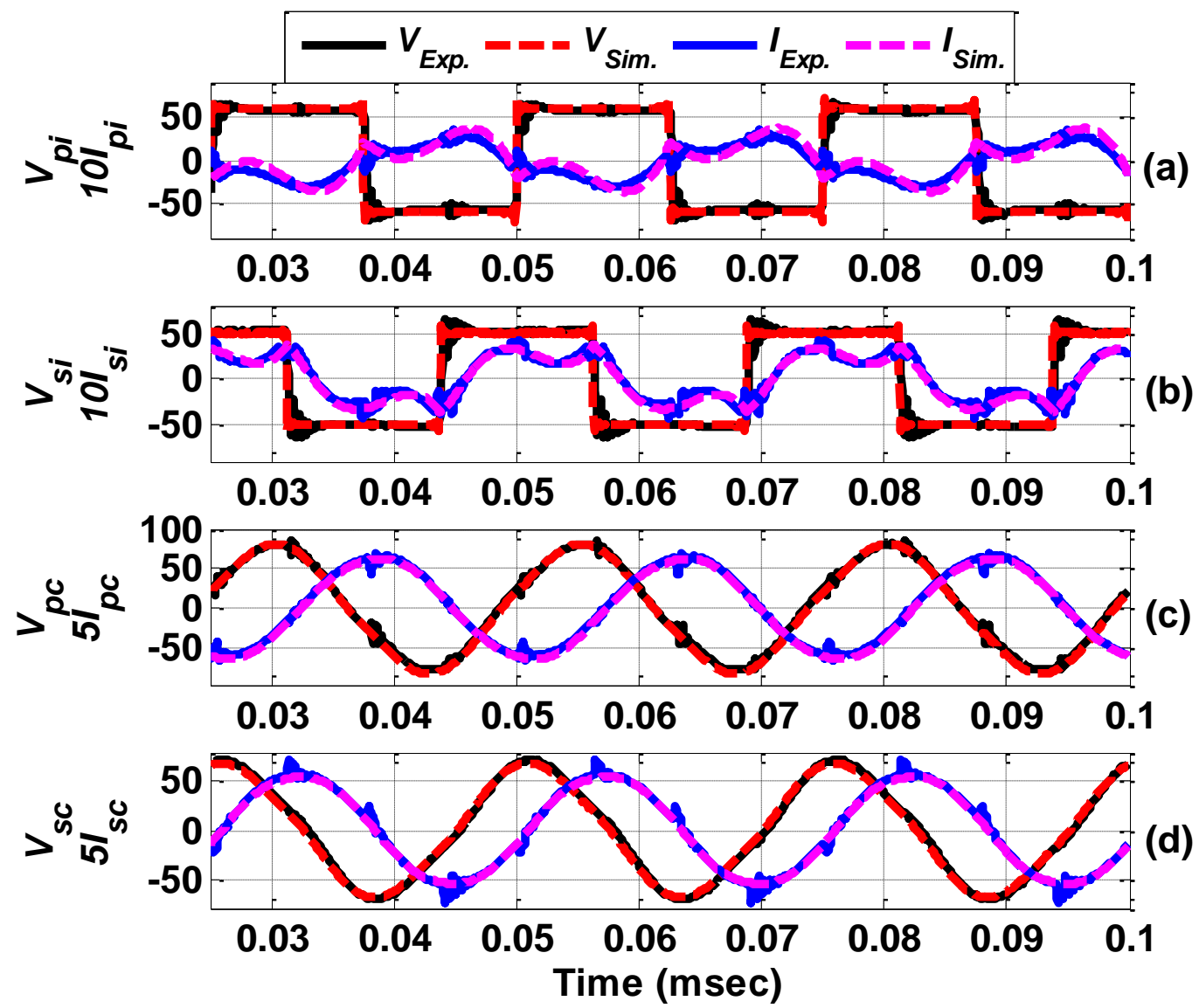

Figure 5.26. Experimental and simulated BIWPTS's performance under full supply voltage for V2G operation $\left(\alpha=\beta=180^{\circ}, \delta=90^{\circ}, V_{d c}=60 \mathrm{~V}\right)$. (a) Primary inverter variables. (b) Secondary inverter variables. (c) Primary coil variables. (d) Secondary coil variables. 


\subsubsection{Reduced Supply Capacity Test}

To verify the power level adjustment capability, the system is analyzed during the charging $(\mathrm{G} 2 \mathrm{~V})$ operation with quasi-square voltage waveform. This situation is achieved by setting the phase-shift between the inverter's legs as $\alpha=\beta=90^{\circ}$, and $\delta=-90^{\circ}$. The system performance under these conditions is described in Figure 5.27. As it can be observed, $V_{p i}$ and $V_{s i}$ waveforms exhibit the zero level, which causes the reduction of the transferred currents and power, in consequence.
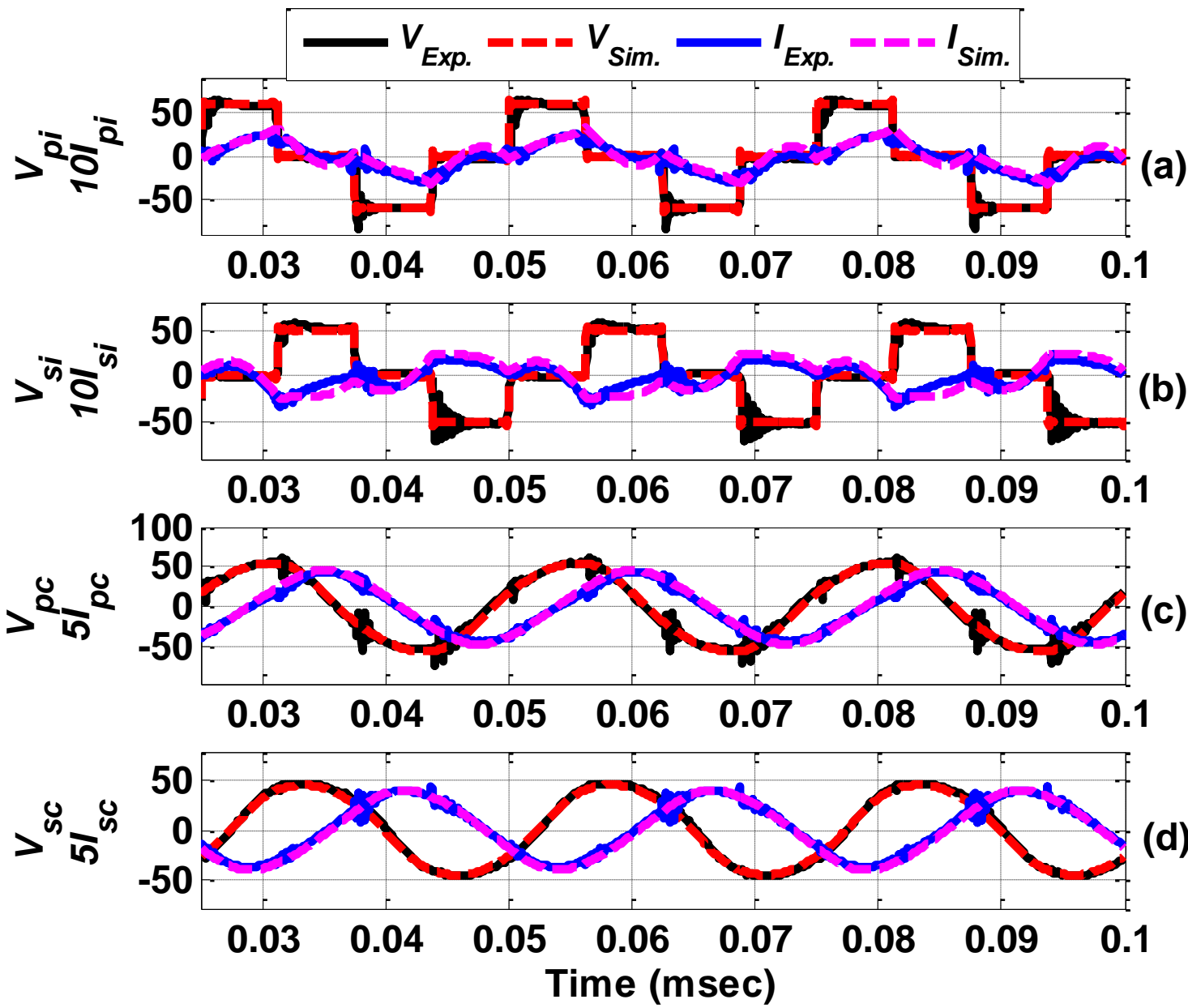

Figure 5.27. Experimental and simulated BIWPTS performance under reduced supply voltage for G2V operation ( $\left.\alpha=\beta=90^{\circ}, \delta=-90^{\circ}, V_{d c}=60 \mathrm{~V}\right)$. (a) Primary inverter variables. (b) Secondary inverter variables. (c) Primary coil variables. (d) Secondary coil variables. 
For a more clear comparison between the simulation and experimental results, the normalized root mean square error (NRMSE) is evaluated, based on Equation (5-7), for the presented cases and the results are indicated in Table 5.4. Most of the predicted variables show less than $5 \%$ errors. The error in $I_{p i}$ and $I_{s i}$ estimation is a little high due to the existence of a DC-current offset in the measurements, which results from the very small asymmetry in the inverter outputs.

$$
N M S E=\sqrt{\sum_{i=1}^{m}\left(f\left(t_{i}\right)-g\left(t_{i}\right)\right)^{2} / \sum_{i=1}^{m}\left(f\left(t_{i}\right)\right)^{2}}
$$

where, $f\left(x_{i}\right)$ and $g\left(x_{i}\right)$ are the value of the variable at sampling index $(i)$, which are calculated from experimental and simulation, respectively.

Table 5.4. NRMSE between Simulation and Experimental Results.

\begin{tabular}{lcccccccc}
\hline Mode & $\boldsymbol{I}_{\boldsymbol{p} i}$ & $\boldsymbol{I}_{\boldsymbol{s i}}$ & $\boldsymbol{I}_{\boldsymbol{p} \boldsymbol{c}}$ & $\boldsymbol{I}_{\boldsymbol{s c}}$ & $\boldsymbol{V}_{\boldsymbol{p} \boldsymbol{}}$ & $\boldsymbol{V}_{\boldsymbol{s i}}$ & $\boldsymbol{V}_{\boldsymbol{p c}}$ & $\boldsymbol{V}_{\boldsymbol{s c}}$ \\
\hline Full G2V & 8.5 & 9 & 0.57 & 0.73 & 1.3 & 1.3 & 0.36 & 0.32 \\
Full V2G & 3.9 & 4.9 & 0.75 & 0.61 & 1.8 & 1.4 & 0.23 & 0.24 \\
Reduced G2V & 11.7 & 10 & 2 & 0.66 & 1 & 1.3 & 0.67 & 0.07 \\
\hline \hline
\end{tabular}

\subsubsection{Practical Considerations during Testing the BIWPTS}

The experimental tests and measurements of the BIWPTS are challenge and need to be done carefully. Some practical issues are examined during testing the LCL BIWPTS prototype, which are concluded below: 
- The HF inverters' switches and driver circuits need to be well designed to supply symmetrical voltage waveform. Any small asymmetry in the voltage generates a large DC current component, due to the very small DC impedance at resonance. This asymmetry may be due to a small difference in dead-time of the inverter switches. This problem does not exist, if there is a series capacitor in the system, such as $L C$-series or $C L C L$ compensation topology, since the series capacitor behaves as a high-pass filter.

- The polarities of the power pad coils must be well defined and connected correctly with the inverters' outputs, to provide the proper power-flow direction, based on the assigned phase-shift between the two inverters' voltages $(\delta)$.

- There is another design problem related to the compensation network elements due to the errors in parameters' estimation and the parasitic elements of both converters and power pads. This problem was addressed in the prototype by initially evaluating the compensation elements based on the resonant frequency and the estimated pads' selfinductances, as given in Equation (4-2). Then, these parameters were corrected by trial and error, until the system shows pure resistive behavior and hits the resonant condition.

- The measuring sensors need to be carefully chosen with wide bandwidth to handle the HF signals.

\subsection{Conclusion}

This chapter presented an effective design and implementation considerations for the different components of the bidirectional inductive wireless power transfer system in EVs applications. The design of HF voltage-source H-bridge converter is investigated in detail. 
The details of the proposed design are presented including the parameters' estimation, components' selection and PCB layout design. Moreover, two protection schemes were introduced to the inverter circuit. Snubber circuits were designed for minimizing the ringing effect. All the inverter components were embedded in one PCB using the commercially available standard size (9"x6"), which results in size and weight reduction. The proposed inverter design performance was tested under different operating and control conditions. In addition, the analog implementation of the phase-shift control technique was performed and tested. The circuit was able to supply the switching activities for two synchronized inverters with the associated dead-times at different modulation conditions. Practical design considerations for the magnetic power pads and the compensation capacitors are addressed. Finally, two identical inverters were built and used in a LCL bidirectional inductive wireless power transfer system, to achieve the two-way power-flow operation between the EV and the power-grid. The entire system performance was analyzed under different modes of operation ( $\mathrm{G} 2 \mathrm{~V}$ and $\mathrm{V} 2 \mathrm{G})$, by simulation and experimentally for verification purposes. The tests show very good correlation between the simulation and the experimental results. 


\section{Chapter 6 Assessment Analysis of Different Compensation Topologies of BIWPTS}

\subsection{Introduction}

In a BIWPTS, the pads are loosely coupled with a large leakage inductance. A compensation network is usually used to reduce the Volt-Ampere (VA) rating in the coil and power supply. In early inductive charging designs, the compensation is set on primary or secondary side only [172]. In the current IPT technology, where the coupling coefficient is reduced to less than 0.3 for $\mathrm{EV}$ wireless charging, compensation at both the primary and secondary side is recommended, to have a more flexible and advanced characteristic [82]. The simplest way to compensate the system inductance, is to add a capacitor at each side. For a symmetrical BIWPTS, there are four basic compensation topologies, which are LCseries, LC-parallel, LCL, and CLCL topology, as was discussed in chapter (4). Based on the analytical models that were presented in chapter (4), an assessment analysis for the steady-state performance of different topologies in BIWPTS for EV implementations is investigated in this chapter. The analysis is presented for the three main compensation configurations: LC-series, LC-parallel and LCL-topology. The study presents evident criteria to pick among the different BIWPTS structures in the diverse applications. The proposed analyses are verified using simulation and experimental tests. The results demonstrate the ability of the proposed models to provide accurate estimation for BIWPTS's performance under various operating and control conditions. Also, the evaluation analysis shows that LCL-topology is more appropriate for the bidirectional 
operation in EV, due to the simple design and control requirements and being less sensitive to the misalignment.

The contributions in this work are summarized as follows: 1) verifying the developed theoretical models in chapter (4) by means of simulation and experimental tests, 2) utilizing these models to assess the performance of the different topologies during the bidirectional operation and present the merits and demerits of each structure, 3) achieving comparative analysis for the BIWPTS's performance parameters (power, power factor and efficiency) among all topologies, under both full and light loading conditions, and 4) evaluating the sensitivity of the different topologies against the misalignment in the system based on theoretical models and measurements.

\subsection{Assessment Criteria of Compensation Topologies in BIWPTS.}

In unidirectional IPT systems, the primary side is connected to the grid and represents the source of energy. The secondary side is coupled to the EV's battery and behaves as a load. Thus, in these systems, there is a specific source side and load side. In this case, the choice of the compensation topology in the secondary side depends on various factors, such as the output type (voltage/current) and the load. The choice of primary topology, on the other hand, depends on other factors, such as the type of inverter used. In bidirectional applications, the BIWPTS will be utilized to charge (G2V) and discharge (V2G) the EV's battery. During G2V operation, the primary side represents the source of energy, while the secondary side acts as a load. In V2G service, the primary side behaves as a load, while the secondary side supplies the required energy. Thus, in this case, there is neither a specific source side nor load side. Hence, from the author's point of view, the two sides need to be 
treated equally. In addition, different considerations during the choice of the compensation topology need to be taken into account. Due to these reasons, it is assumed that the bidirectional system under consideration is symmetrical, in which the compensation topologies in both sides are identical.

In general, as was reported in many works in the literature, the choice of the compensation topology plays a very important role in the performance of IPT system. Regardless of the mode of operation ( $\mathrm{V} 2 \mathrm{G}$ or $\mathrm{G} 2 \mathrm{~V}$ ), the compensation topology in the system helps to reduce the VA rating of power electronics converters, achieve UPF operation (zero-phase-angle) condition, achieve soft switching [zero-voltage switching (ZVS) or zero-current switching (ZCS)] in the power converters, enhance the power transfer capability and increase the system efficiency [82], [89].

In BIWPTS, the compensation topologies need to be evaluated based on the following requirements [139], [86], [82], [89], [146]:

- Providing two-way power-flow operation. The compensation topology needs to be tested during both G2V and V2G operation. It should be able to facilitate a bidirectional and controlled power-flow. In this case, the topology, which needs less complex controllers, is preferred.

- Minimizing VA rating of power converters. In this case, two HF converters are adopted in the system. Thus, the compensation structure on both sides needs to compensate the reactive power requirements, such that the converters carry the active power only.

- Maximizing power transfer capability by compensating the inductive reactance. 
- Enhancing the system efficiency. The ability of the compensation network to achieve soft-switching and minimize the reactive power flow through the converters and feeder leads to higher system efficiency.

- Offering more stability and less insensitivity to the variation of system parameters and loading conditions. The resonant network must be able to realize constant current, constant voltage or constant power under parameters' variation (especially the mutual inductance) and different loading conditions.

\subsection{Performance Evaluation for Different Topologies.}

The proposed mathematical models in chapter (4) are implemented and solved in MATLAB environment. These models are based on the harmonic components and all the parameters and variables are solved at each harmonic component and added together. The results from these models were compared with Simulink models for the different compensation configurations (Figure 4.3 and 5.24), for verification purposes. For fair comparison and assessment among the various structures, the same design parameters, given in Table 5.3, are considered in all of them and only the compensation topology varies. The framework is designed to resonate at $40 \mathrm{kHz}$ with $150 \mathrm{~mm}$ air-gap length between the primary and secondary coils, which is the typical case in EV situation. The DC voltage levels that are used in the Simulink models are $V_{d c}=240 \mathrm{~V}$ and $V_{b}=200 \mathrm{~V}$. In addition, power-flow assessment analysis for all topologies is presented for the same power-flow level $(1.5 \mathrm{~kW})$. Each configuration is analyzed during both $\mathrm{G} 2 \mathrm{~V}$ and V2G operation, considering the full and light loading conditions. 


\subsubsection{Assessment of LC-Series Topology.}

As it was mentioned before, the power-flow direction and the operation mode (either $\mathrm{V} 2 \mathrm{G}$ or $\mathrm{G} 2 \mathrm{~V}$ ) is controlled by adjusting the sign of $\delta$, while the power-flow magnitude is controlled by adjusting $\alpha$ and/or $\beta$. Figure 6.1 describes the system performance with LCseries topology under full charging $(\mathrm{G} 2 \mathrm{~V})$ operation, which means that the inverters transmit the full supply capacity. This mode is achieved by setting $\left(\alpha=\beta=180^{\circ}\right.$ and $\left.\delta=90^{\circ}\right)$. The primary and secondary side variables are indicated in Figure 6.1(a) and 6.1(b), respectively. The theoretical waveforms are plotted for the first 150 harmonics and they show very good matching with the Simulink model results. As it can be noticed, the inverter output voltages are full-square waves with amplitudes equal to the DC voltages.

Also, it can be seen from the figure that, $V_{p i}$ and $I_{p i}$ are in-phase, but $V_{s i}$ and $I_{s i}$ are anti-phase, which means that both the primary and secondary circuits are working almost at UPF and the system is hitting the resonant frequency. Moreover, there is a phase-shift between the primary and secondary voltages $\left(V_{p i}\right.$ lags $V_{s i}$ by $\left.90^{\circ}\right)$, which allows the power to flow from the grid to charge the EV. In addition, both inverters' and coils' currents are equal and sinusoidal, with no DC current components due to the series capacitors, which behave as high-pass filters. It is worth mentioning that the series resonance tank magnifies the pad and capacitor voltages $\left(V_{p c}\right.$ and $\left.V_{c p}\right)$ much higher than the inverter voltages due to the capacitive reactance, as was indicated in Equation (4-8). It can be seen in Figure 6.1(c) and 6.1(d) that the peak of the coil voltage $\left(V_{p c}\right)$ is almost equal to the peak of the capacitor voltage $\left(V_{c p}\right)$, and both are almost five times the peak of the inverter voltage $\left(V_{p i}\right)$. 

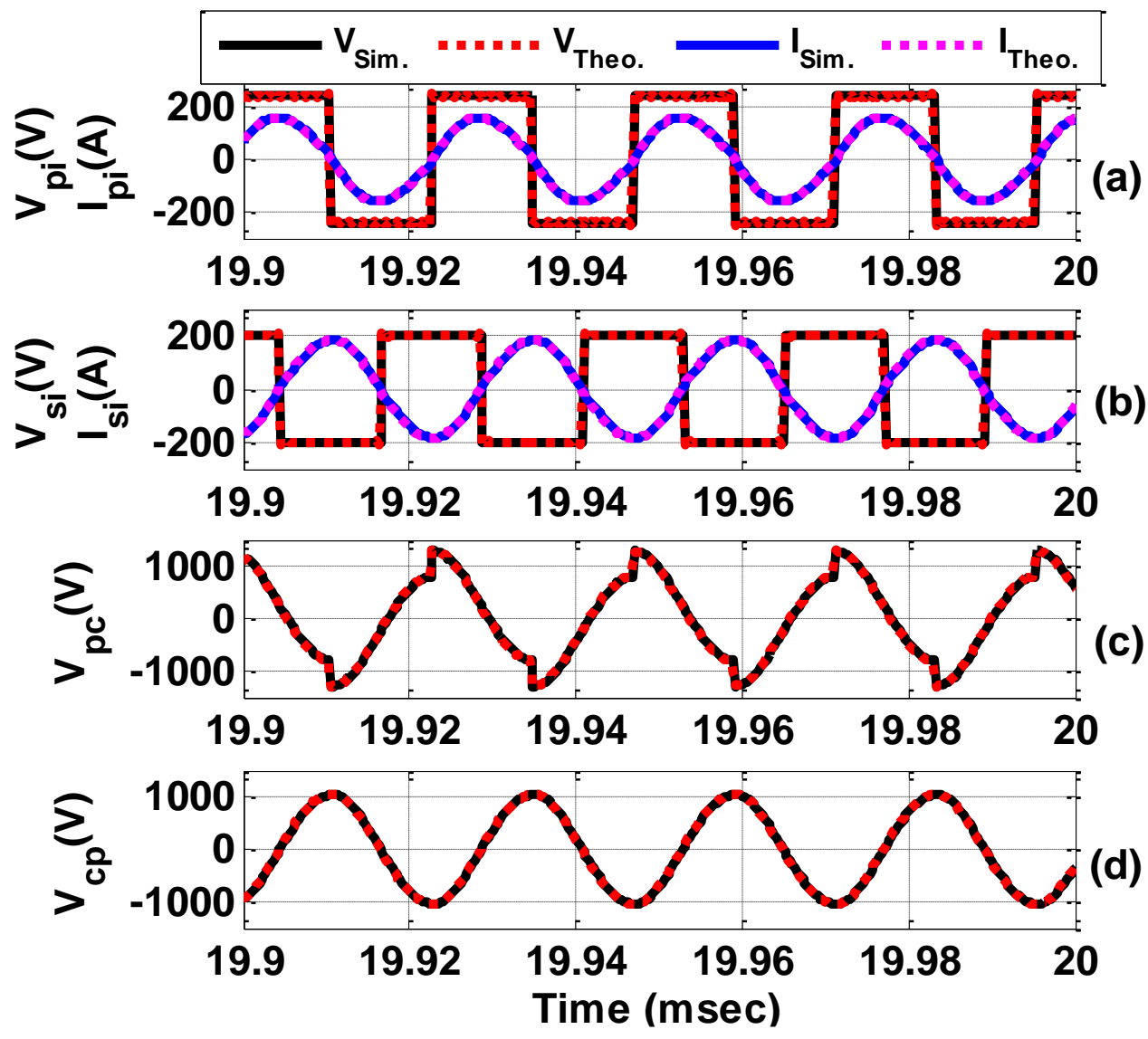

Figure 6.1. LC-series topology performance under full supply capacity for G2V operation ( $\alpha=\beta=180^{\circ}, \delta=90^{\circ}, V_{d c}=240 \mathrm{~V}$ and $V_{b}=200 \mathrm{~V}$ ). (a) Primary inverter variables. (b) Secondary inverter variables. (c) Primary pad voltage. (d) Primary capacitor voltage.

Figure 6.2 describes the G2V operation with reduced power level, by adjusting $\alpha$ and $\beta$ to be $90^{\circ}$ instead of $180^{\circ}$. A zero-voltage level appears in the inverter voltages to reduce the RMS voltages, currents, and accordingly the power. The V2G operation under reduced power level is depicted in Figure 6.3. In this case, the secondary voltage lag the primary one by $90^{\circ}$, which allows the power to flow from the EV to the power grid. Also, $V_{p i}$ and $I_{p i}$ are anti-phase and $V_{s i}$ and $I_{s i}$ are in-phase, which means that the system is still working at UPF, but with the reverse power flow direction. 

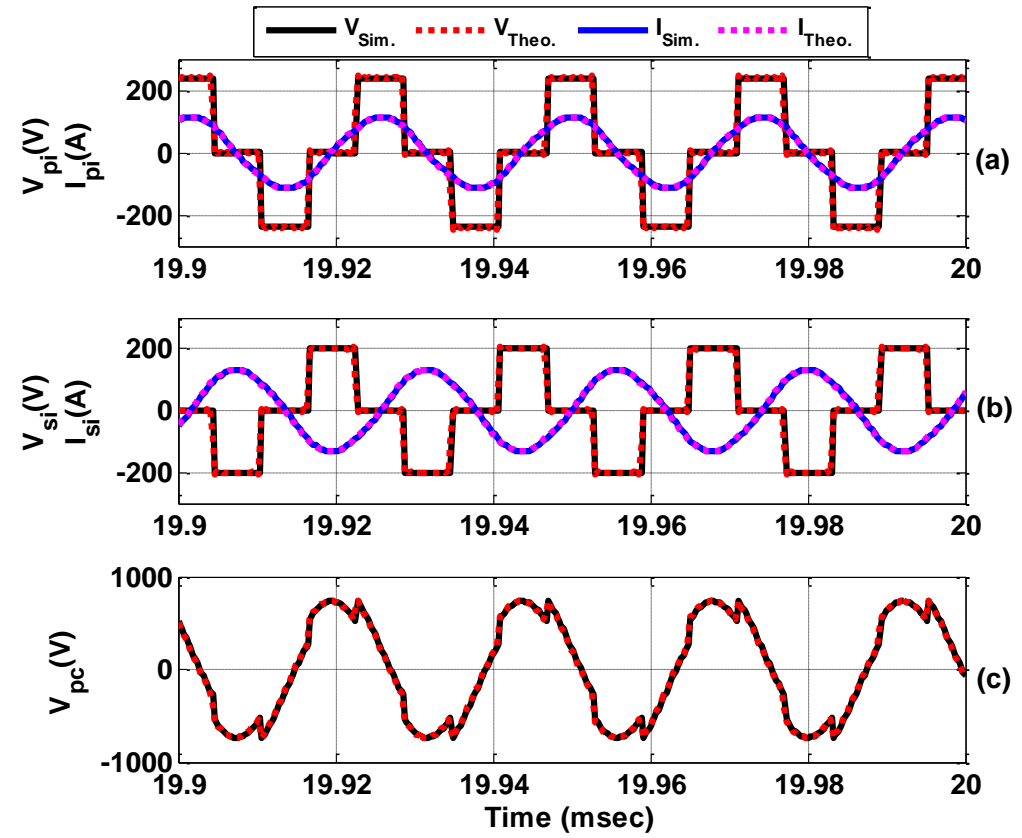

Figure 6.2. LC-series topology performance under reduced supply capacity for charging operation (G2V) $\left(\alpha=\beta=90^{\circ}, \delta=90^{\circ}, V_{d c}=240 \mathrm{~V}\right.$ and $\left.V_{b}=200 \mathrm{~V}\right)$. (a) Primary inverter variables. (b) Secondary inverter variables. (c) Primary pad voltage.
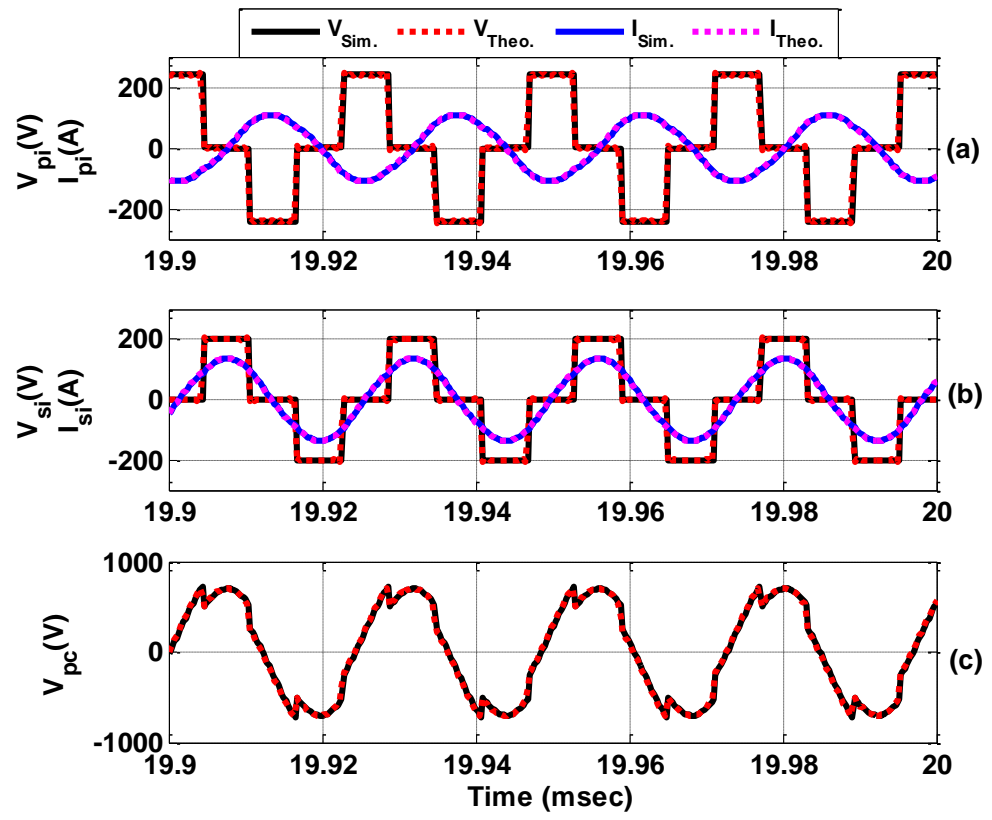

Figure 6.3 LC-series topology performance under reduced supply capacity for discharging operation (V2G) $\left(\alpha=\beta=90^{\circ}, \delta=-90^{\circ}, V_{d c}=240 \mathrm{~V}\right.$ and $\left.V_{b}=200 \mathrm{~V}\right)$. (a) Primary inverter variables. (b) Secondary inverter variables. 
From the presented results, it can be observed that in LC-series topology, huge voltages appear across the power pads, which lead to large current-flow to achieve the desired power-transfer. The inverter currents are sinusoidal, which facilitates the implementation of soft-switching activities, such as ZVS or ZCS. In addition, the same sinusoidal currents are passing in the wireless pad, which results in minimum radio frequency interference on the nearby devices. However, LC-series structure exhibits several limitations in EVs applications, as follows:

- The large pad current must be carried by the inverter. Therefore, the inverter needs to be designed to handle this current, which is a challenge, especially in high-power and HF applications.

- The wireless pad design requires many turns of winding with thick conductors to handle the high-voltage and current operation.

- The compensation capacitors must be capable of supporting these high-voltages and currents.

- The system experiences significant conduction (in power converters, passive elements and feeders) and dielectric (capacitors) losses.

- Current controllers are necessary in this configuration to limit the pad currents in both the system's sides to match with the rated values.

- The high voltage on the pad may become very pronounced if the secondary circuit was left open.

- It is very sensitive to misalignment.

Typically, this topology is used in low-power applications, and when the inverter is located near the coupler, in order to minimize the feeder losses and voltage-drop. 


\subsubsection{Assessment of LC-Parallel Topology}

BIWPTS with LC-parallel configuration is analyzed in this section during G2V operation using the theoretical and Simulink models, as given in Figure 6.4. In this configuration, the capacitor is connected in parallel with the pad to supply the required reactive power. In this case, the inverter provides only the real component that is required to handle the system losses in the two circuits and the load requirements. The square-wave voltages generated by the VSHBCs are rich in HF harmonics, as well as the desired fundamental frequency. The direct connection of such source to a parallel resonant circuit would cause excessive currents to flow at all higher harmonics of the drive frequency.

This is because the capacitor tank in the parallel resonant circuit would present a progressively lower capacitive reactance to increasing frequencies. This results in large current spikes at the switching transitions as the inverter tries to rapidly charge and discharge the capacitor on rising and falling edges of the square-wave, as can be seen in Figure 6.4(a), 6.4 (b) and 6.4 (c). These spikes are potentially very damaging to the VSHBC and lead to large reactive power to be derived from the supply associated with poor PF operation. The inclusion of L-filter between the inverter and capacitor negates this problem, as is demonstrated in the next section. The pad current is almost sinusoidal and does not show these spikes, which are absorbed by the parallel capacitor. In this test, the theoretical model is solved for the first 150 harmonics orders and exhibits good correlation with the Simulink model. 


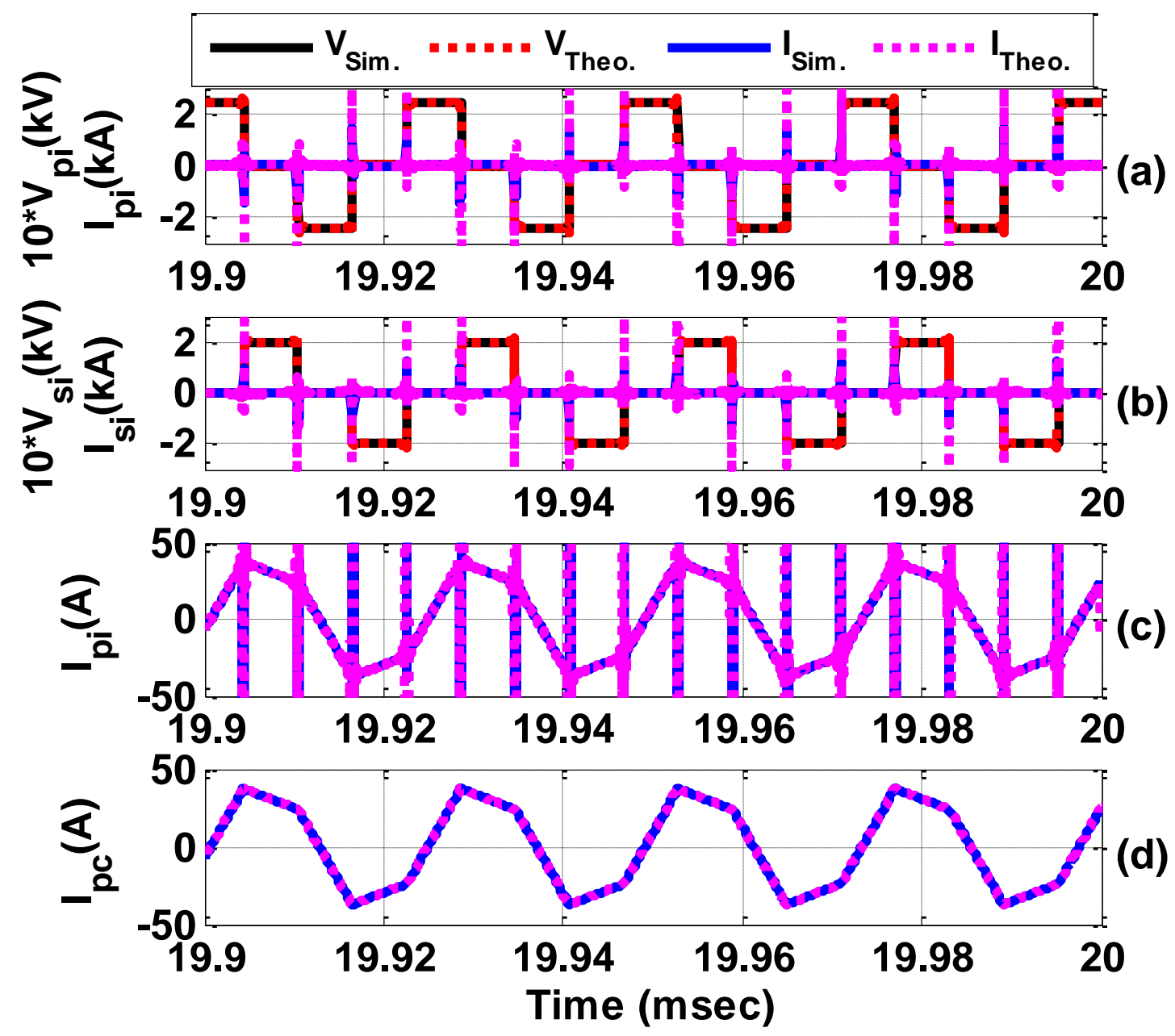

Figure 6.4. LC-parallel topology performance under reduced supply capacity for G2V operation $\left(\alpha=\beta=90^{\circ}, \delta=-90^{\circ}, V_{d c}=240 \mathrm{~V}\right.$ and $\left.V_{b}=200 \mathrm{~V}\right)$. (a) Primary inverter variables. (b) Secondary inverter variables. (c) Zoomed primary inverter current. (d) Primary pad current.

\subsubsection{Assessment of LCL Topology.}

The LCL structure is an improved version of LC-parallel topology by adding an impedance matching coil between the inverter and parallel resonant tank. This topology is investigated during $\mathrm{G} 2 \mathrm{~V}$ operation using the proposed theoretical (for the first 15 harmonics) and Simulink models in this section. LCL-based BIWPTS's performance for full supply capacity operation is described in Figure 6.5. The figure shows all the primary 
$\left(V_{p i}, I_{p i}, V_{p c}\right.$ and $\left.I_{p c}\right)$ and secondary $\left(V_{s i}, I_{s i}, V_{s c}\right.$ and $\left.I_{s c}\right)$ variables. As can be seen, the inverter currents are limited to reasonable values and the spikes disappear after adding the L-filters. Also, the coil currents become pure sinusoidal, leading to lower electromagnetic interference. The inverter current is very small compared with the coil current, since it represents only the active current component, while the reactive part is supplied from the parallel capacitor. The peak inverter and capacitor voltages are almost equal to the DC voltage levels, as the parallel resonance does not magnify the voltage like the series one.
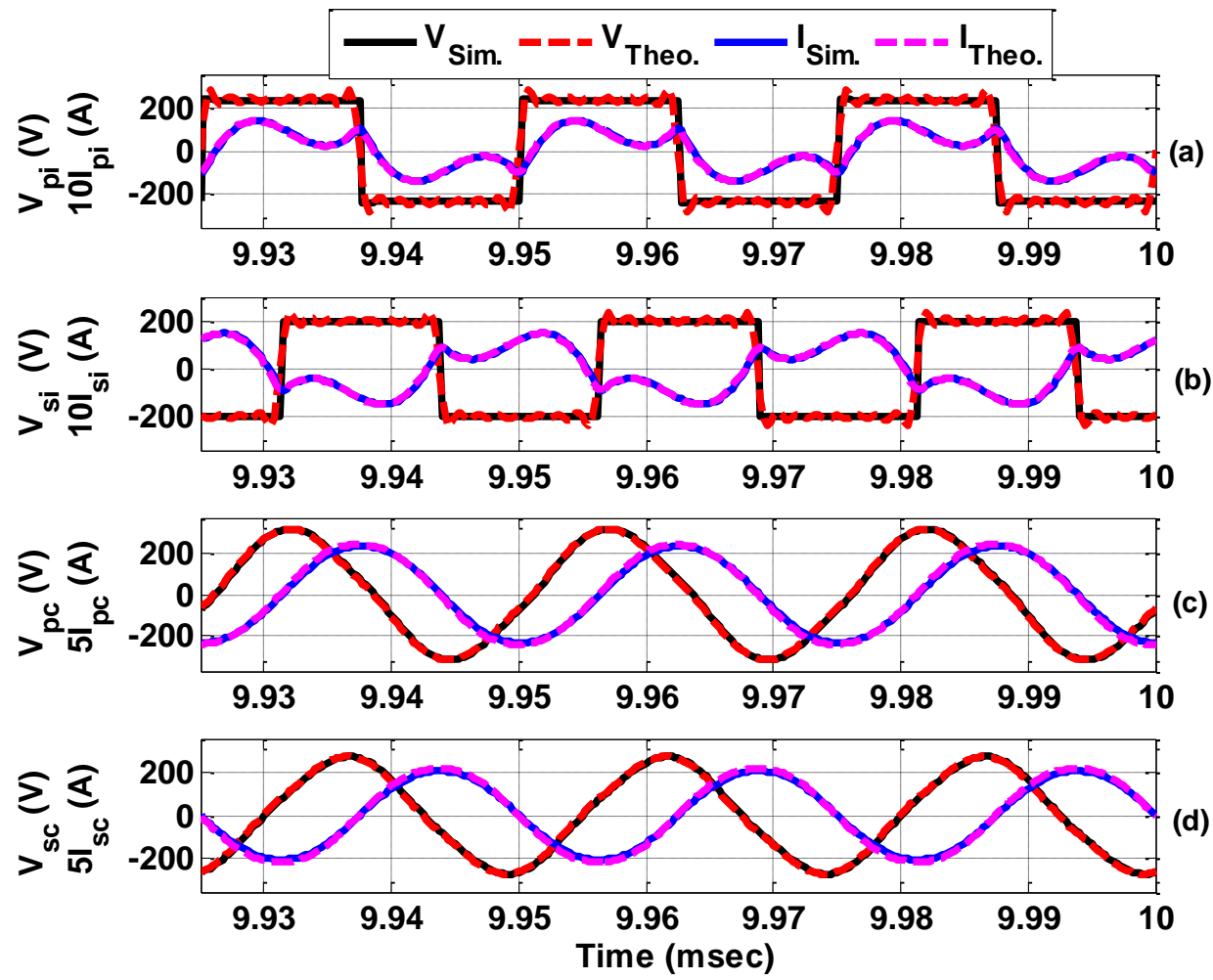

Figure 6.5. LCL-topology performance under reduced supply capacity for charging operation (G2V) ( $\alpha=\beta=180^{\circ}, \delta=-90^{\circ}, V_{d c}=240 \mathrm{~V}$ and $\left.V_{b}=200 \mathrm{~V}\right)$. (a) Primary inverter variables. (b) Secondary inverter variables. (c) Primary coil variables. (c) Secondary coil variables. 


\subsubsection{Power-Flow Assessment for Different Configuration.}

Performance evaluation parameters (power, efficiency and PF) for the three different configurations during G2V operation are analyzed in this section. For fair comparative analysis, the DC voltage levels $\left(V_{d c}\right.$ and $\left.V_{b}\right)$ for the three systems are adjusted, such that they provide the same rated power $(1.5 \mathrm{~kW})$. These voltages are varied with a fixed ratio for all topologies in order to deliver the same current distribution. Each configuration is investigated at full and light (half) loading conditions. The full loading condition is obtained by setting the control parameters $\left(\alpha\right.$ and $\beta$ ) to be $180^{\circ}$, as indicated in Table 6.1. The light loading condition is achieved by applying $\alpha=\beta=90^{\circ}$, as presented in Table 6.2. The given efficiency calculations represent only the passive components' efficiency and do not consider the inverters', feeders' and supplies' losses. The tables show that LC-series topology provides the highest efficiency and PF in both the full and light load conditions, due to the sinusoidal waveforms. LC-parallel exhibits very poor PF and the lowest efficiency operation due to the large current spikes. LCL-topology gives higher PF and efficiency than LC-parallel but less than LC-series, due to the non-sinusoidal inverter currents, which increase the reactive power and the losses in the system.

Table 6.1. Power-Flow Performance for all Topologies under Full-Loading Conditions.

\begin{tabular}{|c|c|c|c|c|}
\hline$\overline{\overline{\text { Topology }}}$ & $\overline{P_{p}(k W)}$ & $\overline{P_{s}(k W)}$ & $\% \eta$ & $\overline{P F}$ \\
\hline$\overline{\mathrm{LC}}$-series $\left(V_{d c}=60 \mathrm{~V}\right.$ and $\left.V_{b}=50 \mathrm{~V}\right)$ & -1.536 & 1.479 & 96.33 & 0.999 \\
\hline LC-parallel $\left(V_{d c}=236 \mathrm{~V}\right.$ and $\left.V_{b}=196 \mathrm{~V}\right)$ & 1.532 & -1.466 & 95.65 & 0.108 \\
\hline LCL $\left(V_{d c}=240 \mathrm{~V}\right.$ and $\left.V_{b}=200 \mathrm{~V}\right)$ & 1.534 & -1.473 & 96 & 0.9654 \\
\hline
\end{tabular}

Evaluated at $\alpha=\beta=180^{\circ}$. 
Table 6.2. Power-Flow Performance for all Topologies under Light-Loading Conditions.

\begin{tabular}{lcccc}
\hline \hline Topology & $\mathrm{P}_{\mathrm{p}}(\mathrm{kW})$ & $\mathrm{P}_{\mathrm{s}}(\mathrm{kW})$ & $\% \eta$ & $\mathrm{PF}$ \\
\hline LC-series $\left(V_{d c}=60 \mathrm{~V}\right.$ and $\left.V_{b}=50 \mathrm{~V}\right)$ & -0.768 & 0.740 & 96.3 & 0.999 \\
LC-parallel $\left(V_{d c}=236 \mathrm{~V}\right.$ and $\left.V_{b}=196 \mathrm{~V}\right)$ & 0.766 & -0.733 & 95.6 & 0.108 \\
LCL $\left(V_{d c}=240 \mathrm{~V}\right.$ and $\left.V_{b}=200 \mathrm{~V}\right)$ & 0.767 & -0.736 & 96 & 0.9654 \\
\hline \hline
\end{tabular}

Evaluated at $\alpha=\beta=90^{\circ}$.

Despite LCL-topology having two more passive components, it is more appropriate for high-power applications (e.g. EVs) and for the situations where the inverters are installed far from the pads, due to the following:

- The parallel capacitors behave as PF correction and supply the required reactive (magnetizing) power.

- Only the real power component is driven from the supply, which leads to simple inverter design and operation.

- The inverter currents are very small compared to the pad currents, which means low conduction and feeder losses.

- The wireless pads and capacitors require simple design since they do not have to handle high voltage operation.

- The system behaves as a current-source, therefore the bidirectional power-flow can be easily achieved by controlling the inverter voltages.

- It is less sensitive to the misalignment than LC-series topology.

- No current spikes appear due to the filtration process. 


\subsection{Experimental Evaluation for Different Topologies.}

In this section, the BIWPTS prototype presented in Figure 5.24 with the design parameters in Table 5.3 is utilized to evaluate the performance of the different topologies based on experimental tests. In this prototype, the DC voltage levels ( $V_{d c}$ and $\left.V_{b}\right)$ are scaled down (1/4 time the Simulink model values) to fit the available Li-ion battery module in the laboratory. The used DC voltage levels are $V_{d c}=60 \mathrm{~V}$ and $V_{b}=50 \mathrm{~V}$.
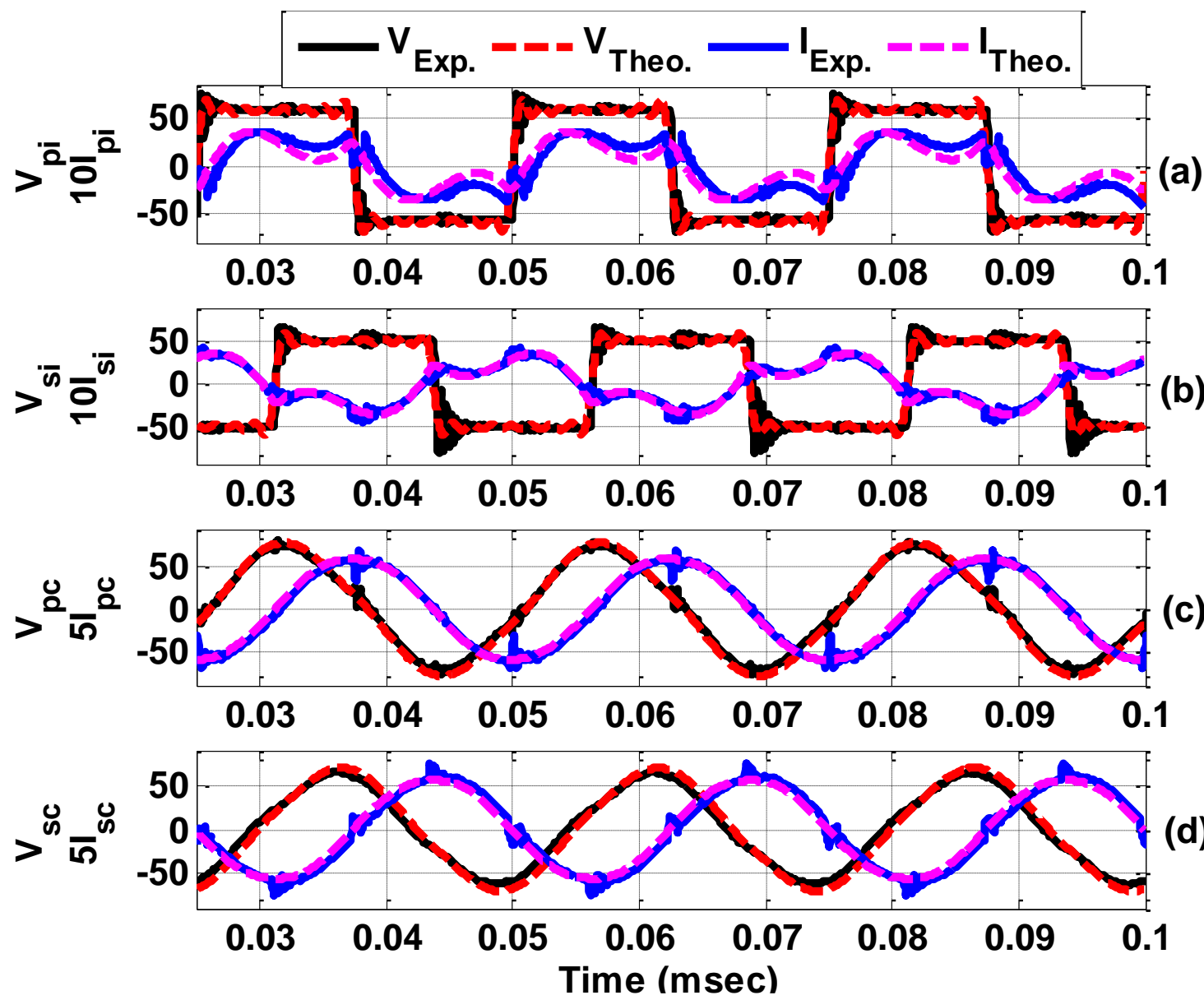

Figure 6.6. Experimental and theoretical LCL-topology performance under full supply capacity for $\mathrm{G} 2 \mathrm{~V}$ operation $\left(\alpha=\beta=180^{\circ}, \delta=-90^{\circ}, V_{d c}=60 \mathrm{~V}, V_{b}=50 \mathrm{~V}\right)$. (a) Primary inverter variables. (b) Secondary inverter variables. (c) Primary coil variables. (d) Secondary coil variables. 


\subsubsection{Experimental Validation of LCL Topology Model.}

In this section, the LCL topology is tested and the instantaneous voltage and current waveforms are recorded. These measured waveforms are compared with the theoretical ones, estimated from the proposed analytical model, for verification purposes. The response of LCL BIWPTS under the full supply capacity during G2V and V2G operation is indicated in Figures 6.6 and 6.7, respectively. The figures present comparison between experimental tests and theoretical model results. Good agreement is observed between the two models.
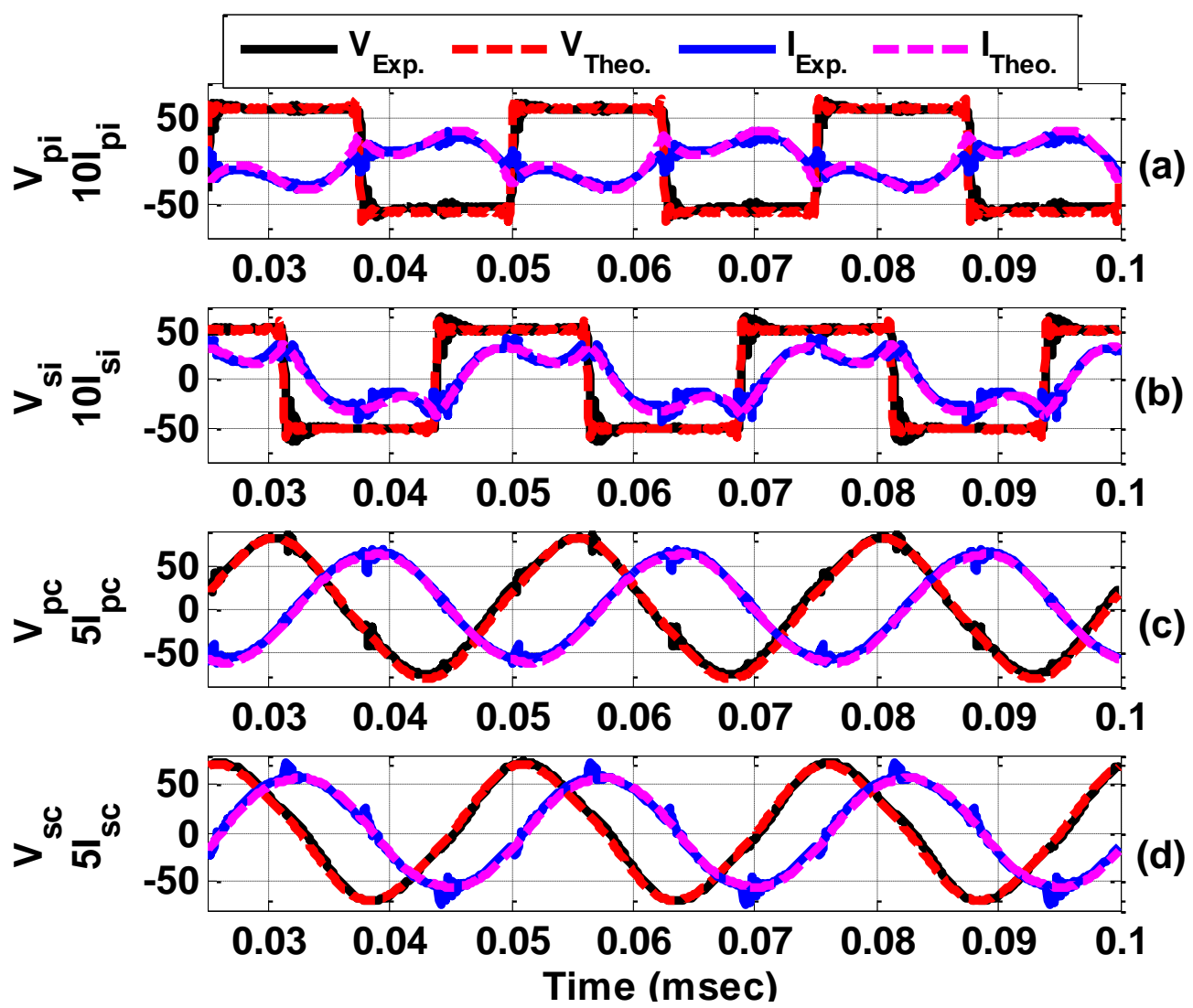

Figure 6.7. Experimental and theoretical LCL-topology performance under full supply capacity for V2G operation ( $\alpha=\beta=180^{\circ}, \delta=90^{\circ}, V_{d c}=60 \mathrm{~V}, V_{b}=50 \mathrm{~V}$ ). (a) Primary inverter variables. (b) Secondary inverter variables. (c) Primary coil variables. (d) Secondary coil variables. 
Moreover, the same prototype is analyzed to verify the effect of the phase-shift parameters on the power-flow magnitude. In this case, the effect of changing $\alpha$ and $\beta$ on the system power-flow is investigated. The system power is reduced by applying $\alpha=\beta=90^{\circ}$, while $\delta= \pm 90^{\circ}$ to control the power flow direction. The results of G2V operation with a reduced power level are described in Figure 6.8. It can be observed that the two inverters' voltages show zero-voltage level to reduce the RMS voltages, currents and, in consequence, the power. This effect is clear in the systems' currents and voltages. The system performance under the V2G operation is shown in Figure 6.9. Also, the results show good agreement between the theoretical and experimental data.
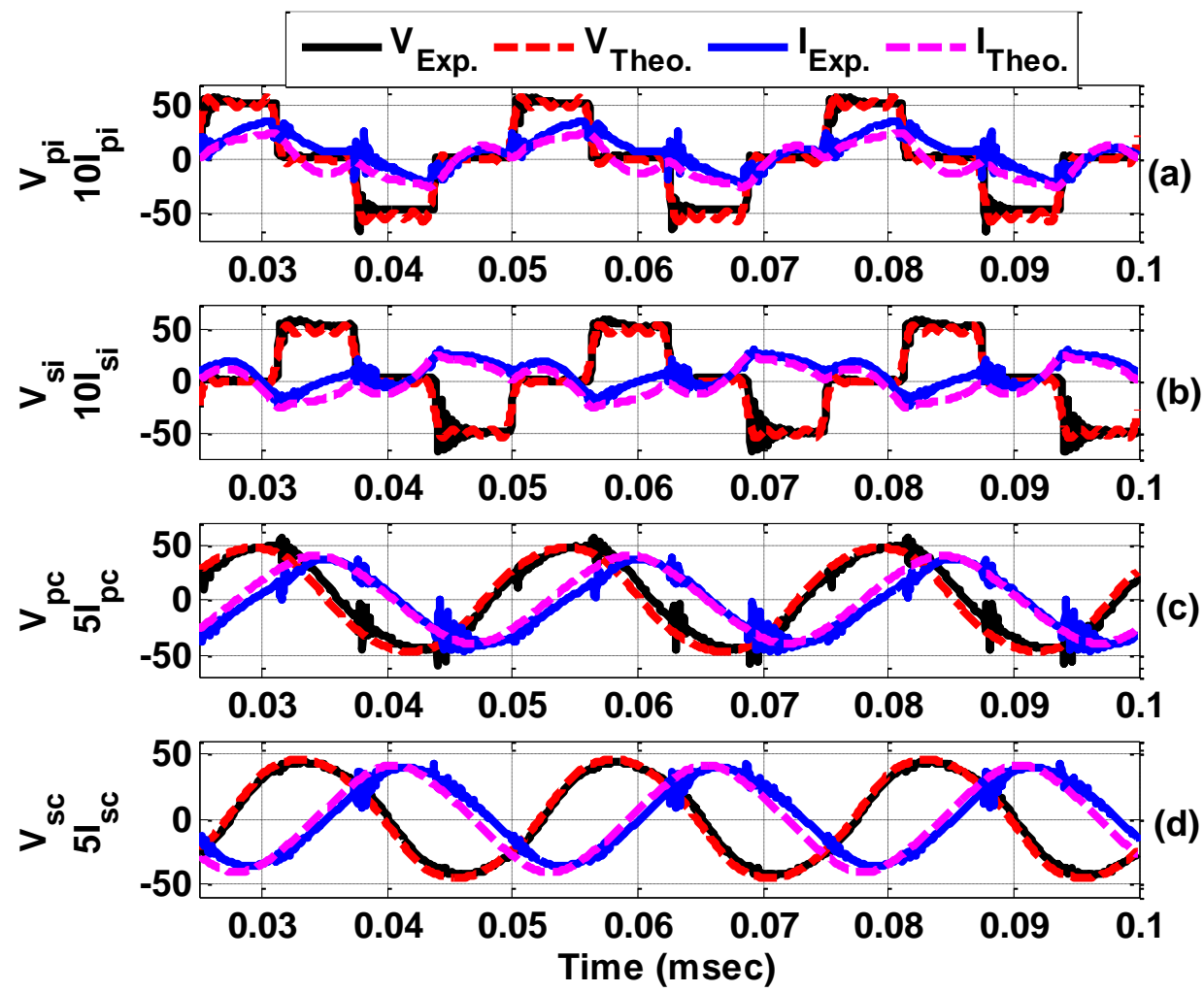

Figure 6.8. Experimental and theoretical LCL-topology performance under reduced supply capacity for G2V operation ( $\left.\alpha=\beta=90^{\circ}, \delta=-90^{\circ}, V_{d c}=60 \mathrm{~V}, V_{b}=50 \mathrm{~V}\right)$. (a) Primary inverter variables. (b) Secondary inverter variables. (c) Primary coil variables. (d) Secondary coil variables. 

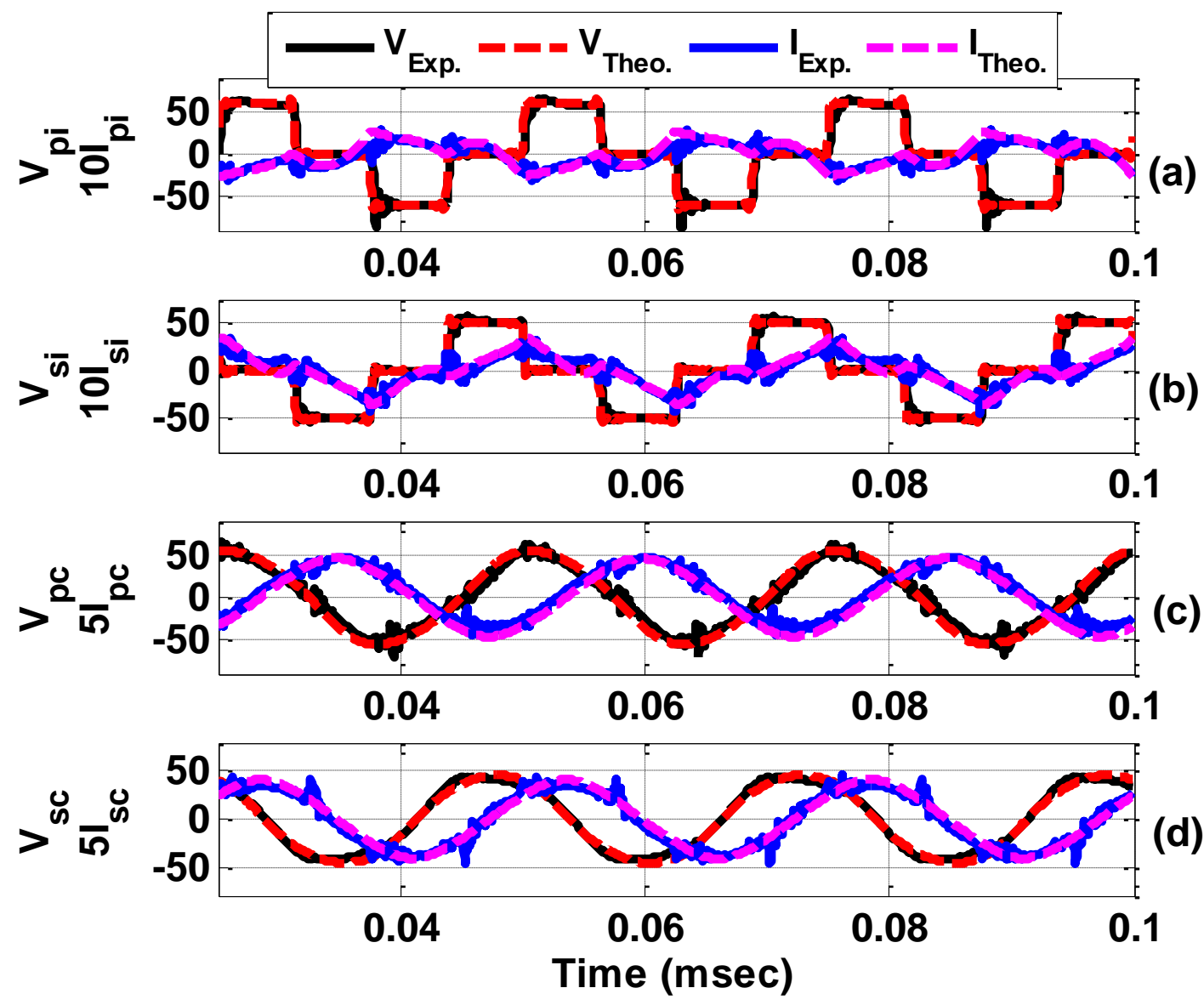

Figure 6.9. Experimental and theoretical LCL-topology performance under reduced supply capacity for V2G operation $\left(\alpha=\beta=90^{\circ}, \delta=90^{\circ}, V_{d c}=60 \mathrm{~V}, V_{b}=50 \mathrm{~V}\right)$. (a) Primary inverter variables. (b) Secondary inverter variables. (c) Primary coil variables. (d) Secondary coil variables.

In this analysis, two scales of BIWPTS are utilized. The large-scale is used in the Simulink model, with $V_{d c}=240 \mathrm{~V}, V_{b}=200 \mathrm{~V}$. The small-scale is considered in the prototype, with $V_{d c}=60 \mathrm{~V}, V_{b}=50 \mathrm{~V}$. In order to ensure the system scalability, the powerflow is evaluated for both large and small-scale models during G2V operation, as presented in Table 6.3. As can be noticed, both scales provide the same efficiency and PF. The ratio between the power levels is about 16 , which is the square of the scaling factor 4 . Thus, it 
can be concluded that the system is scalable, and small-scale prototypes can be utilized to verify its performance.

Table 6.3. Large and Small-Scale Power-Flow Analysis.

\begin{tabular}{lllll}
\hline Mode & $\boldsymbol{P}_{\boldsymbol{p}}(\boldsymbol{W})$ & $\boldsymbol{P}_{\boldsymbol{s}}(\boldsymbol{W})$ & $\boldsymbol{\eta}(\%)$ & $\boldsymbol{P F}(\boldsymbol{p u})$ \\
\hline Large-scale G2V $\left(\boldsymbol{V}_{\boldsymbol{d c}}=\mathbf{2 4 0} \mathbf{V} \& \boldsymbol{V}_{\boldsymbol{b}}=\mathbf{2 0 0} \mathrm{V}\right)$ & 1534 & -1473 & 96 & 0.965 \\
Small-scale G2V $\left(\boldsymbol{V}_{\boldsymbol{d}}=\mathbf{6 0} \mathbf{V} \& \boldsymbol{V}_{\boldsymbol{b}}=\mathbf{5 0} \mathrm{V}\right)$ & 95.855 & -92 & 96 & 0.965 \\
\hline \hline
\end{tabular}

Evaluated at $\alpha=\beta=180^{\circ}$ and $\delta=-90^{\circ}$.

\subsubsection{Evaluating the Sensitivity to Misalignment for all Topologies.}

Current IPT technology requires a perfect alignment between coils in order to keep the transferred power within the desired limits. However, this condition is practically impossible. Typically, different types of misalignment are expected between the two sides during the practical implementation. Thus, the SAE J2954A international standard has defined accepted limits and criteria for the different misalignments that the system may be subjected to. The system must operate within a range of these misalignment to be able to transfer the required power wirelessly. Two different categories of misalignments are accurately defined in J2954A standard [149]. The first class is associated with the planar

offset in $\mathrm{X}, \mathrm{Y}$ and $\mathrm{Z}$ directions. The second category is related to the angles between the two sides. Three different angle misalignments are defined: Rotation, Roll and Yaw angles, as indicated in Figure 6.10. 


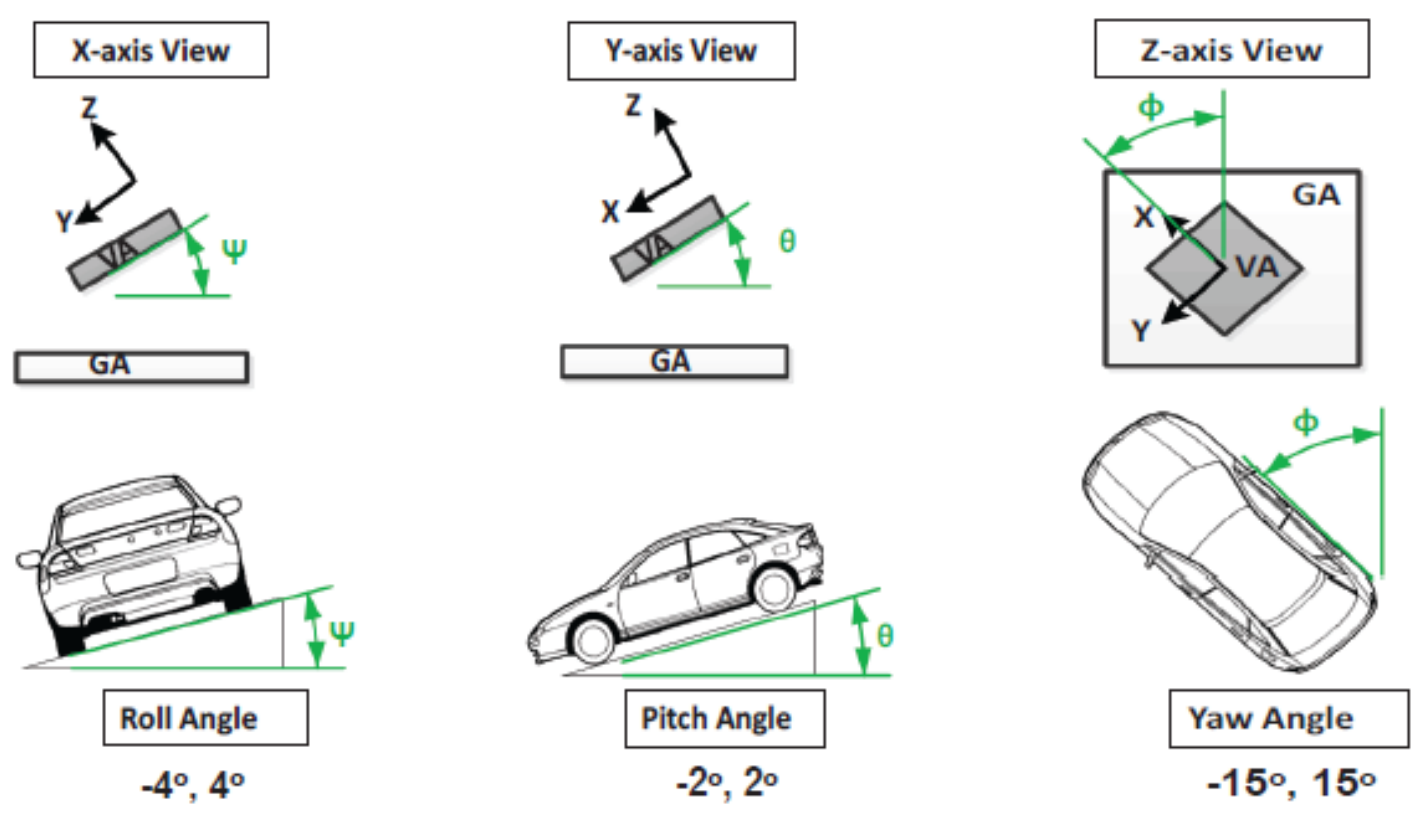

Figure 6.10. Angle misalignment in IPT system [149].

Various solutions are presented in the literature to minimize the misalignment issues, such as installing electro-mechanic alignment systems. This system causes the charging process to be slow and mechanically complex, or multiple primary systems (coil array structures) are required [173]. Another method to mitigate the misalignment problems and achieve fast charging process without the need for electro-mechanic positioning mechanisms is to implement a very precise, complex, closed-loop frequency control [174] that keeps the system operating at resonance, even with varying coupling factor. A cheap and simple way to deal with the misalignment issues is to choose and design the compensation network, such that the power-flow in the system becomes insensitive to the misalignment [175]. Therefore, one important aspect to evaluate the performance of a compensation network is to measure its sensitivity against the misalignment. 
Therefore, the performance of different topologies with the variation of the magnetic coupling between the system's sides, due to misalignment, is evaluated and compared in this section. In this analysis, the system was operated to transfer the maximum power during G2V mode $\left(\alpha=\beta=180^{\circ}\right)$. All the investigated performance measurement parameters are normalized with respect to their values at coupling factor $(k)$ equal 0.2 , as an intermediate value. Only theoretical results are provided for LC-parallel topology, in order not to damage the HF converters. The primary coil RMS current is measured and compared with the theoretical (estimated) value under different coupling factors, as presented in Figure 6.11. The figure shows that the coil current in LCL topology is independent from the coupling factor. This proves that this structure is able to behave as a current-source regardless of the misalignment. In contrast, in LC-series, the current is inversely proportional with the coupling factor.

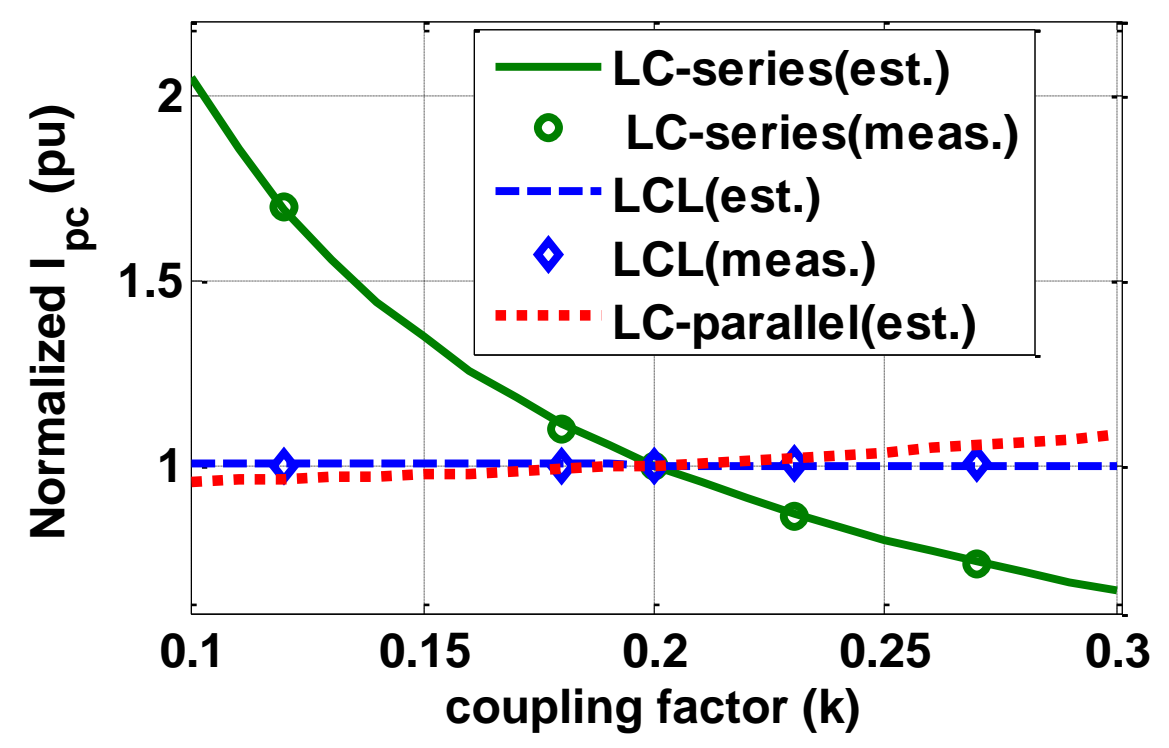

Figure 6.11. Measured and estimated normalized primary coil current under misalignment for all topologies $\left(\mathrm{G} 2 \mathrm{~V}, \alpha=\beta=180^{\circ}\right)$. 
In Figure 6.12, the active and reactive primary power for all topologies are evaluated. The measured powers are extracted from the experimental data using Fast-Fourier transform (FFT) to find the magnitude of each harmonic component. The fundamental component is used for active power estimation, while the higher-order harmonics are considered for reactive power calculation. LC-parallel and LCL show linear and similar increase in active power as $k$ increases [Figure 6.12(a)]. However, LC series exhibits significant reduction of power, as the coupling increases due to the sensitivity to the variation of the reflected impedance. Also, Figure 6.12(b) indicates that the reactive power of LCL topology is fixed regardless of the misalignment, unlike the other two configurations.
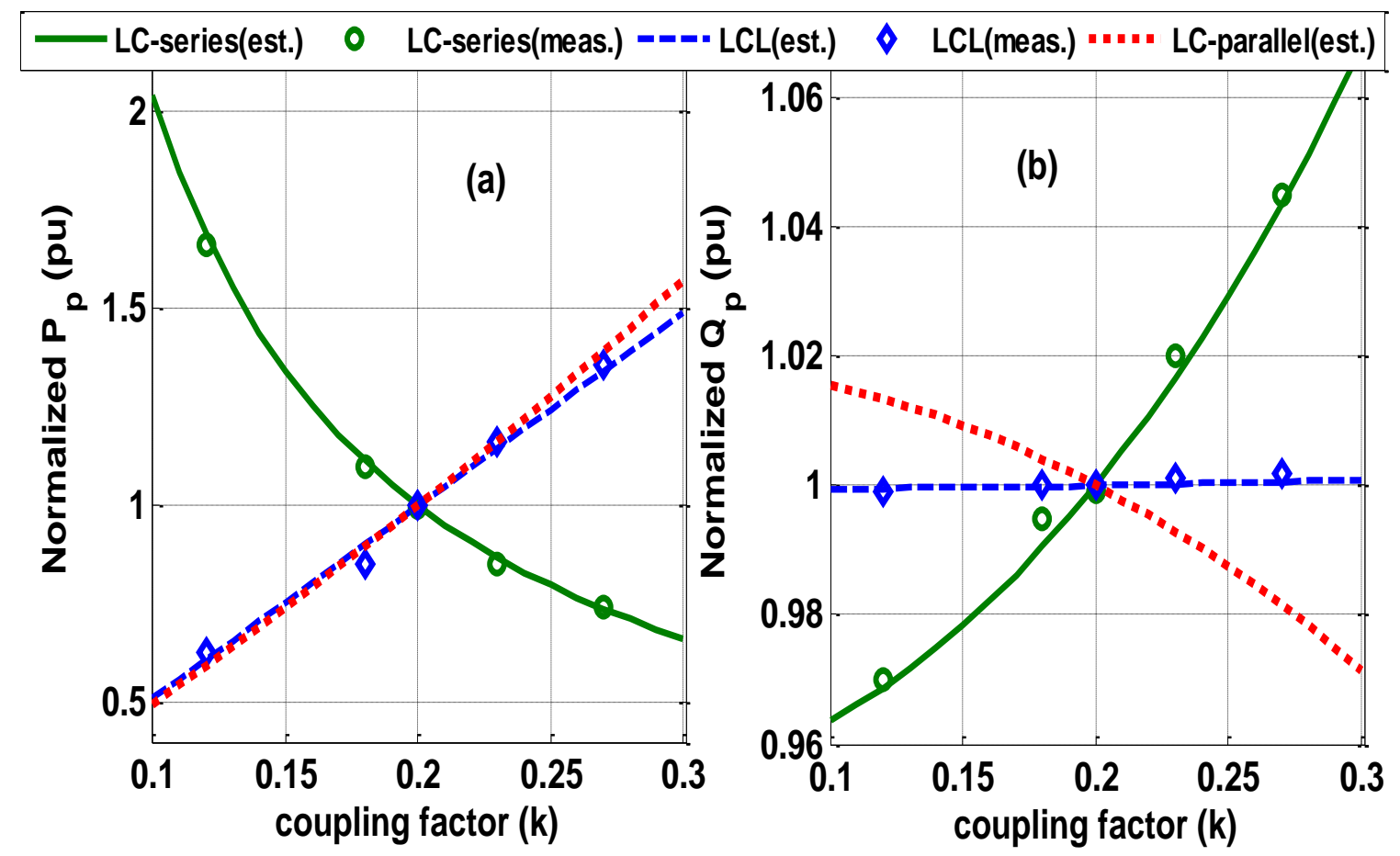

Figure 6.12. Measured and estimated normalized power under misalignment for all topologies (G2V, $\alpha=\beta=180^{\circ}$ ). (a) Active power. (b) Reactive power. 
Finally, the equivalent fundamental primary impedance for all topologies is estimated based on the impedance model in Equation (4-6). This parameter is analyzed versus the coupling factor in Figure 6.13. It can be observed that the equivalent impedance of LCL topology is less sensitive to the misalignment, unlike that of LC-series and LCparallel, which nonlinearly increases and decreases, respectively as the coupling factor increases. From Figure 6.13(b), both LC-series and LCL topology provide zero-impedance angle (UPF), as was discussed before, while LC-parallel shows lagging inductive impedance.
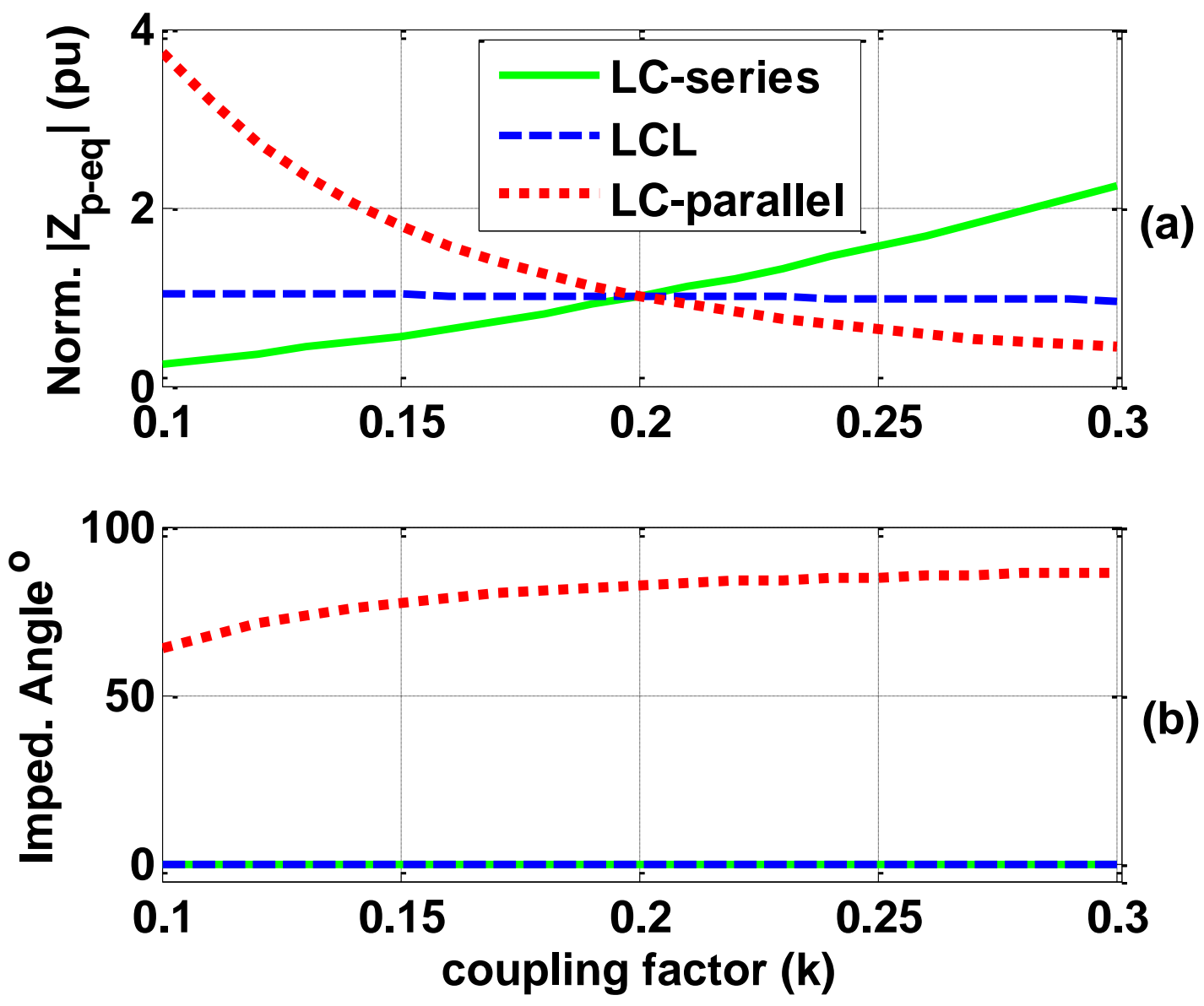

Figure 6.13. Theoretical fundamental equivalent impedance under misalignment for all topologies (G2V, $\alpha=\beta=180^{\circ}$ ). (a) Impedance magnitude. (b) Impedance angle. 


\subsubsection{Summary for the Assessment Analysis.}

The outcomes from the comparative analyses among the different topologies are summarized in Table 6.4. The table presents the advantages and disadvantages of the different topologies, in terms of many factors, such as passive components, control complexity, power factor, etc. It can be noticed that LCL-structure is better in terms of system's design, control implementation, physical installation and the robustness against misalignment, as is clarified below:

- As was discussed in section 6.3.1, in LC series configuration, the inverter should be designed to handle both active and reactive current components, which represents huge challenges in the semiconductor switches, especially in high-power and HF applications. This item not only will represent a problem during the design, but also it leads to significant conduction losses in the power converter and in the feeder, which will reduce the overall system efficiency. Although the coupler efficiency in LC-series is similar to that of LCL topology, the overall system efficiency, including the power electronic converters and the feeders (which was not included in this study), will be less in case of LC series.

- Regarding the control complexity, as was mentioned in section 6.3.3, LCL topology behaves as a current-source, which is the desired operation for EV charging. In this case, the power-flow can be easily controlled by adjusting the output voltage of the inverters. However, in the case of the LC-series structure, the system behaves as a voltage-source and current controllers are necessary, in addition to power-flow controllers, which means more complex control.

- For the physical installation, in LCL topology, the inverter can be installed near to the 
power pad or far from it. This is because, in this case, the current and losses in the feeder are small. On the other hand, in LC-series, it will be impractical to install the inverter far from the power pad, since the feeder current and losses will be significant.

- Concerning the sensitivity to misalignment, LCL topology shows more robustness against the misalignment, in contrast with LC-series network. This feature is very important for IPT system in EV applications, in which the system is usually subjected to the misalignment during the normal operation. The system may become completely useless if it was too sensitive to the misalignment.

Table 6.4. Summary of the Assessment Analysis.

\begin{tabular}{llll}
\hline Topology & LC-series & LC-parallel & LCL \\
\hline $\begin{array}{l}\text { No. of passive components } \\
\text { Bidirectional control }\end{array}$ & 4 & 4 & 6 \\
$\begin{array}{l}\text { Complexity } \\
\text { PF }\end{array}$ & High & ---- & Low \\
Radio interference & High & Low & Medium \\
Coupler efficiency & Low & High & Medium \\
Design requirements & High & Low & High \\
Sensitivity to misalignment & High & High & Low \\
Coupler position & close to & close to inverter & $\begin{array}{l}\text { close to or far } \\
\text { from inverter }\end{array}$ \\
Soft switching capability & High & Low & Medium \\
DC blocking capacitor & No need & Need & Need \\
\hline
\end{tabular}


Thus, it can be concluded that LCL-topology is the most practical and appropriate choice for bidirectional operation of EV. Even though LCL structure exhibits some deficiencies in PF, radio interference and soft switching capability, all these problems can be mitigated by adding DC-blocking capacitors with the proper design for both the capacitors and filter inductors, which can be an open research point.

\subsection{Conclusion}

A comprehensive, steady-state performance analysis for various configurations of bidirectional inductive power transfer system in EVs applications is presented in this chapter. The analysis was based on the theoretical harmonics representation for each topology that was presented in chapter (4). The proposed theoretical models were utilized to study the control parameters' effect on the steady-state system response and power flow. Moreover, the BIWPTS's performance evaluation parameters (active and reactive power, efficiency and power factor) were estimated and compared. For validation purposes, a BIWPTS model was built in Simulink/MATLAB for each configuration, using the same design parameters, and its outcomes were compared with the theoretical models' results. The results demonstrate good correspondence between the theoretical and the simulated models. In addition, the small-scale experimental prototype that was presented in chapter (5) is tested and analyzed to verify the theoretical and simulation results. The proposed analysis could predict the systems' steady-state performance precisely as the correlation between the simulation and experimental results manifest. The presented analysis concludes that, among the different configurations, LCL-topology is more appropriate for 
EV applications and in the situations where the inverter is a long way from the pad. It shows current-source characteristics, power-factor correction capability, simple design and control, high overall efficiency and power factor, and more robustness to misalignment. 


\section{Chapter 7 Dynamic and Physics-based Models of LCL BIWPTS}

\subsection{Introduction}

Inductive wireless power transmission is a promising technology in the charging of EVs. This technology is mainly based on the magnetic coupling between two or more coils. The coupling coefficient $(k)$ ranges from 0 to 1 , based on the magnetic design and the distance between the coils. In the conventional transformer, $k$ is about 0.95 and it is called strongly coupled technology, but in IPT systems $k$ ranges from 0.01 to 0.4 , and it is called loosely coupled technology [176]. The electrical isolation in IPT systems provides safe, reliable, maintenance free operation in harsh environment. For these features, IPT technology has been approved to be convenient and reliable interface for charging and discharging the EV's battery, during long-term parking (stationary), short-term stops (quasi-dynamic) or movement (dynamic) [97]. As was reported in chapter (6), among the different topologies of BIWPTS, LCL-based system provides better performance, especially for EVs applications. Precise model for such system can help the designers and researchers to anticipate, optimize and evaluate the system's behavior amid the development. Thus, this chapter deals with developing accurate nonlinear dynamic model for LCL BIWPTS in EVs applications.

According to the literature, several studies have been focused on BIWPTS modeling analysis. In [142], [75] steady-state mathematical models for LCL BIWPTS were developed. An analytical dynamic model based on state-space representation was investigated in [143], [177] for BIWPTS with CLCL topology. In these works, the coils of the wireless coupler were ideally modeled as linear inductors and the mutual coupling was 
represented by the T-model, as in [75], or the reflected impedance, as in [142]. In these models, the core losses and the nonlinear magnetic characteristics of ferrite materials of the power pads were neglected. These approximations may lead to inaccurate prediction and evaluation for the system performance under different control and operating conditions. Considering such kind of features necessitates physics-based models for the wireless coupler, such as FEMs. Several FEA for different structures of power pads in EV applications were presented in the literature [84], [178]. In these works, FEMs were built and pure sinusoidal currents were injected to emulate the system's performance. In this case, the effect of the nonlinearities due the power electronic converters and the dynamic controllers on the system were neglected. For precise evaluation of BIWPTS, the impacts of both the power electronics and the magnetic material characteristics need to be considered in the same simulation environment.

Thus, this chapter presents a physics-based co-simulation platform for LCL BIWPTS in EVs applications. The platform is established through the coupling between finite element and circuit analysis. The power electronic converters and controllers are developed in Simulink and the power pads are modeled in Magnet environment. The two parts are linked together through the compatible Simulink Plug-in tool. In addition, a state-space dynamic mathematical model for the same LCL BIWPTS is derived and implemented in MATLAB environment. A $1.2 \mathrm{~kW}$ LCL BIWPTS is analyzed under different dynamics by both models, and the results are compared. The effect of the nonlinearities and the magnetic material characteristics on the system performance is assessed in terms of error and harmonics analysis. The analysis considered both the full and the light loading operating conditions in the system. The proposed co-simulation could provide accurate prediction for 
the system dynamics, during both charging and discharging operation. The scheme is generic and can be easily expanded to different pad structures, compensation networks and inverter topologies.

The main contributions in this work are summarized below:

- A dynamic analytical model for LCL BIWPTS based on state-space representation is derived, implemented and analyzed.

- A 2D-FEM for wireless coupler with DD pad structure is built considering the magnetic characteristics of $\mathrm{N} 87$ ferrite material.

- Detailed analysis and explanation for the coupling between the power converters and controller in Simulink and the 2D-FEM in Magnet is presented.

- The impact of the nonlinearities due to the magnetic material and the power electronics on the BIWPTS's performance based on error and harmonics analysis is investigated, during different dynamics in the system.

- The system power flow under both light and full loading conditions is evaluated, during G2V and V2G operations.

- The effectiveness of the platform is verified by means of analytical and experimental results.

\subsection{Analytical Dynamic Model of BIWPTS}

Similar to the steady-state models, presented in chapter (4), the DC-bus is modeled as a fixed DC source $\left(V_{d c}\right)$, and the EV's battery is represented by a fixed DC source $\left(V_{b}\right)$, in the nonlinear model. A dynamic mathematical model, based on state-space representation, is presented in this section to predict the entire BIWPTS's performance. 


\subsubsection{HF VSHBC Dynamic Model}

As was discussed before, two HF VSHBCs are utilized in the BIWPTS to supply the wireless coupler with controlled square-wave voltages at the resonant frequency, as indicated in Figure 7.1. These square-waves are controlled to achieve the required bidirectional power-flow in the system. The phase-shift control technique, depicted in Figure 4.4, is considered for this purpose. In this method, the controller adjusts the magnitude and the phase angle of the two inverters' voltages to manage the power flowmagnitude and direction between the two sides of the system. The switching activities for the primary inverter driven by the phase-shift control method are described in Figure 7.2. The switching signals for the second leg $\left(\mathrm{S}_{13}\right.$ and $\left.\mathrm{S}_{14}\right)$ are shifted by an angle $\alpha$ from the first leg signals $\left(S_{11}\right.$ and $\left.S_{12}\right)$. This parameter $(\alpha)$ varies between 0 to $180^{\circ}$ to change the RMS inverter's output voltage $\left(v_{p i}\right)$ from zero to maximum, respectively. The switching signals of the secondary inverter follow the same activities in Figure 7.1. There is a phaseshift $\beta$ between the second leg signals $\left(S_{23}\right.$ and $\left.S_{24}\right)$ and the first leg signals $\left(S_{21}\right.$ and $\left.S_{22}\right)$. Also, the four secondary signals are shifted by an angle $\delta$ from the first inverter switching signals. Typically, the angle $\delta$ is used to manage the direction of the power-flow from the power grid to the $\mathrm{EV}$, and vice versa. The negative $\delta$ means performing G2V operation, while positive $\delta$ provides V2G service. This parameter is theoretically set to $\pm 90^{\circ}$ to allow maximum active power flow with almost UPF operation. 


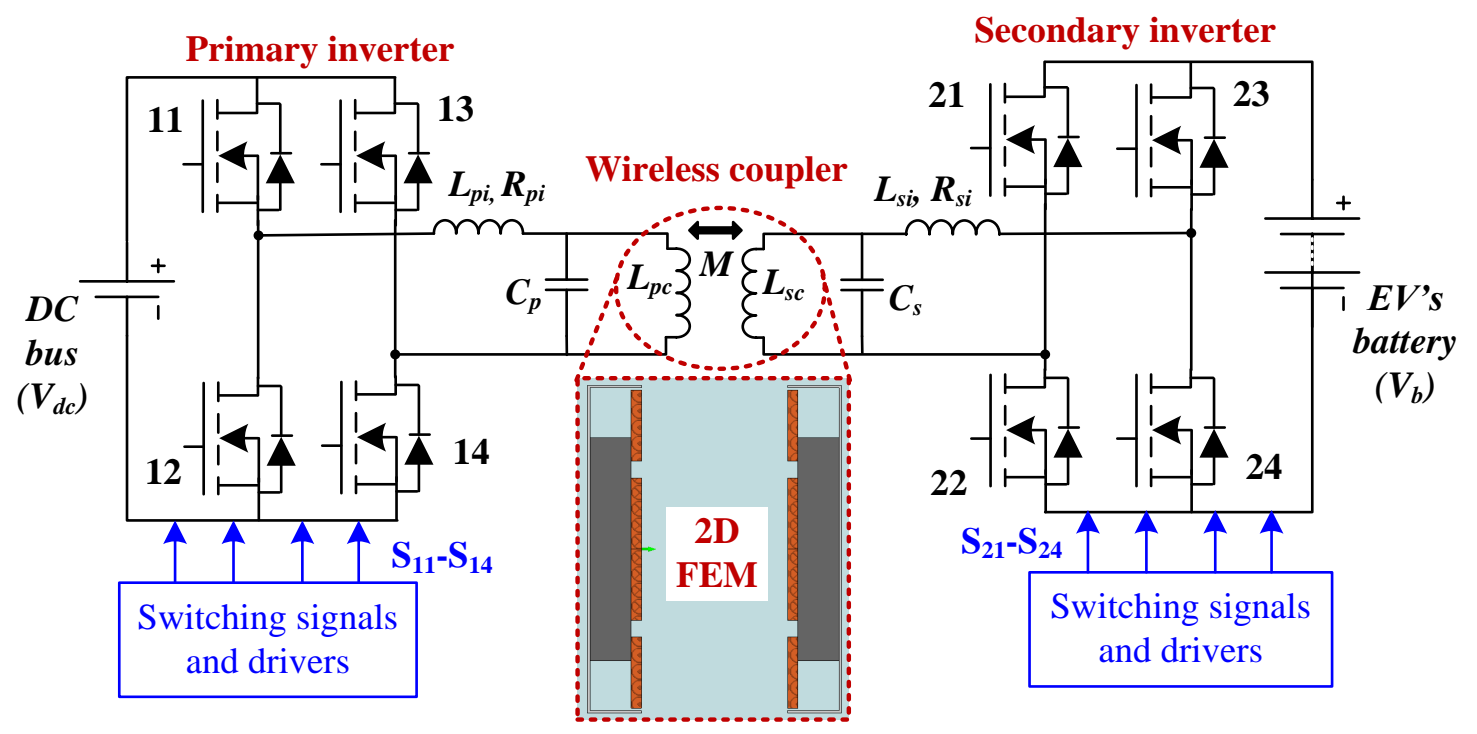

Figure 7.1. Power electronics circuit diagram of LCL BIWPTS.

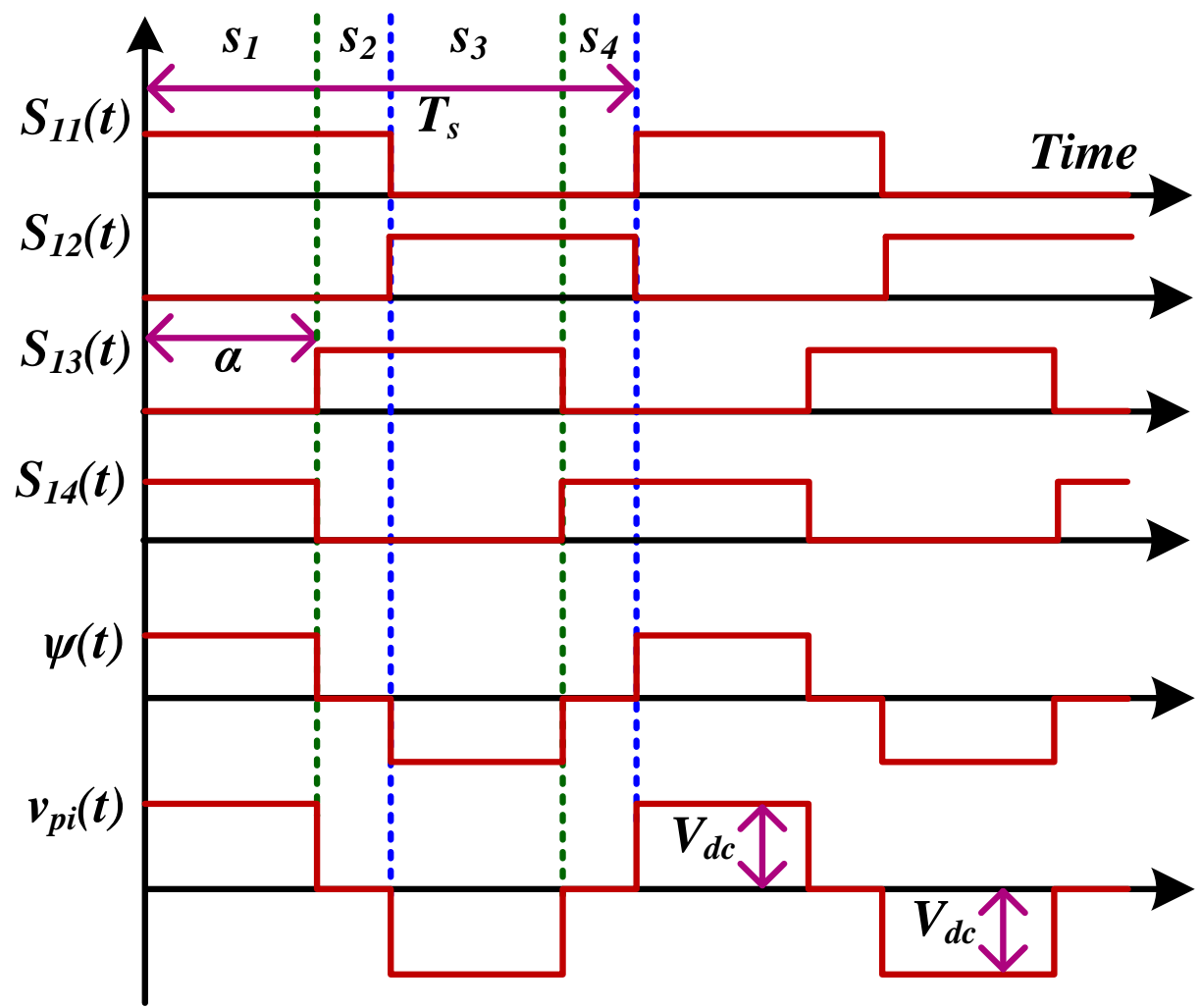

Figure 7.2. Switching activities and output voltage waveform of primary inverter. 
Depending on the switching actions in Figure 7.2, four switching states for the primary inverter are stated in Table 7.1. The tri-state function $\left[\Psi_{p}(t)\right]$ is evaluated from the individual switching function using Equation (7-1) and indicated in Figure 7.2 and Table 7.1 .

$$
\Psi_{p}(t)=S_{11}(t) S_{14}(t)-S_{12}(t) S_{13}(t)
$$

The instantaneous output voltage of the primary inverter $\left[v_{p i}(t)\right]$ is expressed in terms of the tri-state function and the DC-link voltage $\left(V_{d c}\right)$, as in Equation (7-2).

$$
v_{p i}(t)=\Psi_{p}(t) \times V_{d c}
$$

Table 7.1. Switching States of the Primary HF VSHVC.

\begin{tabular}{llllllll}
\hline \hline State & Period & $\boldsymbol{S}_{11}$ & $\boldsymbol{S}_{\mathbf{1 2}}$ & $\boldsymbol{S}_{\mathbf{1 3}}$ & $\boldsymbol{S}_{\mathbf{1 4}}$ & $\boldsymbol{\Psi}_{\boldsymbol{p}}$ & $\boldsymbol{V}_{\boldsymbol{p i}}$ \\
\hline $\boldsymbol{S}_{\boldsymbol{1}}$ & $0<t \leq \alpha / 2 \pi f_{s}$ & 1 & 0 & 0 & 1 & 1 & $V_{d c}$ \\
$\boldsymbol{S}_{\mathbf{2}}$ & $\alpha / 2 \pi f_{s}<t \leq T_{s} / 2$ & 1 & 0 & 1 & 0 & 0 & 0 \\
$\boldsymbol{S}_{3}$ & $T_{s} / 2<t \leq\left(T_{s} / 2+\alpha / 2 \pi f_{s}\right)$ & 0 & 1 & 1 & 0 & -1 & $-V_{d c}$ \\
$\boldsymbol{S}_{4}$ & $\left(T_{s} / 2+\alpha / 2 \pi f_{s}\right)<t \leq T_{s}$ & 0 & 1 & 0 & 1 & 0 & 0 \\
\hline \hline
\end{tabular}

For the secondary inverter, the switching states are the same as the primary ones, but shifted in time by the angle $\delta$, and the phase shift parameter is $\beta$ instead of $\alpha$. Thus, the tristate function $\left[\Psi_{s}(t)\right]$ and the instantaneous output voltage of the secondary inverter $\left[v_{s i}(t)\right]$ are expressed, as in Equation (7-3). 


$$
\begin{aligned}
& \Psi_{s}\left(t_{2}\right)=S_{21}\left(t_{2}\right) S_{24}\left(t_{2}\right)-S_{22}\left(t_{2}\right) S_{23}\left(t_{2}\right) \\
& v_{s i}\left(t_{2}\right)=\Psi_{s}\left(t_{2}\right) \times V_{b}
\end{aligned}
$$

where, $t_{2}=t+\delta / 2 \pi f_{s}, f_{s}$ is the switching frequency $\left(f_{s}=1 / T_{s}\right)$, which is the same as the resonant frequency $\left(f_{r}\right)$ in both sides.

\subsubsection{Magnetic Coupler and Compensation Network Dynamic Model}

In LCL BIWPTS structure, the compensation capacitor is connected in parallel with each coil of the wireless coupler for PFC and reactive power compensation. Also, an Lfilter is introduced between the inverter and the capacitor for providing current-source characteristics. The time-domain equivalent circuit of LCL-based symmetrical BIWPTS is depicted in Figure 7.3. The HF inverters are represented by AC sources with square or quasi-square waves. The system is designed for fixed frequency operation, and the two sides operate at the same resonant frequency $\left(\omega_{r}\right)$. The compensating capacitors are tuned to resonate with the coil self-inductances based on Equation (4-2), to be independent from the misalignment between the ground and the vehicle pads.

The filter inductances $\left(L_{p i}\right.$ and $\left.L_{s i}\right)$ are designed to match the pad self-inductances

(i.e. $L_{p i}=L_{p c}$ and $L_{s i}=L_{s c}$ ) in order to provide fixed pad currents and to make the operating frequency match the design resonant frequency, as was discussed in chapters (4) and (6).

The system dynamics are modeled based on the state-space representation, as given in Equation (7-4).

$$
\dot{x}=A x+B u
$$

where, $x$ is the state vector and $u$ is the control vector. 


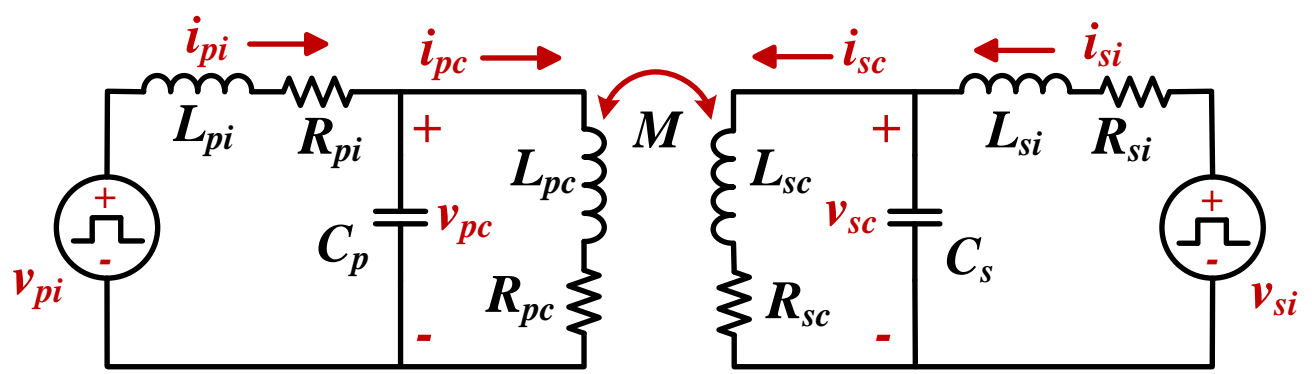

Figure 7.3. Dynamic electric equivalent circuit of symmetrical LCL BIWPTS.

These vectors, $x$ and $u$, are stated for the LCL BIWPTS, in Equation (7-5).

$$
\begin{aligned}
& x=\left[\begin{array}{llllll}
i_{p i} & v_{p c} & i_{p c} & i_{s c} & v_{s c} & i_{s i}
\end{array}\right]^{T} \\
& u=\left[\begin{array}{lll}
v_{p i} & v_{s i}
\end{array}\right]^{T}
\end{aligned}
$$

where, the subscript $p$ stands for the primary side variables and $s$ identifies the secondary side, $i_{p i}$ and $i_{s i}$ are the instantaneous inverter currents, $i_{p c}$ and $i_{s c}$ are the instantaneous coil pad currents, and $v_{p c}$ and $v_{s c}$ are the instantaneous coil voltages.

Following the basic principles of circuit theory, the dynamic model is expressed by six ODEs, as given in Equation (7-6).

$$
\begin{aligned}
i_{p l} & =\left(\frac{1}{L_{p i}}\right)\left(v_{p i}-R_{p i} i_{p i}-v_{p c}\right) \\
v_{p c} & =\left(\frac{1}{c_{p}}\right)\left(i_{p i}-i_{p c}\right) \\
\dot{l}_{p c} & =M \gamma\left(v_{s c}-R_{s c} i_{s c}-\left(\frac{L_{s c}}{M}\right) v_{p c}+\left(\frac{R p c L_{s c}}{M}\right) i_{p c}\right) \\
i_{s c} & =L_{p c} \gamma\left(-v_{s c}+R_{s c} i_{s c}+\left(\frac{M}{L_{p c}}\right) v_{p c}-\left(\frac{R_{p c} M}{L_{p c}}\right) i_{p c}\right) \\
v_{S c} & =\left(\frac{1}{c_{s}}\right)\left(i_{s i}-i_{s c}\right) \\
i_{s l} & =\left(\frac{1}{L_{s i}}\right)\left(v_{s i}-R_{s i} i_{s i}-v_{s c}\right)
\end{aligned}
$$

where, $\gamma=1 /\left(M^{2}-L_{s c} L_{p c}\right)$. 
According to Equations (7-4)-(7-6), the system matrix $A$ and the input matrix $B$ are deduced, as in Equation (7-7).

$$
\mathrm{A}=\left[\begin{array}{cccccc}
\frac{-R_{p i}}{L_{p i}} & \frac{-1}{L_{p i}} & 0 & 0 & 0 & 0 \\
\frac{1}{C_{p}} & 0 & \frac{-1}{C_{p}} & 0 & 0 & 0 \\
0 & -\gamma L_{s c} & \gamma L_{s c} R_{p c} & -\gamma M R_{s c} & \gamma M & 0 \\
0 & \gamma M & -\gamma M R_{p c} & \gamma L_{p c} R_{s c} & -\gamma L_{p c} & 0 \\
0 & 0 & 0 & \frac{-1}{C_{s}} & 0 & \frac{1}{C_{s}} \\
0 & 0 & 0 & 0 & \frac{-1}{L_{s i}} & \frac{-R_{s i}}{L_{s i}}
\end{array}\right] ; \mathrm{B}=\left[\begin{array}{cc}
\frac{1}{L_{p i}} & 0 \\
0 & 0 \\
0 & 0 \\
0 & 0 \\
0 & 0 \\
0 & \frac{1}{L_{s i}}
\end{array}\right]
$$

The system's ODEs are programmed and solved in MATLAB script using the fourth order Runge-Kutta (RK4) numerical technique, and utilized to predict the BIWPTS's dynamics.

\subsection{Physics-Based Co-simulation Platform}

A typical BIWPTS for EV connection consists of the power electronics converters, passive compensation elements and power pads. The power pads are made of copper litz wire to minimize the skin and proximity effects, and magnetic ferrite material to direct the flux lines and reduce the magnetic path reluctance. In typical circuit models, the magnetic coupler is modeled by self and mutual-inductances using T-model mutual representation [75], or using the reflected impedance theory [142]. In this case, the parameters are extracted or estimated at one operating point, and the nonlinearities due to the magnetic material and the magnetic losses are not considered. Thus, relying on such approximate models to simulate the system's performance under different control and operating conditions is not accurate and may lead to significant errors. In order to precisely simulate 
the system's performance, the magnetic characteristics need to be considered in the model. Thus, combining the physics-based model for the power pads, such as FEM and the circuit simulation is necessary. The main limitation for such coupling is the long computation time. For minimizing this problem, compatible simulation tools are considered in this work. More details about the FEM and the co-simulation are presented in this section.

\subsubsection{Finite-Element Model in Magnet}

Among the different pad structures presented in the literature, the polarized DD design is considered in this work. It is simple in design and provides better coupling, greater tolerance and cost effectiveness. Moreover, it offers a charge zone five times larger than that possible with the typical circular pads for a similar material cost [84]. The typical structure of one DD power pad is depicted in Figure 7.4.

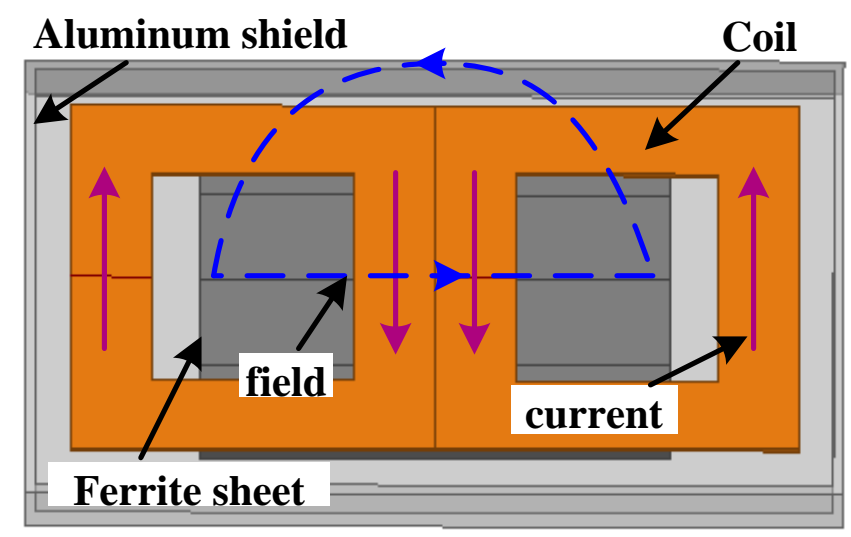

Figure 7.4. Double-D power pad structure using sheet of ferrite core.

It comprises of two or more copper coils magnetically connected in series (flux from one coil passes through the other) and electrically connected in parallel, to lower the 
equivalent inductance seen by the power supply. The coils are placed on top of ferrite strips or a sheet, to direct the flux streamlines from one coil to the other. Thus, there is no magneto-motive force (MMF) produced that can drive the flux out of the back. This allows aluminum shielding to be placed underneath with little impact on the coils' quality factor, creating a truly single-sided flux pad with a path height proportional to half of the pad length [144], [176]. A 2D-FEM for the wireless coupler is developed in Magnet environment. It consists of two identical DD power pads to satisfy the symmetrical bidirectional operation, in which each side can behave as a source and a load at the same time. Each pad consists of two Litz wire coils. The coils are attached to a ferrite sheet with the commercial ferrite material N87. This material is the most commonly used in IPT systems. It is composed of a combination of MnZn magnetic material. It offers good magnetic characteristics, as shown in Figure 7.5 , with mass density of $4850 \mathrm{~kg} / \mathrm{m}^{3}$ [179]. These characteristics are considered in the developed 2D-FEM. The 2D-FEM for the two identical DD power pads is shown in Figure 7.6, and the dimensions are presented in Table 7.2 .
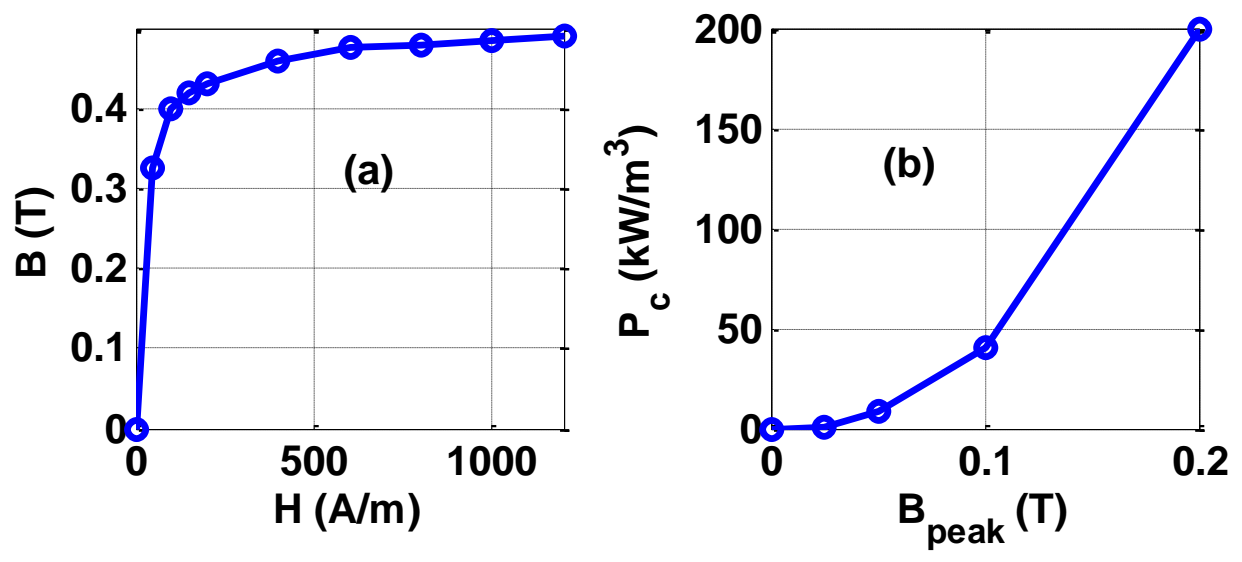

Figure 7.5. Characteristics of N87 ferrite material. (a) Magnetization characteristic @ $10 \mathrm{kHz}$ and $25^{\circ} \mathrm{C}$. (b) Core losses characteristic @ 40kHz and $25^{\circ} \mathrm{C}$. 


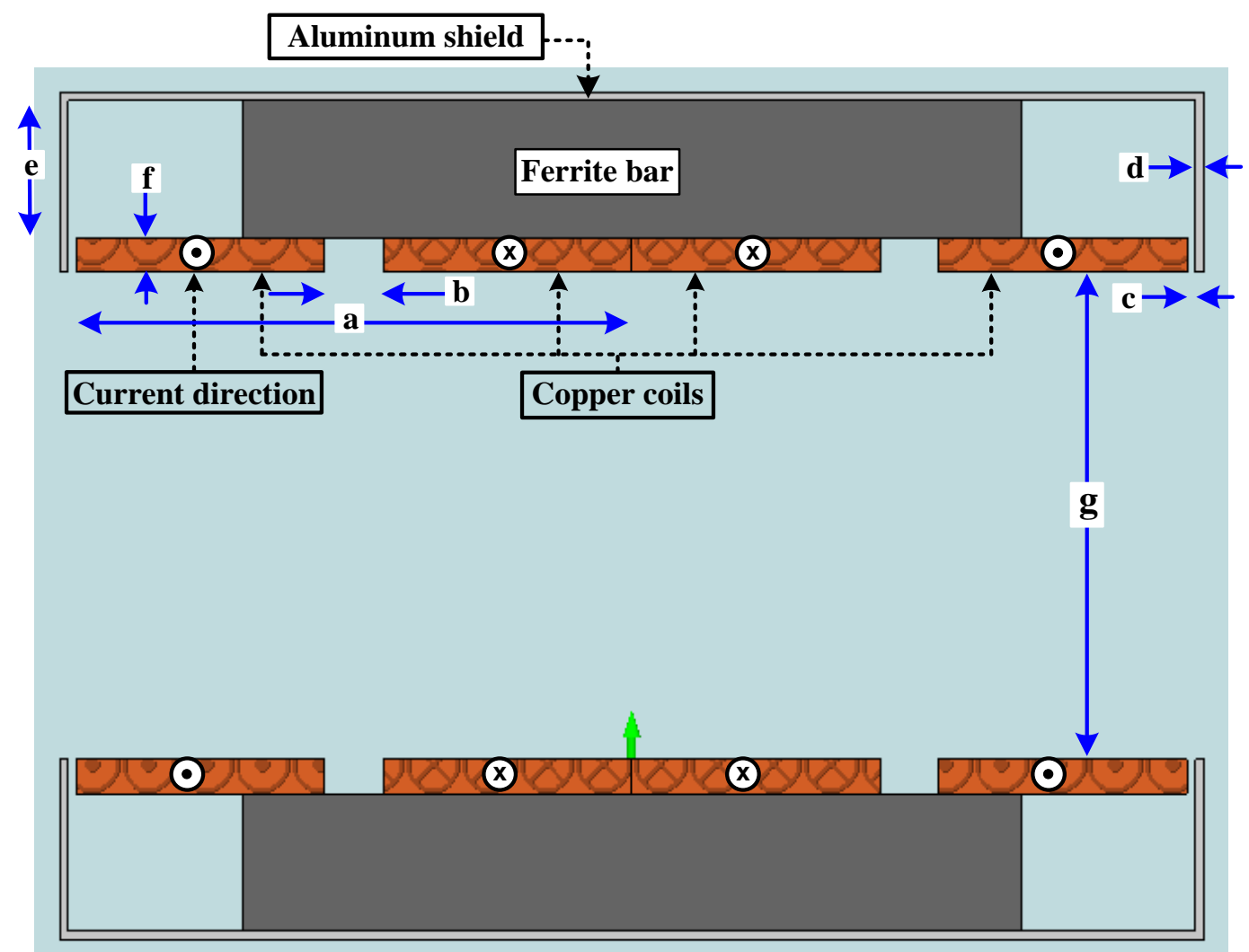

Figure 7.6. 2D-FEM of one power pad of the wireless coupler.

Table 7.2. Dimensions of the Power Pad in 2D-FEM

\begin{tabular}{llll}
\hline \hline Parameter & Value & Parameter & Value \\
\hline $\mathrm{a}$ & $67 \mathrm{~mm}$ & $\mathrm{c}$ & $1 \mathrm{~mm}$ \\
$\mathrm{~b}$ & $7 \mathrm{~mm}$ & $\mathrm{~d}$ & $1 \mathrm{~mm}$ \\
$\mathrm{e}$ & $16 \mathrm{~mm}$ & $\mathrm{f}$ & $5 \mathrm{~mm}$ \\
$\mathrm{~g}$ & $100 \mathrm{~mm}$ & & \\
\hline \hline
\end{tabular}


The model was analyzed based on the 2D FEA for solving a time-varying field, using the magnetic vector potential field formulation given in Equation (7-8) [180], [181].

$$
\nabla \times\left[\mu^{-1}(\nabla \times A)\right]=-\sigma\left(\frac{\partial A}{\partial t}+\nabla V\right)
$$

where, $\mu$ is the magnetic permeability of the medium $(\mathrm{H} / \mathrm{m})$, and $\sigma$ is the electric conductivity of the medium (A/V.m), $A$ is the magnetic vector potential (V.s/m), and $V$ is the scalar potential voltage function.

The nonlinearities that are investigated in this work are associated with two elements: 1) the nonlinear characteristics of the magnetic material, and 2) the nonlinearities in the sources due to the power electronic components. The magnetic material nonlinearity is represented in Equation (7-8) by $\mu$. For the nonlinear magnetic materials (e.g. ferrite material), the permeability $\mu$ depends on the local value of $B$, which changes nonlinearly based on $H$, according to the $B-H$ characteristic. Thus, iterative technique is required to solve Equation (7-8) until the element permeability values have converged. The second nonlinearity (associated to the sources) is characterized by the right-side of Equation (7-8), which represents the current density vector of the source.

Dirichlet (Flux Tangential) boundary conditions are applied to the surrounding area around the model. In this case, the entire outer boundary behaves as a flux line. This choice is the best for this case, since it is equivalent to putting the model in a cavity of a material with zero permeability, so that no flux can escape from the model. In addition, these boundaries are taken sufficiently far away from the components of the model $(\approx 10$ times the model dimensions), thus it represents a good approximation to the open boundaries. Therefore, the model can provide accurate solution with minimum computational effort. 
Transient magnetic field analysis is performed in Magnet environment for the 2DFEM. The coil currents during this analysis are given in Figure 7.7. They are typically sinusoidal, with a $90^{\circ}$ phase-shift between them. Five time instants are defined in the waveforms $\left(t_{1}, t_{2}, t_{3}, t_{4}\right.$ and $\left.t_{5}\right)$, and the magnetic flux distribution at these instants, when the two power pads are perfectly aligned, are indicated in Figure 7.8. These positions almost cover all the possibilities and are repeated periodically with the time.

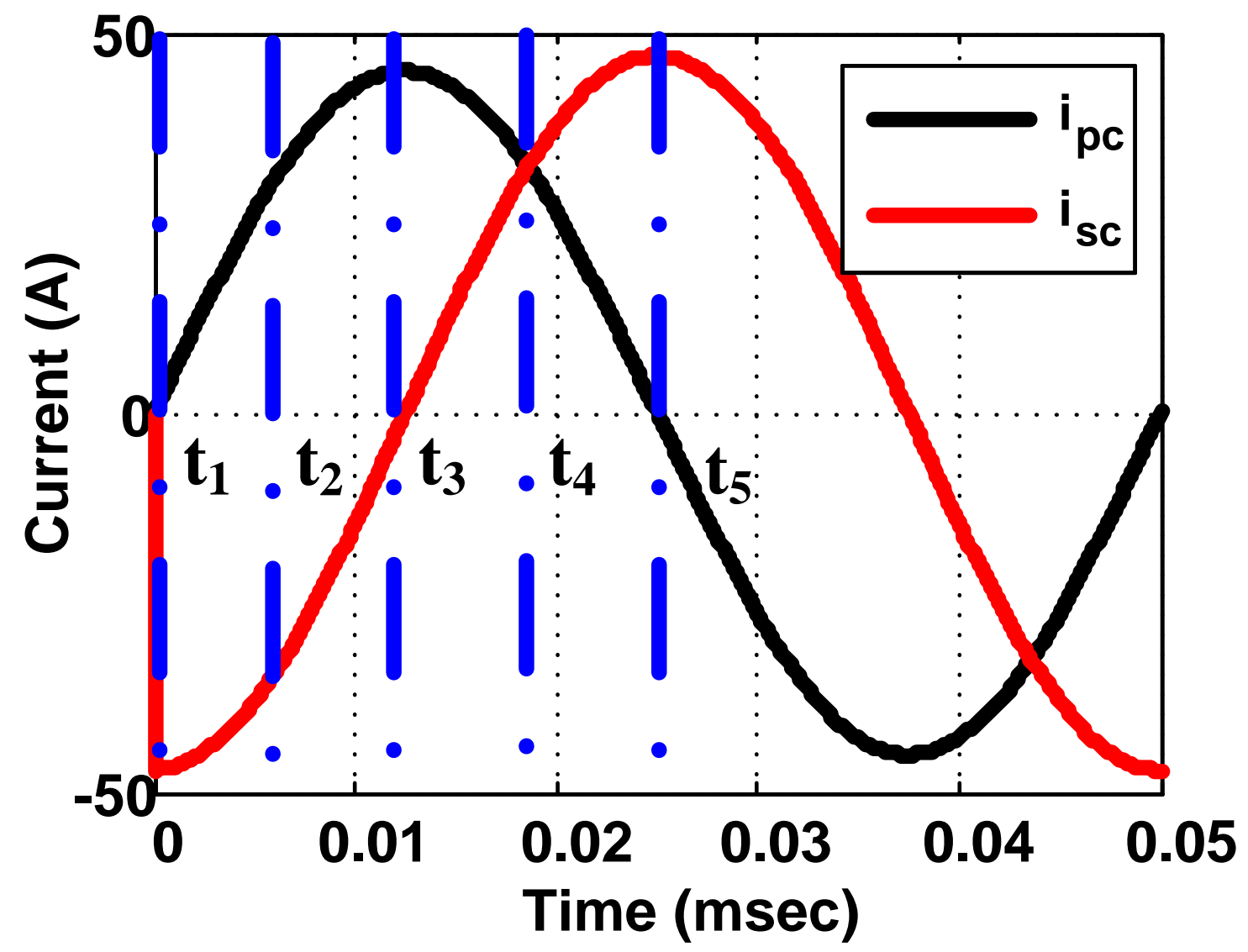

Figure 7.7. Instantaneous wireless pads currents waveforms. 

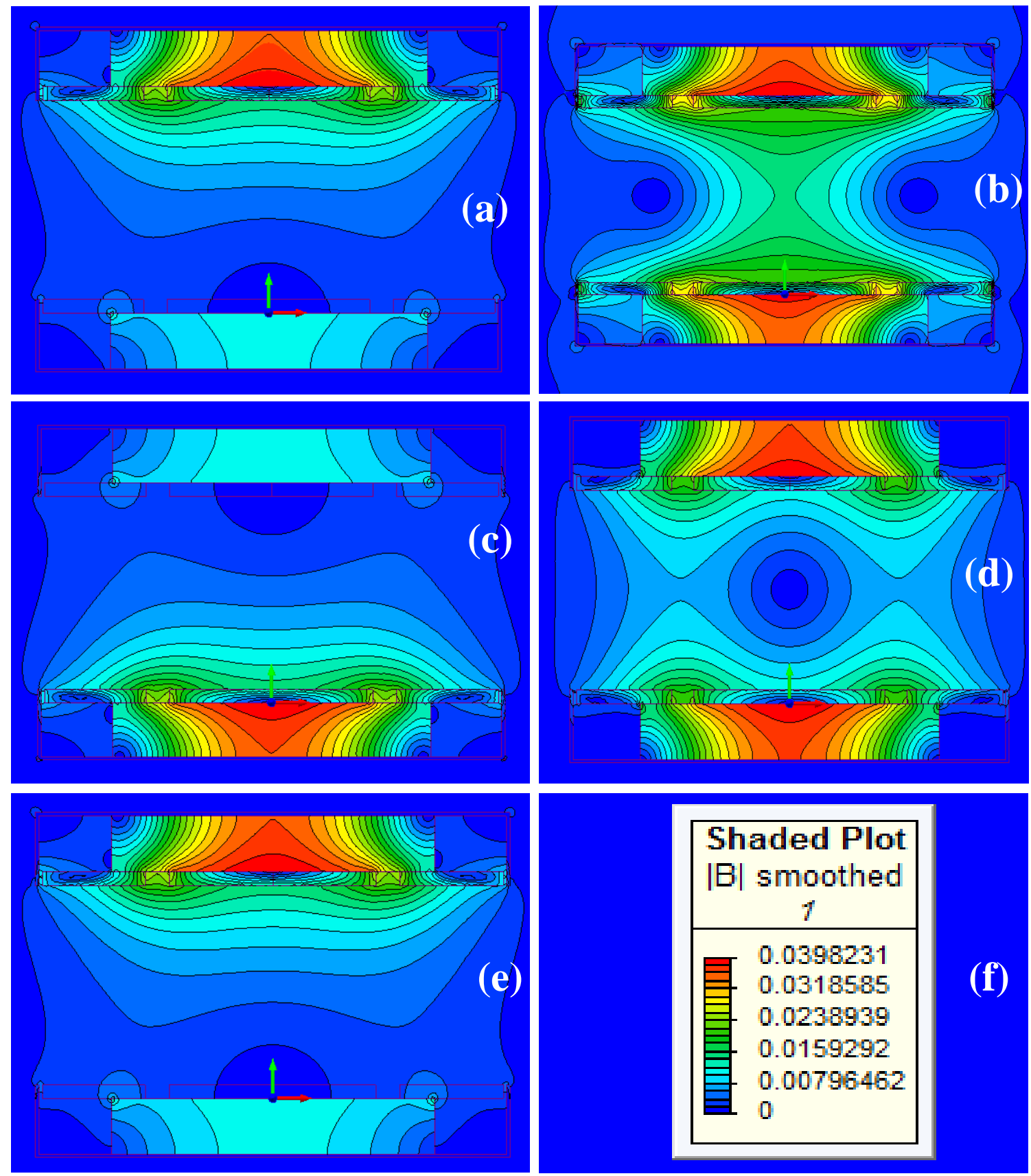

Figure 7.8. Magnetic flux density distribution of 2D-FEM. (a) At $t_{1}$. (b) At $t_{2}$. (c) At $t_{3}$. (d) At t4. (e) At t5. (f) Color map scale. 


\subsubsection{FE-Simulink Co-simulation}

For accurate evaluation of the magnetic material's effect on the BIWPTS's performance, the 2D-FEM of the wireless coupler is coupled with the power converters' simulation. For reasonable simulation time, two compatible tools are used in this platform, as depicted in Figure 7.9. Simulink tool is used to simulate the compensation network, the power electronic converters, and the controllers. Magnet software is considered to model the power pads. The two models are coupled through the Simulink Plug-in tool. This tool allows Simulink to control the sources of the coils in Magnet environment [145].

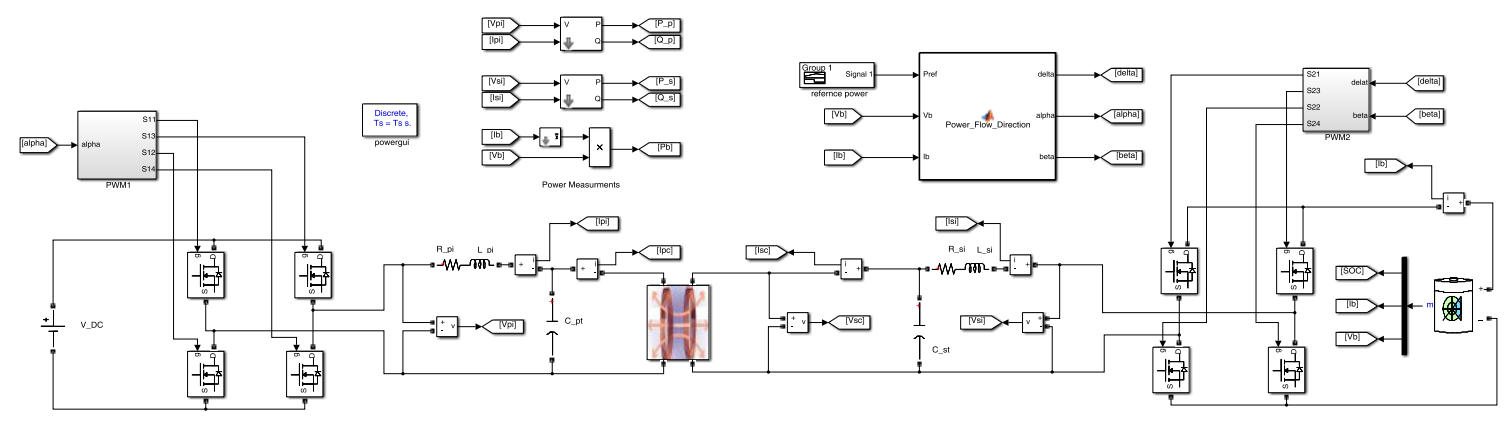

Figure 7.9. Co-simulation Simulink-Magnet Platform.

The details of the coupling between the circuit and finite element environments are described in Figure 7.10. In Magnet environment, each coil is connected to a controlledvoltage or current-source in the Circuit window. Voltage-source is preferred, as it results in a more stable coupling for magnetic components and is less likely to yield unphysical conditions [182]. In Simulink medium, each pad coil is denoted as a controlled currentsource. The voltages across the current-sources represent the control signals of the 
controlled voltage-sources in Magnet. After analyzing the FEM, the coil currents are calculated and used as control signals for the controlled current-sources in Simulink environment, as indicated in Figure 7.10. The Simulink Plug-in tool has been designed to operate properly when the Simulink step-size is fixed. It also provides the ability to set the MagNet step-size to be different from that of Simulink. In this case, the step size of the FEA can be much larger than that of the circuit analysis, to accelerate the simulation time.

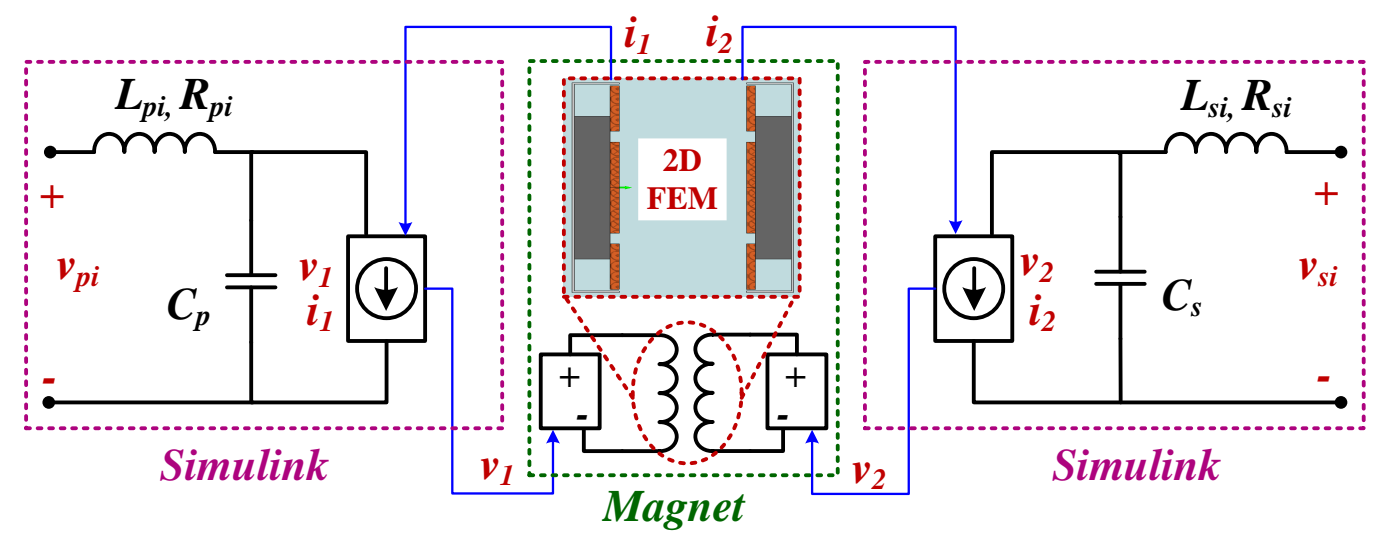

Figure 7.10. Coupling between Simulink and Magnet software.

\subsection{Model Verification and Discussion}

A $1.2 \mathrm{~kW}$ LCL BIWPTS model (shown in Figure 7.1) is analyzed by both the dynamic analytical model and the co-simulation platform. The framework is designed to resonate at $40 \mathrm{kHz}$, with an air-gap length between the primary and secondary coils of 100 $\mathrm{mm}$, and design parameters given in Table 7.3. The BIWPTS's model comprises of two DC supplies to emulate the DC-bus and the EV's battery, two HF full-bridge inverters, two identical DD power pads, two parallel compensation capacitors, and two L-filters. Each power pad consists of two copper coils attached to a ferrite sheet of N87 magnetic material 
from EPCOS. The analytical and co-simulation results during both $\mathrm{G} 2 \mathrm{~V}$ and V2G operation are presented and discussed in this section.

Table 7.3. Design Parameters of the BIWPTS Model

\begin{tabular}{llllll}
\hline Parameter & Value & Parameter & Value & Parameter & Value \\
\hline$L_{p i}$ & $26.1 \mu \mathrm{H}$ & $L_{s i}$ & $26.1 \mu \mathrm{H}$ & $C_{p}$ & $0.61 \mu \mathrm{F}$ \\
$R_{p i}$ & $38 \mathrm{~m} \Omega$ & $R_{s i}$ & $38 \mathrm{~m} \Omega$ & $C_{s}$ & $0.61 \mu \mathrm{F}$ \\
$L_{p c}$ & $26.1 \mu \mathrm{H}$ & $L_{s c}$ & $26.1 \mu \mathrm{H}$ & $M$ & $5.2 \mu \mathrm{H}$ \\
$R_{p c}$ & $38 \mathrm{~m} \Omega$ & $R_{s c}$ & $38 \mathrm{~m} \Omega$ & $F$ & $40 \mathrm{kHz}$ \\
\hline \hline
\end{tabular}

\subsection{Error Analysis}

To show the nonlinearities in the system, selected dynamics are introduced to the BIWPTS, such as starting, power-flow level variation and switching between the different modes of operation ( $\mathrm{G} 2 \mathrm{~V}$ and $\mathrm{V} 2 \mathrm{G})$. The system under these disturbances is analyzed in time-domain, using both the co-simulation platform and the ODEs presented in section 7.2. Both models are solved in MATLAB environment for $0.04 \mathrm{sec}$ with $20 \mathrm{MHz}$ sampling frequency. The transient FEA is achieved at $2 \mathrm{MHz}$ sampling frequency in Magnet environment. The analyzed scenario, including the different dynamics, is indicated in Figure 7.10. The BIWPTS starts with G2V operation to charge the EV at full power level $\left(\alpha=\beta=180^{\circ}\right.$ and $\left.\delta=-90^{\circ}\right)$, as depicted in Figure 7.11(a). In this period, the two inverters generate full-square wave output voltages, with $90^{\circ}$ phase-shift $\left(v_{s i}\right.$ lags $\left.v_{p i}\right)$, as shown in Figure 7.11(b) and 7.11(c). 

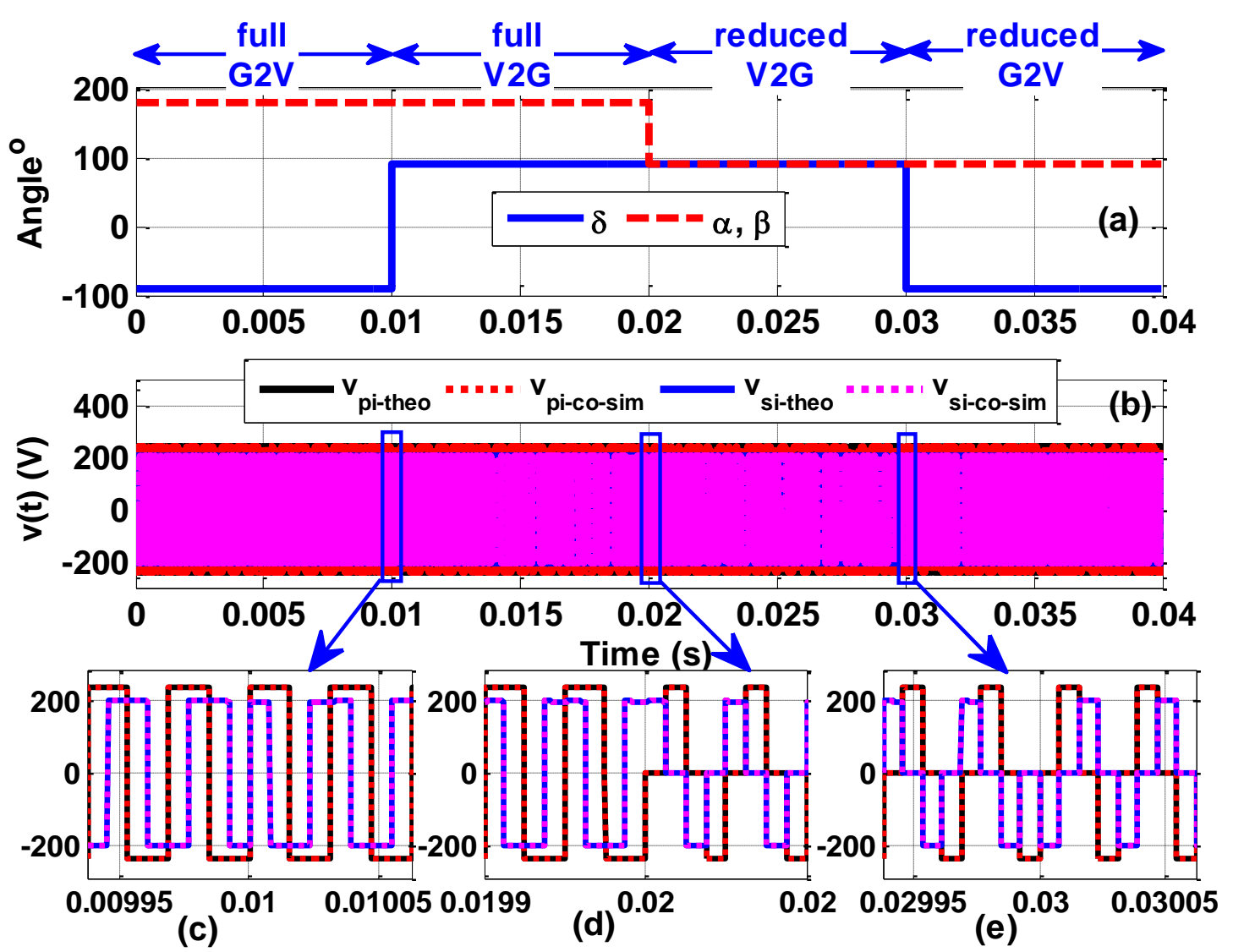

Figure 7.11. Introduced dynamics in BIWPTS ( $V_{d c}=240 \mathrm{~V}$ and $\left.V_{b}=200 \mathrm{~V}\right)$. (a) Phase shift control parameters $(\alpha, \beta$ and $\delta$ ). (b) Inverter output voltages. (c) Zoom for G2V to V2G transition at full capacity. (d) Zoom for power level reduction in V2G mode. (e) Zoom for V2G to G2V transition at reduced power level.

At $0.01 \mathrm{sec}$, the control parameter $\delta$ varies from $-90^{\circ}$ to $90^{\circ}$ to discharge the EV and realize $\mathrm{V} 2 \mathrm{G}$ service. At this instant, the phase of $v_{s i}$ changes to lead $v_{p i}$ by $90^{\circ}$, while both inverters still transfer the full power capacity [see Figure 7.11(c)]. The system keeps working at $\mathrm{V} 2 \mathrm{G}$ operation with full capacity until the control parameters $\alpha$ and $\beta$ are reduced from $180^{\circ}$ to $90^{\circ}$ at $0.02 \mathrm{sec}$. After $0.02 \mathrm{sec}$, the inverters generate quasi-square waves with zero level to reduce the RMS voltages and the power flow, in consequence [see 
Figure 7.11(d)]. Another mode switching action in applied at $0.03 \mathrm{sec}$ to charge the EV with a reduced power level [see Figure 7.11(e)].
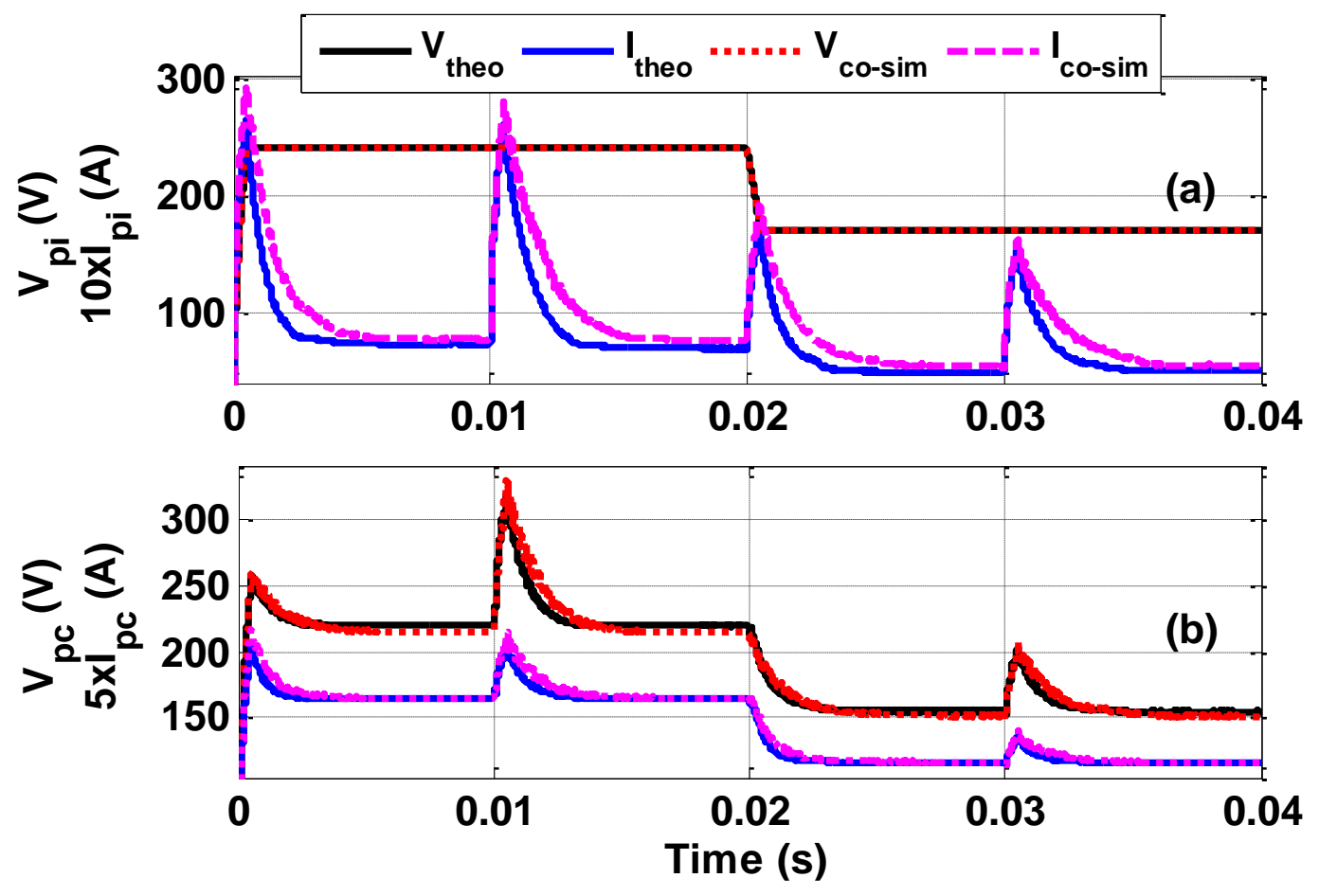

Figure 7.12. RMS values of BIWPTS's variables ( $V_{d c}=240 \mathrm{~V}$ and $V_{b}=200 \mathrm{~V}$ ). (a) Primary inverter variables. (b) Primary coil variables.

The RMS value of the BIWPTS's variables during the presented dynamics are indicated in Figure 7.12. The figure compares between these variables from both cosimulation and analytical model results. Large transients appear during the transition for both power level variations and switching the mode of operation. The results show significant mismatch between the analytical and FE co-simulation platform. The reasons for these mismatches are the actual characteristics of the magnetic material, which are neglected 
in the analytical model. The errors between the two results are evaluated and presented in Figure 7.13. These errors are significant during the transient period and decrease at steadystate region. Also, it can be noticed that the errors in the different modes of operation are not the same. For example, $\Delta I_{p i}$ in $\mathrm{V} 2 \mathrm{G}$ operation is slightly larger than that in $\mathrm{G} 2 \mathrm{~V}$ operation. The reason for this behavior is the nonlinearities in the system.
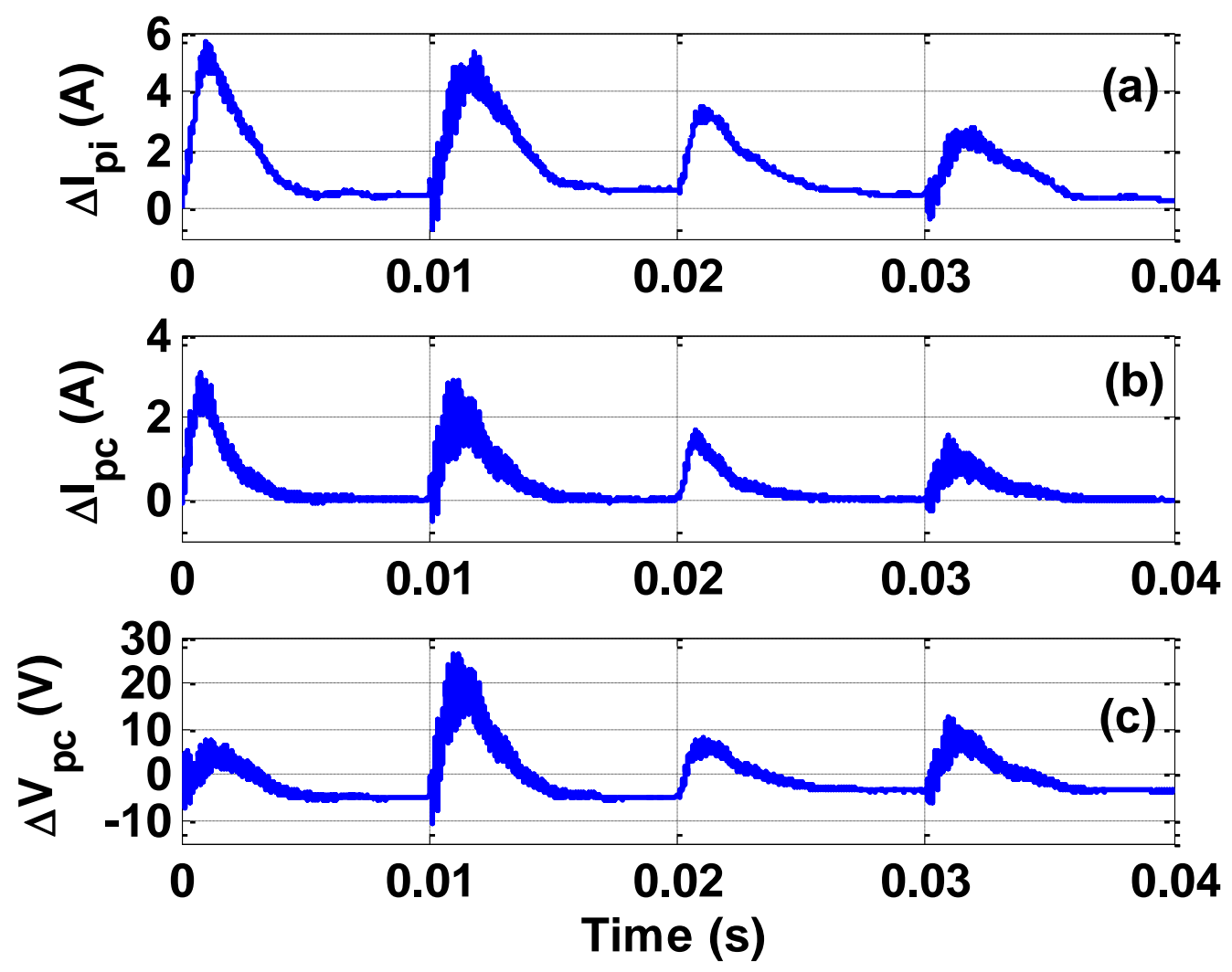

Figure 7.13. Error between analytical and FE co-simulation platform results. (a) Primary inverter current. (b) Primary coil current. (c) Primary coil voltage.

The deviations in the system variables reflect errors in the power flow analysis. The primary inverter power $\left(P_{p}\right)$, during the same operating scenario from both the analytical 
and co-simulation, is given in Figure 7.14(a). The error between the two results is depicted in Figure 7.14(b). As can be seen, a large and non-homogeneous error is experienced between the two data.
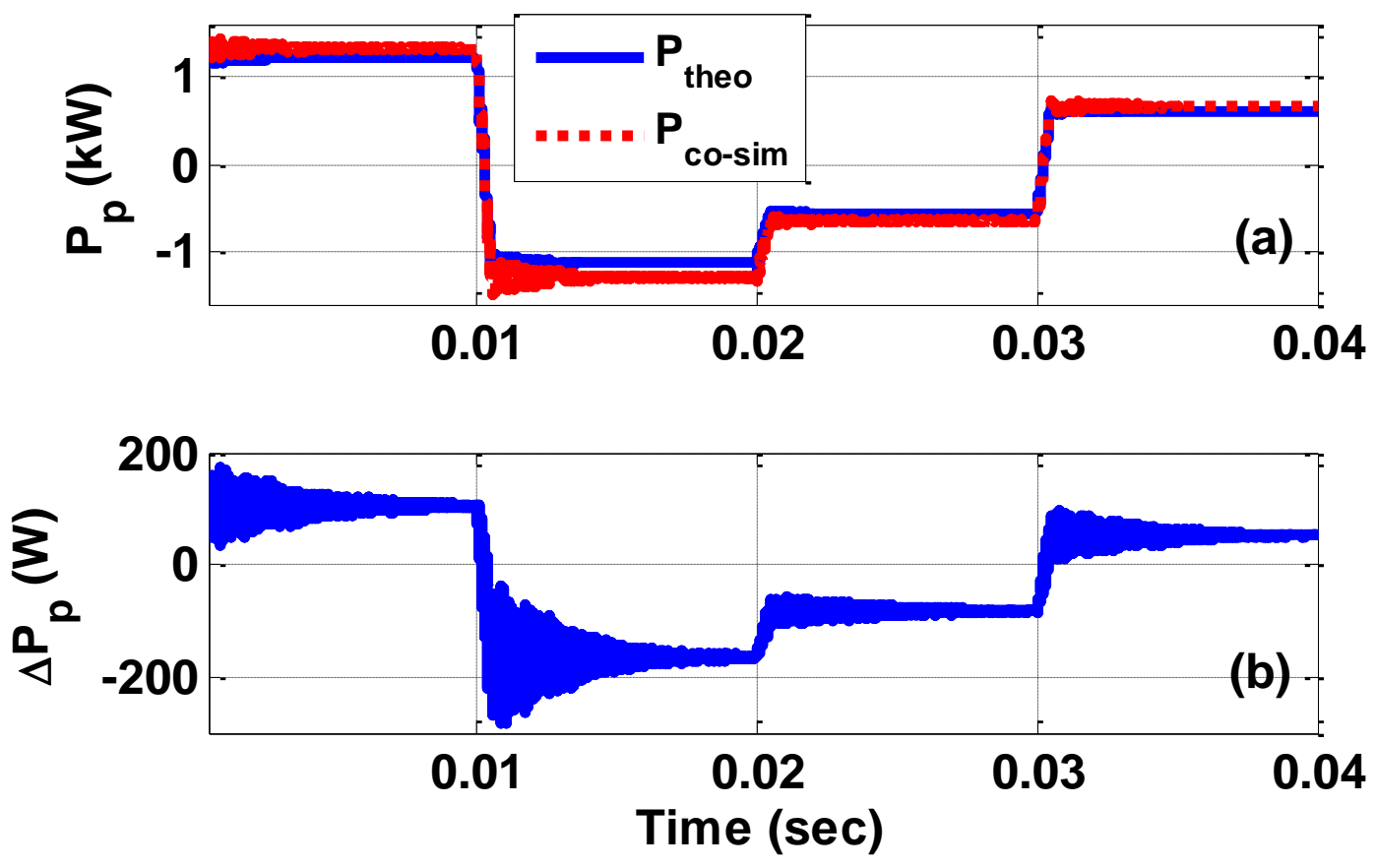

Figure 7.14. Active power flow. (a) Primary power (b) Primary power error.

Different performance is expected at different power levels (loading conditions) of BIWPTS. In order to examine that, the same BIWPTS is analyzed at lower DC voltage levels ( $V_{d c}$ and $V_{b}$ are $60 \mathrm{~V}$ and $50 \mathrm{~V}$ instead of $240 \mathrm{~V}$ and $200 \mathrm{~V}$, respectively). These voltage levels allow only $1 / 16$ of the full-power to be transferred. The NRMSE is evaluated, based on Equation (7-9), for all the system's variables, during both full and light loading conditions, and presented in Table 7.4. 


$$
N R M S E=\sqrt{\sum_{i=1}^{m}\left(f\left(t_{i}\right)-g\left(t_{i}\right)\right)^{2} / \sum_{i=1}^{m}\left(f\left(t_{i}\right)\right)^{2}}
$$

where, $f\left(t_{i}\right)$ and $g\left(t_{i}\right)$ are the value of the variable at sampling index $(i)$, which are calculated from the analytical and FEA co-simulation, respectively.

Table 7.4. \%NRMSE at Full and Light Loading Conditions.

\begin{tabular}{lcccccccc}
\hline \hline Mode & $\boldsymbol{i}_{\boldsymbol{p}}$ & $\boldsymbol{i}_{\boldsymbol{s i}}$ & $\boldsymbol{i}_{\boldsymbol{p c}}$ & $\boldsymbol{i}_{\boldsymbol{s c}}$ & $\boldsymbol{v}_{\boldsymbol{p}}$ & $\boldsymbol{v}_{\boldsymbol{s c}}$ & $\boldsymbol{p}_{\boldsymbol{p}}$ & $\boldsymbol{p}_{\boldsymbol{s}}$ \\
\hline Full load $\left(V_{d c}=240 \mathrm{~V}, V_{b}=200 \mathrm{~V}\right)$ & 17.8 & 17.57 & 2.3 & 2.3 & 2.64 & 2.64 & 11.4 & 11.2 \\
Light load $\left(V_{d c}=60 \mathrm{~V}, V_{b}=50 \mathrm{~V}\right)$ & 14.9 & 14.6 & 1.6 & 1.6 & 2.65 & 2.65 & 10.7 & 10.5
\end{tabular}

\subsection{Harmonics Analysis}

The nonlinearities in the arrangement due to the magnetic characteristics can be identified by performing harmonics analysis in the system. A FFT study is implemented for the primary inverter current $\left(i_{p i}\right)$, during both $\mathrm{G} 2 \mathrm{~V}$ and $\mathrm{V} 2 \mathrm{G}$ operation. The inverter voltage and current waveforms during G2V operation with full supply capacity are indicated in Figure 7.15(a). As can be observed, $i_{p i}$ is in-phase with $v_{p i}$, which means that the system is hitting the resonance and working at UPF. The magnitude of FFT analysis for $i_{p i}$ from both the analytical and finite element (FE) co-simulation models are presented in Figure 7.15(b). Only odd harmonics appear in the current waveform due to the square-wave voltages. The co-simulation results show large fundamental current and slight increase in the third harmonic compared with the analytical results. 

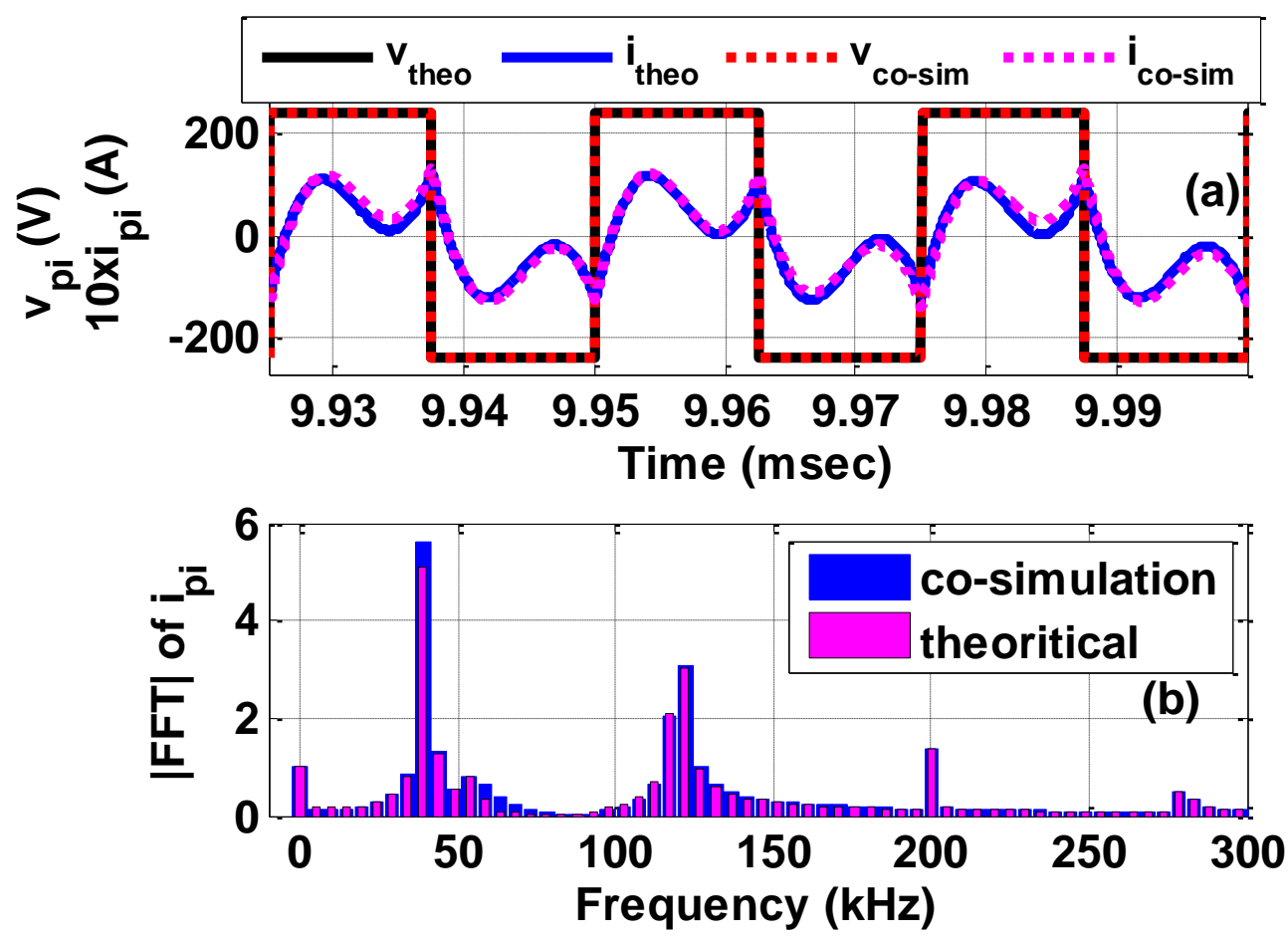

Figure 7.15. FFT analysis during full G2V operation ( $V_{d c}=240 \mathrm{~V}, V_{b}=200 \mathrm{~V}, \alpha=\beta=180^{\circ}$ and $\delta=-90^{\circ}$ ). (a) Primary inverter variables. (b) FFT magnitude of primary inverter current.

For better evaluation, the total harmonic distortion (THD) for all the system's variables is estimated, and presented in Table 7.5. The table shows the THD at both full and light loading conditions. In general, THDs in co-simulation results are smaller than that from the analytical results due to the larger fundamental components in the co-simulation data. The differences diminish in the light loading conditions, since the magnetic core becomes less saturated and offers linear characteristics. The secondary inverter current $\left(i_{s i}\right)$ exhibits less THD than that of $i_{p i}$. Also, it can be seen that THD of the coils' variables $\left(I_{p c}\right.$, $I_{s c}, V_{p c}$ and $\left.V_{s c}\right)$ are very small, which means that these variables are almost sinusoidal due to the filtration in the compensation network. Similar results are noticed during V2G operation, as presented in Figure 7.16 and Table 7.6. 
Table 7.5. \% THD during G2V Operation for Full and Light Loading

\begin{tabular}{|c|c|c|c|c|c|c|c|}
\hline \multicolumn{2}{|c|}{ "Mode } & \multirow{2}{*}{$\begin{array}{l}i_{p i} \\
62.84\end{array}$} & \multirow{2}{*}{$\begin{array}{l}\boldsymbol{i}_{\boldsymbol{s i}} \\
34.32\end{array}$} & \multirow{2}{*}{$\begin{array}{l}\boldsymbol{i}_{p c} \\
0.029\end{array}$} & \multirow{2}{*}{$\begin{array}{l}\boldsymbol{i}_{\boldsymbol{s c}} \\
0.029\end{array}$} & \multirow{2}{*}{$\begin{array}{l}v_{p c} \\
0.23\end{array}$} & \multirow{2}{*}{$\begin{array}{l}v_{s c} \\
0.23\end{array}$} \\
\hline & Full load & & & & & & \\
\hline 1 & Light load & 58.61 & 36.21 & 0.03 & 0.03 & 0.23 & 0.23 \\
\hline \multirow{2}{*}{ Co-simulation } & Full load & 52.34 & 26.34 & 0.035 & 0.035 & 0.25 & 0.25 \\
\hline & Light load & 52.28 & 26.44 & 0.035 & 0.035 & 0.25 & 0.25 \\
\hline
\end{tabular}
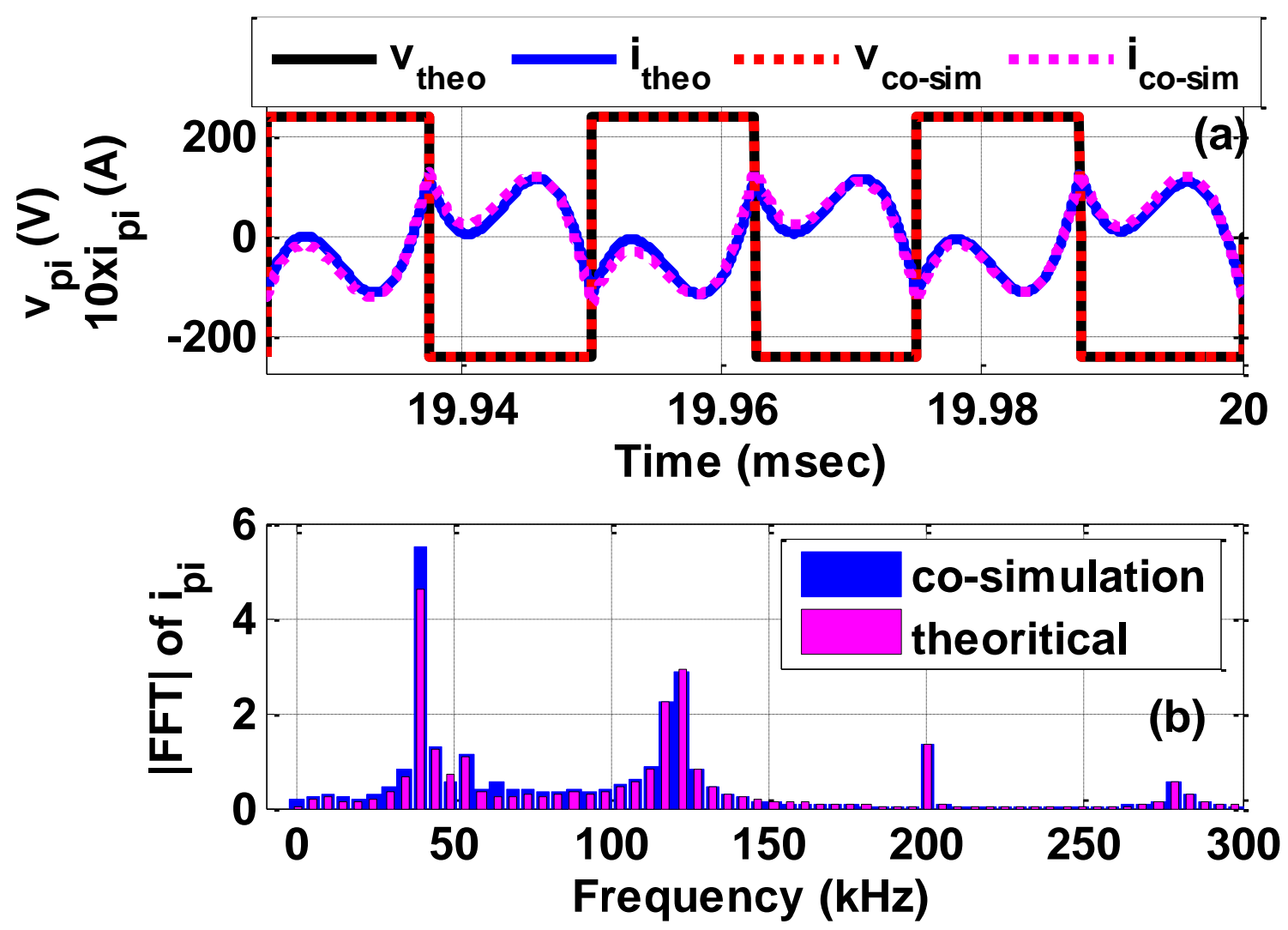

Figure 7.16. FFT analysis during full V2G operation $\left(V_{d c}=240 \mathrm{~V}, V_{b}=200 \mathrm{~V}, \alpha=\beta=180^{\circ}\right.$ and $\delta=90^{\circ}$ ). (a) Primary inverter variables. (b) FFT magnitude of primary inverter current. 
Table 7.6. \% THD during V2G Operation for Full and Light Loading

\begin{tabular}{|c|c|c|c|c|c|c|c|}
\hline \multicolumn{2}{|c|}{ Mode } & \multirow{2}{*}{$\begin{array}{l}\boldsymbol{i}_{p i} \\
72.82\end{array}$} & \multirow{2}{*}{$\begin{array}{l}i_{s i} \\
30.96\end{array}$} & \multirow{2}{*}{$\begin{array}{l}\boldsymbol{i}_{p c} \\
0.029\end{array}$} & \multirow{2}{*}{$\begin{array}{l}\boldsymbol{i}_{\boldsymbol{s c}} \\
0.029\end{array}$} & \multirow{2}{*}{$\begin{array}{l}\boldsymbol{v}_{p c} \\
0.23\end{array}$} & \multirow{2}{*}{$\begin{array}{l}v_{s c} \\
0.23\end{array}$} \\
\hline Theoretical & Full load & & & & & & \\
\hline & Light load & 78.77 & 29.48 & 0.029 & 0.029 & 0.23 & 0.23 \\
\hline \multirow[t]{2}{*}{ Co-simulation } & Full load & 54.25 & 25.62 & 0.034 & 0.034 & 0.25 & 0.25 \\
\hline & Light load & 54.46 & 25.6 & 0.034 & 0.034 & 0.25 & 0.25 \\
\hline
\end{tabular}

\subsection{Conclusion}

This chapter presents an accurate platform for predicting the performance of a bidirectional inductive wireless power transfer system during G2V and V2G services. The proposed co-simulation considered the nonlinearities in the system due to the power electronic converters, the power flow controllers and the magnetic characteristics of the power pads. The platform is developed using two compatible softwares based on circuits and finite element analysis. The system is implemented and analyzed in MATLAB environment. The results from the co-simulation are compared with the outcomes from a six order state-space dynamic model for $1.2 \mathrm{~kW}$ LCL BIWPTS, with double-D pad structure. The comparison shows the impact of the magnetic material characteristics in terms of errors and harmonics. The analysis indicated the effectiveness of the proposed platform, and its ability to be extended for different coupler designs, inverter topologies and compensation configurations. 


\section{Chapter 8 Magnetic Design Strategy of High-Power Symmetrical LCL BIWPTS}

\subsection{Introduction}

WPT is a technology, which could set the human free from the inconveniences of wires. It is capable of transferring the electric power through a relatively large air-gap without physical connection. WPT are intended to deliver power efficiently from a stationary primary source to one or more movable secondary loads by magnetic coupling. Bidirectional operation of this technology is exceptionally encouraging, particularly in EV ancillary services. The proper magnetic design is one of the most difficult and critical phases in developing a BIWPTS, especially for high-power (i.e. EVs) applications. Thus, this chapter presents detailed analysis for the choice and design of the passive elements in a BIWPTS. A clear methodology for achieving the best design for all the passive elements in a symmetrical LCL BIWPTS is developed. The proposed strategy depends on the electromagnetic field computations joined with the steady-state analytical model, presented in chapter (4). A 3D-FEM for an $8 \mathrm{~kW}$ polarized DD power pad is developed and optimized intuitively. The design is accomplished to meet with the WPT2 power level and Z2 class in J2954A standard [149]. Moreover, the choice of the PFC capacitors and the impedance matching coils was investigated. The best combination of the components' parameters, including the power pads, compensation capacitors and L-filters, was investigated. Following the proposed design considerations, a LCL BIWPTS model is developed in MATLAB Simulink, and its outcomes are compared with the theoretical model results. Additionally, a small-scale experimental prototype was assembled, using the same design 
specifications with reduced DC voltage levels. The models were tested during charging (G2V) and discharging (V2G) operation, and good correlation is observed for all of them.

\subsection{The Proposed Design Strategy}

As was indicated in Figure 7.1, the LCL BIWPTS contains six passive components: $L_{p i}, L_{s i}, C_{p}, C_{s}, L_{p c}$, and $L_{s c}$; in addition to the mutual inductance $(M)$ between the primary and secondary sides, which is directly related to the magnetic coupling factor $(k)$. The performance of a BIWPTS depends mainly on the values of these elements. They are governing the system's power transfer capability, efficiency and cost. Thus, these elements must be decided and designed carefully, to accomplish the desired system's operation. A flow-chart for a clear design strategy to configure these parameters is depicted in Figure 8.1. The algorithm describes five different phases (I-V), which need to be followed, to accomplish the BIWPTS's design [178], [183].

The first phase in the design, $\mathrm{I}$, is to decide the operating frequency $\left(f_{r}\right)$, the desired power level $\left(P_{\text {des }}\right)$, the air-gap range and the compensation configuration. The SAE J2954A standard defines the frequency range of $81.38 \mathrm{kHz}$ to $90 \mathrm{kHz}$ [149]. Also, four power levels (WPT1, WPT2, WPT3 and WPT4) were established by the same standard to support 3.7, 7.7, 11.1 and $22 \mathrm{kVA}$, respectively. In addition, the air-gap between the two sides is characterized by three different classes $(\mathrm{Z} 1, \mathrm{Z} 2$ and $\mathrm{Z} 3)$ that cover most of the ranges in the light-duty EVs. Thus, the operating frequency, power level and air-gap length can be decided based on this standard. 


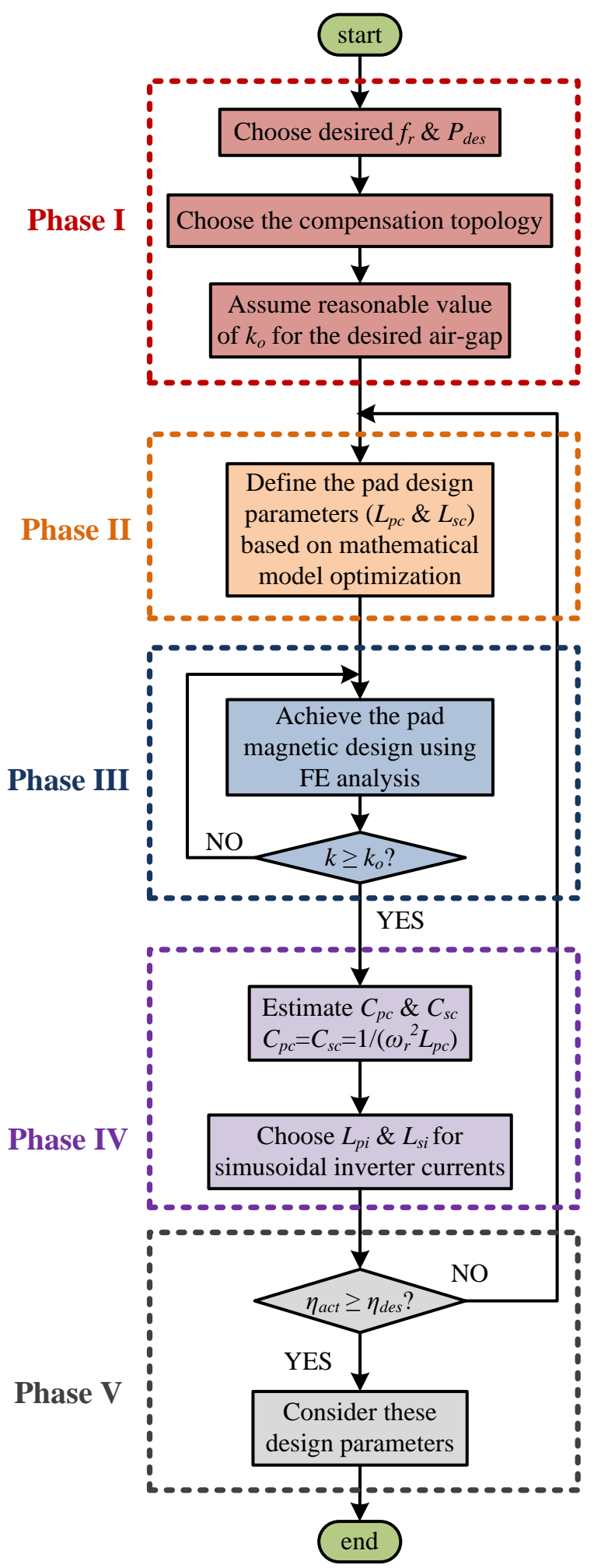

Figure 8.1. Flow-chart for the proposed design methodology. 
Also, in this phase, the designer needs to choose the appropriate compensation configuration, which is decided based on the assessment and comparative analyses presented in Chapter (6) and published in [76]. According to the study, the most appropriate network for EVs' applications is the LCL-topology, which shows high efficiency, simple design and control, and current-source characteristics. Due to these reasons, the LCL compensation topology is considered in this work. The last step in phase I is to assume a reasonable initial value for the coupling coefficient $\left(k_{o}\right)$, based on the provided ranges in the J2954A standard. In this work, WPT2 power level $(7.7 \mathrm{kVA})$, Z2 class $(95-165 \mathrm{~mm}$ air-gap) are chosen as design targets, since there are no specific characteristics for this category and only proposals were presented in the J2954A standard. Also, the initial desired coupling factor is assumed as $k_{o}=0.3$; and the DC-bus and the EV's battery voltage are chosen as $V_{d c}=420 \mathrm{~V}$ and $V_{b}=350 \mathrm{~V}$.

The second phase, II, of the design is to find the wireless power pad magnetic parameters ( $L_{p c}$ and $L_{s c}$ ), which satisfy the desired power, frequency and magnetic coupling requirements, previously defined in phase I. These parameters are decided based on the developed steady-state mathematical model in chapter (4) for the LCL BIWPTS. This model is utilized to predict the effect of the self-inductances of the power pads ( $L_{p c}$ and $L_{s c}$ ) on the system's performance. The BIWPTS's performance is evaluated based on the active power transfer capability, efficiency and power factor. The relations among these evaluation parameters and the pads' self-inductances are described in Figure 8.2. In this figure, the system's primary power $\left(P_{p}\right)$, efficiency $(\eta)$ and primary power factor $\left(P F_{p}\right)$ are analyzed with respect to the self-inductance. In this analysis, it is assumed that the two power pads are identical $\left(L_{p c}=L_{s c}\right)$, due to the symmetry in the system. It can be noticed 
that the inductance value that handles about $7.8 \mathrm{~kW}$ (WPT2), with $92.61 \%$ efficiency and 0.955 power factor is $19 \mu \mathrm{H}$. Thus, this value is considered in the design.

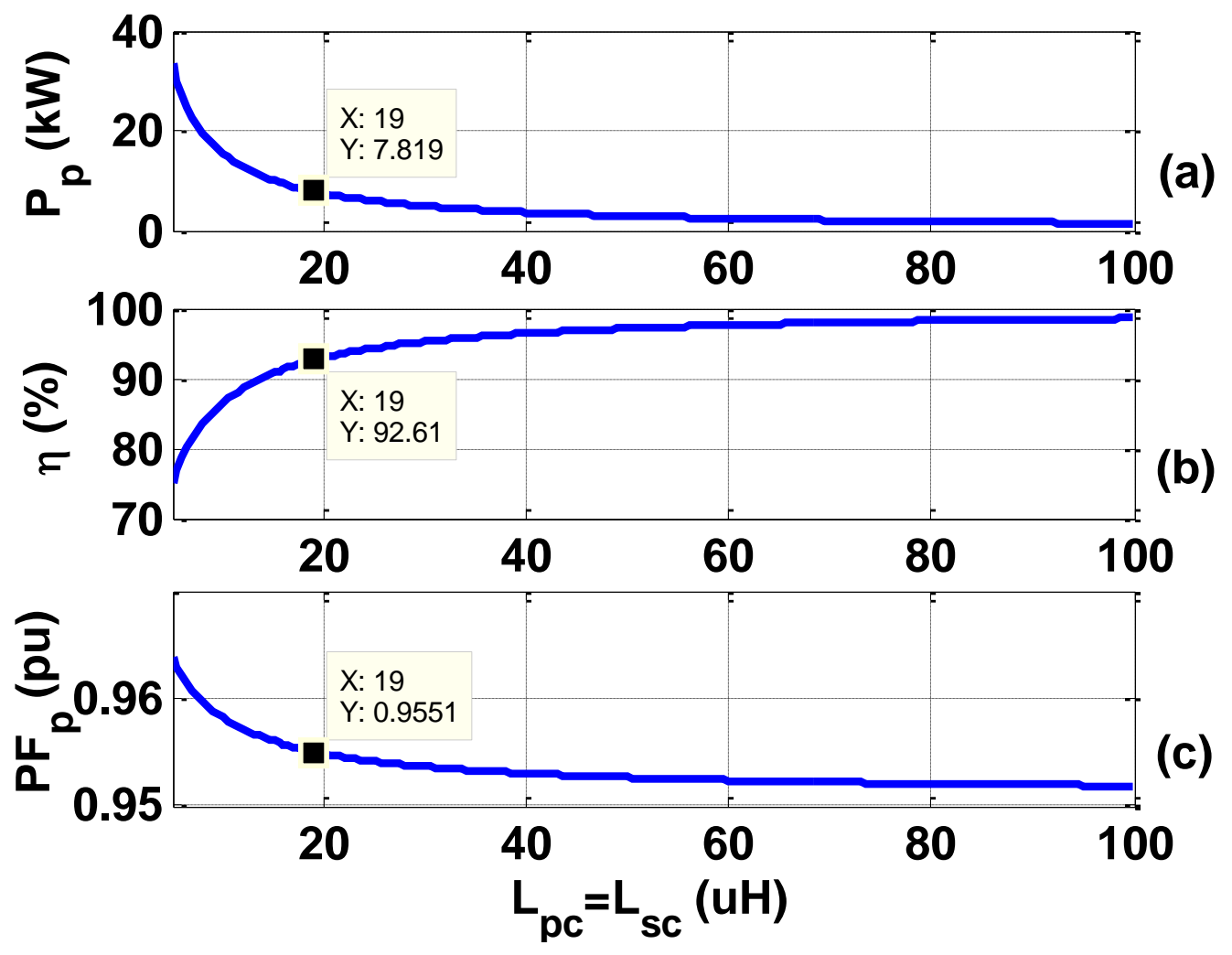

Figure 8.2. Effect of the pad self-inductance on the BIWPTS's performance $\left(V_{d c}=420 \mathrm{~V}\right.$ and $V_{b}=350 \mathrm{~V}$ ). (a) Primary active power. (b) System's efficiency. (c) Primary power factor.

Once the self-inductances of the power pads are defined, the following step is to achieve the magnetic design that is able to provide these values. Then, this design needs to be optimized to fulfill the desired magnetic coupling requirement. This task is usually achieved, using a 2D or 3D FEA. More details about the power pads' magnetic design and optimization are presented in the next section. 


\subsection{Magnetic Design of the Power Pads}

Several planar structures of the power pad for EVs applications are presented in the literature, such as circular or rectangular [184], DD [176], bi-polar [185], [186], DDQ [176] and tripolar [187]. The typical structure of all these configurations contains copper coils, ferrite material and aluminum shield, as depicted in Figure 8.3. Among these different configurations, only the circular/rectangular and the DD shapes are recommended by the J2954A standard. By comparing between the circular and DD structure, the DD provides better coupling, greater tolerance, greater charging zone, and cost effectiveness [84], [85], [169], [176].

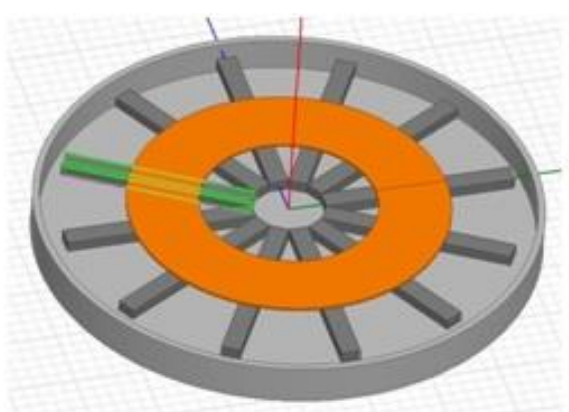

(a)

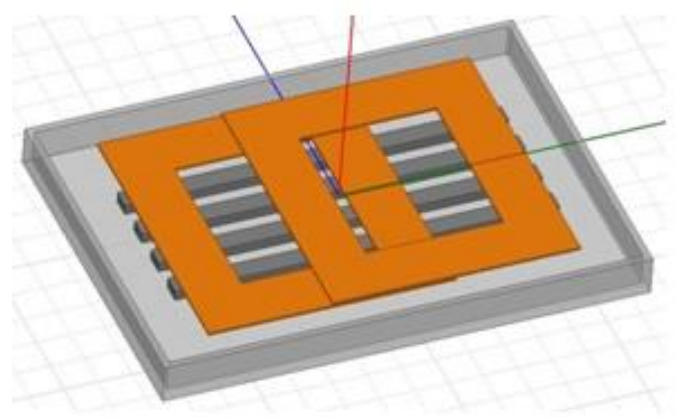

(c)

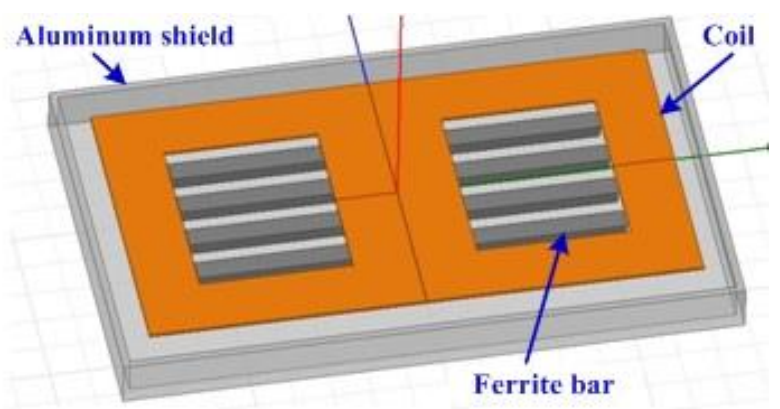

(b)

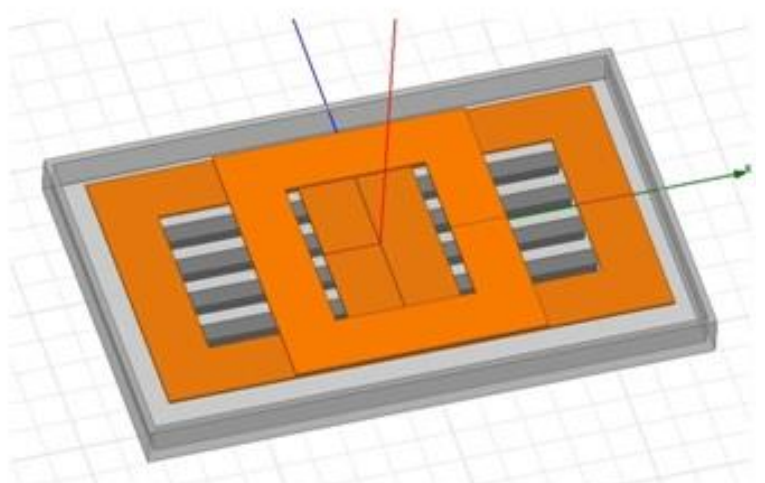

(d)

Figure 8.3. Different structures of power pads in EVs applications. (a) Circular. (b) DoubleD. (c) Bipolar. (d) Double-DQ. 
Because of the advantages of the polarized DD design, it is considered in this work. As was discussed in chapter (7), each pad consists of two or more coils, electrically connected in parallel, to reduce the equivalent inductance; aluminum shield and magnetic ferrite cores. The ferrite cores can be arranged to form a solid sheet, as indicated in Figure 7.4, or multiple parallel bars, as depicted in Figure 8.3. Using the solid sheet guarantees better magnetic coupling and shielding than those from the bars. However, it results in a higher cost and weight for pads.

\subsubsection{D Finite Element Model of DD Power Pad}

Due to the complex structure of the power pad, finding accurate analytical solutions for electromagnetic field distribution may not be conceivable. However, distinctive numerical analysis techniques can be utilized for electromagnetic field analysis. In this study, static 3D-FEA was considered. The commercial tool ANSYS Maxwell software is utilized for electromagnetic field calculation and magnetic parameters' estimation. A 3DFEM is built for the DD power pad, as depicted in Figure 8.4. In this model, the bars' core option is considered to present the most cost-effective solution; and to be able to be built with the available material in the laboratory. This model is optimized in order to achieve the desired requirements, defined in phases I and II. For design simplicity, the standard ferrite core (193x28x16) of N87 magnetic material from EPCOS is utilized, without any special cutting. In this structure, many different variables need to be defined in the design, such as coil length (Lc), coil width (Wc), number of ferrite bars, number of I-cores in each bar, spacing between bars (Df), shield dimensions (Ls, Ws, Ts and Gs), and coil side (CS), 
which is a function of number of turns $(\mathrm{N})$ and the distance between two adjacent turns (pitch), as indicated in Figure 8.5.

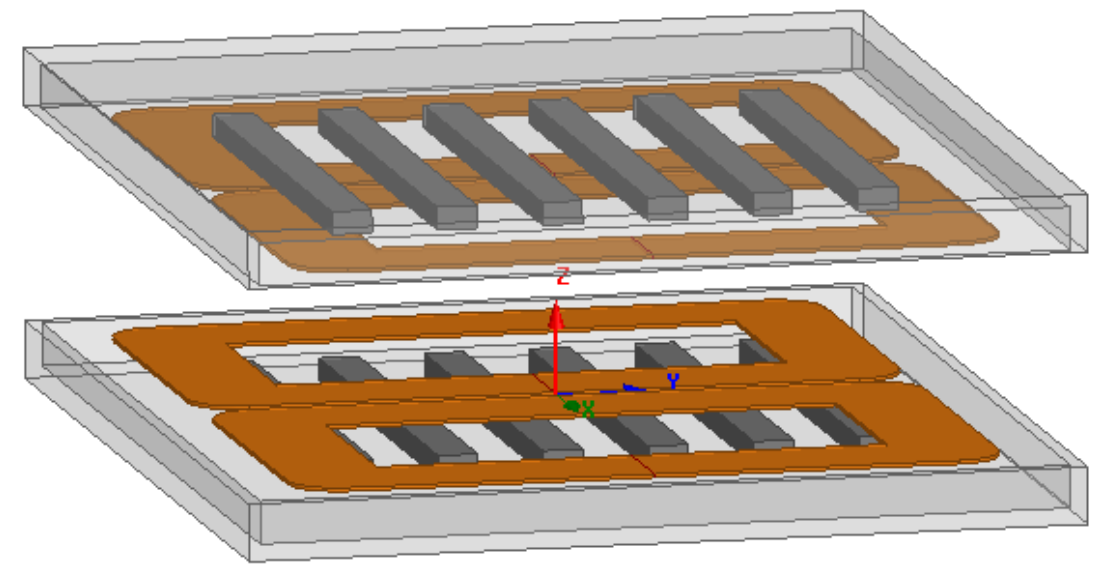

Figure 8.4. 3D-FEM for DD wireless coupler based on ferrite bars.

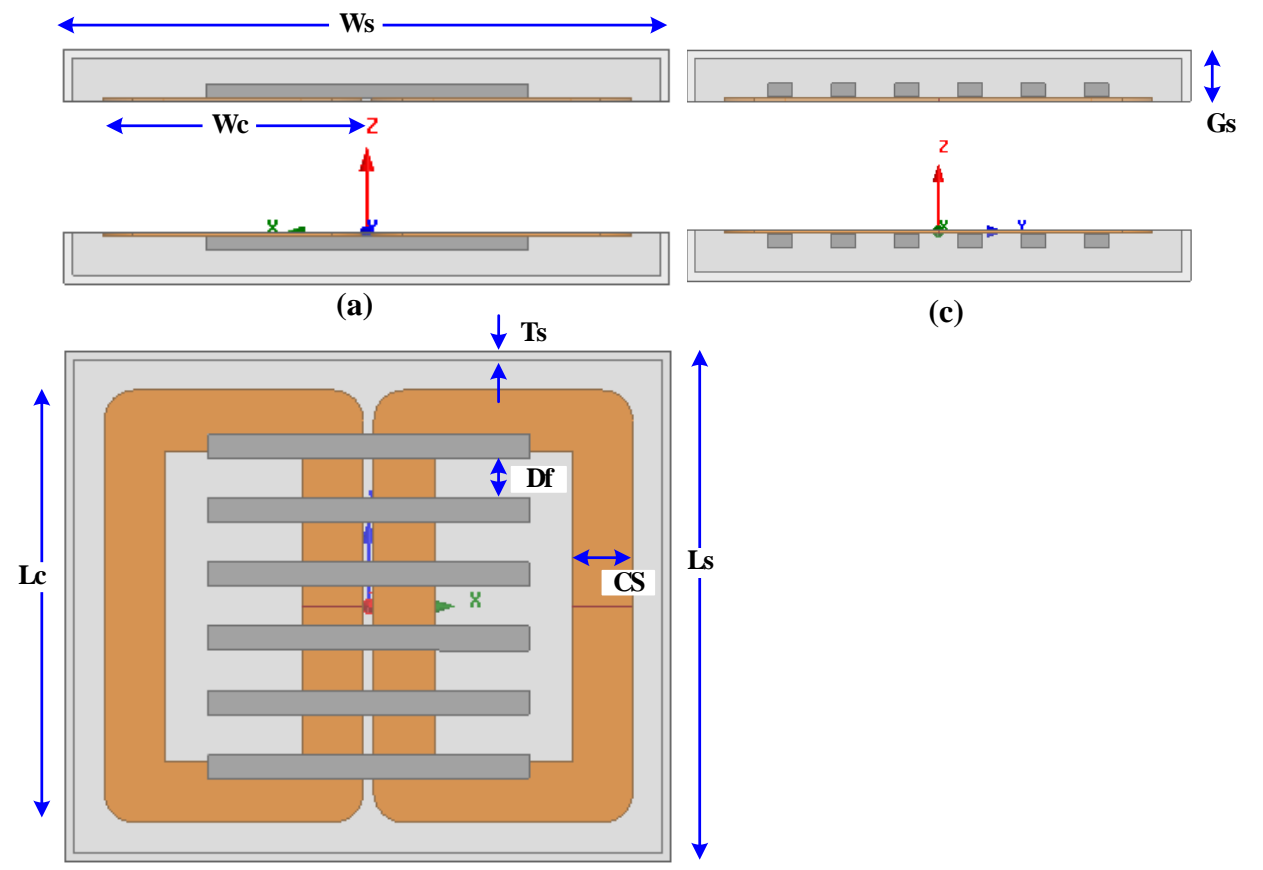

(b)

Figure 8.5. Different views of DD wireless coupler. (a) Front. (b) Top. (c) Side. 


\subsubsection{D Finite Element Analysis and Optimization of DD Power Pad}

The developed model was analyzed based on the 3D FEA for solving the static problem, using the static-magnetic vector potential partial differential equation given in Equation (8-1). The magnetic field distribution, though a vertical section in the middle of the model, while the currents in the two sides are equal to 23 A, is shown in Figure 8.6. Also, the distribution of the magnetic flux density in the ferrite bars is depicted in Figure 8.7 .

$$
\nabla \times(v(\nabla \times \bar{A}))=\overline{J_{e}}
$$

where, $v$ is the magnetic reluctivity $(\mathrm{m} / \mathrm{H}), A$ is the magnetic vector potential $(\mathrm{V} . \mathrm{s} / \mathrm{m})$, and $J_{e}$ is the current density vector.

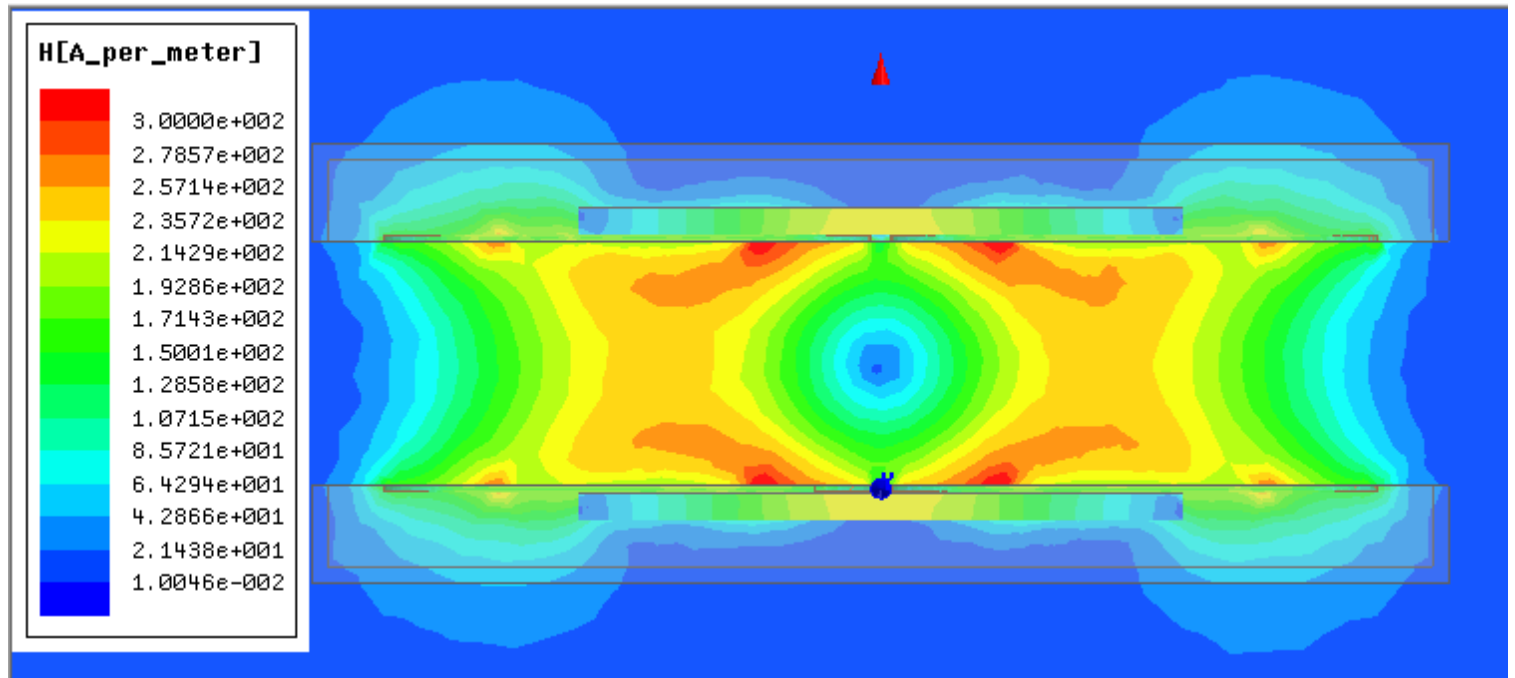

Figure 8.6. Magnetic field distribution across a vertical section in the middle of the 3D FEM of the DD power pad. 

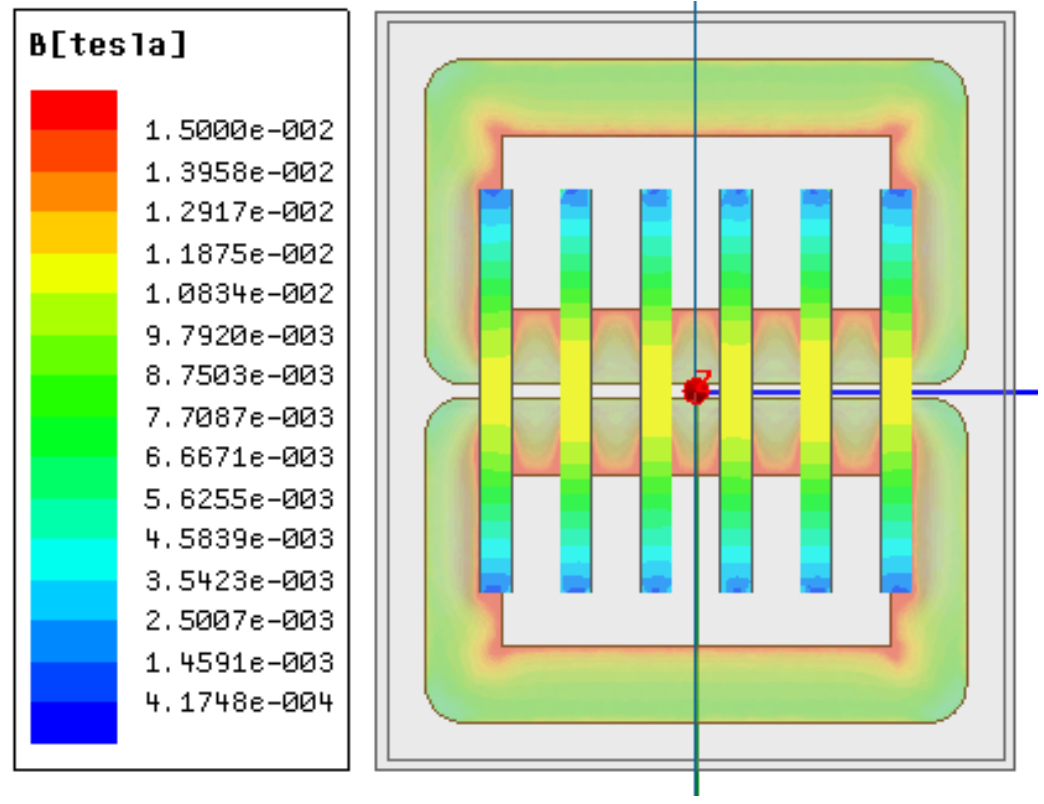

Figure 8.7. Magnetic flus density distribution in the ferrite bars of the DD power pad model.

The 3D static FEA was performed to evaluate the design and obtain the magnetic parameters ( $k$ and $L_{p c}, L_{s c}$ ), then these parameters were introduced inside the mathematical model, to assess the BIWPTS's power $\left(P_{p}\right)$ and efficiency $(\eta)$ [178]. Selected results for optimizing the magnetic design are presented in Figures 8.8, 8.9 and 8.10. In these analyses, the perfect alignment is assumed between the two power pads. In Figure 8.8, the coil-width (Wc) is investigated at fixed ferrite bars (six bars with four I-cores/bar). In this case, the number of turns was set to six turns in each coil. As can be noticed, for Wc that nears the half of the ferrite bars' length $(186 \mathrm{~mm})$, expanding Wc increases $L_{p c}, k, P_{p}$ and $\eta$, significantly. Greater increase in Wc demonstrates a small increase in $L_{p c}, k$ and $\eta$, but $P_{p}$ experiences a maximum point at $\mathrm{Wc}=260 \mathrm{~mm}$. Increasing $\mathrm{Wc}$ over this value leads to a drop in the power transfer. Thus, $\mathrm{Wc}=260 \mathrm{~mm}$ is considered in this design and this value is kept fixed in the flowing analysis. 

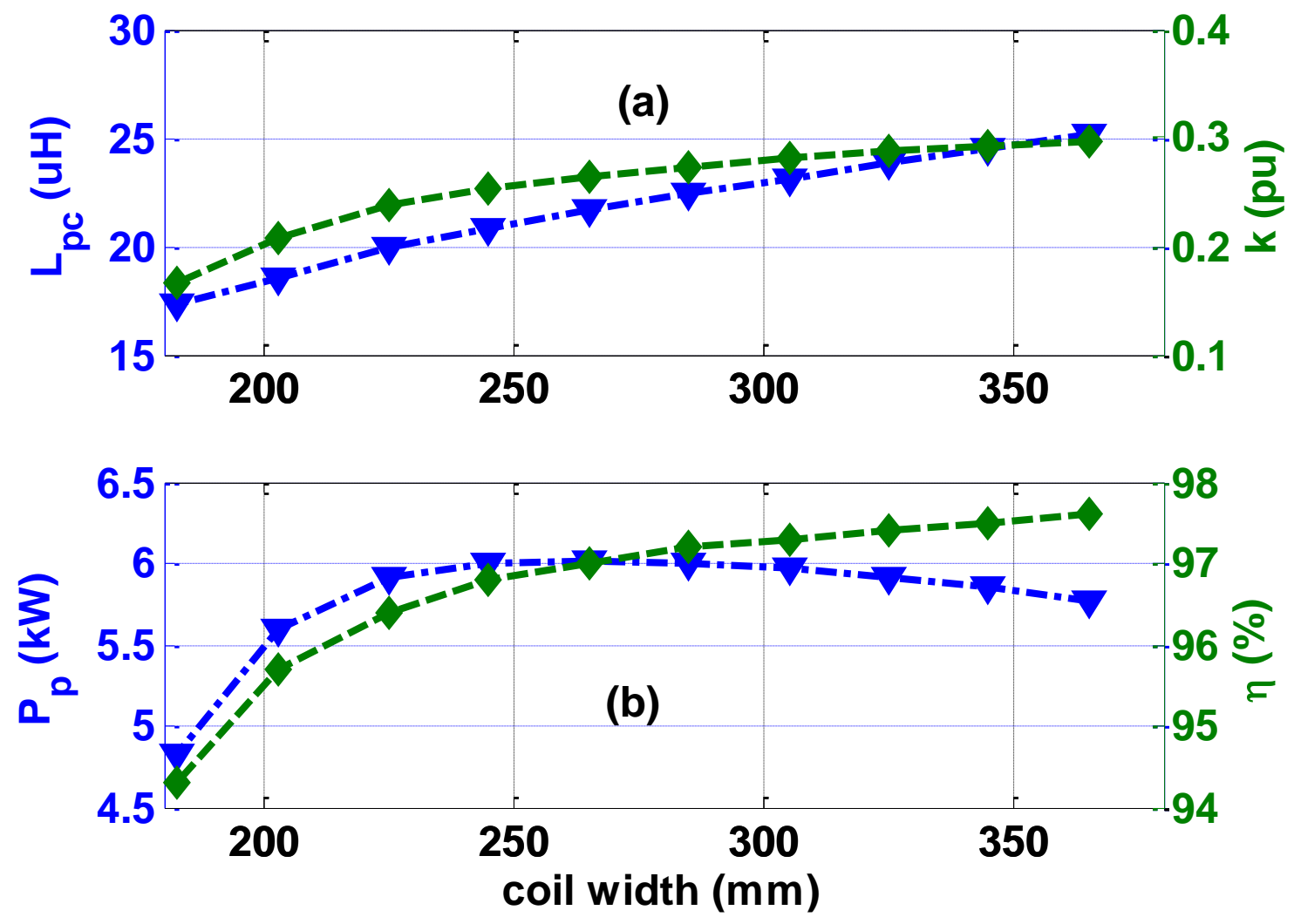

Figure 8.8. Effect of coil-width on the evaluation parameters $\left(V_{d c}=420 \mathrm{~V}\right.$ and $\left.V_{b}=350 \mathrm{~V}\right)$. (a) Primary self-inductance and coupling factor. (b) Primary power and efficiency.

The coil-width is kept fixed at $260 \mathrm{~mm}$, and the spacing between the ferrite bars (Df) is studied in Figure 8.9. The figure shows that a slight increase in Df increases $L_{p c}, k$ and $\eta$, but decreases $P_{p}$. Once Df becomes more than twice the bar width (2x28 mm), all the parameters drop dramatically. The best Df is chosen at $46 \mathrm{~mm}$, at which the model provides the highest coupling factor. 

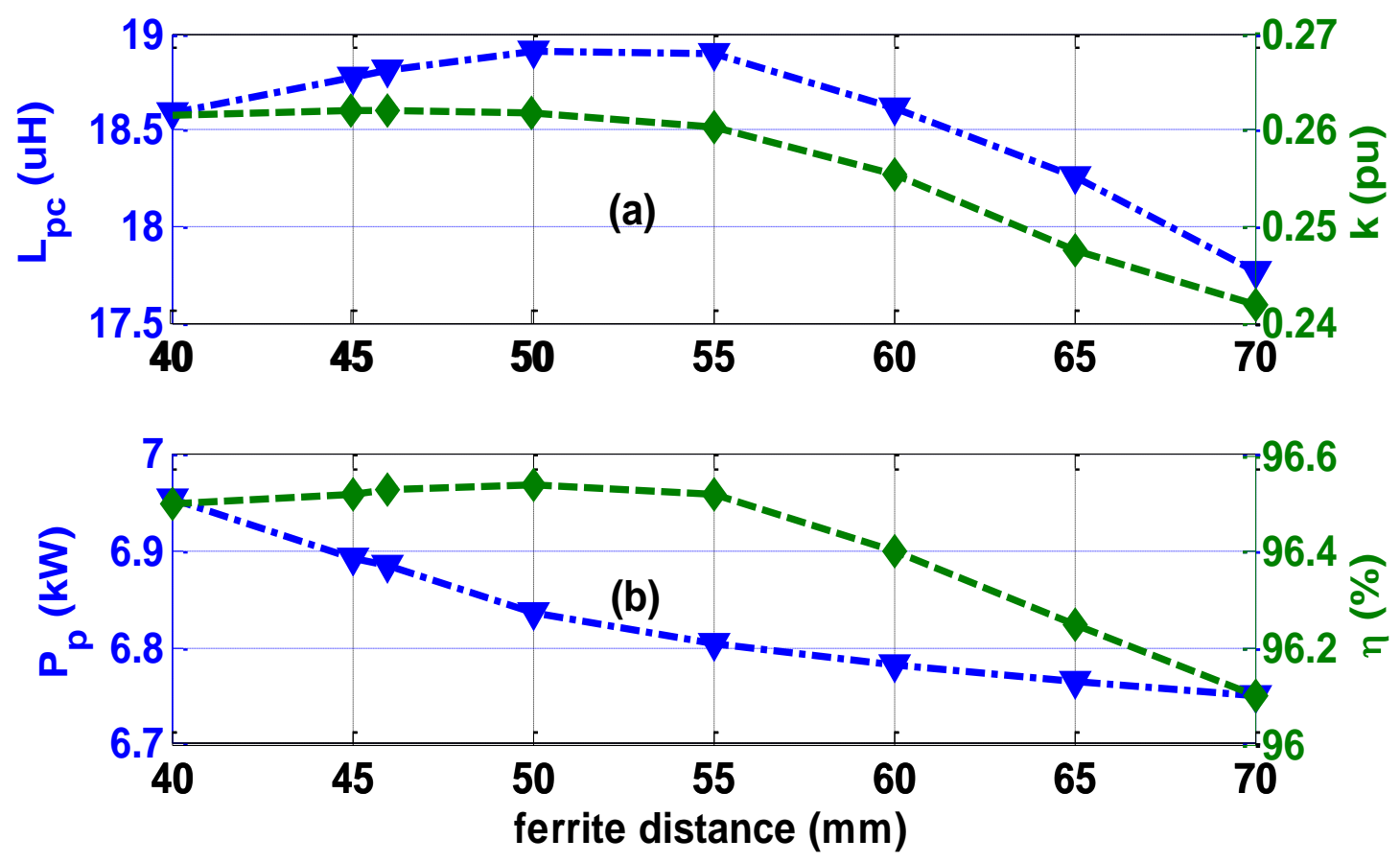

Figure 8.9. Effect of Df on the evaluation parameters $\left(V_{d c}=420 \mathrm{~V}\right.$ and $\left.V_{b}=350 \mathrm{~V}\right)$. (a) Primary self-inductance and coupling factor. (b) Primary power and efficiency.

Another variable [spacing between turns (pitch)] is optimized, as described in Figure 8.10. In this case, it is assumed that the pitch is fixed for all the coils in the DD power pad. The figure demonstrates that expanding the pitch leads to a better coupling factor and higher power transfer, but less system's efficiency. Thus, the value of the pitch was decided to achieve the required power level, with reasonable efficiency and coupling factor. This parameter was set to $11 \mathrm{~mm}$. Similarly, the entire pad's design parameters are investigated and the final parameters are presented in Table 8.1. 

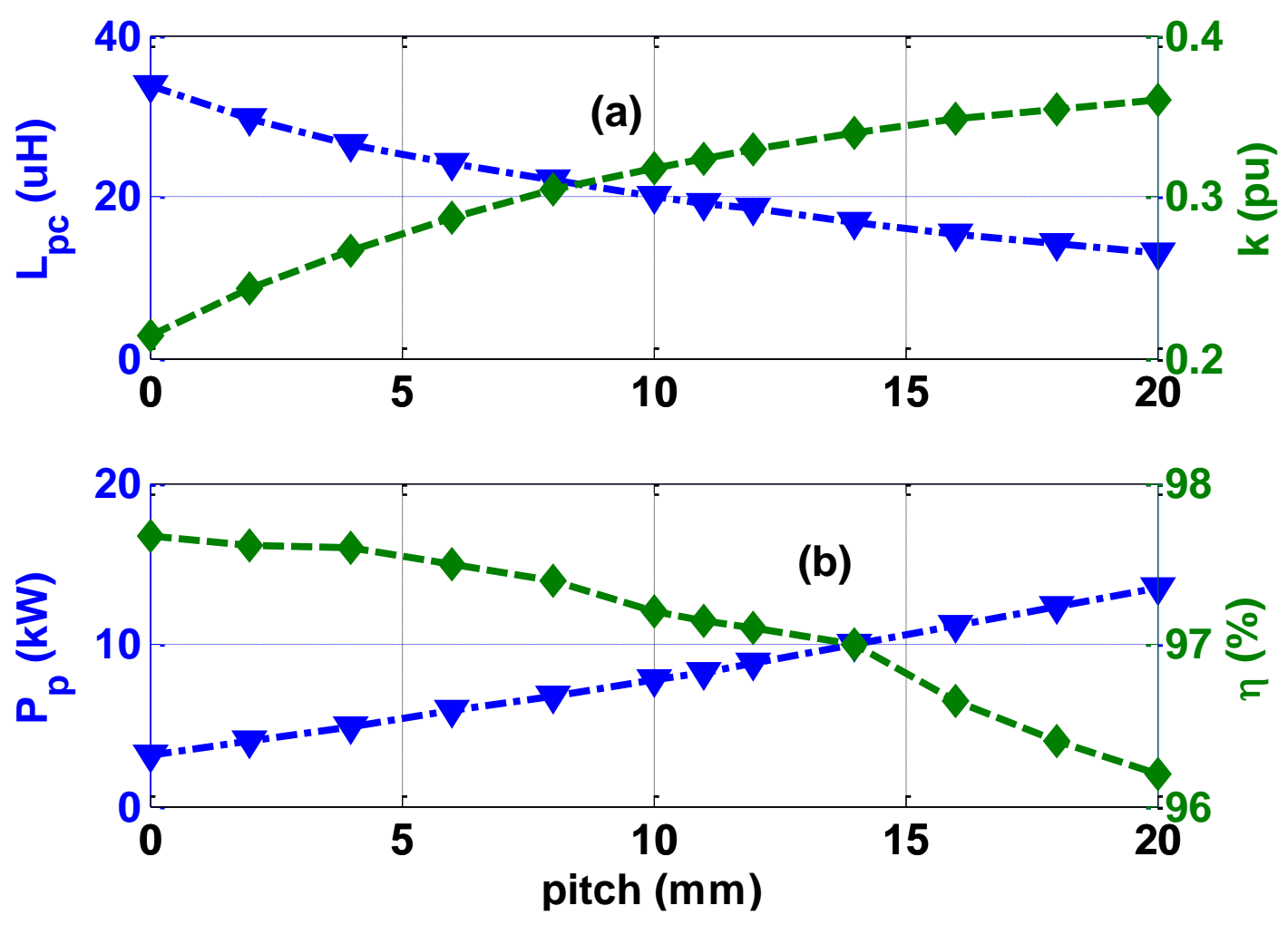

Figure 8.10. Effect the pitch on the evaluation parameters ( $V_{d c}=420 \mathrm{~V}$ and $\left.V_{b}=350 \mathrm{~V}\right)$. (a) Primary self-inductance and coupling factor. (b) Primary power and efficiency.

Table 8.1. Final Design Parameters of the DD Power Pad.

\begin{tabular}{cccc}
\hline \hline Parameter & value & Parameter & Value \\
\hline Wc & $260 \mathrm{~mm}$ & Ls & $700 \mathrm{~mm}$ \\
Lc & $500 \mathrm{~mm}$ & Ws & $600 \mathrm{~mm}$ \\
Df & $46 \mathrm{~mm}$ & Ts & $10 \mathrm{~mm}$ \\
CS & $70 \mathrm{~mm}$ & Strings & 6 \\
Turns & 6 & Bars & 4 \\
Pitch & $11 \mathrm{~mm}$ & & \\
\hline \hline
\end{tabular}




\subsubsection{Design of LCL Compensation Configuration}

In the wake of completing the magnetic design and evaluation, the following phase, IV in Figure 8.1, is to estimate the LCL compensation topology parameters $\left(L_{p i}, L_{s i}, C_{p}\right.$ and $\left.C_{s}\right)$. For LCL topology, the capacitors are connected in parallel, and are tuned to resonate with the self-inductances of the wireless pads, as was indicated in Equation (4-2). In this case, the system behaves as a parallel-resonance circuit, with high equivalent impedance at the resonant frequency to provide current-source characteristics.

Typically, the filters' parameters $\left(L_{p i}\right.$ and $\left.L_{s i}\right)$ are assigned based on the power transfer capabilities. However, the BIWPTS's performance is very sensitive to the variation of the filter inductance with respect to the pad self-inductance, as was depicted in Figure 4.12. For a $40 \mathrm{kHz}$ resonant frequency, the operating frequency matches with $40 \mathrm{kHz}$ only when $L_{p i}=L_{p c}$ and $L_{s i}=L_{s c}$. At these conditions, the system shows UPF operation (zero impedance angle), as indicated in Figure 4.12(b) [102]. A small deviation of $L_{p i}$ from $L_{p c}$ leads to deviation in the resonant frequency. In this case, the operating frequency needs to be adjusted to match with the resonant frequency, which requires more complex controllers. The last phase, $\mathrm{V}$, before considering the design, is to check the system efficiency, using the developed mathematical model. If it achieves the desired efficiency, then the design will be considered, otherwise different magnetic design needs to be tried, by repeating phases II to $\mathrm{V}$.

\subsection{Design Verification and Results}

For confirming the adequacy of the proposed design methodology, two $8 \mathrm{~kW}$ identical DD power pads were built, according to the parameters shown in Table 8.1. These 
pads were developed using litz wire Type 2, 660/38 AWG 10, and the standard ferrite core I93x28x 16 of N87 magnetic material from EPCOS. The ferrite I-cores are attached together to form the ferrite bars. Each pad contains six bars, with four cores per bar, as shown in Figure 8.11. Also, it contains two litz wire coils, connected electrically in parallel and magnetically in series. The final shape of the DD power pad is indicated in Figure 8.12.

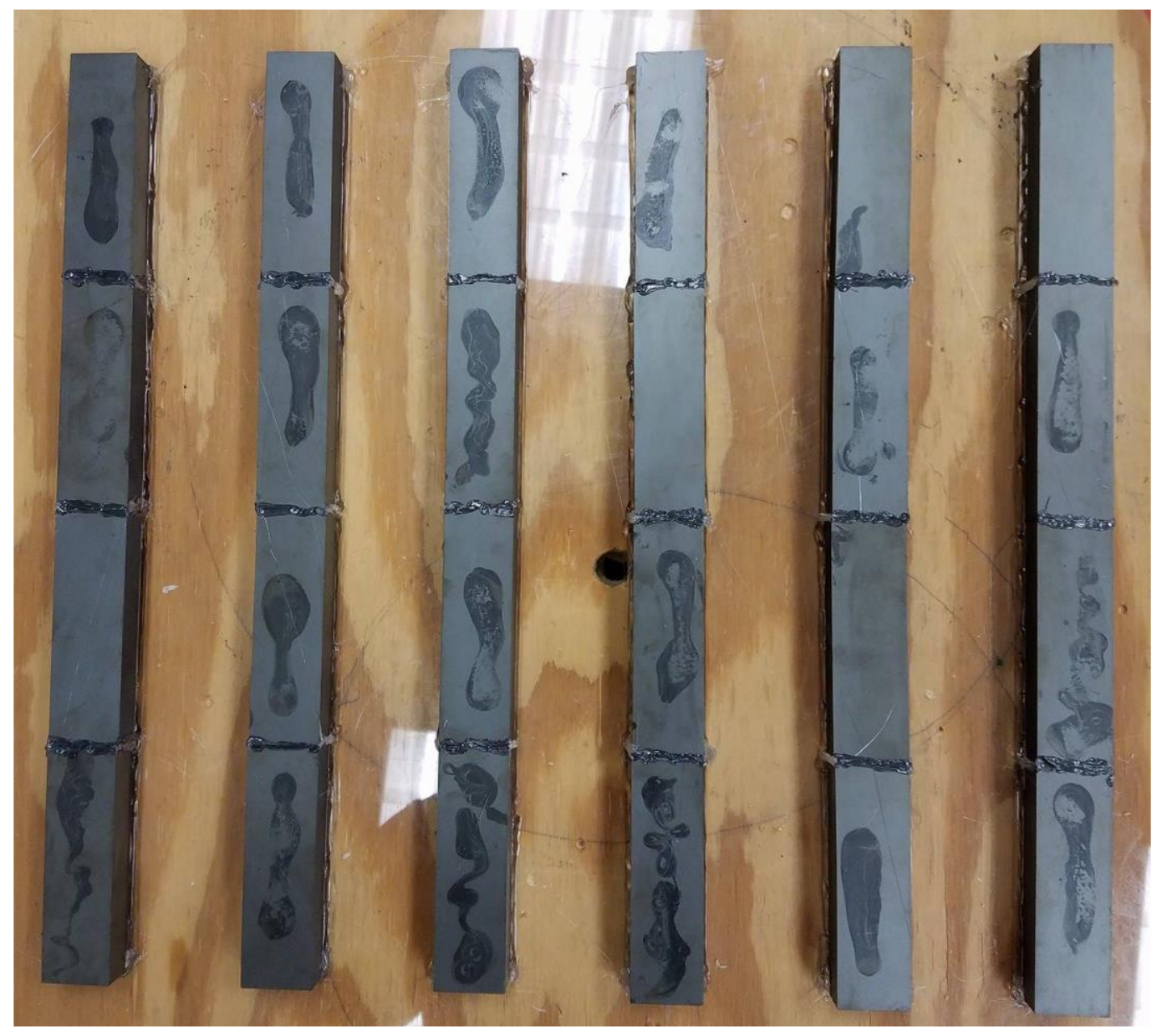

Figure 8.11. Ferrite bars of N87 material from EPCOS for one DD power pad. 


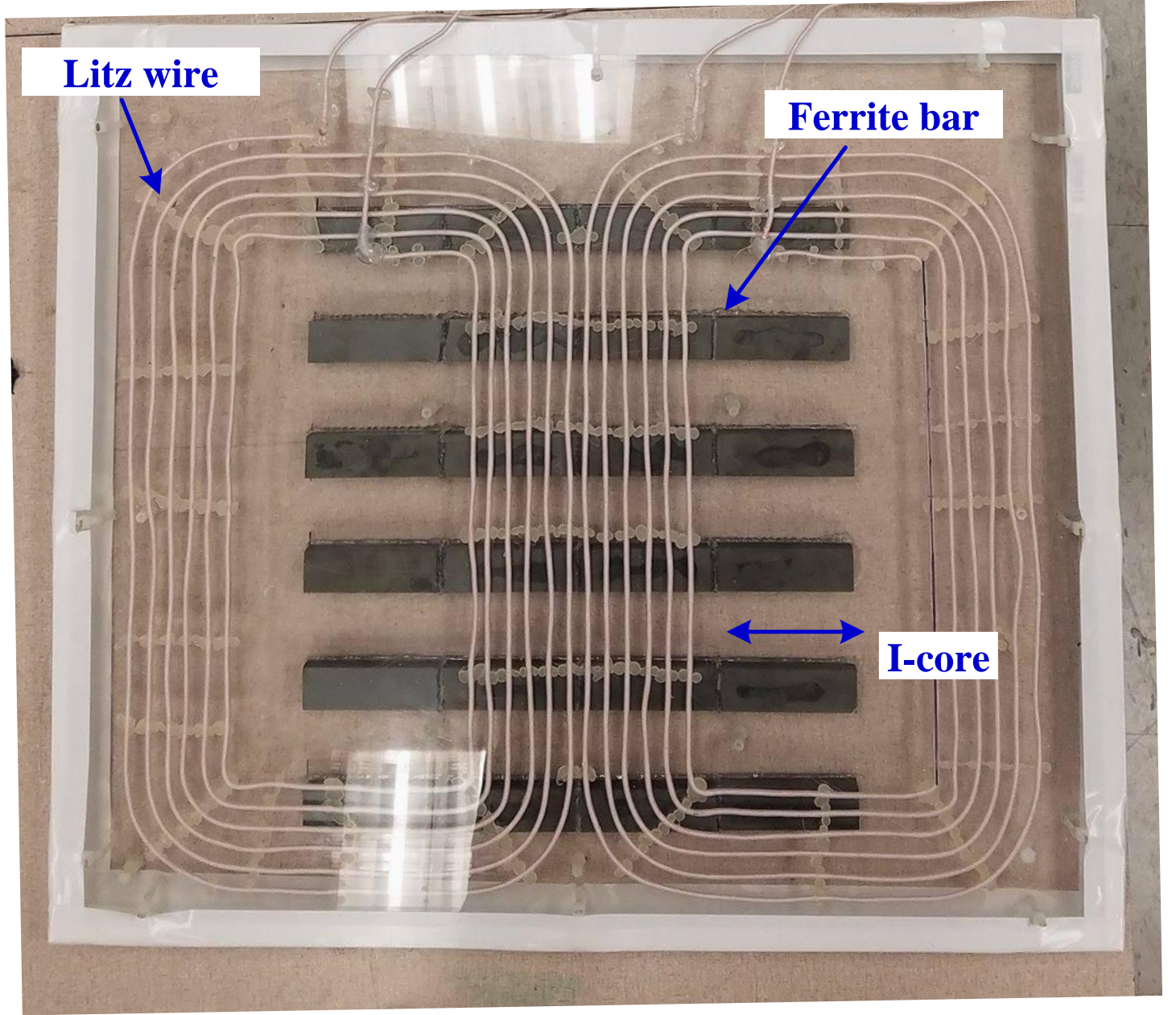

Figure 8.12. Final shape of the developed $8 \mathrm{~kW}$ DD power pad.

The compensation networks are built using Metallized Film capacitors and handmade filters. The power pads, the compensation networks and the HF VSHBCs [described in chapter (5)] are gathered, to form a prototype for an $8 \mathrm{~kW} \mathrm{LCL} \mathrm{BIWPTS,} \mathrm{as} \mathrm{indicated}$ in Figure 8.13. It comprises of two identical DD power pads, two $40 \mathrm{kHz}$ VSHBCs, a firing board to generate synchronized switching signals for the converters using the PPM technique, a $50 \mathrm{~V}$ Li-ion battery module to emulate the EV battery behavior, a programmable MAGNA power supply to emulate the DC-bus, and sensors' board with 
LEM voltage and current transducers and oscilloscopes. The final BIWPTS's design parameters are described in Table 8.2.

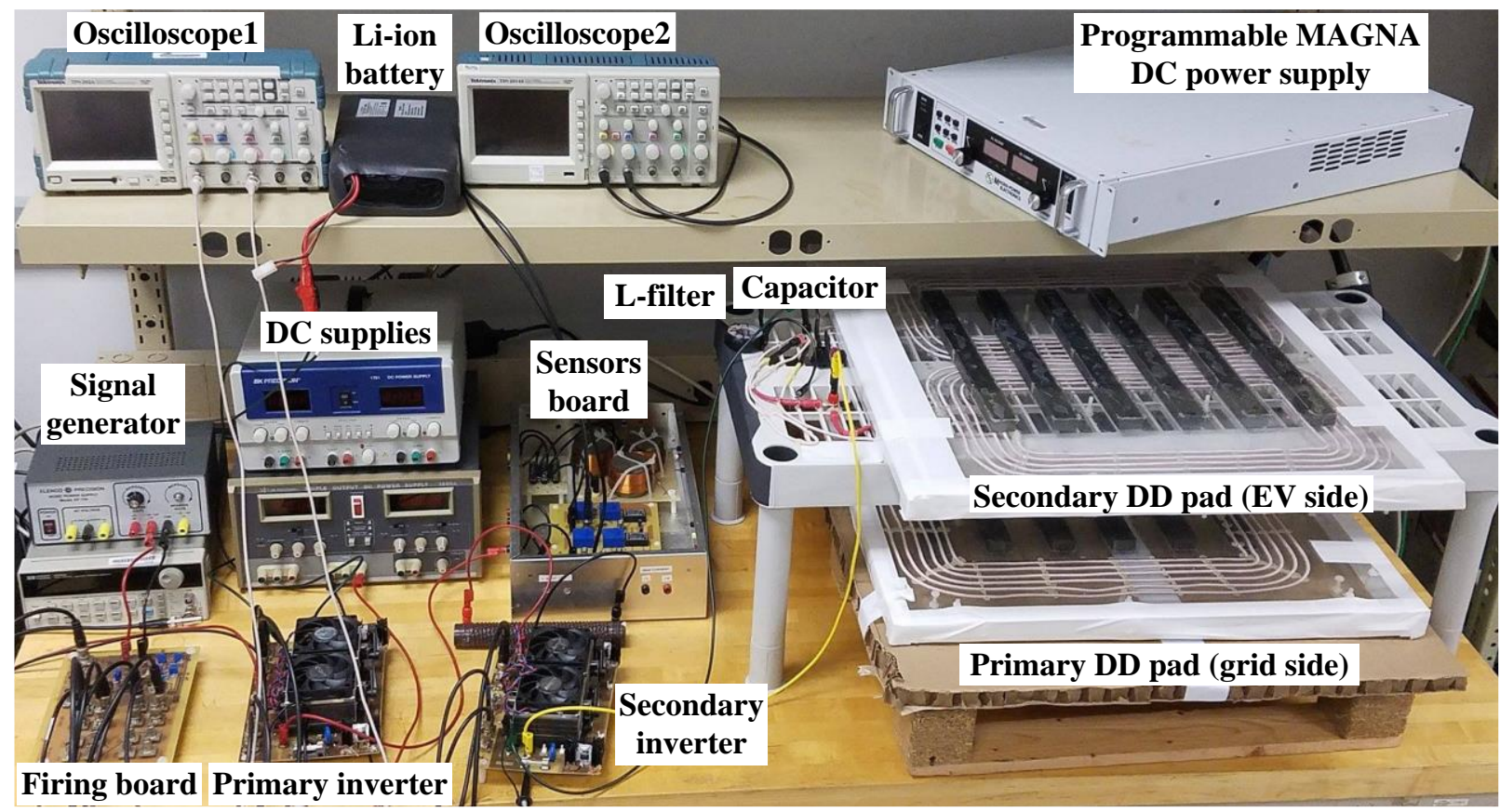

Figure 8.13. Test-bed for a symmetrical LCL BIWPTS based on the DD power pads.

Table 8.2. Design Parameters of LCL BIWPTS with DD pads structure.

\begin{tabular}{llllll}
\hline Parameter & Value & Parameter & Value & Parameter & Value \\
\hline$L_{p i}$ & $19.8 \mu \mathrm{H}$ & $L_{s i}$ & $19.6 \mu \mathrm{H}$ & $C_{p}$ & $0.79 \mu \mathrm{F}$ \\
$R_{p i}$ & $40 \mathrm{~m} \Omega$ & $R_{s i}$ & $40 \mathrm{~m} \Omega$ & $C_{s}$ & $0.8 \mu \mathrm{F}$ \\
$L_{p c}$ & $20.1 \mu \mathrm{H}$ & $L_{s c}$ & $19.8 \mu \mathrm{H}$ & $k$ & 0.32 \\
$R_{p c}$ & $60 \mathrm{~m} \Omega$ & $R_{s c}$ & $60 \mathrm{~m} \Omega$ & $f$ & $40 \mathrm{kHz}$ \\
\hline \hline
\end{tabular}


In addition, these parameters are utilized inside the Simulink model (presented in Figure 5.24), and the steady-state analytical model [presented in chapter (4)]. The two models are analyzed and compared for $8 \mathrm{~kW}$ power level, during G2V (charging) operation, as depicted in Figure 8.14. Very good agreement can be observed between the analytical and simulated results.

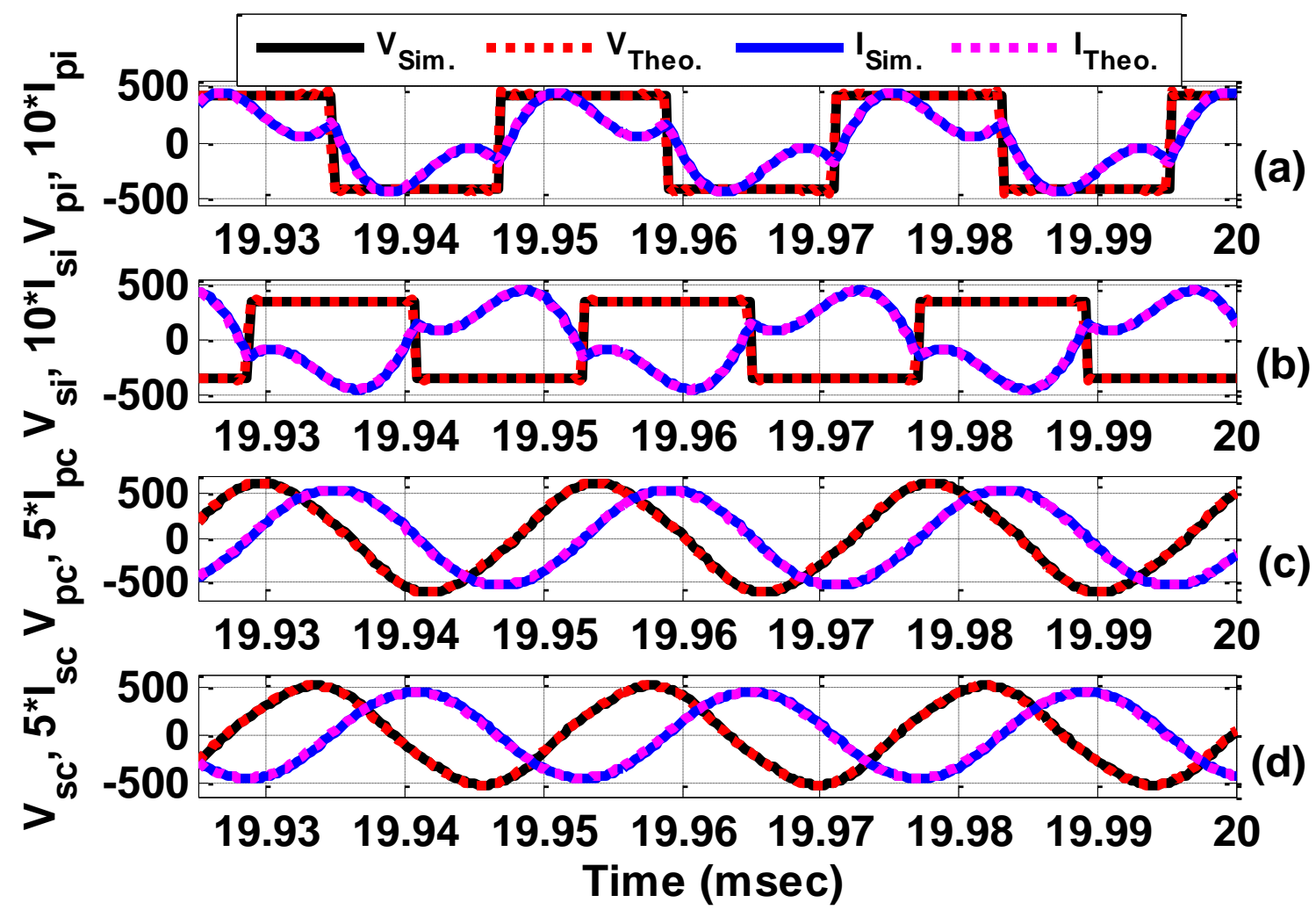

Figure 8.14. Performance of an $8 \mathrm{~kW}$ LCL BIWPTS, during G2V operation $\left(\alpha=\beta=180^{\circ}\right.$, $\delta=-90^{\circ}, V_{d c}=420 \mathrm{~V}$ and $V_{b}=350 \mathrm{~V}$ ), using Simulink and analytical models. (a) Primary inverter variables. (b) Secondary inverter variables. (c) Primary coil variables. (d) Secondary coil variables. 
Moreover, the DC voltage levels $\left(V_{d c}\right.$ and $\left.V_{b}\right)$ are scaled down (1/7 time the design values, to fit the available Li-battery module in the laboratory), and the experimental prototype is analyzed and compared with the analytical model's results, during both G2V and $\mathrm{V} 2 \mathrm{G}$ operation, as indicated in Figure 8.15 and 8.16, respectively. Also, good agreement can be observed between the two results. In order to check the system scalability, the power-flow is evaluated for both the large and small-scale system, during G2V operation, as presented in Table 8.3. As can be noticed, both scales provide the same efficiency and power factor. The ratio between the power levels is about 49 , which represents the square of the scaling factor 7. In addition, the power, efficiency and power factor results match with the desired values, defined by the proposed design strategy.

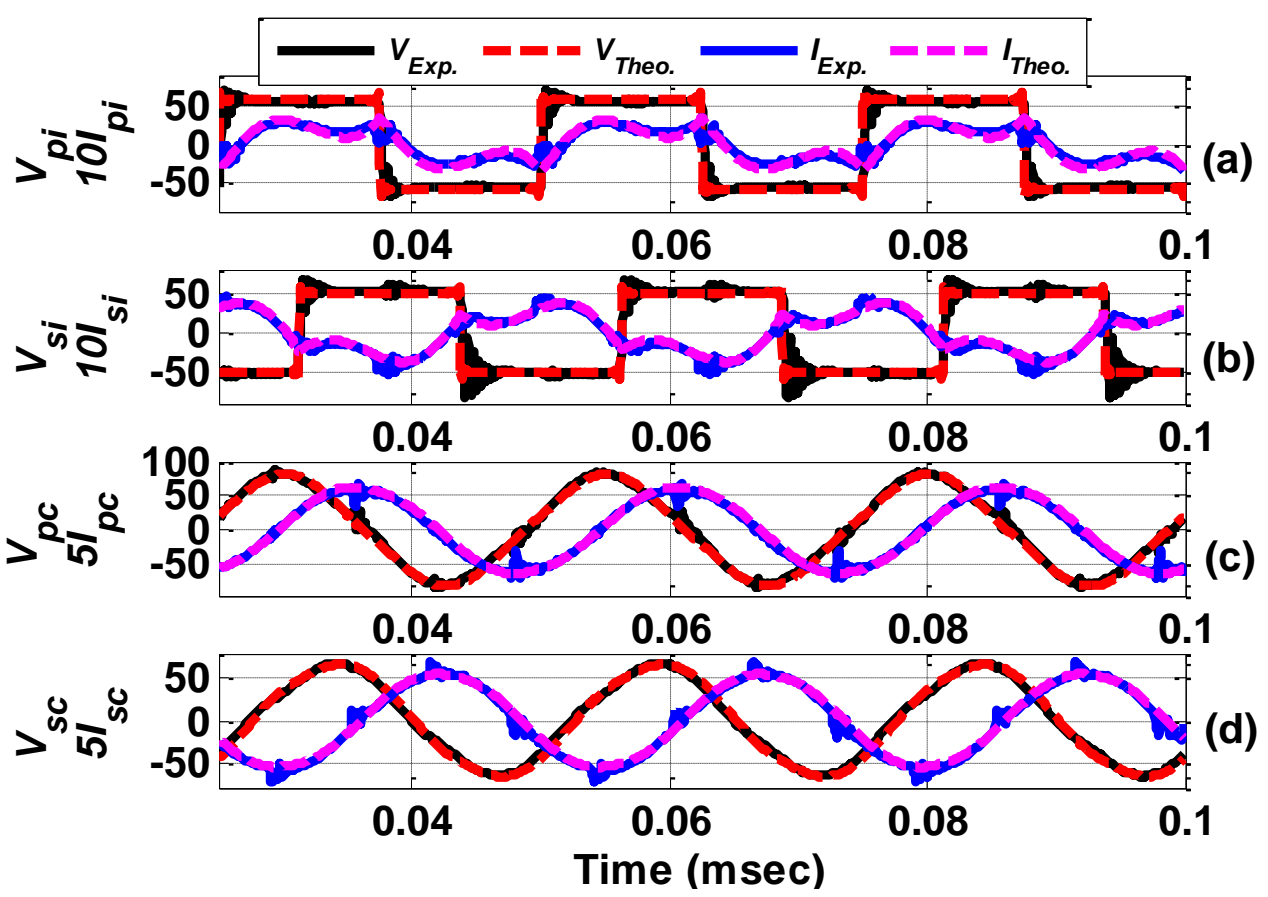

Figure 8.15. Performance of the small scale LCL BIWPTS, during G2V operation ( $\alpha=\beta=180^{\circ}, \delta=-90^{\circ}, V_{d c}=60 \mathrm{~V}$ and $V_{b}=50 \mathrm{~V}$ ), using experimental and analytical models. (a) Primary inverter variables. (b) Secondary inverter variables. (c) Primary coil variables. (d) Secondary coil variables. 


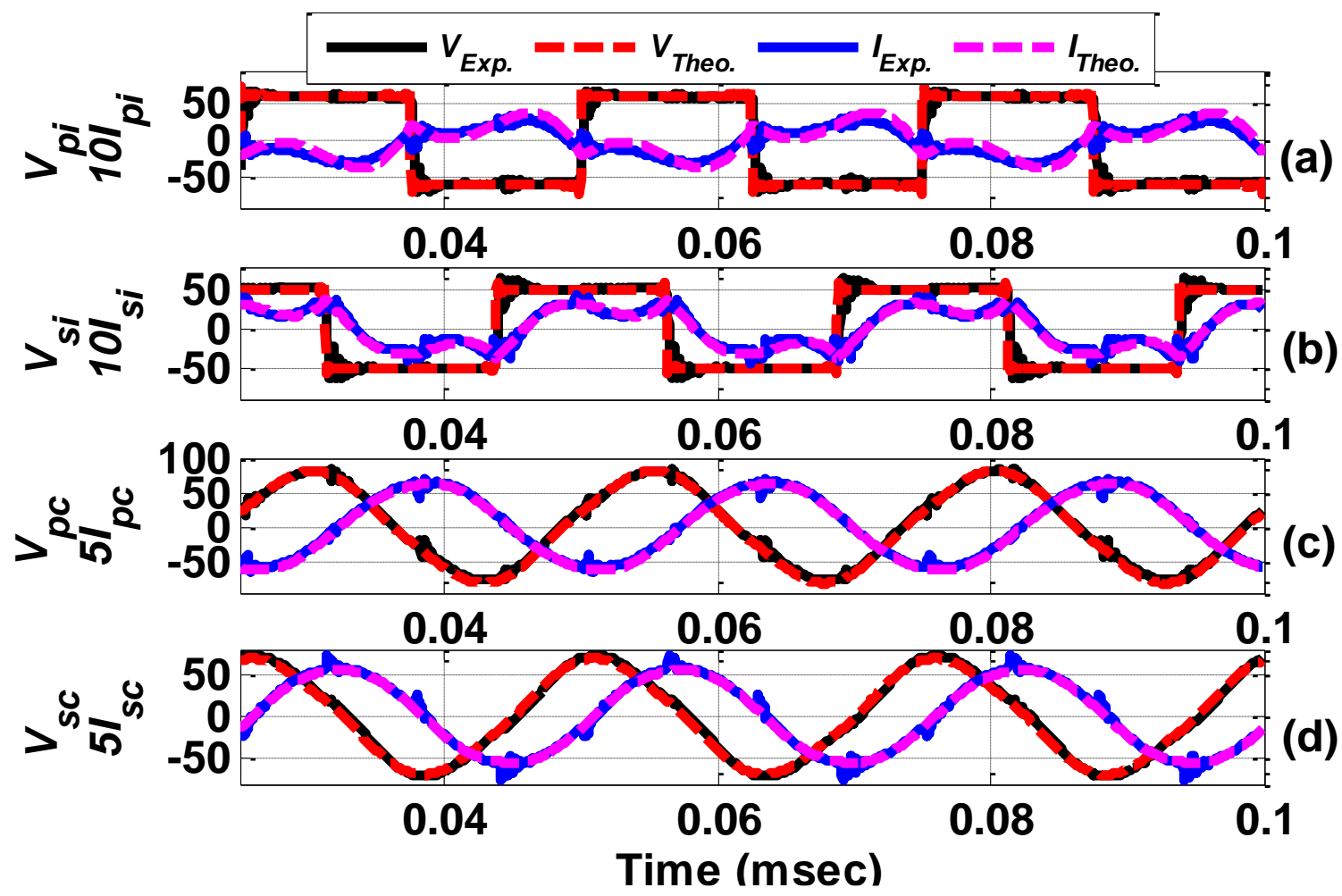

Figure 8.16. Performance of the small scale LCL BIWPTS, during V2G operation $\left(\alpha=\beta=180^{\circ}, \delta=90^{\circ}, V_{d c}=60 \mathrm{~V}\right.$ and $\left.V_{b}=50 \mathrm{~V}\right)$, using experimental and analytical models. (a) Primary inverter variables. (b) Secondary inverter variables. (c) Primary coil variables. (d) Secondary coil variables.

Table 8.3. The Power Flow of the Large and Small Scale Models

\begin{tabular}{lcccc}
\hline Mode & $P_{p}(W)$ & $P_{s}(W)$ & $\eta(\%)$ & $P F(p u)$ \\
\hline Large scale G2V & 8050 & 7424 & 92.2 & 0.978 \\
Small scale G2V & 164 & 151 & 92.2 & 0.978 \\
\hline \hline
\end{tabular}




\subsection{Conclusion}

In this chapter, an efficient design methodology for a symmetrical LCL BIWPTS was presented. An $8-\mathrm{kW}$ polarized DD pad magnetic design was investigated, and intuitively optimized based on finite-element analysis, using ANSYS Maxwell tool. The proposed procedure is utilized to design a LCL BIWPTS that satisfies the standard requirements of WPT2 power level and Z2 class in the J2954A standard. This methodology is generic, and can be utilized for different power levels, air-gap classes, resonant frequencies and power pad structures. Also, the choice and design of the compensation capacitors and the impedance matching parameters were investigated. The proposed design technique was assessed based on simulation, analytical as well as experimental analysis and results. The results confirm the effectiveness of the proposed strategy with good agreement between the different models' results. 


\section{Chapter 9 Coil Design Multi-Objective Optimization in the DD Power Pad Structure}

\subsection{Introduction}

The magnetic design of the power pads is crucial for the IPT system's performance. Several studies for investigating the design of the DD power pad structure in EVs applications are presented in the literature [176], [178], [188]. In these works, the coil turns were modeled as a rectangular block of copper (single-turn and single-layer), as was discussed in chapter (8) and indicated in Figures 8.3 and 8.4. This way of modeling does not represent the actual coil, which contains several turns with air-gaps among them. The single-turn single-layer (STSL) model helps to reduce the computational time and effort, during FEA. However, it introduces some errors in the analysis due to this approximation. These errors are negligible, as long as the turns are close enough to each other. However, if the separations between the turns are noticeable, this approximation will be incorrect and may lead to significant errors. Moreover, in these presented studies, the authors picked up some design variables, and analyzed them manually, within a specific range, as was explained in section 8.3.2. This manual design optimization technique is acceptable in the systems having few and independent design parameters. However, in complex situations, like IPT systems, many design parameters need to configured, and these parameters are related to each other. Thus, applying the manual optimization in IPT systems' design is very difficult, and requires a wide knowledge and experience, even though finding the optimum solution is not guaranteed. 
Some automatic optimization algorithms for IPT systems' design were presented in the literature, based on Pareto multi-objective optimization [189]-[191]. These algorithms were solved, using genetic algorithm, as in [191], and particle swarm, as in [190]. These evolutionary techniques are very time consuming, especially when FEA becomes a part of the optimization process. Moreover, the simplified coil model (STSL) was considered in these works as well.

Different from what was presented in the literature, this chapter presents an automatic multi-objective optimization for the design parameters of the DD power pad structure. A detailed 2D FEM for the power pad, considering the separations among the turns, is developed. The developed model is coupled with a fast and an efficient improved Tabu search (ITS) algorithm, for optimization purposes. In the proposed algorithm, several objectives are evaluated, such as magnetic coupling, misalignment (horizontal, vertical and rotational), as well as the cost of the power pads. For verification purposes, the system coupling performance and cost are investigated, using the optimum design parameters, and compared with three other designs that are presented in the literature. The proposed optimized design shows the best coupling performance with moderate cost. The results proved the validity and advantages of the proposed design optimization methodology.

\subsection{A Detailed 2D FEM of the DD Power Pad}

In this study, the FE modeling and analysis are achieved, using ANSYS Maxwell software for electromagnetic field calculation, and magnetic parameters' estimation. A detailed 2D quasi-static electromagnetic FEM is built and analyzed in this study. The coil 
turns are exactly modeled, to accurately investigate the effect of the separations among them, as shown in Figure 9.1.

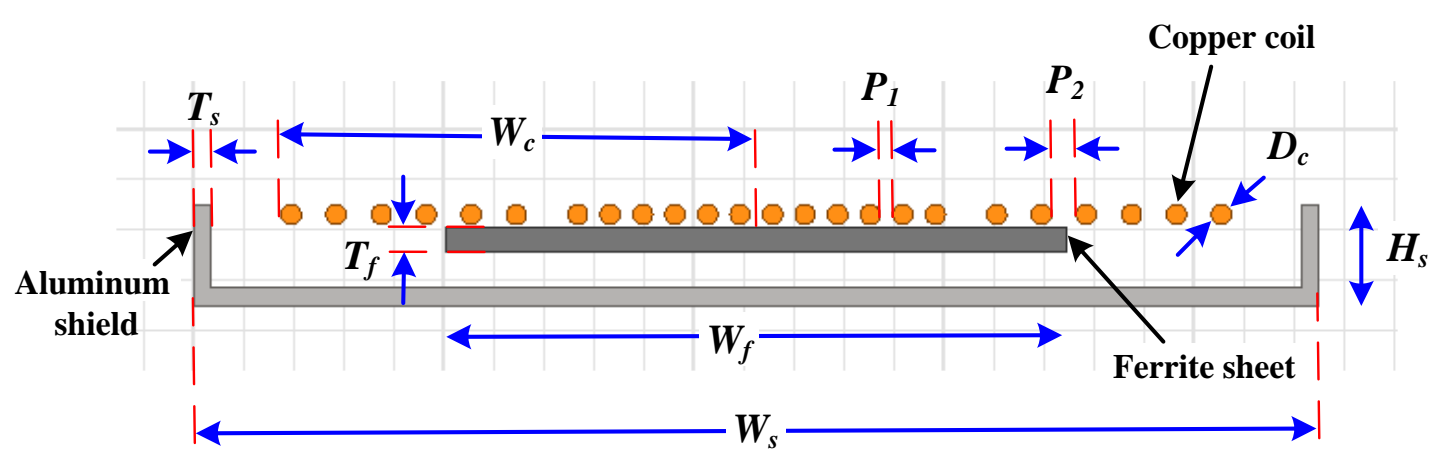

Figure 9.1. A 2D-FEM of the DD power pad, showing the optimization variables.

Each turn is modeled as a stranded coil domain to emulate the litz wire performance, which is commonly used in IPT systems. In this model, the separation among the turns (pitch) in the middle sides is assigned to a variable $P_{1}$, while the pitch in the outer sides is associated to a variable $P_{2}$. This way provides more freedom and flexibility for the search algorithm to find the optimum design solution. In addition, the dimensions of the power pad's components (indicated in Figure 9.1) are assigned to different variables, to facilitate their modification and optimization. The model was analyzed using the 2D FEA for solving the static problem, based on the static magnetic vector potential partial differential equation, given in Equation (8-1). The dimensions of the developed 2D FEM's components are indicated in Table 9.1. These dimensions are kept fixed during the optimization process, and only the separations $\left(P_{1}\right.$ and $\left.P_{2}\right)$ and the ferrite bars' variables [width $\left(W_{f}\right)$ and thickness $\left.\left(T_{f}\right)\right]$ vary. 
Table 9.1. Dimensions of the 2D FEM for the DD pad structure.

\begin{tabular}{llll}
\hline \hline Parameter & Value & Parameter & Value \\
\hline Coil width $\left(W_{c}\right)$ & $190 \mathrm{~mm}$ & Shield thickness $\left(T_{s}\right)$ & $5 \mathrm{~mm}$ \\
Coil diameter $\left(D_{c}\right)$ & $5.6 \mathrm{~mm}$ & Shield width $\left(W_{s}\right)$ & $500 \mathrm{~mm}$ \\
Turns per coil $(N)$ & 6 turns & Shield edge $\left(H_{s}\right)$ & $50 \mathrm{~mm}$ \\
\hline \hline
\end{tabular}

\subsection{The Multi-objective Optimization Problem}

Regardless of the compensation topology, the power transfer between the primary to the secondary side of an IPT system is a function of the supply characteristics (frequency and current), secondary pad magnetic parameters (self and mutual inductances) and load characteristics (quality factor), as given in Equation (9-1) [85].

$$
P_{o}=\omega I_{1}^{2}\left(M^{2} / L_{2}\right) Q_{2}
$$

where, $\omega$ is the frequency of the primary current, $I_{1} ; M$ is the mutual inductance; $L_{2}$ is the secondary pad self-inductance; and $Q_{2}$ is the secondary circuit quality factor.

Under the assumptions of 1) implementing parallel compensation at the secondary circuit, which is the common case in EVs applications, 2) operating the system at fixed resonant frequency in both the primary and secondary sides, and 3) considering identical pads on both sides $\left(L_{1}=L_{2}=L\right)$, the secondary quality factor $\left(Q_{2}\right)$ and mutual inductance (M) can be written as in Equation (9-2).

$$
\begin{aligned}
& Q_{2}=R_{L} / \omega L \\
& M=\sqrt{L_{1} L_{2}}=k L
\end{aligned}
$$

where, $R_{L}$ is the load equivalent resistance. 
By substituting Equation (9-2) into (9-1), the output power at the secondary side can be written, as in Equation (9-3).

$$
P_{o}=I_{1}^{2} k^{2} R_{L}
$$

From Equation (9-3), it can be noted that the power transfer can be increased by increasing the supply current $\left(I_{1}^{2}\right)$, the load equivalent resistance $\left(R_{L}\right)$ and the magnetic coupling $\left(k^{2}\right)$. The term $I_{1}^{2} R_{L}$ is limited by the power electronic converters' capabilities and the system's losses and load. The coupling factor is related to the magnetic design of the power pad and alignment condition between the two sides of the system. An IPT system with a higher coupling factor means that the system is able to transfer more power efficiently. Thus, the higher coupling factor, the better pad design. Generally speaking, the coupling factor can be increased by extending the size and weight of materials used in the power pad. However, this design will result in an expensive and impractical IPT system, which is not acceptable, due to the limitations in EV's manufacturing related to space, weight and cost. Thus, the design optimization of the power pad exhibits a tradeoff between coupling performance and cost. Therefore, in this work, both the coupling coefficient and system's cost are considered as objectives in the optimization problem. These objectives are stated as functions of the geometry parameters given in Figure 9.1.

\subsubsection{Optimization Objectives}

In this study, the main concern is to investigate the effect of the separations among the adjacent turns $\left(P_{1}\right.$ and $\left.P_{2}\right)$ on the system performance, and to find the best combination of these pitches and the dimensions of ferrite sheet [width $\left(W_{f}\right)$ and thickness $\left(T_{f}\right)$ ] in the 
DD pad structure. This combination must achieve the highest coupling performance and the least cost. In this case, the power pad size and number of turns are kept fixed, with the values given in Table 9.1.

\subsubsection{Coupling Performance with Misalignment}

One of the main objectives of the optimization problem is to maximize the mutual coupling between the system's sides. However, the coupling performance varies based on the alignment between the two sides. The coupling factor is maximum, when the two sides are perfectly aligned, and decreases with misalignment. The good pad design must be able to achieve the best coupling, during both the perfect alignment and misalignments. Thus, in this work, the average coupling factor $\left(k_{\text {ave }}\right)$ is considered inside the cost function. This factor $k_{\text {ave }}$ represents the average coupling factor of four different positions for the coupler, which cover the different possible misalignments in the system, as shown in Figure 9.2. The first position [Figure 9.2(a)] denotes the perfect aligned situation. The second and third positions [Figure 9.2(b) and (c)] represent the vertical and horizontal misalignment, respectively. The fourth position investigates the rotational misalignment case [see Figure 9.2(d)]. For each combination of the optimization variables $\left(P_{1}, P_{2}, W_{f}\right.$ and $\left.T_{f}\right)$, the coupling factor is evaluated at the four positions, using FEA. By knowing the individual factors, the

average value is estimated, using Equation (9-4), and considered as an optimization objective. The misalignment parameters, in Figure $9.2(g, v, h$ and $\theta)$, are set to match with the maximum acceptable misalignments defined in the J2954A standard [149].

$$
k_{\text {ave }}=\left(k_{a}+k_{h}+k_{v}+k_{r}\right) / 4
$$


where, $k_{a}, k_{h}, k_{v}$ and $k_{r}$ represnt the coupling factor, during the perfect alignmnet, horizontal misalignment, vertical misalignment and rotational misalignment, respectively.

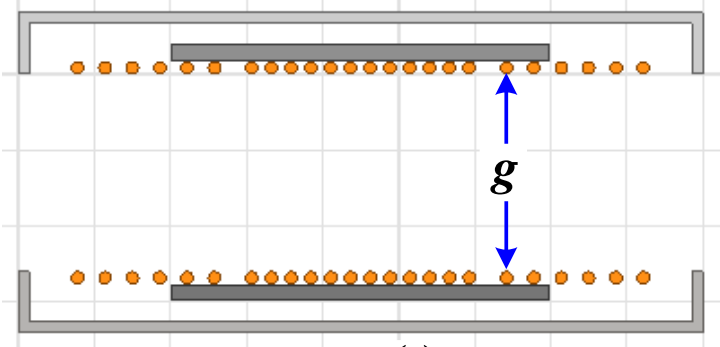

(a)

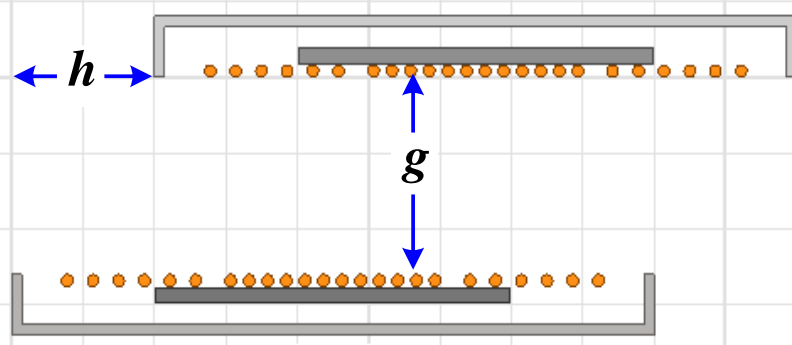

(c)

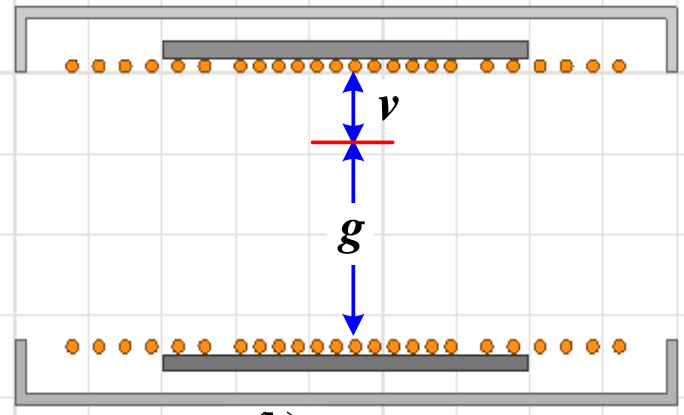

(b)

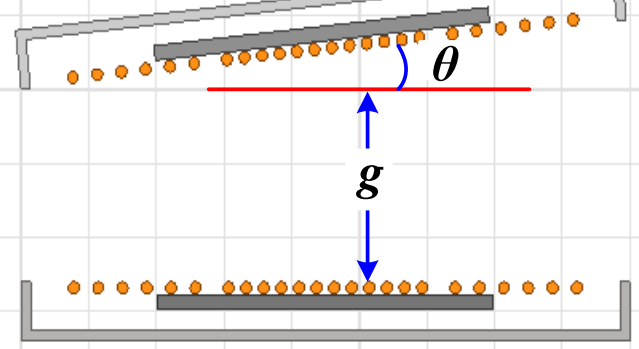

(d)

Figure 9.2. Different positions of misalignments. (a) Aligned. (b) Vertical misalignment. (c) Horizontal misalignment. (d) Rotational misalignment.

\subsubsection{Material Cost of the DD Power Pad}

The total material cost of the wireless coupler is represented by the cost of three main parts: copper windings $\left(C_{c}\right)$, ferrite material $\left(C_{f}\right)$ and aluminum shield $\left(C_{s}\right)$. The estimation of each of these costs in terms of the pad's dimensions is described in Equation (9-5).

$$
\begin{aligned}
& C_{f}=L_{f} \times W_{f} \times T_{f} \times c_{f} \\
& C_{s}=L_{s} \times W_{s} \times c_{s h} \\
& C_{c}=4\left[N\left(L_{c} \times W_{c}\right)-\left(P_{1}+3 P_{2}\right) \sum_{m=1}^{N} m\right] \times c_{c}
\end{aligned}
$$


where, $N$ is the number of turns per coil; $W_{f}, L_{f}$ and $T_{f}$ are the dimensions of the ferrite bars; $W_{s}$ and $L_{s}$ are the dimensions of the aluminum shield; $W_{c}$ and $L_{c}$ are the dimensions of the copper coil; and $c_{f}, c_{s h}$ and $c_{c}$ are the cost coefficients of ferrite sheet, aluminum shield and copper coil, respectively.

The dimensions of the pad's components are indicated in Figure 9.1 and 9.3. Although only the width dimensions are included in the 2D FEM, the entire components' dimensions are required for the cost calculation. The width information is presented in Table 9.1, and the length data are configured as ratios of them, according to the J2954A standard. The length of each component is set to $76 \%$ of its width (i.e. $L_{f}=0.76 W_{f}, L_{s}=0.76$ $W_{s}$ and $\left.L_{c}=0.76 \times 2 W_{c}\right)$.

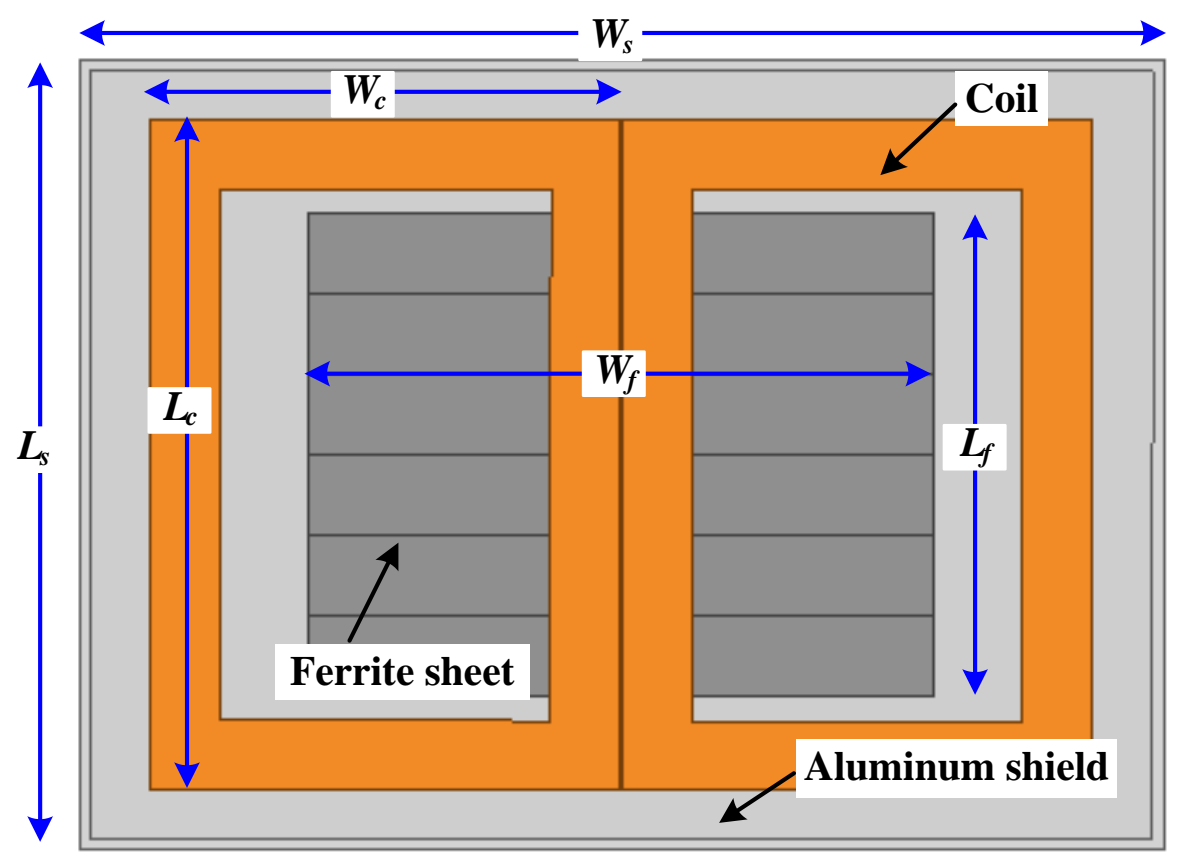

Figure 9.3. Top view of DD power pad with components' dimensions. 
For each combination of the optimization variables, the three types of costs are evaluated and added together to find the total cost of the power pad, as given in Equation (9-6). For the identical pads' case, the total material cost of the wireless coupler, which represents one of the optimization objectives, is double each pad's cost.

$$
C_{t}=C_{f}+C_{s h}+C_{c}
$$

\subsubsection{Multi-objective Cost Function and Constraints}

The basic objectives of the optimization algorithm are to minimize the coupler's material cost and maximize the magnetic coupling. The multi-objective optimization function $(M O F)$ is stated mathematically in Equation (9-7). For simplicity, the weighted sum method is used to evaluate the two objectives, in which two different weights ( $w_{l}$ and $\left.w_{2}\right)$ are assigned to the two objectives.

$$
\operatorname{Min} \operatorname{MOF}\left(P_{1}, P_{2}, W_{f}, T_{f}\right)=w_{1} C_{t}+w_{2}\left(1-k_{\text {ave }}\right)
$$

The optimization constraints are defined by the limits of each optimization variable, as given in Equation (9-8). These limits are decided according to the physical structure limitations and the designer experience.

$$
\text { Constraints }=\left\{\begin{array}{l}
P_{1_{\text {min }}} \leq P_{1} \leq P_{1_{\max }} \\
P_{2_{\text {min }}} \leq P_{2} \leq P_{2_{\max }} \\
W_{f_{\text {min }}} \leq W_{f} \leq W_{f_{\max }} \\
T_{f_{\text {min }}} \leq T_{f} \leq T_{f_{\text {max }}}
\end{array}\right.
$$

\subsection{Solving of the Optimization Problem}

After formulating the multiobjective optimization problem, including defining the 
optimization variables, cost function and constraints, the next step is to solve this problem, using one of the search algorithms. In this work, a modified version of the Tabu search algorithm is considered, and linked with the developed 2D FEM, to find the optimum design parameters. The details of the proposed search algorithm and the coupling with FEA are presented in this section.

\subsubsection{The Proposed Improved Tabu Search (ITS) Algorithm}

An improved version of the Tabu search (ITS) algorithm is utilized to solve the proposed optimization problem. The proposed algorithm is mainly based on the universal Tabu search (UTS) approach for global optimization, with continuous variables in electromagnetics [192]. There are two searching phases in the UTS: diversification and intensification. The task of the diversification phase is to search the objective space widely, while the function of the intensification phase is to locate the global optimal solution precisely. In the ITS algorithm, the sampling points are generated with the Latin Hypercube Sampling (LHS) method [193], which provides more uniform sampling in the objective space than the random sampling used in the UTS technique. The advantages of the

proposed ITS method are: 1) the ability to jump out of the local optimal solutions, 2) high convergence speed, and 3) simplicity of implementation and realization. The procedure of the proposed ITS algorithm execution is described with the flowchart shown Figure 9.4, and explained as follows:

Step 1: The algorithm generates the initial values of its parameters and defines the range of the variables. It also states the iteration number; the maximum iteration number; the iteration number for diversification and intensification, respectively. 


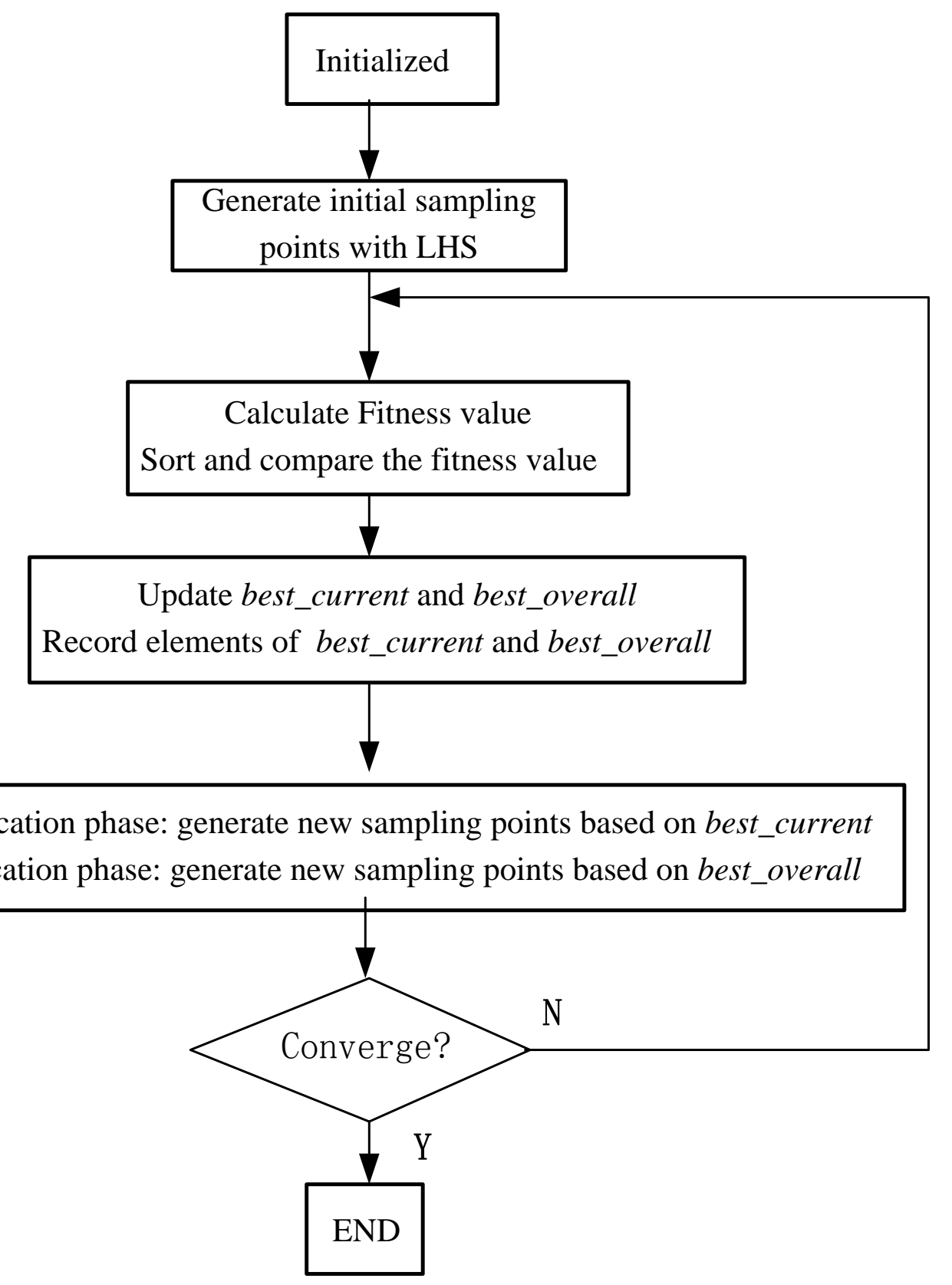

Figure 9.4. Flowchart of the proposed ITS algorithm.

Step 2: It generates initial sampling points with LHS.

Step 3: It calculates the fitness value, using FEA, sorts and compares the fitness value to 
get the best_current and best_overall. The best_current is the best objective function value in the current iteration, and the best_overall is the best solution up till now.

Step 4: It generates new sampling points based on the rules in different phases.

Step 5: It checks the iteration number, if it meets the maximum iteration number, then it goes to step 6; otherwise it goes to step 3 .

Step 6: Stop and end the algorithm.

\subsubsection{The Link between the ITS algorithm and FEA}

The proposed ITS optimization algorithm is implemented in MATLAB script, and coupled with the 2D-FEM of the inductive coupler in Maxwell environment. This link is achieved using Visual Basic commands, as in Figure 9.5. The ITS algorithm generates the populations, which contain information about the optimization variables $\left(P_{1}, P_{2}, W_{f}\right.$ and $\left.T_{f}\right)$. These populations are passed to Maxwell environment, which contains four different projects, I-IV. These projects represent the four different misalignment positions, shown in Figure 9.2. The algorithm opens project I, and modifies its parameters based on the current population. Then, it solves the project and estimates the coupling factor $\left(k_{a}\right)$. This factor is saved in a text file, then the project is closed. The same steps are repeated for the

other three projects, to evaluate and save $k_{v}, k_{h}$ and $k_{r}$. The coupling factors are passed to the MATLAB code, which evaluates the average coupling, total cost and the current $M O F$. This value of $M O F$ is passed to the ITS algorithm, to sort and compare the fitness values to get the best_current and best_overall. These steps are repeated for each population until the optimum solution is obtained, or the maximum number of iterations is reached. 


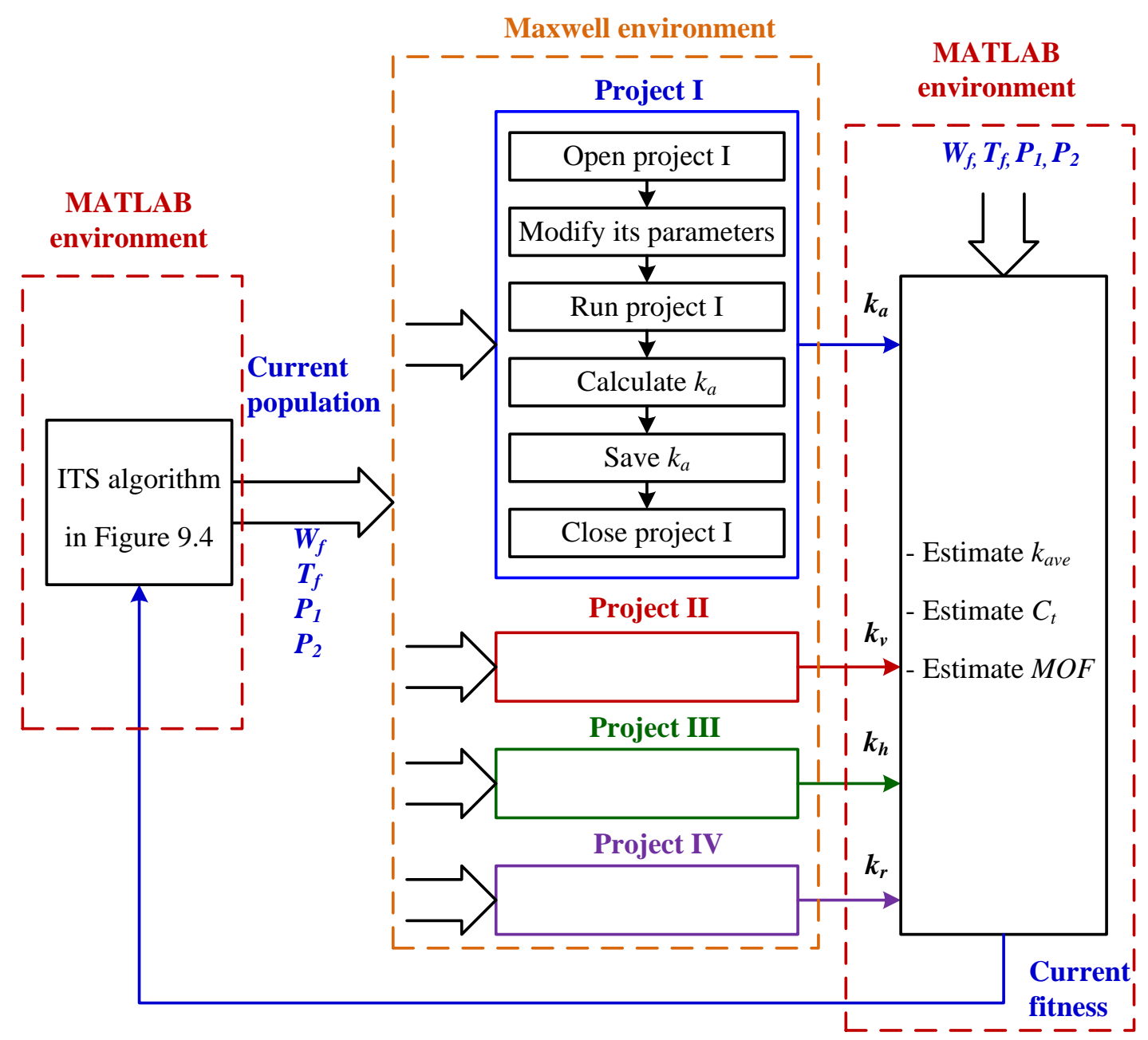

Figure 9.5. Block diagram for the link between ITS algorithm in MATLAB and FEA in Maxwell.

\subsection{Results and Discussion}

The proposed off-line multi-objective design optimization was achieved for an IPT system with two identical DD power pads. The settings and results of the optimization process are discussed in this section. Also, the coupling performance and cost of the wireless coupler, considering the optimization results, are investigated. 


\subsubsection{Optimization Results}

The proposed ITS algorithm is implemented in MATLAB environment and linked with the 2D-FEM of the inductive coupler, as discussed in section 9.4. The different misalignment parameters $(g, v, h$ and $\theta$ ), shown in Figure 9.2, are chosen to represent the maximum acceptable misalignments in the J2954A standard for the Z2 class, as indicated in Table 9.2. The Z2 class covers an air-gap range between the two sides of the system of 95-165 $\mathrm{mm}$. Thus, the avaerage of this range is considered as the normal vertical air-gap $(g=130 \mathrm{~mm})$. Also, the table shows the cost coefficients that are utilized for material cost calcualtion. These values represent the current cost in the market.

Table 9.2. The Misalignment Parameters and the Cost Coefficients during the Optimization.

\begin{tabular}{llll}
\hline \hline Parameter & Value & Parameter & Value \\
\hline$G$ & $130 \mathrm{~mm}$ & $c_{f}$ & $0.18 \$ / \mathrm{cm}^{3}$ \\
$H$ & $100 \mathrm{~mm}$ & $c_{c}$ & $2.04 \$ / \mathrm{m}$ \\
$V$ & $35 \mathrm{~mm}$ & $c_{s h}$ & $333.68 \$ / \mathrm{m}^{2}$ \\
$\Theta$ & $6^{0}$ & ------- & ----- \\
\hline \hline
\end{tabular}

The boundaries of the optimization variables (constraints) are indicated in Table 9.3. These limits are chosen, according to the physical limitations of the DD structure, and the designer experience. The lower limits of the pitches $\left(P_{1 \min }\right.$ and $\left.P_{2 \min }\right)$ are set to zero, to include the case, in which the windings are perfectly attached together without separations. 
The upper limits are chosen to be twice the conductor diameter $\left(P_{1 \max }=P_{2 \max }=2 D_{c}\right)$, in order to avoid the overlap between the coil-sides for the same coil. The boundaries of the width of ferrite bars are decided to cover the whole range of the power pad width $\left(2 W_{c}\right)$. Thus, $W_{\text {fmin }}<<W_{c}$ and $W_{\text {fmax }}>>2 W_{c}$, as given in Table 9.3. Finally, the limits of the ferrite bars' thickness ( $T_{f \min }$ and $\left.T_{f m a x}\right)$ are configured by trial and error.

Table 9.3. Boundaries of the Optimization Variables.

\begin{tabular}{llll}
\hline \hline Parameter & Value & Parameter & Value \\
\hline$P_{\text {Imin }}$ & $0 \mathrm{~mm}$ & $W_{\text {fmin }}$ & $100 \mathrm{~mm}$ \\
$P_{1 \max }$ & $11.2 \mathrm{~mm}$ & $W_{\text {fmax }}$ & $450 \mathrm{~mm}$ \\
$P_{2 \min }$ & $0 \mathrm{~mm}$ & $T_{f \min }$ & $3 \mathrm{~mm}$ \\
$P_{2 \max }$ & $11.2 \mathrm{~mm}$ & $T_{f \max }$ & $20 \mathrm{~mm}$ \\
\hline \hline
\end{tabular}

The setting of the ITS algorithm, during the optimization process, are indicated in Table 9.4. These parameters are chosen based on expeience, and trial and error. The optimization process is repeated three times to confirm the optimum solution. The progression of the multi-objective optimization algorithm is indicated in Figure 9.6. As can be noticed, the algorithm contains two phases: phase I (diversification) and phase II (intensification). In phase I, the best fitness decreases significantly, due to the wide search feature in this stage. In phase II, the algorithm searches the global optimum accurately, thus the reduction in the best fitness is very small. The final optimum design variables are presented in Table 9.5. 
Table 9.4. Settings of the ITS Optimization Algorithm's Parameters.

\begin{tabular}{ll}
\hline \hline Parameter & Value \\
\hline Number of iteration in phase I & 200 \\
Number of iteration in phase II & 100 \\
Number of populations in phase I & 8 \\
Number of populations in phase II & 5 \\
Number of initial populations & 20 \\
Number of runs & 3 \\
\hline \hline
\end{tabular}

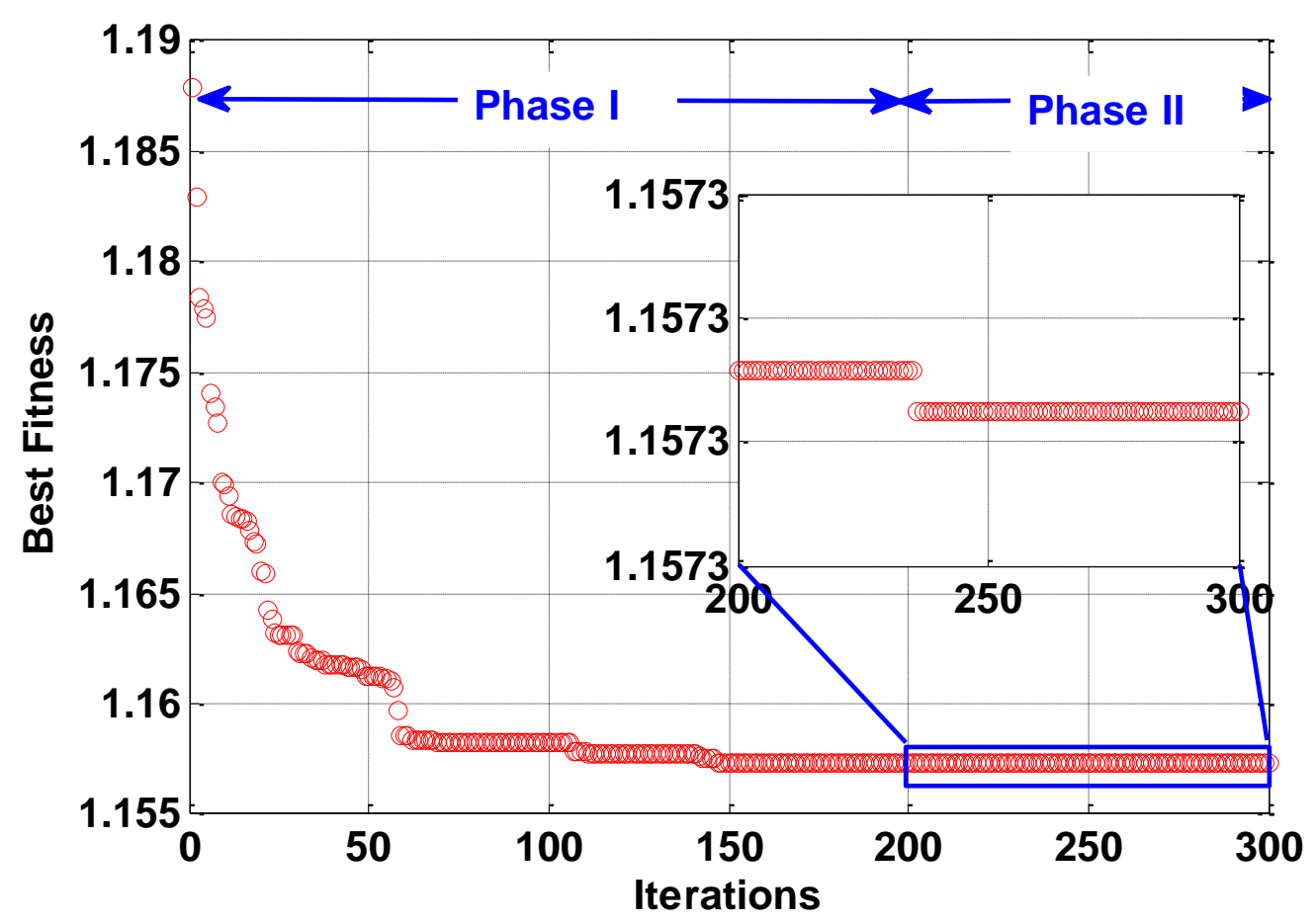

Figure 9.6. Progression of ITS optimization algorithm. 
Table 9.5. The Optimum Design Parameters.

\begin{tabular}{llll}
\hline \hline Parameter & Value & Parameter & Value \\
\hline$P_{\text {lopt }}$ & $8.09574409917262 \mathrm{~mm}$ & $W_{f o p t}$ & $248.292970599325 \mathrm{~mm}$ \\
$P_{\text {opt }}$ & $4.06555885383175 \mathrm{~mm}$ & $T_{f o p t}$ & $5.00004272074758 \mathrm{~mm}$ \\
\hline \hline
\end{tabular}

\subsubsection{Assessment of the IPT System's Performance}

The optimum values of the separations among the turns are introduced to the FEM to evaluate the system's performance. The optimum system performance is compared with three other scenarios that exist in the literature. The power pad structure for the four different scenarios is shown in Figure 9.7. In the Optimum scenario [Figure 9.7(a)], the variables' values in Table 9.5 are considered. In the Minimum scenario [Figure 9.7(b)], the separations were set to zero, which represent the case in which the turns are attached together without separations, as in [188]. For the Maximum one [Figure 9.7(c)], the pitches were set to the maximum limits $\left(P_{l}=P_{2}=11.2 \mathrm{~mm}\right)$, which was considered in [194]. The Different scenario [Figure 9.7(d)] was proposed in [176], in which the separation in the middle sides was set to maximum $\left(P_{l}=11.2 \mathrm{~mm}\right)$, while that for the outer sides was set to zero $\left(P_{2}=0 \mathrm{~mm}\right)$.

Quasi-Static magnetic analysis is performed in Maxwell for each scenario, and the magnetic flux distribution for all the cases are indicated in Figure 9.8. The figure shows that the Optimum scenario provides higher magnetic flux link between the two pads than the Maximum and Different scenarios. Also, the magnetic flux is directed perfectly between the two pads, with minimum leakage, compared with the other cases. 


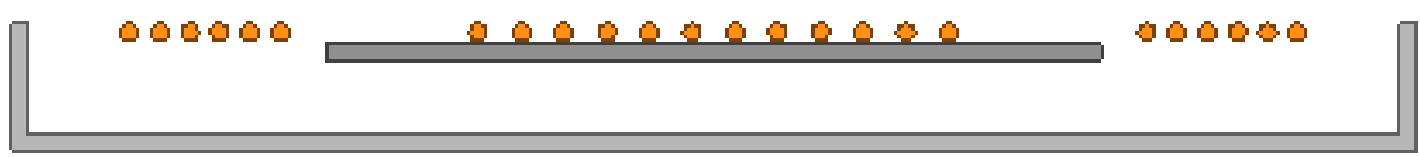

(a)

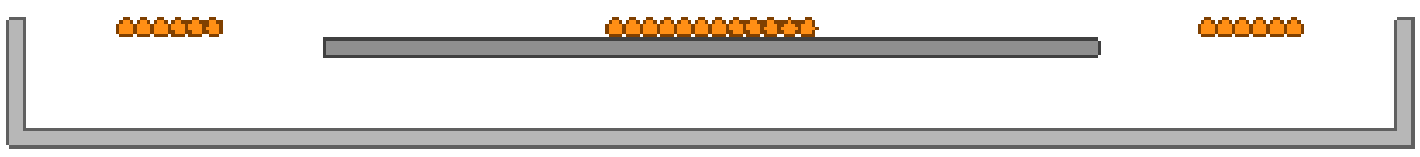

(b)

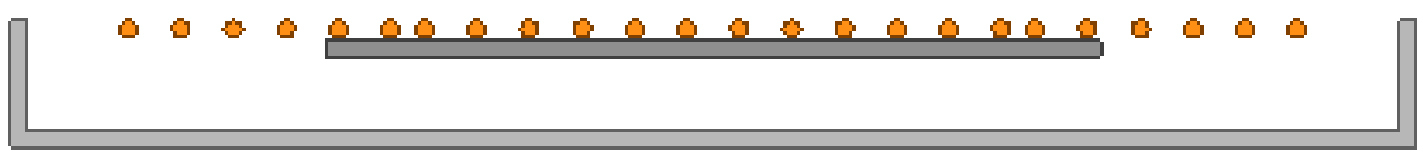

(c)

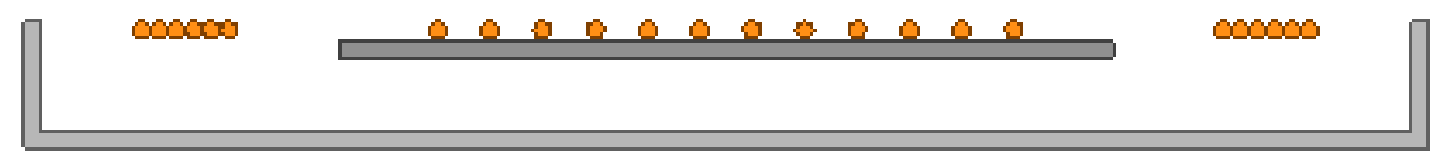

(d)

Figure 9.7. Four different design scenarios. (a) Optimum $\left(P_{1}=P_{1 o p t}, P_{2}=P_{2 o p t}\right)$. (b) Minimum $\left(P_{1}=P_{2}=0\right)$. (c) Maximum $\left(P_{1}=P_{2}=11.2 \mathrm{~mm}\right)$. (d) Different $\left(P_{1}=11.2 \mathrm{~mm}, P_{2}=0\right)$.

In addition, in the Optimum case, the flux density in the magnetic ferrite material is better distributed, with less saturation effect, than the Minimum case. The magnetic flux in the Minimum and Different scenarios shows overlap with the aluminum shield. This overlap badly affects the coupling performance, and increases the sensitivity of the magnetic parameters to the variation of the air-gap and misalignments in the system. On the other hand, no overlap can be noticed in the Optimum case. 


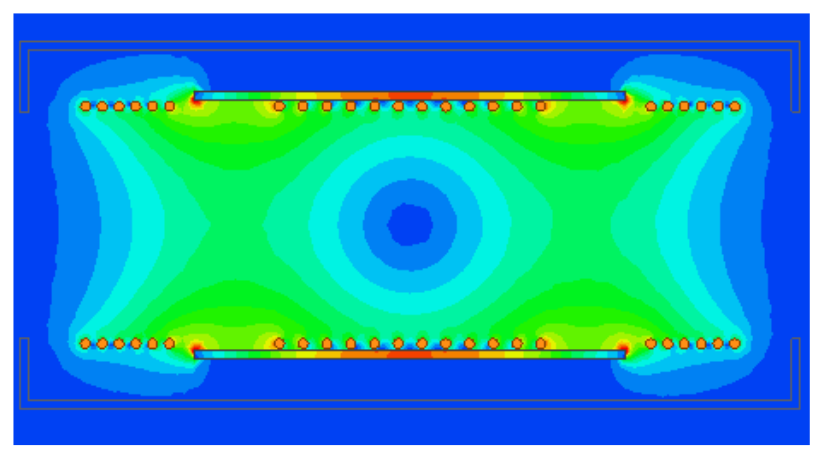

(a)

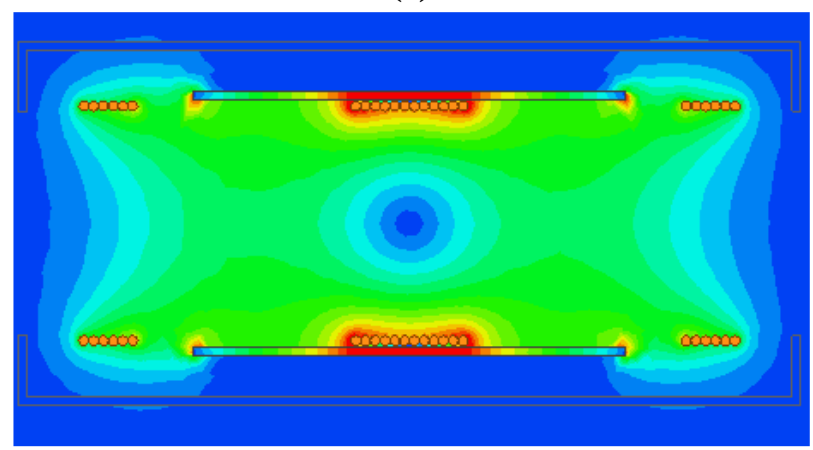

(b)

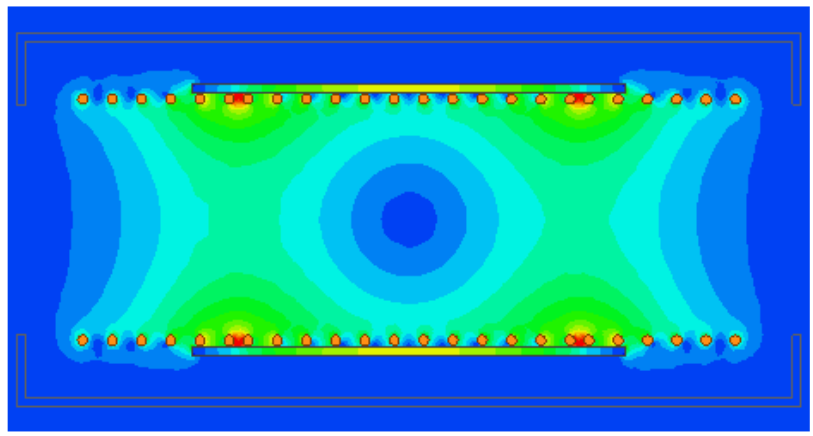

(c)

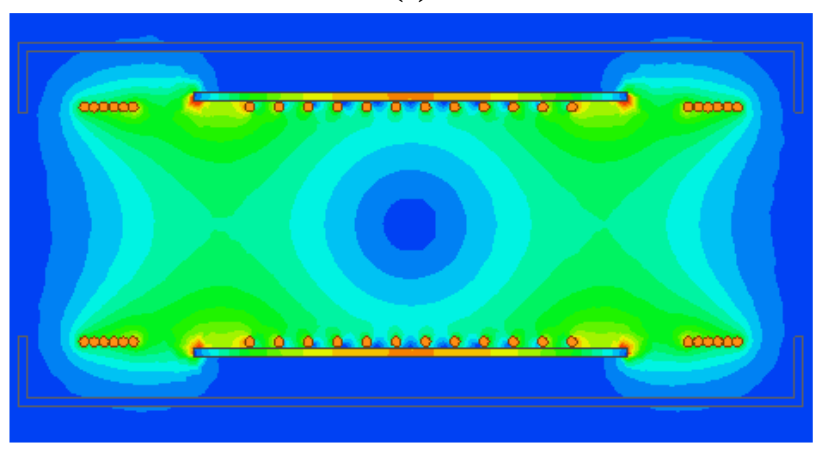

(d)

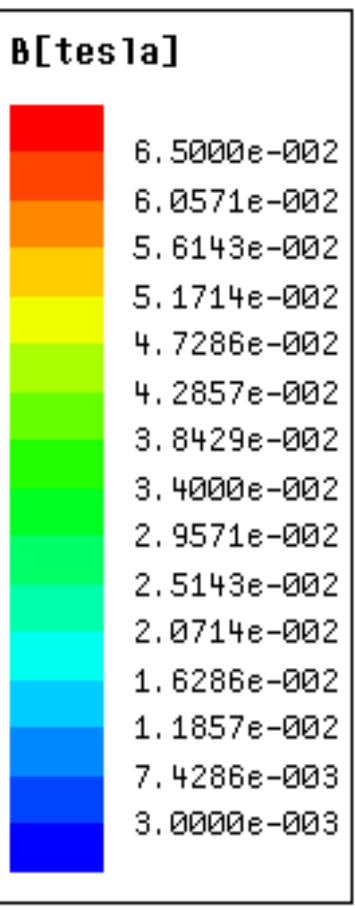

\section{H[A_per_m]}

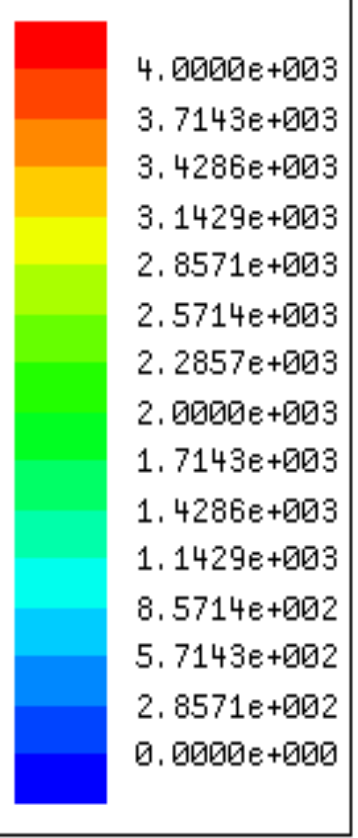

4. $0000 e+003$

3. $7143 e+003$

. $4286 e+003$

3. $1429 e+003$

2. $8571 e+003$

$+003$

2. 000ดe+口0

1. $7143 e+003$

(2)

$1429 e+003$

8. $5714 e+002$

5. $7143 e+002$

2. $8571 e+002$

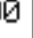


For better performance and design evaluation, the magnetic coupling factor and the coupler's cost are numerically estimated, and compared for all scenarios. The coupling factor under different vertical air-gaps $(g)$ is studied in Figure 9.9. Also, the cost of two identical power pads in all scenarios is evaluated, and compared in Table 9.6.

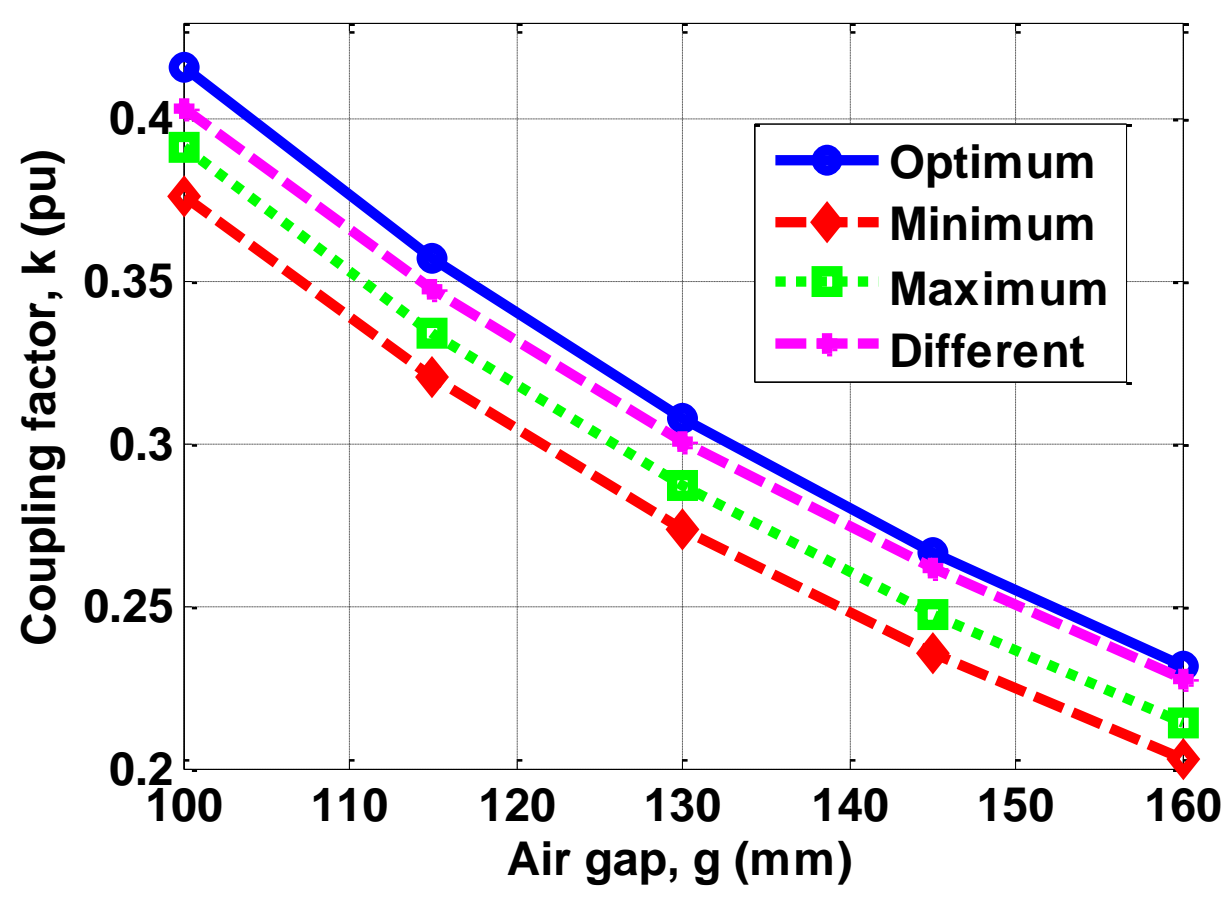

Figure 9.9. Coupling factor vs. air gap length with perfect alignment $(h=\theta=0)$.

As can be noticed, the attached turns with zero separations show the worst coupling performance, and the highest cost. Applying a fixed maximum separation among the turns provides the least cost, and better coupling than the Minimum scenario. Considering different pitches in the system (Optimum and Different) shows the best coupling performance, with moderate cost. The Optimum design provides a higher coupling factor 
and less cost than the Different one. The difference in the coupling factor between the Optimum and Different scenarios is large for small air-gap, and diminishes with increasing the air-gap. This nonlinear behavior is due to the effect of the aluminum shield with the variation of the air-gap. This impact is significant in the Different scenario [Figure 9.8(d)], and almost disappears in the Optimum case [Figure 9.8(a)].

Table 9.6. Cost of the Wireless Coupler (Two Pads) in all Scenarios.

\begin{tabular}{llll}
\hline Scenario & Cost (\$) & Scenario & Cost (\$) \\
\hline Optimum & 384 & Maximum & 375.6 \\
Minimum & 391 & Different & 387.1 \\
\hline
\end{tabular}

Finally, the coupling factor under different horizontal and rotational misalignments is investigated in Figure 9.10 and Figure 9.11, respectively. Similar behavior to that in Figure 9.9 is noticed for all scenarios. For large horizontal misalignment $(h>75 \mathrm{~mm})$, the Different and Optimum scenarios show very similar coupling performance, due to the wide spread of the middle turns, which provides long flux pipe. The Different case shows slight improvement in the coupling factor, higher than that from the Optimum one, at very large $h(>100 \mathrm{~mm})$. However, at this level of misalignment, which exceeds the maximum acceptable limits, it will be impractical and inefficient to operate the system. In addition, still the Optimum one provides less cost than the Different case. 


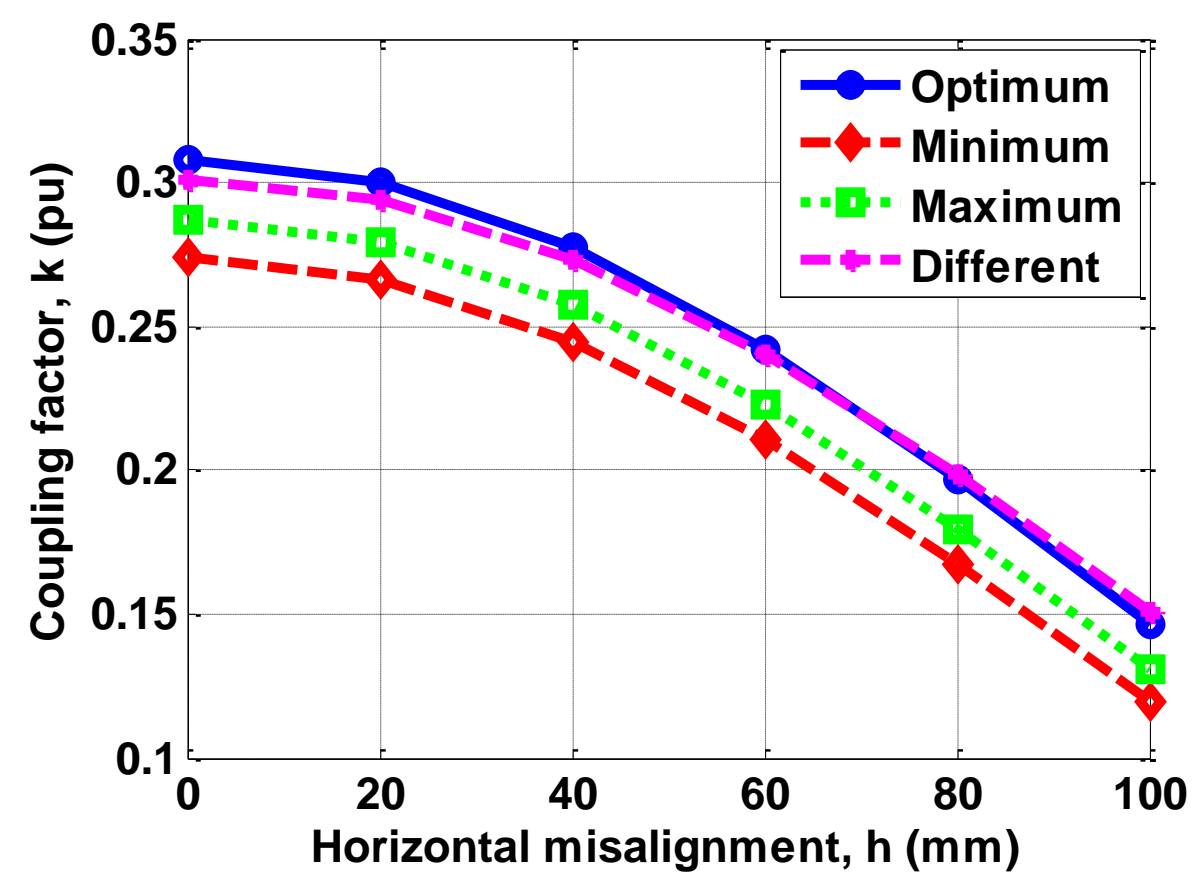

Figure 9.10. Coupling factor vs. horizontal misalignment with $g=130 \mathrm{~mm}$ and $\theta=0$.

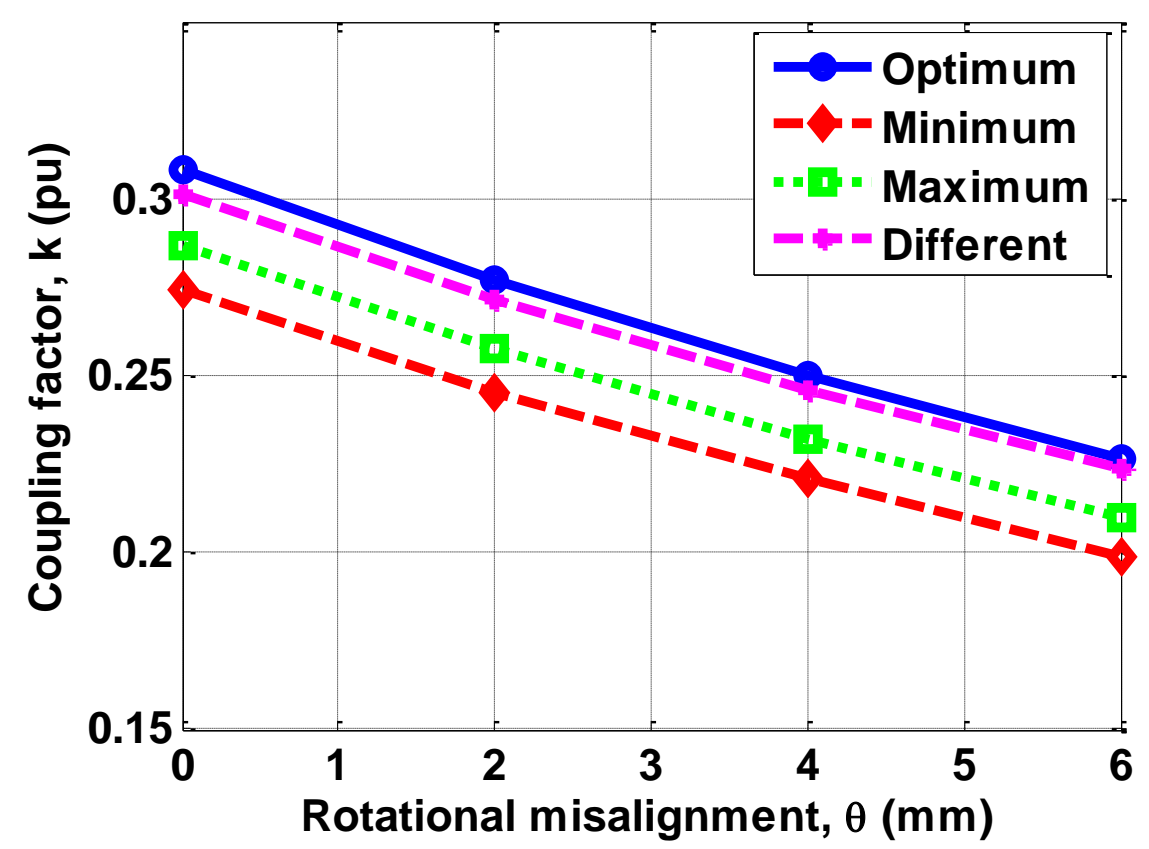

Figure 9.11. Coupling factor vs. rotational misalignment with $g=130 \mathrm{~mm}$ and $h=0$. 


\subsection{Conclusion}

This chapter presents a fast and an efficient multi-objective optimization technique, for the magnetic design of the power pads in IPT systems, for EV applications. The algorithm is based on an improved Tabu search technique, with the link of a 2D static finiteelement analysis. The study investigates the effect of the separations among the turns, and the ferrite martial dimensions on the IPT system's performance and cost. The results show that using different separations for the middle and the outer coils' sides leads to better coupling performance and less cost. Increasing the separation on the middle sides to be larger than that on the outer sides provides better performance. Minimizing the separation on the outer sides does not provide the best coupling, as was claimed in the literature. The outcomes of the proposed design optimization provides the best coupling performance under different misalignments in the system, and shows moderate material cost. 


\section{Chapter 10 Autonomous Two-Layer Predictive Active Power-Flow Control for BIWPTS}

\subsection{Introduction}

Accomplishing effective $\mathrm{G} 2 \mathrm{~V}$ and $\mathrm{G} 2 \mathrm{~V}$ benefits, through wireless power interface requires accurate and quick control reaction. Power-flow controllers are fundamental in the BIWPTSs to manage the magnitude and direction of the power-flow between the EV and power grid. Thus, this chapter proposes a new approach of active power-flow control for a LCL BIWPTS in EVs' ancillary services. The controller allows an EV to autonomously charge and discharge its battery wirelessly, during long term parking (stationary) and/or the transient stops (quasi-dynamic). According to the literature, power-flow controllers can be grouped in terms of the location (primary, secondary or dual) [88], the HF resonant inverter topology [195], and the modulation technique, such as pulse-width [161], [162], pulse-phase [163], [164], pulse-frequency modulation [163], [165], [166] and a mix between them [163]. Diverse sorts of control hypothesis, such as classical PI [165], [166], proportional-integral-derivative (PID) [196], and FLC [162] have been used to drive the above modulation variables. Classic PI and PID controllers encounter large settling time, overshoots, and oscillations, and may not resist the uncertainties and disturbances. Fuzzy controllers are more robust, however they show high computational processing, noisy outcome and complexity in the design [132]. In addition, the design of these controllers should be versatile with variation of the system's parameters, which depend on the relative position of the power pads. Model-based predictive active power-flow (PAPF) controllers 
have all the earmarks of being promising in these applications, in terms of simplicity and accuracy.

This chapter presents a new two-layer PAPF controller for LCL BIWPTS in EVs' applications. The proposed controller has the ability to manage the bidirectional powerflow between the EV and surrounding infrastructures, such as power grid, home microgrid, building micro-grid, road or another vehicle. The main contributions in the proposed controller are:

- It consists of two levels of control:

- The first is responsible for communicating with the surrounding infrastructures and gathering information from the driver, charging station, power grid and battery management system (BMS). Then, based on these information, it takes a decision to charge, discharge or abstain, and estimates the rate of charge or discharge.

- The second control layer receives the reference signal from the first one, and predicts the control parameters for the two HF resonant converters (in the primary and secondary sides) to achieve the desired power-flow.

- It offers intelligent autonomous performance that satisfies both the EV's owner and power grid operator.

- It guarantees the best driving performance with a minimum energy consumption cost and minimum side effects on the power grid.

- It estimates EV's psychological price, as a function of its battery state of charge (SOC), and compares it with the energy price to decide whether to charge, discharge or abstain, and how much the charging or discharging rate. 
- It exists on the vehicle side, to allow the autonomous operation, so the EV can be charged and discharged, independently, without the need of a centralized controller on houses, parking garage, public parking or traffic signal.

- The proposed controller is adaptively estimating the system's parameters to consider the misalignment and the environmental conditions' impacts on the system's performance. The parameter estimation is achieved using only one voltage sensor.

- The second layer control is designed based on an accurate analytical modeling for the LCL BIWPTS. It provides very fast and stable response, during both the transient and steady-state operation, in stationary and quasi-dynamic wireless power transfer operation.

\subsection{Detailed Power-Flow Modeling}

The proposed controller is mainly depends on the analytical modeling for the LCL BIWPTS, which provides rigorous prediction for the system power-flow. This precise prediction helps to achieve the proper control design. Thus, this section presents accurate mathematical representations for the active and reactive power-flow in the BIWPTS, during EVs' charging and discharging in V2G operation. Four different power models with different levels of accuracy are developed. The developed formulas consider the design and control parameters' effect on the BIWPTS's performance. Moreover, the impact of the losses and harmonics in the system, due to the HF resonant converters, on the power-flow performance is considered. In addition, practical limitations for the two-way power-flow are inspected, during different modes of operation. The conditions for achieving maximum 
active and reactive power, and unity and zero power factor operation are investigated and verified.

\subsubsection{Analytical Models of Active and Reactive Power-Flow}

The proposed power-flow models are mainly depend on the steady-state mathematical model, which is developed for LCL BIWPTS and presented in sections 4.3 and 4.4. Based on this model, four different active and reactive power-flow equations with different levels of precision are developed, analyzed and compared [197]. According to the assumption that most of the power-flow controllers exist on the vehicle side, all the powerflow analyses are achieved for the secondary side. However, the system is symmetrical and both sides have similar performance.

\subsubsection{Total Power-Flow Model $\left(P_{A P M}\right.$ and $\left.Q_{R P M}\right)$}

This model represents the fundamental and harmonics power components. It is developed using the exact $V_{s i}$ and $I_{s i}$ formulas in Equations (4-1) and (4-4), respectively, and substituting in Equation (10-1) for secondary power estimation.

$$
\begin{aligned}
& P_{A P M}=\sum_{n=1,3,5, \ldots} \operatorname{Real}\left(V_{s i \_n} I_{s i_{\_} n}{ }^{*}\right) \\
& Q_{R P M}=\sum_{n=1,3,5, \ldots} \operatorname{Imag}\left(V_{s i \_n} I_{s i \_n}{ }^{*}\right)
\end{aligned}
$$

For implementing this model, the power is estimated at each harmonic component and added together to represent the total power. This model provides accurate estimation for the system's power-flow, but it needs iterative solution, and it is not appropriate for a fast control design. Thus, simpler models are developed rely on some reasonable assumptions. 


\subsubsection{Fundamental Power-Flow Model $\left(P_{F A P M}\right.$ and $\left.Q_{F R P M}\right)$}

This model predicts the fundamental power component only. It is derived by neglecting the harmonic components of $I_{s i}$ and $V_{s i}$, to find a simple formula for the fundamental secondary inverter current $\left(I_{s i \_}\right)$, as in Equation (10-2).

$$
I_{s i_{-} 1}=\frac{j M \gamma}{\omega_{r} L_{p i} L_{s i}} V_{p i_{-} 1}+\frac{L_{p i} L_{s i} C_{s} R_{S i}+j \gamma \omega_{r} M^{2} R_{s i}{ }^{2} C_{p}{ }^{2}}{L_{p i} L_{s i}\left(L_{s c}+C_{s} R_{s i}{ }^{2}\right)} V_{s i_{-} 1}
$$

where, $\gamma$ is a real number, which is very close to unity, and is given as $\gamma=$ $\frac{L_{p i} L_{s i}}{\left(L_{p i}+C_{p} R_{p c} R_{p i}\right)\left(L_{s i}+C_{S} R_{s c} R_{s i}\right)+\omega_{r}^{2} M^{2} C_{p} C_{s} R_{p i} R_{s i}} ; V_{s i_{-} l}$ and $V_{p i_{-} l}$ are the fundamental components of $V_{s i}$ and $V_{p i}$, respectively.

Therefore, exact formulas for calculating the fundamental secondary powers are obtained, by substituting Equation (10-2) into Equation (10-1), as given in Equation (103). The active and reactive power are represented by two terms: the first term characterizes the bulk power transfer, and the second one refers to the power losses. The losses' term is effective for active power-flow, since it is proportional with $R_{s i}$, however, it is negligible for reactive power-flow, which depends on $R_{s i}{ }^{2}$, where $R_{s i}$ is typically small $(<0.5 \Omega)$.

$$
\begin{aligned}
& P_{F A P M}=\left(\frac{8}{\pi^{2}}\right)\left[\frac{M \gamma V_{d c} V_{b}}{\omega_{r} L_{p i} L_{s i}} \sin \left(\frac{\alpha}{2}\right) \sin \left(\frac{\beta}{2}\right) \sin (\delta)+\frac{C_{s} R_{s i} V_{b}{ }^{2}}{L_{s c}+C_{s} R_{s i}{ }^{2}} \sin ^{2}\left(\frac{\beta}{2}\right)\right] \\
& Q_{F R P M}=\left(\frac{-8}{\pi^{2}}\right)\left[\frac{M \gamma V_{d c} V_{b}}{\omega_{r} L_{p i} L_{s i}} \sin \left(\frac{\alpha}{2}\right) \sin \left(\frac{\beta}{2}\right) \cos (\delta)+\frac{\gamma \omega_{r} M^{2} R_{p i}{ }^{2} C_{p}{ }^{2} V_{b}{ }^{2}}{L_{p i} L_{s i}\left(L_{p c}+C_{p} R_{p i}{ }^{2}\right)} \sin ^{2}\left(\frac{\beta}{2}\right)\right]
\end{aligned}
$$

\subsubsection{Approximate Fundamental Power-Flow Model $\left(P_{A F A P M}\right.$ and $\left.Q_{A F R P M}\right)$}

It provides simple formulas for the fundamental power, by neglecting the losses terms in Equation (10-3). This will end up with the simple power-flow model in Equation (10-4), which matches with the presented one in Equation (4-17), for the primary power. 


$$
\begin{aligned}
P_{A F A P M} & =\left(\frac{8}{\pi^{2}}\right)\left[\frac{M \gamma}{\omega_{r} L_{p i} L_{s i}} V_{d c} V_{b} \sin \left(\frac{\alpha}{2}\right) \sin \left(\frac{\beta}{2}\right) \sin (\delta)\right] \\
Q_{A F R P M} & =\left(\frac{8}{\pi^{2}}\right)\left[\frac{-M \gamma}{\omega_{r} L_{p i} L_{s i}} V_{d c} V_{b} \sin \left(\frac{\alpha}{2}\right) \sin \left(\frac{\beta}{2}\right) \cos (\delta)\right]
\end{aligned}
$$

By analyzing the abovementioned three models, we could get accurate and fast prediction for the active power-flow, as is discussed in section 10.2.2, however, for reactive power prediction, better model needs to be developed, as is presented in the next section.

\subsubsection{Approximate Power-Flow Model $\left(Q_{A R P M}\right)$}

An accurate reactive power model can be developed by considering the effect of the third and the fifth harmonic components on the reactive power analysis. A simple equation for the reactive power-flow at each harmonic component is derived in Equation (10-5). This formula is evaluated for the third and fifth harmonics, and added to the approximate fundamental reactive power model in Equation (10-4). This results in an accurate and explicit model for the reactive power-flow, as given in Equation (10-6).

$$
\begin{gathered}
Q_{S_{-} h a r m}=\left(\frac{8}{\pi^{2}}\right) \frac{V_{b}^{2}}{\omega_{r} L_{s i}} \frac{1}{n^{3}} \sin ^{2}\left(\frac{n \beta}{2}\right) \\
Q_{A R P M}=\left(\frac{8}{\pi^{2}}\right)\left[\begin{array}{c}
\frac{-M \gamma V_{d c} V_{b}}{\omega_{r} L_{p i} L_{s i}} \sin \left(\frac{\alpha}{2}\right) \sin \left(\frac{\beta}{2}\right) \cos (\delta) \\
+\frac{V_{b}{ }^{2}}{\omega_{r} L_{s i}}\left(\frac{1}{3^{3}} \sin ^{2}\left(\frac{3 \beta}{2}\right)+\frac{1}{5^{3}} \sin ^{2}\left(\frac{5 \beta}{2}\right)\right)
\end{array}\right]
\end{gathered}
$$

\subsubsection{Models Verification and Comparison}

The performance of the above developed models is investigated and compared in this section. In this analysis, the active and reactive power are analyzed with respect to the 
variation of the control parameter $\delta$, which is responsible for managing the power-flow in the BIWPTS. The powers are estimated in per-unit, considering their peak values $\left(P_{s_{-} \max }\right.$ and $\left.Q_{s_{-} \max }\right)$ as bases.

Figure 10.1 shows the secondary active power using the first three models $\left(P_{A P M}\right.$, $P_{F A P M}$ and $\left.P_{A F A P M}\right)$. As can be noticed, the fundamental active power model $\left(P_{F A P M}\right)$ provides very accurate prediction with $0.2 \%$ normalized mean square error (NMSE), with respect to the total power model $\left(P_{A P M}\right)$. However, the approximate fundamental model $\left(P_{A F A P M}\right)$ shows $2.44 \%$ NMSE. Moreover, the fundamental model is simple and explicit, thus it does not require iterative solution. Thus, this model is considered for active powerflow control design analysis.

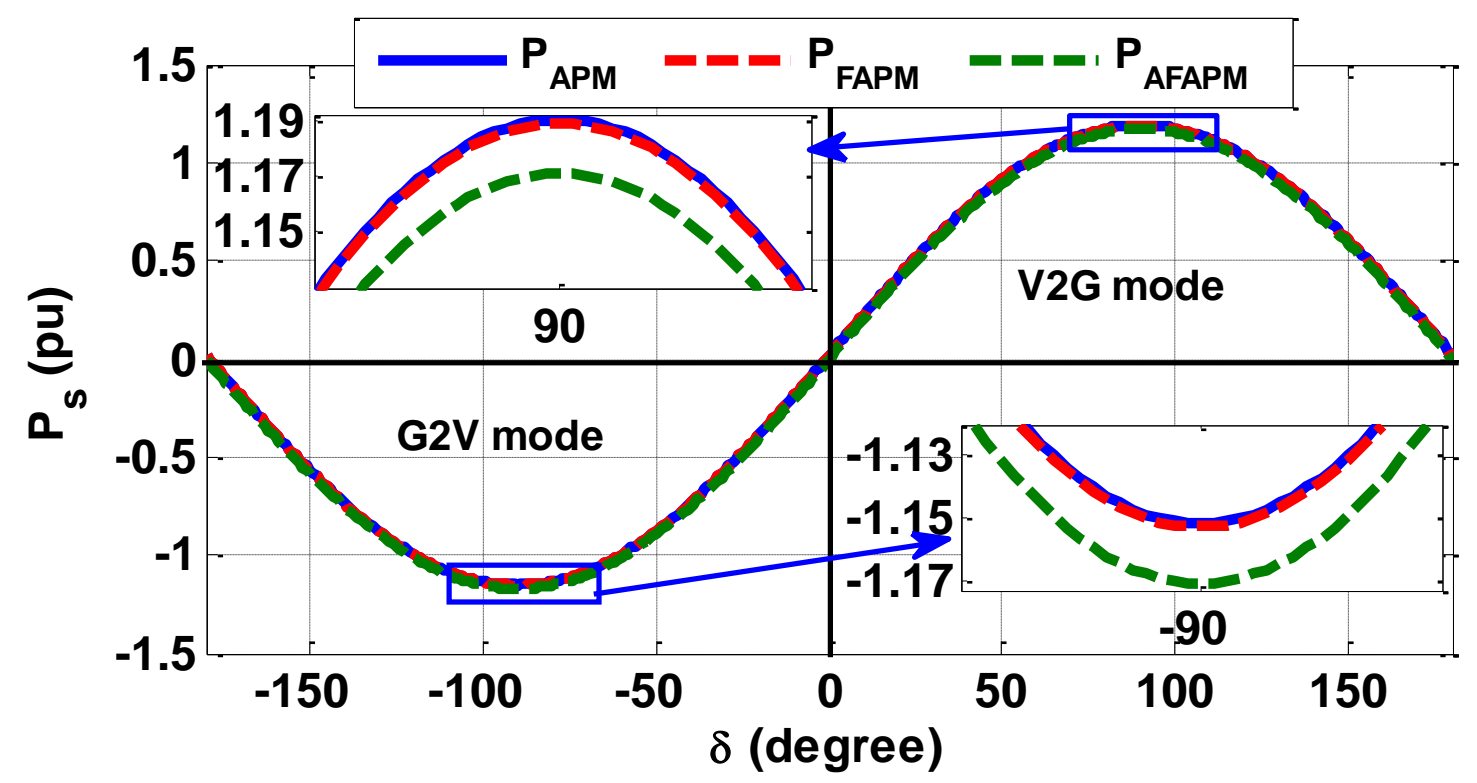

Figure 10.1. Comparison between the different active power models $\left(\alpha=\beta=180^{\circ}\right.$, and $P_{s \_ \text {max }}$ is the base). 
The performance of the reactive power models is described in Figure 10.2. The fundamental $\left(Q_{F R P M}\right)$ and approximate fundamental $\left(Q_{A F R P M}\right)$ models show very similar and ideal symmetrical operation, due to neglecting the harmonics effect. However, they provide poor reactive power prediction, with more than 30\% NMSE with respect to the total power model $\left(Q_{R P M}\right)$. This large mismatch makes them inappropriate for a precise reactive power-flow analysis. On the other hand, it can be observed that the approximate reactive power model $\left(Q_{A R P M}\right)$ is able to predict the system's reactive power accurately, with $6.22 \%$ NMSE. Thus, this model is more applicable for reactive power-flow analysis.

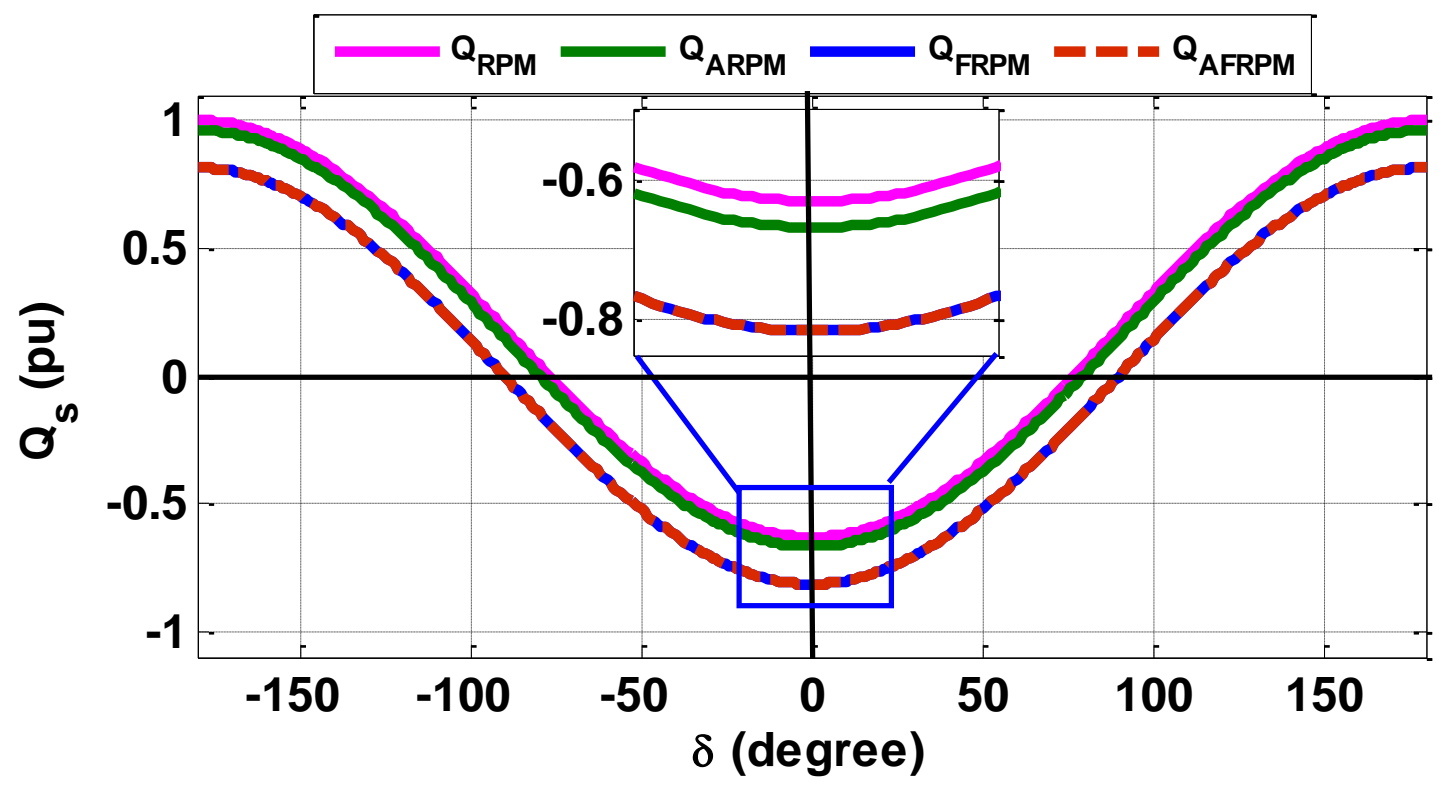

Figure 10.2. Comparison between the different reactive power models $\left(\alpha=\beta=180^{\circ}\right.$ and $Q_{s_{-} \max }$ is the base).

For better numerical evaluation, the powers are estimated by the four presented models, and compared with measured values in Table 10.1, for the LCL BIWPTS shown 
in Figure 5.3, with the parameters in Table 5.23 at lower air-gap. The power-flow analysis is achieved, during G2V and V2G operations with full and light-loading conditions. The measured power is extracted from the recorded voltage and current waveforms, using FFT to get the magnitude of each harmonic component. The results indicate that the models $P_{F A P M}$ and $Q_{A R P M}$ provide the most accurate prediction after the total models, with respect to the measured power. In addition, they are represented in simple and explicit formulas, which do not require iterative solution.

Table 10.1. Numerical Power-Flow Evaluation using the Different Models.

\begin{tabular}{c|cc|cc|cc|cc|cc}
\hline Mode & $P_{A P M}$ & $\boldsymbol{Q}_{R P M}$ & $P_{F A P M}$ & $\boldsymbol{Q}_{F R P M}$ & $P_{A F A P M}$ & $\boldsymbol{Q}_{A F R P M}$ & $P_{F A P M}$ & $\boldsymbol{Q}_{A R P M}$ & $P_{M E A S}$ & $\boldsymbol{Q}_{M E A S}$ \\
\hline Full G2V & -102.5 & 21.3 & -102.7 & 0 & -104.5 & 0 & -102.7 & $\mathbf{1 7}$ & -99.7 & 30.4 \\
Full V2G & 106.4 & 21.3 & 106.3 & 0 & 104.5 & 0 & $\mathbf{1 0 6 . 3}$ & $\mathbf{1 7}$ & 110.5 & 31.1 \\
Light G2V & -51.2 & 10.6 & -51.3 & 0 & -52.2 & 0 & $\mathbf{- 5 1 . 3}$ & $\mathbf{8 . 5}$ & -49.4 & 13.6 \\
Light V2G & 53.2 & 10.6 & 53.1 & 0 & 52.2 & 0 & $\mathbf{5 3 . 1}$ & $\mathbf{8 . 5}$ & 57.3 & 14.8 \\
\hline
\end{tabular}

Active powers are in Watt and reactive powers are in VAR. Full means $\alpha=\beta=180^{\circ}$ and light means $\alpha=\beta$ $=90^{\circ}$.

\subsection{Power-Flow Criteria}

In G2V and V2G concept the EV will not only inject active power to the grid, but also can provide reactive power support. The change among the different modes of operation (inject $P$, inject $Q$, absorb $P$, and absorb $Q$ ) can be achieved, by adjusting the control parameter $(\delta)$, as indicated in Figure 10.3. Considering the fundamental power only, 
the power-flow performance is symmetrical. However, due to the losses and the injected harmonics from the HF converters, the system shows asymmetrical operation, as indicated in Figures 10.1 and 10.2. The harmonic contents increase the absorbed reactive power from the grid, during G2V operation, and limit the ability of the EV to inject $Q$ to the grid, during V2G mode [197]. This impact can be noticed by observing the maximum values of $Q_{s}$ in Figure 10.3 ( 1 and 0.6947 pu at G2V and V2G operation, respectively).

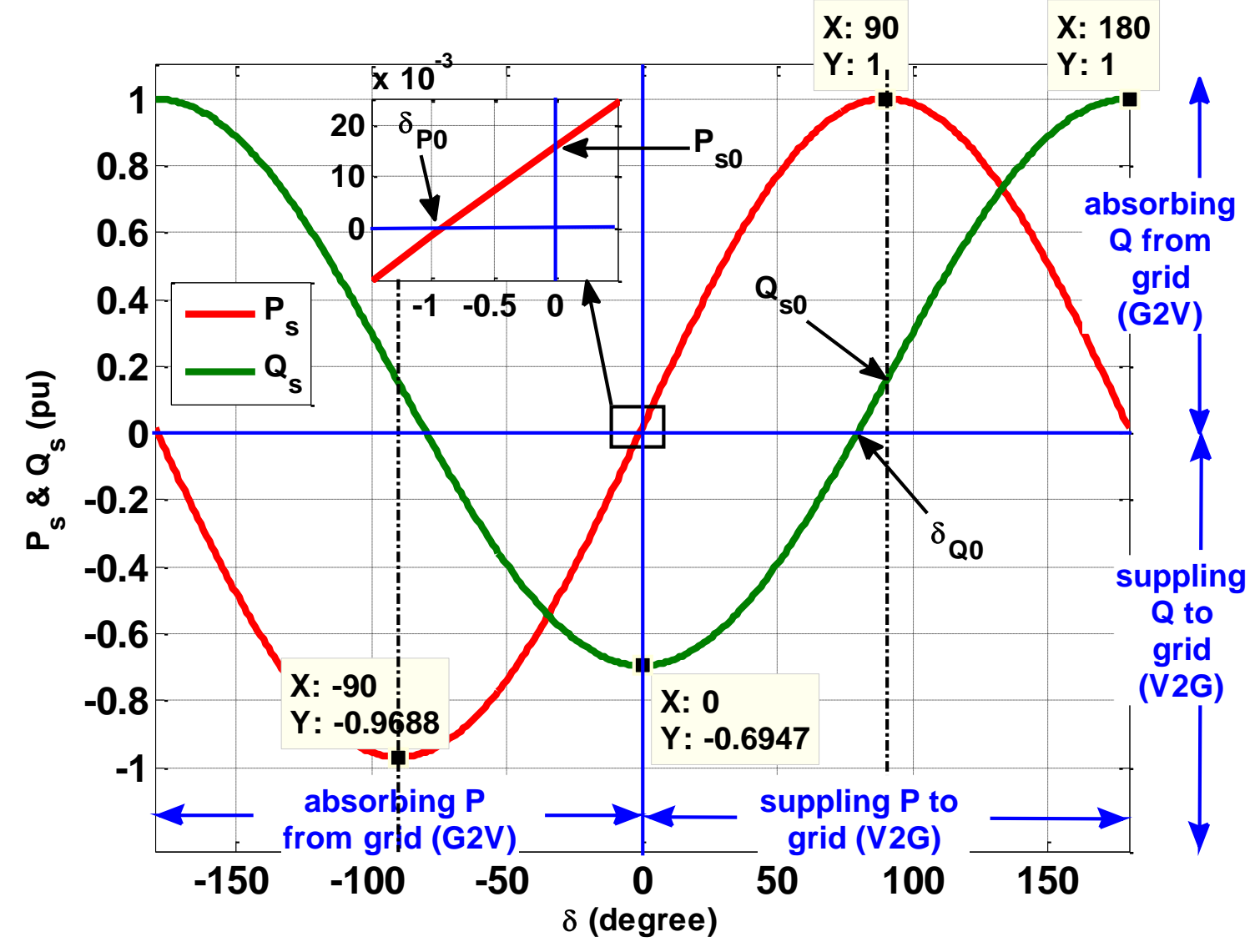

Figure 10.3. BIWPTS's active and reactive power-flow criteria. 
On the other hand, the system's losses introduce small asymmetric effect on the active power-flow ( 1 and 0.9688 pu at $\mathrm{V} 2 \mathrm{G}$ and $\mathrm{G} 2 \mathrm{~V}$ operation, respectively). This asymmetry performance results in some constraints and limitations for the system powerflow, which need to be considered, during the control system design. These limitations are stated mathematically by four novel formulas for power-flow criteria. These formulas describe the conditions for achieving maximum active and reactive power, and unity and zero power factor system operation [197].

\subsubsection{Active Power-Flow Criteria}

During the symmetrical operation by using the approximate fundamental power model $\left(P_{A F P M}\right)$, the active power is zero, when $\delta$ equals zero. However, in the real operation, the system withdraws a small amount of active power $\left(P_{s 0}\right)$ to supply the losses, as described in Figure 10.3. This amount of power is stated mathematically in Equation (107). In order to force the secondary active power to be zero, the angle $\delta$ must start from a negative value $\left(\delta_{p 0}\right)$, which can be calculated from Equation (10-8).

$$
\begin{gathered}
P_{s 0}=\left(\frac{8}{\pi^{2}}\right) \frac{C_{s} R_{s i} V_{b}{ }^{2}}{L_{s c}+C_{s} R_{s i}{ }^{2}} \sin ^{2}\left(\frac{\beta}{2}\right) \\
\delta_{p 0}=-\sin ^{-1}\left(\frac{\omega_{r} L_{p i} L_{s i} \mathrm{C}_{\mathrm{s}} \mathrm{R}_{\mathrm{si}} \mathrm{V}_{\mathrm{b}} \sin \left(\frac{\beta}{2}\right)}{\mathrm{M} \gamma \mathrm{V}_{\mathrm{dc}}\left(\mathrm{L}_{\mathrm{sc}}+\mathrm{C}_{\mathrm{s}} \mathrm{R}_{\mathrm{si}}{ }^{2}\right) \sin \left(\frac{\alpha}{2}\right)}\right)
\end{gathered}
$$

\subsubsection{Reactive Power-Flow Criteria}

Due to the harmonics effect, the system consumes more reactive power than that is

able to inject. This effect makes the actual system deviates from the fact that at $\delta= \pm 90^{\circ}$, the 
reactive power is zero, as indicated in Figure 10.3. This deviation of reactive power $\left(Q_{s}\right)$ is stated in Equation (10-9). The value of $\delta$ that achieves zero reactive power (unity power factor) operation $\left(\delta_{Q 0}\right)$ can be calculated from Equation (10-10).

$$
\begin{gathered}
Q_{s 0}=\frac{V_{b}{ }^{2}}{\omega_{r} L_{s i}}\left(0.03 \sin ^{2}\left(\frac{3 \beta}{2}\right)+0.0065 \sin ^{2}\left(\frac{5 \beta}{2}\right)\right) \\
\delta_{Q 0}=\cos ^{-1}\left(\frac{L_{p i} V_{b}\left[0.037 \sin ^{2}\left(\frac{3 \beta}{2}\right)+0.008 \sin ^{2}\left(\frac{5 \beta}{2}\right)\right]}{M \gamma V_{d c} \sin \left(\frac{\alpha}{2}\right) \sin \left(\frac{\beta}{2}\right)}\right)
\end{gathered}
$$

\subsubsection{Verification for the Power-Flow Criteria}

It can be noticed that the power-flow criteria formulas (10-7)-(10-10) are functions of the design and control parameters. For the system parameters presented in Table 5.23, these criteria are evaluated at different control conditions ( $\alpha$ and $\beta$ ), as shown in Figure 10.4. Figure $10.4(\mathrm{a})$ shows the active power at $\delta=0$ or $180^{\circ}\left(P_{s 0}\right)$, this value does not depend on $\alpha$, and increases gradually as increasing $\beta$, showing the worst (maximum) at $\beta=180^{\circ}$. Figure 10.4(b) describes the criterion $\delta_{Q 0}$, which rises as increasing $\beta$, and decreases as $\alpha$ increases. The reactive power at maximum active power operation $\left(Q_{s 0}\right)$ is given in Figure 10.4(c). It shows fluctuating performance against $\beta$, due to the strong coupling between the shape of the inverter voltage (which is controlled by $\beta$ ) and the harmonic contents in the system. The maximum value of $Q_{s 0}$ occurs also at $\beta=180^{\circ}$. The criterion of UPF operation $\left(\delta_{P 0}\right)$ is given in Figure $10.4(\mathrm{~d})$. At low power level $\left(\beta<80^{\circ}\right)$, it shows large deviation from $90^{\circ}$, but at $\beta>80^{\circ}, \delta_{Q 0}$ becomes closer to $90^{\circ}$, and the worst value appears at $\beta=180^{\circ}$. This deviation is getting worse as $\alpha$ decreases. Based on these analysis, the 
worst criteria almost appear at the full capacity operation $\left(\alpha=\beta=180^{\circ}\right)$, which can be considered as design limitations. The worst values of these criteria are estimated for the LCL BIWPTS and presented in Table 10.2.
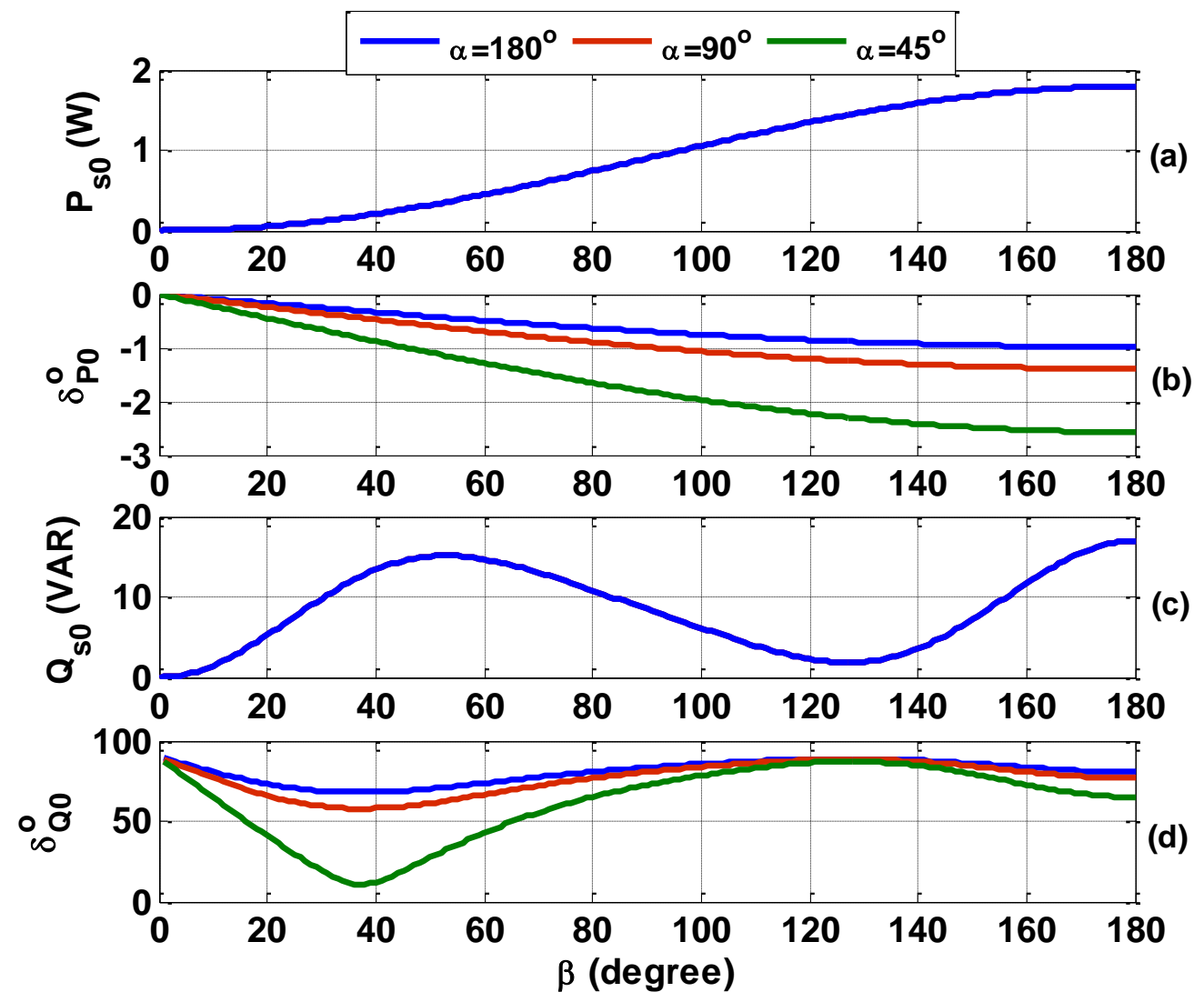

Figure 10.4. Power flow criteria with different control parameters $\left(V_{d c}=60 \mathrm{~V}\right)$.

Table 10.2. Worst Power-Flow Criteria $\left(\alpha=\beta=180^{\circ}\right)$ for LCL BIWPTS.

\begin{tabular}{llll}
\hline Criterion & Value & Criterion & Value \\
\hline $\boldsymbol{P}_{\boldsymbol{s} 0}$ & $1.8 \mathrm{~W}$ & $\boldsymbol{Q}_{\boldsymbol{s} 0}$ & $16.94 \mathrm{VAR}$ \\
$\boldsymbol{\delta}_{\boldsymbol{P} 0}$ & $-0.985^{\circ}$ & $\boldsymbol{\delta}_{\boldsymbol{Q} 0}$ & $80.67^{\circ}$ \\
\hline \hline
\end{tabular}


By applying the criteria presented in Table 10.2 on the system, four different powerflow modes of operation are obtained: 1) maximum active power (MAP), 2) unity power factor (UPF), 3) maximum reactive power (MRP) and 4) zero power factor operation (ZPF). The four modes are investigated and compared in Table 10.3.

Table 10.3. Different Modes of Power-Flow $\left(\alpha=\beta=180^{\circ}\right)$ in BIWPTS.

\begin{tabular}{|c|c|c|c|c|c|}
\hline Mode & Direction & $P_{A P M}$ & $\boldsymbol{P}_{\text {FAPM }}$ & $Q_{R P M}$ & $Q_{A R P M}$ \\
\hline \multirow{2}{*}{ (1) MAP } & $\mathrm{G} 2 \mathrm{~V}\left(\delta=-90^{\circ}\right)$ & -102.5 & -102.7 & 21.3 & 17 \\
\hline & $\mathrm{V} 2 \mathrm{G}\left(\delta=90^{\circ}\right)$ & 106.4 & 106.3 & 21.3 & 17 \\
\hline \multirow{2}{*}{ (2) UPF } & $\mathrm{G} 2 \mathrm{~V}\left(\delta=-\delta_{Q 0}=-80.67^{\circ}\right)$ & -101.1 & -101.3 & 4.4 & 0 \\
\hline & $\operatorname{V} 2 \mathrm{G}\left(\delta=\delta_{Q 0}=80.67^{\circ}\right)$ & 105 & 104.9 & 4.4 & 0 \\
\hline \multirow{2}{*}{ (3) MRP } & $\mathrm{V} 2 \mathrm{G}\left(\delta=0^{\circ}\right)$ & 1.9 & 1.8 & -83.3 & -87.6 \\
\hline & $\mathrm{G} 2 \mathrm{~V}\left(\delta=180^{\circ}\right)$ & 1.9 & 1.8 & 126 & 121.4 \\
\hline \multirow{2}{*}{ (4) $\mathrm{ZPF}$} & $\mathrm{V} 2 \mathrm{G}\left(\delta=\delta_{P 0}=-0.985^{\circ}\right)$ & 0.14 & 0 & -83.3 & -87.5 \\
\hline & $\mathrm{G} 2 \mathrm{~V}\left(\delta=180^{\circ}-\delta_{Q 0}=180.985^{\circ}\right)$ & 0.14 & 0 & 126 & 121.3 \\
\hline
\end{tabular}

In MAP mode, the system shows the maximum active power-flow in both G2V and V2G operation (-102.7 and $106.3 \mathrm{~W}$, respectively), but consumes a small amount of reactive power due to the harmonic contents (17 VAR), which means that the power factor is less than unity. By applying $\delta_{Q 0}$ instead of $90^{\circ}$ (UPF mode), the active power slightly drops and the reactive power almost vanishes, which means that the UPF operation is 
realized. In the MRP mode, the system supplies or absorbs the maximum reactive power, with absorbing a tiny amount of active power $(1.8 \mathrm{~W})$ to feed the losses. This small amount can be avoided by applying $\delta_{P 0}$ criterion, to provide zero power factor operation [ZPF mode]. Moreover, by comparing the peak power value, during V2G and G2V operation, the asymmetry operation can be observed. The choice between the four modes of operation depends on the applications and the operator's priority. For example, if the operator is interested in the magnitude of the power only, thus modes (1) and (3) are the best choice. However, if the interest was for the system's power factor, then modes (2) and (4) are preferred. Anyway, regardless of the mode of operation, these limitations must be considered, while designing the power-flow control system, as discussed in the next sections.

\subsection{Proposed Two-Layer Predictive Active Power-Flow Controller}

The proposed controller is located on the vehicle side to manage the magnitude and direction of the power-flow between the EV and power grid. The power-flow control is achieved by implementing the phase shift modulation technique in both the primary and the secondary sides. The proposed predictive controller is responsible for generating the control parameters $(\alpha, \beta$ and $\delta)$, to accomplish the desired power-flow magnitude and direction. It consists of two control layers, as indicated in Figure 10.5. The first control layer is responsible for gathering data from the BMS, EV's driver/operator desire, charging station and power grid. Then, it decides the mode of operation [discharge (V2G), charge (G2V) or abstain], and estimates the required rate of discharge or charge. 


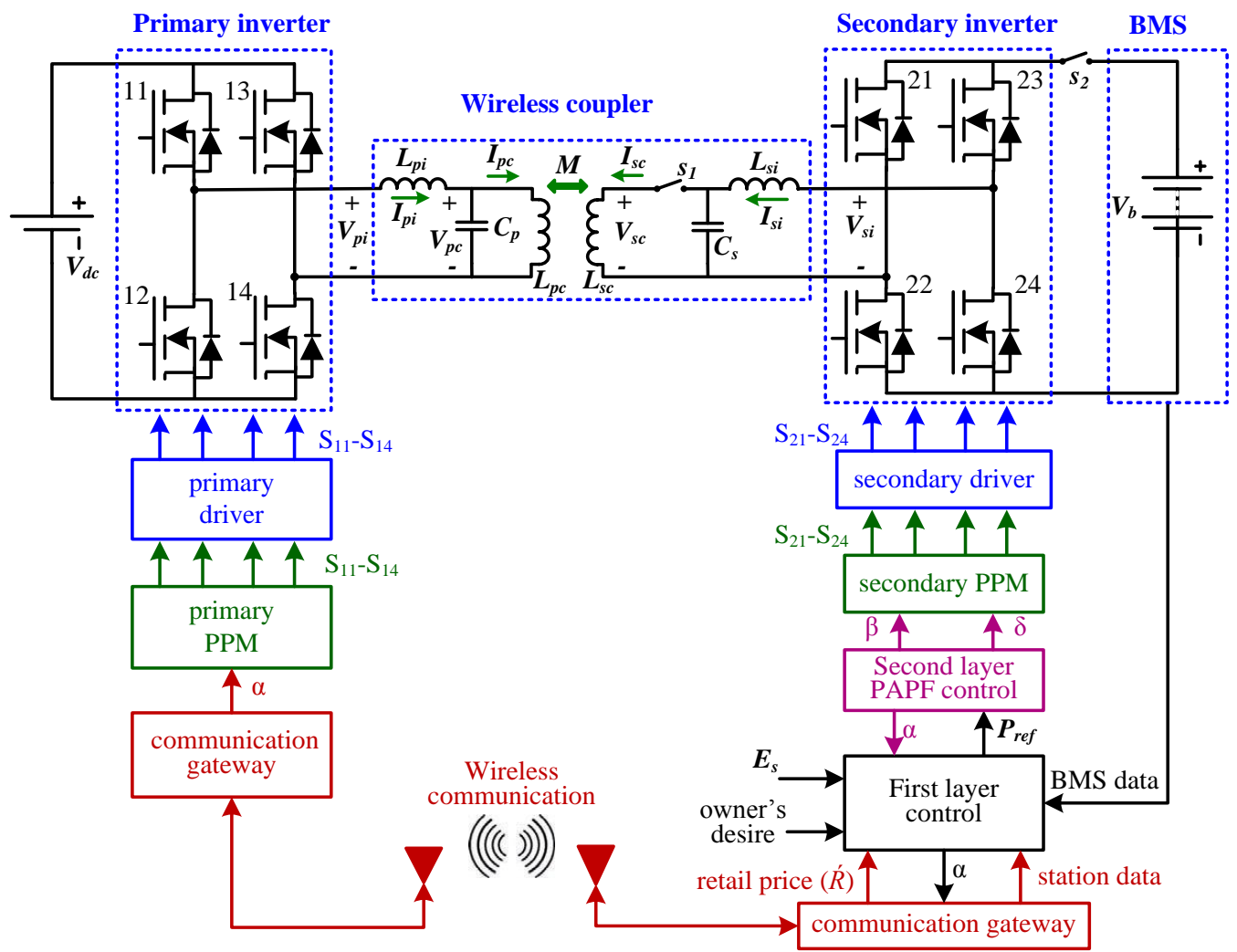

Figure 10.5. The proposed two-layer predictive active power-flow control.

The outcomes from this layer are passed to the second layer PAPF control. This controller predicts the phase-shift modulation parameters $(\alpha, \beta$ and $\delta)$, for both the primary and secondary sides, which are responsible for generating the switching activities of the two resonant converters (primary and secondary). The generated signals are boosted through the driving circuits to the gate's voltage and power level of the power semiconductor switch.

\subsubsection{Control Parameters' Design}

The proposed two-layer control is designed based on the power-flow models and 
criteria presented in sections 10.2 and 10.3. These analytical models are utilized to predict the control parameters $(\alpha, \beta$ and $\delta)$, which are responsible for achieving the desired powerflow. The parameter $(\delta)$ is utilized to provide the wanted power-flow direction and mode of operation ( $\mathrm{V} 2 \mathrm{G}$ or $\mathrm{G} 2 \mathrm{~V}$ ). A negative $\delta$ provides $\mathrm{G} 2 \mathrm{~V}$ (charging) operation, but a positive $\delta$ allows V2G (discharging) service. The value of $\delta$ is decided according to the operator's priority, whether it is the power magnitude or power factor. Keeping $\delta= \pm 90^{\circ}$ will achieve maximum active power-flow (MAP) operation, while putting $\delta= \pm \delta_{Q 0}$ [given in Equation (10-10)] will accomplish UPF operation, as discussed in section 10.3.

The other two control parameters $(\alpha$ and $\beta$ ) are decided to provide the desired rate of charge or discharge (active power magnitude). These parameters are predicted based on $P_{F A P M}$ in Equation (10-3). By assuming that both the HF converters will be driven by the same parameter (i.e $\alpha=\beta$ ) to control the active power-flow magnitude, the two parameters are estimated using Equation (10-11) [198].

$$
\begin{aligned}
& \alpha=\beta=\cos ^{-1}\left[1-\left(2 P_{\text {ref }} / P_{\text {max }}\right)\right] \\
& P_{\text {max }}=\left(\frac{8}{\pi^{2}}\right) \frac{M \gamma V_{d c} V_{b}}{\omega_{r} L_{p i} L_{s i}} \sin (\delta)+\left(\frac{8}{\pi^{2}}\right) \frac{C_{s} R_{s i} V_{b}{ }^{2}}{L_{s c}+C_{s} R_{s i}{ }^{2}}
\end{aligned}
$$

where, $P_{r e f}$ is the desired active power, which is estimated during the first control layer and passed to the second one.

\subsubsection{Online Mutual Inductance Estimation}

As indicated in the previous section, the control parameters are functions of the system's design parameters. For the controller to be able to achieve the proper prediction, the system's parameters need to be known. The LCL BIWPTS under consideration is 
designed for fixed resonant frequency $\left(\omega_{r}\right)$ operation, on both the primary and secondary sides. Also, the compensation capacitors $\left(C_{p}, C_{s}\right)$ are tuned to resonate with the selfinductance of the coils $\left(L_{p c}, L_{s c}\right)$, which are identified after the design stage, and are relatively fixed [184]. Therefore, the compensation capacitors are fixed as well. Under these constraints, only the mutual inductance $(M)$ varies in light of the vehicle position and the misalignment between the power pads. Thus, this parameter needs to be adaptively estimated, before applying the control algorithm. In this work, a modified online mutual inductance estimation technique has been proposed. It depends on utilizing the developed analytical model besides one direct measurement on the vehicle side. A known value for the primary control parameter $(\alpha)$ is applied to the primary converter, while the secondary circuit is left open, by opening the ideal switches $S_{1}$ and $S_{2}$ in Figure 10.5. The open circuit secondary voltage $\left(E_{s}\right)$ is measured, and the primary coil current at open circuit secondary (I $\left.I_{p c_{-} O C S}\right)$ is calculated, using Equation (10-12). Using these two recognized values, the mutual inductance is estimated based on Equation (10-13) [199].

$$
\begin{gathered}
I_{p c_{-} O c S}=-j V_{p i_{-} 1} /\left[\omega_{r}\left(L_{p c}+C_{p} R_{p c} R_{p i}\right)\right] \\
M=E_{S} /\left(\omega_{r} I_{p c_{-} o c s}\right)
\end{gathered}
$$

\subsubsection{First Control Layer}

As depicted in Figure 10.5, the first control layer receives the BMS's data, EV's driver desire and the open circuit voltage $\left(E_{s}\right)$ measurement, directly from the vehicle side. Also, it receives information about the grid retail price $(R)$ and the charging station, through the wireless communication link. Based on these data, it generates the reference active 
power $\left(P_{r e f}\right)$ to be passed to the second control layer (PAPF). The charging and discharging decision and rate is defined, by comparing the EV's psychological price $(R)$ with the grid retail price of the wireless charging $(R)$ [200]. The EV's psychological price depends on the battery's SOC, and is defined in this work using the first-order polynomial cost function, as indicated in Figure 10.6(a). The EV's psychological price, in terms of the battery SOC, is stated mathematically in Equation (10-14).

$$
R=\frac{R_{\min }-R_{\max }}{S O C_{\max }-S O C_{\min }} S O C+R_{\text {max }}+\frac{R_{\max }-R_{\min }}{S O C_{\max }-S O C_{\min }} S O C_{\min }
$$

where, $S O C_{\min }$ and $S O C_{\max }$ are the limits of the linear operating region of the EV's battery, which is typically $20-80 \% ; R_{\min }$ and $R_{\max }$ are the limits of EV psychological price, which are chosen to cover the range of variation of the wireless charger retail price changes $(\dot{R})$, as depicted in Figure 10.6(a). The retail price for wireless charging of a road is available for the EV's drivers based on the current traffic information systems of Intelligent Transportation Systems (ITSs) [200].

The charging and discharging benefit is evaluated, as $\Delta R=R$ - $R$. The reference powerflow direction and rate $\left(P_{r e f}\right)$ is assessed in terms of $\Delta R$, as described in Figure 10.6(b) and Equation (10-15).

$$
P_{\text {ref }}=\left\{\begin{array}{lr}
P_{\max , d} & \Delta R \geq \Delta R_{\max , d} \\
m_{d} \Delta R+k_{d} & 0<\Delta R<\Delta R_{\max , d} \\
m_{c} \Delta R+k_{c} & \Delta R_{\max , c}<\Delta R \leq 0 \\
P_{\max , c} & \Delta R \leq \Delta R_{\max , c}
\end{array}\right.
$$

where, $m_{d}=\frac{P_{\max , d}-P_{\min , d}}{\Delta R_{\max , d}}, k_{d}=P_{\min , d}, m_{c}=\frac{P_{\max , c}-P_{\min , c}}{\Delta R_{\max , c}}, k_{c}=P_{\min , c}$.

$P_{\max , c}$ and $P_{\max , d}$ are the maximum rate of charge and discharge, respectively, which are estimated from $P_{F A P M}$ in Equation (10-11), by applying $\alpha=\beta=180^{\circ} . P_{\min , c}$ and $P_{\min , d}$ are 
the minimum rate of charge and discharge, respectively, which are defined based on the EV's battery manufactures and charging system's limits.

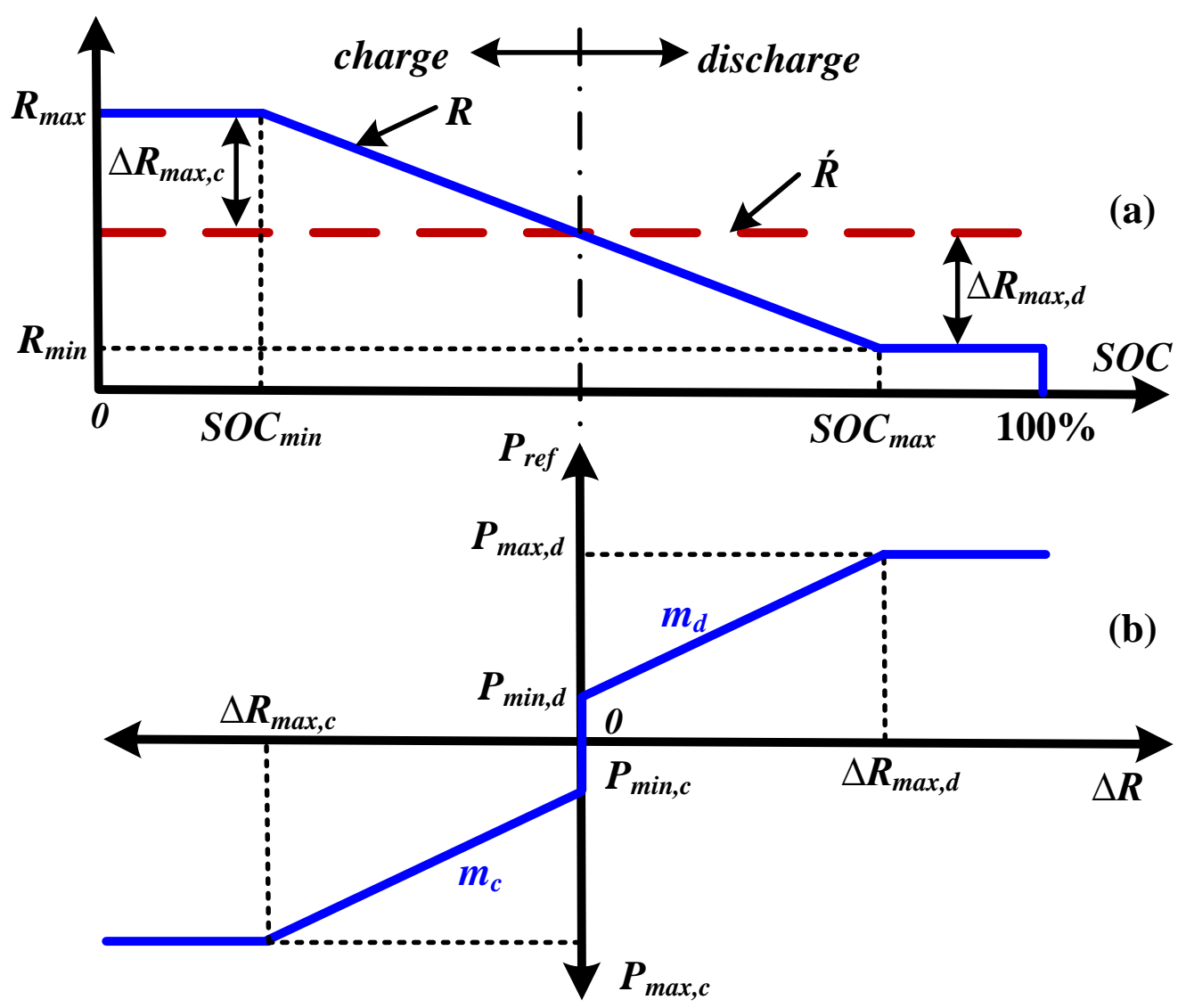

Figure 10.6. First layer control principle. (a) EV psychological price and grid retail price. (b) Reference power vs. cost benefits.

Over $S O C_{\max }$, the benefit is maximum $\left(\Delta R_{\max , d}=\dot{R}-R_{\min }\right)$, and the EV will discharge at the maximum limit $\left(P_{\max , d}\right)$, in order to avoid overcharging situation. Below $S O C_{\min }$, the EV will keep charging at the maximum capacity $\left(P_{\max , c}\right)$, to help the vehicle to reach its 
destination. In this case, the benefit is maximum with negative sign $\left(\Delta R_{\max , c}=\dot{R}\right.$ $\left.R_{\text {mmax }}\right)$.When the two prices $(R$ and $R$ ) match, i.e $\Delta R=0$, the EV will charge at the minimum limit $\left(P_{\min , c}\right)$. When $\Delta R$ lies between 0 and $\Delta R_{\max , d}$, the EV will discharge following a linear profile with a slop $m_{d}$. When $\Delta R$ ranges between 0 and $\Delta R_{\max , c}$, the EV will charge through another linear profile with a slop $m_{c}$.

If the EV's driver has some restrictions, during the integration with the power grid, the controller allows him/her to introduce these limitations, in terms of the desired final SOC $\left(S O C_{f}\right)$, integration (connection) time $\left(t_{c}\right)$ and required operation (Mode). Then, it calculates the required rate $\left(P_{r e s}\right)$ to satisfy these limitations using Equation (10-16).

$$
P_{r e s}=\mp \frac{S O C_{f} \cdot Q_{n}}{\eta_{b} \cdot t_{c}}
$$

where, $Q_{n}$ is the nominal battery capacity, and $\eta_{b}$ is the charging and discharging efficiency [201], [202].

The proposed first control layer provides five different modes of operation: Mode I means abstain (no interaction);

Mode II provides restricted operation by manually entering $M o d e, S O C_{f}$ and $t_{c}$; Mode III allows charge only service, without considering the grid price; Mode IV enables charge only, with considering the grid price; and Mode $\mathrm{V}$ activates the interaction operation, in which the system will be able to charge and discharge automatically, based on the grid price and EV's psychological price.

The sequence of the algorithm implementation linked with the available wireless communication system is indicated in Figure 10.7 and described as follows: 


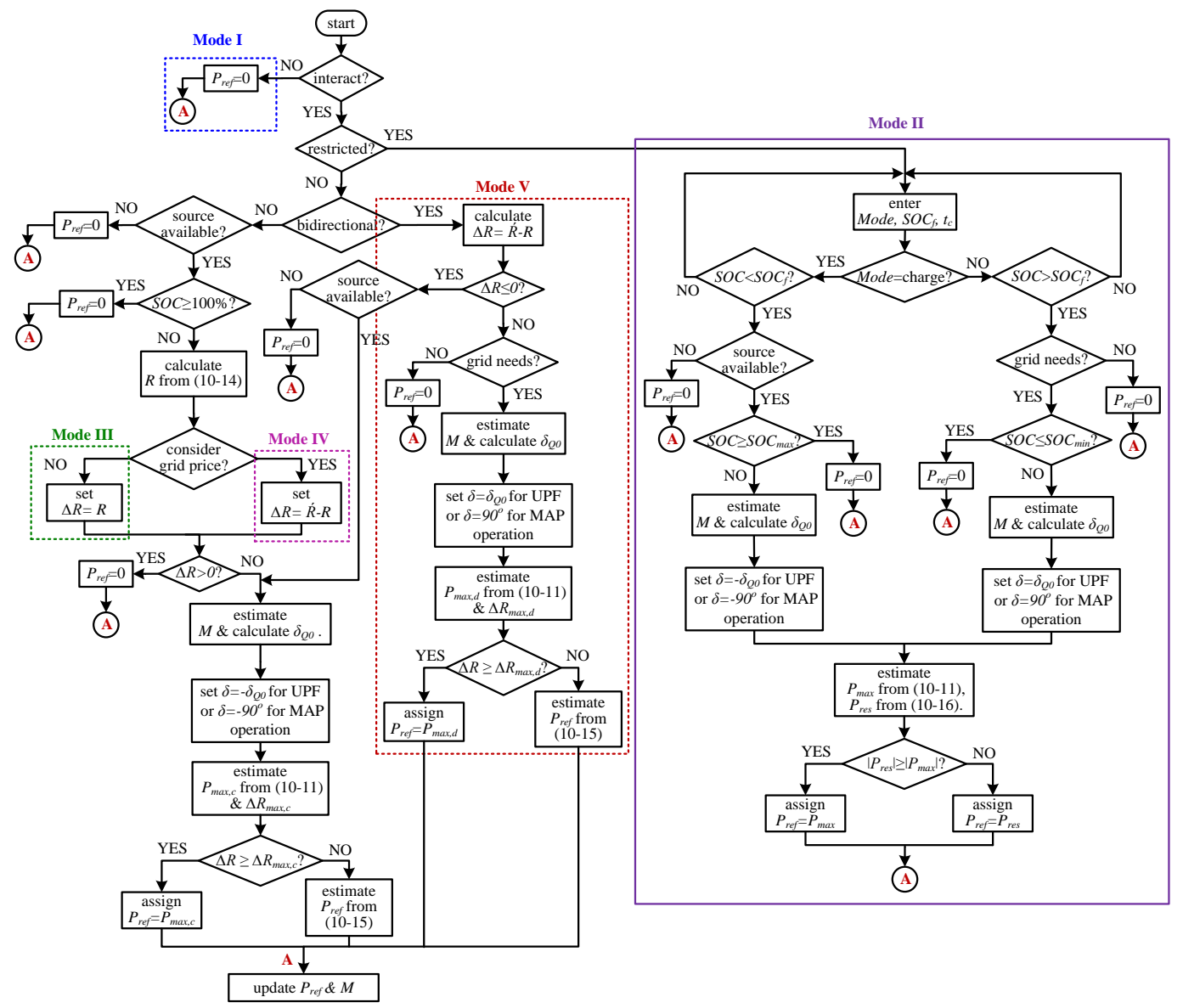

Figure 10.7. The proposed first layer control algorithm.

- A vehicle enters the coverage area of the wireless communication system, and receives a notification that there is a WPT service available in the area.

- If the vehicle wants to interact, it will send a request of service to the grid side unit, otherwise it will ignore the notification.

- Based on the driver's desire, EV battery SOC, grid price and the BIWPTS's specifications, the first control layer checks the grid resources availability, estimates the mutual inductance, decides the mode of operation (among modes I to V), and calculates $P_{r e f}$, depending on Figures 10.6 and 10.7, and using Equations (10-12)-(10-16). 
- The first layer updates the variables $M$ and $P_{r e f}$, and passes them to the second control layer (PAPF), which predicts the phase-shift parameters $(\alpha, \beta$ and $\delta)$, as is explained in the next section.

\subsubsection{Second Control Layer}

This control layer receives the reference power from the first stage, and predicts the modulation parameters $(\alpha, \beta$ and $\delta)$ that direct the system to transfer this power. The operation procedure of this control layer is explained in Figure 10.8. The control gets $M$, $P_{\max }$ and $P_{r e f}$, from the first stage, and checks the sign of $P_{r e f}$. If $P_{r e f}=0$, then it keeps the control parameters as zero. In this case, there is no power transfer, which can be due to many reasons, such as limited resources, the grid does not need extra power, the driver does not want to sell energy, while the EV fully charged or $\Delta R>0$, or the driver does not want to interact with the grid at all [199]. On the other hand, if $P_{\text {ref }}$ is positive, the controller sets $\delta=-\delta_{Q}$ or $-90^{\circ}$, to charge the battery; and if it is negative, the controller assigns $\delta=\delta_{Q 0}$ or $90^{\circ}$, for discharging operation. Finally, the controller checks the charger limit $\left(P_{\max }\right)$, if $P_{\text {ref }}$ is exceeding of this limit, then $\alpha, \beta$ will be set to the maximum, i.e $\alpha=\beta=180^{\circ}$, otherwise they will be estimated, using Equation (10-11). Finally, the controller updates the new parameters, and passes $\delta$ and $\beta$ to the secondary PPM system, and sends $\alpha$ to the primary PPM, through the wireless communication, as indicated in Figure 10.5. The PPM generates the switching signals for both the primary and secondary inverters, which are boosted through the driving circuits to the gate voltage and power level of the semiconductor switch. 


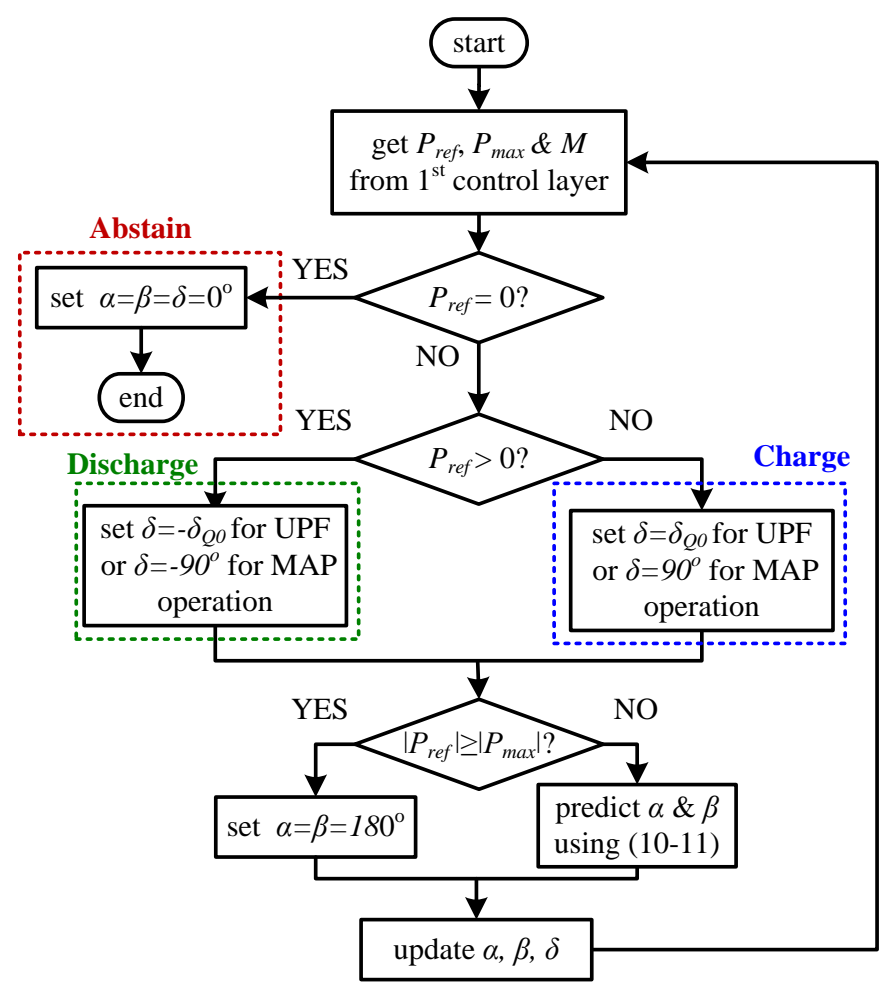

Figure 10.8. The proposed second control layer algorithm.

\subsection{Control Implementation and Results}

The prototype for LCL BIWPTS, shown in Figure 8.13 with the parameters in Table 8.2 , is driven by the developed controller and analyzed by means of simulation and experimental tests. The proposed control is implemented using Cmod S6 FPGA board, shown in Figure 10.9. The implementation of the PPM inside the FPGA board for both inverters is depicted in Figure 10.10. The figure shows that the dead-time between the switches in the same legs are digitally implemented inside the board, using logic AND gates. The signals from the FPGA are boosted up to the MOSFETs' voltage and power level, using FAN7391 ICs. The proposed PAPF controller is implemented and its performance is compared with a PI controller, for verification purposes. 


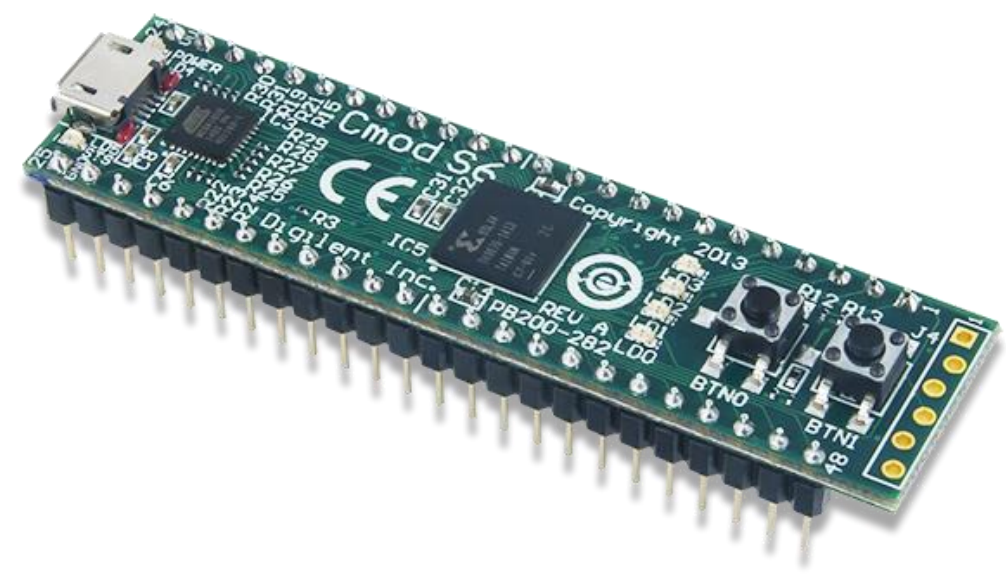

Figure 10.9. Cmod S6 FPGA board.

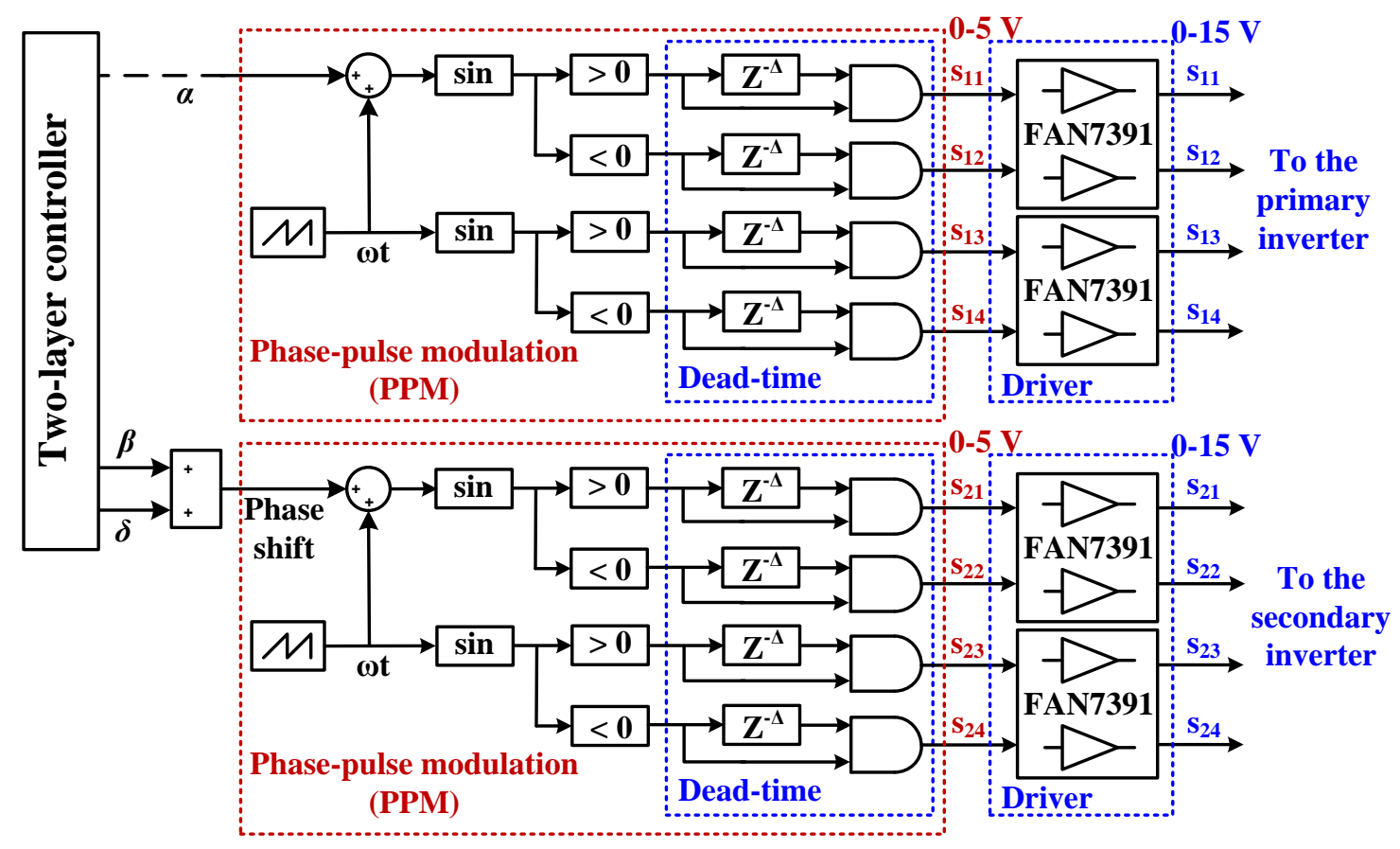

Figure 10.10. Implementation of FPGA-based PPM including the dead-time and the driver. 

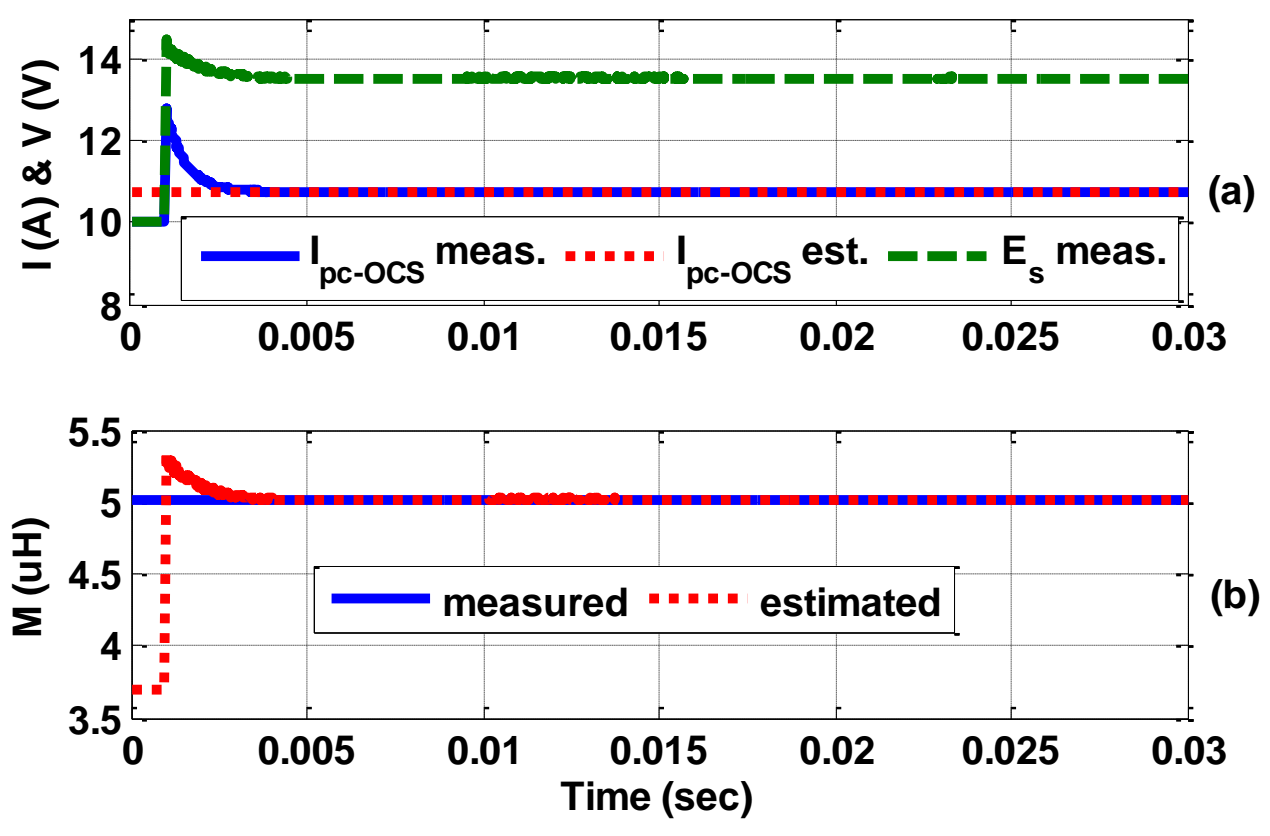

Figure 10.11. Mutual inductance estimation performance $\left(V_{d c}=60 \mathrm{~V}\right)$. (a) $E_{s}$ and $I_{p c_{-} O C S \text {. (b) }}$ Mutual inductance.

\subsubsection{Testing the Proposed Mutual Inductance Estimation}

The performance of the proposed mutual inductance estimation procedure is described in Figure 10.11. The parameter $\alpha$ is set to $180^{\circ}$, and the open circuit secondary voltage $\left(E_{s}\right)$ is measured, as indicated by the green dashed line in Figure 10.11(a). The primary coil current is calculated, using Equation (10-12), and also measured for comparison purpose. As can be seen in Figure 10.11(a), the estimated and the measured values show good agreement. Depend on Equation (10-13), the mutual inductance is estimated and compared with the real actual value in Figure 10.11(b). It can be noted that the proposed technique could reach to the correct value of $M$ accurately, in less than 5 msec, which proves the real-time implementation of this technique. 


\subsubsection{Verifying the Proposed Controller Performance}

The proposed PAPF control is enforced and tested in this section. The LCL BIWPTS's performance, during the Abstain and Discharging operation, is described in Figure 10.12. The system starts with the Abstain mode, and switches to the Discharge mode at $0.04 \mathrm{sec}$. During the Abstain operation, all the control parameters were set to zero, and no power transfer occurs. In the wake of applying the Discharge, $\delta$ was changed in accordance with $90^{\circ}$ to accomplish V2G service, while $\alpha$ and $\beta$ were adjusted to match with the desired power $\left(P_{r e f}\right)$, as shown in Figure 10.12(a) and 10.12(b). At $0.08 \mathrm{sec}, P_{r e f}$ exceeds the power limits, accordingly the controller conforms $\alpha$ and $\beta$ to $180^{\circ}$ to provide the maximum available power. Then, at $0.12 \mathrm{sec}$, the required power diminished, and the control framework takes after the new value. During these transitions of power-flow, the variation of $V_{s i}$ can be seen in Figure 10.12(c) and 10.12(d).
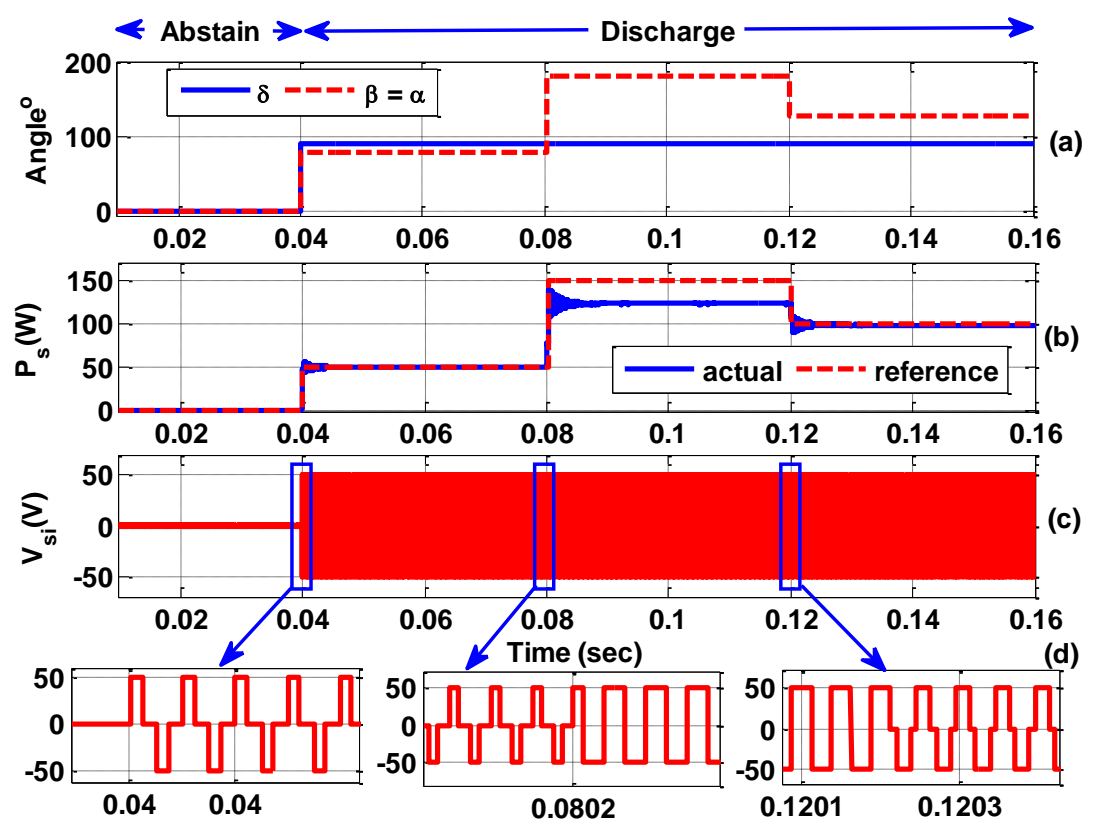

Figure 10.12. PAPF control performance during Abstain and Discharge modes. (a) Control parameters. (b) Secondary power. (c) Secondary voltage. (d) Zoomed secondary voltage. 
The performance of the proposed controller is compared with the classical proportional integral (PI) control, as delineated in Figure 10.13. The figure demonstrates the reference active power, and the actual system power-flow due to both the proposed PAPF and PI controllers. During the Abstain mode, the PI controller couldn't keep the power at zero, which prompts to unwanted inverters' switching. Additionally, it shows noisy performance, during both the transient and steady-state response. Also, a large delay is experienced in the PI transient response (at $0.12 \mathrm{sec}$ ). The proposed controller shows more precise and faster tracking performance for the reference power.

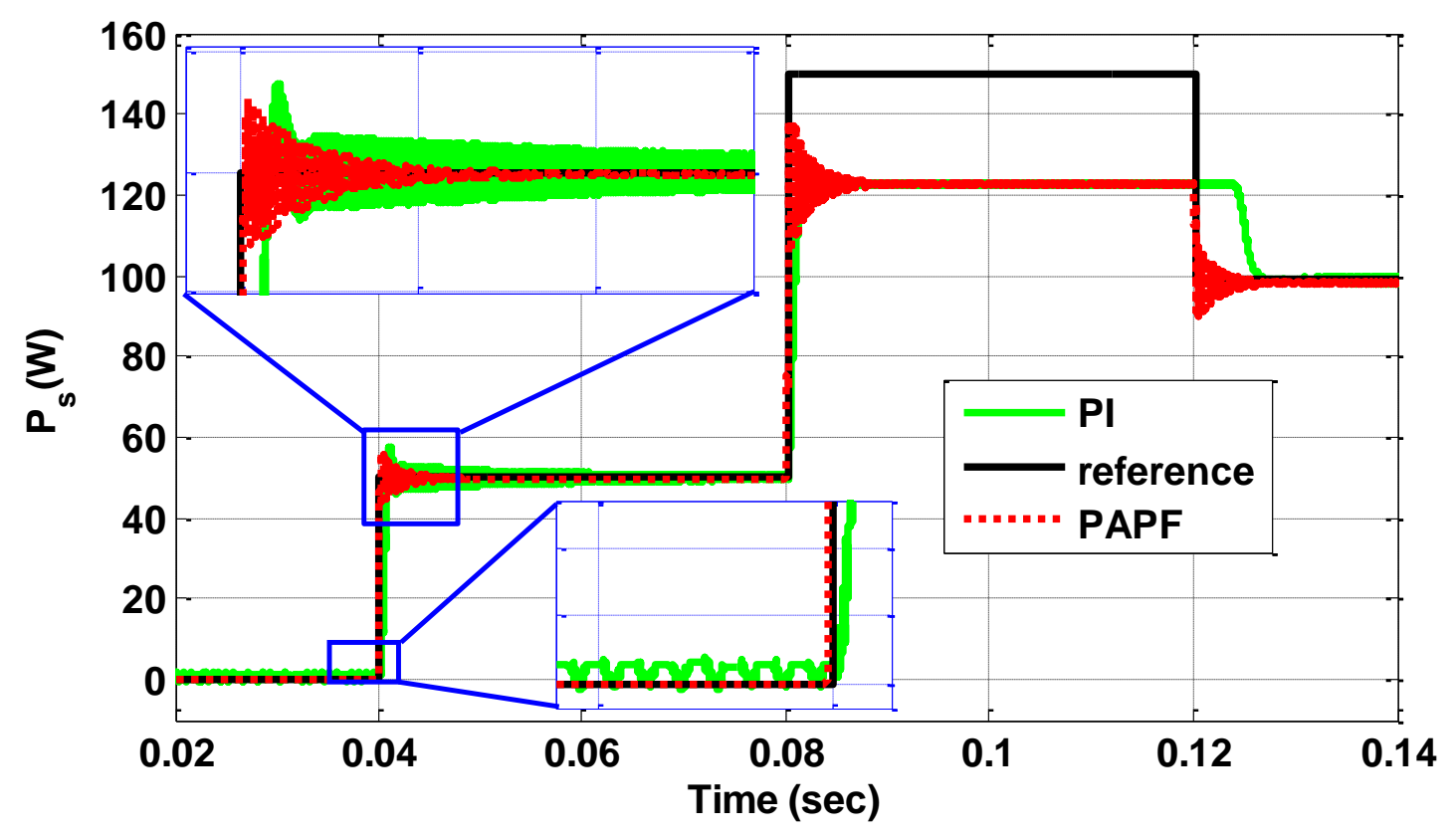

Figure 10.13. Comparison between the performance of PI and the proposed PAPF control during Abstain and Discharge modes. 
The behavior of the proposed controller, during the transition from Discharge to Charge modes, is examined in Figure 10.14. In this test, the controller changes the framework from discharging (V2G) to charging $(\mathrm{G} 2 \mathrm{~V})$ operation at $0.06 \mathrm{sec}$, by adjusting $\delta$ from $90^{\circ}$ to $-90^{\circ}$ [see Figure 10.14(a)]. As a result of $\delta$ variation, the power-flow is reversed from positive to negative, following the new reference value. The system remains working in the Charging mode the rest of the time with various power levels. The inverter secondary voltage variations, due to the control actions, are indicated in Figure 10.14(c) and 10.14(d).
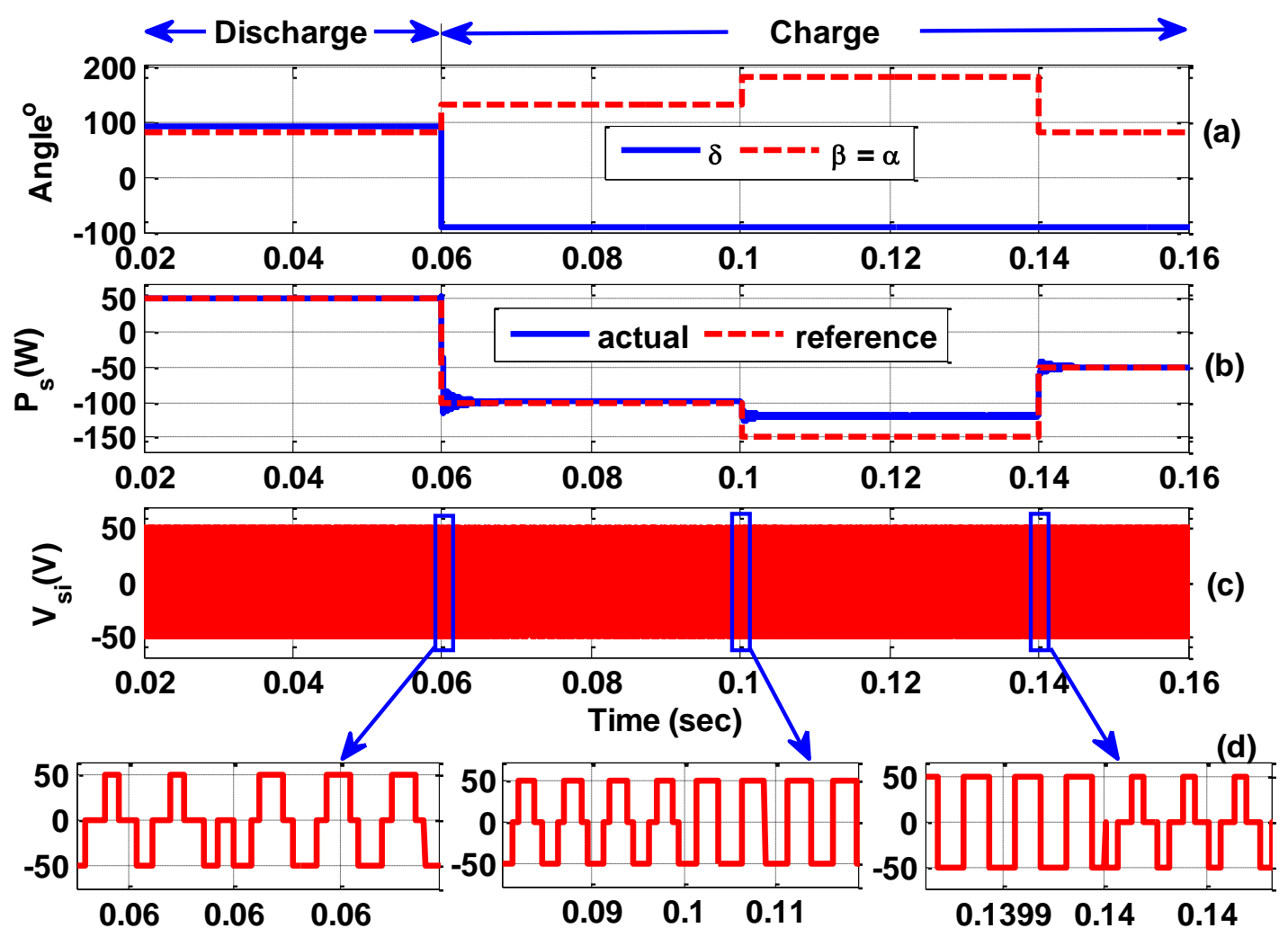

Figure 10.14. PAPF control performance during Discharge and Charge modes. (a) Control parameters. (b) Secondary power. (c) Secondary voltage. (d) Zoomed secondary voltage. 
During the entire period of operation, the controller succeeded to follow the reference power value, and the system was capable of transferring the required power. A similar test is applied, while utilizing the PI controller, and compared with the proposed PAPF control, as indicated in Figure 10.15. The proposed controller demonstrates fast transient response, with accurate steady-state attitude, compared with the PI controller.

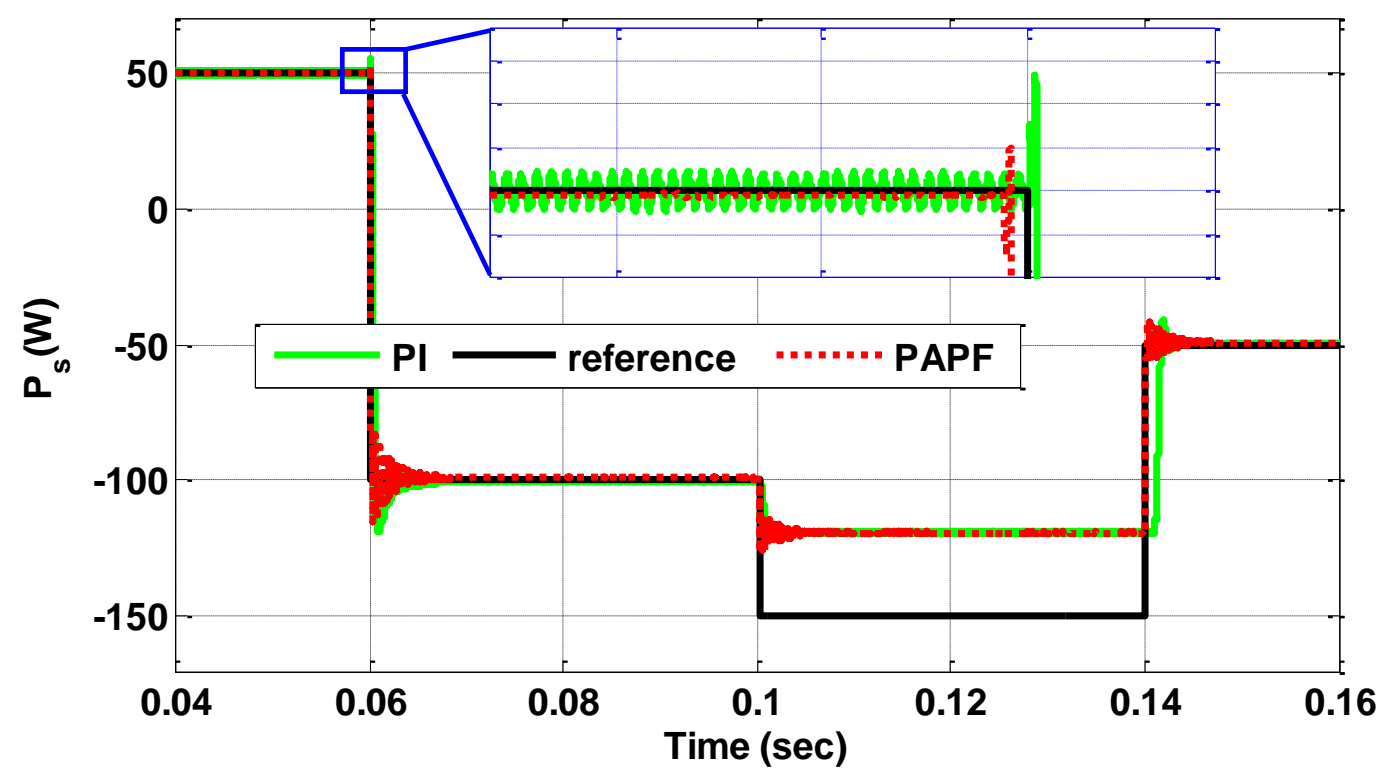

Figure 10.15. Comparison between the performance of PI and the proposed PAPF control during Discharge and Charge modes.

\subsection{Conclusion}

A two-layer vehicle-side predictive active power-flow controller for the LCL BIWPTS in V2G applications is presented in this chapter. The controller is suitable for 
stationary and quasi-dynamic interaction between the EV and power grid. It allows the EV's driver to choose among five different modes of operation. Also, it considers the grid retail price to achieve the maximum profits for the EVs' owners. The proposed controller is based on the system analytical modeling, and it has the ability to predict the modulation parameters for both the primary and secondary inverters, to achieve the desired active power-flow. Moreover, it considers the misalignment in WPT system, by adaptively estimating the mutual inductance before applying the proposed algorithm. The practical implementation of the proposed controller evidenced its performance's accuracy and fast response, during both the transient and steady-state operation, compared to the available controller in the literature. 


\section{Chapter 11 Feasibility Analysis of Quasi-dynamic BIWPTS for EV Applications}

\subsection{Introduction}

Wireless charging methods present a new revolution in the EV industry [203]. Unlike conventional plug-in EVs tethered to a charger, no power connection is needed. Two different visions for implementing wireless charging in EVs' applications are intensively investigated in the literature: stationary and dynamic. In both modes, a primary coil is buried in the ground underneath the vehicle, where in stationary mode, the energy is transferred to a parked vehicle using a single coil, while in dynamic mode, the energy is transferred to a moving vehicle using a series of coils. The removal of cables, autonomy for the driver, and relatively low maintenance has improved the practicality of this technology. Stationary system topologies were demonstrated as early as the General Motors EV1 in 1998 through the implementation of IPT [204]. In [83], a review is presented noting that current stationary applications are beginning to reach their maturity, as the SAE has already established industry-wide specification guidelines in the SAE J2954A standard [149]. Current research has focused on the optimization and design of coupled coils, which have a profound impact on the system's efficiency. Despite the ability to transfer energy more efficiently using stationary WPT since it offers better alignment between pads, it is still limited by its requirement for the EV to park in a specific position.

As wireless charging technology continues to grow, dynamic (online) WPT (DWPT) charging has been introduced accompanied by a number of new advantages [205]. An online EV has already been tested at the Korea Advanced Institute of Science and 
Technology demonstrating a commercially available DWPT charging system for busses [206]. A review of DWPT technologies was conducted in [82], identifying that it could provide the foundation for mass market penetration of EVs regardless of the battery technology. In 2015 , a comprehensive study was conducted evaluating the feasibility of installing DWPT charging lanes on UK roadways [207]. Through the introduction of WPT in EVs, concerns associated with traditional plug-in EVs including charging duration and range limitations could be alleviated.

DWPT is not limited to benefiting the EV's driver, but can also provide enhanced V2G services as well. In [73], a bi-directional WPT (BWPT) V2G scheme was proposed including the potential inclusion of mobile EVs. The inclusion of DWPT added flexibility to V2G schemes but despite a number of advantages, several high level challenges exist in its deployment where cost becomes the center of attention [208]. Charging EVs consumes a large amount of energy and with the inclusion of mobile loads at this magnitude, the economic toll is unclear, which has incited feasibility studies on this topic [209]. In [200], the authors quantified some of these issues through the development of an analytical model, predicting variations in the locational marginal price of energy. However, massive initial cost associated with constructing a robust embedded coil network along the road was a serious concern. Furthermore, reliable, high speed bi-directional communications are required to handle the control. However, this is the most practical solution to support highway driving.

For city driving, an intermediate solution could provide a balance between the infrastructure costs while still enabling a majority of the same advantages dynamic charging has to offer. For city driving, an alternative, such as a quasi (semi)-DWPT 
(QDWPT) system, could provide a balance between the advantages seen in the stationary versus a dynamic system. Using QDWPT, an EV could charge during transient stops on the city roads, such as bus stops for electric buses and traffic signals for EVs. Little work has been presented in the literature that analyzes the QDWPT concept. In [97], the effect of implementing QDWPT over one driving cycle with different profiles was briefly studied. To the author's knowledge, detailed modeling with comprehensive performance and feasibility analysis based on the J2954A standardized power levels have not yet been studied for a QDWPT system.

In this chapter, the feasibility of implementing the QDWPT system at traffic signals is explored. The comprehensive charging and EV system architecture has been modeled and exercised, through the integration of multiple driving scenarios. The novelties of this work are as follows: 1) modeling analysis has been conducted for the entire wirelessly connected EV, including BIWPTS, EV's battery, and traction system with experimental verification, 2) feasibility assessments in implementing a QDWPT system at traffic signals is investigated under three distinct WPT scenarios: fixed power charging, variable power charging and fixed power charging and discharging , 3) a comparative analysis over the maximum driving range and duration per cycle has been examined, 4) quantification of the additional distance gained over all charging scenarios for each consumed $\mathrm{kWh}$ is calculated, and 5) the effect of coil misalignment in the WPT system over the driving performance is investigated.

\subsection{Quasi-Dynamic BIWPTS Description}

The implementation of a QDWPT system at traffic signals could provide a promising 
solution for EV charging. This concept can not only take advantage of dynamic charging features, but would also significantly reduce the cost of infrastructure, simplify control complexity, and potentially increase the transfer efficiency by enabling better alignment. As shown in Figure 11.1, the primary wireless string of pads are placed beneath the pavement in each travel lane at each direction of the intersection and are depicted in blue. Each pad can be driven by an independent power converter or one converter can be utilized to drive a few of them, while controlling the current in each pad. Thus, the primary pads can be selectively excited based on the EV's position, such that the energized pads are covered by the vehicle [82]. To determine the optimal number of WPT coils to support each lane, a traffic-flow analysis can be conducted to define the minimum coverage distance. In this system, it is assumed that over the course of a full traffic light cycle across all directions, wireless coils are available for all stopped traffic (under a red signal). While stopped, authentication with a charging controller can activate either V2G or G2V services. Conversely, the wireless coils fixed in directions with a green signal will have their coils de-energized. Apart from the upfront installation cost to the system, BIWPTSs' activation can be coordinated with existing traffic light controllers and installed in combination with common inductive loops used for traffic detection [210].

The integration between EVs and the surrounding infrastructures at traffic signal can be represented by the DC-bus configuration shown in Figure 1.1. In this structure, the AC grid connection is established using a bi-directional grid-tied converter responsible for regulating a common DC-bus voltage. Each EV at the traffic signal is then connected to the DC-bus through its own BIWPTS to facilitate charging or discharging operations. As an added benefit, the introduction of a localized DC-bus at the traffic signal would help 
ease the integration of RESs, such as PV generations, as shown in Figures 1.1 and 11.1. In this case, the PV panels can be distributed along the road sides, and coupled with the DCbus through a unidirectional DC-DC converter. In addition, external DC loads in and around the traffic signal area can be connected to the same DC-bus through another DCDC converter. Through the inclusion of renewable energy, the bulk load demand can reduce grid stress when EVs are in G2V mode, while in V2G mode, the EV battery can aid in smoothening the PV generation.

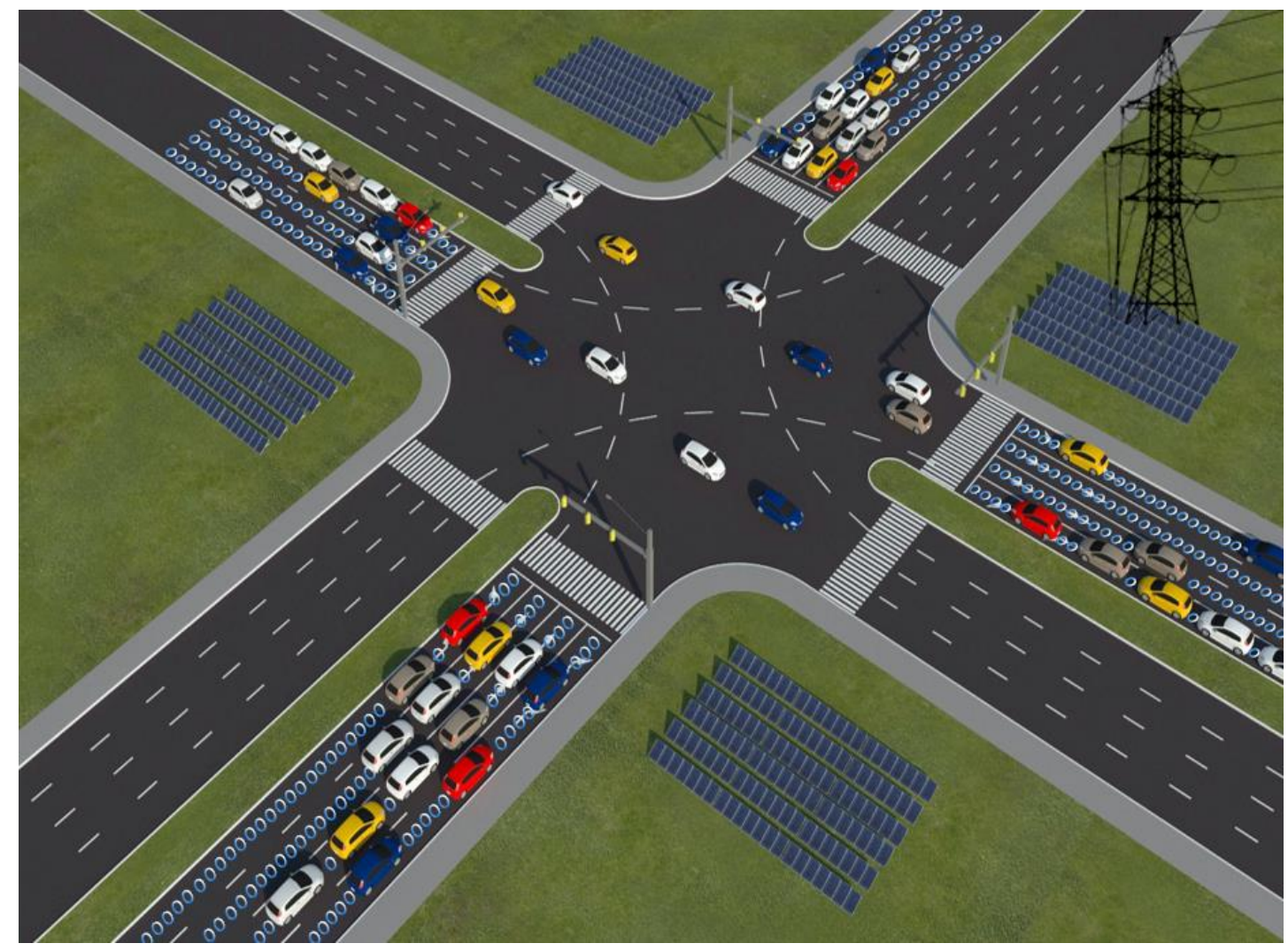

Figure 11.1. The proposed implementation of Quasi-dynamic WPT system at traffic signal. 


\subsection{Modeling of Wirelessly Connected Light-Duty EV}

A block diagram of the wirelessly connected EV configuration is indicated in Figure 11.2 [92]. The structure consists of three main parts: a BIWPTS, EV's battery and EV's drive system. The system has two isolated sides: the grid and vehicle. The two sides are talking to each other through radio communication. The power-flow between these sides is managed by the secondary controller. The modeling of each part in the system is described in this section.

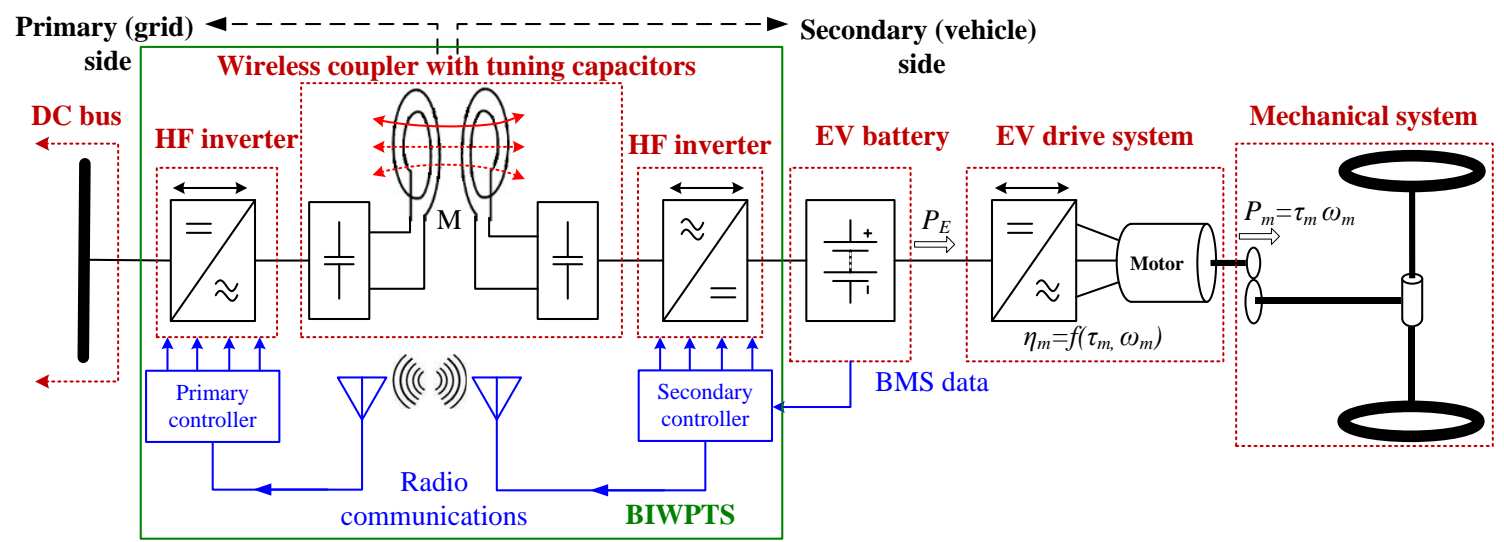

Figure 11.2. Block diagram of a wirelessly connected EV through the BIWPTS.

\subsubsection{Lithium-Ion EV's Battery Model}

Present-day EVs are subjected to heavy power and energy demands, which are not limited to high transient discharge currents, but also sporadic regenerative braking charge currents. Furthermore, with the inclusion of QDWPT, a battery model accounting for multiple time-constants is needed to accurately depict the dynamic response of the battery 
system. Simulation of the EV requires an advanced battery model capable of depicting precise SOC, $I-V$ characteristics, and accurate dynamic behavior. In this study, a $21 \mathrm{Ah}$ lithium-ion base module has been utilized to represent the EV's battery pack, as depicted in Figure 11.3(a). This module contains 14 PL8048168 cells in series at a nominal voltage of 51.8 V, as shown in Figure 11.3(b) [211]. In this section, a model is developed for simulation purposes, where seven modules are placed in series and three in parallel to reach a standard EV pack voltage and capacity of $362.6 \mathrm{~V}$ and $63 \mathrm{Ah}$, respectively.

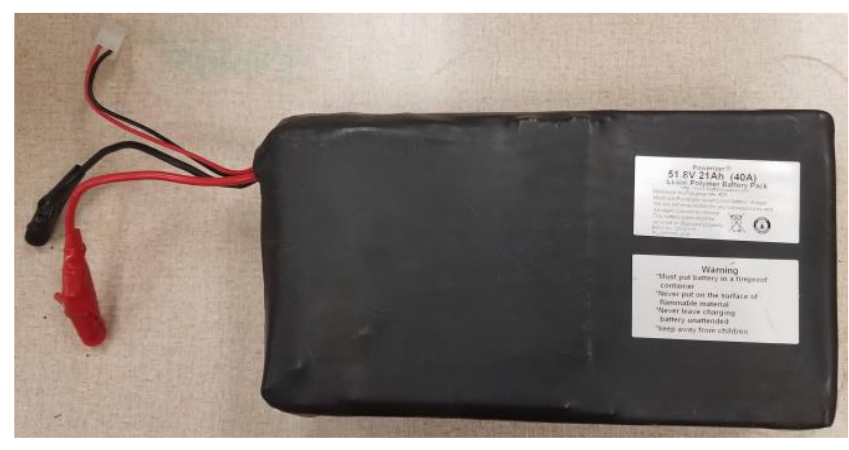

(a)

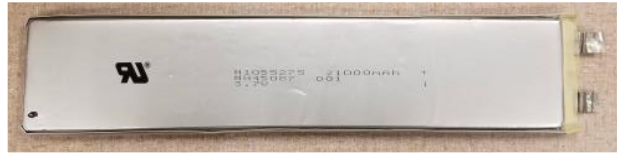

(b)

Figure 11.3. Lithium-ion test battery. (a) Battery Module. (b) PL8048168 battery cell.

In [212], a procedure for obtaining a $3^{\text {rd }}$ order dynamic battery model for EV simulations was presented, where a series of standardized charge and discharge current pulses are administered throughout the full SOC range. High charge and discharge C-rates are utilized to push the battery out of equilibrium highlighting its dynamic response. To differentiate between the voltage recovery governed by multiple time constants, test pulses 
are administered over second, minute, and hour ranges. An analysis of the voltage deviation and an exponential curve fit of the voltage recovery extracts the ohmic resistance $R_{0}$ and the associated impulse response components. The driving profile and quasi-dynamic wireless charging simulations fall within the second and minute response periods, thus the hour time constant was omitted, reducing the model to $2^{\text {nd }}$ order, as shown in Figure 11.4. To obtain the open circuit voltage $(\mathrm{OCV}) V_{o c}$, long rest periods were featured between each SOC step, during the charging and discharging cycles. Following a comprehensive test, final measurements for each component were plotted over the full SOC range and curve fitted, as shown in Figure 11.5.

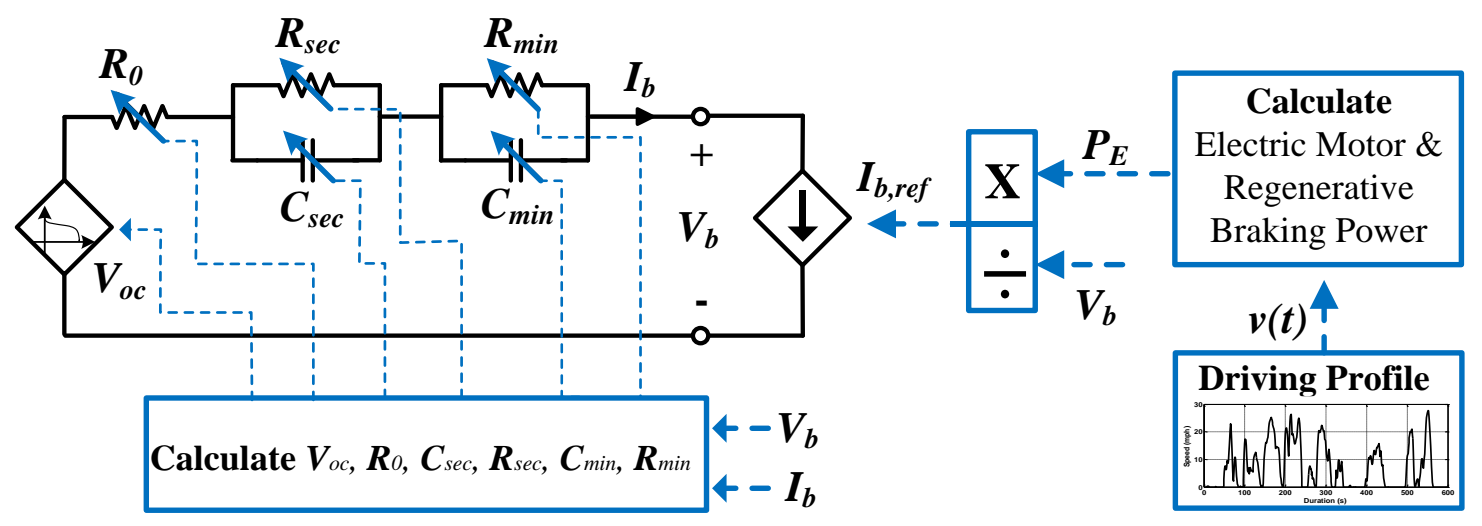

Figure 11.4. $2^{\text {nd }}$ order dynamic battery equivalent circuit model with EV traction system.

The OCV $\left(V_{o c}\right)$ and both minutes $\left(R_{\min }\right.$ and $\left.C_{\text {min }}\right)$ and seconds $\left(R_{\text {sec }}\right.$ and $\left.C_{s e c}\right)$ impulse response components produce different values dependent on whether the battery is in charging or discharging mode. The charging experimental result and best curve fits 
are shown in blue and discharging in red. $R_{0}$ does not significantly vary between charging and discharging, thus the dataset was combined. The closest fit is consistent with a $4^{\text {th }}$ order polynomial given in Equation (11-1).

$$
R_{0}(S O C)=a_{1} S O C^{4}+a_{2} S O C^{3}+a_{3} S O C^{2}+a_{4} S O C+a_{5}
$$

where, $a_{1}-a_{5}$ are the $R_{0}$ equation's coefficients, which are the same for both charging and discharging modes.
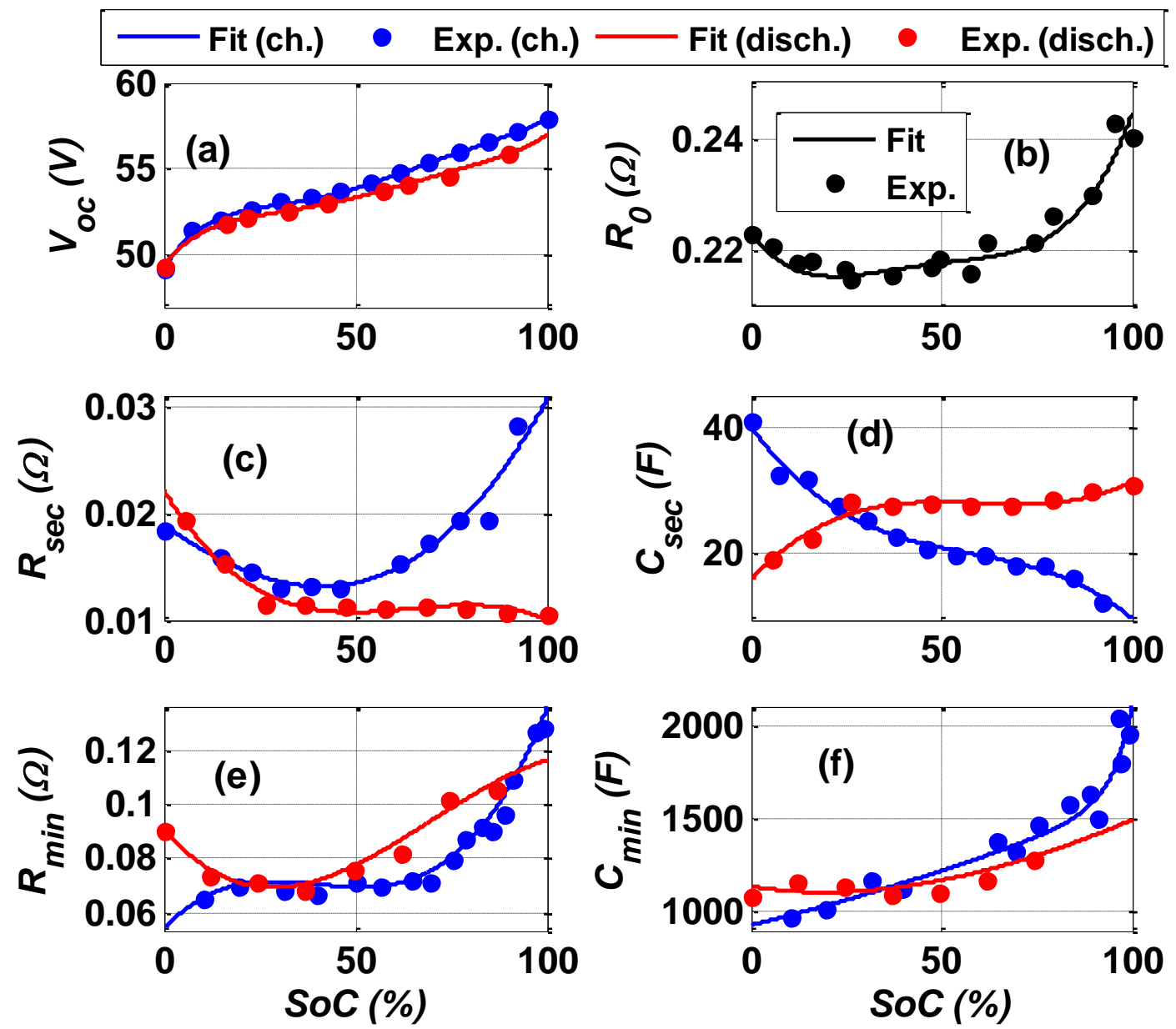

Figure 11.5. Curve fitted battery model coefficients. (a) OCV ( $\left.V_{o c}\right)$. (b) $R_{0}$. (c) $R_{s e c}$. (d) $C_{\text {sec }}$. (e) $R_{\min }$. (f) $C_{\min }$. 
For the OCV, the SOC to voltage relationship complexity warrants the need of a $5^{\text {th }}$ order polynomial, as given in Equation (11-2).

$$
V_{O C}(S O C)=a_{6} S_{O C C}^{5}+a_{7} S_{O C C}^{4}+a_{8} S_{O C C}^{3}+a_{9} S_{O C}{ }^{2}+a_{10} S O C+a_{11}
$$

where, $a_{6}-a_{11}$ represent the $V_{o c}$ 's equation's coefficients, which have different values in both the charging and discharging operation.

The curve fits of the remaining impulse response parameters over the second and minute time spans are accomplished through the formulas given in Equations (11-3)-(11$6)$.

$$
\begin{aligned}
& R_{\text {sec }}(S O C)=a_{12} S O C^{3}+a_{13} S O C^{2}+a_{14} S O C+a_{15} \\
& C_{\mathrm{sec}}(S O C)=a_{16} S O C^{3}+a_{17} S O C^{2}+a_{18} S O C+a_{19} \\
& R_{\min }(S O C)=a_{20} S O C^{3}+a_{21} S O C^{2}+a_{22} S O C+a_{23} \\
& C_{\min }(S O C)=a_{24} e^{a_{25} S O C}+a_{26} e^{a_{27} S O C}
\end{aligned}
$$

where, $a_{12}-a_{27}$ represent the parameters' coefficients, which are different between charging and discharging operation.

The model's coefficients $\left(a_{1}-a_{27}\right)$ are extracted by curve fitting the measured data, during both the charging and discharging operation, as indicated in Figure 11.5. The extracted coefficients for the chosen li-ion battery module are presented in Table 11.1. The battery module's model shown in Figure 11.4, is implemented in MATLAB Simulink, where dynamic values for each of the components are passed to variable resistances, capacitances, and a controlled voltage-source. The SOC input to each is calculated through 
the combination of the traditional current integration technique and an adjustment factor for the discharge rate $f_{\text {rate }}$, as described in Equation (11-7). This factor accounts for the adjusted capacity, as a result of the internal losses observed at high discharge currents.

Table 11.1. Charging and Discharging Coefficients of the Battery Module's Analytical Model.

\begin{tabular}{|c|c|c|c|c|c|}
\hline Coefficient & Charge & Discharge & Coefficient & Charge & Discharge \\
\hline$a_{1}$ & \multicolumn{2}{|c|}{0.29500} & $a_{15}$ & 0.01895 & 0.02218 \\
\hline$a_{2}$ & \multicolumn{2}{|c|}{-0.5399} & $a_{16}$ & -69.94 & 63.22 \\
\hline$a_{3}$ & \multicolumn{2}{|c|}{0.35810} & $a_{17}$ & 121 & -113.6 \\
\hline$a_{4}$ & \multicolumn{2}{|c|}{-0.09226} & $a_{18}$ & -81.3 & 66 \\
\hline$a_{5}$ & \multicolumn{2}{|c|}{0.22350} & $a_{19}$ & 40.12 & 15.64 \\
\hline$a_{6}$ & 78.99 & 70.98 & $a_{20}$ & 0.3397 & -0.1734 \\
\hline$a_{7}$ & -237.5 & -202.2 & $a_{21}$ & -0.4096 & 0.3648 \\
\hline$a_{8}$ & 266.3 & 218.2 & $a_{22}$ & 0.1516 & -0.1666 \\
\hline$a_{9}$ & -133.2 & -107.3 & $a_{23}$ & 0.05377 & 0.09134 \\
\hline$a_{10}$ & 34.03 & 27.89 & $a_{24}$ & $2.184 \times 10^{-6}$ & 720.8 \\
\hline$a_{11}$ & 49.24 & 49.36 & $a_{25}$ & 19.25 & 0.6925 \\
\hline$a_{12}$ & 0.01715 & -0.04911 & $a_{26}$ & 928.7 & 419.6 \\
\hline$a_{13}$ & 0.01964 & 0.09523 & $a_{27}$ & 0.5496 & -2.012 \\
\hline$a_{14}$ & -0.0251 & -0.05824 & - & - & - \\
\hline
\end{tabular}




$$
\operatorname{SOC}(t)=\operatorname{SOC}\left(t_{0}\right)-\int_{0}^{t} \frac{f_{\text {rate }}\left[I_{b}(\tau)\right]}{3600 C_{b}} d \tau
$$

where, $C_{b}$ is the nominal battery capacity $(\mathrm{Ah})$.

The final terminal voltage at the battery $\left(V_{b}\right)$ is estimated as in Equation (11-8).

$$
V_{b}=V_{o c} \pm I_{b}(t)\left[R_{0}+R_{s e c}\left(1-e^{\frac{-t}{\tau_{s e c}}}\right)+R_{\min }\left(1-e^{\frac{-t}{\tau_{\min }}}\right)\right]
$$

where, $\tau_{\text {sec }}=R_{\text {sec }} C_{\text {sec }}$, is the seconds time-constant, and $\tau_{\min }=R_{\min } C_{\min }$, is the minutes time-constant.

To verify the accuracy of the model, short (1.5 s) and long (6 min) charge and discharge current pulses were applied and compared at four different SOC levels, where the results are shown in Figure 11.6. The top plots depict the voltage response during and following discharge current pulses of $0.75 \mathrm{C}$, where the measured values at multiple SOC levels are shown in black solid lines compared to the simulation in colored dotted lines. The model reveals a close match to the experimental values, where the most variation is observed when charging from a low SOC or discharging from a high SOC. In the case of the $\mathrm{EV}$, the practical SOC operating range is between $20-80 \%$ SOC to preserve the lifespan, thus extremely low and high SOC levels are avoided in this simulation.

Using the single module model as a reference, the final configuration is expanded to the EV testing level placing seven models in series and three in parallel. Two versions were developed for simulation: one for conducting in real-time and a second time-scaled version $(0.001 \mathrm{x})$ to assist in reducing processing times for longer driving simulations. The timescaled version includes a scaling of the capacitance to scale the time constants $\tau_{\sec }, \tau_{\min }$, as well as a revised time base for the SOC calculation. 

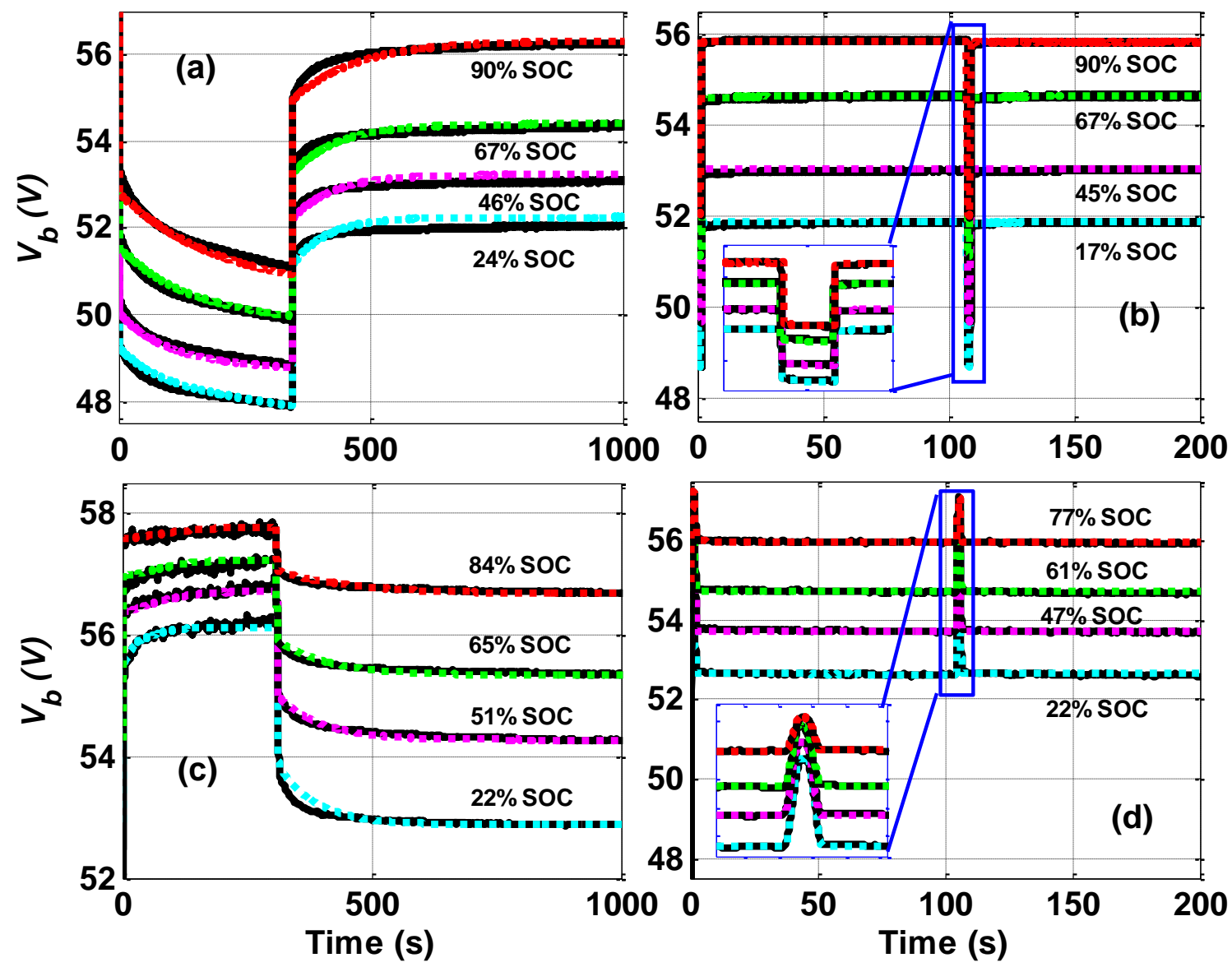

Figure 11.6. Battery model verification tests. (a) Long discharge. (b) Short discharge. (c) Long charge. (d) Short charge.

\subsubsection{EV's Drive System Model}

In this section, the specifications of the EV under test are discussed, as well as the selected driving cycles and powertrain model used to calculate the power exchange in the EV's battery system. The EV modeled in this study aligns to provisions published by the US National Highway Traffic Safety Administration (NHTSA) classified as a medium passenger car similar to the small-sized sedan with a curb weight of $1680 \mathrm{~kg}$. 
To evaluate the strength of the proposed EV BIWPT architecture, the Federal Test Procedure (FTP-72) dynamometer driving profile was selected for use in simulation. FTP72, sometimes referred to as LA4, represents a typical city driving test conditions [213]. A 7.5 mile commute is run over an approximately 22 minute period, at an average speed of $19.6 \mathrm{mi} / \mathrm{h}$ and is shown in Figure 11.7(a). The FTP-72 consists of two phases: 1) $505 \mathrm{sec}$ "cold start cycle" taking the vehicle up to a high speed (56.7 mi/h), and 2) an $867 \mathrm{sec}$ "transient" phase representing stop-and-go city driving. In the case of the EV, phase 1 presents the greatest challenge tests on the battery pack in terms of power and energy output, as the high speed driving portion reduces the availability of quasi-dynamic WPT. Phase 2 subjects the EV to frequent stop-and-go at traffic signals, where WPT will be initiated. The FTP-72 speed profile is then passed to the EV powertrain model to produce the power profile.

The EV under test is modeled to include both drive power applied to the motor, as well as account for regenerative braking recovery power. First, the resistance force $F_{v}$ of the EV at speed $v(t)$ can be calculated by the summation of aerodynamic drag, rolling resistance, and grading resistance at angle $\vartheta$, as given in Equation (11-9) [214].

$$
F_{v}(v(t))=\frac{1}{2} \rho_{a} c_{D} A_{f} v(t)^{2}+F_{R}(v(t))+g m_{t} \sin \vartheta
$$

where, $\rho_{a}, c_{D}, A_{f}$, and $m_{t}$ represent the air density $\left(1.205 \mathrm{~kg} / \mathrm{m}^{3}\right)$, drag coefficient ( 0.32$)$, frontal $\mathrm{EV}$ area $\left(2.31 \mathrm{~m}^{2}\right)$, and vehicle mass, respectively.

The rolling resistance function $\left[F_{R}(v(t))\right]$ is derived from a fifth order polynomial function, which can be found in detail with a complete list of its associated coefficients in [214]. To calculate the motor torque and speed, the wheel resistance and dynamic torque 
for acceleration are passed through the gearbox $G_{r}(6.45)$, as given in Equation (11-10).

$$
\begin{aligned}
\tau_{m}(t) & =\frac{r_{w h} F_{v}(t)}{G_{r}}+\frac{\theta_{v}}{G_{r} r_{w h}} \frac{d v(t)}{d t} \\
\omega_{m}(t) & =\frac{v(t)}{r_{w h} G_{r}}
\end{aligned}
$$

where, $r_{w h}$ and $\theta_{v}$ represent the radius of the wheels $(0.29 \mathrm{~m})$ and total vehicle inertia (145 $\mathrm{kg} \cdot \mathrm{m}^{2}$ ), respectively.

The resulting bi-directional battery power $\left(P_{E}\right)$ (see Figure 11.2) is then calculated as in Equation (11-11).

$$
P_{E}(t)= \begin{cases}\tau_{m} \omega_{m} / \eta_{m}(t), & \tau_{m}(t) \geq 0 \\ \tau_{m} \omega_{m} \eta_{m}(t), & \text { otherwise }\end{cases}
$$

where, $0<\eta_{m}(t)<1$ is the motor-inverter efficiency, which is a function of the motor speed and torque interpolated from [214].

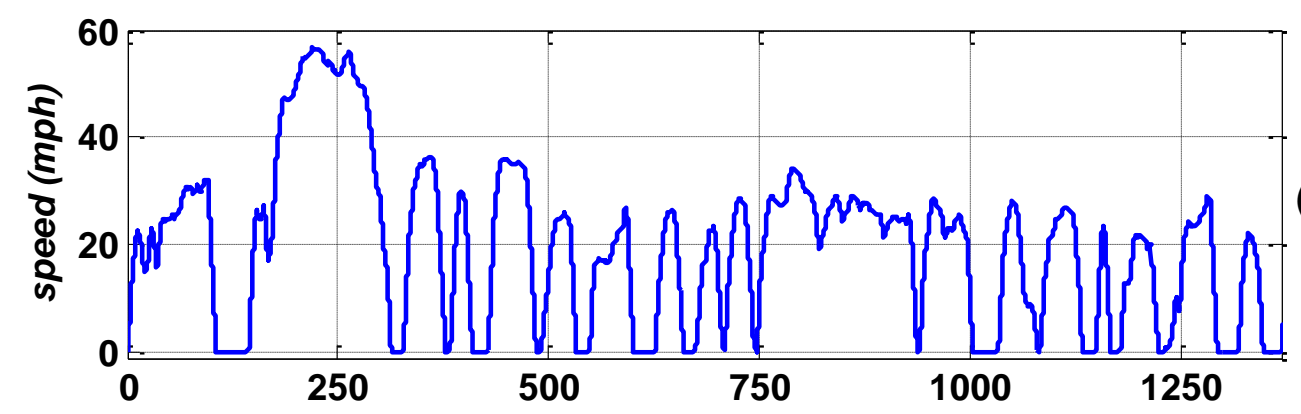

(a)

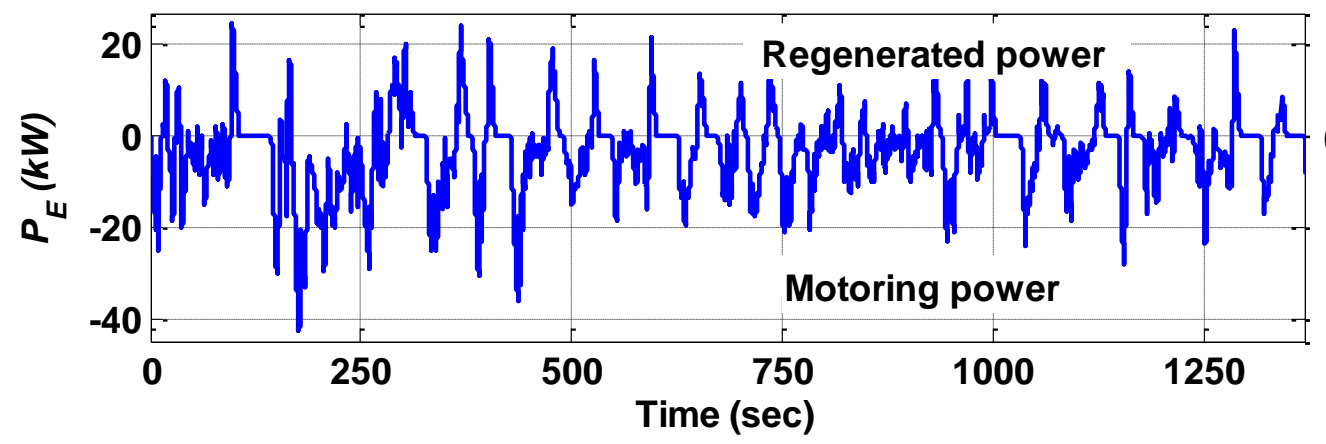

(b)

Figure 11.7. FTP-72 Driving profile. (a) Speed. (b) Electric power. 
The FTP-72 Driving profile is passed through the traction system's model given in Equations (11-9)-(11-11) to generate the resulting motoring and regenerated power profile indicated in Figure 11.7(b). This power profile is divided by the battery voltage $\left(V_{b}\right)$ to estimate the reference battery current $\left(I_{b}\right.$, ref $)$, as depicted in Figure 11.4. The reference battery current is utilized as a control signal to manage a controlled current-source that emulates the EV's traction system.

\subsubsection{Model Verification}

The Simulink model of an LCL BIWPTS presented in Figure 5.25 is utilized and linked with the battery and the drive system models presented above, to form a comprehensive simulation platform for the wirelessly connected EV shown in Figure 11.2. The small-scale LCL BIWPTS prototype shown in Figure 5.23 is utilized to verify the proposed simulation platform. The prototype contains one module of the $51.8 \mathrm{~V} 21 \mathrm{Ah}$ lithium-ion battery pack to emulate the EV's behavior, which is the same module that is considered in the model. The system is analyzed in both charging $(\mathrm{G} 2 \mathrm{~V})$ and discharging (V2G) operation and compared with the simulated model where the results are presented in Figures 5.25-5.27.

The experimental and simulated models of the BIWPTS are designed and analyzed for $40 \mathrm{kHz}$ resonant frequency, which is different from the defined frequency in the SAE J2954A standard $(81.38-90 \mathrm{kHz})$ [149]. However, this study focused on the impact of implementing a QDWPT system on the driving performance in terms of driving miles and hours. This performance is affected only by the power level of charging and discharging. As long as the WPT system was able to transfer the required power level, at any frequency, 
these results will not change. A different resonant frequency for the same power level leads to different design parameters. In order to clarify this point, a simulation-based analysis is conducted. The analysis compares between the performances of two LCL BIWPTSs with two different resonance frequencies: $40 \mathrm{kHz}$ and $85 \mathrm{kHz}$. The two systems are designed to supply the same power level $(11.1 \mathrm{~kW})$. As can be noticed in Table 11.2 , the two systems have different design parameters, in order to be able to transfer the same amount of power.

Table 11.2. Design Parameters of an $11.1 \mathrm{~kW}$ LCL BIWPTS at 40 and $85 \mathrm{kHz}$.

\begin{tabular}{lc|lc}
\hline \hline \multicolumn{1}{c|}{ 40 kHz design } & \multicolumn{2}{c}{ 85 kHz design } \\
\hline Parameter & Value & Parameter & Value \\
\hline$L_{p c}=L_{p i}=L_{s i}=L_{s c}$ & $25.5 \mu \mathrm{H}$ & $L_{p c}=L_{p i}=L_{s i}=L_{s c}$ & $12 \mu \mathrm{H}$ \\
$R_{p c}=R_{p i}=R_{s i}=R_{s c}$ & $30 \mathrm{~m} \Omega$ & $R_{p c}=R_{p i}=R_{s i}=R_{s c}$ & $20 \mathrm{~m} \Omega$ \\
$C_{p}=C_{s}$ & $0.62 \mu \mathrm{F}$ & $C_{p}=C_{s}$ & $0.292 \mu \mathrm{F}$ \\
\hline \hline
\end{tabular}

The higher frequency system $(85 \mathrm{kHz})$ provides simpler passive components' design (lower pad self-inductances, filter inductances and compensation capacitors). The performance of both designs ( $40 \mathrm{kHz}$ and $85 \mathrm{kHz}$ ) is analyzed during $\mathrm{G} 2 \mathrm{~V}$ operation and compared in Figures 11.8 and 11.9, respectively. As can be observed, the two systems show very similar voltage and current waveforms. Thus, it can be concluded that using the 40 $\mathrm{kHz}$ system's design to evaluate the simulation platform is applicable since the amount of power-transfer is only matter. 


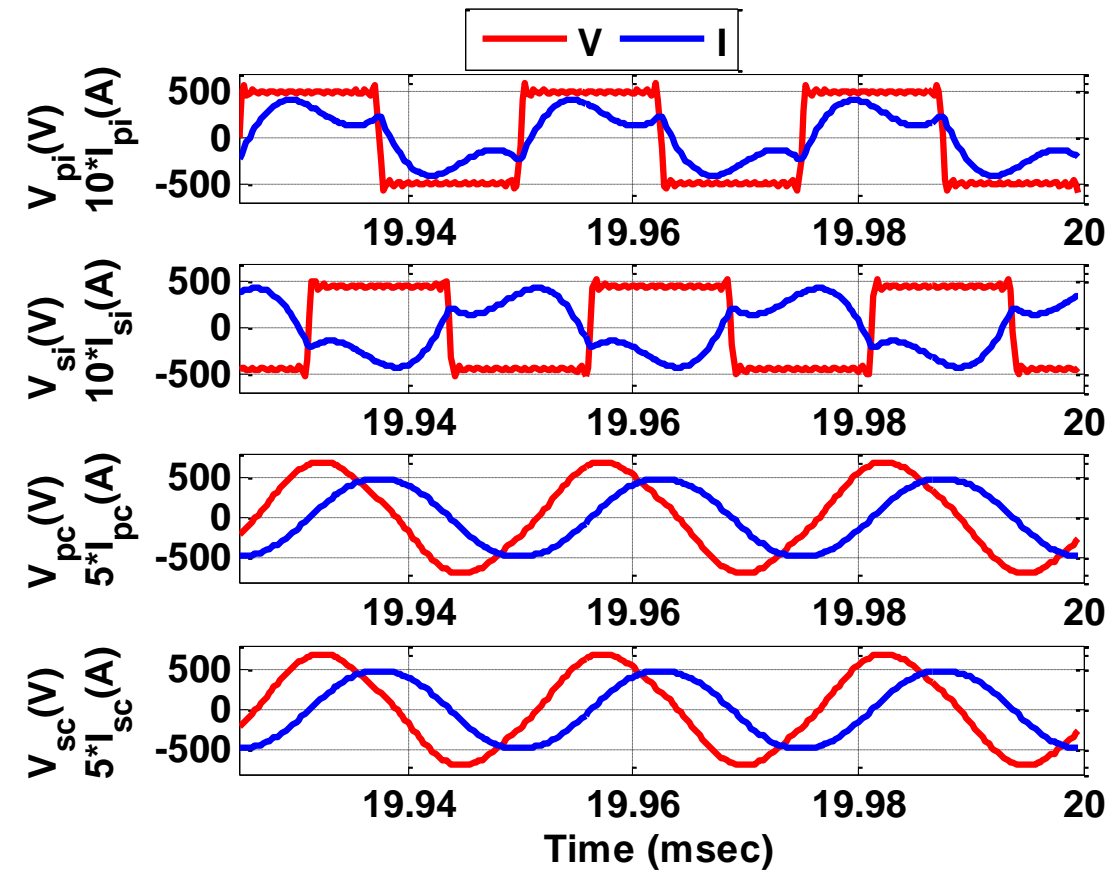

Figure 11.8. BIWPTS's performance at $40 \mathrm{kHz}$ resonant frequency, during G2V operation $\left(\alpha=\beta=180^{\circ}, \delta=-90^{\circ}\right)$.

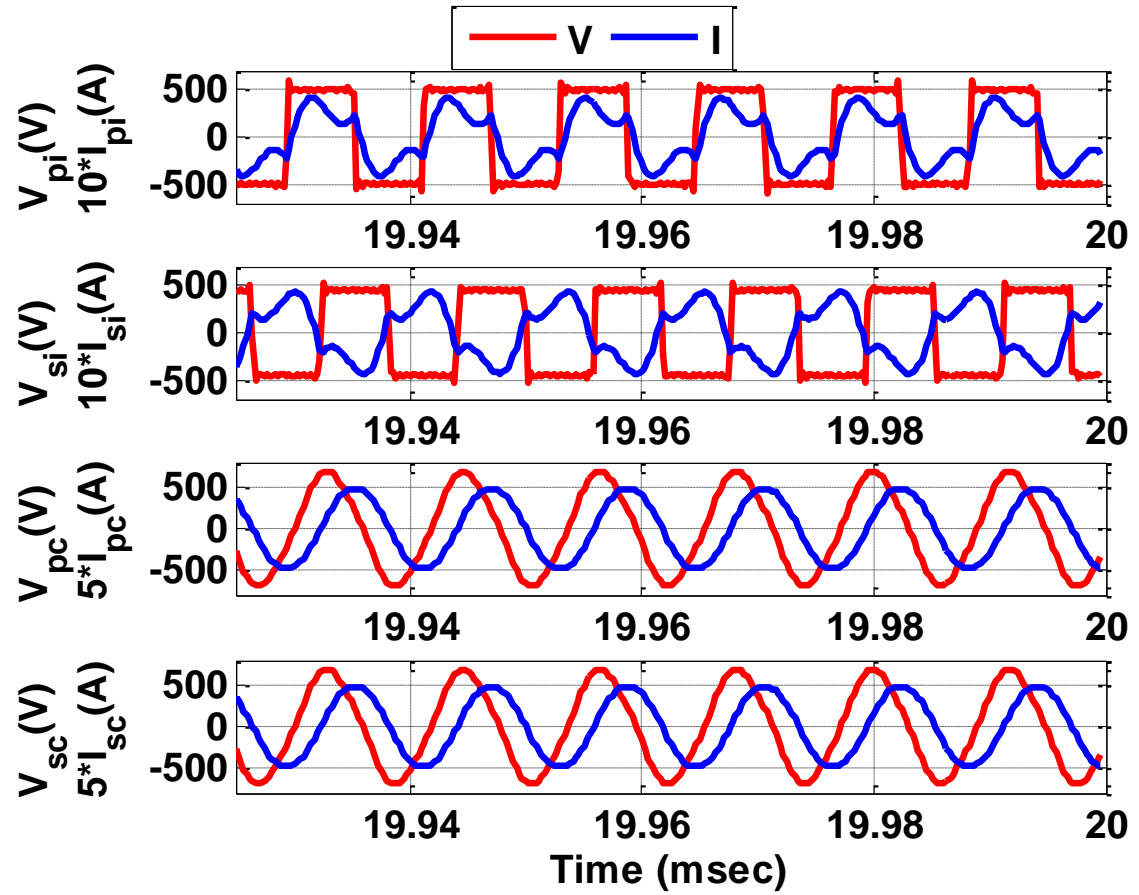

Figure 11.9. BIWPTS's performance at $85 \mathrm{kHz}$ resonant frequency, during G2V operation $\left(\alpha=\beta=180^{\circ}, \delta=-90^{\circ}\right)$. 


\subsection{Feasibility Analysis of Implementing QDWPT Systems at Traffic Signals}

After modeling and verification of the small scale prototype of the wirelessly connected EV, a large scale model has been used to evaluate the effectiveness of implementing the QDWPT system at traffic signals. This implementation will allow EVs to charge and discharge during the stop time. The feasibility of this project from the driving performance and the consumed energy point of view is presented in this section. The FTP72 driving profile is considered in this analysis. Three different charging scenarios have been investigated: fixed power charging, variable power charging, and fixed power charging and discharging operation.

\subsubsection{Fixed Power Charging Scenario}

In this scenario, it is assumed that the EV charging will start automatically once the vehicle stops at the traffic signal. The charging power is kept fixed by the power-flow controller of the BIWPTS. The control system incrementally adjusts the battery current to maintain the target power, while keeping the system power factor very close to unity due to the resonance operation. Four different standard charging levels are analyzed: WPT1=3.7 kVA, WPT2=7.7 kVA, WPT3=11.1 kVA, and WPT4=22 kVA, based on the SAE J2954A international standard. The whole driving performance during the WPT2 charging level is indicated in Figure 11.10. As can be noticed, the charging operation is initiated during the stop time of the vehicle only (zero speed period). In this level, the charging power is about $7.7 \mathrm{~kW}$ [Figure 11.10(c)]. The behavior of the EV's battery SOC during the entire driving profile is indicated in Figure 11.10(d). The EV starts with $80 \%$ initial SOC $\left(S O C_{i}\right)$, which decreases with driving. Due to the charging energy during the 
stops, the reduction in the SOC is less than what is expected, as will be indicated later. The EV's battery current, including the motoring, regenerated and charging current is shown in Figure 11.10(e). Both the charging and the regenerative currents are negative, while the motoring current is positive.

A second test is analyzed for a fixed charging scenario with WPT4 $(22 \mathrm{~kW})$ power transfer, and the results are indicated in Figure 11.11. The figure shows the speed profile, battery voltage, WPT charger current, WPT charger power and SOC, over the entire FTP72 driving cycle. Also, a zoom of all fields, during a 25-second traffic intersection stop occurring between 1005 and 1030 seconds, is depicted in the same figure. It can be noticed that the battery voltage steadily rising, while the current is being incrementally reduced to maintain a constant power charging level of $22 \mathrm{~kW}$. Also, the figure shows that after a driving cycle, the reduction in the SOC is less than the case of WPT2 level in Figure 11.10.

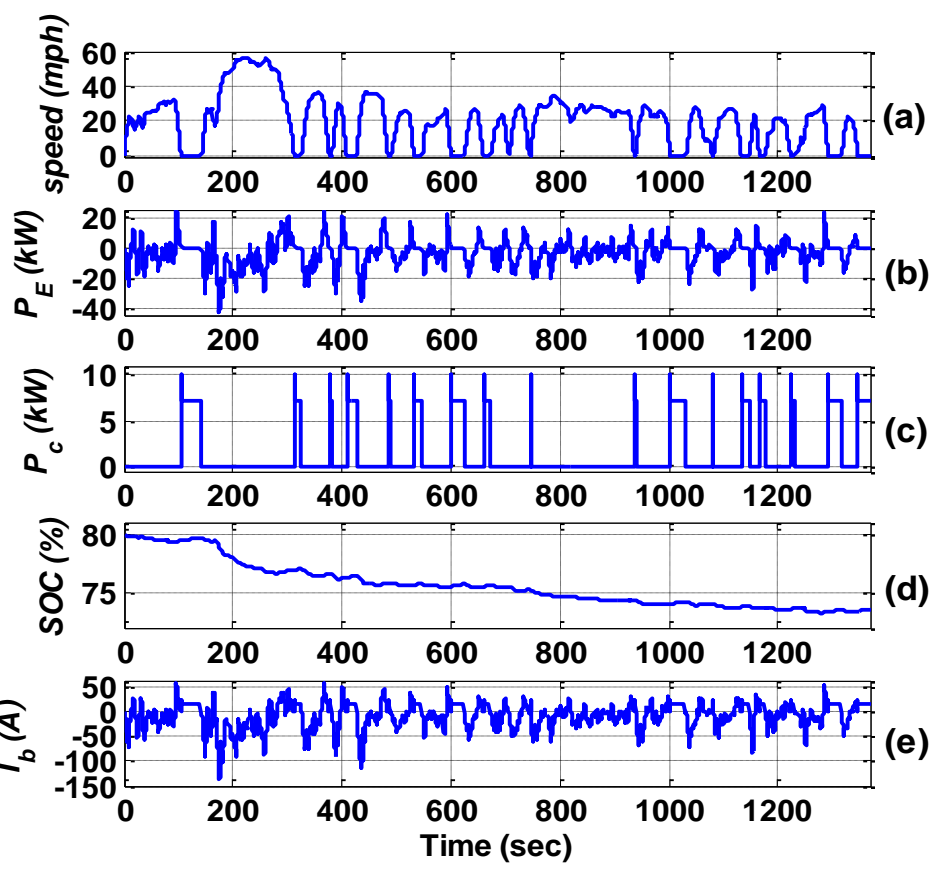

Figure 11.10. Driving performance under fixed power charging WPT2 $\left(S O C_{i}=80 \%\right)$. (a) Speed. (b) Vehicle power. (c) Charger power. (d) SOC. (e) Battery current. 

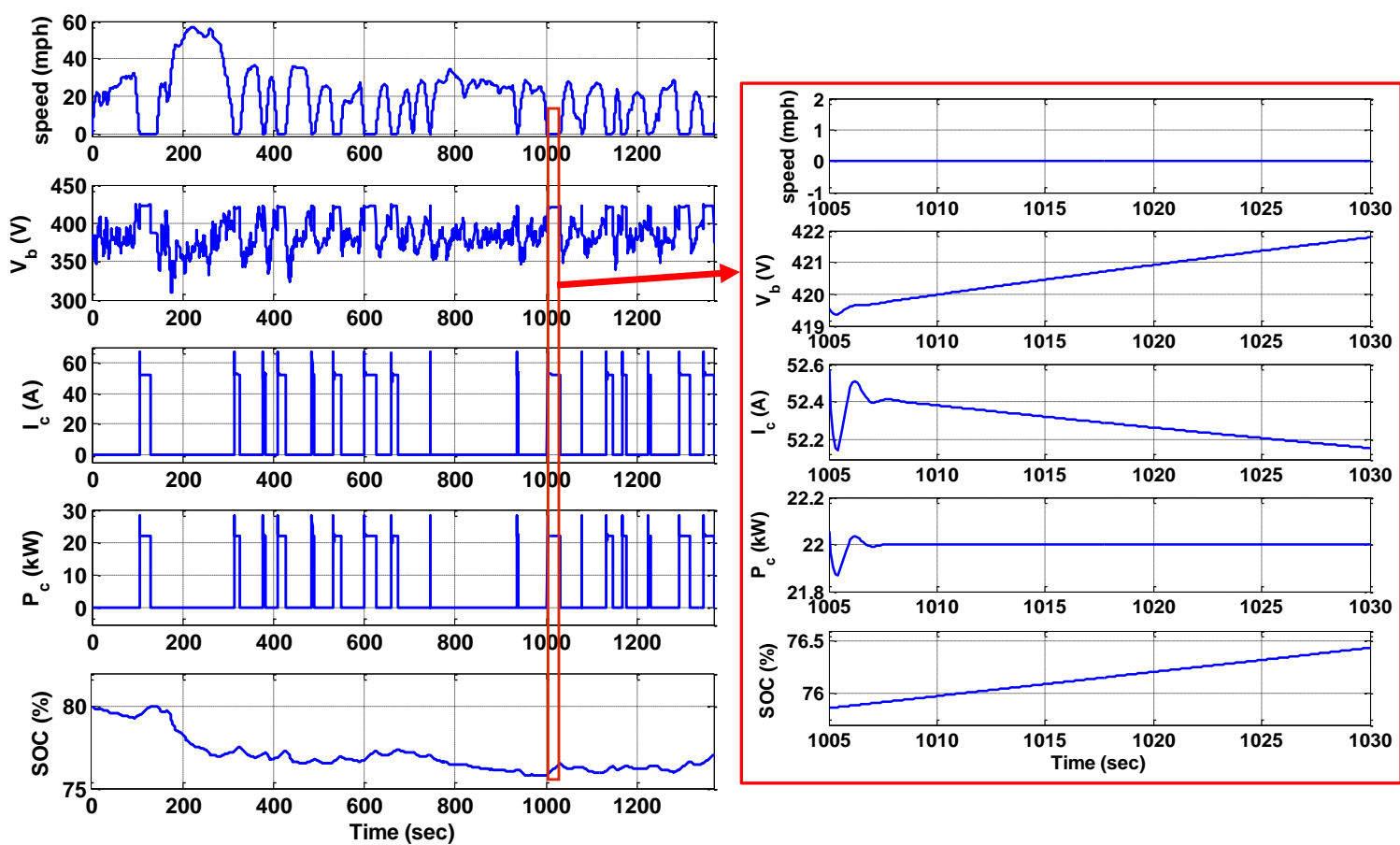

Figure 11.11. Driving performance under fixed power charging WPT4 (SOC $i=80 \%)$. (a) Speed. (b) Battery voltage. (c) Charger current. (c) Charger power. (d) SOC.

Similar to the analyses in Figures 11.10 and 11.11, this test has been conducted for the other standard charging levels, and the driving performance is compared with the case while there is no QDWPT charging, as shown in Figure 11.12. The figure shows the EV's battery SOC through the driving period without and with implementing the WPT charger at the traffic signal. It can be observed that through the utilization of QDWPT charging, the driving range is extended as a function of the charging power level for the same stop time. All charging levels exhibit a shallower reduction in the SOC, where WPT4 results in only a $3 \%$ SOC reduction at the end of the driving cycle. WPT4 and higher levels appear promising for these applications since the vehicle may recover the initial SOC by the end of the driving profile. 


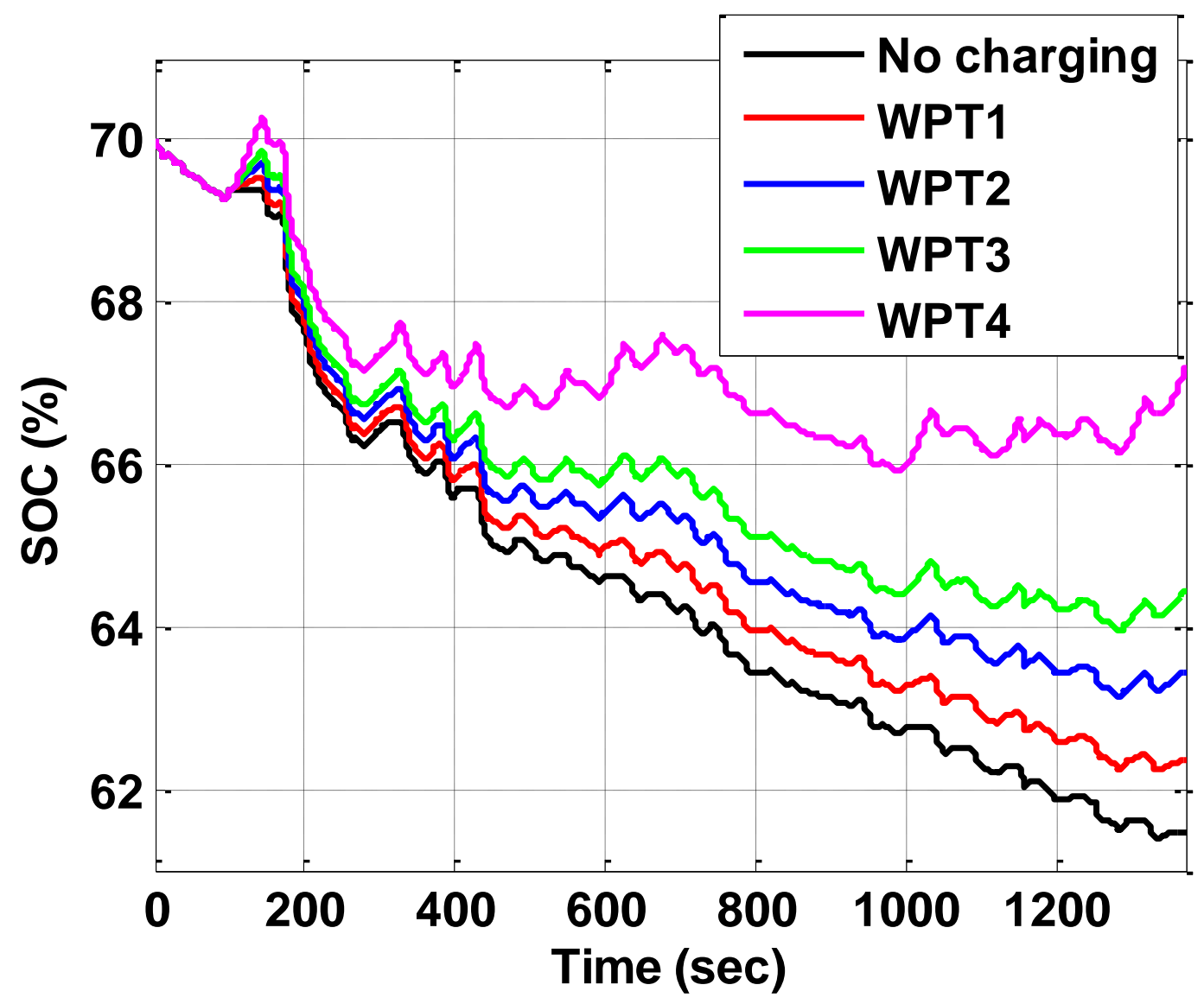

Figure 11.12. Driving performance at different charging levels $\left(S O C_{i}=70 \%\right)$.

\subsubsection{Variable Power Charging Scenario}

In this case, the charging power rate varies automatically based on the EV's SOC. It is desirable that an EV that has a lower SOC should be permitted to charge at a higher charging rate, while as the SOC increases, the rate decreases linearly, as depicted in Figure 11.13. In this figure, $P_{\min }$ and $P_{\max }$ represent the minimum and maximum acceptable limits of the charging rate, which are defined based on the charger limits. $S O C_{\min }$ and $S O C_{\max }$ denote the SOC range, in which the power is adjusted where below $S O C_{\min }$, a constant 
$P_{\text {max }}$ is supplied while above $S O C_{\max }$, charging is deactivated. This behavior would help aid in limiting utility grid stress, while simultaneously prioritizing charging amongst EVs and preserving their battery lifetimes, by greatly reducing the charging rate when the battery approaches the maximum SOC level [215].

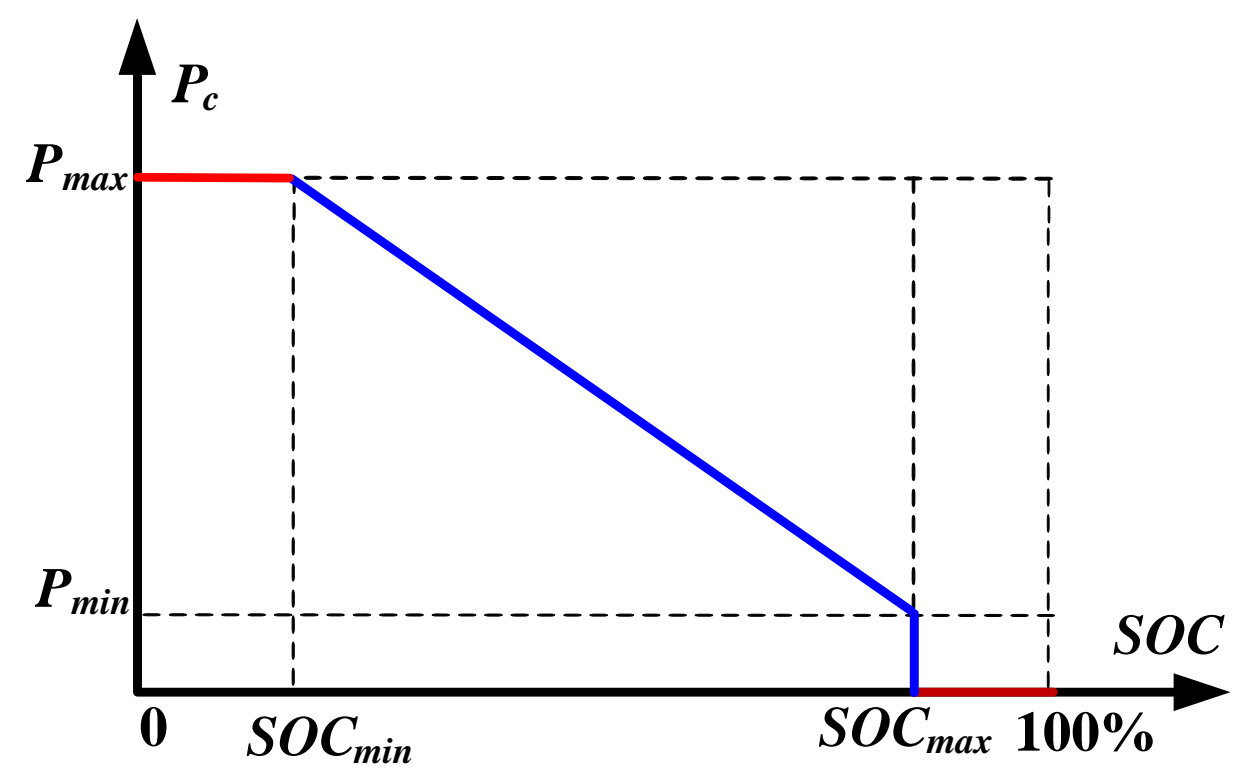

Figure 11.13. Charging rate decision based on SOC.

In this study, we have considered a safe linear $\mathrm{SOC}$ operating range of $S O C_{\min }=$ $30 \%$ to $S O C_{\max }=80 \%$, where $P_{\max }$ is set to the WPT4 level, and the minimum supported charging level $\left(P_{\min }\right)$ is set to $30 \%$ of $P_{\max }$. WPT4 is chosen since it has shown promising performance in this application, as was indicated in Figure 11.12. The charging rate adjustment is achieved by the secondary side controller shown in Figure 11.2. The driving performance under this scenario is indicated in Figure 11.14. In this case, the FTP-72 
profile is extended by multiple repetitions to cover the full operating SOC range (80-20\%). It can be noticed that the charging rate starts with $P_{\min }$ at $80 \%$ SOC increasing linearly as the SOC decreases. Once the SOC reaches $30 \%$, the charger supplies $P_{\max }$ for the remaining period. By applying this algorithm, the driving time is extended by more than 5 hours.

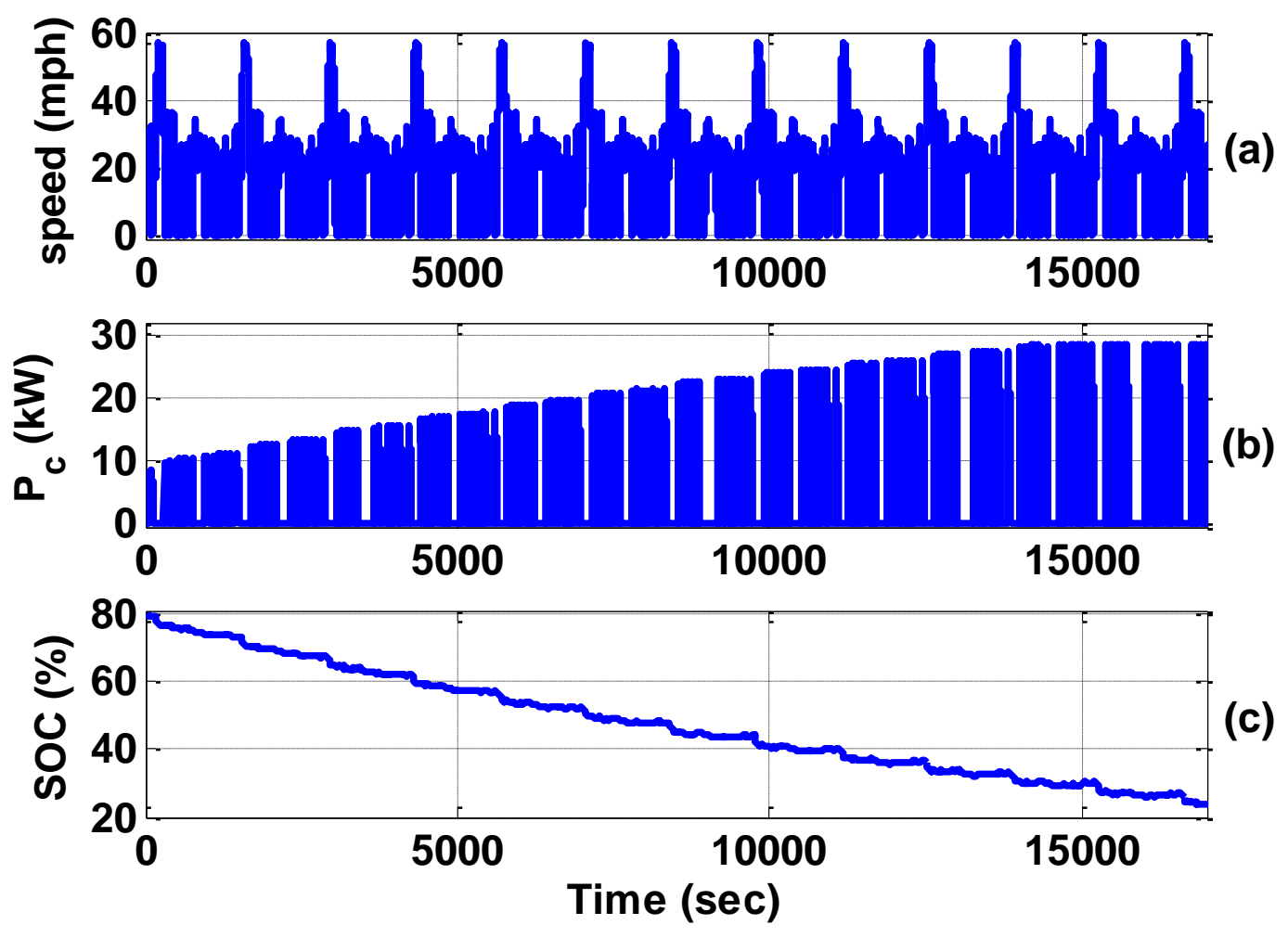

Figure 11.14. Driving performance under variable power charging level $\left(S O C_{i}=S O C_{\max }\right.$ $=80 \%, S O C_{\min }=30 \%$ ). (a) Speed. (b) Charger power. (c) Battery SOC.

\subsubsection{Fixed Power Charging and Discharging Scenario}

This scenario is applied to identify the ability of the QDWPT system to achieve V2G 
and G2V operation. In this case, the EV can charge and discharge during the stop time at the traffic signal with a fixed level of WPT4. The charging and discharging decision is made by comparing the EV's psychological price $(R)$ (the maximum acceptable price) with the grid retail price of the wireless charger $\left(R^{\prime}\right)$, where $R$ depends on the EV's battery SOC, as was presented in Figure 10.6(a) and Equation (10-14).

The wireless charger retail price $\left(R^{\prime}\right)$, should be more than that of the base load, which is 4 cents $/ \mathrm{kWh}$ [200]. Thus, $R^{\prime}$ is set to change from 4.5 to 6.5 cents $/ \mathrm{kWh}$. The retail price for wireless charging of a road is available for the EV owners based on the current traffic information systems of Intelligent Transportation Systems (ITS) [200]. The switching between charging and discharging operation is achieved by the vehicle-side controller after the EV's driver accepts. The controller compares the psychological price with the retail price of the road. If $R$ is higher than $R^{\prime}$, the EV charges the battery; and if $R$ is less than $R^{\prime}$, the EV discharges the battery. This procedure is applied using the same SOC range as was established in the previous section. Over $80 \%$, the EV will discharge in order to avoid overcharging, and below 30\% the EV will keep charging.

The driving performance during this scenario is indicated in Figure 11.15. These results are obtained by assuming the retail price $R^{\prime}=5.5$ cents $/ \mathrm{kWh}$, and the maximum and the minimum accepted EV psychological price is 6.5 and 4.5 cents $/ \mathrm{kWh}$, respectively. It can be noticed, at high SOC ( $\geq 55 \%), R>R$ ', thus the vehicle discharges and the SOC drops dramatically [Figure 11.15(c)]. When the SOC goes below a certain value, $R$ becomes less than $R^{\prime}$ and the BIWPTS starts to charge the battery. These results will vary dynamically as the retail price changes. 

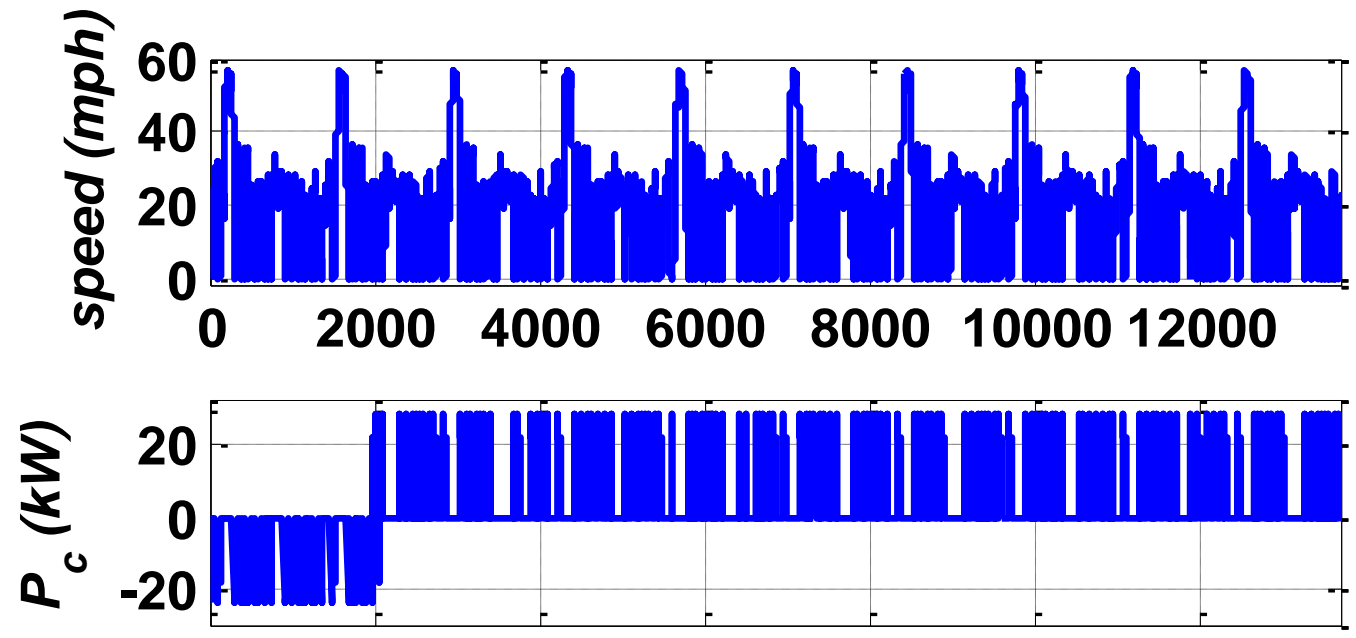

\section{0}

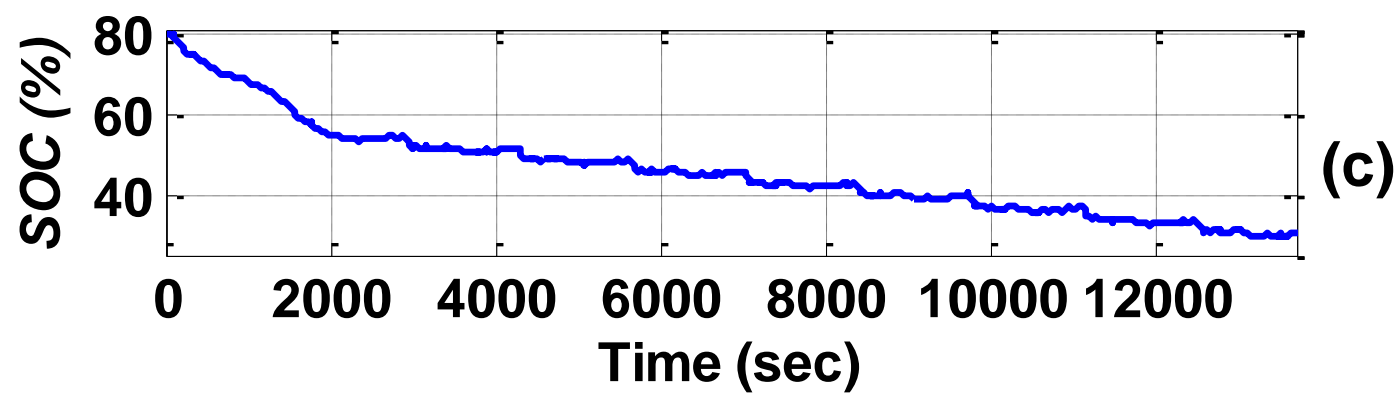

Figure 11.15. Driving performance under charging and discharging operation (WPT4, $S O C_{i}=80 \%, P^{\prime}=5.5$ cents/kWh). (a) Speed. (b) Charger power. (c) SOC.

\subsubsection{Driving Cycle Performance Evaluation}

For a more clear driving performance assessment, the driving range for all the different scenarios are evaluated and compared with the case where there is no charging at all. This range is estimated based on two factors: full cycle time $(F C T)$ [maximum continuous driving time per battery cycle (80-20\%)], and miles per cycle (mpc) (maximum driving distance per cycle). Since these factors are functions of the initial SOC $\left(S O C_{i}\right)$, they are estimated at different $S O C_{i}$, as indicated in Figure 11.16. As can be shown, 
implementing the QDWPT technology at the traffic signal extends the driving range (time and distance) for the whole range of $S O C_{i}$. Using the WPT4 fixed charging level, the driving range is tripled compared to the no-charging case. The automatic variable charging (WPTAC) and the automatic charging and discharging (WPTACD) scenarios show significant improvement in the driving range as well. This analysis is achieved under the assumption that the WPT system is working with perfect alignment between the two power pads, and the system is able to transfer the full power capacity.
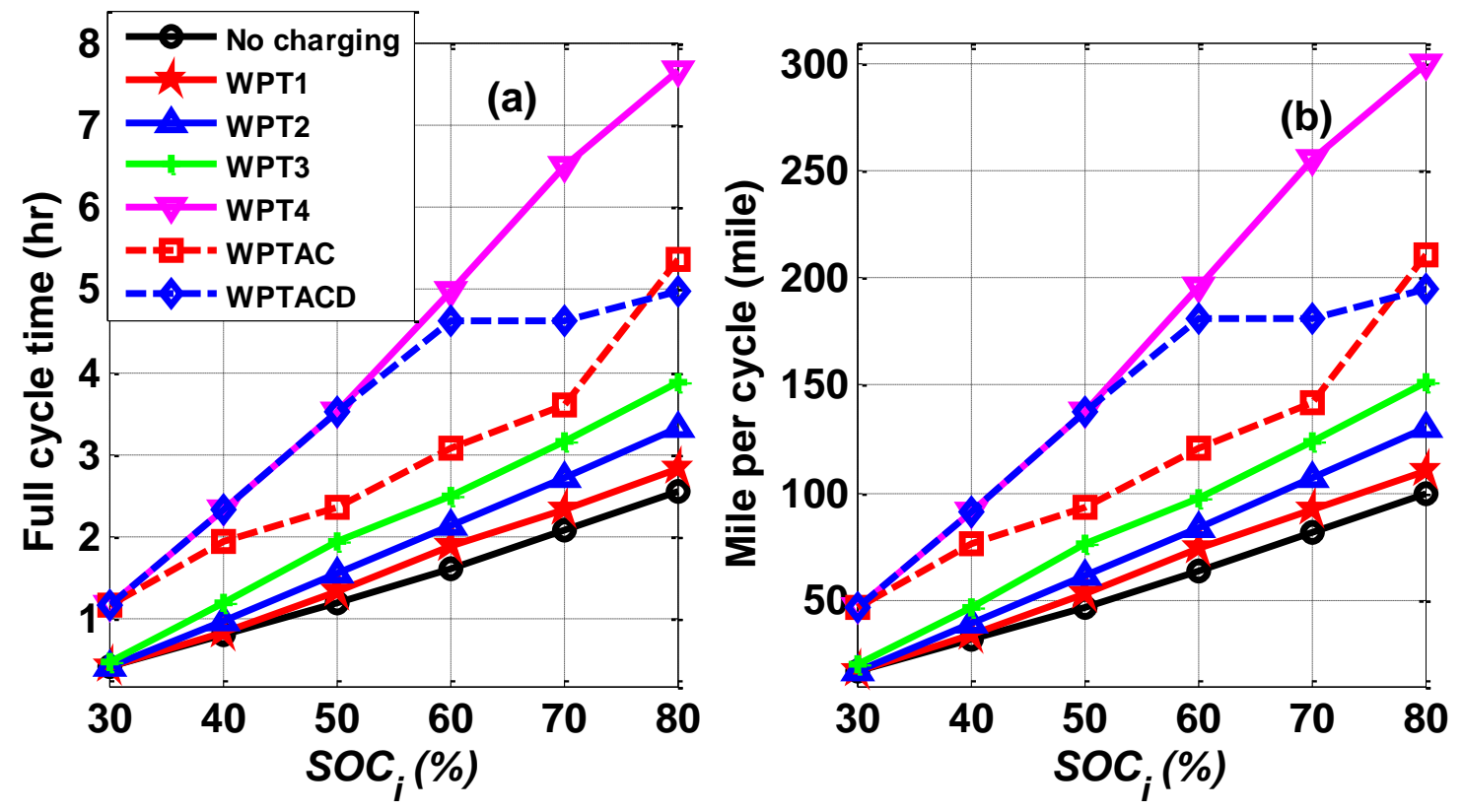

Figure 11.16. Driving range for all different scenarios. (a) Full cycle time $(F C T)$. (b) Maximum miles per cycle ( $m p c)$.

Logically, increasing the charging power level will result in increasing the stored energy in the battery for the same charging period, resulting in an improvement in the driving range. However, at the same time, the energy consumption and cost will increase. 
Thus, for fair evaluation among the different scenarios, a performance index is introduced in this work. A benefit measure factor has been deduced $(b . f)$ for all scenarios. This factor evaluates the extra $m p c$ that the wireless charging adds to the no-charging case over the average consumed energy per cycle (kWh), as given in Equation (11-12).

$$
b . f=\frac{\left.m p c\right|_{\text {with charger }}-\left.m p c\right|_{\text {without charger }}}{\text { Average Consumed Energy per cycle }} \quad(\text { miles } / k W h)
$$

This factor is estimated for all scenarios and compared at different $S O C_{i}$, as presented in Table 11.3.

Table 11.3. Benefit Factor Evaluation for all Scenarios (miles/kWh).

\begin{tabular}{lllllll|l}
\hline \hline \multirow{2}{*}{ Scenario } & $\mathbf{8 0 \%}$ & $\mathbf{7 0 \%}$ & $\mathbf{6 0 \%}$ & $\mathbf{5 0 \%}$ & $\mathbf{4 0 \%}$ & $\mathbf{3 0 \%}$ & Average \\
& $\boldsymbol{S O C}_{\boldsymbol{i}}$ & $\boldsymbol{S O C}_{\boldsymbol{i}}$ & $\boldsymbol{S O C}_{\boldsymbol{i}}$ & $\boldsymbol{S O C}_{\boldsymbol{i}}$ & $\boldsymbol{S O C}_{\boldsymbol{i}}$ & $\boldsymbol{S O C}_{\boldsymbol{i}}$ & (mile/kWh) \\
\hline WPT1 & 6.5583 & 7.5684 & 9.7528 & 7.4983 & 3.4248 & 1.2626 & 6.0109 \\
WPT2 & 7.1885 & 7.2134 & 7.1898 & 7.3160 & 5.7584 & 1.3605 & 6.0044 \\
WPT3 & 6.8806 & 6.8902 & 6.9911 & 7.7204 & 6.4914 & 3.1659 & 6.3566 \\
WPT4 & 7.1200 & 6.8886 & 6.8183 & 6.6669 & 6.6056 & 6.5272 & 6.7711 \\
WPTAC & 6.7995 & 6.7651 & 7.0121 & 6.6713 & 6.7812 & 6.4574 & 6.7478 \\
WPTACD & 6.1799 & 6.4199 & 6.6774 & 6.6357 & 6.5294 & 6.4116 & 6.4755 \\
\hline \hline
\end{tabular}

For WPT1, WPT2 and WPT3, $b$. $f$ changes dramatically with different $S O C_{i}$. Smaller benefits are observed at low $S O C_{i}$, and high benefits at high $S O C_{i}$. On the other hand, 
WPT4, WPTAC, and WPTACD show flat and high benefits over the entire $S O C_{i}$ range. By calculating the average $b . f$, these three scenarios provide the highest benefits, regardless of $S O C_{i}$. Notice the benefit factor in the case of WPTACD is dynamic based on the retail price, which is 5.5 cents/kWh in this case. These results can be supplied to the power-flow controller to decide the most beneficial scenario based on the initial SOC.

\subsection{Impact of Misalignment on the Feasibility Analysis}

One of the main practical limitations of the WPT system is the alignment between the two sides, which is significantly dependent on the primary pad structure. Based on the literature [82], there are two main visions for implementing the primary pad in dynamic WPT systems. The first vision is by burying a long track coupler in the road, in which an EV is running along with the track and continues power is transferred, as shown in Figure 11.17 [216]. The problem of the long track design is that the pickup coil only covers a small portion of the track, which makes the coupling coefficient very small. Poor coupling generates efficiency and EMI concerns. In addition, this structure shows serious issues related to the safety of the creatures crossing the road, while the primary circuit is being energized.

The second vision is to implement a string of pads near to each other similar to the one utilized in the stationary charging, as depicted in Figure 11.18 [217]. Each pad can be driven by an independent power converter, or one converter can be utilized to drive a number of pads, with controlling the current in each pad. Thus, the primary pads can be selectively excited without a high-frequency common current. Also, the energized primary pads are covered by the vehicle, thus safer operation is experienced. The efficiency and 
EMI performance can be as good as that in a stationary charging application, however, with a tradeoff in higher cost.

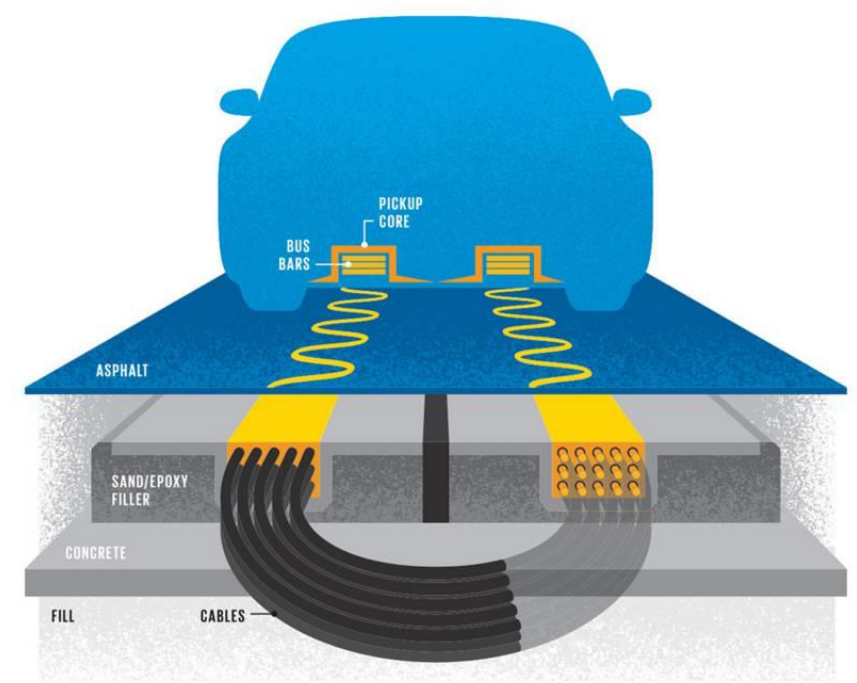

Figure 11.17. Long track primary coupler.

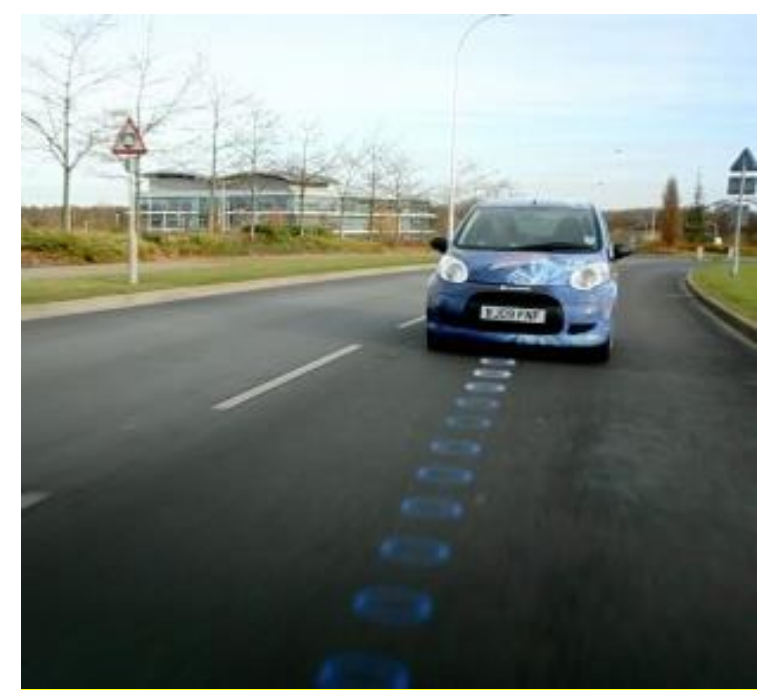

Figure 11.18. String of pads primary coupler. 
The author believes that the second vision is more appropriate for the quasi-dynamic WPT system, since it can handle both the stationary and dynamic operation. By considering the second vision, it can be concluded that different sizes and geometries of the vehicle should not introduce significant misalignment effect. The expected misalignments will result from the stop position, which can be minimized by implementing an automatic guiding system.

Therefore, during the transient stops, it is expected that the EV will experience some misalignments, during charging and discharging operation, resulting in a considerable deviation from the ideal case that is assumed in the previous feasibility analysis. This phenomenon has been considered and presented in this section. The misalignment range is defined based on the J2954A standard, in which the accepted range of variation of coupling factor in the $\mathrm{Z} 2$ class is defined as 0.082 to 0.215 [149]. A reflection of this range on the power transfer is evaluated using the system model to be from approximately $40 \%$ to $100 \%$ of the associated power level capacity. Therefore, the misalignment is expected to vary randomly within this range.

The driving performance considering the misalignment is investigated during fixed power charging at the WPT4 level in Figure 11.19. This figure shows the charging power and the battery SOC over the whole driving profile for three cases: (1) Aligned, when the two sides are perfectly aligned and $P_{c}=22 \mathrm{~kW}$, (2) Worst Misalignment, when the EV experiences the maximum misalignment and $P_{c}=0.4 \times 22 \mathrm{~kW}$, and (3) Random Misalignment, in which the degree of misalignment is haphazardly assigned at each stop, based on a uniform distribution random variable. As can be noticed, the misalignment negatively affects the driving performance. For clear evaluation of this effect, the $F T C$, 
$m b c$ and $b . f$ are estimated for all previously mentioned scenarios at $S O C_{i}=80 \%$, as presented in Table 11.4. Considering the misalignment, WPT1 is revealed to be impractical in terms of performance for $F T C$ and $m b c$, since it gives similar results to the case with no charging. With WPT4, a significant drop is experienced in the driving range, however, it still provides almost double the case without charging. With implementing an automatic guiding system, it is expected that the driving performance to be very similar to the perfect alignment case.

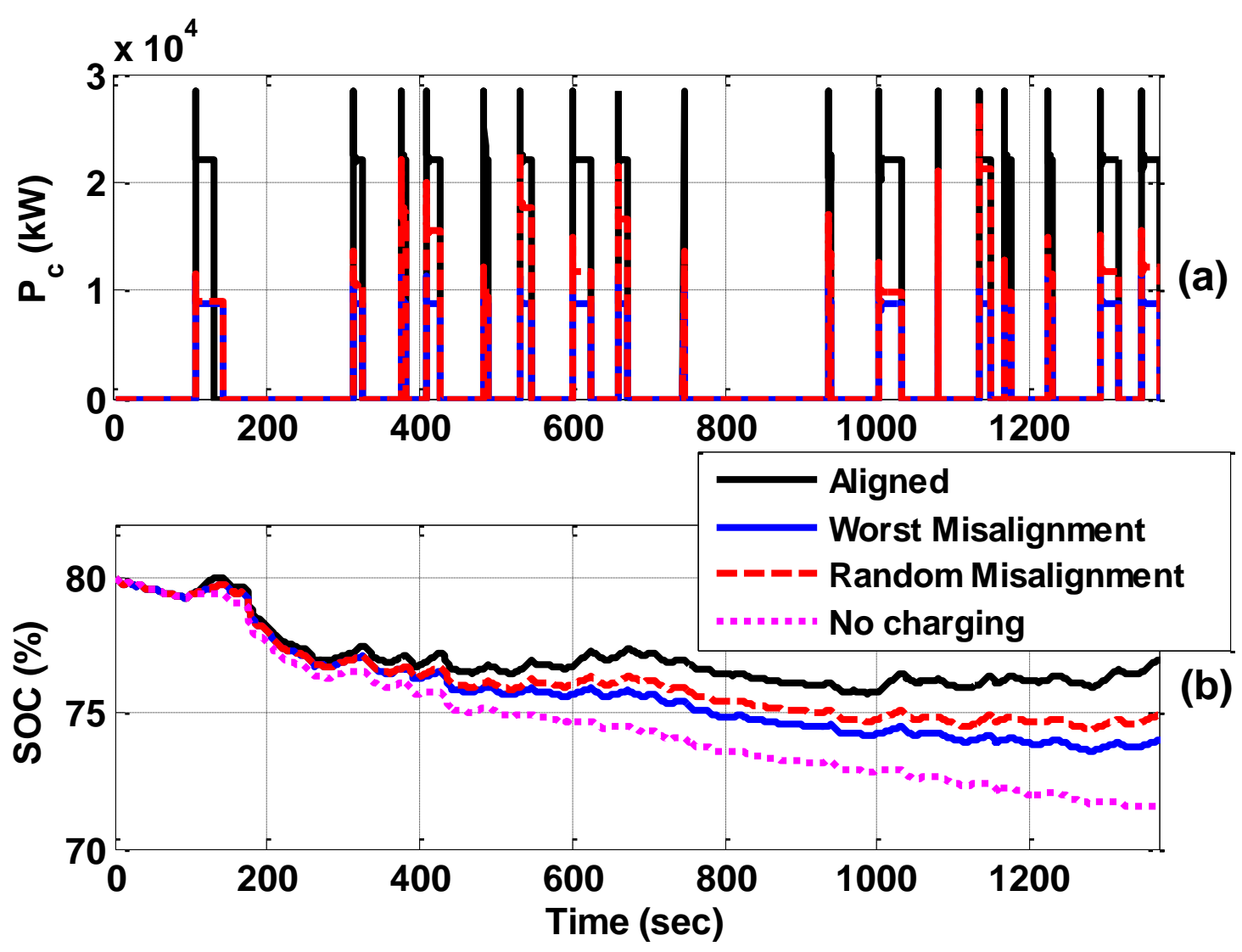

Figure 11.19. Driving performance considering a misalignment (WPT4, $S O C_{i}=80 \%$ ). (a) Charging power. (b) Battery SOC. 
Table 11.4. Driving Performance Considering Misalignment.

\begin{tabular}{|c|c|c|c|c|c|c|c|c|c|}
\hline \multirow[b]{2}{*}{ Scenario } & \multicolumn{3}{|c|}{ Aligned } & \multicolumn{3}{|c|}{ Worst Misalignment } & \multicolumn{3}{|c|}{ Random Misalignment } \\
\hline & $\begin{array}{l}F C T \\
(\mathbf{h r})\end{array}$ & $\begin{array}{c}\text { mpc } \\
\text { (miles) }\end{array}$ & $b . f$ & $\begin{array}{l}F C T \\
(\mathbf{h r})\end{array}$ & $\begin{array}{c}\text { mpc } \\
\text { (miles) }\end{array}$ & $b . f$ & $\begin{array}{r}F C T \\
(\mathbf{h r})\end{array}$ & $\begin{array}{l}\text { mpc } \\
\text { (miles) }\end{array}$ & $b . f$ \\
\hline WPT1 & 2.82 & 110.5 & 6.56 & 2.71 & 106.2 & 6.14 & 2.72 & 106.7 & 6.44 \\
\hline WPT2 & 3.33 & 130.5 & 7.18 & 2.77 & 108.64 & 6.21 & 3.1 & 121.14 & 7.81 \\
\hline WPT3 & 3.87 & 151.84 & 6.88 & 3.01 & 117.88 & 7.73 & 3.375 & 132.25 & 7.28 \\
\hline WPT4 & 7.67 & 300.4 & 7.12 & 3.492 & 136.8 & 6.814 & 4.99 & 195.37 & 7.01 \\
\hline WPTAC & 5.375 & 210.6 & 6.7995 & 3.164 & 124 & 6.78 & 3.994 & 156.5 & 6.79 \\
\hline WPTACD & 4.98 & 195.3 & 6.18 & 2.872 & 112.54 & 6.04 & 3.48 & 136.3 & 6.13 \\
\hline
\end{tabular}

\subsection{Conclusion}

This chapter proposed, modeled, and analyzed the feasibility of installing the qusidynamic WPT systems at traffic signals to improve the range and driving time constraints present in the EV. A precise model for wirelessly connected EV, including BIWPTS, a lithium-ion battery array and drive system was developed and verified by means of simulation and experimentally. Three different scenarios of QDWPT system implementation were evaluated based on the FTP-72 city driving profile. Under the first scenario, the EV charges at fixed power levels during stops at the traffic intersections. As expected, driving range is increased as the charging rate increases until reaching WPT4, where energy recovery from charging is close to that of which was consumed by the electric 
motor. In the second scenario, a variable power charging profile is applied to reduce the grid stress, while dramatically extending the driving range. In the final scenario, V2G and G2V operation was tested considering the grid retail price and EV's psychological price. The performance of these scenarios were evaluated based on the continuous driving time per battery cycle and miles per cycle. A benefit measure factor was introduced to quantify the tradeoff between energy consumption and extra miles added, due to QDWPT charging. At fixed charging rates below WPT4, the benefit measure factor changed dramatically when driving started at different initial SOC values, revealing that starting at a low SOC yielded less benefit than starting at a high SOC. However, at WPT4, WPTAC and WPTACD, high benefits were revealed regardless of the initial SOC. Finally, the effect of coil misalignment during WPT system operation was investigated for all scenarios questioning the feasibility of WPT1 charging given a misalignment. This study concluded that QDWPT at traffic signals could provide a promising solution to dramatically extend the driving range of EVs, while increasing the operating time between traditional charging cycles, especially with high power levels. 


\section{Chapter 12 Conclusions}

This dissertation presented modeling, design and control analyses for wirelessly connected electric vehicles with the power grid to accomplish grid-to-vehicle and vehicleto-grid services, in the presence of Photovoltaic generation. Developing accurate analytical and physics-based models that are able to predict the system's dynamics, during transient and steady-state operation, utilizing these models to design and optimize the system's components, and then evaluating the entire system's performance, is the general goal of this research. How to choose the wireless power pads and optimize their design parameters; how to choose, design and build the appropriate resonant converter topology; and how to design and implement the appropriate controller that is able to manage the bidirectional power-flow in the system, are the central questions that drive most of these research activities.

In this work, the power conversion systems that integrate the EVs, PV generations and power grid through a common DC-bus, to achieve $\mathrm{G} 2 \mathrm{~V}$ and $\mathrm{V} 2 \mathrm{G}$ services, are researched. First, the coupling between the PV power system and the DC-link is investigated. An accurate nonlinear dynamic model for the grid-connected PV system is developed and utilized to predict the system's performance during the different dynamics. Then, this model is linearized to obtain the small signal model for the same system. Based on this model, clear design considerations for the maximum power point tracking algorithm in the system are stated and applied for the different MPPT algorithms. In addition, an advanced intelligent MPPT algorithm based on fuzzy logic is proposed and its design parameters are optimized using both the developed small signal model and genetic 
algorithm search technique. The proposed intelligent MPPT algorithm is simulated and experimentally tested under fast variation of irradiance and temperature conditions, and compared with the $\mathrm{P} \& \mathrm{O}$ and INC algorithms. The proposed controller shows faster transient response, less steady state error and robust tracking performance.

Another part in the $\mathrm{V} 2 \mathrm{G}$ system that is researched in this work is the bidirectional wireless integration between the EV and DC-bus. In this topic, many aspects have been explored, analyzing practical issues in the system. The themes that have been examined in this area are summarized as follows:

- Developing accurate mathematical and physics-based models for the bidirectional inductive wireless power transfer system in EVs applications. The models were able to predict the system's performance and power-flow, and novel practical limitations for active and reactive power-flow during G2V and V2G operations are stated.

- Designing, building and testing experimental prototypes and computer based models for the BIWPTS to verify the proposed theoretical analyses.

- Performing comparative and assessment analyses for the main compensation topologies in the symmetrical BIWPTS: LC-series, LC-parallel and LCL topology. The BIWPTSs' performance is evaluated by studying the active and reactive power-flow, efficiency, power factor, physical installation, design and control complexity, and sensitivity to the misalignment. The results demonstrate that LCL-topology is more appropriate for EVs applications and in the situations where the inverter is a long way from the pad. This topology shows current-source characteristics, power factor correction capability, simple design and control, high overall efficiency and power factor, and more robustness to misalignment. 
- Building an accurate physics-based co-simulation platform for the LCL BIWPTS that links the circuit model of the power electronic converters and controllers in Simulink, with the finite-element model of the electro-magnetic coupler. The effectiveness of the platform is clarified by investigating the nonlinearities in the system's performance due to the magnetic material and power electronics converters, in terms of error and harmonics analysis.

- Proposing a clear design methodology for the different components of the LCL BIWPTS in EVs applications. The proposed strategy depends on electromagnetic field computations, joined with analytical models. A 3-D finite-element model for an 8-kW polarized double-D power pad was modeled, optimized, and built. Moreover, the choice of the power factor correction and the impedance matching elements was investigated.

- Accomplishing an automatic magnetic design multi-objective optimization for the double-D power pad's parameters. The design parameters are chosen, such that the system shows the best coupling performance under different misalignments with minimum cost. The optimization analysis is achieved by linking the efficient improved Tabu search algorithm in the MATLAB environment with a 2D FEM in Maxwell. For verification purposes, the system coupling performance and cost are investigated using the optimum design parameters and compared with three other designs presented in the literature. The proposed optimized design shows the best coupling performance with moderate cost.

- Developing a new two-layer predictive power-flow controller to manage the bidirectional power-flow between the EV and power grid, during the long-term parking (stationary) and transient stops at traffic signals (quasi-dynamic). The controller is 
designed to be placed on the vehicle's side to allow the autonomous charging and discharging operation for the EV's battery. It gives the capability to the EV's operator to choose among five different modes of operation, considering the grid's retail price and EV's battery SOC. The proposed controller is able to predict the control parameters for both the primary and secondary converters based on the system's analytical model. Moreover, it considers the misalignment in the system, by adaptively estimating the mutual inductance before applying the proposed algorithm. The practical implementation of the proposed controller evidenced its performance's accuracy and fast response, during both the transient and steady-state operation, compared to the available controller in the literature.

- Building a comprehensive simulation model for a light-duty wirelessly connected EV, including BIWPTS, EV's battery and the traction system. The model was utilized to investigate the feasibility of implementing the quasi-dynamic wireless charging systems at the traffic signals on the road, to charge the EV during the transient stops at the intersections. This study demonstrated that implementing the quasi-dynamic wireless charging systems at the traffic signals could provide a promising solution to dramatically extend the driving range of EVs while increasing the operating time between traditional charging cycles, especially with high power levels. 


\section{Chapter 13 Recommendations and Future Works}

The main focus of this dissertation is to present modeling, design and control analyses for the power conversion systems between the EVs, PV generations and powergrid, to accomplish G2V and V2G services. More focus was provided to the bidirectional wireless power transfer system between each EV and the DC-bus. However, to further improve the quality of the power conversion systems, the following recommendations are stated:

- Utilizing the wide-band gap switching devices for building the HF resonance converters.

- Considering the switching frequency range recommended by the J2954A standard $(81.38-90 \mathrm{kHz})$ in the prototype design instead of $40 \mathrm{KHz}$.

- Performing EMI and EMC analysis for both the wireless coupler and the power electronics converters.

- Investigating the design of the aluminum shield and its impact on the system's performance.

- Including the wireless data communication between the two sides of the BIWPTS and exploring its impact on the autonomous controller's performance.

- Modifying the physics-based co-simulation platform to represent the BIWPTS in dynamic and quasi-dynamic WPT operation besides the stationary situation.

Following the presented study, several future research works can be performed, as follows:

- Developing and optimizing new power pad structures for high-power inductive 
stationary, dynamic and quasi-dynamic charging systems for EVs.

- Proposing new arrangements for the compensation networks in the inductive charger that provide interoperable and in-sensitive power-flow operation.

- Researching the high frequency resonant power converters for the wireless charger, involving the wide-band gap switching devices, such as $\mathrm{SiC}$ and GaN.

- Developing intelligent and adaptive controllers for managing the bidirectional active and reactive power-flow operation.

- Building an accurate simulation platform to test and evaluate the different implementation visions of inductive power charging for EVs (stationary, dynamic and quasi-dynamic).

- Developing autonomous and centralized high-level intelligent controllers that achieve the integration among a fleet of EVs, RESs and power grid based on EV's operator desire, energy price, EV's psychological price, grid voltage and frequency.

- Implementing the wireless data communication for the inductive charging system and investigating the cyber and physical security issues in both power and data transfer.

- Investigating the rule of implementing different energy storage technologies, such as Li-ion battery, super-capacitor and flywheel, in the EV's power train, with the wirelessly connected EVs. 


\section{LIST OF REFERENCES}

[1] M. Yilmaz and P. T. Krein, "Review of the Impact of Vehicle-to-Grid Technologies on Distribution Systems and Utility Interfaces," IEEE Trans. Power Electron., vol. 28, no. 12, pp. 5673-5689, Dec. 2013.

[2] M. Duvall and E. Knipping, "Environmental assessment of plug-in hybrid electric vehicles. Volume 1: Nationwide Greenhouse Gas Emissions," EPRI/NRDC, Palo Alto, CA, 1015325, 2007.

[3] C. Li, Y. Cao, Y. Kuang, and B. Zhou, Influences of Electric Vehicles on Power System and Key Technologies of Vehicle-to-Grid. Springer, 2016.

[4] E. L. Karfopoulos, E. M. Voumvoulakis, and N. Hatziargyriou, "Steady-state and dynamic impact analysis of the large scale integration of plug-in EV to the operation of the autonomous power system of Crete Island," in MedPower 2014, pp. 1-9.

[5] H. Xiao, Y. Huimei, W. Chen, and L. Hongjun, "A survey of influence of electrics vehicle charging on power grid," in 2014 9th IEEE Conference on Industrial Electronics and Applications, 2014, pp. 121-126.

[6] N. Hartmann and E. D. Özdemir, "Impact of different utilization scenarios of electric vehicles on the German grid in 2030," J. Power Sources, vol. 196, no. 4, pp. 23112318, Feb. 2011.

[7] S. Hadley, "Impact of Plug-in Hybrid Vehicles on the Electric Grid," Oak Ridge National Laboratory, Oak Ridge, TN, 2006.

[8] M. K. Meyers, K. Schneider, and R. Pratt, "Impacts Assessment of Plug-in Hybrid Vehicles on Electric Utilities and Regional US Power Grids," Pacific Northwest National Laboratory, State of Washington, Technical Analysis, 2007.

[9] S. W. Hadley and A. Tsvetkova, "Potential Impacts of Plug-in Hybrid Electric Vehicles on Regional Power Generation," Oak Rige National Lab, Tennessee, 2008.

[10] A. H. Hajimiragha, C. A. Canizares, M. W. Fowler, S. Moazeni, and A. Elkamel, “A Robust Optimization Approach for Planning the Transition to Plug-in Hybrid Electric Vehicles," IEEE Trans. Power Syst., vol. 26, no. 4, pp. 2264-2274, Nov. 2011.

[11] A. Hajimiragha, C. A. Canizares, M. W. Fowler, and A. Elkamel, "Optimal Transition to Plug-In Hybrid Electric Vehicles in Ontario, Canada, Considering the ElectricityGrid Limitations," IEEE Trans. Ind. Electron., vol. 57, no. 2, pp. 690-701, Feb. 2010.

[12] P. Denholm and W. Short, "An Evaluation of Utility System Impacts and Benefits of Optimally Dispatched Plug-in Hybrid Electric Vehicles," National Renewable Energy Laboratory, Colorado, 2007. 
[13] A. Ipakchi and F. Albuyeh, "Grid of the future," IEEE Power Energy Mag., vol. 7, no. 2, pp. 52-62, Mar. 2009.

[14] J. C. Gomez and M. M. Morcos, "Impact of EV battery chargers on the power quality of distribution systems," IEEE Trans. Power Deliv., vol. 18, no. 3, pp. 975-981, Jul. 2003.

[15] A. M. Leite da Silva, I. P. Coutinho, A. C. Zambroni de Souza, R. B. Prada, and A. M. Rei, "Voltage collapse risk assessment," Electr. Power Syst. Res., vol. 54, no. 3, pp. 221-227, Jun. 2000.

[16] A. Dubey and S. Santoso, "Electric Vehicle Charging on Residential Distribution Systems: Impacts and Mitigations," IEEE Access, vol. 3, pp. 1871-1893, 2015.

[17] S. Rahman and G. B. Shrestha, "An investigation into the impact of electric vehicle load on the electric utility distribution system," IEEE Trans. Power Deliv., vol. 8, no. 2, pp. 591-597, Apr. 1993.

[18] Y. Chen, A. Oudalov, and J. S. Wang, "Integration of electric vehicle charging system into distribution network," in 8th International Conference on Power Electronics ECCE Asia, 2011, pp. 593-598.

[19] J. Taylor, J. W. Smith, and R. Dugan, "Distribution modeling requirements for integration of PV, PEV, and storage in a smart grid environment," in 2011 IEEE Power and Energy Society General Meeting, 2011, pp. 1-6.

[20] J. Taylor, A. Maitra, M. Alexander, D. Brooks, and M. Duvall, "Evaluation of the impact of plug-in electric vehicle loading on distribution system operations," in 2009 IEEE Power Energy Society General Meeting, 2009, pp. 1-6.

[21] S. Shao, M. Pipattanasomporn, and S. Rahman, "Challenges of PHEV penetration to the residential distribution network," in 2009 IEEE Power Energy Society General Meeting, 2009, pp. 1-8.

[22] S. W. Hadley, "Evaluating the impact of Plug-in Hybrid Electric Vehicles on regional electricity supplies," in 2007 iREP Symposium - Bulk Power System Dynamics and Control - VII. Revitalizing Operational Reliability, 2007, pp. 1-12.

[23] O. Warweg, F. Schaller, S. Ritter, and P. Bretschneider, "Technical and regulatory challenges for the integration of electric vehicles into the energy system," in 2011 2nd IEEE PES International Conference and Exhibition on Innovative Smart Grid Technologies, 2011, pp. 1-8.

[24] K. Clement-Nyns, E. Haesen, and J. Driesen, "The Impact of Charging Plug-In Hybrid Electric Vehicles on a Residential Distribution Grid," IEEE Trans. Power Syst., vol. 25, no. 1, pp. 371-380, Feb. 2010. 
[25] A. G. Boulanger, A. C. Chu, S. Maxx, and D. L. Waltz, "Vehicle Electrification: Status and Issues," Proc. IEEE, vol. 99, no. 6, pp. 1116-1138, Jun. 2011.

[26] A. Dubey, S. Santoso, and M. P. Cloud, "Understanding the effects of electric vehicle charging on the distribution voltages," in 2013 IEEE Power Energy Society General Meeting, 2013, pp. 1-5.

[27] A. Dubey, S. Santoso, and M. P. Cloud, "Comparative analysis of effects of electric vehicle loads on distribution system voltages," in 2014 IEEE PES T D Conference and Exposition, 2014, pp. 1-5.

[28] "Transportation Electrification: A Technology Overview," Electric Power Research Institute, Technical Results 1021334, Jul. 2011.

[29] C. Weiller, "Plug-in hybrid electric vehicle impacts on hourly electricity demand in the United States," Energy Policy, vol. 39, no. 6, pp. 3766-3778, Jun. 2011.

[30] J. Cook, C. Churchwell, and S. George, "Final Evaluation of San Diego Gas \& Electric Plug-In Electrical Vehicle TOU," San Diego Gas \& Electric, San Diego, 2014.

[31] S. L. Schey, J. Smart, and D. R. Scoffield, "A First Look at the Impact of Electric Vehicle Charging on the Electric Grid in the EV Project," vol. 5, Jan. 2012.

[32] S. Shao, T. Zhang, M. Pipattanasomporn, and S. Rahman, "Impact of TOU rates on distribution load shapes in a smart grid with PHEV penetration," in IEEE PES T D 2010, 2010, pp. 1-6.

[33] Y. Gao, C. Wang, Z. Wang, and H. Liang, "Research on time-of-use price applying to electric vehicles charging," in IEEE PES Innovative Smart Grid Technologies, 2012, pp. 1-6.

[34] A. Dubey, S. Santoso, M. P. Cloud, and M. Waclawiak, "Determining Time-of-Use Schedules for Electric Vehicle Loads: A Practical Perspective," IEEE Power Energy Technol. Syst. J., vol. 2, no. 1, pp. 12-20, Mar. 2015.

[35] C. Gerkensmeyer, M. KintnerMeyer, and J. DeSteese, "Technical Challenges of Plugin Hybrid Electric Vehicles and Impacts to the US Power System: Distribution System Analysis,” PNNL, Technical Report, Jan. 2010.

[36] S. Han, S. Han, and K. Sezaki, "Development of an Optimal Vehicle-to-Grid Aggregator for Frequency Regulation," IEEE Trans. Smart Grid, vol. 1, no. 1, pp. 65-72, Jun. 2010.

[37] S. Shao, M. Pipattanasomporn, and S. Rahman, "Demand Response as a Load Shaping Tool in an Intelligent Grid With Electric Vehicles," IEEE Trans. Smart Grid, vol. 2, no. 4, pp. 624-631, Dec. 2011. 
[38] Y. Cao et al., "An Optimized EV Charging Model Considering TOU Price and SOC Curve," IEEE Trans. Smart Grid, vol. 3, no. 1, pp. 388-393, Mar. 2012.

[39] Z. Li, Q. Guo, H. Sun, S. Xin, and J. Wang, "A New Real-Time Smart-Charging Method Considering Expected Electric Vehicle Fleet Connections," IEEE Trans. Power Syst., vol. 29, no. 6, pp. 3114-3115, Nov. 2014.

[40] S. Deilami, A. S. Masoum, P. S. Moses, and M. A. S. Masoum, "Real-Time Coordination of Plug-In Electric Vehicle Charging in Smart Grids to Minimize Power Losses and Improve Voltage Profile," IEEE Trans. Smart Grid, vol. 2, no. 3, pp. 456467, Sep. 2011.

[41] S. Shao, M. Pipattanasomporn, and S. Rahman, "Grid Integration of Electric Vehicles and Demand Response With Customer Choice," IEEE Trans. Smart Grid, vol. 3, no. 1, pp. 543-550, Mar. 2012.

[42] E. Sortomme, M. M. Hindi, S. D. J. MacPherson, and S. S. Venkata, "Coordinated Charging of Plug-In Hybrid Electric Vehicles to Minimize Distribution System Losses," IEEE Trans. Smart Grid, vol. 2, no. 1, pp. 198-205, Mar. 2011.

[43] Y. He, B. Venkatesh, and L. Guan, "Optimal Scheduling for Charging and Discharging of Electric Vehicles," IEEE Trans. Smart Grid, vol. 3, no. 3, pp. 10951105, Sep. 2012.

[44] C. Jin, J. Tang, and P. Ghosh, "Optimizing Electric Vehicle Charging: A Customer's Perspective," IEEE Trans. Veh. Technol., vol. 62, no. 7, pp. 2919-2927, Sep. 2013.

[45] J. de Hoog, T. Alpcan, M. Brazil, D. A. Thomas, and I. Mareels, "Optimal Charging of Electric Vehicles Taking Distribution Network Constraints Into Account," IEEE Trans. Power Syst., vol. 30, no. 1, pp. 365-375, Jan. 2015.

[46] K. Mets, T. Verschueren, W. Haerick, C. Develder, and F. D. Turck, "Optimizing smart energy control strategies for plug-in hybrid electric vehicle charging," in 2010 IEEE/IFIP Network Operations and Management Symposium Workshops, 2010, pp. 293-299.

[47] B. W. Zhou, T. Littler, and H. F. Wang, "The impact of vehicle-to-grid on electric power systems: A review," in 2nd IET Renewable Power Generation Conference (RPG 2013), 2013, pp. 1-4.

[48] X. E. Yu, Y. Xue, S. Sirouspour, and A. Emadi, "Microgrid and transportation electrification: A review," in 2012 IEEE Transportation Electrification Conference and Expo (ITEC), 2012, pp. 1-6.

[49] S. Habib and M. Kamran, "A novel vehicle-to-grid technology with constraint analysis-a review," in 2014 International Conference on Emerging Technologies (ICET), 2014, pp. 69-74. 
[50] G. Xiao, C. Li, Z. Yu, Y. Cao, and B. Fang, "Review of the impact of electric vehicles participating in frequency regulation on power grid," in 2013 Chinese Automation Congress, 2013, pp. 75-80.

[51] Y. Jang and M. M. Jovanović, "Contactless electrical energy transmission system," US6301128 B1, 09-Oct-2001.

[52] S. B. Peterson, J. F. Whitacre, and J. Apt, "The economics of using plug-in hybrid electric vehicle battery packs for grid storage," J. Power Sources, vol. 195, no. 8, pp. 2377-2384, Apr. 2010.

[53] P. M. de Quevedo, G. Muñoz-Delgado, and J. Contreras, "Impact of Electric Vehicles on the Expansion Planning of Distribution Systems considering Renewable Energy, Storage and Charging Stations," IEEE Trans. Smart Grid, vol. PP, no. 99, pp. 1-1, 2017.

[54] M. Moeini-Aghtaie, H. Farzin, M. Fotuhi-Firuzabad, and R. Amrollahi, "Generalized Analytical Approach to Assess Reliability of Renewable-Based Energy Hubs," IEEE Trans. Power Syst., vol. 32, no. 1, pp. 368-377, Jan. 2017.

[55] J. Ugirumurera and Z. J. Haas, "Optimal Capacity Sizing for Completely Green Charging Systems for Electric Vehicles," IEEE Trans. Transp. Electrification, vol. 3, no. 3, pp. 565-577, Sep. 2017.

[56] H. N. T. Nguyen, C. Zhang, and J. Zhang, "Dynamic Demand Control of Electric Vehicles to Support Power Grid With High Penetration Level of Renewable Energy," IEEE Trans. Transp. Electrification, vol. 2, no. 1, pp. 66-75, Mar. 2016.

[57] R. Wang, P. Wang, and G. Xiao, "Two-Stage Mechanism for Massive Electric Vehicle Charging Involving Renewable Energy," IEEE Trans. Veh. Technol., vol. 65, no. 6, pp. 4159-4171, Jun. 2016.

[58] A. Y. S. Lam, K. C. Leung, and V. O. K. Li, "Capacity Estimation for Vehicle-toGrid Frequency Regulation Services With Smart Charging Mechanism," IEEE Trans. Smart Grid, vol. 7, no. 1, pp. 156-166, Jan. 2016.

[59] E. L. Karfopoulos and N. D. Hatziargyriou, "Distributed Coordination of Electric Vehicles Providing V2G Services," IEEE Trans. Power Syst., vol. 31, no. 1, pp. 329338, Jan. 2016.

[60] S. Sahoo, S. Prakash, and S. Mishra, "Handshaking V2G strategy for grid connected PV assisted charging station," IET Renew. Power Gener., vol. 11, no. 11, pp. 1410$1417,2017$.

[61] N. Rahbari-Asr, M. Y. Chow, J. Chen, and R. Deng, "Distributed Real-Time Pricing Control for Large-Scale Unidirectional V2G With Multiple Energy Suppliers," IEEE Trans. Ind. Inform., vol. 12, no. 5, pp. 1953-1962, Oct. 2016. 
[62] R. Hara, H. Kita, T. Tanabe, H. Sugihara, A. Kuwayama, and S. Miwa, "Testing the technologies," IEEE Power Energy Mag., vol. 7, no. 3, pp. 77-85, May 2009.

[63] A. Woyte, V. V. Thong, R. Belmans, and J. Nijs, "Voltage fluctuations on distribution level introduced by photovoltaic systems," IEEE Trans. Energy Convers., vol. 21, no. 1, pp. 202-209, Mar. 2006.

[64] J. A. Short, D. G. Infield, and L. L. Freris, "Stabilization of Grid Frequency Through Dynamic Demand Control," IEEE Trans. Power Syst., vol. 22, no. 3, pp. 1284-1293, Aug. 2007.

[65] U. C. Chukwu and S. M. Mahajan, "V2G Parking Lot With PV Rooftop for Capacity Enhancement of a Distribution System," IEEE Trans. Sustain. Energy, vol. 5, no. 1, pp. 119-127, Jan. 2014.

[66] M. J. E. Alam, K. M. Muttaqi, and D. Sutanto, "Effective Utilization of Available PEV Battery Capacity for Mitigation of Solar PV Impact and Grid Support With Integrated V2G Functionality," IEEE Trans. Smart Grid, vol. 7, no. 3, pp. 1562-1571, May 2016.

[67] M. Falahi, H. M. Chou, M. Ehsani, L. Xie, and K. L. Butler-Purry, "Potential Power Quality Benefits of Electric Vehicles," IEEE Trans. Sustain. Energy, vol. 4, no. 4, pp. 1016-1023, Oct. 2013.

[68] M. E. Ropp, "Similarities between vehicle-to-grid interfaces and photovoltaic systems," in 2009 IEEE Vehicle Power and Propulsion Conference, 2009, pp. 12211225 .

[69] N. K. Kandasamy, K. Kandasamy, and K. J. Tseng, "Loss-of-life investigation of EV batteries used as smart energy storage for commercial building-based solar photovoltaic systems," IET Electr. Syst. Transp., vol. 7, no. 3, pp. 223-229, 2017.

[70] K. W. Hu and C. M. Liaw, "Incorporated Operation Control of DC Microgrid and Electric Vehicle,” IEEE Trans. Ind. Electron., vol. 63, no. 1, pp. 202-215, Jan. 2016.

[71] A. A. A. Elgammal and A. M. Sharaf, "Self-regulating particle swarm optimised controller for (photovoltaic-fuel cell) battery charging of hybrid electric vehicles," IET Electr. Syst. Transp., vol. 2, no. 2, pp. 77-89, Jun. 2012.

[72] J. Tomić and W. Kempton, "Using fleets of electric-drive vehicles for grid support," J. Power Sources, vol. 168, no. 2, pp. 459-468, Jun. 2007.

[73] P. Javanbakht, S. Mohagheghi, B. Parkhideh, S. Dutta, R. Chattopadhyay, and S. Bhattacharya, "Vehicle-to-grid scheme based on inductive power transfer for advanced distribution automation," in 2013 IEEE Energy Conversion Congress and Exposition, 2013, pp. 3250-3257. 
[74] M. Ş. Kuran, A. C. Viana, L. Iannone, D. Kofman, G. Mermoud, and J. P. Vasseur, "A Smart Parking Lot Management System for Scheduling the Recharging of Electric Vehicles," IEEE Trans. Smart Grid, vol. 6, no. 6, pp. 2942-2953, Nov. 2015.

[75] A. Mohamed, A. Berzoy, and O. Mohammed, "Experimental Validation of Comprehensive Steady-state Analytical Model of Bidirectional WPT System in EVs Applications," IEEE Trans. Veh. Technol., vol. PP, no. 99, pp. 1-1, 2016.

[76] A. A. S. Mohamed, A. Berzoy, F. G. N. de Almeida, and O. Mohammed, "Steadystate performance assessment of different compensation topologies in two-way IWPT system for EV ancillary services," in 2016 IEEE Industry Applications Society Annual Meeting, 2016, pp. 1-8.

[77] G. Lempidis, Y. Zhang, M. Jung, R. Marklein, S. Sotiriou, and Y. Ma, "Wired and wireless charging of electric vehicles: A system approach," in Electric Drives Production Conference (EDPC), 2014 4th International, 2014, pp. 1-7.

[78] A. A. S. Mohamed, A. Berzoy, F. G. N. de Almeida, and O. Mohammed, "Modeling and Assessment Analysis of Various Compensation Topologies in Bidirectional IWPT System for EV Applications," IEEE Trans. Ind. Appl., vol. 53, no. 5, pp. 49734984, Sep. 2017.

[79] A. A. S. Mohamed and O. Mohammed, "Physics-Based Co-simulation Platform with Analytical and Experimental Verification for Bidirectional IPT System in EV Applications," IEEE Trans. Veh. Technol., vol. P.P, no. P.P, 2017.

[80] N. Tesla, "System of transmission of electrical energy.," US645576 A, 20-Mar-1900.

[81] A. Kurs, A. Karalis, R. Moffatt, J. D. Joannopoulos, P. Fisher, and M. Soljačić, "Wireless Power Transfer via Strongly Coupled Magnetic Resonances," Science, vol. 317, no. 5834, pp. 83-86, Jul. 2007.

[82] S. Li and C. C. Mi, "Wireless Power Transfer for Electric Vehicle Applications," IEEE J. Emerg. Sel. Top. Power Electron., vol. 3, no. 1, pp. 4-17, Mar. 2015.

[83] S. Lukic and Z. Pantic, "Cutting the Cord: Static and Dynamic Inductive Wireless Charging of Electric Vehicles," IEEE Electrification Mag., vol. 1, no. 1, pp. 57-64, Sep. 2013.

[84] G. A. Covic and J. T. Boys, "Modern Trends in Inductive Power Transfer for Transportation Applications," IEEE J. Emerg. Sel. Top. Power Electron., vol. 1, no. 1, pp. 28-41, Mar. 2013.

[85] G. A. Covic and J. T. Boys, "Inductive Power Transfer," Proc. IEEE, vol. 101, no. 6, pp. 1276-1289, Jun. 2013. 
[86] B. Esteban, M. Sid-Ahmed, and N. C. Kar, "A Comparative Study of Power Supply Architectures in Wireless EV Charging Systems," IEEE Trans. Power Electron., vol. 30, no. 11, pp. 6408-6422, Nov. 2015.

[87] M. Kamar Uddin, G. Ramasamy, S. Mekhilef, K. Ramar, and Y.-C. Lau, “A review on high frequency resonant inverter technologies for wireless power transfer using magnetic resonance coupling," in 2014 IEEE Conference on Energy Conversion (CENCON), 2014, pp. 412-417.

[88] R. Bosshard, U. Badstubner, J. W. Kolar, and I. Stevanovic, "Comparative evaluation of control methods for Inductive Power Transfer," in 2012 International Conference on Renewable Energy Research and Applications (ICRERA), 2012, pp. 1-6.

[89] W. Zhang and C. C. Mi, "Compensation Topologies of High-Power Wireless Power Transfer Systems," IEEE Trans. Veh. Technol., vol. 65, no. 6, pp. 4768-4778, Jun. 2016.

[90] F. Musavi and W. Eberle, "Overview of wireless power transfer technologies for electric vehicle battery charging," IET Power Electron., vol. 7, no. 1, pp. 60-66, Jan. 2014.

[91] T. W. Ching and Y. S. Wong, "Review of wireless charging technologies for electric vehicles," in 2013 5th International Conference on Power Electronics Systems and Applications (PESA), 2013, pp. 1-4.

[92] A. A. S. Mohamed, C. R. Lashway, and O. Mohammed, "Modeling and Feasibility Analysis of Quasi-Dynamic WPT System for EV Applications," IEEE Trans. Transp. Electrification, vol. 3, no. 2, pp. 343-353, Jun. 2017.

[93] Inductive Powering - Basic Theory and Application | Koenraad van Schuylenbergh | Springer.

[94] B. I. Ashraf, Wireless Power Transfer: Fundamentals and Technologies, 1st edition. S.1.: Auris Reference, 2017.

[95] K. Y. Kim, Wireless Power Transfer: Principles and Engineering Explorations. Intech, 2012.

[96] A. G. Batres, A. Moghe, and J. Taiber, "A communication architecture for wireless power transfer services based on DSRC technology," in 2016 IEEE Transportation Electrification Conference and Expo (ITEC), 2016, pp. 1-8.

[97] S. Chopra and P. Bauer, "Driving Range Extension of EV With On-Road Contactless Power Transfer \#x2014;A Case Study,” IEEE Trans. Ind. Electron., vol. 60, no. 1, pp. 329-338, Jan. 2013. 
[98] A. K. Swain, S. Devarakonda, and U. K. Madawala, "Modeling, Sensitivity Analysis, and Controller Synthesis of Multipickup Bidirectional Inductive Power Transfer Systems," IEEE Trans. Ind. Inform., vol. 10, no. 2, pp. 1372-1380, May 2014.

[99] M. Kabalo, F. Berthold, B. Blunier, D. Bouquain, S. Williamson, and A. Miraoui, "Efficiency comparison of wire and wireless battery charging: Based on connection probability analysis," in 2014 IEEE Transportation Electrification Conference and Expo (ITEC), 2014, pp. 1-6.

[100] X. Huang, H. Qiang, Z. Huang, Y. Sun, and J. Li, "The Interaction Research of Smart Grid and EV Based Wireless Charging," in 2013 IEEE Vehicle Power and Propulsion Conference (VPPC), 2013, pp. 1-5.

[101] A. A. S. Mohamed, A. Berzoy, and O. Mohammed, "Power Flow Modeling of Wireless Power Transfer for EVs Charging and Discharging in V2G Applications," in 2015 IEEE Vehicle Power and Propulsion Conference (VPPC), 2015, pp. 1-6.

[102] A. A. S. Mohamed, F. G. N. de Almeida, and O. Mohammed, "Harmonics-based steady-state mathematical model of bidirectional inductive wireless power transfer system in V2G applications," in 2016 IEEE Transportation Electrification Conference and Expo (ITEC), 2016, pp. 1-6.

[103] Y. Mahmoud and E. F. El-Saadany, "A Photovoltaic Model With Reduced Computational Time," IEEE Trans. Ind. Electron., vol. 62, no. 6, pp. 3534-3544, Jun. 2015.

[104] Y. A. Mahmoud, W. Xiao, and H. H. Zeineldin, “A Parameterization Approach for Enhancing PV Model Accuracy," IEEE Trans. Ind. Electron., vol. 60, no. 12, pp. 5708-5716, Dec. 2013.

[105] L. H. I. Lim, Z. Ye, J. Ye, D. Yang, and H. Du, “A Linear Identification of Diode Models from Single \#x2013; Characteristics of PV Panels," IEEE Trans. Ind. Electron., vol. 62, no. 7, pp. 4181-4193, Jul. 2015.

[106] M. A. Farahat, H. M. B. Metwally, and A. Abd-Elfatah Mohamed, "Optimal choice and design of different topologies of DC-DC converter used in PV systems, at different climatic conditions in Egypt," Renew. Energy, vol. 43, pp. 393-402, Jul. 2012.

[107] R. Middlebrook and S. Cuk, "A general unified approach to modelling switchingconverter power stages," in 1976 IEEE Power Electronics Specialists Conference, 1976, pp. 18-34.

[108] A. A. S. Mohamed, A. Berzoy, and O. A. Mohammed, "Design and Hardware Implementation of FL-MPPT Control of PV Systems Based on GA and Small-Signal Analysis," IEEE Trans. Sustain. Energy, vol. 8, no. 1, pp. 279-290, Jan. 2017. 
[109] X. Li, Y. Li, J. E. Seem, and P. Lei, "Detection of Internal Resistance Change for Photovoltaic Arrays Using Extremum-Seeking Control MPPT Signals," IEEE Trans. Control Syst. Technol., vol. 24, no. 1, pp. 325-333, Jan. 2016.

[110] A. Ahmed, L. Ran, S. Moon, and J.-H. Park, "A Fast PV Power Tracking Control Algorithm With Reduced Power Mode," IEEE Trans. Energy Convers., vol. 28, no. 3, pp. 565-575, Sep. 2013.

[111] Y. Mahmoud and E. F. El-Saadany, "A Photovoltaic Model With Reduced Computational Time," IEEE Trans. Ind. Electron., vol. 62, no. 6, pp. 3534-3544, Jun. 2015.

[112] Y. A. Mahmoud, W. Xiao, and H. H. Zeineldin, "A Parameterization Approach for Enhancing PV Model Accuracy," IEEE Trans. Ind. Electron., vol. 60, no. 12, pp. 5708-5716, Dec. 2013.

[113] G. M. Masters, Renewable and Efficient Electric Power Systems, 2 edition. Hoboken, New Jersey: Wiley, 2013.

[114] A. Mohamed, H. Metwally, and M. Farahat, Photovoltaic Water Pumping Systems. LAP LAMBERT Academic Publishing, 2012.

[115] M. Fazzolari, R. Alcala, Y. Nojima, H. Ishibuchi, and F. Herrera, "A Review of the Application of Multiobjective Evolutionary Fuzzy Systems: Current Status and Further Directions," IEEE Trans. Fuzzy Syst., vol. 21, no. 1, pp. 45-65, Feb. 2013.

[116] A. A. Ramadan, M. T. Faheem, A. F. Amer, and S. A. Sallam, "New genetic-based design of a Pi-like fuzzy logic speed controlter for an induction motor," in Second IEEE International Conference on Computational Cybernetics, 2004. ICCC 2004, 2004, pp. 25-32.

[117] “bp 4175t pv module datasheet - Google Search.” [Online]. Available: https://www.google.com/?gws_rd=ssl\#q=bp+4175t+pv+module+datasheet. [Accessed: 20-Oct-2015].

[118] B. Subudhi and R. Pradhan, "A Comparative Study on Maximum Power Point Tracking Techniques for Photovoltaic Power Systems," IEEE Trans. Sustain. Energy, vol. 4, no. 1, pp. 89-98, Jan. 2013.

[119] M. A. G. de Brito, L. Galotto, L. P. Sampaio, G. de Azevedo e Melo, and C. A. Canesin, "Evaluation of the Main MPPT Techniques for Photovoltaic Applications," IEEE Trans. Ind. Electron., vol. 60, no. 3, pp. 1156-1167, Mar. 2013.

[120] A. A. S. Mohamed, A. Berzoy, and O. Mohammed, "Optimized-fuzzy MPPT controller using GA for stand-alone photovoltaic water pumping system," in IECON 2014 - 40th Annual Conference of the IEEE Industrial Electronics Society, 2014, pp. 2213-2218. 
[121] N. Femia, G. Petrone, G. Spagnuolo, and M. Vitelli, "Optimization of perturb and observe maximum power point tracking method," IEEE Trans. Power Electron., vol. 20, no. 4, pp. 963-973, Jul. 2005.

[122] N. Femia, G. Petrone, G. Spagnuolo, and M. Vitelli, “A Technique for Improving P \#x00026;O MPPT Performances of Double-Stage Grid-Connected Photovoltaic Systems," IEEE Trans. Ind. Electron., vol. 56, no. 11, pp. 4473-4482, Nov. 2009.

[123] M. A. Elgendy, B. Zahawi, and D. J. Atkinson, "Assessment of Perturb and Observe MPPT Algorithm Implementation Techniques for PV Pumping Applications," IEEE Trans. Sustain. Energy, vol. 3, no. 1, pp. 21-33, Jan. 2012.

[124] A. A. S. Mohamed, A. Berzoy, and O. Mohammed, "Control Parameters Optimization for PM DC Motor in Photovoltaic Applications," presented at the IEEE International Electric Machines and Drives Conference (IEMDC) 2015, 2015.

[125] S. K. Kollimalla and M. K. Mishra, "Variable Perturbation Size Adaptive P amp;O MPPT Algorithm for Sudden Changes in Irradiance," IEEE Trans. Sustain. Energy, vol. 5, no. 3, pp. 718-728, Jul. 2014.

[126] Y. Jiang, J. A. A. Qahouq, and T. A. Haskew, “Adaptive Step Size With AdaptivePerturbation-Frequency Digital MPPT Controller for a Single-Sensor Photovoltaic Solar System," IEEE Trans. Power Electron., vol. 28, no. 7, pp. 3195-3205, Jul. 2013.

[127] A. K. Abdelsalam, A. M. Massoud, S. Ahmed, and P. Enjeti, "High-Performance Adaptive Perturb and Observe MPPT Technique for Photovoltaic-Based Microgrids," IEEE Trans. Power Electron., vol. 26, no. 4, pp. 1010-1021, Apr. 2011.

[128] A. Berzoy, E. Baethge, J. Restrepo, and J. Viola, "Fuzzy Control System for Maximum Power Point Tracking in Solar Panels Based on DC-DC Converter PI Current Control," in Andean Region International Conference (ANDESCON), 2012 VI, 2012, pp. 119-122.

[129] T. L. Kottas, Y. S. Boutalis, and A. D. Karlis, "New maximum power point tracker for PV arrays using fuzzy controller in close cooperation with fuzzy cognitive networks," IEEE Trans. Energy Convers., vol. 21, no. 3, pp. 793-803, Sep. 2006.

[130] A. El Khateb, N. Abd Rahim, J. Selvaraj, and M. N. Uddin, "Fuzzy-LogicController-Based SEPIC Converter for Maximum Power Point Tracking," IEEE Trans. Ind. Appl., vol. 50, no. 4, pp. 2349-2358, Jul. 2014.

[131] M. A. A. Mohd Zainuri, M. A. Mohd Radzi, A. C. Soh, and N. A. Rahim, "Development of adaptive perturb and observe-fuzzy control maximum power point tracking for photovoltaic boost dc-dc converter," IET Renew. Power Gener., vol. 8, no. 2, pp. 183-194, Mar. 2014. 
[132] G. J. Klir and B. Yuan, Fuzzy Sets and Fuzzy Logic: Theory and Applications, 1st edition. Upper Saddle River, N.J: Prentice Hall, 1995.

[133] G. J. Klir and B. Yuan, Fuzzy Sets and Fuzzy Logic: Theory and Applications, 1st edition. Upper Saddle River, N.J: Prentice Hall, 1995.

[134] T. D. Vrionis, X. I. Koutiva, and N. A. Vovos, "A Genetic Algorithm-Based Low Voltage Ride-Through Control Strategy for Grid Connected Doubly Fed Induction Wind Generators," IEEE Trans. Power Syst., vol. 29, no. 3, pp. 1325-1334, May 2014.

[135] D. Sera, L. Mathe, T. Kerekes, S. V. Spataru, and R. Teodorescu, "On the Perturband-Observe and Incremental Conductance MPPT Methods for PV Systems," IEEE J. Photovolt., vol. 3, no. 3, pp. 1070-1078, Jul. 2013.

[136] H. Liu, H. Ning, Y. Zhang, and L. T. Yang, "Aggregated-Proofs Based PrivacyPreserving Authentication for V2G Networks in the Smart Grid," IEEE Trans. Smart Grid, vol. 3, no. 4, pp. 1722-1733, Dec. 2012.

[137] U. K. Madawala and D. J. Thrimawithana, "Current sourced bi-directional inductive power transfer system," IET Power Electron., vol. 4, no. 4, pp. 471-480, Apr. 2011.

[138] J. G. Meins and J. D. Sinsley, "Method and apparatus for supplying contactless power," US6515878 B1, 04-Feb-2003.

[139] U. K. Madawala and D. J. Thrimawithana, "A Bidirectional Inductive Power Interface for Electric Vehicles in V2G Systems," IEEE Trans. Ind. Electron., vol. 58, no. 10, pp. 4789-4796, Oct. 2011.

[140] D. J. Thrimawithana and U. K. Madawala, "A contactless bi-directional power interface for plug-in hybrid vehicles," in IEEE Vehicle Power and Propulsion Conference, 2009. VPPC '09, 2009, pp. 396-401.

[141] C.-S. Wang, G. A. Covic, and O. H. Stielau, "Investigating an LCL load resonant inverter for inductive power transfer applications," IEEE Trans. Power Electron., vol. 19, no. 4, pp. 995-1002, Jul. 2004.

[142] D. J. Thrimawithana and U. K. Madawala, "A Generalized Steady-State Model for Bidirectional IPT Systems," IEEE Trans. Power Electron., vol. 28, no. 10, pp. 46814689, Oct. 2013.

[143] A. K. Swain, M. J. Neath, U. K. Madawala, and D. J. Thrimawithana, "A Dynamic Multivariable State-Space Model for Bidirectional Inductive Power Transfer Systems," IEEE Trans. Power Electron., vol. 27, no. 11, pp. 4772-4780, Nov. 2012. 
[144] A. A. S. Mohamed, A. Berzoy, and O. A. Mohammed, "Physics-based FE model and analytical verification of bi-directional inductive wireless power transfer system," in 2016 IEEE/ACES International Conf. on Wireless Information Techn. and Systems (ICWITS) and Applied Computational Electromagnetics (ACES), 2016, pp. 1-2.

[145] A. A. S. Mohamed, A. Berzoy, and O. A. Mohammed, "Physics-Based CoSimulation Platform for EMC Analysis of Two-Way Inductive WPT System in EV Applications," in 2016 IEEE Vehicle Power and Propulsion Conference (VPPC), 2016, pp. 1-6.

[146] L. Zhao, D. J. Thrimawithana, and U. K. Madawala, "A comparison of LCL and LC bi-directional inductive power transfer systems," in Electronics and Application Conference and Exposition (PEAC), 2014 International, 2014, pp. 766-771.

[147] A. A. S. Mohamed, A. Berzoy, F. G. N. N. de Almeida, and O. Mohammed, "Steady-State Performance Assessment of Different Compensation Topologies in Two-way IWPT System for EV Ancillary Services," IEEE Trans. Ind. Appl., vol. PP, no. 99, pp. 1-1, 2017.

[148] L. Tan, B. Wu, and S. Rivera, "A bipolar-DC-bus EV fast charging station with intrinsic DC-bus voltages equalization and minimized voltage ripples," in IECON 2015 - 41st Annual Conf. of the IEEE Indus. Electro. Soc., 2015, pp. 002190-002195.

[149] “J2954A (WIP) Wireless Power Transfer for Light-Duty Plug-In/ Electric Vehicles and Alignment Methodology - SAE International." [Online]. Available: http://standards.sae.org/wip/j2954/. [Accessed: 10-Jul-2016].

[150] W. Zhang, S.-C. Wong, C. K. Tse, and Q. Chen, "Design for Efficiency Optimization and Voltage Controllability of Series \#x2013;Series Compensated Inductive Power Transfer Systems," IEEE Trans. Power Electron., vol. 29, no. 1, pp. 191-200, Jan. 2014.

[151] W. Zhang, S.-C. Wong, C. K. Tse, and Q. Chen, "Design for Efficiency Optimization and Voltage Controllability of Series \#x2013;Series Compensated Inductive Power Transfer Systems," IEEE Trans. Power Electron., vol. 29, no. 1, pp. 191-200, Jan. 2014.

[152] V. PALTANEA, G. PALTANEA, H. GAVRILĂ, and G. IONESCU, "MAGNETIC PROPERTIES OF N87 MnZn (EPCOS TYPE) FERRITE," UPB Sci Bull, vol. 75, no. 3, 2013.

[153] K. L. Kaiser, Electromagnetic Compatibility Handbook. CRC Press, 2004.

[154] J. T. Boys, G. A. J. Elliott, and G. A. Covic, "An Appropriate Magnetic Coupling Co-Efficient for the Design and Comparison of ICPT Pickups," IEEE Trans. Power Electron., vol. 22, no. 1, pp. 333-335, Jan. 2007. 
[155] H. Yoo, E. Shim, J. Kang, G. Choi, C. Lee, and B. Bang, "100kHz IGBT inverter use of LCL topology for high power induction heating," in 2011 IEEE 8th International Conference on Power Electronics and ECCE Asia (ICPE ECCE), 2011, pp. $1572-1575$.

[156] S. Dieckerhoff, M. J. Ruan, and R. W. D. Doncker, "Design of an IGBT-based LCL-resonant inverter for high-frequency induction heating," in Conference Record of the 1999 IEEE Industry Applications Conference, 1999. Thirty-Fourth IAS Annual Meeting, 1999, vol. 3, pp. 2039-2045 vol.3.

[157] "IXFB110N60P3 - IXYS SEMICONDUCTOR - Power MOSFET, N Channel, 110 A, $600 \mathrm{~V}, 0.056 \mathrm{ohm}, 10 \mathrm{~V}, 5 \mathrm{~V}$ | Farnell element14 Israel." [Online]. Available: http://il.farnell.com/ixys-semiconductor/ixfb110n60p3/mosfet-n-ch-600v-110a-to264/dp/2429703. [Accessed: 14-Feb-2016].

[158] "FAN7391 PDF Datasheet 625V," Shell. [Online]. Available: https://www.fairchildsemi.com/products/power-management/mosfet-igbt-gatedrivers/high-side-drivers/FAN7391.html. [Accessed: 14-Feb-2016].

[159] "AN-6076 Design and Application Guide of Bootstrap Circuit for High-Voltage Gate-DriveIC-AN-6076.pdf.”[Online]. Available: https://www.fairchildsemi.com/ application-notes/AN/AN-6076.pdf. [Accessed: 14-Feb-2016].

[160] "Ringing Reduction Techniques for NexFET High Performance MOSFETs slpa010.pdf." [Online]. Available: http://www.ti.com/lit/an/slpa010/slpa010.pdf. [Accessed: 14-Feb-2016].

[161] J.-Y. Lee and B.-M. Han, "A Bidirectional Wireless Power Transfer EV Charger Using Self-Resonant PWM,” IEEE Trans. Power Electron., vol. 30, no. 4, pp. 17841787, Apr. 2015.

[162] K. Thirugnanam, T. P. E. R. Joy, M. Singh, and P. Kumar, "Modeling and Control of Contactless Based Smart Charging Station in V2G Scenario," IEEE Trans. Smart Grid, vol. 5, no. 1, pp. 337-348, Jan. 2014.

[163] M. Bojarski, K. K. Kutty, D. Czarkowski, and F. De Leon, "Multiphase resonant inverters for bidirectional wireless power transfer," in Electric Vehicle Conference (IEVC), 2014 IEEE International, 2014, pp. 1-7.

[164] L. Zhao, D. J. Thrimawithana, and U. K. Madawala, "A hybrid bi-directional IPT system with improved spatial tolerance," in Future Energy Electronics Conference (IFEEC), 2015 IEEE 2nd International, 2015, pp. 1-6.

[165] M. J. Neath, U. K. Madawala, and D. J. Thrimawithana, "A new controller for bidirectional inductive power transfer systems," in 2011 IEEE International Symposium on Industrial Electronics (ISIE), 2011, pp. 1951-1956. 
[166] U. K. Madawala, M. Neath, and D. J. Thrimawithana, "A Power \#x2013;Frequency Controller for Bidirectional Inductive Power Transfer Systems," IEEE Trans. Ind. Electron., vol. 60, no. 1, pp. 310-317, Jan. 2013.

[167] O. C. Onar, S. L. Campbell, L. E. Seiber, C. P. White, and M. Chinthavali, "A highpower wireless charging system development and integration for a Toyota RAV4 electric vehicle," in 2016 IEEE Transportation Electrification Conference and Expo (ITEC), 2016, pp. 1-8.

[168] "Guidelines for limiting exposure to time-varying electric, magnetic, and electromagnetic fields (up to $300 \mathrm{GHz}$ ). International Commission on Non-Ionizing Radiation Protection," Health Phys., vol. 74, no. 4, pp. 494-522, Apr. 1998.

[169] S. Kim, G. Covic, and J. Boys, "Tripolar Pad for Inductive Power Transfer Systems for EV Charging," IEEE Trans. Power Electron., vol. PP, no. 99, pp. 1-1, 2016.

[170] "CHE Series Metallized Film Capacitors On High Energy Corp." [Online]. Available: http://highenergycorp.thomasnet-navigator.com/viewitems/metallizedfilm-capacitors/che-series-metallized-film-capacitors. [Accessed: 02-Jul-2017].

[171] A. A. S. Mohamed, D. Allen, T. Youssef, and O. Mohammed, "Optimal design of high frequency $\mathrm{H}$-bridge inverter for wireless power transfer systems in EV applications," in 2016 IEEE 16th International Conference on Environment and Electrical Engineering (EEEIC), 2016, pp. 1-6.

[172] N. H. Kutkut and K. W. Klontz, "Design considerations for power converters supplying the SAE J-1773 electric vehicle inductive coupler," in Proceedings of APEC 97 - Applied Power Electronics Conference, 1997, vol. 2, pp. 841-847 vol.2.

[173] J. Achterberg, E. A. Lomonova, and J. de Boeij, "Coil Array Structures Compared for Contactless Battery Charging Platform," IEEE Trans. Magn., vol. 44, no. 5, pp. 617-622, May 2008.

[174] A. P. Sample, D. T. Meyer, and J. R. Smith, "Analysis, Experimental Results, and Range Adaptation of Magnetically Coupled Resonators for Wireless Power Transfer," IEEE Trans. Ind. Electron., vol. 58, no. 2, pp. 544-554, Feb. 2011.

[175] J. L. Villa, J. Sallan, J. F. S. Osorio, and A. Llombart, "High-Misalignment Tolerant Compensation Topology For ICPT Systems," IEEE Trans. Ind. Electron., vol. 59, no. 2, pp. 945-951, Feb. 2012.

[176] M. Budhia, J. T. Boys, G. A. Covic, and C.-Y. Huang, "Development of a SingleSided Flux Magnetic Coupler for Electric Vehicle IPT Charging Systems," IEEE Trans. Ind. Electron., vol. 60, no. 1, pp. 318-328, Jan. 2013.

[177] B. Liangu, T. Houjun, and L. Chao, "Bi-directional Contactless Inductive Power Transfer System Modeling and verifying," Przeglad Elektrot., vol. R. 89, nr 1b, 2013. 
[178] A. A. S. Mohamed, A. A. Marim, and O. A. Mohammed, "Magnetic Design Considerations of Bidirectional Inductive Wireless Power Transfer System for EV Applications," IEEE Trans. Magn., vol. 53, no. 6, pp. 1-5, Jun. 2017.

[179] "Ferrites and Accessories - Data Book 2013 - ferrites-and-accessories-db130501.pdf." [Online]. Available: https://en.tdk.eu/download/519704/069c210d0363d7b4682d9ff22c2ba503/ferritesand-accessories-db-130501.pdf. [Accessed: 05-Mar-2017].

[180] D. K. Cheng, Field and Wave Electromagnetics, 2nd edition. Reading, Mass: Addison-Wesley, 1989.

[181] "MagNet."

[Online].

Available: https://www.infolytica.com/secured/customer/elite/livedocs/MagNet/. 18-Aug-2017].

[182] "Simulink."

[Online]. Available: http://www.infolytica.com/secured/customer/elite/livedocs/MagNet/\#07Circuits/SpecializedTopics/Simulink.htm?Highlight=simulink. [Accessed: 05-Mar2017].

[183] A. A. S. Mohamed, A. Berzoy, and O. Mohammad, "Magnetic design considerations of bidirectional inductive wireless power transfer system for EV applications," in 2016 IEEE Conference on Electromagnetic Field Computation (CEFC), 2016, pp. 1-1.

[184] M. Budhia, G. A. Covic, and J. T. Boys, "Design and Optimization of Circular Magnetic Structures for Lumped Inductive Power Transfer Systems," IEEE Trans. Power Electron., vol. 26, no. 11, pp. 3096-3108, Nov. 2011.

[185] G. A. Covic, M. L. G. Kissin, D. Kacprzak, N. Clausen, and H. Hao, "A bipolar primary pad topology for EV stationary charging and highway power by inductive coupling," in 2011 IEEE Energy Conversion Congress and Exposition, 2011, pp. 1832-1838.

[186] A. Zaheer, D. Kacprzak, and G. A. Covic, "A bipolar receiver pad in a lumped IPT system for electric vehicle charging applications," in 2012 IEEE Energy Conversion Congress and Exposition (ECCE), 2012, pp. 283-290.

[187] S. Kim, G. A. Covic, and J. T. Boys, "Tripolar Pad for Inductive Power Transfer Systems for EV Charging," IEEE Trans. Power Electron., vol. 32, no. 7, pp. 50455057, Jul. 2017.

[188] M. Budhia, G. A. Covic, J. T. Boys, and C.-Y. Huang, "Development and evaluation of single sided flux couplers for contactless electric vehicle charging," in 2011 IEEE Energy Conversion Congress and Exposition (ECCE), 2011, pp. 614-621. 
[189] R. Bosshard, J. W. Kolar, J. Mühlethaler, I. Stevanović, B. Wunsch, and F. Canales, "Modeling and \$eta \$ - \$alpha \$ -Pareto Optimization of Inductive Power Transfer Coils for Electric Vehicles," IEEE J. Emerg. Sel. Top. Power Electron., vol. 3, no. 1, pp. 50-64, Mar. 2015.

[190] S. Bandyopadhyay, V. Prasanth, P. Bauer, and J. A. Ferreira, "Multi-objective optimisation of a 1-kW wireless IPT systems for charging of electric vehicles," in 2016 IEEE Transportation Electrification Conf. and Expo (ITEC), 2016, pp. 1-7.

[191] M. Moghaddami, A. Anzalchi, A. Moghadasi, and A. Sarwat, "Pareto optimization of circular power pads for contactless electric vehicle battery charger," in 2016 IEEE Industry Applications Society Annual Meeting, 2016, pp. 1-6.

[192] Y. Shiyou, N. Guangzheng, L. Yan, T. Baoxia, and L. Ronglin, “A universal tabu search algorithm for global optimization of multimodal functions with continuous variables in electromagnetics," IEEE Trans. Magn., vol. 34, no. 5, pp. 2901-2904, Sep. 1998.

[193] K. R. Davey, "Latin Hypercube Sampling and Pattern Search in Magnetic Field Optimization Problems," IEEE Trans. Magn., vol. 44, no. 6, pp. 974-977, Jun. 2008.

[194] A. A. S. Mohamed, A. A. Marim, and O. A. Mohammed, "Magnetic Design Considerations of Bidirectional Inductive Wireless Power Transfer System for EV Applications," IEEE Trans. Magn., vol. 53, no. 6, pp. 1-5, Jun. 2017.

[195] F. Turki and U. Reker, "Further design approaches of the standardization: Inductive charging of electric vehicles," in Electric Drives Production Conference (EDPC), 2012 2nd International, 2012, pp. 1-6.

[196] M. J. Neath, A. K. Swain, U. K. Madawala, and D. J. Thrimawithana, “An Optimal PID Controller for a Bidirectional Inductive Power Transfer System Using Multiobjective Genetic Algorithm," IEEE Trans. Power Electron., vol. 29, no. 3, pp. 1523-1531, Mar. 2014.

[197] A. A. S. Mohamed, A. Berzoy, and O. Mohammed, "Experimental Validation of Comprehensive Steady-state Analytical Model of Bidirectional WPT System in EVs Applications," IEEE Trans. Veh. Technol.

[198] A. A. S. Mohamed, A. Berzoy, and O. Mohammed, "Predictive active power-flow control of two-way wireless power transfer system in V2G services," in 2016 IEEE 2nd Annual Southern Power Electronics Conference (SPEC), 2016, pp. 1-6.

[199] A. A. S. Mohamed, T. Youssef, and O. Mohammed, "Vehicle side predictive power-flow control of bidirectional WPT system for EV ancillary services," in 2017 IEEE Applied Power Electronics Conference and Exposition (APEC), 2017, pp. 3211-3217. 
[200] C. H. Ou, H. Liang, and W. Zhuang, "Investigating Wireless Charging and Mobility of Electric Vehicles on Electricity Market," IEEE Trans. Ind. Electron., vol. 62, no. 5, pp. 3123-3133, May 2015.

[201] R. Xiong, H. He, F. Sun, and K. Zhao, "Evaluation on State of Charge Estimation of Batteries With Adaptive Extended Kalman Filter by Experiment Approach," IEEE Trans. Veh. Technol., vol. 62, no. 1, pp. 108-117, Jan. 2013.

[202] C. R. Lashway and O. A. Mohammed, "Adaptive Battery Management and Parameter Estimation Through Physics-Based Modeling and Experimental Verification," IEEE Trans. Transp. Electrification, vol. 2, no. 4, pp. 454-464, Dec. 2016.

[203] F. Musavi and W. Eberle, "Overview of wireless power transfer technologies for electric vehicle battery charging," IET Power Electron., vol. 7, no. 1, pp. 60-66, Jan. 2014.

[204] J. G. Hayes, "Battery charging systems for electric vehicles," in 1998/262), IEE Colloquium on Electric Vehicles - A Technology Roadmap for the Future (Digest No, 1998, p. 4/1-4/8.

[205] S. Jeong, Y. J. Jang, and D. Kum, "Economic Analysis of the Dynamic Charging Electric Vehicle," IEEE Trans. Power Electron., vol. 30, no. 11, pp. 6368-6377, Nov. 2015.

[206] "Korean Electric Buses Go Wireless - Korea Real Time - WSJ." [Online]. Available: http://blogs.wsj.com/korearealtime/2013/08/08/korean-electric-buses-gowireless/. [Accessed: 29-Jul-2016].

[207] "Feasibility study — powering electric vehicles on England's major roads," 01Jan-1970. [Online].

Available: http://www.highways.gov.uk/knowledge/publications/1902/. [Accessed: 18-Jan2017].

[208] J. M. Miller, P. T. Jones, J. M. Li, and O. C. Onar, "ORNL Experience and Challenges Facing Dynamic Wireless Power Charging of EV's," IEEE Circuits Syst. Mag., vol. 15, no. 2, pp. 40-53, Secondquarter 2015.

[209] A. Shekhar, V. Prasanth, P. Bauer, and M. Bolech, "Economic Viability Study of an On-Road Wireless Charging System with a Generic Driving Range Estimation Method," Energies, vol. 9, no. 2, p. 76, Jan. 2016.

[210] L. Bhaskar, A. Sahai, D. Sinha, G. Varshney, and T. Jain, "Intelligent traffic light controller using inductive loops for vehicle detection," in 2015 1st International Conference on Next Generation Computing Technologies (NGCT), 2015, pp. 518522. 
[211] "Polymer Li-Ion Cell: 3.7V 21Ah (1055275-2C, 77.7Wh, 42A rate) - UN38.3 Passed(DGR)," BatterySpace.com/AA Portable Power Corp. Tel: 510-525-2328. [Online]. Available: http://www.batteryspace.com/polymer-li-ion-cell-3-7v-21ah1055275-2c-77-7wh-42a-rate---un38-3-passed-dgr.aspx. [Accessed: 29-Jul-2016].

[212] Y. Cao, R. C. Kroeze, and P. T. Krein, "Multi-Timescale Parametric Electrical Battery Model for Use in Dynamic Electric Vehicle Simulations," IEEE Trans. Transp. Electrification, vol. PP, no. 99, pp. 1-1, 2016.

[213] O. US EPA, "Dynamometer Drive Schedules." [Online]. Available: https://www.epa.gov/vehicle-and-fuel-emissions-testing/dynamometer-driveschedules. [Accessed: 30-Jul-2016].

[214] M.-E. Choi, J.-S. Lee, and S.-W. Seo, "Real-Time Optimization for Power Management Systems of a Battery/Supercapacitor Hybrid Energy Storage System in Electric Vehicles," IEEE Trans. Veh. Technol., vol. 63, no. 8, pp. 3600-3611, Oct. 2014.

[215] A. T. Al-Awami, E. Sortomme, G. M. A. Akhtar, and S. Faddel, "A Voltage-Based Controller for an Electric-Vehicle Charger," IEEE Trans. Veh. Technol., vol. 65, no. 6, pp. 4185-4196, Jun. 2016.

[216] "Electric Vehicle and Roadway." [Online]. Available: http://evr.usu.edu/news/press-releases/2014/jul17-test-track-approved. [Accessed: 09-Aug-2017].

[217] “Charged EVs | What's up with wireless EV charging." [Online]. Available: https://chargedevs.com/features/whats-wireless-ev-charging/. [Accessed: 09-Aug2017]. 


\section{AHMED A S MOHAMED}

01 January 1986

2002-2007

2008-2011

2014-2016

Spring 2017

Summer and Fall 2017

Fall 2017

2008-2014
Born, Dakahliya, Egypt.

B.Sc. in Electrical Engineering, Zagazig University, Zagazig, Egypt.

M.S. in Electrical Engineering, Zagazig University, Zagazig, Egypt.

Research Assistant, Florida International University, Miami, Florida, USA.

Doctoral Evidence Acquisition (DEA) Fellow, Florida International University, Miami, Florida, USA.

Dissertation Year Fellow (DYF), Florida International University, Miami, Florida, USA.

The Outstanding PhD Graduate for College of Engineering and Computing

Research and Teaching Assistant, Zagazig University, Zagazig, Egypt.

\section{PUBLICATION AND PRESENTATIONS}

$\left[\mathrm{P}_{1}\right]$ A. A. S. Mohamed, and Osama Mohammed, "Autonomous Two-layer Predictive Controller for Bidirectional Inductive Power Transfer in EV Applications," pending patent.

$\left[\mathrm{P}_{2}\right]$ A. A. S. Mohamed, and Osama Mohammed, "Integrated Bidirectional Inductive/Conductive Electrical Apparatus," pending patent.

$\left[\mathrm{J}_{1}\right]$ A. A. S. Mohamed, A. Berzoy, and O. Mohammed, "Design and Hardware Implementation of FL MPPT Control for PV Systems Based on GA and Small-Signal Analysis," IEEE Trans. Sustain. Energy, vol. 8, no. 1, pp. 279-290, Jan. 2017.

$\left[\mathrm{J}_{2}\right]$ A. A. S. Mohamed, A. Berzoy, and O. Mohammed, "Experimental Validation of Comprehensive Steady-state Analytical Model of Bidirectional WPT System in EVs Applications," IEEE Trans. Veh. Technol., vol. 66, no. 7, pp. 5584-5594, July 2017.

$\left[\mathrm{J}_{3}\right]$ A. A. S. Mohamed, A. A. Marim and O. A. Mohammed, "Magnetic Design Considerations of Bidirectional Inductive Wireless Power Transfer System for EV Applications," in IEEE Transactions on Magnetics, vol. 53, no. 6, pp. 1-5, June 2017.

[J $\left.\mathrm{J}_{4}\right]$ A. A. S. Mohamed, C. R. Lashway and O. Mohammed, "Modeling and Feasibility Analysis of QuasiDynamic WPT System for EV Applications," in IEEE Transactions on Transportation Electrification, vol. 3, no. 2, pp. 343-353, June 2017.

[J $\mathrm{J}_{5}$ ] A. A. S. Mohamed, A. Berzoy, F. G. N. de Almeida, and O. Mohammed, "Modeling and Assessment Analysis of Various compensation topologies in Bidirectional IWPT system for EV Applications," IEEE Transaction on Industry Applications , vol. 53, no. 5, pp. 4973-4984, Sept.-Oct. 2017. 
$\left[\mathrm{J}_{6}\right]$ A. A. S. Mohamed and O. Mohammed, "Physics-Based Co-simulation Platform with Analytical and Experimental Verification for Bidirectional IPT System in EV Applications," IEEE Trans. Veh. Technol., vol. pp, no. 99, pp. 1-1, 2017.

$\left[\mathrm{J}_{7}\right]$ A. A. S. Mohamed, Siguang An, and O. Mohammed, "Coil Design Multi-Objective Optimization of Power Pad in IPT System for Electric Vehicle Applications," IEEE Transaction on Magnetics, vol. pp, no. 99, pp. 1-1, 2017.

$\left[\mathrm{J}_{8}\right]$ A. Berzoy, A. A. S. Mohamed and O. Mohammed, "Complex-Vector Model of Interturn Failure in Induction Machines for Fault Detection and Identification," in IEEE Transactions on Industry Applications, vol. 53, no. 3, pp. 2667-2678, May-June 2017.

[ $\left.\mathrm{J}_{9}\right]$ Alberto Berzoy, A. A. S. Mohamed, and Osama Mohammed, "Optimizing power converter PCB design for lower EMI," COMPEL - Int. J. Comput. Math. Electr. Electron. Eng., vol. 34, no. 5, pp. 13641380, Sep. 2015.

[ $\left.\mathrm{J}_{10}\right]$ Hany F. Habib, A. A. S. Mohamed, Mohamad El Hariri, Osama A. Mohammed, "Utilizing Supercapacitors for Resiliency Enhancements and Adaptive Microgrid Protection Against Communication Failures", Electric Power Systems Research, vol. 145, pp. 223-233, Apr. 2017.

$\left[\mathrm{J}_{11}\right]$ A. Berzoy, A. A. S. Mohamed and O. Mohammed, "Impact of Inter-Turn Short-Circuit Location on Induction Machines Parameters Through FE Computations," in IEEE Transactions on Magnetics, vol. 53, no. 6, pp. 1-4, June 2017.

[J $\left.{ }_{12}\right]$ Samy Fadel, A. A. S. Mohamed, and Osama A. Mohammed, "Fuzzy Logic-Based Autonomous Controller for Electric Vehicles Charging in Residential Distribution Systems," Electric Power Systems Research, vol. 148, pp. 48-58, July 2017.

[ $\left.\mathrm{J}_{13}\right]$ A. Berzoy, A. A. S. Mohamed, and O. Mohammed, "Design considerations and predictive direct current control of Active Regenerative Rectifiers for harmonic and current ripple reduction," under review in IEEE Transaction on Power Electronics.

$\left[\mathrm{C}_{1}\right]$ A. A. S. Mohamed, A. Berzoy, F. G. N. de Almeida, and O. Mohammed, "Steady-state performance assessment of different compensation topologies in two-way IWPT system for EV ancillary services," in 2016 IEEE Industry Applications Society Annual Meeting, 2016, pp. 1-8.

$\left[\mathrm{C}_{2}\right]$ A. A. S. Mohamed, A. Berzoy, and O. A. Mohammed, "Physics-Based Co-Simulation Platform for EMC Analysis of Two-Way Inductive WPT System in EV Applications," in 2016 IEEE Vehicle Power and Propulsion Conference (VPPC), 2016, pp. 1-6.

$\left[\mathrm{C}_{3}\right]$ A. A. S. Mohamed, D. Allen, T. Youssef, and O. Mohammed, "Optimal design of high frequency Hbridge inverter for wireless power transfer systems in EV applications," in 2016 IEEE 16th International Conference on Environment and Electrical Engineering (EEEIC), 2016, pp. 1-6.

$\left[\mathrm{C}_{4}\right]$ A. A. S. Mohamed, A. Berzoy, and O. Mohammed, "Power Flow Modeling of Wireless Power Transfer for EVs Charging and Discharging in V2G Applications," in 2015 IEEE Vehicle Power and Propulsion Conference (VPPC), 2015, pp. 1-6.

$\left[\mathrm{C}_{5}\right]$ A. A. S. Mohamed, T. Youssef and O. Mohammed, "Vehicle side predictive power-flow control of bidirectional WPT system for EV ancillary services," 2017 IEEE Applied Power Electronics Conference and Exposition (APEC), Tampa, FL, USA, 2017, pp. 3211-3217. 